\title{
Ermüdungsversuche an
}

Stahlbetonbalken mit und ohne

\section{Schubbewehrung}

\section{Working Paper}

\section{Author(s):}

Frey, Rudolf; Thürlimann, Bruno

Publication date:

1983

Permanent link:

https://doi.org/10.3929/ethz-a-000305080

\section{Rights / license:}

In Copyright - Non-Commercial Use Permitted

\section{Originally published in:}

Bericht / Institut für Baustatik und Konstruktion ETH Zürich 7801(1) 
Ermüdungsversuche an Stahlbetonbalken mit und ohne Schubbewehrung

Rudolf Frey

Bruno Thürlimann 


\section{CIP-Kurztitelaufnahme der Deutschen Bibliothek}

\section{Frey, Rudolf:}

Ermüdungsversuche an Stahlbetonbalken mit und ohne Schubbewehrung / von Rudolf Frey; Bruno Thürlimann. Inst. für Baustatik u. Konstruktion, Eidgenöss. Techn. Hochsch. Zürich; Boston; Stuttgart:

Birkhäuser, 1983.

(Bericht / Institut für Baustatik und Konstruktion ETH Zürich; Nr. 7801-1)

ISBN 3-7643-1575-X

NE: Thürlimann, Bruno: ; Institut für Baustatik und Konstruktion <Zürich > : Bericht

(c) Birkhäuser Verlag Basel

ISBN 3-7643-1575-X 


\title{
Ermüdungsversuche an Stahlbetonbalken mit und ohne Schubbewehrung
}

\author{
von \\ Dipl. Ing. Rudolf Frey \\ Prof. Dr. Bruno Thürlimann \\ Institut für Baustatik und Konstruktion \\ Eidgenössische Technische Hochschule Zürich
}


1. EINLEITUNG

1.1 Problemstellung

1.2 Zielsetzung

2. VERSUCHSPROGRAMM

2.1 Versuchsparameter

2.1.1 Balken mit Schubbewehrung

2.1.2 Balken ohne Schubbewehrung

3. VERSUCHSKOERPER

3.1 Beschreibung

3.1.1 Abmessungen und Bewehrung

3.1.2 Herstellung und Lagerung

3.2 Baustoffe

3.2.1 Bewehrungsstah1

3.2.2 Beton

4. VERSUCHSDURCHFUEHRUNG

4.1 Versuchsanlage

4.1.1 Belastungseinrichtung

4.1.2 Messeinrichtung

4.2 Messstellenplan

4.3 Belastungsgeschichte, Messvorgang

4.3.1 Balken mit Schubbewehrung

4.3.2 Balken ohne Schubbewehrung

5. VERSUCHSERGEBNISSE

5.1 Ermudungsverhal ten

5.1.1 Balken mit Schubbewehrung

5.1 .2 Bügelbrïche

5.1.3 Balken ohne Schubbewehrung

5.1 .4 Schubbrüche

5.2 Traguerhalten

5.2.1 Balken mit Schubbewehrung

5.2.2 Balken ohne Schubbewehrung

5.3 Rissverhalten

5.3.1 Balken mit Schubbewehrung

VERDANKUNGEN 


\section{$1.1 \quad$ Problemstellung}

Das statische Verhalten von Stahlbeton- und Spannbetonbauteilen bis zum Bruch, auch unter kombinierter Beanspruchung, ist eingehend untersucht worden. Die praktischen und theoretischen Ergebnisse führten zu neuen Bemessungsregeln und Normen, welche eine höhere Materialausnützung erlauben (CEB-Mustervorschrift, 1976; Norm SIA 162, Richtlinie 34, 1976, [1], [2]). Mit der Entwicklung neuer Bewehrungs- und Spannstähle erhöhten sich ausserdem die statischen Materialfestigkeiten.

Diese Entwicklung machte die Untersuchung des Verhaltens von Stahlbeton- und Spannbetonbauteilen im Gebrauchszustand notwendig. In besonderem Mass gilt dies für Bauwerke wie Eisenbahn- und Strassenbrücken mit ermüdungswirksamen Betriebslasten.

Die Ermittlung des Ermüdungsverhaltens der Bauwerke erfolgt einerseits aufgrund der durch Versuche an Prüfkörpern (Bewehrungs- und Spannstahlproben, Betonprüfkörper) erhaltenen Kenntnisse der Ermüdungsfestigkeiten der verwendeten Materialien und andererseits mit der Uebertragung dieser Materialkennwerte auf den Bauteil. Wichtig zur Beurteilung des Ermüdungsverhaltens sowie zur Ableitung von Bemessungsregeln ist auch die Kenntnis des Spannungszustandes und dessen Geschichte, der er während der geforderten Lebensdauer unterworfen ist.

Ermüdungsversuche an ungerissenen und gerissenen Stahlbetonträgern unter reiner Biegung sind in grösserer Zahl bekannt und Bemessungsregeln dazu sind hergeleitet worden. Die Kenntnisse des Spannungszustandes und Aenderungen desselben im Laufe der Lebensdauer unter kombinierter Biege- und Schubbeanspruchung weisen jedoch noch grössere Lücken auf. Von Interesse sind vor allem bei schubbewehrten Bauteilen die Stahldehnungen und Stahldehnungsdifferenzen in den Bügeln und in der Längsbewehrung in Funktion des Querkraft/MomentenVerhältnisses, des Belastungsniveaus und des Bewehrungsgehaltes. Nicht zuletzt interessieren auch die Rissgrössen unter Gebrauchslast für die Beurteilung einer möglichen Korrosionsgefahr.

Um im unteren Schubbereich auf eine Schubbewehrung verzichten zu können, ist die Kenntnis der Ermüdungsfestigkeit des Betons unter Druck- und Schubbeanspruchung nötig.

Mit wenigen Ausnahmen wurden Balken im Schubversuch bisher meist unter monoton wachsender Belastung geprüf, mit gelegentlich wenigen Lastwiederholungen zur Kontrolle zuvor untersuchter Laststufen. Die uns bekannten Schubermüdungsversuche dienten oft mehr einer phänomenologischen Betrachtungsweise und der Bestimmung des Ermuidungswiderstandes im Verhältnis zum statischen Schubbruch als der Erfassung des inneren Kräftespiels [3], [4], [5]. Lediglich der Bericht [6] informiert über eine Versuchsserie mit füf Balken, an denen unter wiederholter Belastung detaillierte Messungen der Stahl- und Betondehnungen durchgefüht worden sind. Diese Versuche dienten hauptsächlich zur Deutung des inneren Tragmechanismus, der Rissentwicklung sowie der Ermüdungsbeanspruchung der Schubbüge 1 unter Schwell beanspruchung.

\subsection{Zielsetzung}

Die Zielsetzung des Forschungsprogrammes ist, aufgrund eigener Versuchsresultate und ausländischer Untersuchungen ein mechanisch-mathematisches Model1 für das Tragverhalten unter Biege- und Schubbeanspruchung bei wiederholter Belastung auf dem Niveau des Gebrauchszustandes zu entwickeln. Mit dem Model1 soll ein Verfahren für die Ermüdungsbemessung von Stahlbeton- und Spannbetonbauteilen erarbeitet werden, das in die Neufassung der Norm SIA 162 Eingang finden soll. 


\section{Versuchsprogramm}

Die experimentellen Arbeiten wurden Anfang 1979 aufgenommen und konnten Ende Juli 1981 abgeschlossen werden. Das Versuchsprogramm umfasste zwei Versuchsserien:

- Serie BI : Balken mit Schubbewehrung (Bild 2)

- Serie BII : Balken ohne Schubbewehrung (Bild 3 )

\subsection{Versuchsparameter}

\subsubsection{Balken mit Schubbewehrung}

An sechs bügelbewehrten Balken wurde das Schub- und Biegetragverhalten unter wiederholter Belastung experimentell untersucht. Als Grundlage für die Versuchsplanung diente die Richtlinie 34 der Norm SIA 162 (1968) (Bild 1).

Um die Anzahl Versuchsparameter möglichst gering zu halten, waren folgende Grössen für a) le Versuchsträger gleich:

- Spannweite

- Trägerhöhe

- Beton- und Stahlqualität

- Betonüberdeckung

- Belastung (mit Ausnahme von BI/1)

Folgende Grössen wurden als Versuchsparameter gewählt:

- Stegstärke

- Biegebewehrungsgehalt

- Schubbewehrungsgehalt

Folgende Beobachtungsgrössen sind bei den Versuchen erfasst worden:

- Das Verhalten im ungerissenen und im gerissenen Zustand unter monotoner Laststeigerung bis zur oberlast.

- Das Verhalten unter zyklischer Belastung bei konstanter Ober- und Unterlast. (Beim Versuch BI/1 unter Lastblöcken mit blockweise gesteigerter oberlast.)

Das Messprogramm umfasste die aufgebrachten Kräfte, die globalen und lokalen Verschiebungen, die Verzerrungen, das Rissbild und die Rissweiten.

\subsubsection{Balken ohne Schubbewehrung}

Zur Erfassung des Ermüdungswiderstandes von Balken ohne Schubbewehrung wurden Versuche an elf Versuchskörpern durchgeführt. Folgende Grössen wurden für alle Versuchsträger gleich gewählt:

- Verhältnis Spannweite/Trägerhöhe

- Längsbewehrungsgehalt bezogen auf den nominellen Schubquerschnitt (mit Ausnahme von BII/1)

- Betonüberdeckung

- Zementgehalt des Betons (mit Ausnahme von BII/10) 
Versuchsparameter:

- Belastungsniveau (Oberlast)

- Trägerabmessungen (zwei Balkengrössen)

- Zuschlagstoffe (zwei verschiedene maximale Korngrössen)

Folgende Beobachtungsgrösse wurde erfasst:

- Das Tragverhalten unter zyklischer Belastung im Ein- und im Mehrstufenversuch bis zum Ermuidungsbruch.

Das Messprogramm umfasste die aufgebrachten Kräfte, die globalen und lokalen Verschiebungen, das Rissbild und die Rissweiten.

\section{Versuchskörper}

3.1 Beschreibung

Die Versuchskörper wurden in der Forschungshalle der Bauwissenschaften (HIF) der ETHHönggerberg in Zürich hergestellt.

\subsubsection{Abmessungen und Bewehrung}

Die Abmessungen der Versuchsbalken der ersten Versuchsserie gehen aus den Bildern 5 bis 7 hervor. Die Balken BI/1 bis BI/4 wurden mit der gleichen Schalung hergestellt und hatten bezüglich Stege eine unsymmetrische Ausbildung. Die Stegstärken erlaubten die Verwendung von in der Praxis noch ublichen Bügeldurchmessern, sie stellen jedoch für viele Anwendungsbereiche eine untere Grenze dar. Die Ausbildung der relativ starken Unterflansche war notwendig für das Unterbringen der starken Längsbewehrung in den Balken BI/I und $B I / 3$. Dadurch konnte ein zu grossser Verlust an statischer Höhe vermieden werden.

Die Träger $B I / 5$ und $B I / 6$ erfuhren eine symmetrische Ausbildung mit einem gegenüber den ersten vier Versuchskörpern verbreiterten, im Querschnitt jedoch flächengleichen, Oberflansch. Alle Balken erhielten eine Verstärkung in den Krafteinleitungsbereichen. Die geforderten Betonabmessungen konnten sehr gut eingehalten werden. Die Betonüberdeckung betrug für die Längsbewehrung $20 \mathrm{~mm}$ und für die Bügelbewehrung $10 \mathrm{~mm}$.

Die verwendeten Bügel vom Durchmesser $d=8 \mathrm{~mm}$ und $d=10 \mathrm{~mm}$ waren gemäss Norm SIA 162 (1968) mit einem Biegerollendurchmesser von 4d abgebogen. Dank der vom Stahllieferanten sehr gut eingehaltenen Bewehrungsabmessungen konnte eine hohe Verlegegenauigkeit erreicht werden. Schweissungen wurden keine ausgeführt. Die gegenseitigen Abstände der Längsbewehrungsstäbe waren genügend gross, um das ungestörte Einbringen des Betons und somit einen guten Verbund zu gewährleisten.

Die Abmessungen der Versuchskörper ohne Schubbewehrung sind in den Bildern 8 bis 10 enthalten. Vier der sieben Träger mit den kieineren Abmessungen hatten Rechteckquerschnitte. Die Balken BII/3, BII/4 und BII/5 waren bei gleichem nominellem Stegquerschnitt mit einem oberflansch versehen, der konstruktiv bewehrt war. Die Balken BII/8 bis BII/11 (mit den grösseren Abmessungen) besassen alle einen T-Querschnitt. Alle Versuchskörper der zweiten Serie waren in der Krafteinleitungszone mit einer Querrippe versehen.

\subsubsection{Herstellung und Lagerung}

Die Versuchskörper wurden in Holzschalungen betoniert, die bis viermal verwendet werden konnten. Der Beton wurde in einem 380/300 1 Zwangsmischer aufbereitet. Für die grossen Träger waren neun Chargen zu 2501 notwendig, für die kleineren Balken inkl. Probekörper genügten deren 2 wei. Mit Ausnahme des Balkens BII/6 wurden alle Versuchsbalken mit hoch- 
wertigem Beton $B H$ PC $300 \mathrm{~kg} / \mathrm{m}^{3}$ hergestellt. Beim Balken BII/10 wurden $350 \mathrm{~kg} / \mathrm{m}^{3} \mathrm{vom}$ gleichen Zement beigegeben. Der Beton wurde mit Hilfe von Hochfrequenz-Vibriernadeln verdichtet. Nachdem die Betonoberfläche abgerieben worden war, wurde sie mit Plastikfolien abgedeckt.

Das Ausschalen der Versuchskörper erfolgte sechs bis acht Tage nach dem Betonieren. Anschliessend wurden sie in der Forschungshalle bei einer Raumtemperatur von ca. $20{ }^{\circ} \mathrm{C}$ gelagert. In der Zeitspanne zwischen Herstellung und Lagerung erfolgten die Versuchsvorbereitungen oder es wurde der zuvor hergestellte Versuchsträger geprüft. Die Betonprüfkörper hatten die gleichen Lagerungsbedingungen wie die zugehörigen Balken.

\subsection{Baustoffe}

\subsubsection{Bewehrungsstah1}

Bei allen Balken bestand die schlaffe Bewehrung aus dem kaltverformten Stahl TOR 50 , der der Kategorie IIIb der Norm SIA 162 (1968) entspricht. Die experimentell ermittelten Materialkennwerte sind im Bild 16 tabellarisch zusammengestellt. Die Festigkeitswerte wurden mit einer $1600 \mathrm{kN}$ Universalprüfmaschine (Schenck) verformungsgesteuert mit einer Dehngeschwindigkeit von $\dot{\varepsilon}=\mathrm{d} \varepsilon \mathrm{e}^{/ \mathrm{dt}}=1.2 \cdot 10^{-2} / \mathrm{min}$ bestimmt.

Die verwendeten Stähle zeigten ein einheitliches Verhalten. Die gemessenen Streckgrenzen übertragen durchwegs die in der Norm geforderten Nennwerte. Bild 16 zeigt ein aus einem Zugversuch gewonnenes Spannungs-Dehnungs-Diagramm eines Stabes vom Durchmesser $10 \mathrm{~mm}$.

Die Elastizitätsmoduli $E_{e}$ stammen aus den $\sigma-\varepsilon-D i a g r a m m e n$, die mit Hilfe eines am Probestab angebrachten $100 \mathrm{~mm}-$ Feindehnmessgerätes und der Kraftmessung der Prüfmaschine resultierten.

Die verwendeten Bügel mit gleichen Durchmessern sind für die Balken $B I / 1$ bis $B I / 4$ sowie $B I / 5$ und $B I / 6$ jeweils der gleichen Charge entnommen worden.

\subsubsection{Beton}

Die Zusammensetzung der Zuschlagstoffe war für die Versuchskörper $B I / 1$ bis $B I / 6$ und $B I I / 1$ bis BII/7 gleich. Der maximale Korndurchmesser betrug $16 \mathrm{~mm}$. Für die Balken BII/8 bis $B I I / 11$ wurde ein Maximalkorn vom Durchmesser $32 \mathrm{~mm}$ verwendet. Die Siebkurven des rolligen gewaschenen Materials lagen zwischen den Kurven A und B der Norm SIA 162 (1968), Art. 2.02. Die Anlieferung der Zuschlagstoffe erfolgte in den Komponenten 0 bis $4 \mathrm{~mm}, 4$ bis $8 \mathrm{~mm}$, 8 bis $16 \mathrm{~mm}$ und 16 bis $32 \mathrm{~mm}$ und wurde in silos zwischengelagert. Als Bindemittel wurde normaler Portlandzement $P C$, der ebenfalls in Silos gelagert worden war, verwendet.

Zur Herstellung des Betons steht im Betonierraum des Forschungsgebäudes ein 380/300 1 Zwangsmischer zur Verfügung. Die Mischdauer beträgt ca. Zwei Minuten. Dem unterschiedlichen Feuchtigkeitsgrad der Zuschlagstoffe wurde bei der Beigabe des Anmachwassers Rechnung getragen. Das Setzmass ergab einen steifen bis plastischen Beton. Zusätzlich erfolgte eine visuelle Kontrolle der Konsistenz. Auf eine Beigabe chemischer Zusatzmittel wurde verzichtet.

Zur Ermittlung der Betonkennwerte wurden pro Balken neun Zylinder vom Durchmesser $150 \mathrm{~mm}$, $h=300 \mathrm{~mm}$, zwei bis sechs Würfe 1 150/150/150 mm und vier Prismen 120/120/360 mm betoniert. Die Angaben in bezug auf die Anzahl Proben, die Probenabmessungen, das Prüfalter, die Festigkeitswerte (Mittelwerte) und die Standardabweichungen sind in den Bildern 17 bis 19 tabellarisch zusammengeste11t. Die Würfel- und Prismendruckfestigkeit sowie die Querzugfestigkeit (Brasilianerversuch) und die Biegezugfestigkeit wurden im Versuchsalter der Balken bestimmt. Gleichzeitig mit den Druck- und Zugfestigkeiten wurde auch der 
E-Modul an Zylindern vom Durchmesser $150 \mathrm{~mm}, h=300 \mathrm{~mm}$, ermittelt. Die Dehnungen wurden jeweils mit drei induktiven Wegaufnehmern mit einer Basislänge von $100 \mathrm{~mm}$ in halber Zylinderhöhe gemessen.

Zusätzlich zu den üblichen Betonprüfungen wurden pro Balken vier bis sechs Stempelversuche an Zylindern vom Durchmesser $150 \mathrm{~mm}, h=150 \mathrm{~mm}$, durchgeführt. Bei diesen Versuchen werden die Betonzylinder beidseitig über einen Stahlstempel mit $2 \mathrm{a}=37.5 \mathrm{~mm}$ belastet. Der unter dem Stahistempel in den Zylinder eindringende Betonkegel verdrängt das radial aufgerissene Material seitwärts. Unter der Annahme, der Beton sei ein starr-ideal plastisches Material und folge der Fliessbedingung von Coulomb, kann mit einem oberen Grenzwert die Traglast $P$ berechnet werden [17]. Der Mechanismus ist in Bild 21 dargestellt. Aus der Traglast kann die Betonzugfestigkeit wie folgt berechnet werden:

$$
\beta_{S Z}=\frac{P}{\pi\left(1.2 \cdot b \cdot h-a^{2}\right)}
$$

Diese Formel ist für einen Beton mit normaler Korngrösse gültig, wenn die Probeabmessungen mit den im Bild 21 angegebenen Werten ungefähr übereinstimmen. Die Werte von $B_{s z}$ sind ebenfalls in den Bildern 17 bis 19 enthalten.

\subsubsection{Materialkennwerte}

Zur Bestimmung der statischen Materialkennwerte wurden die gleichen Versuchsparameter gewählt, wie bei gleichzeitig oder kurz zuvor durchgeführten Versuchsprogrammen am Institut für Baustatik und Konstruktion. Dies erlaubt eine gesamthaft bessere Kenntnis des Materials und ist einer kleinen Anzahl Ermüdungsversuche an Materialkomponenten mit der ihnen anhaftenden Streuung vorzuziehen.

Da das hauptsächliche Ziel des Forschungsprojektes in der Erfassung des inneren Kräftezustandes unter zyklischer Belastung liegt, und weil zudem nur eine beschränkte Möglichkeit zur Durchführung von Ermüdungsversuchen an Materialkomponenten bestand, wurde gänzlich darauf verzichtet.

\section{Versuchsdurchfuhrung}

Alle Versuche wurden auf dem Aufspannboden der Forschungshalle der Bauwissenschaften (HIF) der ETH-Hönggerberg in Zürich durchgeführt.

\subsection{Versuchsanlage}

Die Versuchsanlage wurde für das gesamte Versuchsprogramm konzipiert. Mit wenigen Ausnahmen konnten alle Balken mit der gleichen Anlage geprüft werden. Bild 14 zeigt eine Ansicht der gesamten Anlage. Sie bestand aus folgenden zwei Teilen:

- Belastungseinrichtung

- Messeinrichtung.

\subsubsection{Belastungseinrichtung}

Diese bestand aus einer Rahmenkonstruktion, die in Balkenlängsrichtung erstellt wurde. Dies erlaubte einen einfachen seitlichen Einbau der Versuchsträger und gab gute Bedingungen für die photographischen Aufnahmen der Rissbilder auf den Stegen der Versuchsbalken.

Die Last wurde in Balkenmitte über eine Verteilplatte bzw. über einen Verteilträger mit 
einem dynamischen Doppelkammerdruckkolben aufgebracht. Dieser war mit einem AMSLERWOLPERT Pulsator verbunden und zusätzlich mit einem servohydraulischen Regelkreis ausgestattet. Alle Versuche wurden in den dynamischen Zyklen lastgesteuert gefahren. Die Servohydraulik erlaubte ein sehr gutes Einhalten der. Soll-Lasten in den dynamischen Beanspruchungsphasen sowie eine sehr gute Deformationssteuerung während der unter statischer Belastung erfolgten Messungen. Unter der zyklischen, sinusförmigen Belastung standen zwei Sicherheitsschaltungen zur Verfügung. Ueber unabhängige Regelkreise konnten die Last und der Weg in Trägermitte begrenzt werden. Für das Ueberschreiten bzw. Unterschreiten der Sol1-Last wurde eine Begrenzung von 2 bis $3 \%$ der Lasten gewählt. Die Wegbegrenzung betrug 0.2 bis $0.3 \mathrm{~mm}$. Durch diese Sicherheitsmassnahmen konnten Ueberlastungen während des Schwingens ausgeschlossen und die vollständige Zerstörung der Versuchsträger ohne Schubbewehrung nach erfolgtem Bruch vermieden werden.

Die Versuchsbalken waren schwimmend auf zwei bewehrten Neoprene-Blöcken mit aufgesetzten Kipplagern abgestützt. Die Kipplager gewährleisteten, dass sich der Angriffspunkt der Auflager infolge der Auflagerverdrehung nicht verschob. Die Neoprene-Blöcke hatten in dieser Ausführung nur Schiebungen zu ertragen (Bilder 11, 15). Sie haben sich unter pulsierender Belastung sehr gut bewährt und zeigten am Ende der Versuchsserie keinerlei Schäden. Ihre leicht dämpfenden Eigenschaften hatten einen günstigen Einfluss auf die Belastungseinrichtung.

\subsubsection{Messeinrichtung}

Die Messeinrichtung bestand aus einer elektronischen Mess- und Datenerfassungsanlage und elektronischen Messgeräten. Zur Kontrolle wurden mechanische Messuhren verwendet. Für die Messung der vertikalen Verschiebungen (Ourchbiegungen und Lagereinsenkungen) wurden induktive Wegaufnehmer, eine versetzbare und mit einem induktiven Wegaufnehmer bestückte Messstange sowie in Trägermitte mechanische Messuhren verwendet. Die Relativverschiebungen auf den Prüfkörpern wurden mit Hilfe induktiver Deformeter verschiedener Basislängen erfasst. Dehnungsmessstreifen dienten zur lokalen Messung von Betonstauchungen. Alle elektronisch erfassten Messgrössen wurden digitalisiert auf einem Lochstreifen gespeichert und konnten mit einer elektronischen Datenerfassungsanlage ausgewertet werden. Die Durchbiegung in Trägermitte konnte während der pulsierenden Belastung kontrolliert werden; die Spitzenwerte wurden gespeichert.

\subsection{Messitellenplan}

Die Anordnung der Messstellen, die Messnummern, die Basislängen der Messstrecken sowie die Messfehler gehen aus den Bildern 22 bis 31 hervor. Da verschiedene Deformeter älterer und neuerer Generation verwendet wurden, sind die absoluten Messfehler in Millimetern verschieden. Ihre Grösse hängt von den verwendeten elektrischen Gebern sowie vom Lagerspiel ab. Die angegebenen Grössen wurden mit Hilfe von Probemessungen mit den verwendeten Geräten bestimmt. Die angegebenen Messfehler entsprechen den Standardabweichungen, die bei 50 Probemessungen festgestellt wurden.

Folgende Messungen wurden an den Versuchsbalken ausgeführt:

\section{Vertikalkraft}

Während der pulsierenden Last wurden laufend die Maximal- und Minimalwerte der Kraft über eine Kraftmessdose und über den 0eldruck kontrolliert. Das Konstanthalten der Oberund der Unterlast während der Ermüdungsbelastung erfolgte über den 0eldruck.

Bei der statischen Belastung während der Messungen wurde die Last mit der Kraftmessdose gemessen. 


\section{Bügeldehnungen}

Bei den bügelbewehrten Balken wurden die Bügeldehnungen auf beiden Stegseiten über $30 \mathrm{~cm}$ Messlänge bestimmt. Dies erlaubte eine globale Kontrolle möglicher Unsymmetrien in der Stegbeanspruchung. Auf einer Stegseite (Seite A) wurden die Bügeldehnungen zusätzlich über drei Messlängen von je $10 \mathrm{~cm}$ erfasst. Diese aufwendigen Messreihen waren notwendig, um die auftretenden maximalen Bügeldehnungen an den Rissufern besser zu erfassen und somit die Ermüdungsbeanspruchung der Bügel abschätzen zu können. Die Verschiebungen wurden auf Messbolzen gemessen, die direkt auf die Bügel aufgeklebt worden waren.

\section{Dehnungen der Längsbewehrung}

Die Dehnungen der Längsbewehrung wurden mit Hilfe einer Messreihe über die ganze Balkenlänge auf dem untersten Bewehrungsstab in Balkenaxe bestimmt. Die Messungen erfolgten auf Bolzen, die auf dem Bewehrungsstab aufgeklebt worden waren.

\section{Betonstauchungen}

Die Betonstauchungen wurden mit Deformetermessungen bestimmt. Die Messbolzen waren auf die Betonoberfläche aufgeklebt worden. Bei den Trägern mit Oberflansch wurden drei Messreihen angebracht, eine in Balkenlängsaxe und zwei auf den Flanschrändern. Die Versuchsbalken mit Rechteckquerschnitt erhielten nur eine zentrale Messreihe. Dehnungsmessstreifen wurden an ausgewählten Stellen zur Bestimmung der lokalen Betonstauchungen verwendet.

\section{Durchbiegungen}

Für die Messung der Durchbiegungen unter der dynamischen Beanspruchung war in Balkenmitte ein induktiver Geber montiert, dessen Spitzenwerte (Maximum, Minimum) zu jeder Zeit abgerufen werden konnten. Dies erlaubte einen globalen Vergleich der statischen und dynamischen Verformung.

\section{Lagereinsenkung}

Durch die Verwendung elastischer Neoprene-Lager ergab sich die Notwendigkeit, ihre Verformungen zu messen. Sie wurden beidseitig am Lager gemessen. So ergab sich pro Lager ein Mittelwert, der bei den Durchbiegungsmessungen ausgeglichen wurde.

\section{Verzerrungen}

Für die Bestimmung der Verzerrungen im Steg wurde an allen Balken, mit Ausnahme von BII/1 bis BII/5, ein Messnetz seitlich an den Stegen angebracht (Bilder 22 bis 26, 30, 31). Bei den Versuchsträgern mit Bügelbewehrung wurden Messbolzen verwendet, die direkt auf die Bügel geklebt waren. Bei den Balken ohne Schubbewehrung waren die Bolzen auf die Betonoberfläche geklebt.

Durch die Ausmessung des Messnetzes waren die Knotenverschiebungen überbestimmt. Um den notwendigen Ausgleich der Messungen zu erreichen, wurde das Messnetz auf dem Steg als Fachwerk aufgefasst. Die aus den Messungen gerechneten Dehnungen wurden als Temperaturdehnungen der Stäbe betrachtet. Mit diesen Zwängungen konnten das statisch unbestimmte Fachwerk belastet und die Verschiebungen der Knotenpunkte berechnet werden. Die Steifigkeit der Fachwerkstäbe, EA/1, wurde konstant angesetzt.

Die Verzerrungen werden aus den Knotenverschiebungen $u$, w eines Messvierecks berechnet. Es gelten folgende Beziehungen:

$$
\varepsilon_{x}=\frac{\partial u}{\partial x}, \quad \varepsilon_{z}=\frac{\partial w}{\partial z}, \quad \gamma_{x z}=\frac{\partial u}{\partial z}+\frac{\partial w}{\partial x}
$$

Da die Verschiebungen nur in diskreten Punkten bekannt sind, werden die Ableitungen durch Differenzen ersetzt, und es ergeben sich folgende, über den Messbereich gemittelte, Verzerrungen:

$$
\varepsilon_{x}=\frac{\Delta u}{\Delta x}, \quad \varepsilon_{z}=\frac{\Delta w}{\Delta z}, \quad \gamma_{x z}=\frac{\Delta u}{\Delta z}+\frac{\Delta w}{\Delta x}
$$


Die Geometrie des Messvierecks und die verwendeten Formeln sind im Bild 32 dargestellt.

\section{Risse}

Nach jeder Laststufe wurden die mit blossem Auge sichtbaren Risse auf der Seite B der Balken mit einem Filzstift nachgezeichnet. Die Messung der Rissbreiten erfolgte mit einem Rissmassstab, der eine Abschätzung der Rissbreite auf ca. $2 \cdot 10^{-2} \mathrm{~mm}$ erlaubte. Die Rissbreite wurde immer an derselben Stelle, senkrecht zur globalen Rissrichtung, gemessen. Dies ist für die Biegerisse relativ eindeutig. Für die Schubrisse wurde eine Stelle auf halber Steghöhe, bei einigen Balken zusätzlich in der Nähe der Uebergänge in die Flansche, gewählt, wo die lokale Rissrichtung mit der globalen übereinstimmte.

\subsection{Belastungsgeschichte, Messvorgang}

\subsubsection{Balken mit Schubbewehrung}

Die Belastungsgeschichten der Versuchskörper mit Schubbewehrung sind in den Bildern 34 bis 39 dargestellt. Sie können wie folgt aufgeteilt werden:

- Statische Belastung bis zur Oberlast des ersten dynamischen Zyklus

- Dynamische Belastungszyklen

- Statische Belastung bis zum Bruch

Die statische Belastung erfolgte mit einer stufenweisen Erhöhung der Last. Messungen wurden auf verschiedenen Stufen durchgefürt. Zu Beginn erfolgten zwei Nulimessungen (ohne Last), die gemittelt als Referenzmessung dienten. Weitere Messungen erfolgten im homogenen Zustand, bei Risslast, bei gerissenem Querschnitt zwischen Riss- und oberlast und unter Oberlast. Nachher wurde entlastet und bis zur Unterlast gefahren. Die letzten beiden beschriebenen Laststufen gelten als Erstbelastung für den darauf folgenden dynamischen Belastungszyklus.

Die pulsierende Last wurde bei den Trägern BI/1 und BI/2 mit einer Servohydraulik mit einer Frequenz von 1 Lastwechsel pro Sekunde aufgebracht. So konnte vom ersten Lastwechse] an die vorgesehene volle Belastung aufgebracht werden, was bei der Verwendung des Pulsators infolge der Einschwingzeit nicht erreicht werden kann. Dieses Vorgehen wurde gewählt, weil das Verhalten der ersten beiden Balken von den ersten Lastwechseln an beobachtet werden sollte.

Die Belastung der weiteren Balken wurde von Anfang an mit einem Pulsator erzeugt, der bei einer Frequenz von 250 Lastwechseln pro Minute eine Einschwingzeit von hundert bis zweihundert Lastwechseln benötigte, um auf die vorgesehenen Lastniveaus zu kommen.

Alle Versuche mit den dynamischen Belastungszyklen wurden lastgesteuert gefahren. Während den dynamischen Zyklen wurden Zwischenmessungen unter statischer Belastung durchgefüht. Mit dem Pulsator wurde auf Null-Last gefahren und hernach statisch auf oberlast, unter welcher eine Messung erfolgte. Nach erfolgter Messung wurde auf Unterlast gefahren und wieder gemessen. Diese Doppelmessungen wurden zu Beginn der dynamischen Zyklen nach einigen hundert bis tausend Lastwechseln durchgeführt. Später erfolgten sie nach grösseren Zeiträumen, die bis zu einer Million Lastwechsel enthielten. Der generelle Verlauf einer Messung ist im Bild 33 dargestellt. Während den Messungen, die jeweils 30 bis 40 Minuten dauerten, wurde die Durchbiegung in Trägermitte konstant gehalten. Die dabei auftretende Relaxation zeigte sich im Abfallen der Last vom Anfangswert $P_{A}$ auf den Endwert $P_{E}$. Der Abfall unter oberlast betrug maximal 1.5\%. Bei der Versuchsauswertung wurde den Messwerten stets die Anfangslast $\mathrm{P}_{\mathrm{A}}$ zugeordnet.

Die Zunahme der Mittendurchbiegung unter konstanter Oberlast während der zwei Minuten vor Messbeginn betrug im Maximum $0.5 \%$. Der so erhaltene Wert stimmte mit den beobachteten 
dynamischen Spitzenwerten überein.

Bei einigen Versuchsträgern konnte am Schluss der dynamischen Lastzyklen noch eine statische Belastung bis zum Bruch aufgebracht werden, sei es, dass der Versuchsträger die dynamische Last nicht mehr aufnehmen konnte, weil er infolge der Bügelbrüche zu weich geworden war, oder weil er keine Ermüdungsschädigung erhalten hatte wie der Balken BI/3. Der Versuch an einem Balken inkl. Messungen nahm ca. einen Monat in Anspruch.

\subsubsection{Balken ohne Schubbewehrung}

Die Belastungsgeschichten der Träger ohne Schubbewehrung sind in den Bildern 43 und 44 dargestellt. Als Unterschied zu den schubbewehrten Trägern, bei denen fünf von sechs Balken als Einstufenversuche gefahren wurden, hatte diese Serie verschiedene Mehrstufenversuche. Hier galt es, den Ermüdungswiderstand des Betons unter Schubbeanspruchung zu bestimmen. Daher wurde eine Anzahl Versuche von tiefen Lastniveaus ausgehend gefahren, um einen Dauerfestigkeitswert abschätzen zu können. Nach 2 bis 2.5 Mio Lastwechsein ohne Bruch erfolgte bei diesen Versuchsläufen eine Laststeigerung, mit Ausnahme von BII/1, bei dem auf dem gleichen Lastniveau 6.6 Mio Lastwechsel gefahren wurden.

\section{Versuchsergebnisse}

Mit der Darstellung der Versuchsresultate soll besonders auch das Verhalten der Versuchsträger während der dynamischen Belastungszyklen beschrieben werden. So wird auf eine Darstellung von Messresultaten aus den statischen Belastungszyklen zu Beginn der Belastungsgeschichten verzichtet. Von den statischen Endbelastungszyklen werden nur die Bruchlasten und die Bruchbilder aufgeführt.

Die Versuchsergebnisse werden in zwei Abschnitte gegliedert. Der erste beinhaltet globale Ergebnisse der einzelnen Versuchsbalken sowie umfassende Versuchsresultate der beiden Versuchsserien. Im zweiten Abschnitt sind für jeden Versuchskörper ausgewählte Messresultate dargestellt, die Auskunft über die Verformungen und Verformungsänderungen im Laufe der Belastungsgeschichte geben. Ebenso sind Rissbilder und die Rissentwicklungen enthalten.

\subsection{Ermüdungsverhalten}

\section{1 .1 Balken mit Schubbewehrung}

Die schubbewehrten Balken zeigten erwartungsgemäss unterschiedliches Ermüdungsverhalten in Abhängigkeit der gewählten Hauptparameter: Schubbewehrung, Längsbewehrung, Stegstärke. Bei allen Versuchsträgern traten nur Ermüdungsbrüche von Bügeln auf. Die Längsbewehrung bl ieb unversehrt.

Die Anzahl Bügelbrïche und der genaue Ort derselben waren nur durch Aufspitzen der Stege und der Unterflanschen der Balken festzustellen. Nur so konnten die Bruchflächen beurteilt und eine Klassifikation in reine Ermüdungsbrüche und Brüche mit Einschnürung erstellt werden. Die in den Bildern 34 bis 39 aufgeführten Brüche sind reine Ermüdungsbrüche. Die unter statischer Endbelastung erfolgten Brüche sind im Bericht nicht enthalten, ebenso wie die letzten Brüche am Schluss des dynamischen Lastzyklus, die infolge vorausgegangener Ermüdungsbrüche im gleichen Querschnitt bzw. zwischen den Rissufern eines Schubrisses keine reinen Ermüdungsbrüche mehr waren und eine Querschnittseinschnürung erfuhren. Einige Ermüdungsbrüche konnten unter der pulsierenden Belastung von blossem Auge festgestellt werden, wenn der Bruch bei einem Messbolzen in der Betonaussparung erfolgte. Die restlichen Brüche, die einer Lastspielzahl zugeordnet sind, wurden bei der Messung der Bügeldehnungen mit dem Deformeter festgestellt. Dies war bei allen Brüchen, die im Bereich des $100 \mathrm{~mm}$ Messnetzes der Bügel erfolgten, eindeutig möglich. Erfolgte der erste 
Bruch eines Bügels auf der Seite des $300 \mathrm{~mm}$ Messnetzes (Seite B), so war dies nicht immer eindeutig möglich, vor allem dann nicht, wenn es der erste Bügelbruch in einem Riss überhaupt war. Da ein Schrägriss im allgemeinen drei Bügel überquerte, war der Verlust eines Sechstels des Stahlquerschnittes zu gering, um über $300 \mathrm{~mm}$ eindeutig gemessen werden zu können. Der Balken konnte die Kraft auf die Nachbarbügel und über die benachbarten Risse abtragen.

Die in den Bildern 34 bis 39 den Brüchen zugeordneten Lastspielzahlen entsprechen dem letzten Beobachtungs- oder Messzeitpunkt, in welchem der Bügel noch nicht gebrochen war. Die tatsächlich ertragenen Lastwechsel bis zum Bruch überschritten somit die im Bericht zugeordneten Werte. Als oberen Grenzwert der ertragenen Lastwechsel kann die Lastspielzahl des folgenden Bügelbruchs angesehen werden.

\section{Balken BI/I}

Beim Balken BI/1 (Bild 34) erfolgten alle festgestellten Bügelbruche im Lastzyklus $F$. Ein Bruch (Seite $B$, Bügel $\mathrm{Nr}$. 4) konnte während des Versuchs nicht festgestellt werden. Es ist möglich, dass er als Folge des beidseitigen Bruchs des Bügels $\mathrm{Nr}$. 5 im gleichen Schubriss eingetreten ist, und dass er somit auch im Lastzyklus $F$ versagt hat. Die Bügel im Steg der Stärke $150 \mathrm{~mm}$ vermochten nur einseitig zu brechen, was als Zeichen für eine bessere Mitwirkung des Stegbetons angesehen werden kann.

\section{Balken BI/2}

Beim Balken BI/2 (Bild 35), der im Vergleich zum ersten Träger BI/1 nur die Hälfte der Schub- und Längsbewehrung enthielt, trat die Mitwirkung des Stegbetons deutlich in Erscheinung. Von insgesamt 16 Ermüdungsbrüchen erfolgten deren 13 im dünneren steg.

Infolge der hohen Beanspruchung erfuhren die Bügel sehr grosse Dehnungen und Dehnungsdifferenzen. Im mittleren Stegbereich wurde die Streckgrenze unter oberlast beträchtlich überschritten.

Nach den ersten beiden Brüchen des Bugels $\mathrm{Nr} .6$ stellten sich im gleichen Schubriss an den Nachbarbügeln weitere Ermüdungsbrüche ein. Erst nach erfolgtem Bruch der Bügel Nr. 5 und $\mathrm{Nr} .7$ traten die weiteren Brüche ausserhalb dieses Schubrisses auf.

In beiden Stegen wurden die ersten Bügelbrüche auf halber Steghöhe festgestellt. Nach 16 Ermüdungsbrüchen im dynamischen Lastzyklus konnte noch eine statische Last von $510 \mathrm{kN}$ bis zur Erschöpfung der statischen Tragfähigkeit aufgebracht werden.

\section{Balken BI/3}

Dieser Balken zeigte erwartungsgemäss das beste Ergebnis bezüglich der Ermüdungsfestigkeit der Bügel, und er ist deshalb mit den beiden weiteren Einstufenversuchen an den Balken $B I / 5$ und $B I / 6$ zu vergleichen. Alle drei Versuchskörper hatten die gleiche Bügelbewehrung, deren Dimensionierung gemäss Richtlinie 14 der Norm SIA 162 (1968) unter Annahme von $\tan \gamma=3 / 5$ erfolgte.

Die Stegstärken des Balkens BI/3 betrugen 100 und $150 \mathrm{~mm}$. Der Steg auf der Seite A links kann somit mit den Stegen von BI/6, und der Steg auf der Seite $A$ rechts mit den Stegen von $B I / 5$ verglichen werden. Als Unterschied $z$ u diesen beiden Balken hatte $B I / 3$ jedoch eine stärkere Biegebewehrung.

Für die Ermüdungsbelastung war das Niveau der Oberlast mit $50 \%$ der rechnerischen Bruchlast gemäss Norm SIA $162, \mathrm{RL} 14$, angesetzt.

Ein einziger einseitiger Bügelbruch erfolgte bei $2.45 \cdot 10^{6}$ Lastwechseln. Die in diesem Fall genaue Lastwechselzahl bis zum Bruch konnte dank der Anwesenheit des Versuchsleiters 
beim Bruch (dumpfer Knall) mit einer sofortigen Messung bestätigt werden. Der Balken erfuhr bis zum Abbruch des dynamischen Lastzyklus nach $5.75 \cdot 10^{6}$ Lastwechseln keine weiteren Ermüdungsbrüche mehr.

Bei der anschliessenden statischen Endbelastung konnte eine Bruchlast von $1050 \mathrm{kN}$ erreicht werden. Diese entspricht unter Berücksichtigung der gemessenen Materialkennwerte und der sich beim Versagen einstellenden Neigung des Druckfeldes (Bild 104) dem rechnerischen Wert.

Der Versuch zeigte deutlich den redundanten Charakter, den ein Stahlbetonträger mit seinen als Einzelzugglieder wirkenden Stahleinlagen haben kann. Insbesondere muss der Ermüdungsbruch eines Einzelstabes, der bei einer vergleichsweise geringen Beanspruchung infolge eines Defektes am Stahl auftreten kann, nicht unbedingt eine Schädigung des Balkens bedeuten. Im vorliegenden Fall zeigte der gebrochene Stahl im Bruchquerschnitt keine äussere Beschädigung. Der Anriss des Ermiudungsbruchs erfolgte wie bei fast allen Bügeln beim Zusammentreffen der durchgehenden Längsrippe mit einer Querrippe.

\section{Balken BI/4}

Der Balken BI/4 (Bild 37) war gleich ausgebildet wie BI/2 und durchlief auch die gleiche Belastungsgeschichte. Von insgesamt 14 Bügelbrüchen wurden 12 im dünen Steg festgestellt. Die Brüche waren über die ganze Stegbreite verteilt und wichen im vertikalen Sinn nur wenig von der Stegmitte ab. Auch hier erfolgten auf gleicher Steghöhe meist Doppelbrüche an den Bügeln. Die ersten Brüche traten im Vergleich mit BI/2 nach der doppelten Anzahl Lastwechsel ein. Nach Beendigung des dynamischen Belastungsvorgangs konnten statisch noch $406 \mathrm{kN}$ aufgebracht werden.

\section{Balken BI/5}

Der Balken BI/5 war ohne Unterflansch ausgebildet. Der mit den ersten vier Balken flächengleiche oberflansch wurde verbreitert und auf eine Stärke von $80 \mathrm{~mm}$ reduziert. Die durchgehende Stegstärke von $150 \mathrm{~mm}$ erlaubte das Unterbringen der mit BI/2 und BI/4 identischen Längsbewehrung im Steg. Die im Bild 38 eingezeichneten Bruchpositionen zeigen deutlich die Ausbildung zweier Bruchzonen längs diskreter Risse. Auf der Seite A rechts verlief der Riss, wo die Ermudungsbruche auftraten, direkt von der Krafteinleitung in Balkenmitte zur Balkenzugseite. Nach dem ersten Bügelbruch schien hier der "Bruchlinienriss" vorgegeben zu sein. Dies könnte auf den geringeren Längsbewehrungsgehalt zurückgeführt werden. Durch die grösseren Dehnungen in der Längsbewehrung und den Wegfall des Unterflansches konnte bei einer Schwächung des Querschnittes keine genügende Umlagerung der kräfte mehr stattfinden.

Auf der Seite A links erfolgte der erste Bügelbruch im Bügel $\mathrm{Nr}$. 4, also gegen das Auflager hin. Auch hier stellten sich die Brüche in einem einzigen diskreten Riss ein. Allerdings konnte eine Verlagerung der Brüche $\mathrm{Nr} .12$ und $\mathrm{Nr} .17$ gegen die Balkenmitte hin beobachtet werden.

Als Sekundärbrüche, d.h. nach vorausgegangenen Bügelbrüchen im gleichen Schubriss, konnten zwei Ermüdungsbrüche ( $\mathrm{Nr}$. 5 und $\mathrm{Nr}$. 13) an Bügelausrundungen beobachtet werden (Bild 109). Im weiteren erfolgten drei Brïche am gleichen Bugel (Nr. 6).

\section{Balken BI/6}

Der gegenüber $B I / 5$ um $40 \%$ erhöhte Längsbewehrungsgehalt und der durchgehende $100 \mathrm{~mm}$ starke Steg bedingten beim Balken BI/6 die Ausbildung eines $200 \mathrm{~mm}$ breiten Unterflansches. Die Längs- und Schubbewehrungen entsprachen dem in der Richtlinie 14 unter Annahme einer Strebenneigung $\operatorname{tany}=3 / 5$ geforderten Wert, was unter statischer Bruchbelastung Fliessen beider Bewehrungen bedeutet. 
Die Belastungsgeschichte und die erfolgten Ermüdungsbrüche sind im Bild 39 dargestellt. Die unsymmetrische Verteilung der Brüche auf die beiden Balkenhälften und deren Anzahl konnte nicht erklärt werden. Eine Kontrolle der Belastungsgeometrie ergab, dass die Lasteinteilung zentrisch erfolgte. Für die Betonkennwerte der beiden Seiten konnten im Rahmen der vorgenommenen Prüfungen keine Abweichungen festgestellt werden.

In der Stegseite A rechts fällt beim Vergleich mit dem Balken BI/5 die breite Verteilung der Brüche auf. Diese kann durch den grösseren Längsbewehrungsgehalt und durch die Verteilwirkung des Unterflansches verursacht worden sein. Auch die einseitigen Brüche an verschiedenen Bügeln bis zu einer Lastwechselzahl von $1.16 \cdot 10^{6}$ können derart gedeutet werden. Erst anschliessend erfolgten die ersten Ermüdungsbrüche auf den anderen Bügelseiten.

\section{1 .2 Bügelbrüche}

Wie bereits einleitend erwähnt wurde, konnten nicht alle Bügelbrüche zeitlich genau erfasst werden. Bei einer kleineren Anzahl Bügel konnte die Dehnungsgeschichte bis zum Bruch verfolgt werden. Unter 'Dehnungsgeschichte' wird die in Funktion der Lastwechsel gemessene maximale Dehnung und Dehnungsifferenz am Ort des erfolgten Bruchs verstanden. Für eine Darstellung der gemessenen Werte in einem $\Delta \sigma-N-D i a g r a m m$ können nur Maximalwerte, die uber den Riss gemessen wurden, wo die Brüche erfolgten, verwendet werden. So kommen nur die Messungen mit der kürzesten Messbasis $(100 \mathrm{~mm})$, die auf der Seite $A$ der Balken erfolgten, in Frage. Es sind nicht die maximal im Bügel auftretenden Werte, sie kommen innen jedoch nahe, da sie immer nur über einen Schubriss gemessen wurden.

Bei den meisten Bügeln erfolgte zu Beginn des dynamischen Lastzyklus eine starke Zunahme der gemessenen Dehnungen und Dehnungsdifferenzen. Sie stabilisierten sich, wenn zu einem späteren Zeitpunkt kein neuer Riss entstand, nach $5 \cdot 10^{3}$ bis $50 \cdot 10^{3}$ Lastwechseln und erfuhren anschliessend nur noch unbedeutende Zunahmen. Beim Bruch eines Bügels in einem Riss stellte sich bei den Nachbarbügeln, die den gleichen Riss durchquerten, eine Erhöhung der Dehnung ein. Diese war nach dem ersten einseitigen Bruch eines Bügels nicht immer messbar, bei beidseitigem Bruch hingegen beträchtlich. Die meisten Bügel waren demnach, ohne den normalen Dehnungszuwachs zu berücksichtigen, auch unter konstanten äusseren Lasten einem 'Mehrstufenversuch' unterworfen.

In den Bildern 40 und 41 sind diejenigen Messungen aufgeführt, die als 'Einstufenversuche' gelten können. Die Tabellen dieser Bilder enthalten Dehnungswerte von Messstellen, an denen bis zum Bruch keine sprunghafte Zunahme der Dehnungen infolge benachbarter Brüche festgestellt worden sind. Diese Bedingung traf für etwa ein Drittel aller Ermüdungsbrüche zu.

Im Bild 42 sind die Dehnungsdifferenzen aus den Tabellen der Bilder 40 und 41 zusammen mit dem einzig aufgetretenen Bügelbruch im Balken BI/3 in einem $\Delta \sigma-N-D i a g r a m m$ eingezeichnet. Als Durchläufer sind die maximal gemessenen Dehnungsdifferenzen in jedem der 19 nicht gebrochenen Bügel des Balkens BI/3 aufgeführt. Das Diagramm setzt sich somit aus verschiedenen Prüfserien zusammen und ist entsprechend vorsichtig zu interpretieren: Die Brüche umfassen nicht alle gebrochenen Bügel und stammen aus drei verschiedenen Balkenversuchen. Die 19 Durchläufer stellen Messwerte aus insgesamt 60 Messungen an einem Balken dar, wobei sie nicht die 19 grössten Dehnungsdifferenzen, sondern die grössten Dehnungsdifferenzen, die an jedem der 19 Bügel ohne Bruch gemessen wurden, darstellen. Es gab mehrere Bügel, bei denen das zweitgrösste $\Delta \sigma$ grösser war als das maximale in einem anderen Bügel.

Die im Diagramm verwendeten $\Delta \sigma$ entsprechen dem gemessenen $\Delta \sigma$ zum Zeitpunkt $N_{1}$. Als Lastwechselzahl wurde $N_{2}-N_{1}$ verwendet. Diese vereinfachte Darsteliung als Einstufenversuche wurde aufgrund der Beobachtung, dass zu Beginn des Lastzyklus die Dehnungen stark an- 
stiegen (bis $N_{1}$ ) und später nur noch wenig zunahmen, gewählt. Der Ansatz berïcksichtigt weder die Vorschädigungen bis zur Lastspielzahl $N_{1}$, noch den späteren Anstieg von $\Delta \sigma$. Die Berücksichtigung dieser beiden Einflussfaktoren hätte nur mit der Zuhilfenahme einer Schadenakkumulationshypothese erfolgen können, die allerdings zusätzliche Annahmen bedingt hätte. In jedem Fall sind die eingezeichneten Werte konservativ. Im weiteren ist noch darauf hinzuweisen, dass die eingetragenen Dehnungsdifferenzen $\Delta \varepsilon$ verschiedenen Maximaldehnungen $\varepsilon_{\max }$ zugeordnet werden. Als Lastspielzahl $N_{1}$ wurde in den meisten Fällen $5 \cdot 10^{3}$ verwendet.

\section{1 .3 Balken ohne Schubbewehrung}

Bei den Balken $B I I / 1$ bis $B I I / 11$ wurden unterschiedliche Ermüdungswiderstände festgestellt. Die Balken $B I I / 8$ bis $B I I / 11$ mit grossen Abmessungen und maximalen Korndurchmessern $32 \mathrm{~mm}$ der Zuschlagstoffe zeigten bei vergleichbaren oder sogar höheren statischen Betonkennwerten eine geringere Ermüdungsfestigkeit als die Versuchsträger mit kleinen Abmessungen und maximalem Korndurchmesser $16 \mathrm{~mm}$. Dies konnte anhand von 19 Schubbrüchen an elf Versuchsträgern festgestellt werden. Die Anzahl Schubbrüche kann damit erklärt werden, dass bei den meisten Balken nach erfolgtem Bruch auf einer Seite eine Reparatur vorgenommen werden konnte. Der Versuch wurde anschliessend jeweils bis zum Bruch der anderen Seite fortgesetzt. Die Reparatur erfolgte mit einer vorgespannten äusseren Schubbewehrung (Bilder 155, 157).

In den Brucharten unterschieden sich die Balken nach ihrer Querschnittsausbildung. Bei allen Balken mit Oberflansch stellte sich ein Ermüdungsbruch des Steges ein. Nach dem Auftreten des Schubrisses, der zum Bruch führte, war die Tragfähigkeit je nach Belastungsniveau nach einigen hundert bis einigen zehntausend Lastwechseln erschöpft. Bei zwei Balken, bei denen sich die kritischen Risse in der Nähe der Krafteinleitung ausbildeten, wurde eine Direktabstützung mit vollständiger Lösung des Verbundes der Längsbewehrung beobachtet. Die Phase der Direktabstützung bis zur Erschöpfung der Tragfähigkeit war in einem Fall sehr kurz, im andern relativ lang.

Bei den Balken mit Rechteckquerschnitt konnten zwei Bruchvorgänge beobachtet werden: Plötzlicher Bruch sowie Bruch nach vorgängiger lang dauernder Direktabstützung mit vollständiger Lösung des Verbundes über eine Balkenhälfte oder die ganze Balkenlänge.

Eine Zusammenstellung der Belastungsgeschichten aller Balken der Serie II ist in den Bildern 43 und 44 gegeben.

\section{Balken BII/1}

Der erste Träger der Serie ohne Schubbewehrung wurde mit einer Oberlast von $110 \mathrm{kN}$, entsprechend einer nominellen Schubspannung von $0.5 \mathrm{~N} / \mathrm{mm}^{2}$ gefahren. Er hatte als einziger Träger einen kleineren Längsbewehrungsgehalt, bezogen auf den nominellen Schubquerschnitt. Nach $6.6 \cdot 10^{6}$ Lastwechseln ohne Aenderung des Rissbildes wurde die Last erhöht. Bei $165 \mathrm{kN}$ oberlast erfolgte nach $5.3 \cdot 10^{5}$ Lastwechseln ein Ermüdungsbruch in einem Längseisen in Trägermitte. Die gemessene Dehnungsdifferenz bei $110 \mathrm{kN}$ Oberlast betrug in Trägermitte $0.85 \cdot 10^{-3}$, bei $165 \mathrm{kN}$ Oberlast $1.3 \cdot 10^{-3}$.

\section{Balken BII/2}

Dieser Balken wurde im ersten dynamischen Zyklus mit einer Oberlast von $165 \mathrm{kN}$, entsprechend einer nominellen Schubspannung von $0.75 \mathrm{~N} / \mathrm{mm}^{2}$ belastet (Bild 43 ). Der Balken hatte wie $B I I / 1$ einen Rechteckquerschnitt, jedoch einen um $2 / 3$ erhöhten Längsbewehrungsgehalt. Nach $2.1 \cdot 10^{6}$ Lastwechseln ohne Bruch wurde die Last auf $220 \mathrm{kN} \mathrm{bzw} .1 .0 \mathrm{~N} / \mathrm{mm}^{2}$ nominelle Schubspannung erhöht. Nach $3 \cdot 10^{3}$ Lastspielen im Lastzyklus $C$ erfolgte der erste Ermuidungsbruch. Dieser ging von einem schon zu Beginn des Lastzyklus $B$ vorhandenen Schrägriss aus. Der Bruchriss stellte sich ohne Vorankündigung ein und durchquerte mit flacher Neigung die ungerissene Druckzone bis zur Balkenoberfläche bei der Krafteinleitung. Zusätzlich bildeten sich im zur Auflagerseite gehörenden abgetrennten Teil zwei 
Risse an der Balkenoberseite aus (Bild 155).

Nach erfolgter Reparatur scherte nach $12.8 \cdot 10^{3}$ Lastwechseln im Lastzyklus $C$ auch die zweite Balkenhälfte ab. Auch dieser Bruch erfolgte ohne Vorankundigung.

\section{Balken BII/3}

Der mit gleicher Längsbewehrung wie BII/2, jedoch mit einem T-Querschnitt ausgebildete Versuchsbalken BII/3 wurde mit einer nominellen Schubspannung $\tau=1.0 \mathrm{~N} / \mathrm{mm}^{2}$ beansprucht. Er versagte schon kurz nach Versuchsbeginn bei $2.58 \cdot 10^{3}$ Lastwechseln. Es erfolgte ein plötzlicher Stegbruch mit anschliessender Trennung des Steges vom Oberflansch und der Längsbewehrung (Biid 156).

\section{Balken BII/4}

Der mit dem Balken $B I I / 3$ identische Balken $B I I / 4$ wurde weniger beansprucht ( $\tau_{\text {nom }}=$ $0.75 \mathrm{~N} / \mathrm{mm}^{2}$ ). Nach $2.57 \cdot 10^{6}$ Lastwechse in wurde die Last um einen Drittel auf das Niveau des zuletzt geprüften Balkens erhöht. Der erste Bruch erfolgte schon nach kurzer Zeit. Der Versuch konnte vor der Zerstörung des Oberflansches gestoppt und der Träger repariert werden. Offenbar war diese Belastung, wie bereits beim Balken BII/3 erstmals festgestellt, eine obere Grenze.

Der zweite Bruch erfolgte im gleichen Steg gegen das Auflager hin, mit einem stärker geneigten Winkel zur Trägerachse nach $3.7 \cdot 10^{3}$ Lastwechseln im gleichen Lastzykius. Es konnte beobachtet werden, dass sich zwischen den Risswurzeln der äussersten leicht geneigten Risse plötzlich ein neuer Riss auftat, der den ganzen Steg durchquerte. Im Gegensatz zu den beiden schon vorher vorhandenen Rissen, die jetzt praktisch nicht mehr arbeiteten, schloss sich der neue Riss unter Minimallast nicht mehr und öffnete sich unter Maximallast immer mehr. Gleichzeitig begann er den Steg vom Oberflansch und von der Längsbewehrung zu trennen. Nach wenigen hundert Lastwechseln wurde der Pulsator gestoppt, um eine weitere Reparatur zu ermöglichen. Ein dritter Ermüdungsbruch stellte sich darauf auf der zweiten Balkenseite ein (Bild 157). Die Bruchlinie im Steg entstand in diesem Fall aus einem bestehenden Riss. Nachdem er sich nicht mehr zu schliessen begann erfolgte binnen weniger hundert Lastwechsel der Bruch.

\section{Balken BII/5}

Nachdem bei den T-Trägern unter einer nominellen Schubspannung $\tau_{\text {nom }}=0.75 \mathrm{~N} / \mathrm{mm}^{2}$ ein Durchläufer resultierte und unter $\tau_{\text {nom }}=1.0 \mathrm{~N} / \mathrm{mm}^{2}$ nach wenigen Lastwechseln Briche erfolgten, wurde der Balken BII/5 mit einer oberlast von $190 \mathrm{kN}$ belastet, entsprechend einer nominellen Schubspannung ${ }_{4}$ nom $=0.85 \mathrm{~N} / \mathrm{mm}^{2}$. Es stellten sich zwei stegbrüche ein. Der erste erfolgte nach $2.43 \cdot 10^{4}$ Lastwechseln, der zweite nach $3.58 \cdot 10^{5}$ Lastwechseln. Die Bruchvorgänge verliefen ähnlich wie beim Balken BII/4. Auf der Trägerseite A rechts entwickelte sich der Bruchriss aus einem bestehenden Riss, auf der anderen Seite durchquerte er mit einer flacheren Neigung zwei bestehende Risse.

Infolge des niedrigeren Belastungsniveaus konnte die Entwicklung des Bruchvorgangs über eine etwas längere Zeit als beim Balken BII/4 verfolgt werden. Der Stegbruch auf der Seite A links erfolgte nach $3 \cdot 10^{3}$ Lastwechseln kurz nach der Ausbildung des Schubrisses (Bi)d 158).

\section{Balken BII/6}

Dieser Träger, der einen Rechteckquerschnitt hatte, war im ersten dynamischen Zyklus ebenfalls mit $190 \mathrm{kN}$ belastet, entsprechend einer nominellen Schubspannung $\tau_{\text {nom }}=$ $0.75 \mathrm{~N} / \mathrm{mm}^{2}$. Nach $60 \cdot 10^{3}$ Lastwechseln stel1te sich auf der Trägerseite $B$ rechts ein mit einer Neigung von etwa $45^{\circ}$ verlaufender Schubriss ein. Er begann unterhalb der Biegedruckzone in Trägermitte. Anschliessend erfolgte die Lösung des Verbundes der Längsbewehrung zum Auflager hin, vorerst langsam, dann immer schneller. Die Biegerisse gegen das Auflager hin hörten mit fortschreitender Verbundlösung zu arbeiten auf. Der Balken 
hatte ein neues Tragsystem ausgebildet, und die Last wurde über eine Direktabstützung zum Auflager abgetragen. Da ein plötzlicher Oruckbruch zu befürchten war, wurde der Versuch abgebrochen und diese Seite durch eine äussere Schubbewehrung verstärkt. Nach 2.6 $10^{5}$ Lastwechseln stellte sich die gleiche Situation auf der anderen Balkenseite, A rechts, ein. Der kritische Riss war hier leicht gekrümmt, und es stellte sich nach erfolgtem Verlust des Verbundes zusätzlich ein Riss auf der Balkenoberseite ein (Bild 159).

Nach $8 \cdot 10^{5}$ Lastspielen wurde die Verstärkung entfernt und hernach weiterbelastet. Mit der Zeit bildeten sich im Druckbereich noch Spaltzugrisse aus, und es konnte eine leichte Erwärmung (handwarm) festgestellt werden. Da sich keine wesentliche Aenderung im nun beidseitig direkt abgestutzten Balken einstellte, wurde der Versuch nach $3.3 \cdot 10^{6}$ Lastwechseln gestoppt und die oberlast um $15 \%$ erhöht.

Unter der neuen Oberlast (entsprechend $\tau_{\text {nom }} \cdot 1.0 \mathrm{~N} / \mathrm{mm}^{2}$ ) versagte der Balken nach $9.7 \cdot 10^{4}$ Lastwechseln einseitig. Er schob schräg über die Druckzone zur Krafteinleitung hin ab (Bild 159).

\section{Balken BII/7}

Der Balken BII/7 wurde auf dem gleichen Belastungsniveau geprüft wie BII/6, und sein Verhalten war demjenigen von BII/6 ähnlich. Der kritische Riss stellte sich auf der Seite B links nach $2.5 \cdot 10^{4}$ Lastwechseln ein, und die Direktabstützung war nach $3.6 \cdot 10^{4}$ Lastspielen ausgebildet. Der Bruch erfolgte nach $6.9 \cdot 10^{4}$ Lastwechseln (Bild 160).

In der anderen Balkenseite bildete sich der Schubriss, der sehr flach verlief, nach $1.8 \cdot 10^{5}$ Lastwechseln aus. Nach ausgebildeter Direktabstützung nach weiteren $10^{4}$ Lastwechseln versagte der Träger nach total 1.96.10 $0^{5}$ yklen (Bild 161).

Beidseitig war wiederum eine Gelenkbildung zu beobachten, die auch durch die sich einstellenden Querrisse an der Balkenoberseite angezeigt wurde. Beide Brüche erfolgten nach kurzen Phasen mit unmittelbarer Abtragung der Kräfte zu den auflagern durch Abschieben der Druckzone.

\section{Balken BII/8}

Der erste Träger mit T-Querschnitt und den grösseren Abmessungen sowie dem Maximalkorn $032 \mathrm{~mm}$ erfuhr eine Belastung von $\tau_{\text {nom }}=0.75 \mathrm{~N} / \mathrm{mm}^{2}$. Dieses Belastungsniveau entsprach der oberen Grenze bei der bei den kleinen Versuchskörpern nach $2.5 \cdot 10^{6}$ Lastwechseln noch keine Brüche erfolgt waren. Der erste Stegbruch erfolgte bereits nach $4.5 \cdot 10^{3}$ Belastungszyklen. Der kritische Schubriss bildete sich als Verlängerung eines bestehenden Risses aus.

Der Bruch auf der anderen Seite erfolgte mit einer flacheren Rissneigung bei $3.3 \cdot 10^{4}$ Lastwechseln. Der kritische Riss war etwas stärker geneigt und verlief durch die Risswurzeln zweier benachbarter, bestehender Risse (Bild 162).

\section{Balken BII/9}

Aufgrund der nach wenigen Lastwechseln erfolgten Schubbrüche beim Balken BII/8 wurde für den Balken $B I I / 9$ ein tieferes Belastungsniveau gewählt. Unter einer Oberlast von $254 \mathrm{kN}$, entsprechend $\tau_{\text {nom }}=0.5 \mathrm{~N} / \mathrm{mm}^{2}$, waren nach $2.5 \cdot 10^{6}$ Lastwechseln weder eine Aenderung des Rissbildes noch ein Ermüdungsbruch erfolgt. Die Last wurde somit auf $\tau_{\text {nom }}=0.65 \mathrm{~N} / \mathrm{mm}^{2}$ erhöht. Auf diesem Niveau stellte sich nach $6.4 \cdot 10^{4}$ Belastungszyklen ein Bruch ein. Der Bruch kündigte sich nach $5 \cdot 10^{4}$ Lastwechseln durch die Verlängerung zweier bestehender Risse in Stegmitte an. Gleichzeitig war eine Verästelung der Risse im kritischen Bereich zu beobachten (Bild 163).

Nach erfolgter Reparatur wurde der Balken auf dem gleichen Belastungsniveau weiter beansprucht. Nach total $2.5 \cdot 10^{6}$ Lastwechseln auf diesem Niveau konnte die Last nochmals ge- 
steigert werden, da kein weiterer Bruch erfolgt war. Unter der oberlast von $\tau_{\text {nom }}=$ $0.75 \mathrm{~N} / \mathrm{mm}^{2}$ erfolgte dann der zweite Bruch nach weiteren $3.5 \cdot 10^{5}$ Lastzyk1en (Bild 164).

\section{Balken BII/10}

Der Balken BII/10 wurde auf dem Lastniveau geprüft, bei dem im vorangegangenen Versuchsbalken der erste Bruch erfolgte. Dies geschah trotz der höheren Betonfestigkeitswerte, die durch eine grössere Zementdosierung $\left(350 \mathrm{~kg} / \mathrm{m}^{3}\right)$ erreicht wurden. Unter der nominellen Schubbelastung $\tau_{\text {nom }}=0.65 \mathrm{~N} / \mathrm{mm}^{2}$ wurden $2.6 \cdot 10^{6}$ Lastwechsel gefahren, ohne dass ein Bruch erfolgte. Nach der Lasterhöhung auf $\tau_{\text {nom }}=0.75 \mathrm{~N} / \mathrm{mm}^{2}$ stellte sich nach $1.445 \cdot 10^{6}$ Lastwechseln ein kritischer Schubriss ein, der nach weiteren $6 \cdot 10^{4}$ Lastwechseln zur Erschöpfung des Tragwiderstandes führte (Bild 165).

\section{Balken BII/11}

Auch beim letzten Träger mit grossen Abmessungen konnte nach 2.76.106 Lastwechseln die nominelle Schubspannung von $\tau_{n o m}=0.65$ auf $0.75 \mathrm{~N} / \mathrm{mm}^{2}$ erhöht werden, da im ersten Belastungszyklus kein Bruch erfolgte. Auf dem höheren Lastniveau traten nach $4 \cdot 10^{5}$ und $8 \cdot 10^{5}$ Lastwechseln zwei Schubbrüche ein.

Auf der Seite B rechts war der Bruchvorgang ähnlich wie beim Balken BII/8. Auch hier konnte ein Teil der Last über den Oberflansch abgetragen werden, da sich der kritische Riss im Steg in der Nähe der Krafteinleitung befand. Im Gegensatz zum Balken BII/8 bildete sich der Bruchriss jedoch aus einem schon zu Beginn der zyklischen Belastungsphase vorhandenen Riss aus. Die Phase der Abschiebung des Steges und der allmählichen Verbundlösung bis zur Erschöpfung des dynamischen Tragwiderstandes dauerte in diesem Fall vergleichsweise lange (Bild 166).

Im Bild 167 sind die Bruchoberflächen des Bruches auf der Seite B rechts dargestellt. Das Abscheren des Oberflansches zur Krafteinleitung hin wurde durch eine statische Belastung nach Abschluss des dynamischen Belastungszyklus unter entsprechend grossen Verformungen erhalten.

\subsubsection{Schubbrüche}

Wie bereits einleitend erwähnt, konnten mit 11 Versuchsträgern insgesamt 19 Brüche erreicht werden. Im Bild 45 sind die Ermüdungsschubbrüche in Funktion der nominellen Schubspannung dargestellt.

Jeder Bruch wird als unabhängiger Einzelversuch betrachtet. Als Durchläufer wird eine Balkenhälfte ohne Bruch bezeichnet. Bei den meisten Trägern wurden Mehrstufenversuche gefahren. Im Diagramm kann also derselbe Balken auf jedem seiner Belastungsniveaus Durchläufer und Brïche aufweisen. Die Resultate aus jedem Belastungsniveau werden wiederum als unabhängige Versuche betrachtet. Die auf dem ersten Belastungsniveau erhaltenen Resultate eines Balkens sind im Diagramm als Einstufenversuche bezeichnet. Die angegebenen Lastwechselzahlen gehören zu der jeweiligen Laststufe. Eine mögliche Vorschädigung aus einer tieferen Laststufe wird somit nicht in Betracht gezogen.

Bei den Trägern, bei denen eine Direktabstützung beobachtet wurde, die im allgemeinen von kurzer oder wie beim Träger BII/6 von langer Dauer war, wurde bereits die vollständige Ab1ösung der Längsbewehrung vom Steg als Bruch betrachtet und die zugehörige Lastwechselzahl im Diagramm eingetragen. Diese Wahl wurde getroffen, da das ursprüngliche Tragsystem erschöpft war und im neuen Tragsystem die Deformationen sehr gross wurden.

Im Gegensatz zu den Stahleinlagen, bei denen für den Ermüdungsbruch die Spannungsdifferenz massgebend ist, wird beim Beton die maximale Spannung massgebend. Im erwähnten Bild entsprechen den eingetragenen ${ }$ nom-Werten allerdings fast gleich grosse $\Delta \tau_{n o m}$, da die Unterlast in den Versuchen knapp grösser als null war. Die zugehörigen nominellen Schub- 
spannungsdifferenzen sind auf dem höchsten Lastniveau $4 \%$ und auf dem tiefsten Lastniveau $9 \%$ kleiner als die eingetragenen nominellen schubspannungen.

\subsection{Tragverhalten}

\subsubsection{Balken mit Schubbewehrung}

Im folgenden wird das. Tragverhalten der einzelnen Versuchsbalken beschrieben. Es werden nur die dynamischen Lastzyklen behandelt.

Das Hauptinteresse dieses Abschnittes gilt der Erfassung des inneren Tragsystems und dessen Aenderungen infolge der eintretenden Umlagerungen. Die aufgezeichneten Durchbiegungen, Dehnungen und Schiebungen in den Bildern 47 bis 97 beschreiben die Verformungsgeschichte ab der Erstbelastung in den dynamischen Lastzyklen. Die Darstellung der Dehnungen und Schiebungen wird nur bis zum Zeitpunkt bzw. zur Lastwechselzahl geführt, wo infolge weiterer Bügelbrüche ein progressives Versagen des Versuchsträgers erfolgte. Für die Herleitung von Bemessungsregeln muss nämlich der Spannungszustand vor dem Beginn des Versagens der Tragfähigkeit bekannt sein. Alle Balken mit Schubbewehrung bildeten das gleiche Tragsystem aus. Nach dem Ueberschreiten der Risslast im statischen Lastzyklus A, die sich durch die Ausbildung von Biegerissen im Zugflansch in Trägermitte oder eines Schubrisses in der Nähe der Krafteinleitungszone anzeigte, bildeten sich gegen die Auflager hin fortschreitend sukzessive weitere Biege- und Schubrisse. Die globale Neigung der Schubrisse verflachte sich etwas gegen die Auflager hin. Der Schubrissabstand bildete sich bei einigen Versuchsträgern regelmässig aus, bei anderen blieben zwischen einzelnen Rissen grössere homogene Betonblöcke bestehen. Das globale Rissbild war nach der statischen Erstbelastung bis zur Oberlast meist ausgebildet. Bildeten sich zusätzlich neue Diagonalrisse aus, geschah dies während der ersten Lastwechsel im dynamischen Belastungszyklus.

Die Messungen zeigten, dass das innere Tragsystem als Fachwerk aufgefasst werden kann, wobei der von Schubrissen durchsetzte Stegbeton als diagonales Druckfeld, die Bugel als Zugstreben, die Längsbewehrung als Zuggurt, und der Oberflansch als Druckgurt aufgefasst werden können. Infolge diskreter Risse sind lokale Dehnungsspitzen in den Bewehrungen zu beobachten. Generell sind unter konstanter Oberlast während der Versuchsdauer grössere Dehnungszunahmen beobachtet worden als Dehnungsdifferenz-Zunahmen unter konstanter Lastdifferenz. Die Zunahme der Dehnungsdifferenzen $k l a n g$ jeweils nach der Einspielung des Tragsystems $a b$, während die Zunahme der Absolutwerte der Dehnungen über grössere Belastungszeiträume bestehen blieb, bis sich im Versuchsträger ein konstanter Eigenspannungsustand ausgebildet hatte.

\section{Balken BI/1}

Die Belastungsgeschichte des ersten Versuchsträgers ist im Bild 34 dargestellt. Die gemessenen Durchbiegungen und Dehnungen sind in den Bildern 46 bis 57 aufgezeichnet. Die Messungen der Durchbiegung in Trägermitte zeigen bei diesem Mehrstufenversuch, dass zu Beginn jedes Lastzyklus eine Zunahme der Verformungen unter Oberlast festgestellt wurde. Die Zunahme verkleinerte sich mit steigender Lastspielzahl. Die Phase der starken Durchbiegungsvergrösserung verkürzte sich mit steigendem Lastniveau. Unter der Minimallast, die konstant gehalten wurde, ist die ausgeprägte Anfangszunahme nur zu Beginn der ersten beiden dynamischen Belastungsphasen festgestellt worden (Bild 47).

Die Asymmetrie des Bildes 47 ist dem Einfluss der unterschiedlichen Stegstärken der linken und rechten Balkenhälfte zuzuschreiben. Der Verlauf der Dehnungen und Dehnungsdifferenzen in der Längsbewehrung (Bilder 48 und 49) zeigt den gleichen Charakter wie derjenige der Durchbiegungen, wobei zwischen benachbarten Messstellen lokal Sprünge in den Dehnungen auftreten. Die folgenden Bilder 50 und 51 enthalten die Dehnungs-Lastwechsel- 
Geschichte eines typischen Bügels. Zu Beginn jedes dynamischen Lastzyklus ist wieder ein Anstieg festzustellen, der beim ersten Zyklus am ausgeprägtesten ist.

In den Bildern 52 und 53, in denen die Zunahme der Dehnungen und der Dehnungsdifferenzen in den einzelnen Lastzyklen dargestellt ist, fällt vor allem die unterschiedliche Grösse der einzelnen gemessenen Werte im gleichen Steg auf. Dies gilt besonders für die Maximaldehnungen unter oberlast, während die Differenzen für die über die Steghöhe gemessenen Werte weniger ausgeprägt sind. Auch in diesen beiden Bildern tritt in Erscheinung, dass im ersten Lastzyklus während der Ausbildung des Tragsystems die Dehnungszunahmen am grössten sind.

Die Betondehnungen im Oberflansch sind für die Messreihe in Trägerachse dargestellt (Bilder 54 und 55). Die grössten Aenderungen unter konstanter dynamischer Belastung sind in den Lastzyklen $B$ und $C z u$ beobachten. Auf der Seite des Steges $t=100 \mathrm{~mm}$ wurden gegen das Auflager hin Zugdehnungen beobachtet, was auf eine weitergehende Ausbildung des Fachwerk-Tragsystems hindeutet als beim dickeren Steg.

\section{Balken BI/2}

Die Belastungsgeschichte des zweiten Versuchsträgers ist im Bild 35 dargestellt. Die gemessenen Durchbiegungen und Dehnungen sind in den Bildern 57 bis 63 aufgezeichnet. Der Balken $B I / 2$, der die gleichen Abmessungen wie $B I / 1$ jedoch nur den halben Bewehrungsgehalt aufwies, erlitt unter der aufgebrachten Belastung erwartungsgemäss sehr grosse Verformungen.

Den Bildern 56 und 57 ist zu entnehmen, dass unter konstanter ober- und Unterlast die Durchbiegungen zu Beginn des dynamischen Lastzyklus während der ersten 2000 Lastwechse 1 ebenfalls sehr stark zunahm und später bis zu den ersten Bügelbrüchen linear mit der Lastwechselzahl wuchs. Die von den zwei verschiedenen Stegstärken herrührende Asymmetrie im Durchbiegungsverlauf war deutlicher als beim ersten Versuchskörper.

Der Dehnungsverlauf in der Längsbewehrung ist für die $\Delta \varepsilon$ deutlich regelmässiger als für die $\varepsilon$, wo lokal ein starker Einbruch in der Nähe der Krafteinleitung auf der schwächeren Stegseite ersichtlich ist (Bild 59). Der bei Verwendung der Fachwerkanalogie von der Querkraft herrührende Dehnungsanteil in der Längsbewehrung ist in der Darstellung der Dehnungsdifferenzen deutich sichtbar.

Die maximalen Bügeldehnungen ( $B i l d e r 60$ und 61 ) unter oberlast erreichten örtlich 2 wischen den Rissufern nahezu $0.4 \%$ und überschritten somit $k 1$ ar den elastischen Bereich. Die maximalen Dehnungsdifferenzen überschritten vereinzelt die $0.2 \%$-Dehngrenze. Deutlich sichtbar ist, dass praktisch die ganze Zunahme der Dehnungen und Dehnungsdifferenzen während der ersten Lastzykien erfolgte.

Die Betondehnungen im Oberflansch sind im Bild 63 aufgezeichnet. Im Gegensatz zum ersten Versuchsträger konnten im Oberflansch auch auf der Seite des Steges $t=150 \mathrm{~mm}$ Zugdehnungen gemessen werden. Zwei ausgeprägte Abstufungen in der Dehnungslinie konnten beidseits der Krafteinleitung festgestellt werden. Auf der Stegseite $t=100 \mathrm{~mm}$ vermochte sich der Dehnungsverlauf infolge der stärkeren Umlagerungen zu glätten.

\section{Balken BI/3}

Bild 36 gibt einen Veberblick der Belastungsgeschichte dieses Versuchsträgers. Die gemessenen Durchbiegungen, Dehnungen und die gerechneten mittleren Schiebungen sind in den Bildern 64 bis 74 aufgezeichnet.

Der Versuchsträger, der identisch mit BI/1 war, konnte über einen sehr langen Zeitraum entsprechend $5.75 \cdot 10^{6}$ Lastwechse1n beobachtet werden. Durch den einzigen einseitigen Bügelbruch nach $2.45 \cdot 10^{6}$ Lastwechseln wurde der Dehnungszustand nicht wesentlich beein- 
flusst. Ein Vergleich der Durchbiegungszunahme während der ersten $2 \cdot 10^{6}$ Lastwechsel und während der folgenden $2 \cdot 10^{6}$ Lastwechsel zeigt, dass sich ein Trägersystem einspielte, das keine grossen Aenderungen mehr erfuhr, und dass die inneren Kräfteumlagerungen zum Stillstand kamen. Dies wird auch durch die Dehnungen und Schiebungen bestätigt (Bilder 66 bis 74 ).

Die bereits beim Balken BI/1 unter gleicher Belastung über eine kürere Lastspielzahl gemachte Beobachtung des gleichzeitigen Anstiegs der Durchbiegungslinie unter ober- und Unterlast wurde beim dritten Versuch über die ganze Versuchsdauer bestätigt. Zusätzlich zu den bisherigen Messungen erfolgten beim Balken BI/3 Durchbiegungsmessungen ohne Belastung. Um Kurzzeiteffekte im Träger auszuschliessen, liess man ihn jeweils ruhen, bis die Erholungsphase abgeschlossen war. Diese war umso kürzer, je mehr Lastwechsel der Balken ertragen hatte. Im Zusammenhang mit der immer grösser werdenden bleibenden Durchbiegung konnte beobachtet werden, dass sich die Risse immer weniger schlossen und sich so eine Art Vorspannung aufbaute. Dies war bedingt durch die Lösung von Feinteilen an den Rissufern, die in Form von hellem Staub auch auf dem Boden der Versuchshalle festgestellt worden waren. Ein Teil dieser Feinteile rührt vom fortschreitenden lokalen Abbau des Verbundes her. Dazu ist zu bemerken, dass in keinem Fall eine Verbundlösung über die Messdistanzen von $100 \mathrm{~mm}$ bei den Bügeln und $200 \mathrm{~mm}$ bei der Längsbewehrung beobachtet wurde.

Bei den Dehnungen der Längsbewehrung (Bild 67) zeigt sich deutlich ein stufenförmiger Verlauf, der durch eine stärkere Ausbildung einzelner Druckdiagonalen erklärt werden kann. Deutlich tritt hier die grosse Zunahme hervor, während bei den Dehnungsdifferenzen in der Längsbewehrung der Anstieg gesamthaft kleiner blieb.

Bei den Bügeldehnungen konnten vereinzelt Dehnungsrückgänge zugunsten von Dehnungszunahmen an Nachbarbügeln beobachtet werden ( $B i l d e r 68$ bis 70 ). Sehr ausgeprägt können aus den Bildern 71 und 72 die starke Zunahme der Dehnungen nach der Erstbelastung sowie die spätere Stabilisierung des Schubdehnungsustandes herausgelesen werden. Dies wird auch durch die mittleren Schiebungen (Bild 74) bestätigt. Bei den Bügeldehnungen ist festzustellen, dass die Zunahme der Dehnungen grösser war als die Zunahme der Dehnungsdifferenzen. Die Bügel mit kleinen Anfangsdehnungen erfuhren grössere Zunahmen als Bügel mit grossen Anfangsdehnungen. Es erfolgte also ein Ausgleich über Kräfteumlagerung. Einzelne Bügel erreichten unter Oberlast, die sich bei diesem Balken auf dem Niveau der Gebrauchslast befindet, die $0.2 \%$-Dehngrenze, während die maximalen Dehnungsdifferenzen auch im Vergleich zur knapp tieferen Belastungsdifferenz alle im elastischen Bereich des Stahls blieben.

Bei den Betondehnungen ( $B i l d 73$ ) ist analog zu den vorher beschriebenen Balken auf den regelmässigen Verlauf der Dehnungsdifferenzen aufmerksam zu machen.

\section{BaIken BI/4}

Die Belastungsgeschichte ist im Bild 37 dargestellt. Die Durchbiegungen, Dehnungen und Schiebungen sind in den Bildern 75 bis 83 aufgeführt.

Der Versuchsablauf sowie die Versuchsträger waren mit dem Versuch BI/2 identisch, und die Versuchsergebnisse wurden bestätigt. Der Verlauf der Dehnungszunahmen in der Längsbewehrung fiel bei diesem Träger etwas regelmässiger aus, und die Zunahme der Dehnungsdifferenzen war über 50'000 Lastwechsel praktisch gleich null (Bild 78). Bei den maximalen Bügeldehnungen wurde lokal am Bügel $\mathrm{Nr}$. 3 ein sehr hoher Wert gemessen, der dann auch zum ersten Bruch nach 96 '000 Lastwechseln führte (Bilder 80 und 81 ).

Der Betondehnungsverlauf war qualitativ ähnlich wie beim Balken BII/2, wenn auch unter der Lastdifferenz praktisch keine Zugdehnungen auftraten (Bild 82). Beim Versuchsträger BI/4 wurden zusätzlich die Schiebungen erfasst (Bild 83). Die Zunahme der Schiebungsdifferenzen ist deutlich kleiner als diejenige der Schiebungen und kam schon nach 5 '000 
Lastwechseln zum Stillstand.

Balken BI/5

Die Belastungsgeschichte dieses Versuchsträgers ist im Bild 38 dargestellt. Die Durchbiegungen, Dehnungen und Schiebungen sind in den Bildern 84 bis 90 aufgeführt.

Der Balken war symmetrisch und mit einer Stegstärke von $150 \mathrm{~mm}$ ausgebildet. Die Schubbewehrung entsprach den $B a 1$ ken $B I / 1$ und $B I / 3$, die Längsbewehrung den $B a 1 k e n ~ B I / 2$ und $B I / 4$. Die verwendete Stegstärke erlaubte eine Ausführung ohne Unterflansch.

Die gemessenen Durchbiegungswerte waren gegenüber dem Balken BI/3 um 45\% grösser, dies bei halbem Längsbewehrungsgehalt ( $B i l d 85$ ). Daraus ist der grosse Anteil der Schubverformungen an der Gesamtdurchbiegung ersichtlich.

Beim Verlauf der Dehnungen in der Längsbewehrung hebt sich in Balkenmitte der Krafteinleitungsbereich durch eine horizontale Dehnungsverteilung hervor, was auf eine mehr oder weniger direkte krafteinleitung hinweist.

In den Bildern 87 und 88 sind die Bügeldehnungen dargestellt, wobei auffällt, dass die maximalen und mittleren Dehnungen in den ersten Bügeln bei der Krafteinleitung in Trägermitte fast verschwinden und in den folgenden Bügeln steil ansteigen. Das gleiche gilt für den Auflagerbereich. Die Reduktion der Längsbewehrung bei gleichbleibender Schubbewehrung bewirkte offenbar eine langsamere Ausbreitung der Verteilkräfte auf die Bügel. Im weiteren scheint die Umlagerungsfähigkeit geringer. In den Bügeln der Auflager und Auflagerzonen beider Stege wurde praktisch keine Zunahme der Dehnungen beobachtet. Erst in den Stegmitten fand ein Ausgleich der mittleren Dehnungsdifferenzen $\Delta \bar{\varepsilon}$ statt.

Die kleinere Umlagerungskapazität wurde auch durch das fortschreitende Auftreten der Ermüdungsbrüche in zwei diskreten Schubrissen bekräftigt.

Im Bild 89 sind die Betondehnungen aufgezeichnet. Der Verlauf der Dehnungen in oberflanschmitte ist unregelmässig und mit sprüngen durchsetzt, die dem Bügeldehnungsverlauf entsprechen, während infolge der Querverteilung im Mittel uber die Flanschbreite sich der Dehnungsverlauf $g l a ̈ t t e t$. Im Gegensatz zu den vorher geprüften Balken wurden an den Balkenenden praktisch keine Betonzugdehnungen gemessen.

Für die Darstellung der mittleren Schiebungen wurden die Mittelwerte aus linker und rechter Balkenseite verwendet (Bild 90). Auch hier wird deutlich, dass sich die Schiebungsdifferenzen $\Delta \gamma$ nach der Einspielphase nicht mehr ändern, während sich die Schiebungen $\gamma$ infolge bleibender Verformungen weiter erhöhen. Etwa in Stegmitte ist eine Einsenkung im Schiebungsverlauf festzustellen, die auch bei den Balken BI/3 und BI/4, allerdings weniger ausgeprägt, zu beobachten war.

\section{Ba]ken BI/6}

Die Belastungsgeschichte des Versuchskörpers ist im Bild 39 dargestellt. Die Durchbiegungen, Dehnungen und Schiebungen sind in den Bildern 91 bis 97 festgehalten.

Der Balken BI/6 mit den beidseitigen Stegstärken von $100 \mathrm{~mm}$ hatte die gleiche Bügelbewehrung wie die Versuchsträger $B I / 1, B I / 3$ und $B I / 5$. Der Längsbewehrungsgehalt, der Zwischen $B I / 1, B I / 3$ und $B I / 51$ ag, machte einen kleinen Unterflansch notwendig.

Das Biegeverhalten (Bild 93) war demjenigen des Balkens BI/6 ähnlich. Bei den Bügeldehnungen ist die Wirkung des grösseren Längsbewehrungsgehaltes und der reduzierten Stegstärke bei gleicher Last deutlich zu sehen. Die Dehnungen sind regelmässiger verteilt, und der Einbruch in der Lasteinleitungszone ist viel weniger ausgeprägt und wird mit steigender Lastspielzahl durch viel stärkere Umlagerungen als bei BI/5 kompensiert 
(Bilder 94 und 95). Dies erklärt auch die breite Verteilung des Auftretens der ersten Ermüdungsbrüche auf mehrere Bügel. Bei den Betondehnungen im oberflansch wurden gegen die Auflager hin wieder Zugdehnungen gemessen. Der Dehnungsverlauf ist mit demjenigen des Balkens BI/5 vergleichbar.

Beim Schiebungsverlauf ist wiederum in Stegmitte eine Einsenkung zu beobachten, die weniger ausgeprägt ist als beim Balken BI/5, jedoch etwas stärker in Erscheinung tritt als beim Balken BI/3. Bei den Schiebungsdifferenzen erfolgte, wie beim Balken BI/5, nach $5 \cdot 10^{3}$ Lastwechseln keine Zunahme mehr (Bild 97).

\subsubsection{Balken ohne Schubbewehrung}

Zur Darstellung des Tragverhaltens in den dynamischen Lastzyklen sind in den Bildern 113 bis 154 für alle Träger der zweiten Versuchsserie, Träger BII/1 bis BII/11, die Mittendurchbiegung sowie der Stahl- und Betondehnungsverlauf über die Trägerlänge dargestellt. Die Lastzyklen gehen aus den Bildern 43 und 44 hervor.

Die bereits im Abschnitt 5.1.3 beschriebenen Beobachtungen wurden durch die Messungen zum grossen Teil bestätigt. Die Messwerte konnten bei den meisten Versuchsbalken nur bis unmittelbar vor Eintritt des ersten Bruchs erfasst werden. Auch bei allen Balken der zweiten Serie wurde eine Zunahme der Mittendurchbiegung unter Ober- und Unterlast im dynamischen Lastzyklus gemessen. Bei den Balken mit Rechteckquerschnitt waren die Zunahmen unter Ober- und Unterlast praktisch gleich. Dagegen war bei den Balken mit Oberflansch in den ersten Lastzyklen der Anstieg unter Oberlast leicht grösser. Es scheint, dass die T-Balken eine grössere Anzahl Lastwechsel benötigten bis ihr Tragsystem ausgebildet war.

In den Dehnungsverläufen der Längsbewehrung fehlt die bei allen Versuchsträgern mit Schubbewehrung beobachtete Fachwerkwirkung praktisch ganz. Es konnten folgende Tragwirkungen

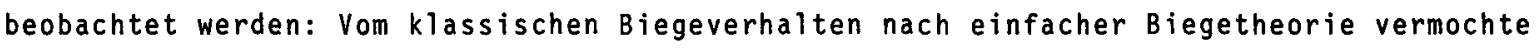
sich über Sekundärbiegung bei einzelnen Trägern auch eine Direktabstützung in Form eines Sprengwerks - entsprechend der Bogenwirkung für verteilte Lasten - auszubilden. Die beobachtete Direktabstützung ergab sich nach dem Auftreten eines diskreten Schubrisses mit anschliessender Ablösung der Längsbewehrung vom Stegbeton, wobei sich der Schubriss in Abhängigkeit des Belastungsniveaus und der Anzahl Lastwechsel ausbildete.

\section{Balken BII/1}

In den Bildern 112 bis 115 sind Messwerte für den Lastzyklus $B$ (Oberlast $\tau_{\text {nom }}=0.5 \mathrm{~N} / \mathrm{mm}^{2}$ ) dargestellt. Die schon bei den Versuchsbalken mit Schubbewehrung gemachte Beobachtung eines gleichen Anstiegs der Mittendurchbiegung unter Maximal- und Minimallast wurde auch hier bestätigt. Bei den Dehnungen in der Längsbewehrung fällt die kleinere Zunahme der Dehnungsdifferenzen auf. Eine klare Tendenz zur Fachwerkbildung ist nicht ersichtlich. Die Zunahme der Betondehnungen erfolgte in ähnlichem Mass wie diejenige der Stahldehnungen und erklärt die grosse Mittendurchbiegungszunahme unter Oberlast, während die Durchbiegungsdifferenz unter der Lastdifferenz konstant blieb.

\section{Balken BII/2}

Bei diesem Versuchsträger zeigte sich schon bei der statischen Erstbelastung unter einer nominellen Schubspannung $\tau_{\text {nom }}=0.75 \mathrm{~N} / \mathrm{mm}^{2}$ eine starke Diskontinuität im Betondehnungsverlauf (Bild 117). Die kontinuierliche Abtragung der Vertikallast über die Betondruckzone war auf diesem Lastniveau offenbar gestört. Dies zeigte sich dann unter zyklischer Belastung auch im Dehnungsverlauf der Stahldehnungen, vermochte aber bis $2 \cdot 10^{6}$ Lastwechsel nicht zum Bruch zu führen. Nach der Lasterhöhung auf $\tau_{\text {nom }}=1.0 \mathrm{~N} / \mathrm{mm}^{2}$ erfolgte der Bruch allerdings nach wenigen Lastwechseln an der von Anfang an vorgezeichneten Stelle, die auch im Rissbild durch einen geneigten Schubriss angezeigt war (Bild 155). 
Die grosse Betonbeanspruchung im zweiachsialen Spannungszustand zusammen mit der Vergrösserung der Schubrissöffnung führte zum plötzlichen Abschieben der Druckzone, da sich eine vollständige Direktabstützung mit Ablösung der Bewehrung vom Stegbeton nicht auszubilden vermochte.

\section{Balken BII/3}

Bei diesem ersten Versuchsbalken mit Oberflansch konnte nur eine Messung unter Erstbelastung (Oberlast $\tau_{\text {nom }}=1.0 \mathrm{~N} / \mathrm{mm}^{2}$ ) gemacht werden, da er schon kurz nach Beginn der dynamischen Belastungsphase versagte. Die Beobachtungen beschränken sich auf das Rissund Bruchbild (Bild 156).

\section{Balken BII/4}

Für den Versuchsträger BII/4 sind die Messwerte in den Bildern 120 bis 123 dargestellt. Die Messwerte erfassen nur den Lastzyklus $B$, in dem unter einer nominellen Schubbeanspruchung $\tau_{\text {nom }}=0.75 \mathrm{~N} / \mathrm{mm}^{2}$ kein Bruch erfolgte. Die Erhöhung der nominellen Schubbeanspruchung auf $1.0 \mathrm{~N} / \mathrm{mm}^{2}$ brachte dann innert kurzer Zeit drei Stegbriche. Im regelmässigen Verlauf der Dehnungsdifferenzen der Längsbewehrung (Bild 122) zeigten sich auf dem tieferen Belastungsniveau keine Anzeichen einer Umlagerung, während im Dehnungsverlauf unter oberlast auf der Seite A rechts von Anfang an ein starker Sprung auftrat und auf der Seite A links lokal eine grosse Dehnungszunahme zu beobachten war (Bild 123). An diesen Stellen traten in der höheren Laststufe auch zwei der drei Brüche auf.

Der Verlauf der Betondehnungen in Flanschmitte war von Anfang an sehr unregelmässig. Aus der Dehnungsverteilung und der Kinematik der ungerissenen Teile lässt sich eine Sekundärbiegung herauslesen. Der erste Stegbruch kündigte sich auf der Seite A links schon am Ende des Lastzyklus B durch eine starke Aenderung des Spannungszustandes in Oberflanschmitte an und hätte vielleicht bei einer genügend grossen Lastspielzahl auch ohne Laststeigerung eintreten können.

\section{Balken BII/5}

Beim Versuchsträger BII/5 erfolgten beide Brüche im ersten dynamischen Lastzyklus. Die Messwerte sind in den Bildern 124 bis 127 dargestellt. Der Bruchvorgang konnte bei diesem Balken unter einer nominellen Schubspannung $\tau_{\text {nom }}=0.85 \mathrm{~N} / \mathrm{mm}^{2}$ über eine vergleichsweise 1 ängere Periode beobachtet werden. Das Abschieben des Steges wurde mit den letzten Messungen, die $200 \mathrm{bzw}$. 300 Lastwechsel vor der endgiltigen Trennung erfolgten, deutlich erfast.

Der Verlauf der Betondehnungen unter Oberlast zeigte auf beiden Balkenseiten eine starke Aenderung des Spannungszustandes. Lokal traten sogar Betonzugdehnungen auf. Die Dehnungsdifferenzen verblieben jedoch immer im Druckbereich. Auf der Seite A links erfuhren sie praktisch keine Aenderung, während auf der Seite A rechts an der Stelle, wo unter oberlast Risse auftraten, unter Lastdifferenz eine grosse Dehnungsdruckdifferenz gemessen wurde.

Der Verlauf der Stahldehnungen und Stahldehnungsdifferenzen zeigt ebenfalls, dass kurz vor dem Bruch durch das immer grössere Oeffnen und nicht wieder Schliessen der kritischen Risse sehr grosse Aenderungen im Eigenspannungszustand eintraten, sich das Tragsystem jedoch nur wenig änderte.

\section{Balken BII/6}

Wie schon im Abschnitt 5.2 beschrieben, vermochte sich bei diesem Versuchsbalken nach der Verbundlösung mit der Längsbewehrung ein vollständig neues Tragsystem in Form einer Direktabstützung auszubilden, was auch aus den Messungen, dargestellt in den Bildern 128 bis 131 , hervorgeht. 
Im Bild 129 ist für die Seite A links die Entwicklung der Betondehnungen bis zur beginnenden Verbundablösung dargestellt. Der Dehnungsverlauf nach $5 \cdot 10^{4}$ Lastwechseln entspricht dem Zeitpunkt zu dem der Verbund bis zur Mitte der Balkenseite A links gelöst war. Im rechten Bildabschnitt ist der Dehnungsverlauf nach vollständiger Lösung des Verbundes vom kritischen Riss bis zum Auflager ersichtlich. Für den gleichen Zeitpunkt ist in den Bildern 130 und 131 der Stahldehnungsverlauf dargestellt. Offensichtlich weist der ziemlich konstante Dehnungsverlauf in Richtung eines Bogenzugbandes hin. Durch die Ausbildung eines flachen Schubrisses stellte sich infolge einer zu Beginn starken Dübelwirkung eine fortschreitende Ablösung der Längsbewehrung vom Stegbeton gegen das Auflager hin ein (Bild 159). Gleichzeitig bildete sich in der Druckzone in Trägermitte ein Gelenk aus, das zum Bewegungszentrum wurde. Nach dem vollständigen Verlust des Verbundes fand die ganze Verformung im immer grösser gewordenen Schubriss statt. Die Dübelwirkung war abgebaut, dafür erfuhren die Bewehrungsstähle am Uebergang vom freigelegten Zugband zum vertikal gehaltenen lokal ein Umlenkung. Diese Krümmung erklärt die gemessenen Dehnungsspitzen in der Längsbewehrung am Fuss der Schubrisse.

\section{Balken BII/7}

Die Durchbiegungs- und Dehnungsverläufe sind in den Bildern 132 bis 135 dargestellt. Das Tragverhalten dieses Versuchsträgers war mit demjenigen von BI/6 identisch. Es bildete sich auch hier beidseitig eine Direktabstützung aus. Die schon beim Balken BI/6 beobachteten Risse auf der Balkenoberseite, die sich nach der Ausbildung des neuen Tragsystems einstellten, waren hier grösser und sind im Verlauf der Betondehnungen erkennbar. Bei dieser zweiten Gelenkausbildung konnte das Arbeiten dieser Risse, die den Druckkeil oberhalb des Schubrisses durchtrennten, gut beobachtet werden. Die Messungen bestätigten, dass sich der Riss unter Oberlast schloss und unter Unterlast öffnete (Bilder 160, 161).

\section{Balken BII/8}

Die Messwerte des Versuchträgers $B I I / 8$ sind in den Bildern 136 bis 139 dargestellt. Wie bei BII/5 konnte das Entstehen des Bruchs beobachtet werden. Der erste Stegbruch bildete sich aus einem schon bei der Erstbelastung entstandenen Schubriss aus. Die kurz vor dem Abschieben auf der Seite A gemachten Messungen zeigen im Verlauf der Betondehnung eine starke Spannungsumlagerung unter Oberlast, die auch im Dehnungsverlauf der Längsbewehrung sichtbar ist. Im Verlauf der Dehnungsdifferenzen ist hingegen keine Aenderung festzustellen. Das Tragverhalten ist direkt mit demjenigen des Balkens BII/5 zu vergleichen.

Der Bruch auf der Seite A links erfolgte unter Lösung des Verbundes mit der Längsbewehrung (Bild 162). Die Verbundlösung entstand während der Abschiebung des Steges und erfolgte binnen weniger Lastwechsel. Während dieser Belastungsphase wurde die Last teilweise über den oberflansch abgetragen, der zugleich infolge seiner Trennung vom Steg unter Sekundärbiegung beansprucht wurde. Der rasch zunehmende Lastanteil, der auf den oberflansch wirkte, führte dann innert kurzer Zeit zum Erschöpfen des Tragwiderstandes. In diesem Sinn kann von einer kurzen Phase einer Direktabstützung gesprochen werden.

\section{Balken BII/9}

Der Verlauf der Mittendurchbiegung sowie der Beton- und Stahldehnungen während der Lastzyklen $B$ und $C$ sind in den Bildern 140 bis 143 dargestellt. Der erste Bruch erfolgte zu Beginn des Lastzyklus $C$ und war möglicherweise schon durch eine Veränderung im Verlauf der Betondehnungen während des Lastzyklus B auf der Seite A links vorgezeichnet. Im Verlauf der Stahldehnungen fanden sich jedoch keine eindeutigen Anzeichen. Erst nach der Lasterhöhung war der zuvor regelmässige Dehnungsverlauf gestört. Gleichzeitig stellte sich eine neue Dehnungsspitze bei den Betondehnungen ein. Die Entwicklung der Dehnungsdifferenzen gab keinen Hinweis auf den folgenden Bruch.

Die Dehnungsverläufe auf der Seite A rechts erfuhren in den Lastzyklen $B$ und $C$ keine einen Bruch ankündigenden Dehnungsspitzen, was auf keine oder nur geringe Vorschädigung 
für den zweiten Ermijdungsbruch schliessen liess. Dies wurde auch durch die relativ grosse im Lastzyklus 0 erreichte Lastspielzahl von $3.5 \cdot 10^{5}$ bestätigt.

Balken BII/10

Die Messwerte für den Versuchsbalken BII/10 sind in den Bildern 144 bis 149 dargestellt. Im Lastzyklus $B$, der ohne Bruch durchfahren wurde, deutete die Zunahme der Stahldehnung unter Oberlast bei der Messstelle 26 eine Aenderung des Spannungszustandes an. Gleichzeitig wurden bei dieser Messstelle eine Verlängerung und eine stärkere 0effnung des Risses beobachtet, die dann allerdings stationär blieben. Nach $2.6 \cdot 10^{6}$ Lastwechseln ohne weitere Anzeichen eines kommenden Ermüdungsbruches wurde die Last erhöht.

Im Lastzyklus $C$ stellte sich nach $1.445 \cdot 10^{6}$ Lastwechseln plötzlich ein Verbindungsriss von der Krümmung des oben beschriebenen Risses zum Fuss des Nachbarrisses ein; damit war ein ausgeprägter Schubriss vorhanden, der nach weiteren $6 \cdot 10^{4}$ Lastwechseln zum Versagen des Trägers führte (Bild 165). Die Wirkung dieser Tragsystemänderung konnte nun auch in der Ausbildung einer Stufe im Verlauf der Dehnungsdifferenzen in der Längsbewehrung festgestellt werden (Bild 148). Gleichzeitig wurde eine Reduktion der Betonstauchungen in der Trägerachse im Bereich des Schubrisses gemessen (Bild 149).

\section{BaIken BII/11}

Beim Balken BII/11, der die gleiche Belastungsgeschichte erfuhr wie der Balken BII/10, wurde ein ähnliches Tragverhalten beobachtet wie beim Balken BII/8 auf der Seite $A$ links. Auf der Seite A rechts bildete sich ein Schubriss als Verbindung der ersten beiden Biegerisse, von der Balkenmitte her gesehen, aus. Der geknickte Schubriss verlief direkt zur Krafteinlejtung hin. Dies erfolgte nach $3 \cdot 2 \cdot 10^{5}$ Lastwechseln im Lastzyklus C. Nach weiteren $8 \cdot 10^{4}$ Lastwechseln begann die Lösung des Verbundes, die bis zum Auflager fortschritt.

Auf der Seite A links bildete sich der kritische Riss aus einem bestehenden, gegen den Oberflansch hin schon flach geneigten Riss aus (Bild 166). Die eindeutige Lösung des Verbundes zusammen mit einer sukzessiven Trennung des Steges vom oberflansch erfolgte nach ca. $5.8 \cdot 10^{5}$ Lastwechseln. Gleichzeitig öffnete sich der Riss unter oberlast immer mehr, und er schloss sich unter Unterlast nicht mehr. Die Lösung des Verbundes schritt bei dieser Balkenseite langsamer voran als auf der anderen. Der Oberflansch erlitt mit fortschreitender Trennung vom Steg infolge Sekundärbiegung immer grössere Verformungen. Als die Trennung genügend weit fortgeschritten war, schob der steg über den inzwischen fast $2 \mathrm{~mm}$ breiten Riss ab. Dies erfolgte bei $8.8 \cdot 10^{5}$ Lastwechsein.

Auf der anderen Balkenseite hatten sich seit der vollständigen Lösung des Verbundes und somit der vollständigen Direktabstützung bis zu diesem Zeitpunkt keine Anzeichen für ein Abschieben bzw. Versagen des Betongelenks ergeben.

Die Ausbildung des neuen Tragsystems, die Direktabstützung, ist in den Dehnungsverläufen, die auf der Seite A rechts erhalten wurden, deutlich zu erkennen. Auf der Seite A links wurden Kräfteumlagerungen unter Oberlast gemessen. Der Verlauf der Dehnungsdifferenzen in der Längsbewehrung zeigt jedoch, dass das ursprüngliche Tragsystem bis zum Abschieben des Steges erhalten blieb (Bilder 152 bis 154). 


\subsection{Rissverhalten}

\subsubsection{Balken mit Schubbewehrung}

Die Rissbilder mit den gemessenen Rissweiten sind fur die Balken BI/1 bis BI/6 in den Bildern 98 bis 111 (Fotos) dargestellt. Für alle Träger ist das Rissbild nach seiner vollen Entwicklung festgehalten. Für den in sechs Belastungsstufen geprüften Balken BI/1 sind drei Lastniveaus ausgewählt worden, während für BI/6 die Rissentwicklung in funktion der Lastwechselzahl verfolgt werden kann.

Wie schon im Abschnitt 5.2.1 einleitend erwähnt, war das globale Rissbild nach der statischen Erstbelastung bis zur oberlast bei allen Versuchsbalken praktisch ausgebildet. Zusätzliche Schub- und Biegerisse sowie Rissverlängerungen vermochten sich meistens noch zu Beginn der dynamischen Belastungsphase bis etwa 5'000 Lastwechsel auszubilden. Die Ausbildung kleiner Verästelungen an den bestehenden Rissen wurde jedoch über die ganze Versuchsdauer hin beobachtet. Ebenso vergrösserten sich die Rissweiten leicht unter konstanter Last während der dynamischen Belastung. Dies auch, ohne dass Ermüdungsbrüche an Bügeln eingetreten waren.

Beim Balken BI/1 (Mehrstufenversuch) blieb das Rissbild beim Steg $t=150 \mathrm{~mm}$ auch unter Laststeigerung erhalten. Erst unter einer oberlast $P_{0}=660 \mathrm{kN}$ bildete sich im Auflagerbereich ein zusätzlicher Schubriss aus, der aus einem bestehenden Biegeriss hervorging. Beim dünneren Steg wurde das Rissbild unter der Laststeigerung stärker verändert (Bilder 98 und 99).

Beim Versuchsträger BI/3 konnte die Rissentwicklung am längsten beobachtet werden. Mit einem einseitigen Bügelbruch nach $2.45 \cdot 10^{6}$ Lastwechseln konnte die Entwicklung bis $5.75 \cdot 10^{6}$ Lastwechseln beobachtet werden. Das Rissbild war im steg $t=150 \mathrm{~mm}$ nach 11000 Lastwechseln, im Steg $t=100 \mathrm{~mm}$ nach $50 \cdot 10^{3}$ Lastwechseln vollständig ausgebildet. Die Zunahme der Schubrissbreiten unter Oberlast betrug bis zu einer Lastspielzahl $\mathrm{N}=$ $2.45 \cdot 10^{6}$ im Maximum $5 / 100 \mathrm{~mm}$. Eine Zunahme bis $N=5.75 \cdot 10^{6}$ Lastwechsel war mit dem Rissmassstab nicht mehr messbar (Bild 103). Eine bedeutende Aenderung im Rissbild stellte sich bei der statischen Endbelastung bis zum Bruch ein. Kurz vor dem Abschieben der Stegseite $B$ rechts, das unter einer Neigung von etwa $15^{\circ}$ bei einer Belastung von 1 '050 kN erfolgte, stellten sich in beiden Stegen neue und stärker geneigte Risse ein. Sie sind im Bild 104 gestrichelt hervorgehoben. Diese Kräfteumlagerungen fanden im dünneren Steg bei einer Belastung von 1'010 kN, im dicken Steg bei $11030 \mathrm{kN}$, statt, also praktisch gleichzeitig. Die Ausbildung des neuen Rissbildes erfolgte schlagartig. Rissverlängerungen waren unter der monotonen Laststeigerung ab ca. $650 \mathrm{kN}$ beobachtet worden.

Die maximalen Schubrissgrössen waren bei den Balken mit gleicher Schubbewehrung in $A b-$ hängigkeit des Längsbewehrungsgehaltes unterschiedlich. Bei den Trägern BI/5 und BI/6 wurden zusätzlich die Rissgrössen der Biegerisse gemessen. Durch die feinere Verteilung erreichten die Maximalwerte nur etwa $30 \%$ der maximalen Schubrissgrössen (Bilder 107, 110 , 111).

\subsubsection{Balken ohne Schubbewehrung}

Die Rissbilder mit den gemessenen Rissweiten sind für die Balken der Serie II in den Bildern 155 bis 166 festgehalten. Für eine Auswahl Balken ist das Rissbild nach seiner vollen Entwicklung und beim Bruch dargestel1t. Bei den Versuchsbalken BII/6 und BII/7 (Rechteckquerschnitte) sowie bei den Balken BII/10 und BII/11 (T-Querschnitte) kann die Rissentwicklung auf einer Balkenseite in Funktion der Lastwechselzahl verfolgt werden.

Aehnlich wie bei den schubbewehrten Trägern waren auch hier die Rissbilder nach den ersten Belastungszyklen gezeichnet. Auf einem tiefen Belastungsniveau (Durchläufer) er- 
folgte bis zur Lasterhöhung keine Aenderung mehr. Auf den Lastniveaus, wo Ermüdungsbrüche erfolgten, wurde der Bruch oder die Direktabstützung durch die Verlängerung (unter einem flacheren Winkel) eines bestehenden, schon leicht geneigten Risses, oder das Entstehen eines die vorhandenen Risse durchquerenden eindeutigen Schubrisses verursacht.

Bis zur Entstehung der Schubbrüche oder eines neuen Tragsystems betrugen die Rissbreiten im Maximum 15/100 mm. 


\section{Zusammenfassung}

Im Rahmen des Forschungsprojektes "Ermüdung von Stahlbeton- und Spannbetontragwerken" wurden am Institut für Baustatik und Konstruktion der ETH Zürich sechs Versuche an Stahibetonbalken mit Schubbewehrung und elf Versuche an Balken ohne Schubbewehrung durchgeführt. Der vorliegende Versuchsbericht enthält die Planung und die Ergebnisse der Versuche.

Das Ermüdungsverhalten wird durch das Spannungsniveau und die Lastwechselzahl dargestellt. Das Traguerhalten wird mit der Verformungs- und der Rissentwicklung in Funktion der Lastwechsel unter konstanter Ober- und Unterlast beschrieben. Folgende Grössen wurden gemessen: Belastung, Durchbiegungen, Dehnungen der Längs- und Schubbewehrung, Betondehnungen, Schiebungen und Rissbreiten.

\section{Balken mit Schubbewehrung}

Mit Ausnahme eines Versuchsbalkens, der einem Mehrstufenversuch unterzogen wurde, erfuhren alle Balken die gleiche Belastungsgeschichte: lastgesteuerte zyklische Belastung unter konstanter Ober- und Unterlast (Bilder 34 bis 39 ). Alle Balken hatten ein Verhältnis Spannweite/Trägerhöhe $L / H=7.4$. Die Belastung erfolgte konzentriert in Trägermitte. Folgende Grössen wurden als Versuchsparameter gewählt (Bild 2): Stegstärke, Schubbewehrungs gehalt, Biegebewehrungsgehalt. Die Ergebnisse können wie folgt zusammengefasst werden:

\section{Verformungs - und Tragverhalten}

- Bei allen Balken war das Rissbild nach der Erstbelastung ausgebildet. In den folgenden Lastzyklen entstanden nur unbedeutende Rissverlängerungen.

- Zu Beginn der zyklischen Belastungsphase wurde eine starke Zunahme der Durchbiegungen, Dehnungen und Schiebungen unter Ober- und Unterlast festgestellt, die sich mit zunehmender Lastspielzahl verkleinerte. Die Zunahme der Verformungsdifferenzen war hingegen kleiner.

- Lokale Spitzen wurden im Verlauf der Betonstauchungen und der Dehnungen der Längs- und Bügelbewehrung unter Oberlast beobachtet. Demgegenüber zeigten die Dehnungsdifferenzen einen regelmässigeren Verlauf.

- Der unterschiedliche Verlauf der Dehnungen und Dehnungsdifferenzen deutet darauf hin, dass sich zum Teil bedeutende Aenderungen im Tragsystem einstellten.

- Die lokale Ausbildung der Schubrisse im Stegbeton bestimmte die maximal gemessenen Bügeldehnungen und Bügeldehnungsdifferenzen.

- Bügel mit kleinen Anfangsdehnungen erfuhren einen grösseren Dehnungszuwachs als Bügel mit grossen Anfangsdehnungen. Das Umlagerungsvermögen war ausgeprägter bei einem grösseren Verhältnis Längsbewehrung/Schubbewehrung.

- Bei gleicher Schub- und Biegebewehrung ergaben sich bei grösserer Stegstärke kleinere Bügeldehnungen und im Laufe der zyklischen Belastung eine kleinere Zunahme derselben (Bild 71). Dies verursachte immer die Erschöpfung der dynamischen Tragfähigkeit des dünneren Steges (Bilder 35, 37).

- Der Verlauf der Dehnungen in der Biegebewehrung und dem Druckflansch zeigte deutlich die Fachwerkwirkung des inneren Tragsystems (Bilder 93, 96).

\section{Ermüdungsverhalten}

- Bei allen Batken erfolgten Ermüdungsbrüche in der Bügelbewehrung. Versagen der Längsbewehrung trat in keinem Fall ein. 
- Der Ermuidungswiderstand einbetonierter Bügel entspricht demjenigen der nackten Bewehrungsstähle (Bild 42).

- Die Bewehrung war infolge der Dehnungszunahmen auch unter konstanter ober- und Unterlast einem Mehrstufenversuch unterworfen. Lokal wurde unter Gebrauchslast die Streckgrenze erreicht (Bilder 67, 68).

- Von insgesamt 74 Ermüdungsbrüchen erfolgten zwei an der Ausrundung bei der Umfassung der Längsbewehrung als Folge vorausgegangener Brüche im gleichen Schubriss. Die restlichen Brüche erfolgten fast ausnahmslos beim Zusammentreffen einer Querrippe mit einer Längsrippe des Stabes.

- Bei keinem Balken trat infolge Ermüdungsbeanspruchung ein plötzliches Schubversagen ein. Es zeigte sich vielmehr das redundante Verhalten eines Stahlbetonträgers, wenn eine Mehrzahl Bewehrungsstäbe in einem Bruchriss vorhanden ist und eine genügende Umlagerung der Kräfte erfolgen kann. Dies war bei allen Versuchsbalken ohne reduzierte Längsbewehrung auch in der Verteilung der Bügelbrüche deutlich (Bilder 34 bis 37,39 ).

\section{Balken ohne Schubbewehrung}

Alle Balken hatten ein Verhältnis Spannweite/Trägerhöhe $L / H=8.0$. Sie wurden mit einer Einzellast in Trägermitte unter lastgesteuerter zyklischer Belastung im Ein- oder Mehrstufenversuch bis zum Bruch beansprucht (Bilder 43, 44). Folgende Grössen wurden als Versuchsparameter gewählt (Bild 3 ): Belastungsniveau (Oberlast), Bewehrungsgehalt, Trägerabmessungen (zwei Balkengrössen), Querschnittsform (Rechteck- oder T-Querschnitt), Maximalkorn der Zuschlagstoffe. Die Ergebnisse können wie folgt zusammengefasst werden:

\section{Verformungs- und Tragverhalten}

- Bei allen Balken war das Rissbild nach den ersten Lastzyklen ausgebildet. Bis zum Auftreten eines kritischen Schubrisses traten nur unbedeutende Rissverlängerungen ein.

- Versagen wurde durch die Bildung eines kritischen Schubrisses eingeleitet, der bei fast allen Balken die vorhandenen Biegerisse durchquerte und meist zur Erschöpfung der dynamischen Tragfähigkeit führte (Bilder 155 bis 158).

- Bei einigen Versuchsträgern vermochte sich eine kinematisch bedingte Ablösung der Längsbewehrung vom Stegbeton einzustellen, ohne direkt zum Bruch zu führen (Bild 159).

- Zu Beginn der zyklischen Belastungsphasen wurde eine starke Zunahme der Durchbiegungen und Dehnungen unter Ober- und Unterlast festgestellt, die sich mit zunehmender Lastspielzahl verkleinerte. Die Zunahme der Verformungsdifferenzen war hingegen kleiner (Bilder 112, 114, 115).

- Aus den Dehnungsverläufen konnten folgende Tragwirkungen beobachtet werden: $k 1$ assisches Biegeverhalten nach einfacher Biegetheorie (Bilder 113, 114), Sekundärbiegung (Bilder $125,126,127$ ) und Direktabstützung in Form eines Sprengwerks (Bilder 129 bis 131). Eine wie bei den Balken mit Schubbewehrung beobachtete Fachwerkwirkung konnte nicht eindeutig beobachtet werden.

\section{Ermijdungsverhalten}

- Mit Ausnahme eines Versuchsträgers mit reduzierter Längsbewehrung, bei dem ein Stah1bruch erfolgte, wurden bei allen Balken Ermüdungs-Schubbrüche erreicht.

- Bei den Balken mit sehr hohem Belastungsniveau und entsprechend kleiner Lastwechselzahl bis zum Bruch führte der Bruchvorgang nach wenigen Lastwechseln zum Versagen des Trägers. Je tiefer das Belastungsniveau war, desto progressiver erfolgte der Bruchvorgang, was alimähiich zum Versagen des Tragsystems führte. 
- Bei denjenigen Trägern, bei denen sich nach dem Auftreten des kritischen Schubrisses eine Direktabstützung auszubilden vermochte, konnte die Lastwechselzahl bis zum Versagen des neu gebildeten Tragsystems zum Teil noch erheblich gesteigert werden (Bild 159).

- Eine Darstellung aller Ermüdungsbrüche in Funktion der nominellen Schubspannung und der Lastwechselzahl ist im Bild 45 gegeben. 


\section{Résumé}

Dans le cadre du projet de recherche intitulé "Fatigue des structures porteuses en béton armé ou précontraint" et développé à l'institut de statique et construction de l'EPF à Zurich, on a expérimenté 17 poutres en béton armé dont 6 possêdaient une armature à l'effort tranchant. Dans le présent rapport, on trałte de la planification, de l'exécution et des résultats de ces essais.

Le comportement à la fatigue est caractérisé par le niveau de sollicitations et le nombre de cycles. On décrit l'histoire du comportement à la fatigue sur la base de l'accroissement des déformations et du développement des fissures par rapport au nombre de cycles et a l'action de charges maximale et minimale constantes. Lors des essais, on a mesuré les grandeurs suivantes: charge, flëches, allongements spécifiques des armatures de flexion et d'effort tranchant, raccourcissement du béton, déformations de cisaillement et largeur des fissures.

\section{Poutres avec une armature $\$$ l'effort tranchant}

L'histoire du chargement a été la même pour toutes les poutres à l'exception d'une pour laquelle différentes étapes de chargement furent exécutées: chargement cyclique exécuté en fonction de la charge variant entre des valeurs maximale et minimale (fig. 34 à 39 ). Toutes les poutres avaient le méme élancement $L / H=7.4$. Le chargement fut exécuté à l'aide d'une charge concentrée agissant au milieu de la portée. Les paramẽtres choisis pour les essais étaient les suivants: épaisseur de l'âme de la poutre, pourcentage géometrique des armatures à la flexion et à l'effort tranchant. Lors des essais, on a obtenu les résultats suivants:

\section{Déformations et comportement des poutres}

- Pour toutes les poutres, on a observé que la répartition des fissures était déterminée lors du premier cycle de chargement et que durant les cycles suivants, les fissures existantes se prolongeaient d'une façon insignifiante.

- Au début du chargement cyclique, on a mesuré une forte augmentation des flecches, des allongements spécifiques et des déformations de cisaillement qui s'est ralantie au fur et a mesure de l'augmentation du nombre de cycles. L'accroissement des déformations différentielles était par contre plus petite.

- Localement, on a mesuré des valeurs extrêmes pour le raccourcissement du béton et l'allongement spécifique des armatures a l'effort tranchant et a la flexion. Par contre les allongements spécifiques différentiels avaient une distribution plus rêgulière.

- Les différences observées entre la distribution des allongements spécifiques et celle des allongements spécifiques différentiels sont une preuve que des modifications importantes interviennent dans le système porteur durant l'histoire du chargement.

- Les fissures de cisaillement déterminent les valeurs maximales des allongements spécifiques ainsi que celles des allongements spécifiques différentiels de l'armature à l'effort tranchant.

- Plus les allongements spécifiques des êtriers sont petits au début du chargement, plus leur augmentation est grande en cours de chargement. La capacité de redistribution des efforts intêrieurs est particulièrement importante lorsque le rapport entre l'armature à la flexion et celle à l'effort tranchant est grand. 
- Pour une quantité d'armature à la flexion et à l'effort tranchant égale on a observé que les allongements spécifiques des etriers et leur accroissement durant le chargement étaient plus petits dans l'áme la plus épaisse (fig. 71). C'est pourquoi l'épuisement de la capacité portante dynamique de l'áme est déterminante pour les poutres avec une âme mince (figs. 35 et 37 ).

- La distribution des allongements specifiques dans l'armature à la flexion et dans la zone de compression démontre clairement que les poutres ont un système porteur analogue à celui d'un treillis (figs. 93 et 96 ).

\section{Comportement à la fatigue}

- Pour toutes les poutres, on a observé une rupture à la fatigue qui est intervenue dans les étriers. On n'a pas observé de rupture à la fatigue de l'armature longitudinale.

- La résistance à la fatigue d'un étrier ne dépend pas du fait qu'il soit enrobé ou non de béton.

- Même sous l'effet d'un chargement cyclique entre des limites supérieure et inférieure constantes, l'armature est soumise à des sollicitations variables qui sont provoquées par l'accroissement des allongements spécifiques. On a observé que même sous l'effet des charges du domaine d'utilisation les sollicitations dans les barres d'armature pouvaient atteindre la limite d'écoulement (figs. 67 et 68).

- Parmi les 74 cas de rupture à la fatigue observé lors des essais, deux cas de rupture se sont produits dans le coude d'un étrier à la jonction avec l'armature longitudinale. Les autres cas de rupture se sont produits presque exclusivement à l'intersection de deux nervures transversale et longitudinale.

- On n'a pas observé de rupture fragile due à l'effort tranchant. On a plutôt observé une rupture se développant progressivement dans les poutres en béton armé quand plusieurs barres d'armature traversaient une fissure critique et quand une distribution suffisante des efforts intérieurs pouvait se produire. Ce comportement a été également observé dans la répartition des ruptures des etriers dans toutes les poutres qui n'avaient pas une armature réduite.

\section{Poutres sans armature à l'effort tranchant}

Toutes les poutres avaient un élancement $L / H=8.0$. Elles étaient chargées au milieu de la portée et soumise à un chargement cyclique pour différents niveaux de charges (figs. 43 et 44 ). Les paramètres choisis sont les suivants (fig. 3 ): niveau de charge, pourcentage géométrique de l'armature, dimensions de la poutre ( 2 grandeurs), forme de la section (rectangulaire et en $T$ ), diamètre maximal des aggrégats. Les résultats d'essais peuvent être résumés de la façon suivante:

\section{Deformations et comportement des poutres}

- Pour toutes les poutres, on a observé que la répartition des fissures était déterminée lors du premier cycle de chargement. Jusqu' a l'apparition d'une fissure de cisaillement, on a observé que les fissures de flexion se prolongeaient d'une façon insignifiante.

- La rupture se produit au moment de l'apparition d'une fissure de cisaillement inclinée par rapport a l'horizontale. Celle-ci traverse, en général, les fissures de flexion et conduit à l'épuisement de la capacité portante dynamique (figs. 155 à 158).

- Dans certains cas on a observé que, pour des raisons de compatibilité cinématique, 
l'armature longitudinale se séparait de l'áme en béton, sans que la rupture se produise (fig. 159).

- Au début du chargement cyclique, on a observer un accroissement important des flèches et des allongements spécifiques. Ensuite et pour un niveau de charge cyclique donné, on a observé que les déformations se stabilisaient. L'accroissement des déformations différentielles était par contre petit (figs. 112, 114, 115).

- Des figures montrant les répartitions des allongements spécifiques, on peut constater les differents types du comportement des poutres: comportement flexionnel classique (figs. 113 et 114), comportement flexionnel secondaire (figs. 125 a 127) et effet de béquilles (figs. 129 à 131). Un comportement analogue à celui d'un treillis n'a pas ètê observé.

\section{Comportement à la fatigue}

- Pour toutes les poutres à l'exception d'une ayant une armature longitudinale reduite, la rupture a été provoqueé par épuisement de la résistance à l'effort tranchant du a la fatigue.

- La rupture des poutres sollicitées à un niveau de charge élevé s'est produite pour un petit nombre de cycles. Plus le niveau de charge etait bas, plus le procéde de rupture se développait progressivement.

- Les poutres pour lesquelles un effet de béquilles pouvait se développer après la formation d'une fissure de ciscaillement, ont supporté un nombre de cycles élevé avant de se rompre (fig. 159).

- Dans la figure 45, on a representé les ruptures dús à la fatigue en fonction de la contrainte nominale de cisaillement et du nombre de cycles. 
Tests on six reinforced concrete beams with, and eleven beams without shear reinforcement have been carried out at the Swiss Federal Institute of Technology as a part of the research project "Fatigue of Reinforced and Prestressed Concrete Structures". The report contains the planning and results of the tests.

The fatigue behaviour is presented in terms of the stress level and the number of fatigue load cycles. The load carrying behaviour is described in terms of the deformation- and crack-development as a function of load cycles with constant upper and lower load levels. The following quantities were measured: load, deflections, longitudinal and shear strains, concrete strains, shear deformations and crack widths.

\section{Beams with shear reinforcement}

With the exception of one beam which underwent a multistep test, all beams had the same load history: load controlled cyclic loading with constant upper and lower levels (Figs. 34 - 39). All beams had a span to depth ratio $L / H$ of 7.4 and were loaded at midspan. The following quantities were chosen as test parameters (Fig. 2): web thickness, shear reinforcement and longitudinal reinforcement. The results may be summarized as follows:

Deformation and load carrying behaviour

- The crack pattern of all beams was developed at the first cycle. In the following load cycles only small crack elongations took place.

- A large increase in deflections, strains and shear deformations was observed under both the upper and the lower load levels during early phases of cycling. These increases became smaller with time. On the other hand, the increase in deformation range was smaller.

- Local peaks in the variation of concrete compressive strains and of longitudinal strains and stirrup strains were observed under the upper load. However, the strain-range showed a more regular variation.

- The difference between the strain-and the strain-range-variations implies in part, a significant change in load carrying behaviour.

- The local shear crack pattern in the web determined the maximum measured stirrup strains and strain-ranges.

- Stirrups with small initial strains displayed a larger increase than those with larger initial strains. The capacity for redistribution was more pronounced in beams with a larger longitudinal to shear reinforcement ratio.

- With the same shear and longitudinal reinforcement, a larger web thickness led to smaller stirrup strains and smaller increases in the strains during cycling (Fig. 71). This always exhausted the dynamic load carrying capacity of the thinner webs (Figs. 35,37 ).

- The variation of strains in the longitudinal reinforcement and in the compression flange indicated clearly the truss action of the internal load carrying behaviour (Figs. 93, 96).

\section{Fatigue behaviour}

- In all beams fatigue failures took place only in the stirrups and never in the longitudinal reinforcement. 
- The fatigue resistance of the stirrups in the concrete corresponds to that of the bear steel (Fig. 42).

- Although the upper and lower load levels remained constant, the reinforcement was subjected to a multi-step test due to the increase in strain with cycling.

- Out of a total of 74 fatigue breaks only two took place at the lower bend near the longitudinal steel. These were due to previous stirrup failures in the same diagonal crack. The other breaks occurred almost without exception at the meeting point of a transverse and the longitudinal ribs of the reinforcement.

- No beam showed a sudden shear failure due to fatigue. Rather, the redundant characteristic of a reinforced concrete beam in which several reinforcement bars cross a crack and in which sufficient redistribution of the internal forces is possible, was observed. This was clearly visible in the distribution of the stirrup breaks in all beams without a reduction in longitudinal reinforcement (Figs. $34-37,39$ ).

\section{Beams without shear reinforcement}

All beams had a span to depth ratio $L / H$ of 8.0 and were cyclicly loaded at midspan until failure, under load control in one-step and in multi-step tests (Figs. 43, 44). The following quantities were chosen as test parameters (Fig. 3): load level (upper), longitudinal reinforcement, beam dimensions(two sizes), cross-section (rectangular- and $T$ sections) and maximum aggregate size. The results may be summarized as follows:

\section{Deformation and load carrying behaviour}

- The crack pattern of all beams was developed after the first few cycles. Only small crack elongations were observed before a critical shear crack appeared.

- The failure of the beam resulted from the development of this critical shear crack, which in almost all beams crossed the existing bending cracks (Figs. $155-158$ ).

- In some beams, separation of the longitudinal steel from the upper web concrete due to dowel action took place without directly leading to failure (Fig. 159).

- At the beginning of cyclic loading a large increase in deflections and strains under both the upper and the lower load level was observed. These increases became smaller with cycling. The increase in the strain ranges on the other hand was smaller (Figs. $112,114,115)$.

- The following load carrying behaviour could be observed: classical bending behaviour according to simple bending theory (Figs. 113, 114), secondary bending (Figs. 125 - 127) and arch action (Figs. 129 - 131). Truss action as displayed in the beams with shear reinforcement was not clearly observed.

\section{Fatigue behaviour}

- With the exception of one beam with reduced reinforcement in which a longitudinal bar broke, all beams failed in fatigue by shear.

- In beams with very high load levels and correspondingly small number of cycles to failure, collapse took place within a few load cycles. The lower the load level, the longer was the collapse phase.

- In those beams in which arch action developed following the appearance of the critical shear crack, the number of cycles to collapse was in some cases significantly higher (Fig. 159). 
- A plot of the number of cycles to failure for all beams as a function of the nominal shear stress is given in Fig. 45. 


\section{Verdankungen}

Der vorliegende Bericht wurde im Rahmen des Forschungsprojektes "Ermüdung von Stahlbeton- und Spannbetontragwerken" am Institut für Baustatik und Konstruktion der Eidgenössischen Technischen Hochschule Zürich (ETH) ausgearbeitet. Für die grosszügige Unterstitzung dieses Projektes möchten die Verfasser folgenden Institutionen und Gesellschaften aufrichtig danken:

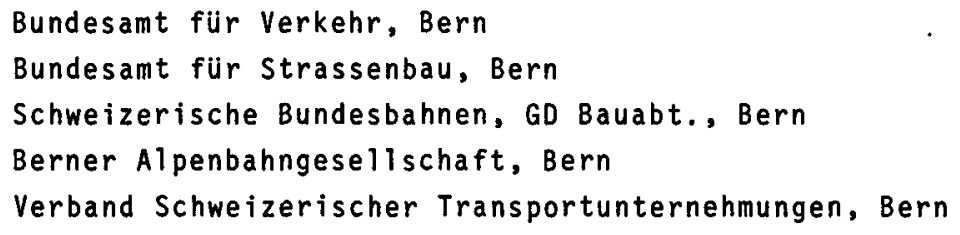

Das Forschungsprojekt wird von einer Beratenden Kommission begleitet, der folgende Herren angehören: U. Graber, dipl. Ing., Dr. J. Grob, Prof. Dr. M.A. Hirt, E. Rey, dipl. Ing., Prof. R. Steiner, M. Tschumi, dipl. Ing., K. Zimmermann, dipl. Ing. Ihnen allen sei für ihr Mitwirken auch an dieser Stelle gedankt.

Ein spezieller Dank gebihrt der

von Moos Stahl $A G$, Luzern,

die uns sämtliche Stähle für die schlaffe Bewehrung der Versuchsbalken fertig bearbeitet gespendet hat.

Im weiteren danken wir besonders der

Proceq SA, Zürich

für die Lieferung der LASTO-BLOCK-Lager.

Bei der Versuchsdurchführung haben die Herren K. Bucher, K. Alpiger, dipl. Ing., D. Breitenmoser, dipl. Ing., Dr. A. Fernandez Canteli mitgewirkt. Herr M. Baumann, dipl. Ing., unterstützte uns bei der Lösung der messtechnischen Probleme. Die Herren G. Göseli und G. Benvenuto fertigten die Zeichnungen an und Frl. S. Burki schrieb die Druckbogen. Für ihre Mitarbejt sei den Genannten wie auch allen anderen, die zum Entstehen dieses Versuchsberichtes beigetragen haben, bestens gedankt. Herrn R. Caflisch, Adm. Institutsleiter, möchten wir speziell für seine Ratschläge und seine organisatorische und administrative Betreuung des Forschungsprojektes bestens danken. 
Bezeichnungen

\section{Geometrische Grössen}

$\begin{array}{ll}x & \text { Koordinatenachse parallel zur Balkenlängsrichtung } \\ z & \text { Koordinatenachse senkrecht zur Balkenlängsrichtung } \\ u & \text { Verschiebung in x-Richtung } \\ \text { w } & \text { Verschiebung in z-Richtung } \\ F_{L} & \text { Querschnittsfläche der Längsbewehrung } \\ F_{B} & \text { Querschnittsfläche der Bügelbewehrung } \\ s_{B} & \text { Bügelabstand } \\ t & \text { Stegstärke } \\ b_{0} & \text { Oberflanschbreite } \\ b_{1} & \text { Unterflanschbreite } \\ H & \text { Trägerhöhe } \\ h & \text { statische Trägerhöhe } \\ D & \text { Durchmesser }\end{array}$

\section{Kraftgrössen}

$\begin{array}{ll}P_{O} & \text { Oberlast } \\ P_{U} & \text { Unterlast } \\ \Delta P & \text { Lastdifferenz }\left(P_{O}-P_{U}\right) \\ P_{A} & \text { Last zu Beginn einer Laststufe } \\ P_{E} & \text { Last am Ende einer Laststufe }\end{array}$

\section{Materialwerte, Spannungen}

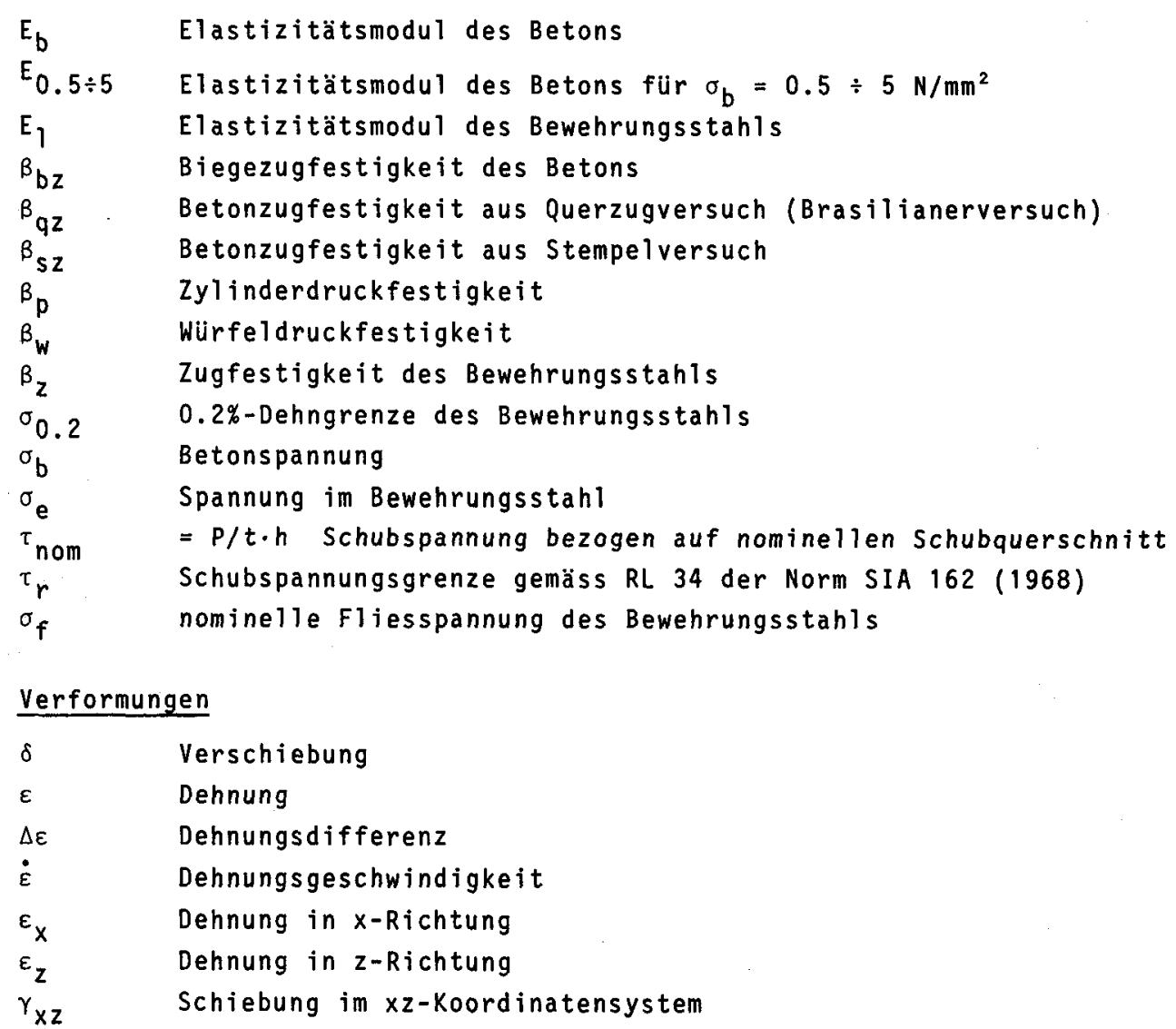




\section{Allgemeine Bezeichnungen}

PC

Portland Zement

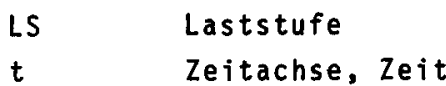

$t_{A} \quad$ Zeit am Anfang einer Laststufe

$t_{E} \quad$ Zeit am Ende einer Laststufe

$t_{I}$ Zeit beim Erreichen eines Lastniveaus

$N$ Lastwechselzahl

\section{Indizes}

$\begin{array}{ll}\text { B } & \text { Bügel } \\ \text { A } & \text { Anfang } \\ \text { E } & \text { Ende } \\ \text { I } & \text { initial } \\ \text { b } & \text { Beton } \\ \text { e } & \text { Bewehrungstahl } \\ \text { f } & \text { Fliessen } \\ \text { o } & \text { oben } \\ \text { u } & \text { unten }\end{array}$




\section{Li teraturverzeichnis}

[1] Comite Euro-International du Béton: "Code modèle pour les structures en béton; Système international de règlementation technique unifiée des structures, Vol. II, Bulletin d'Information, No. 117, Paris, décembre 1976.

[2] Richtlinie 34 zur Norm SIA 162(1968): "Bruchwiderstand und Bemessung von Stahlbeton- und Spannbetontragwerken", Schweizerischer Ingenieur- und ArchitektenVerein, Zürich, 1976.

[3] Chang T.S., Kester C.E.: "Static and Fatigue Strength in Shear of Beams with Tensile Reinforcement", Journal of the American Concrete Institute (ACI), June 1958.

[4] Price K.M., Edwards A.D.: "Fatigue Strength in Shear of Prestressed Concrete I-Beams, Journal of the American Concrete Institute (ACI), April 1971.

[5] Hawkins N.M.: "Fatigue Characteristics in Bond and Shear of Reinforced Concrete Beams, ACI-Publ. SP-41, 1973.

[6] Kupfer H., Ruhnau J.: "Schubversuche an Stahlbetonbalken unter wiederholter Belastung", Technische Universität München, Lehrstuhl für Massivbau, Bericht Nr. 826, München, 1975.

[7]

Chen W.F.: "Limit Analysis and Soil Plasticity", Developments in Geotechnical Engineering 7, Elsevier Scientific Publishing Company, New York, 1975, Chapter 11, pp. 501-541. 

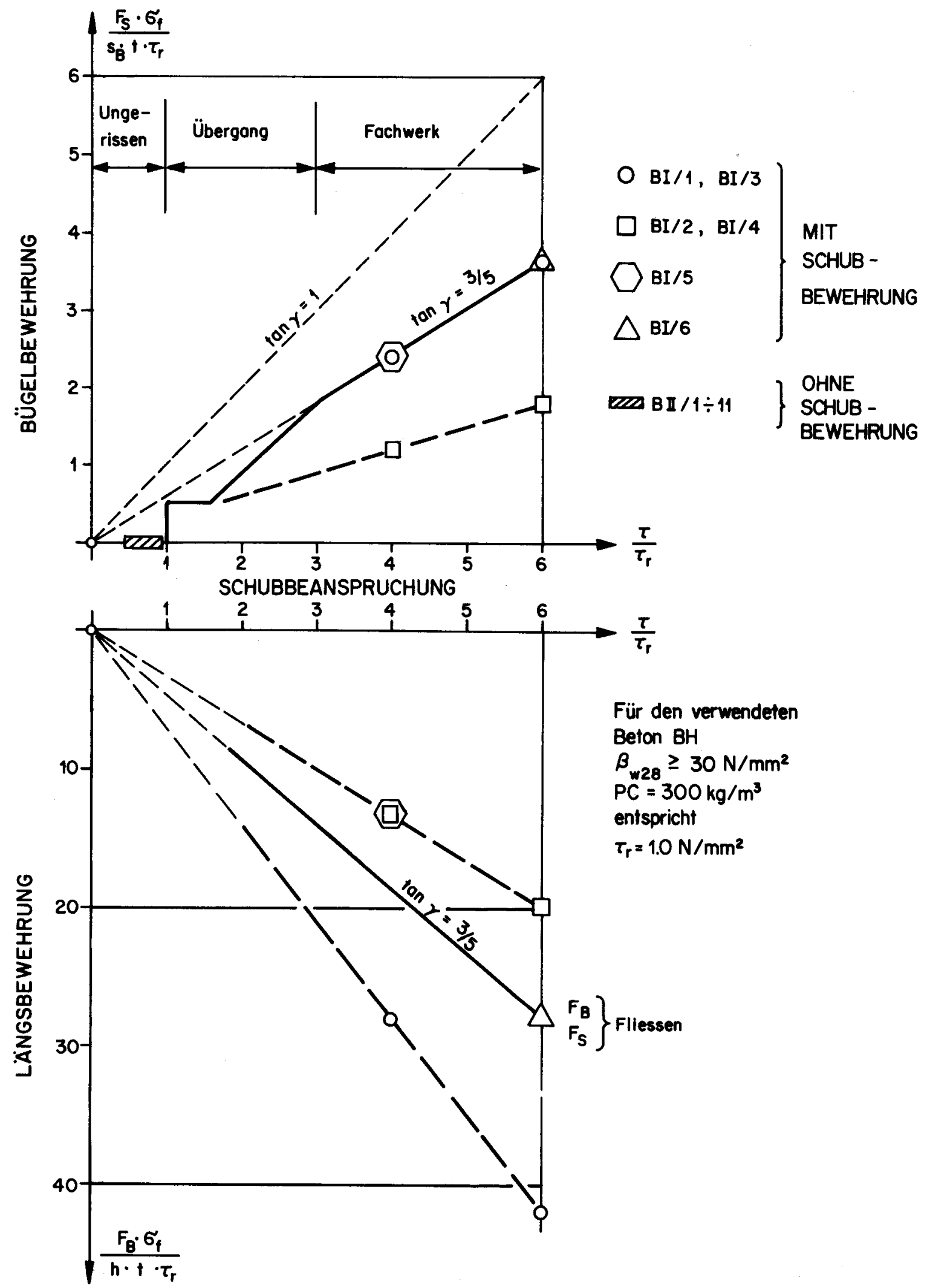

Bild 1: Versuchsplanung $\mathrm{BI} / 1 \div 6$ und $\mathrm{BI} / 1 \div 11$ 


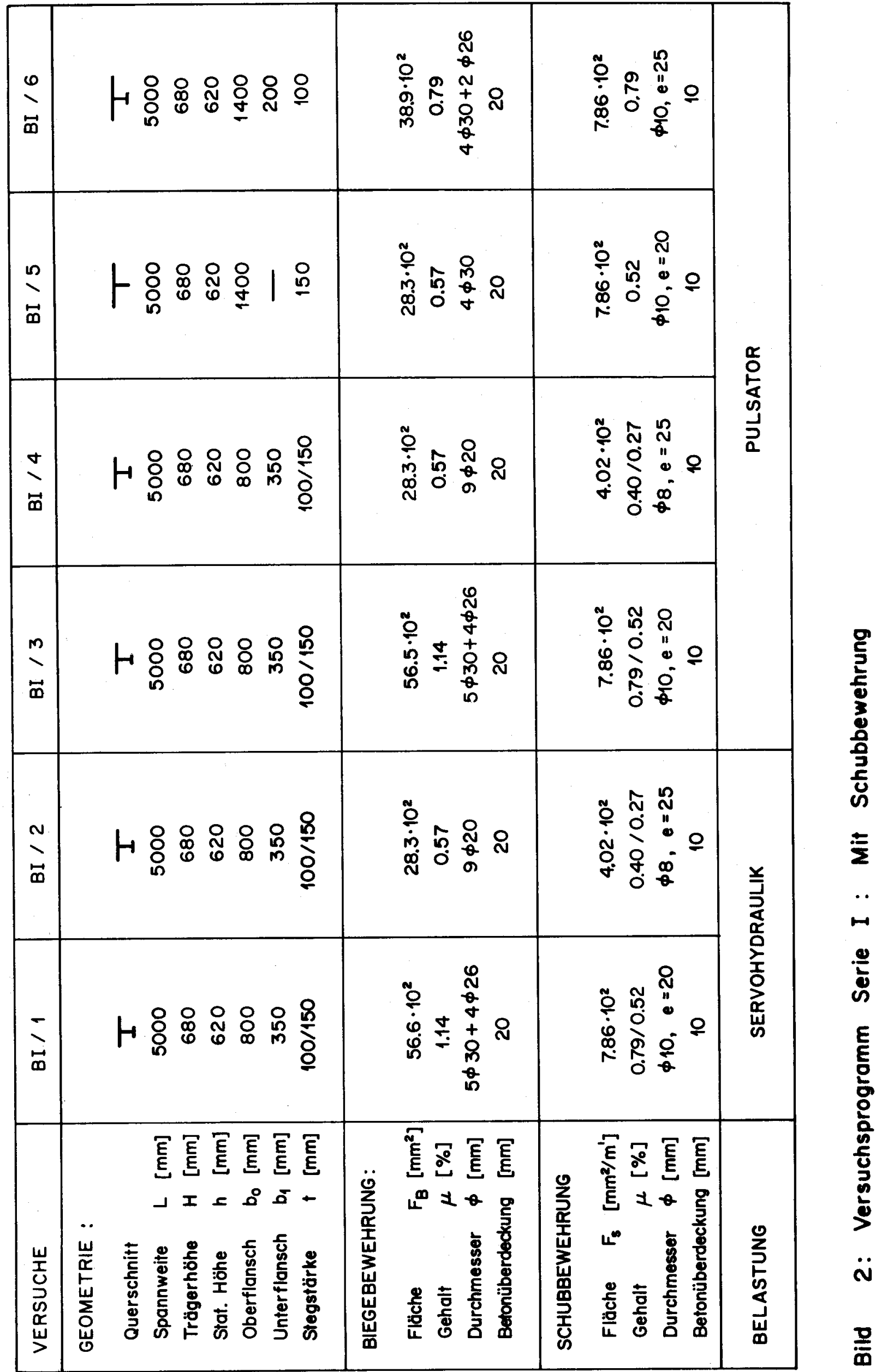




\begin{tabular}{|c|c|c|c|c|c|}
\hline $\begin{array}{l}= \\
\vdots \\
\vdots \\
0 \\
0\end{array}$ & & \$ & & 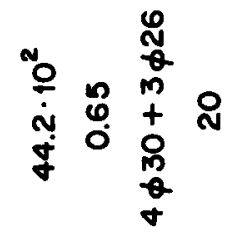 & \multirow{5}{*}{ 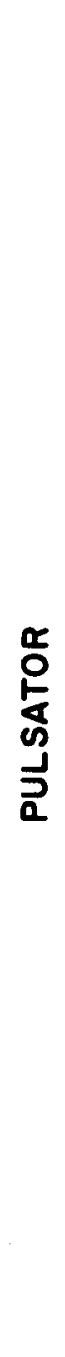 } \\
\hline 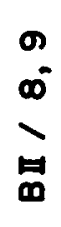 & & 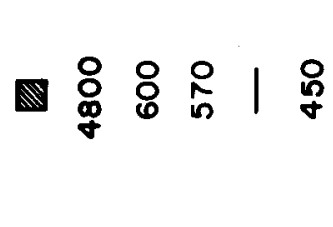 & & 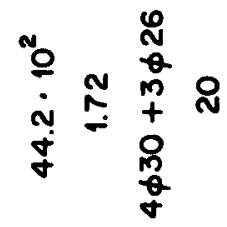 & \\
\hline $\begin{array}{l}n \\
\dot{8} \\
\dot{n} \\
\dot{0}\end{array}$ & & 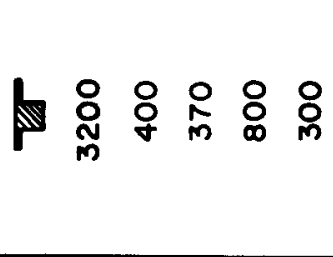 & & 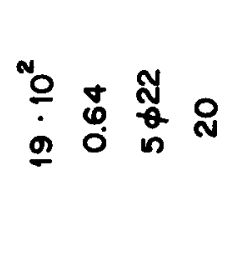 & \\
\hline $\begin{array}{l}n \\
0 \\
\text { i } \\
0 \\
0\end{array}$ & & 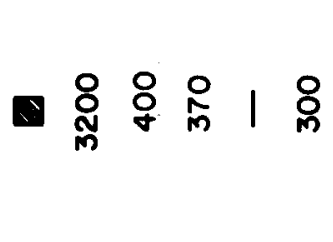 & & 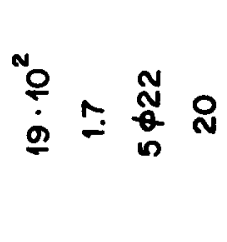 & \\
\hline 三 & & 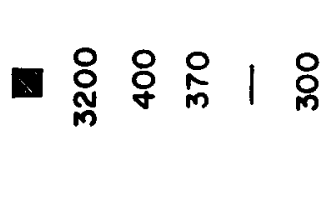 & & 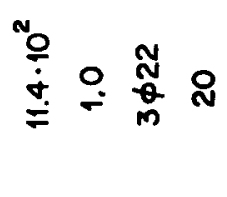 & \\
\hline 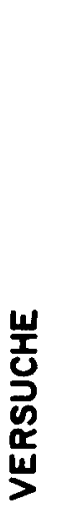 & 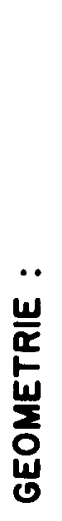 & 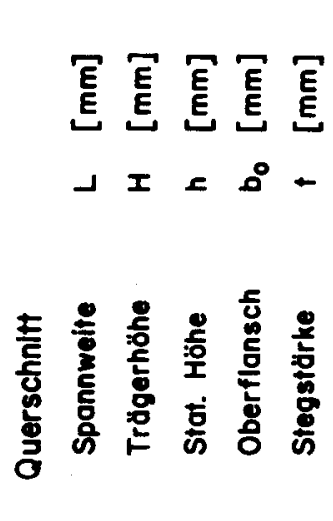 & 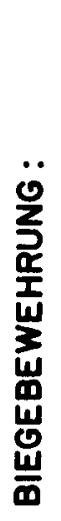 & 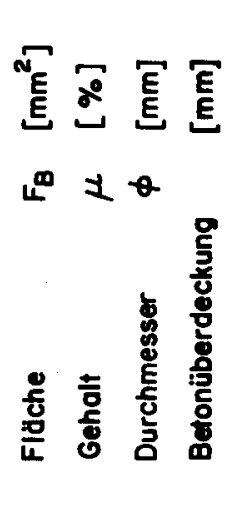 & 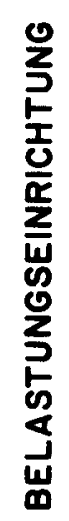 \\
\hline
\end{tabular}

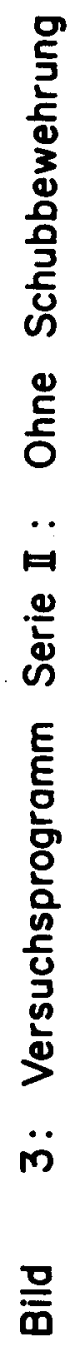



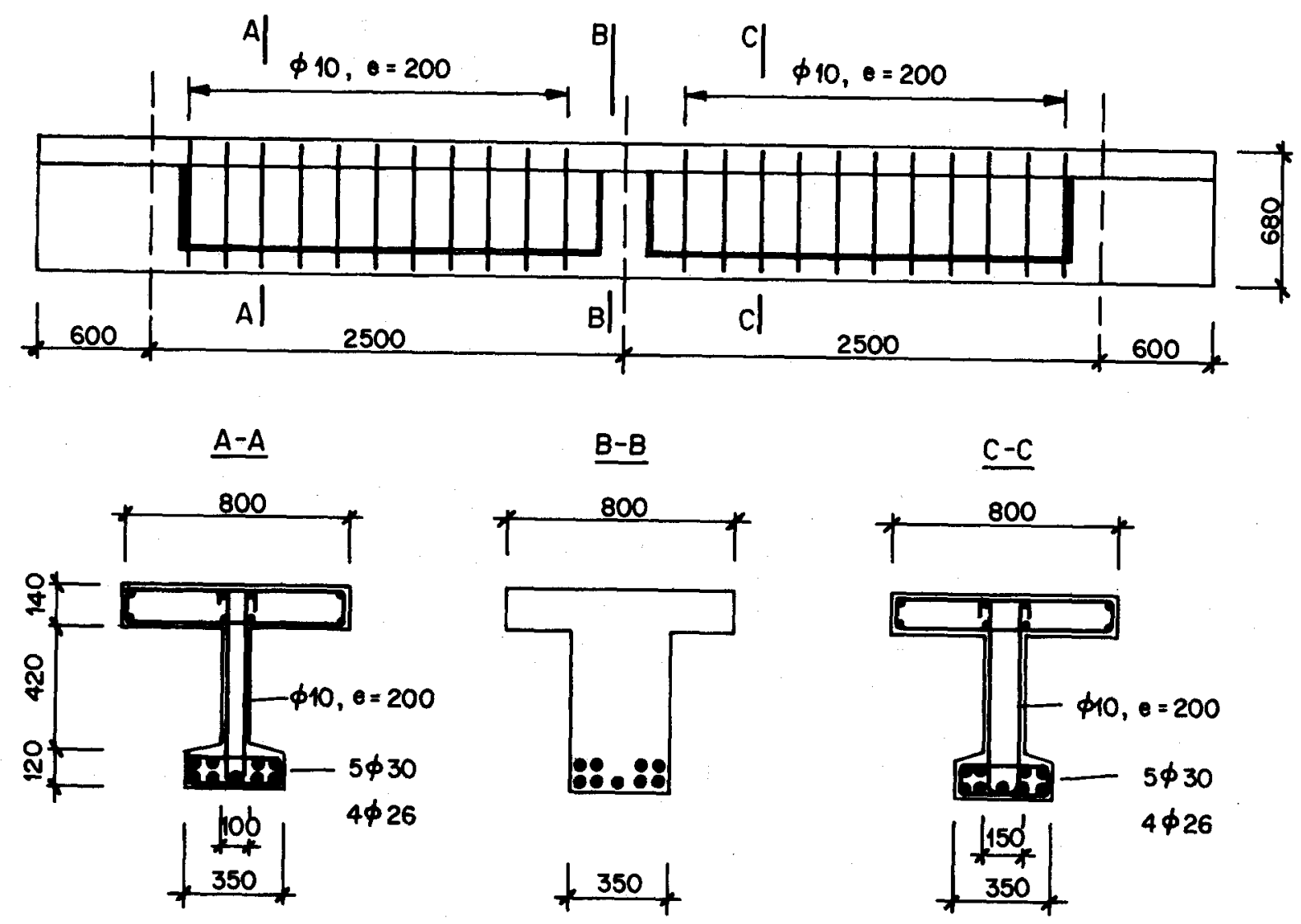

Bild 4: Schalung und Bewehrung BI/1, BI/3

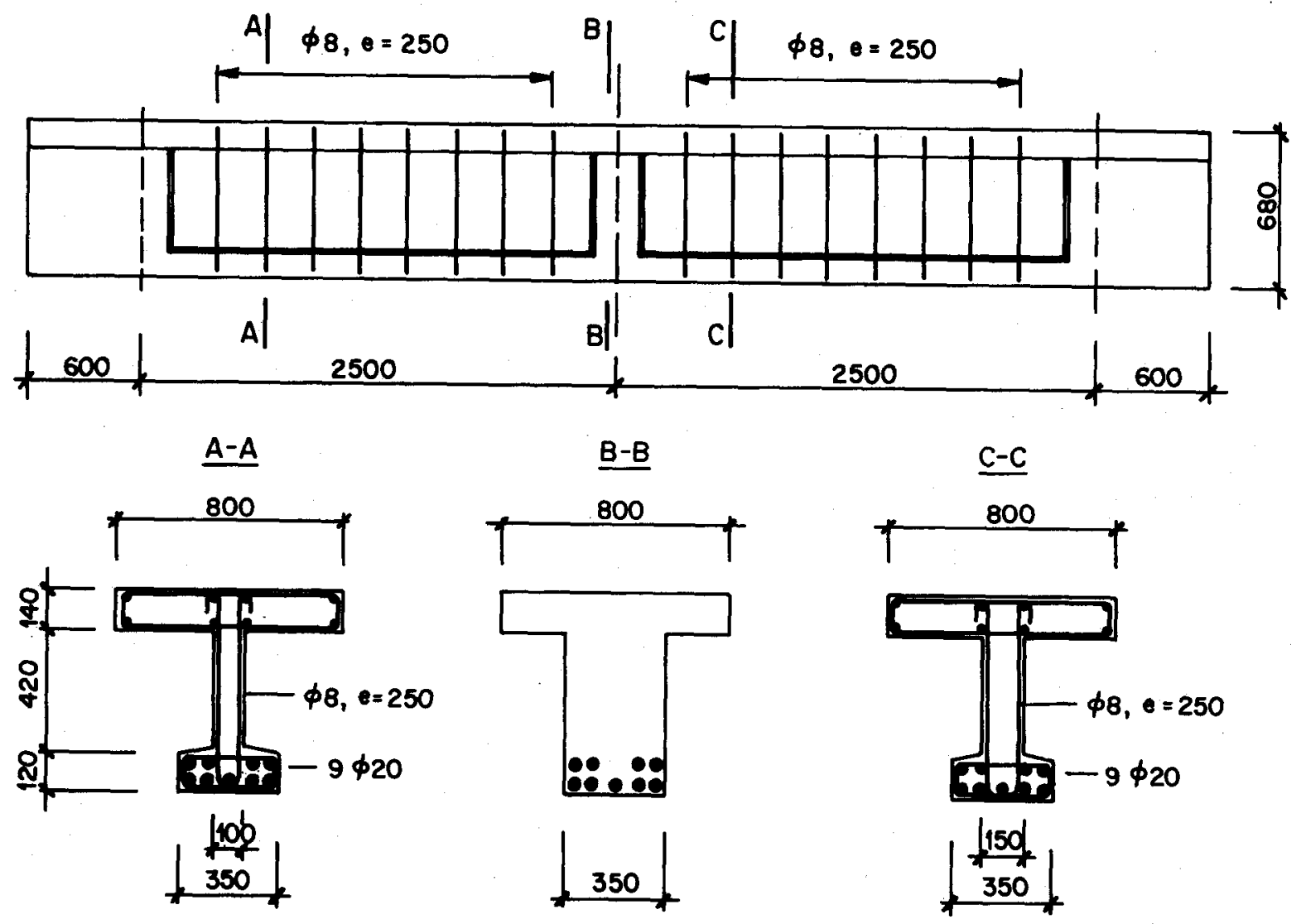

Bild 5: Schalung und Bewehrung BI/2, BI/4 

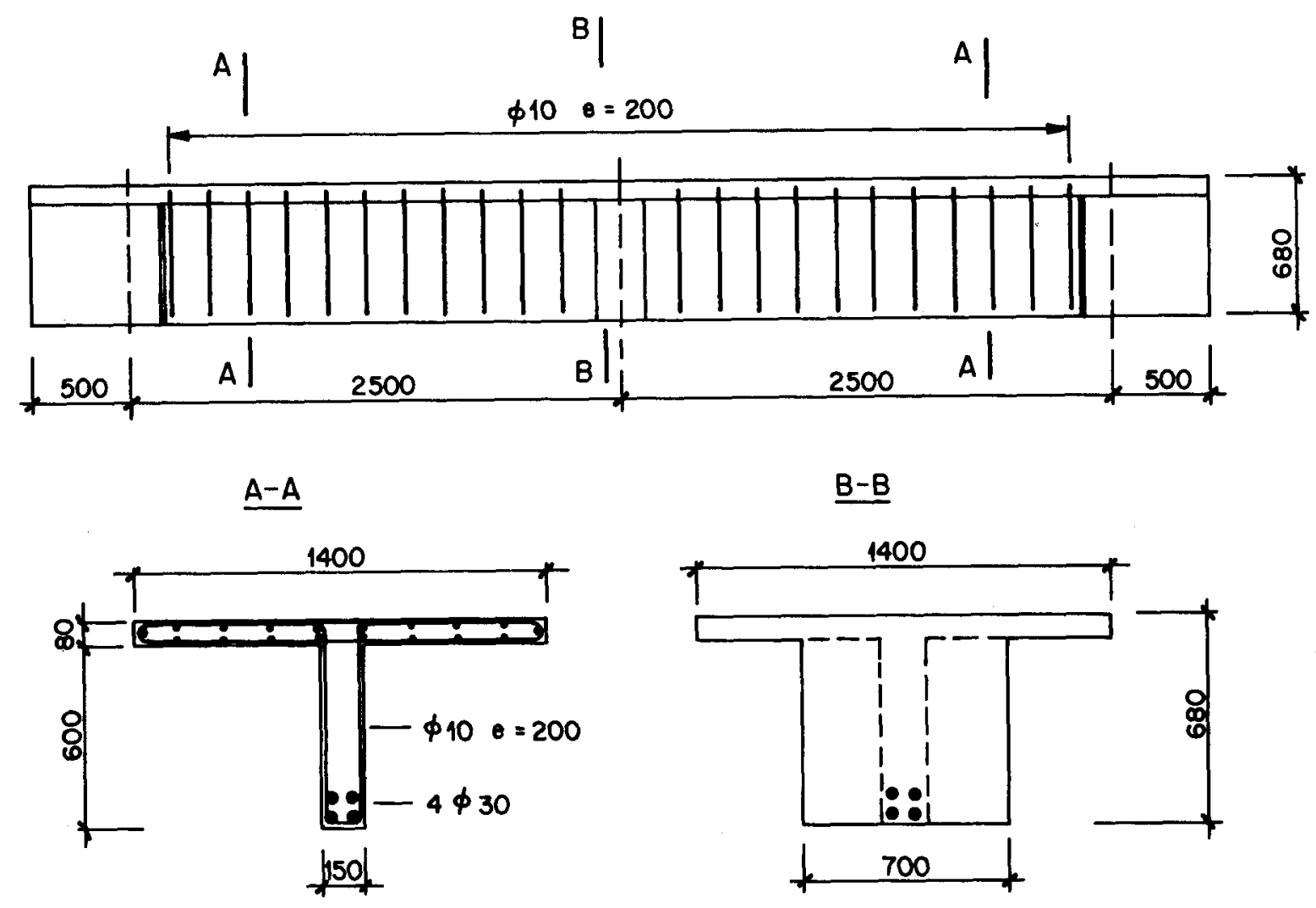

Bild 6: Schalung und Bewehrung BI / 5
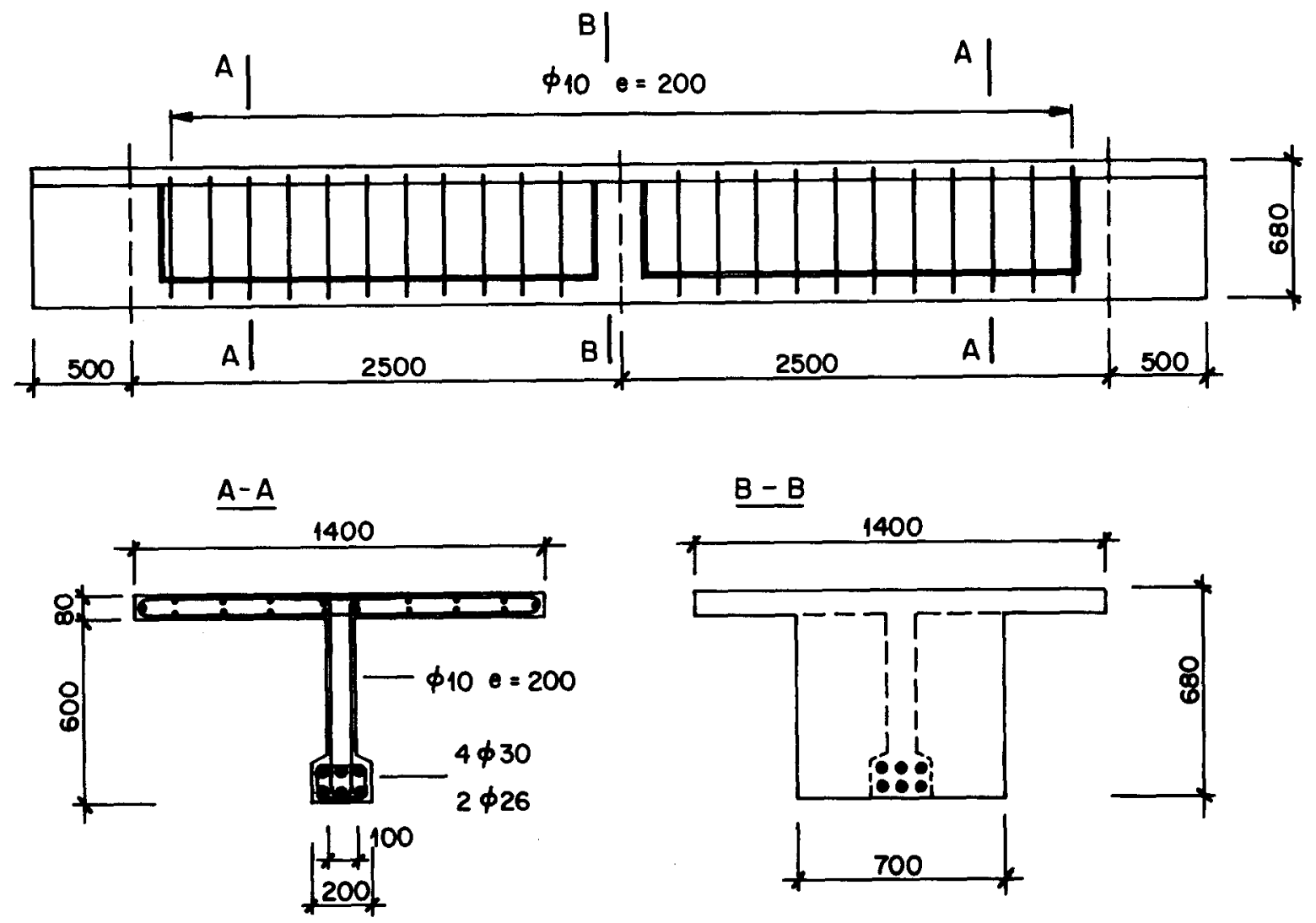

Bild 7: Schalung und Bewehrung BI $/ 6$ 


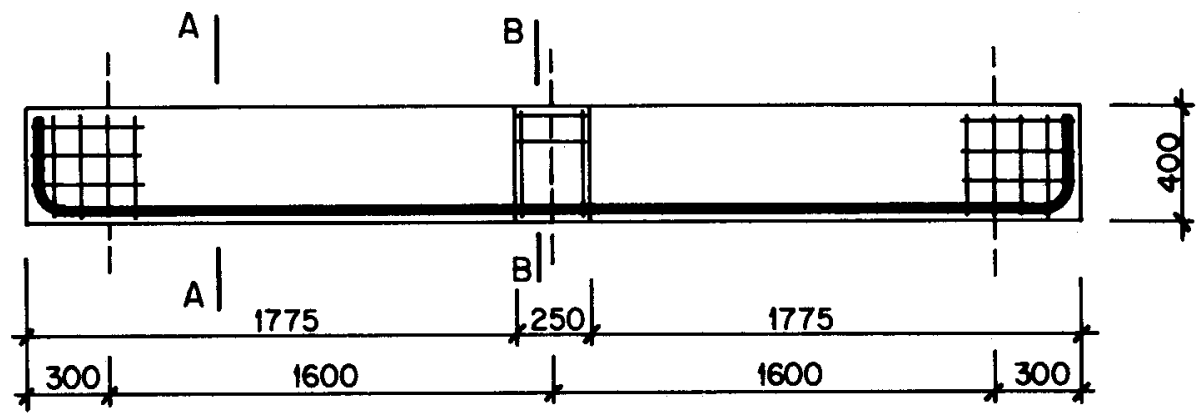

$\underline{A-A}$

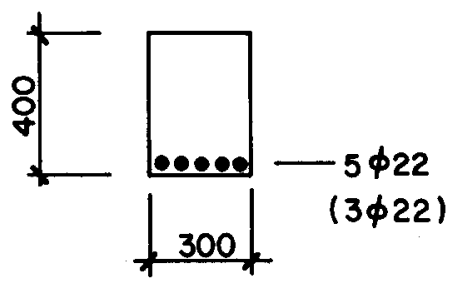

$\underline{B-B}$
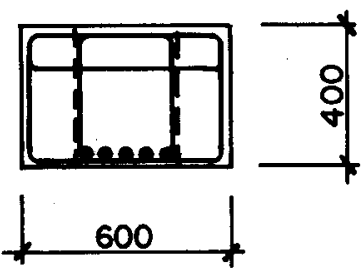

Bild 8: Schalung und Bewehrung BI/2, BII/6, BII/7, (BI/1)
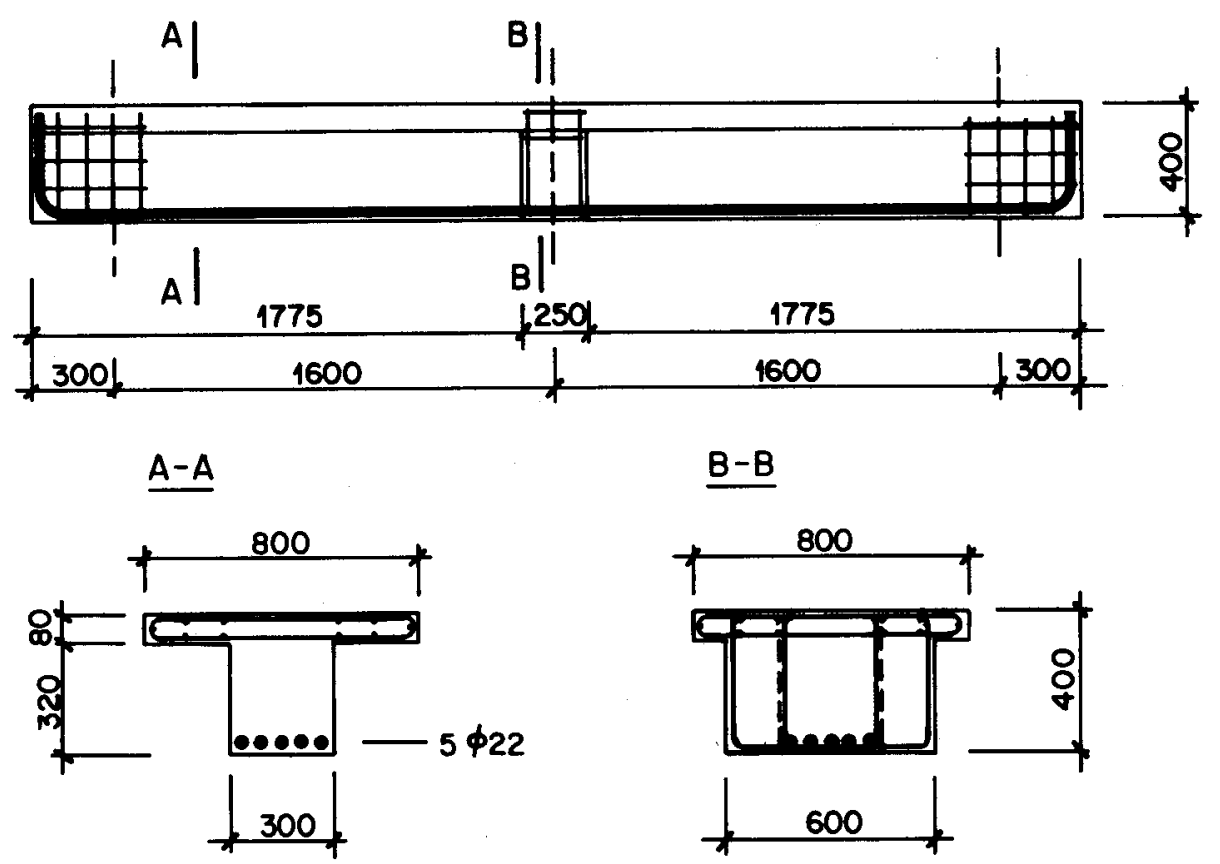

$\underline{B-B}$

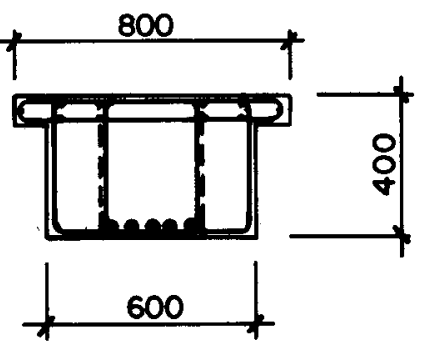

Bild 9: Schalung und Bewehrung BII/3, BII/4, BII/5 


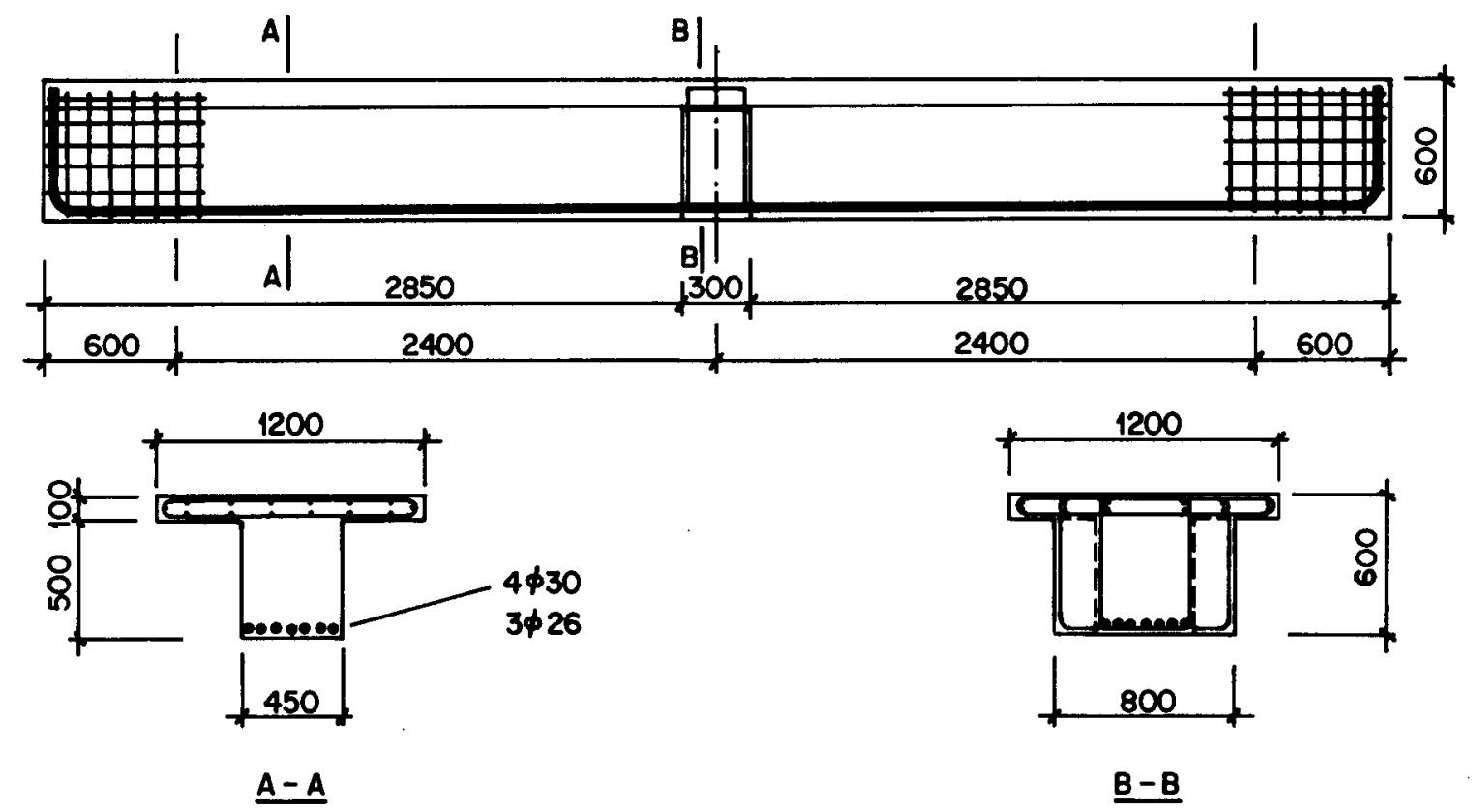

Bild 10: Schalung und Bewehrung $B I / 8$ bis $B I / 11$ 


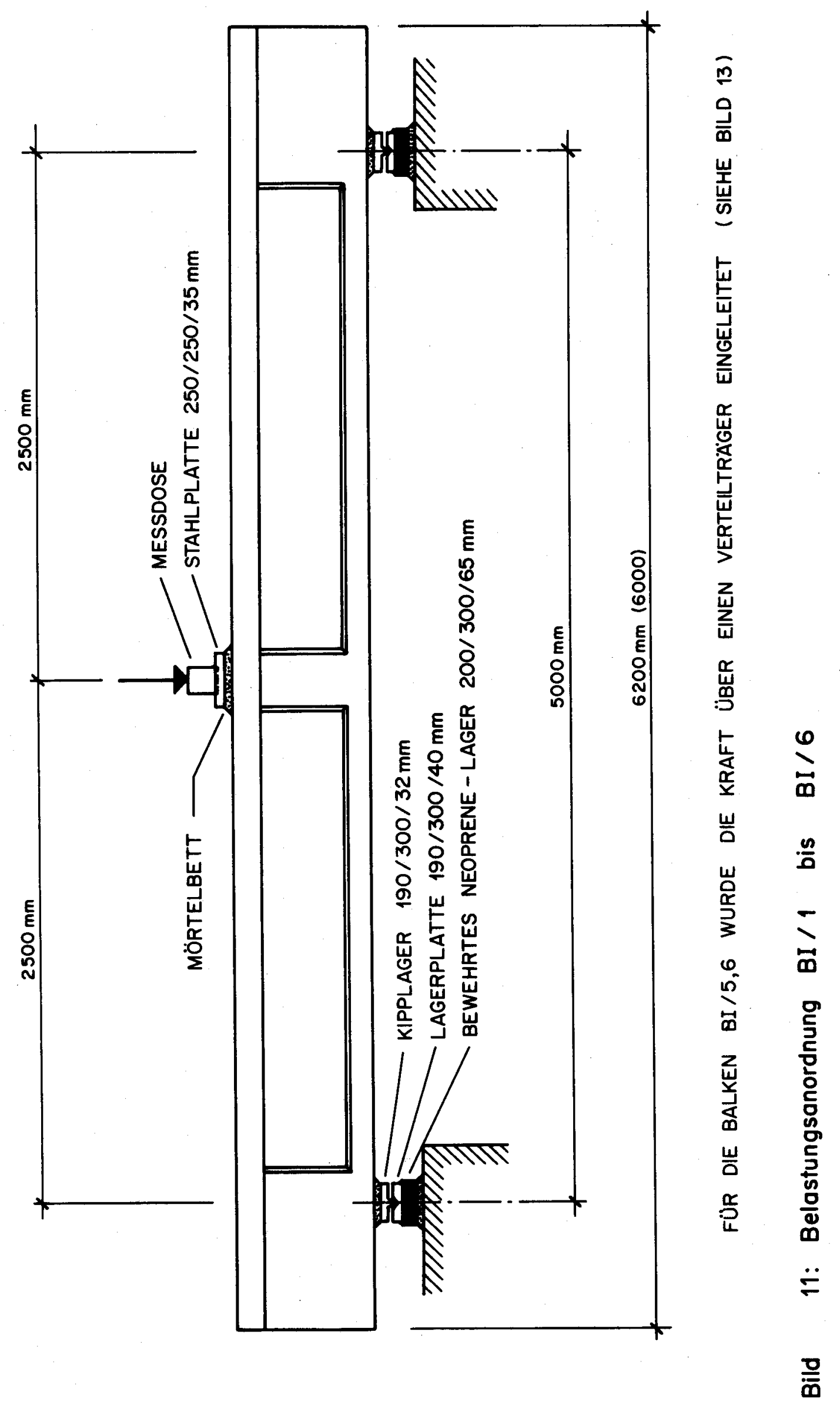




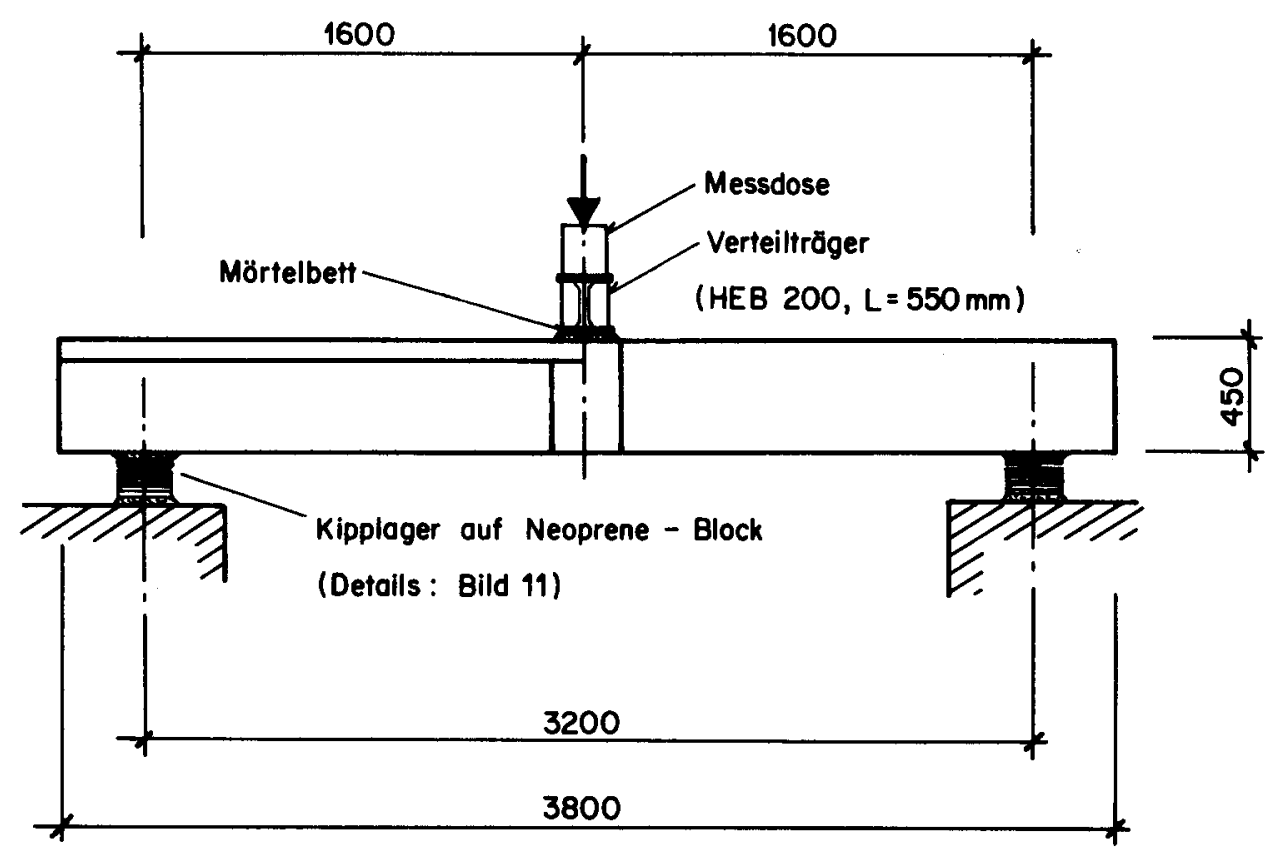

Bild 12: Belastungsanordnung $\mathrm{BI} / 4$ bis $\mathrm{BI} / 7$

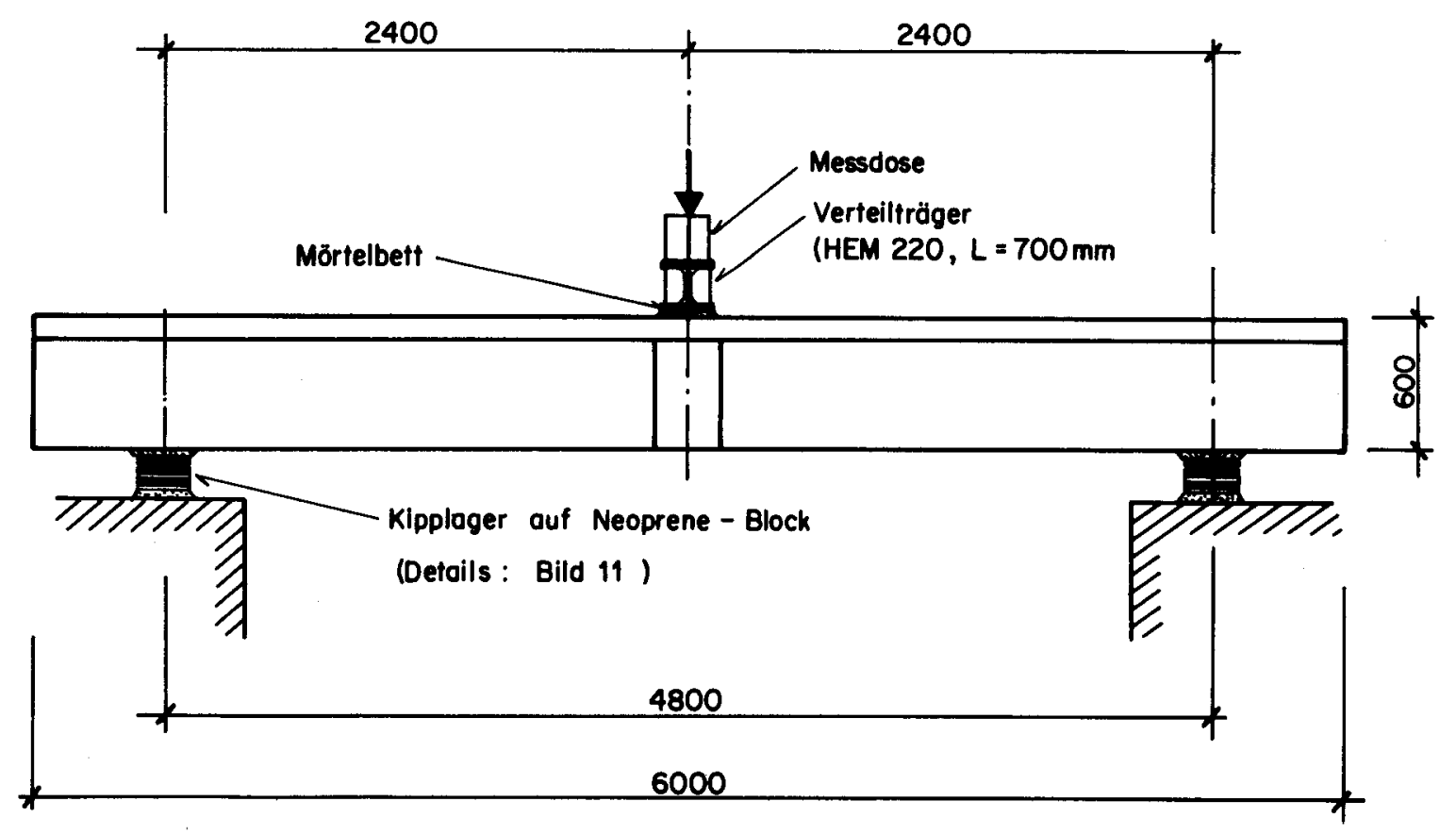

Bild 13: Belastungsanordnung $\mathrm{BI} / 8$ bis $\mathrm{BI} / 11$ 


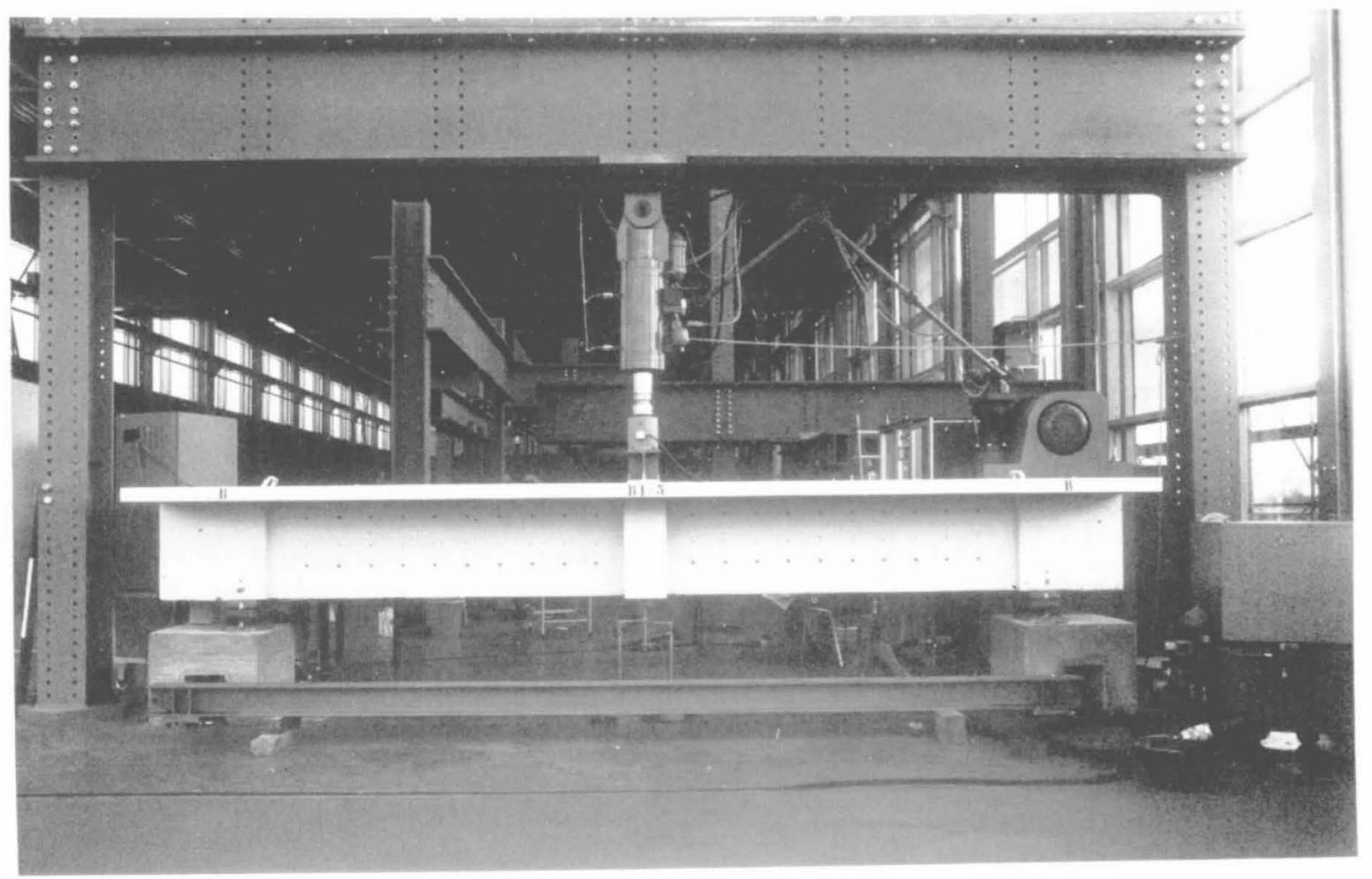

Bild 14: Versuchsanlage

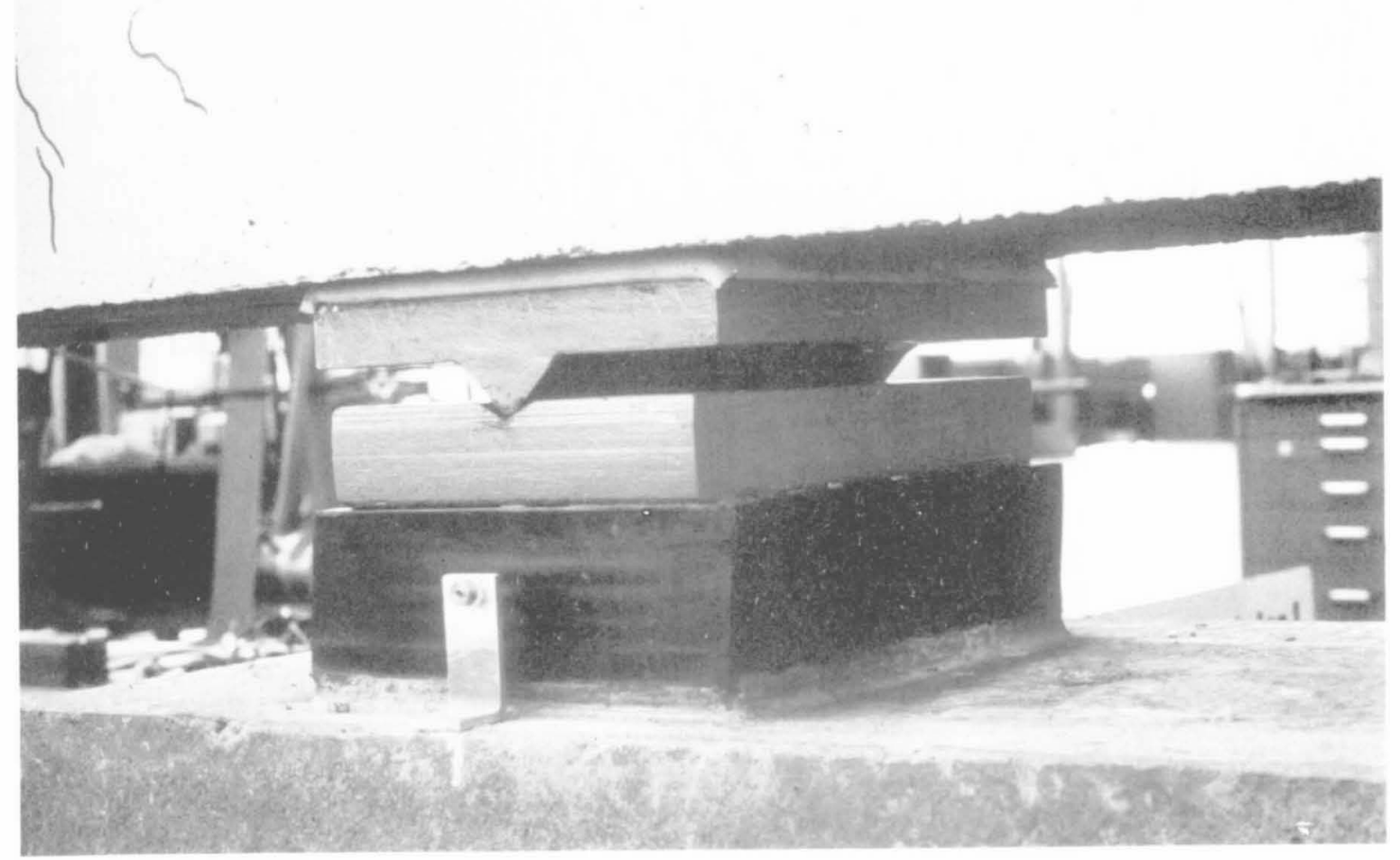

Bild 15: Lagerdetail 


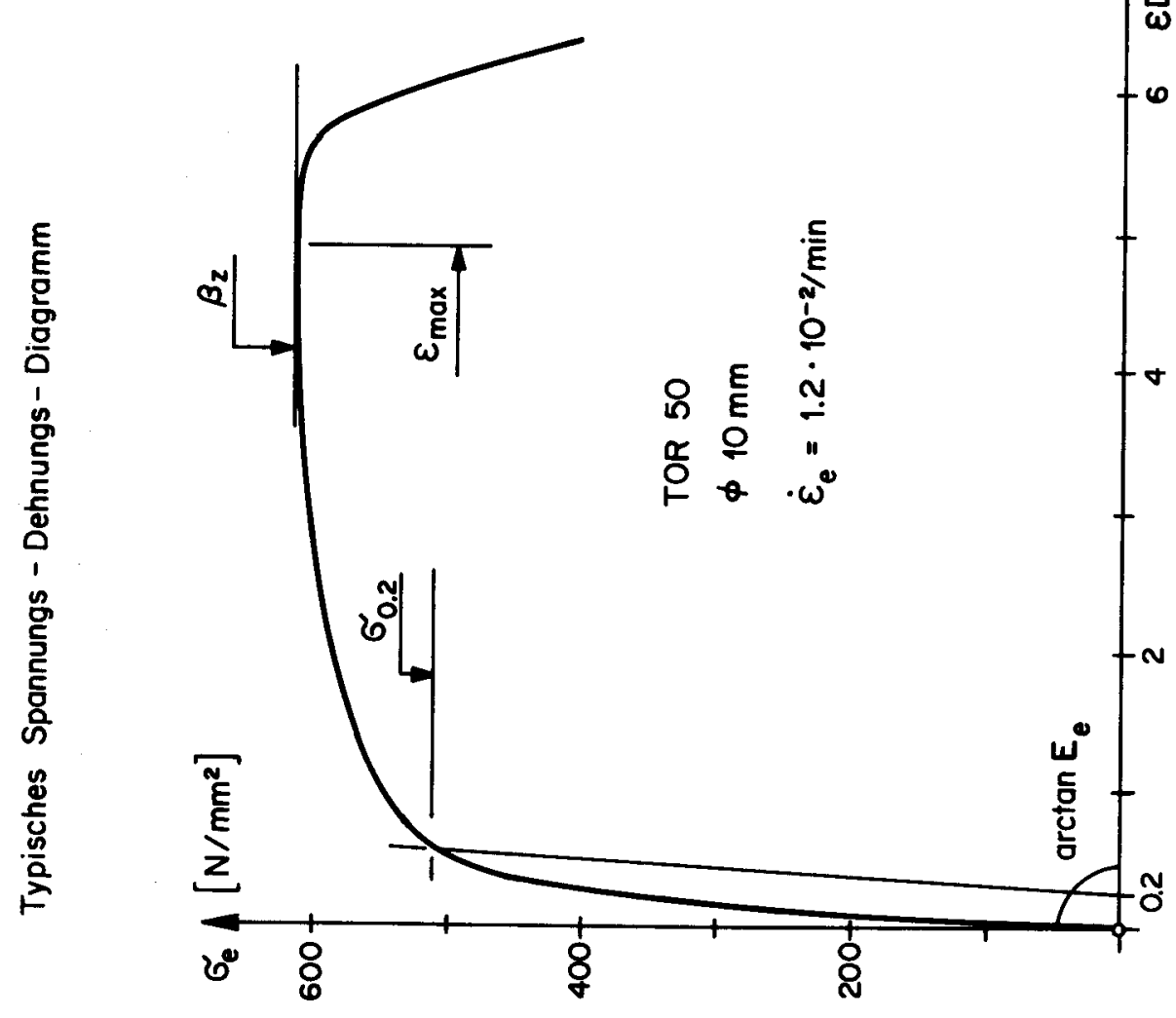

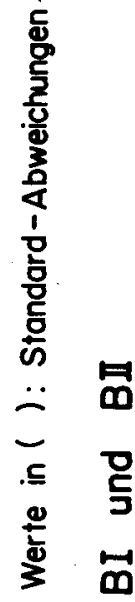

\begin{tabular}{|c|c|c|c|c|c|c|c|c|c|c|c|c|}
\hline ن & 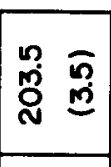 & 依 & $\overline{\mathcal{N}} \overline{\bar{N}}$ & ָ̃ & 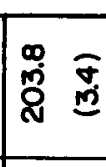 & 앙 & 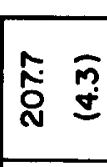 & 雚 & $\begin{array}{ll}\infty & \overline{0} \\
\stackrel{\leftrightarrow}{8} & \stackrel{0}{0}\end{array}$ & ְ̊ & 孞 & 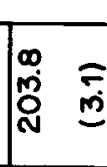 \\
\hline 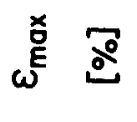 & g. $\overline{\mathscr{g}}$ & $\begin{array}{ll}0 & \bar{Q} \\
i & \stackrel{0}{0}\end{array}$ & 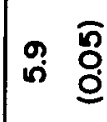 & チे & $\stackrel{\infty}{\circ}$ & : $\overline{\mathscr{Z}}$ & 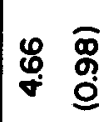 & 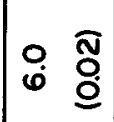 & $\begin{array}{l}\infty \\
\stackrel{\infty}{*}\end{array}$ & 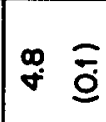 & กิ & $f=\bar{O}$ \\
\hline$Q^{N} \frac{\bar{E}}{\Sigma}$ & ָ̃ & 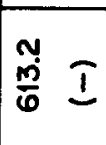 & $\begin{array}{ll}\overline{8} & \bar{\sigma} \\
0 & \dot{ \pm}\end{array}$ & 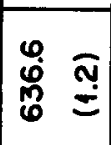 & 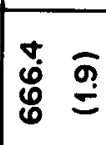 & 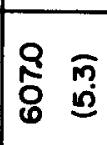 & 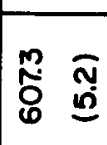 & 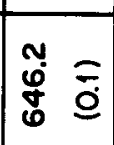 & 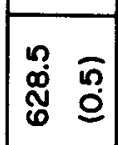 & ${ }_{0}^{2}$ 工 & 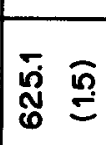 & 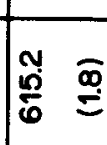 \\
\hline రั & 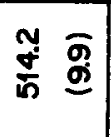 & : & 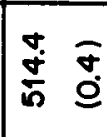 & 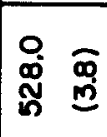 & 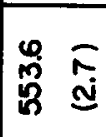 & 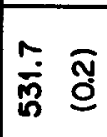 & 움 $\overline{\overline{\hat{m}}}$ & 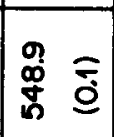 & $\overline{\overline{0}} \overline{\bar{m}}$ & 总 & 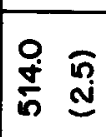 & 品 \\
\hline$\omega^{*} \stackrel{\tilde{\tilde{E}}}{\underline{E}}$ & $\begin{array}{ll}\overline{\hat{N}} & \stackrel{0}{0} \\
\end{array}$ & 吕 I & ఫ̊ & $\begin{array}{c}m \\
\infty \\
\infty\end{array}$ & 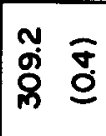 & $\stackrel{n}{i} \overline{\bar{\delta}}$ & 융 & జ్లి & $\begin{array}{ll}8 \\
\frac{\infty}{n} & -1\end{array}$ & $\stackrel{n}{\stackrel{n}{N}}$ I & $\begin{array}{l}\stackrel{0}{\circ}= \\
\text { 足 }\end{array}$ & $\prod_{0}^{10}$ \\
\hline 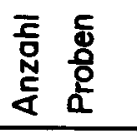 & $m$ & $N$ & $N$ & $m$ & $m$ & $m$ & & $m$ & $m$ & $m$ & $m$ & $m$ \\
\hline 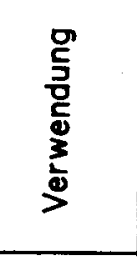 & 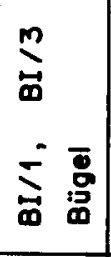 & 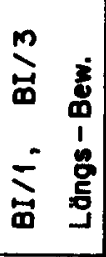 & 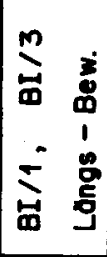 & 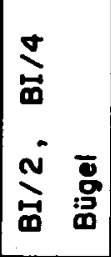 & 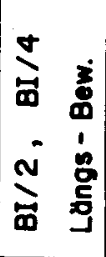 & 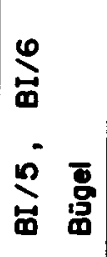 & 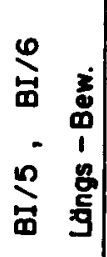 & 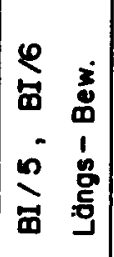 & 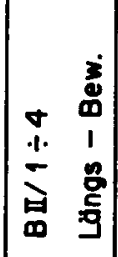 & 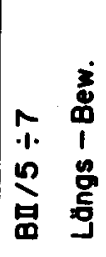 & 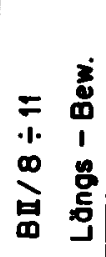 & 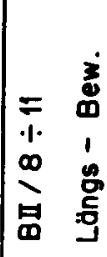 \\
\hline $\begin{array}{l}\stackrel{+}{E} \\
\stackrel{\Phi}{\mathbf{E}} \\
\end{array}$ & 오 & $\mathscr{N}$ & 을 & $\infty$ & 尺) & 으 & পి & $\stackrel{\mathscr{N}}{ }$ & $\mathbb{N}$ & 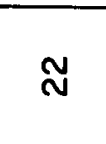 & $\stackrel{\mathscr{N}}{ }$ & 요 \\
\hline
\end{tabular}




\begin{tabular}{|c|c|c|c|c|c|c|c|c|}
\hline VERSUCH & $\begin{array}{c}\text { ABMESS. } \\
\text { [mm] }\end{array}$ & $\begin{array}{l}\text { BELAST. } \\
\text { GESCHW. } \\
\cdot 10^{-3} / \mathrm{min}\end{array}$ & BI/I & $\mathrm{BI} / 2$ & $\mathrm{BI} / 3$ & BI $/ 4$ & $\mathrm{BI} / 5$ & $\mathrm{BI} / 6$ \\
\hline ALTER [TAGE] & & & 40 & 48 & 130 & 80 & 31 & 41 \\
\hline ANZAHL PROBEN & & & 4 & 6 & 6 & 6 & 4 & 4 \\
\hline$\beta_{\mathrm{sz}} \quad\left[\mathrm{N} / \mathrm{mm}^{2}\right]$ & $\begin{array}{l}\phi=150 \\
h=150\end{array}$ & 0.1 & $\begin{array}{c}3.88 \\
(0.36)\end{array}$ & $\begin{array}{c}3.20 \\
(0.55)\end{array}$ & $\begin{array}{c}2.87 \\
(0.44)\end{array}$ & $\begin{array}{c}3.20 \\
(0.23)\end{array}$ & $\begin{array}{c}2.88 \\
(0.22)\end{array}$ & $\begin{array}{c}2.88 \\
(0.40)\end{array}$ \\
\hline ANZAHL PROBEN & & & 3 & 2 & 3 & 3 & 2 & 2 \\
\hline$\beta_{\mathrm{qz}} \quad\left[\mathrm{N} / \mathrm{mm}^{2}\right]$ & $\begin{array}{l}\phi=150 \\
h=300\end{array}$ & 0.1 & $\begin{array}{c}2.43 \\
(0.51)\end{array}$ & $\begin{array}{c}2.30 \\
(0.43)\end{array}$ & $\begin{array}{c}3.47 \\
(0.48)\end{array}$ & $\begin{array}{c}2.72 \\
(0.10)\end{array}$ & $\begin{array}{l}2.89 \\
(0.06)\end{array}$ & $\begin{array}{c}2.56 \\
(-)\end{array}$ \\
\hline ANZAHL PROBEN & & & 4 & 4 & 4 & 4 & 4 & 4 \\
\hline$\beta_{\mathrm{bz}} \quad\left[\mathrm{N} / \mathrm{mm}^{2}\right]$ & $120 / 120 / 300$ & 1.0 & $\begin{array}{c}4.92 \\
(0.36)\end{array}$ & $\begin{array}{l}5.39 \\
(0.63)\end{array}$ & $\begin{array}{c}6.61 \\
(0.38)\end{array}$ & $\begin{array}{c}5.20 \\
(0.33)\end{array}$ & $\begin{array}{l}4.89 \\
(0.34)\end{array}$ & $\begin{array}{l}4.72 \\
(0.47)\end{array}$ \\
\hline ANZAHL PROBEN & & & 3 & 3 & 4 & 4 & 2 & 2 \\
\hline$\beta_{\mathrm{p}} \quad\left[\mathrm{N} / \mathrm{mm}^{2}\right]$ & $\begin{array}{l}\phi=150 \\
h=300\end{array}$ & 0.1 & $\begin{array}{l}37.9 \\
(2.7)\end{array}$ & $\begin{array}{l}34.8 \\
(4.6)\end{array}$ & $\begin{array}{l}36.0 \\
(2.9)\end{array}$ & $\begin{array}{l}34.4 \\
(3.1)\end{array}$ & $\begin{array}{l}28.0 \\
(3.0)\end{array}$ & $\begin{array}{l}28.0 \\
(3.9)\end{array}$ \\
\hline$\varepsilon_{u} \quad\left[10^{-3}\right]$ & & & 1.9 & 2.0 & 2.3 & 2.1 & 2.1 & 20 \\
\hline ANZAHL PROBEN & & & 4 & 3 & 4 & 2 & 6 & 6 \\
\hline$\beta_{w} \quad\left[\mathrm{~N} / \mathrm{mm}^{2}\right]$ & $150 / 150 / 150$ & 0.5 & $\begin{array}{l}38.2 \\
(0.7)\end{array}$ & $\begin{array}{l}42.0 \\
\text { (1.1) }\end{array}$ & $\begin{array}{l}41.9 \\
(0.5)\end{array}$ & $\begin{array}{l}36.7 \\
(1.1)\end{array}$ & $\begin{array}{l}35.4 \\
(2.2)\end{array}$ & $\begin{array}{l}37.6 \\
(3.1)\end{array}$ \\
\hline
\end{tabular}

DRUCK - UND ZUGFESTIGKEITEN

WERTE IN ( ): STANDARD - ABWEICHUNGEN

\begin{tabular}{|c|c|c|c|c|c|c|c|c|}
\hline ANZAHL PROBEN & & & 3 & 3 & 4 & 4 & 2 & 2 \\
\hline$E_{b 0.5 \div 5}\left[\mathrm{~N} / \mathrm{mm}^{2}\right]$ & $\begin{array}{l}\phi=150 \\
h=300\end{array}$ & 0.3 & $\begin{array}{r}43.200 \\
(1930)\end{array}$ & $\begin{array}{l}40^{\circ} 100 \\
(3270)\end{array}$ & $\begin{array}{l}36.200 \\
(1220)\end{array}$ & $\begin{array}{l}36500 \\
(2980)\end{array}$ & $\begin{array}{l}38^{\prime} 500 \\
(1000)\end{array}$ & $\begin{array}{r}41300 \\
(980)\end{array}$ \\
\hline$E_{b 5 \div 0.5} \quad\left[\mathrm{~N} / \mathrm{mm}^{2}\right]$ & $\begin{array}{l}\phi=150 \\
h=300\end{array}$ & 0.3 & $\begin{array}{l}43^{\prime} 100 \\
(2950)\end{array}$ & $\begin{array}{l}39.900 \\
(3050)\end{array}$ & $\begin{array}{l}36.200 \\
(1070)\end{array}$ & $\begin{array}{l}37.700 \\
(2490)\end{array}$ & $\begin{array}{r}39 " 100 \\
(800)\end{array}$ & $\begin{array}{l}41700 \\
(1360)\end{array}$ \\
\hline$E_{b 0.5 \div 10}\left[\mathrm{~N} / \mathrm{mm}^{2}\right]$ & $\begin{array}{l}\phi=150 \\
h=300\end{array}$ & 0.3 & $\begin{array}{l}40.900 \\
(2480)\end{array}$ & $\begin{array}{l}38.700 \\
(2810)\end{array}$ & $\begin{array}{l}34.400 \\
(4890)\end{array}$ & $\begin{array}{l}35 \cdot 300 \\
(2180)\end{array}$ & $\begin{array}{l}36000 \\
(240)\end{array}$ & $\begin{array}{l}39^{\prime} 200 \\
(1680)\end{array}$ \\
\hline$E_{b 10 \div 0.5}\left[\mathrm{~N} / \mathrm{mm}^{2}\right]$ & $\begin{aligned} \phi & =150 \\
h & =300\end{aligned}$ & 0.3 & $\begin{array}{l}41^{\circ} 500 \\
(1950)\end{array}$ & $\begin{array}{l}38.500 \\
(2000)\end{array}$ & $\begin{array}{l}34.900 \\
(1850)\end{array}$ & $\begin{array}{l}35 \cdot 700 \\
(2250)\end{array}$ & $\begin{array}{r}36700 \\
(950)\end{array}$ & $\begin{array}{l}39^{\circ} 100 \\
(1910)\end{array}$ \\
\hline
\end{tabular}

E - MODULI

Bild 17: Betonkennwerte $\mathrm{BI} / 1$ bis $\mathrm{BI} / 6$ 


\begin{tabular}{|c|c|c|c|c|c|c|c|c|c|}
\hline VERSUCH & $\begin{array}{c}\text { ABMESS. } \\
{[\mathrm{mm}]}\end{array}$ & $\begin{array}{l}\text { BELAST. } \\
\text { GESCHW. } \\
\cdot 10^{-3} / \mathrm{min}\end{array}$ & B II / I & B II /2 & BII $/ 3$ & BII /4 & $B I / 5$ & $\mathrm{BI} / 6$ & $\mathrm{BII} / 7$ \\
\hline ALTER [Tage] & & & 41 & 95 & 37 & 41 & 44 & 45 & 46 \\
\hline ANZAHL PROBEN & & & 6 & 4 & 6 & 6 & 6 & 4 & 4 \\
\hline$\beta_{s z}\left[\mathrm{~N} / \mathrm{mm}^{2}\right]$ & $\begin{array}{l}\phi=150 \\
h=150\end{array}$ & 0.1 & $\begin{array}{l}2.90 \\
(0.24)\end{array}$ & $\begin{array}{c}3.89 \\
(0.42)\end{array}$ & $\begin{array}{c}3.35 \\
(0.55)\end{array}$ & $\begin{array}{c}3.59 \\
(0.45)\end{array}$ & $\begin{array}{c}3.40 \\
(0.41)\end{array}$ & $\begin{array}{c}3.06 \\
(0.39)\end{array}$ & $\begin{array}{c}3.08 \\
(0.22)\end{array}$ \\
\hline ANZAHL PROBEN & & & 2 & 2 & 3 & 3 & 3 & 2 & 2 \\
\hline$\beta_{q 2}\left[\mathrm{~N} / \mathrm{mm}^{2}\right]$ & $\begin{array}{l}\phi=150 \\
h=300\end{array}$ & 0.1 & $\begin{array}{c}1.60 \\
(0.06)\end{array}$ & $\begin{array}{c}1.65 \\
(0.16)\end{array}$ & $\begin{array}{c}2.32 \\
(0.38)\end{array}$ & $\begin{array}{c}2.41 \\
(0.16)\end{array}$ & $\begin{array}{l}2.14 \\
(0.26)\end{array}$ & $\begin{array}{r}1.78 \\
(0.10)\end{array}$ & $\begin{array}{c}2.01 \\
(0.10)\end{array}$ \\
\hline ANZAHL PROBEN & & & 4 & 4 & 4 & 4 & 4 & 3 & 3 \\
\hline$\beta_{b z}\left[\mathrm{~N} / \mathrm{mm}^{2}\right]$ & $120 / 120 / 360$ & 1.0 & $\begin{array}{c}5.03 \\
(0.24)\end{array}$ & $\begin{array}{c}7.28 \\
(0.29)\end{array}$ & $\begin{array}{c}5.64 \\
(0.61)\end{array}$ & $\begin{array}{c}5.30 \\
(0.33)\end{array}$ & $\begin{array}{c}5.13 \\
(0.59)\end{array}$ & $\begin{array}{c}6.15 \\
(0.41)\end{array}$ & $\begin{array}{c}5.65 \\
(0.15)\end{array}$ \\
\hline ANZAHL PROBEN & & & 4 & 3 & 3 & 3 & 3 & 2 & 2 \\
\hline$\beta_{P}\left[\mathrm{~N} / \mathrm{mm}^{2}\right]$ & $\begin{array}{l}\phi=150 \\
h=300\end{array}$ & 0.1 & $\begin{array}{l}32.9 \\
(2.0)\end{array}$ & $\begin{array}{c}34.6 \\
(2.95)\end{array}$ & $\begin{array}{c}33.9 \\
(1.35)\end{array}$ & $\begin{array}{l}35.3 \\
(1.12)\end{array}$ & $\begin{array}{c}33.9 \\
(1.74)\end{array}$ & $\begin{array}{c}34.4 \\
(0.51)\end{array}$ & $\begin{array}{c}32.3 \\
(6.89)\end{array}$ \\
\hline$\varepsilon_{u} \quad\left[10^{-3}\right]$ & & & 2.0 & 2.1 & 1.9 & 2.0 & 2.0 & 2.1 & 2.1 \\
\hline ANZAHL PROBEN & & & 4 & 4 & 4 & 4 & 4 & 3 & 4 \\
\hline$\beta_{w}\left[\mathrm{~N} / \mathrm{mm}^{2}\right]$ & $150 / 450 / 450$ & 0.5 & $\begin{array}{l}37.2 \\
(3.2)\end{array}$ & $\begin{array}{l}37.5 \\
(1.3)\end{array}$ & $\begin{array}{l}37.7 \\
(1.5)\end{array}$ & $\begin{array}{l}43.1 \\
(2.7)\end{array}$ & $\begin{array}{l}37.9 \\
(1.7)\end{array}$ & $\begin{array}{l}36.7 \\
(0.2)\end{array}$ & $\begin{array}{l}40.5 \\
(4.7)\end{array}$ \\
\hline
\end{tabular}

DRUCK - UND ZUGFESTIGKEITEN

WERTE IN ( ): STANDARD-ABWEICHUNGEN

\begin{tabular}{|c|c|c|c|c|c|c|c|c|c|}
\hline ANZAHL PROBEN & & & 4 & 3 & 3 & 3 & 3 & 2 & 2 \\
\hline$E_{b 0.5 \div 5}\left[\mathrm{~N} / \mathrm{mm}^{2}\right]$ & $\begin{array}{l}\phi=150 \\
h=300\end{array}$ & 0.3 & $\begin{array}{l}40^{\circ} 100 \\
(1390)\end{array}$ & $\begin{array}{l}37700 \\
(2010)\end{array}$ & $\begin{array}{l}39600 \\
(2950)\end{array}$ & $\begin{array}{r}38100 \\
(640)\end{array}$ & $\begin{array}{l}39^{\circ} 100 \\
(780)\end{array}$ & $\begin{array}{l}39^{\circ} 900 \\
(2780)\end{array}$ & $\begin{array}{l}37700 \\
\text { (4840) }\end{array}$ \\
\hline$E_{b} 5 \div 0.5\left[\mathrm{~N} / \mathrm{mm}^{2}\right]$ & $\begin{array}{l}\phi=150 \\
h=300\end{array}$ & 0.3 & $\begin{array}{l}39700 \\
(1800)\end{array}$ & $\begin{array}{l}38^{\circ} 000 \\
(1740)\end{array}$ & $\begin{array}{l}40.500 \\
(3370)\end{array}$ & $\begin{array}{c}38.300 \\
(700)\end{array}$ & $\begin{array}{c}38.500 \\
(360)\end{array}$ & $\begin{array}{l}39^{\circ} 000 \\
(2430)\end{array}$ & $\begin{array}{l}37 \cdot 100 \\
(4320)\end{array}$ \\
\hline$E_{b 0.5 \div 10}\left[\mathrm{~N} / \mathrm{mm}^{2}\right]$ & $\begin{array}{l}\phi=150 \\
h=300\end{array}$ & 0.3 & $\begin{array}{l}37 \cdot 200 \\
(1200)\end{array}$ & $\begin{array}{l}35600 \\
(1430)\end{array}$ & $\begin{array}{l}37.500 \\
(3160)\end{array}$ & $\begin{array}{r}36300 \\
(240)\end{array}$ & $\begin{array}{l}37700 \\
(490)\end{array}$ & $\begin{array}{l}36600 \\
(1650)\end{array}$ & $\begin{array}{l}35700 \\
(4430)\end{array}$ \\
\hline$E_{b 10 \div 0.5}\left[\mathrm{~N} / \mathrm{mm}^{2}\right]$ & $\begin{array}{l}\phi=150 \\
h=300\end{array}$ & 0.30 & $\begin{array}{l}37 \cdot 300 \\
(1270)\end{array}$ & $\begin{array}{l}36.200 \\
(1140)\end{array}$ & $\begin{array}{l}37 \cdot 700 \\
(3130)\end{array}$ & $\begin{array}{r}36.400 \\
(250)\end{array}$ & $\begin{array}{r}38.000 \\
(380)\end{array}$ & $\begin{array}{l}36.000 \\
(1780)\end{array}$ & $\begin{array}{l}36400 \\
(4510)\end{array}$ \\
\hline
\end{tabular}

E-MODULI

Bild 18: Betonkennwerte $\mathrm{BI} / 1$ bis $\mathrm{BI} / 7$ 


\begin{tabular}{|c|c|c|c|c|c|c|}
\hline VERSUCH & $\begin{array}{c}\text { ABMESS. } \\
{[\mathrm{mm}]}\end{array}$ & $\begin{array}{l}\text { BELAST. } \\
\text { GESCHW. } \\
\cdot 10^{-3} / \mathrm{min}\end{array}$ & $\mathrm{BI} / 8$ & BI $/ 9$ & BII $/ 10$ & BII $/ 11$ \\
\hline ALTER [TAGE] & & & 41 & 37 & 60 & 125 \\
\hline ANZAHL PROBEN & & & 6 & 6 & 6 & 6 \\
\hline$\beta_{\mathrm{sZ}}\left[\mathrm{N} / \mathrm{mm}^{2}\right]$ & $\begin{array}{l}\phi=150 \\
h=150\end{array}$ & 0.1 & $\begin{array}{c}3.03 \\
(0.30)\end{array}$ & $\begin{array}{c}2.90 \\
(0.44)\end{array}$ & $\begin{array}{c}3.47 \\
(0.51)\end{array}$ & $\begin{array}{c}3.56 \\
(0.41)\end{array}$ \\
\hline ANZAHL PROBEN & & & 3 & 3 & 3 & 3 \\
\hline$\beta_{\mathrm{qz}}\left[\mathrm{N} / \mathrm{mm}^{2}\right]$ & $\begin{array}{l}\phi=150 \\
h=300\end{array}$ & 0.1 & $\begin{array}{r}2.80 \\
(0.39)\end{array}$ & $\begin{array}{c}3.00 \\
(0.23)\end{array}$ & $\begin{array}{c}3.26 \\
(0.33)\end{array}$ & $\begin{array}{c}3.12 \\
(0.21)\end{array}$ \\
\hline ANZAHL PROBEN & & & 4 & 4 & 4 & 4 \\
\hline$\beta_{\mathrm{bz}}\left[\mathrm{N} / \mathrm{mm}^{2}\right]$ & $120 / 120 / 360$ & 1.0 & $\begin{array}{c}6.35^{*} \\
(0.54)\end{array}$ & $\begin{array}{c}6.32^{* *} \\
(0.61)\end{array}$ & $\begin{array}{c}6.60 \\
(0.37)\end{array}$ & $\begin{array}{c}5.13 \\
(0.47)\end{array}$ \\
\hline ANZAHL PROBEN & & & 3 & 3 & 3 & 3 \\
\hline$\beta_{\mathrm{p}} \quad\left[\mathrm{N} / \mathrm{mm}^{2}\right]$ & $\begin{array}{l}\phi=150 \\
h=300\end{array}$ & 0.1 & $\begin{array}{r}33.3 \\
(3.38)\end{array}$ & $\begin{array}{r}32.3 \\
(1.38)\end{array}$ & $\begin{array}{c}40.4 \\
(3.35)\end{array}$ & $\begin{array}{l}41.56 \\
(1.78)\end{array}$ \\
\hline$\varepsilon_{u} \quad\left[10^{-3}\right]$ & & & 1.7 & 1.6 & 1.8 & 1.7 \\
\hline ANZAHL PROBEN & & & 5 & 6 & 6 & 6 \\
\hline$\beta_{w}\left[\mathrm{~N} / \mathrm{mm}^{2}\right]$ & $150 / 150 / 150$ & 0.5 & $\begin{array}{r}41.7 \\
(1.31)\end{array}$ & $\begin{array}{r}43.3 \\
(1.89)\end{array}$ & $\begin{array}{c}53.1 \\
(1.28)\end{array}$ & $\begin{array}{c}50.06 \\
(1.8)\end{array}$ \\
\hline
\end{tabular}

"geprüft nach 165 Togen

* geprüft noch 155 Togen

DRUCK - UND ZUGFESTIGKEITEN

WERTE IN ( ): STANDARD-

ABWEICHUNGEN

\begin{tabular}{|c|c|c|c|c|c|c|}
\hline ANZAHL PROBEN & & & 3 & 3 & 3 & 3 \\
\hline$E_{b 0.5 \div 5}\left[\mathrm{~N} / \mathrm{mm}^{2}\right]$ & $\begin{array}{l}\phi=150 \\
h=300\end{array}$ & 0.3 & $\begin{array}{l}39400 \\
(2.320)\end{array}$ & $\begin{array}{l}42 \cdot 100 \\
(2360)\end{array}$ & $\begin{array}{c}44000 \\
(2040)\end{array}$ & $\begin{array}{l}43500 \\
(1700)\end{array}$ \\
\hline$E_{b 5} \div 0.5\left[\mathrm{~N} / \mathrm{mm}^{2}\right]$ & $\begin{array}{l}\phi=150 \\
h=300\end{array}$ & 0.3 & $\begin{array}{c}38900 \\
(1 \cdot 170)\end{array}$ & $\begin{array}{r}40000 \\
(620)\end{array}$ & $\begin{array}{c}43800 \\
(2220)\end{array}$ & $\begin{array}{r}43700 \\
(1730)\end{array}$ \\
\hline$E_{b 0.5 \div 10}\left[\mathrm{~N} / \mathrm{mm}^{2}\right]$ & $\begin{array}{l}\phi=150 \\
h=300\end{array}$ & 0.3 & $\begin{array}{c}37.900 \\
(870)\end{array}$ & $\begin{array}{c}38600 \\
(280)\end{array}$ & $\begin{array}{c}42300 \\
(1700)\end{array}$ & $\begin{array}{l}41800 \\
(1600)\end{array}$ \\
\hline$E_{b 10 \div 0.5}\left[\mathrm{~N} / \mathrm{mm}^{2}\right]$ & $\begin{array}{l}\phi=150 \\
h=300\end{array}$ & 0.3 & $\begin{array}{l}38 \cdot 100 \\
(500)\end{array}$ & $\begin{array}{l}38800 \\
(200)\end{array}$ & $\begin{array}{l}42800 \\
(4470)\end{array}$ & $\begin{array}{l}42500 \\
(2280)\end{array}$ \\
\hline
\end{tabular}

E-MODULI

Bild 19: Betonkennwerte BII/8 bis $\mathrm{BI} / 11$ 


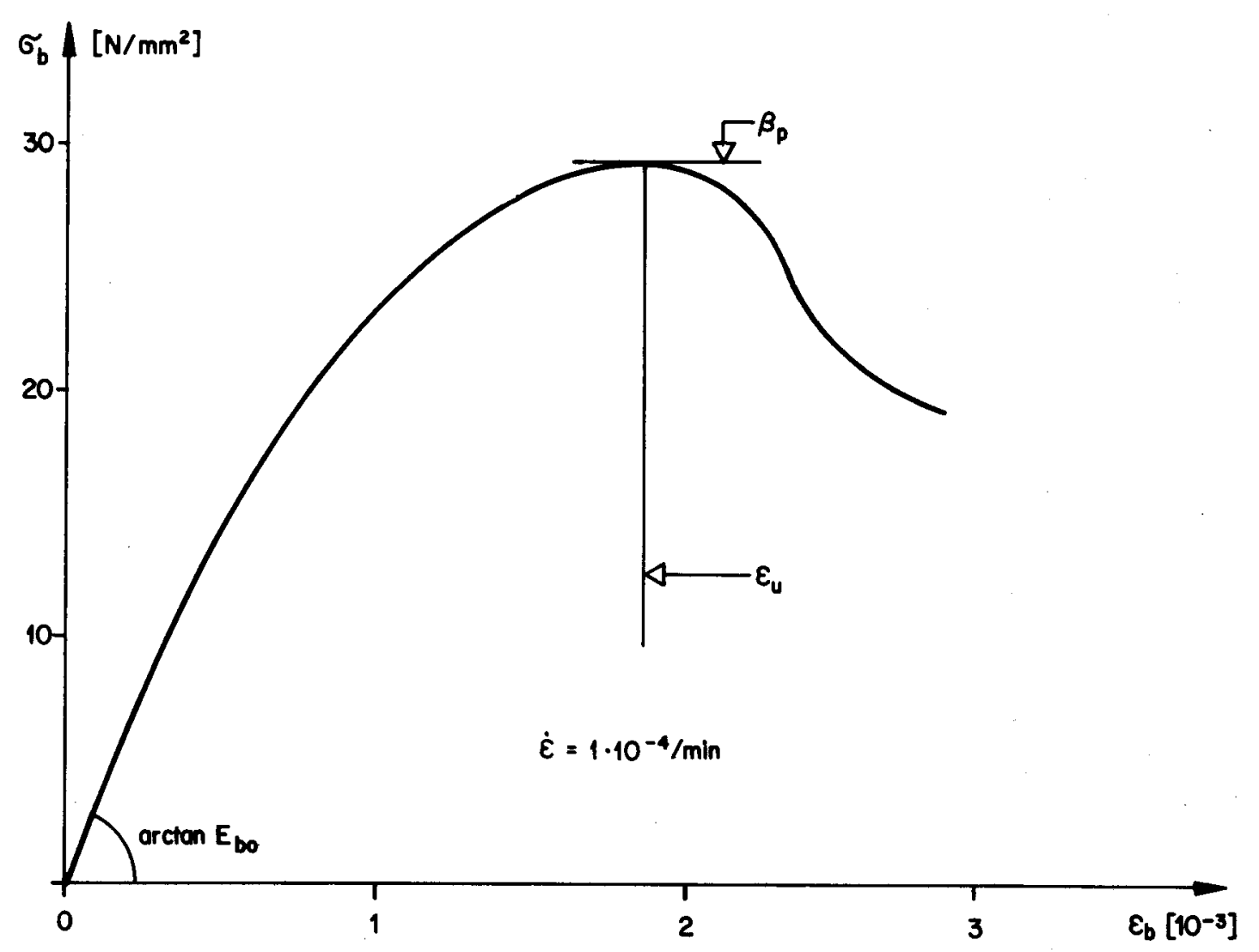

Bild 20: Spannungs - Dehnungs -Diagramm eines Betonzylinders

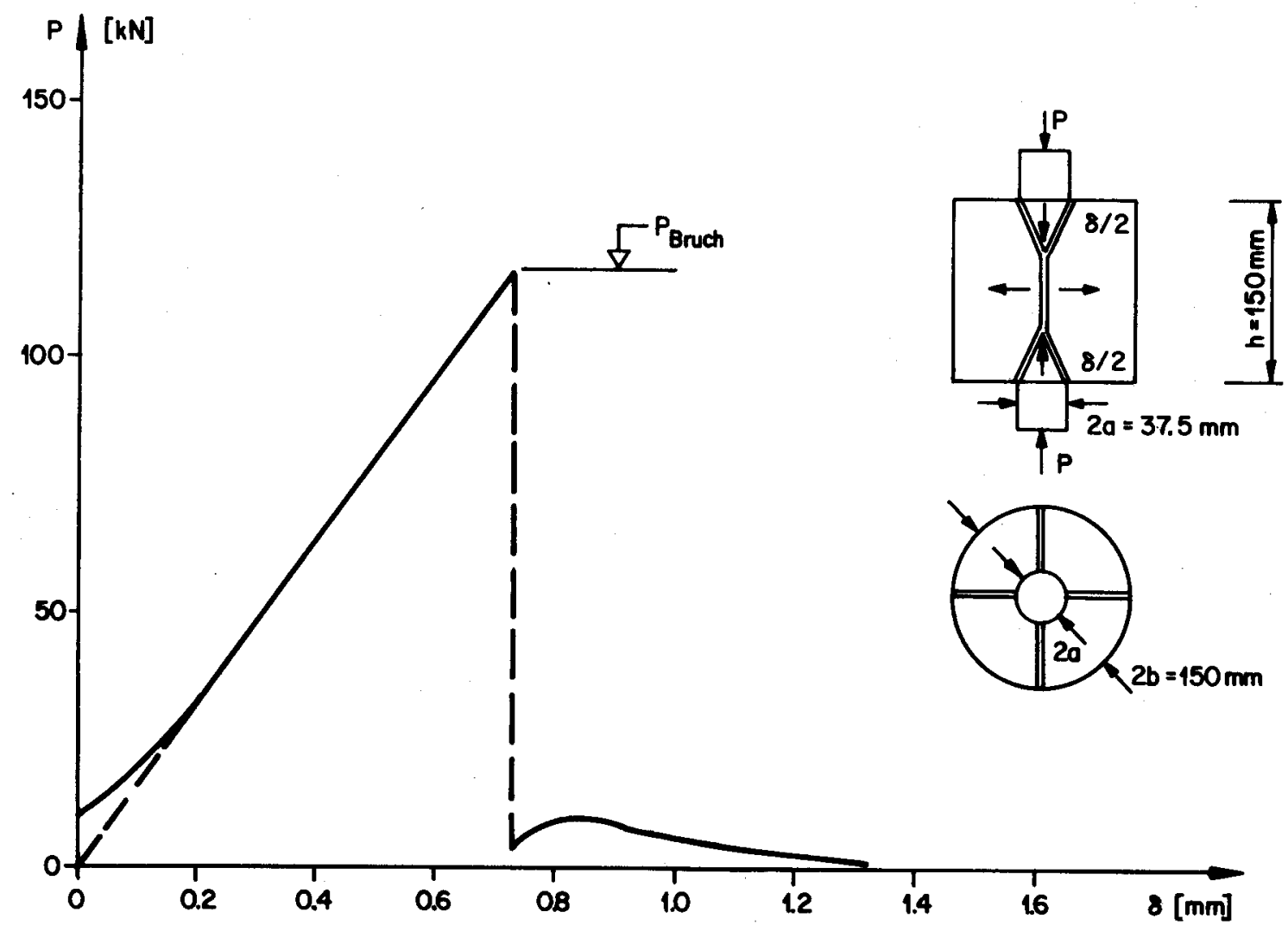

Bild 21: Last - Weg-Diogramm eines Stempelversuchs 


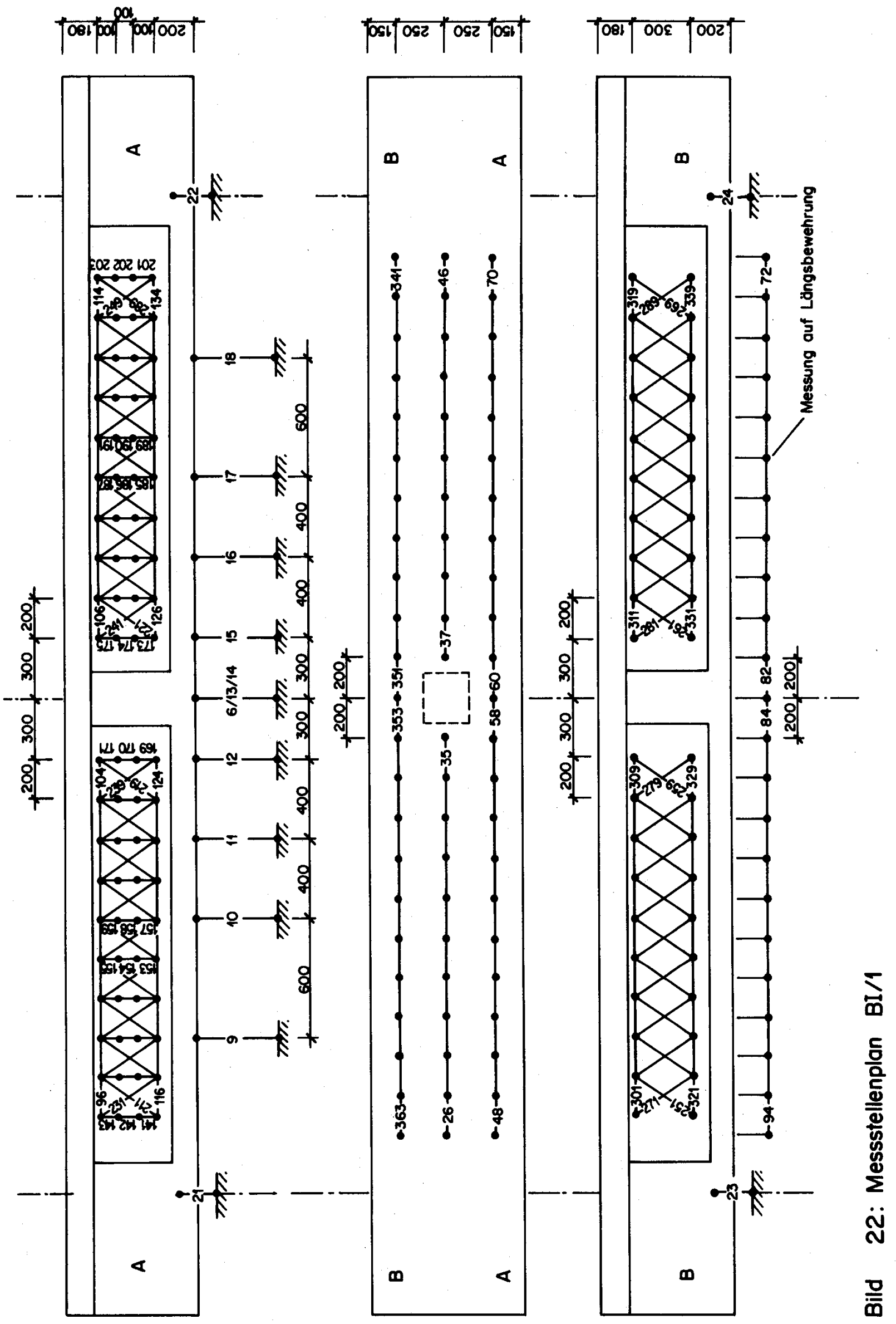




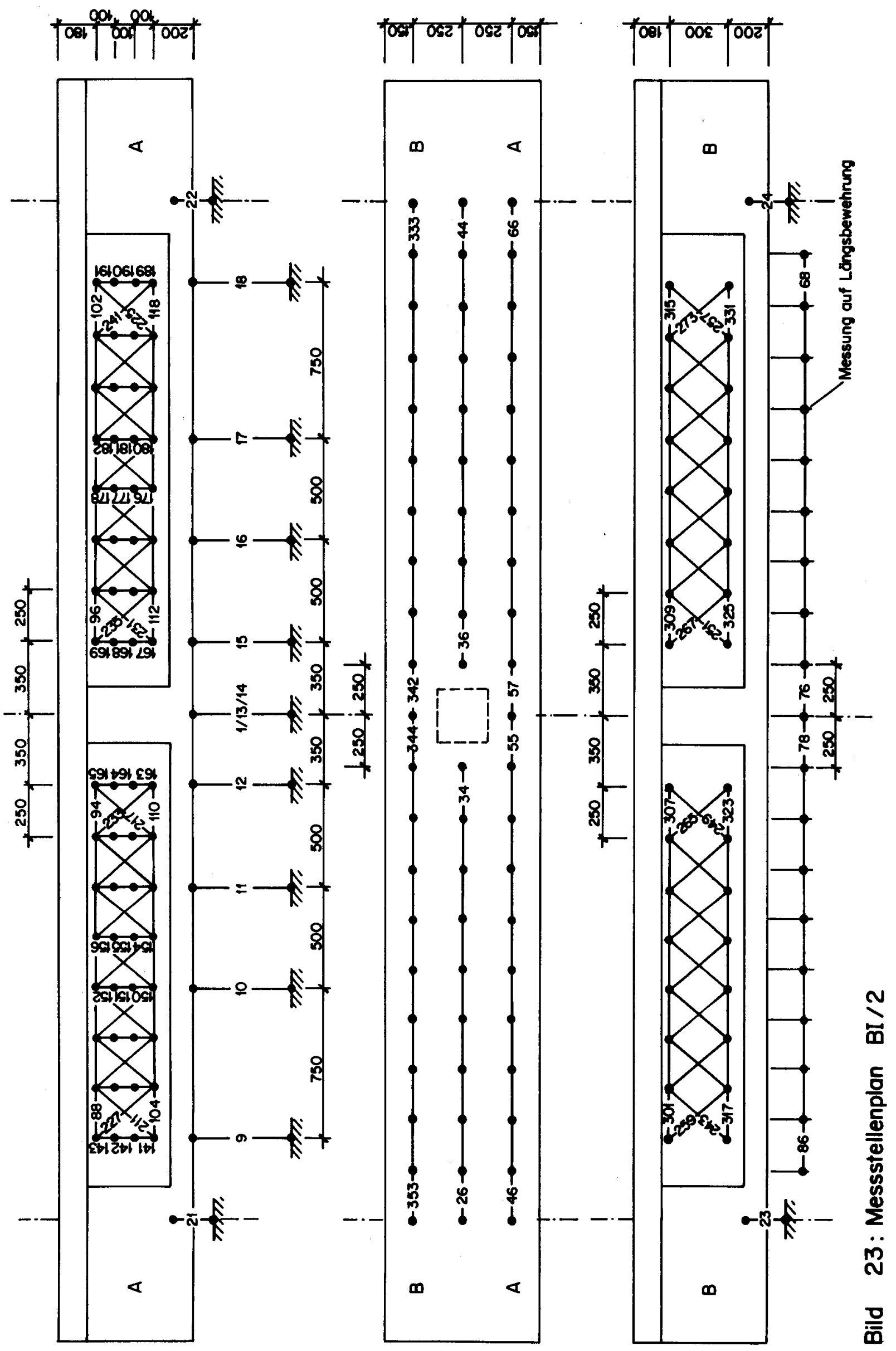




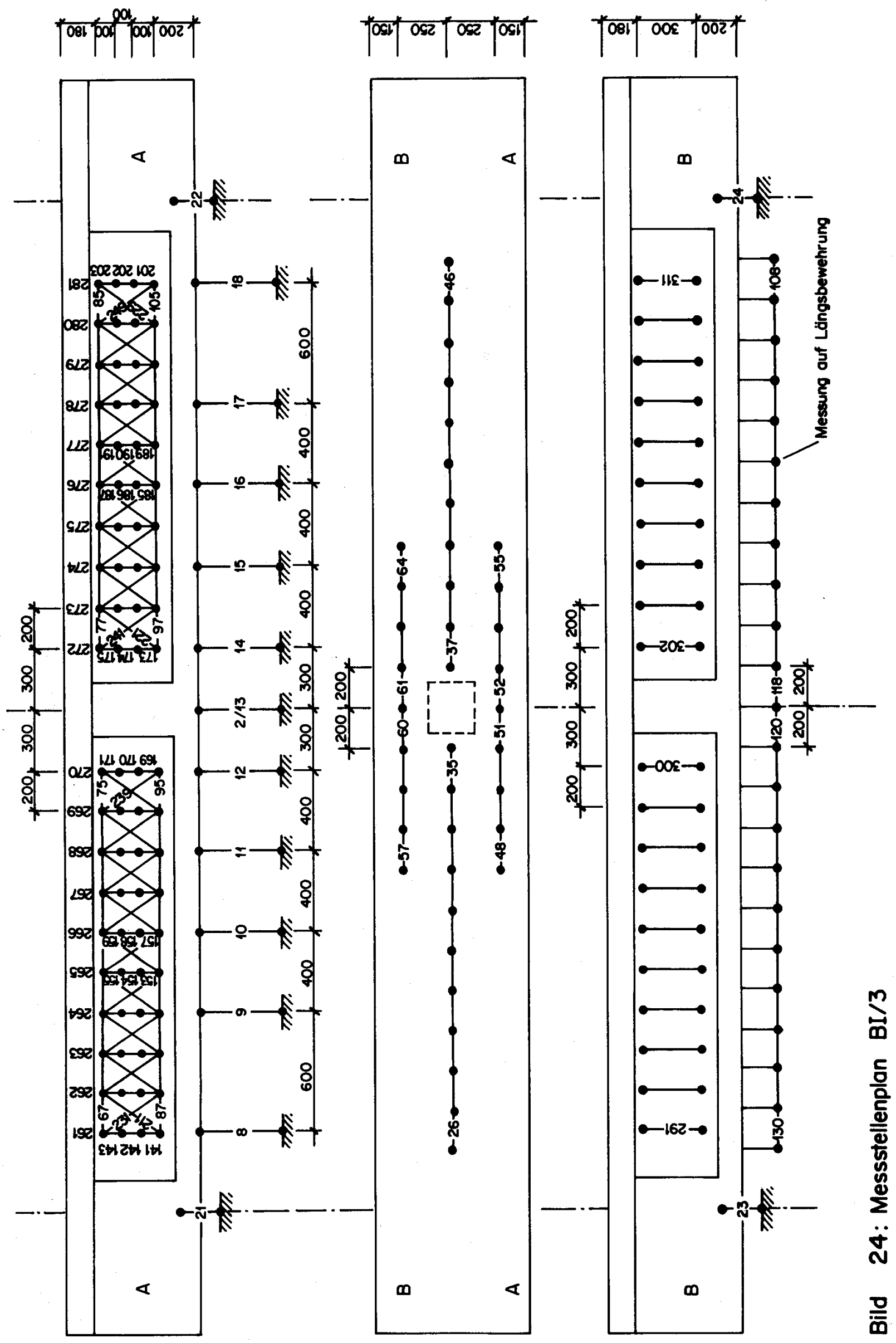




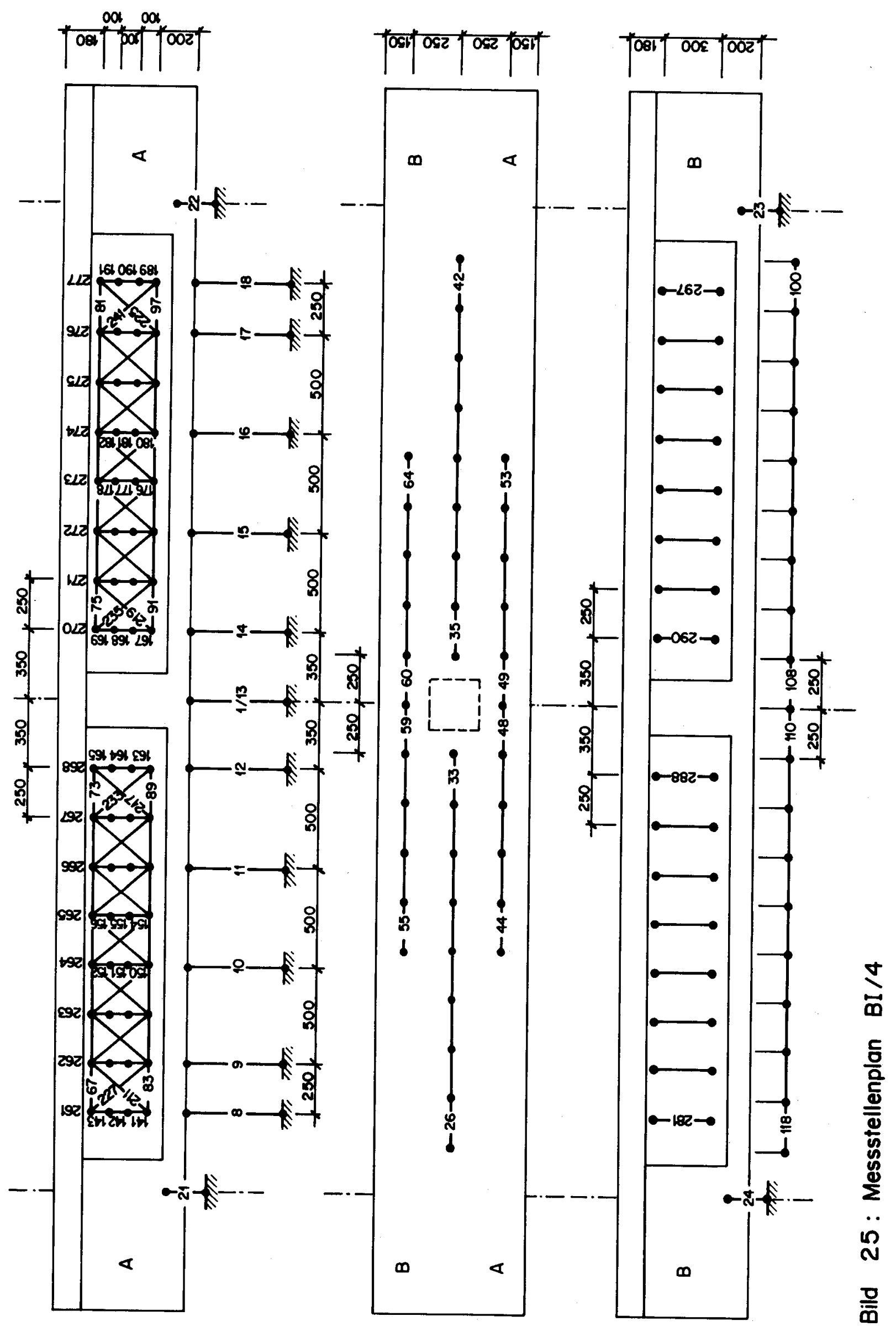




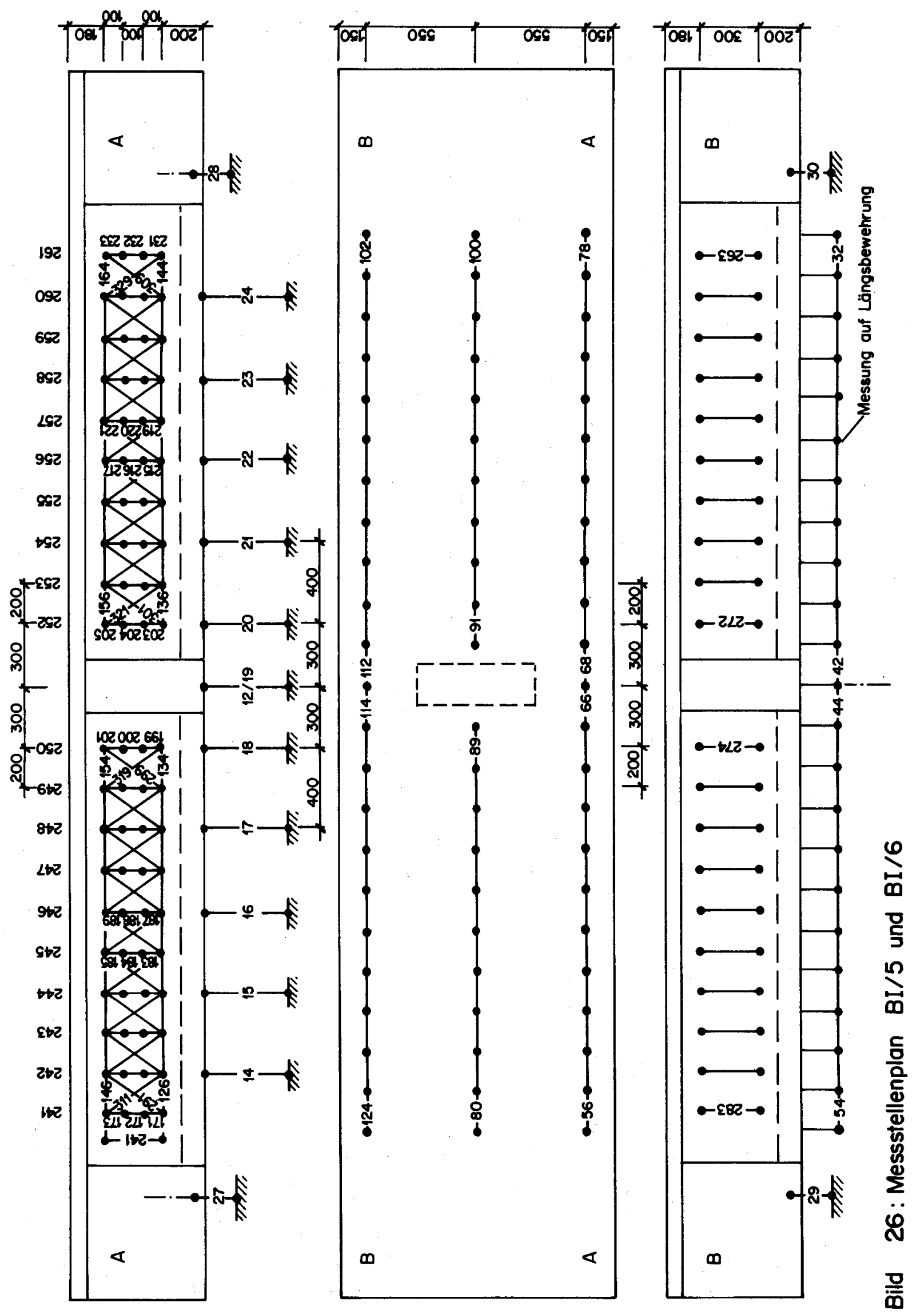




$\begin{array}{cl}6 & \text { Dynamische Durchbiegungsmessung in Trögermitte } \\ 9 \div 18 & \text { Statische Durchbiegungsmessungen } \\ 21 \div 24 & \text { Logereinsenkungen } \\ 141 \div 203 & b=100 \mathrm{~mm} \\ 26 \div 134,301 \div 363 & b=200 \mathrm{~mm} \\ 211 \div 289 & b=360.6 \mathrm{~mm}\end{array}$

$\pm 0.01$

0.04

0.004

0.004

0.004

0.01

Balken BI/2

$\begin{array}{cl}1 & \text { Dynamische Durchbiegungsmessung in Trägermitte } \\ 9 \div 18 & \text { Statische Durchbiegungsmessungen } \\ 21 \div 24 & \text { Lagereinsenkungen } \\ 141 \div 191 & b=100 \mathrm{~mm} \\ 26 \div 118,301 \div 353 & b=250 \mathrm{~mm} \\ 211 \div 273 & b=390.5 \mathrm{~mm}\end{array}$

0.04

0.015

0.004

0.01

0.01

\section{Balken BI/3}

$$
\begin{gathered}
2 \\
8 \div 18 \\
21 \div 24 \\
141 \div 203 \\
26 \div 130 \\
211 \div 249 \\
261 \div 311
\end{gathered}
$$

\section{Balken BI/4}

$$
\begin{gathered}
1 \\
8 \div 18 \\
21 \div 24 \\
141 \div 191 \\
26 \div 118 \\
211 \div 241 \\
261 \div 297
\end{gathered}
$$$$
b=300 \mathrm{~mm}
$$$$
0.004
$$

\section{Balken $\mathrm{BI} / 5$ und $\mathrm{BI} / 6$}

$$
\begin{gathered}
14 \div 24 \\
27 \div 30 \\
32 \div 164 \\
171 \div 233 \\
241 \div 283 \\
291 \div 329
\end{gathered}
$$

- Nach 4 bis 11 Messungen in einer Messreihe erfolgt jemoits eine Eichmesung.

- Doppelt ousgeführte Messreiten (Kontrollmessungen) sind nur einmal oufgefíhrt.

- Die Messung der dynamischen und statischen Vertikalkräfte erfolgt mittch einer Messdose mit einer Auf lösung von OS kN 

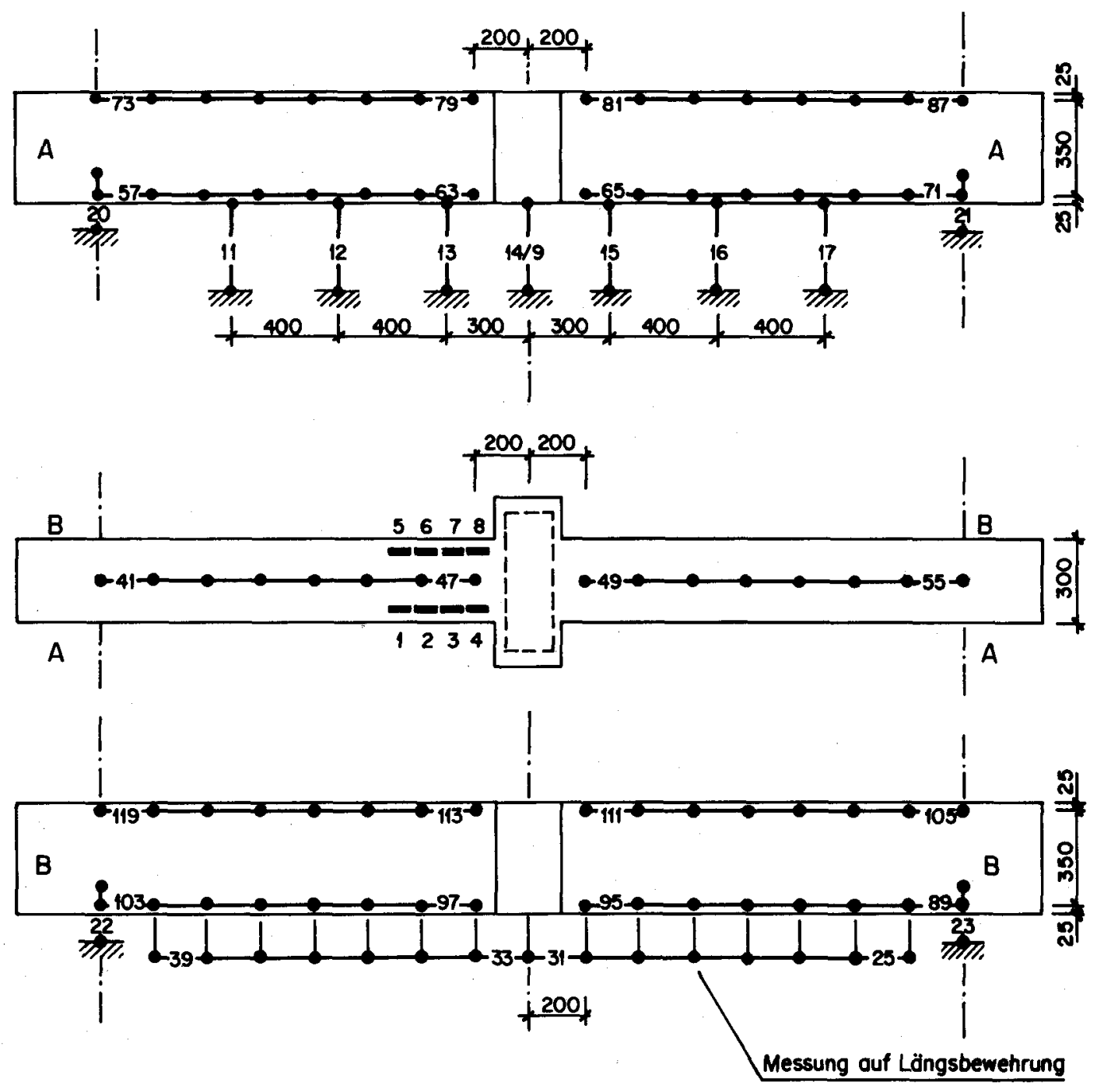

Messnummern, Messbosen, Messfehler

$\begin{array}{clc} & & \text { Messfehler [mm] } \\ 1 \div 8 & \text { b }=60 \mathrm{~mm} \quad \text { Dehnungsmessstreifen } & \\ 9 & \text { Dynamische Durchbiegungsmessung in Trägermitte } & \pm 0.01 \\ 11 \div 17 & \text { Statische Durchbiegungsmessungen } & 0.04 \\ 20 \div 23 & \text { Lagereinsenkungen } & 0.004 \\ 25 \div 119 & \mathrm{~b}=200 \mathrm{~mm} & 0.004 \\ \text { Vertikalkraft: } & \begin{array}{l}\text { Dynamische und statische Messung mittels einer Messdose } \\ \end{array} & \\ & \text { mit einer Auflösung von } 0.1 \mathrm{kN} & \end{array}$

Nach 4 bis 8 Messungen in einer Messreihe erfolgt jeweils eine Eichmessung

Bild 28: Messstellenplan $\mathrm{BI} / 1$ und $\mathrm{BI} / 2$ 


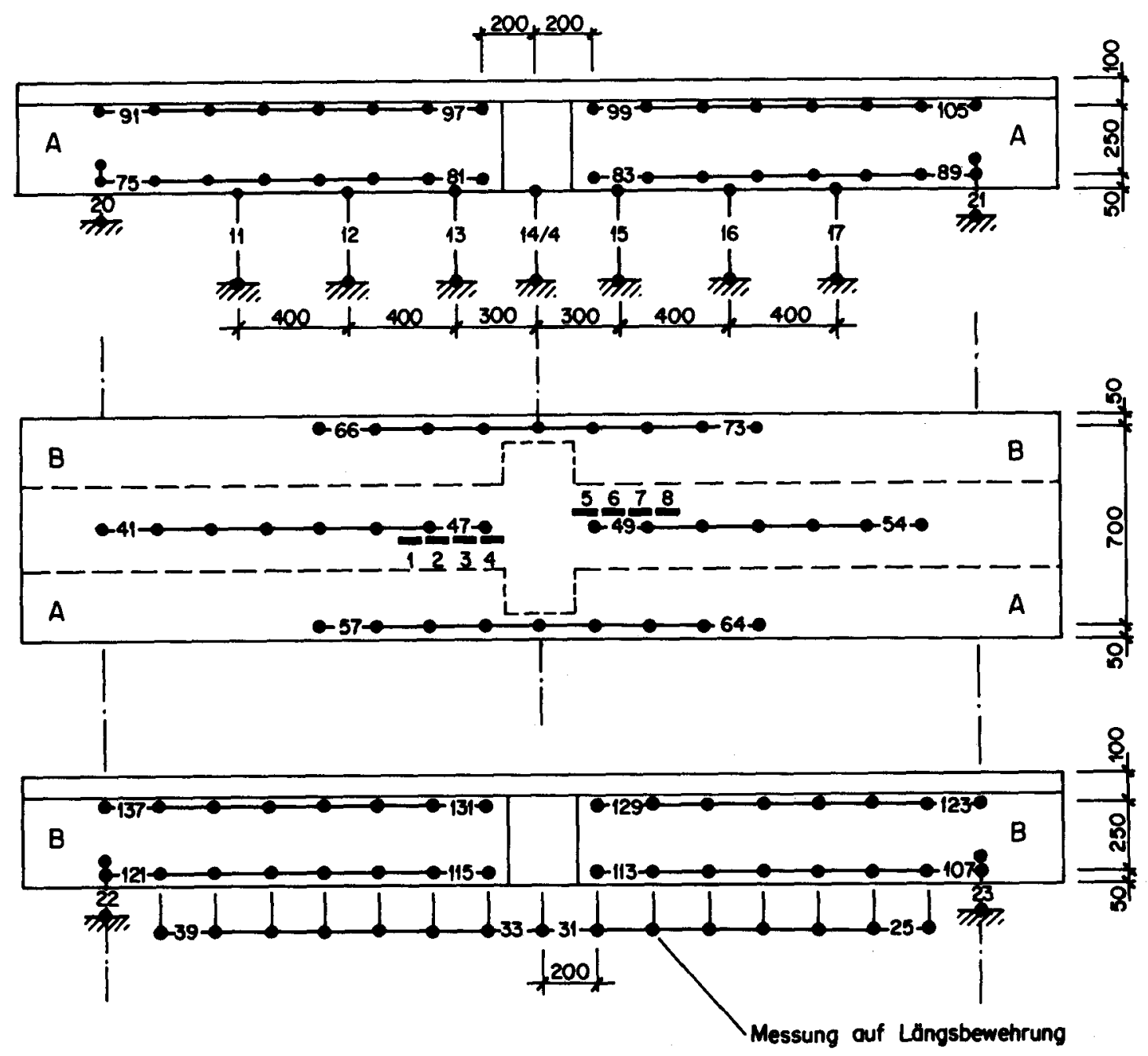

Messnummern, Messbasen b, Messfehier

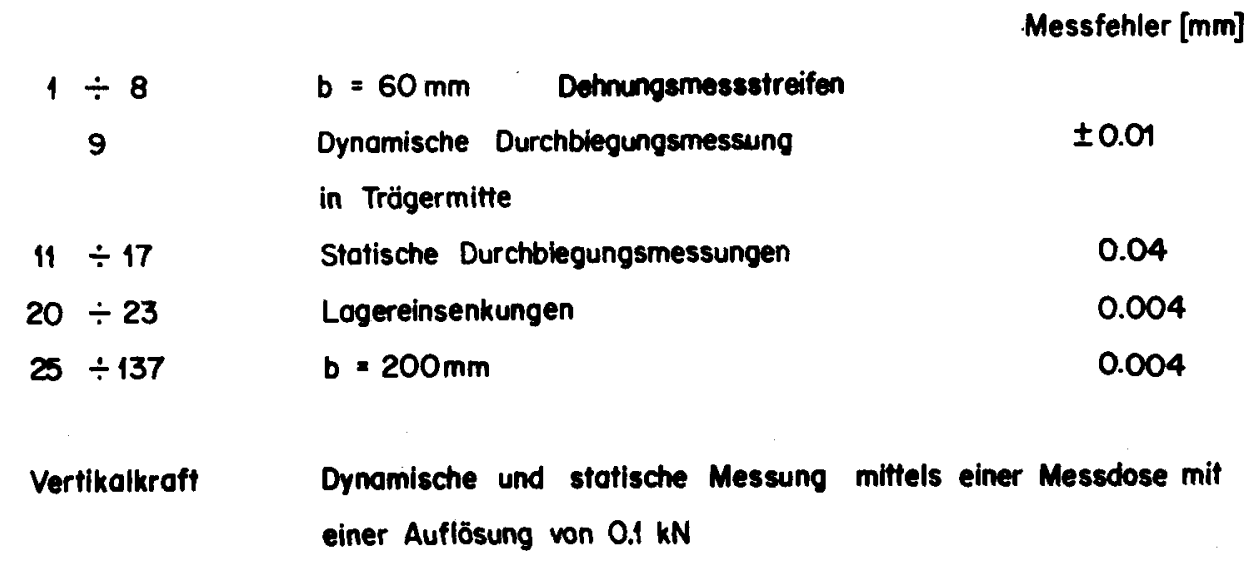

Nach 4 bis 8 Messungen in einer Messreihe erfolgt jeweils eine Eichmessung. 

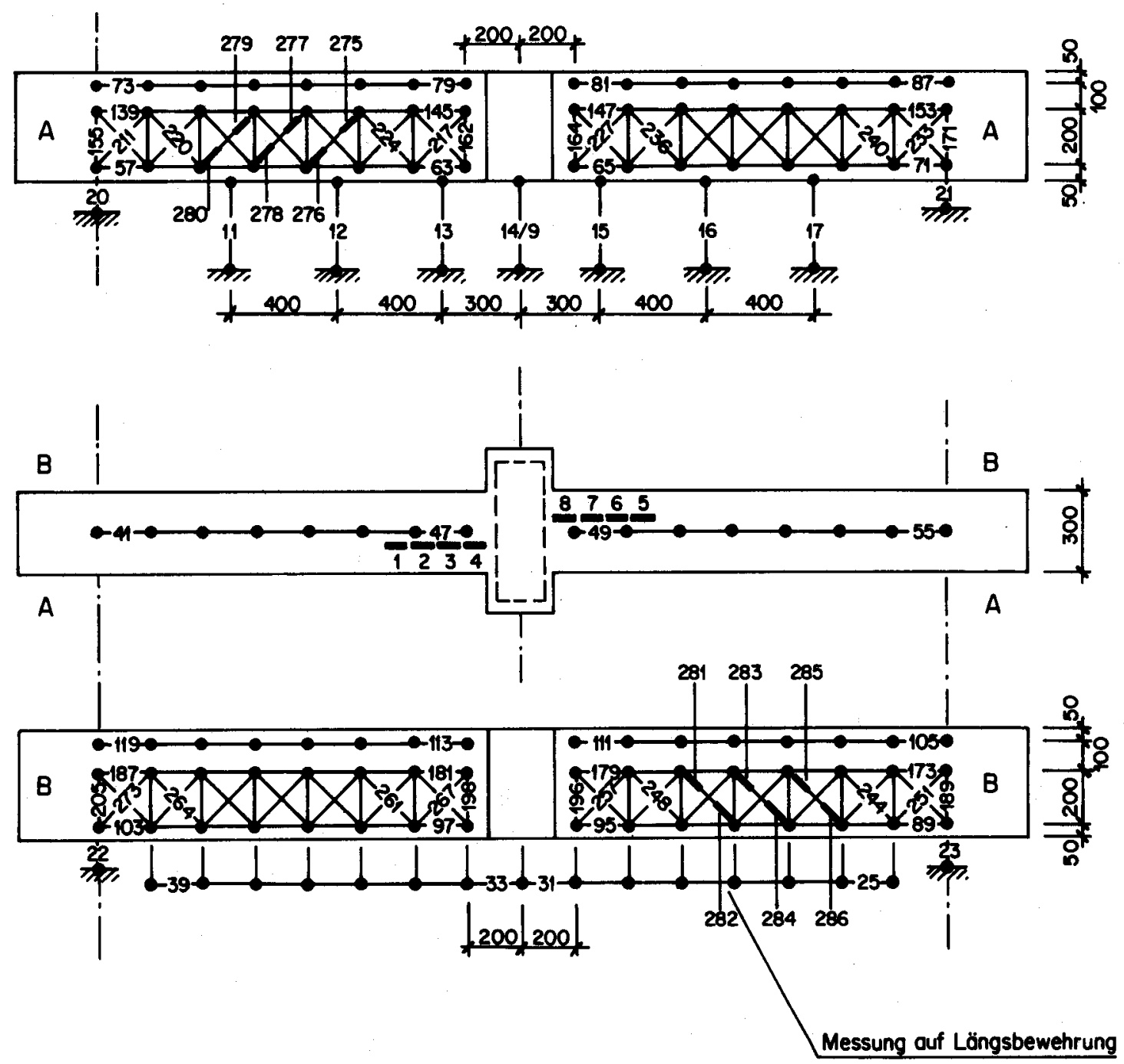

Messnummern, Messbasen, Messfehler

$\begin{array}{clc}1 \div 8,275 \div 286 & b=60 \mathrm{~mm} \quad \text { Dehnungsmessstreifen } \\ 9 & \begin{array}{l}\text { Dynamische Durchbiegungsmessung } \\ \text { in Trägermitte }\end{array} & \pm 0.01 \\ 11 \div 17 & \text { Statische Durchbiegungsmessungen } & 0.04 \\ 20 \div 23 & \text { Lagereinsenkungen } & 0.004 \\ 25 \div 205 & b=200 \mathrm{~mm} & 0.004 \\ 211 \div 273 & b=282.8 \mathrm{~mm} & 0.004 \\ \text { Vertikalkroft: } & \text { Dynamische und stotische Messung mittels einer Messdose } \\ & \text { mit einer Auflosung von } 0.1 \mathrm{kN} & \end{array}$

Noch 4 bis 8 Messungen in einer Messreihe erfolgt jeweils eine Eichmessung.

Bild 30: Messstellenplan BI/6 und BI/7 


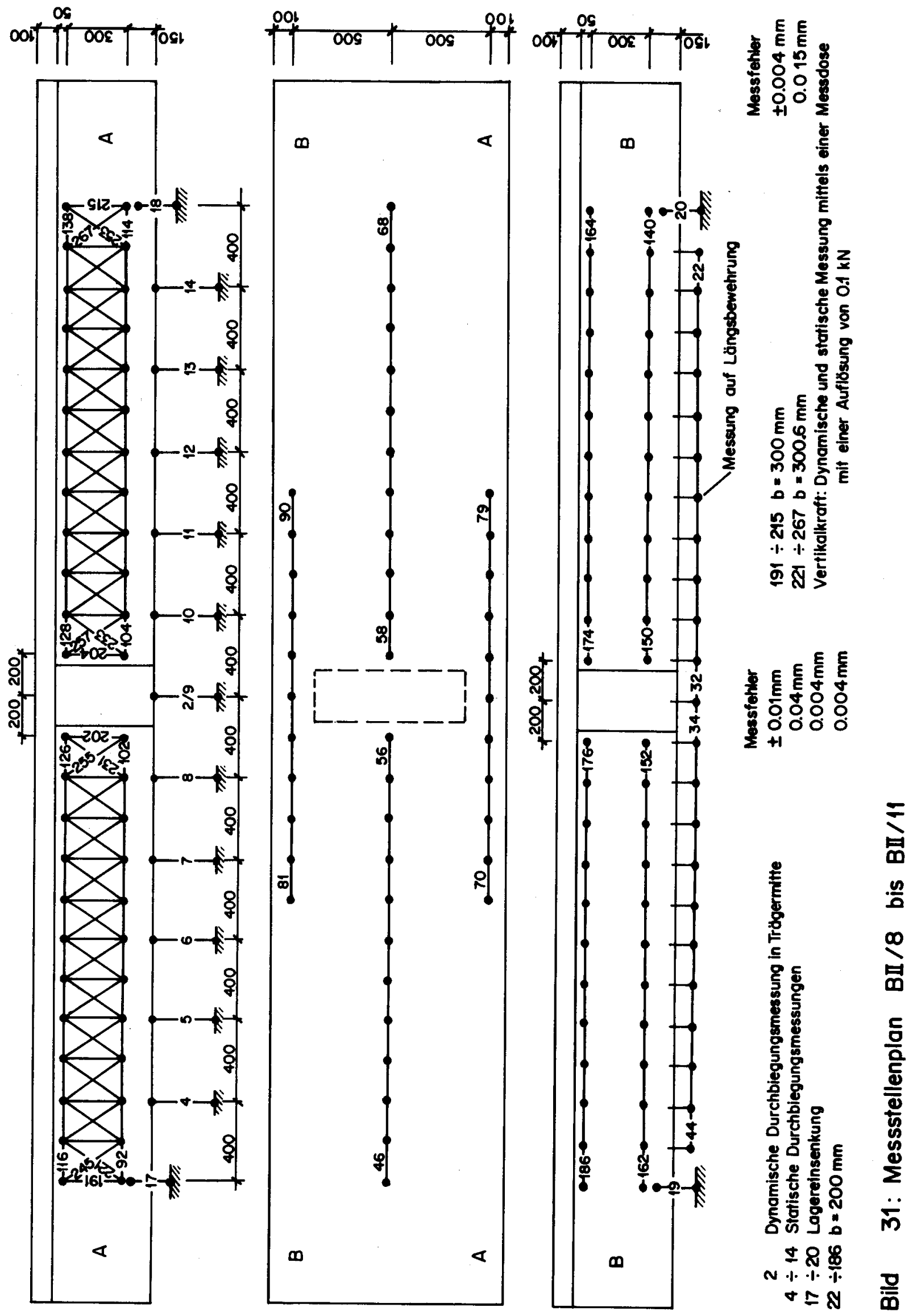




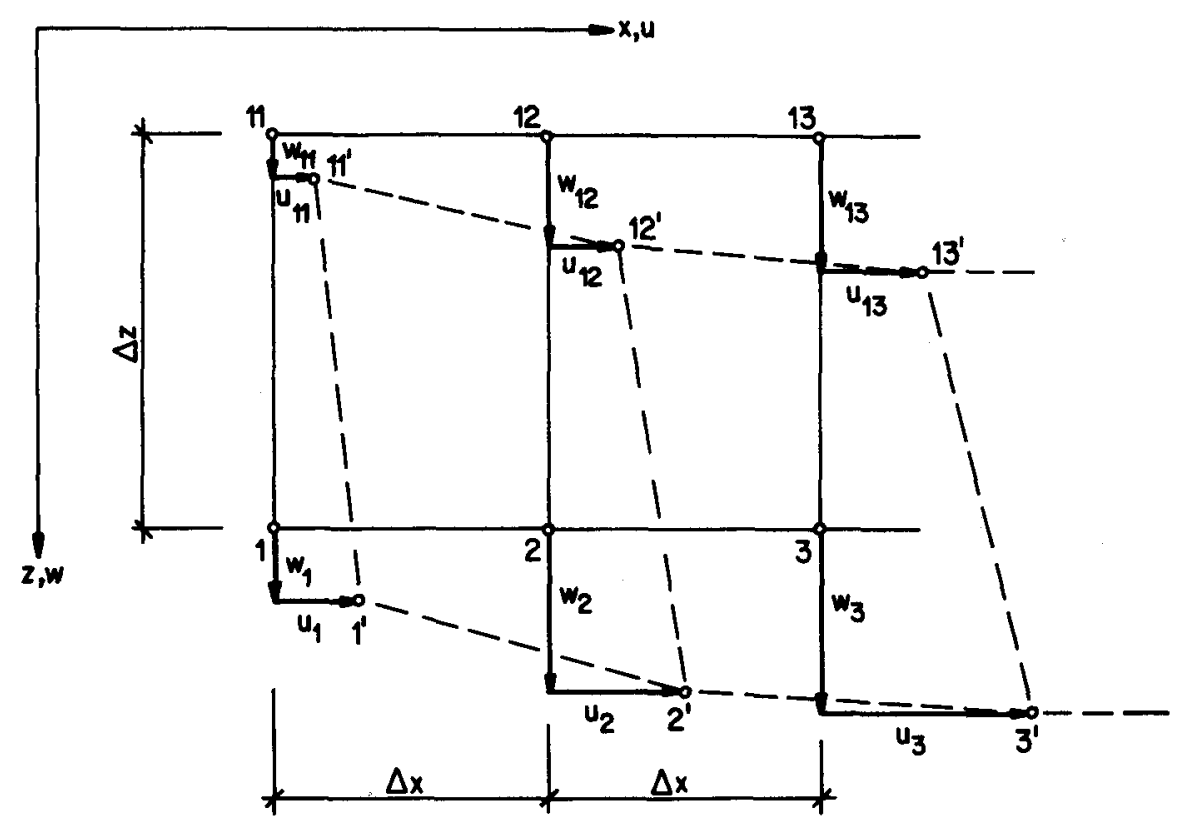

Mittlere Dehnungen und Schiebungen im Messnetz ouf den Bakkenstegen

$$
\begin{aligned}
& \varepsilon_{x}=\frac{1}{2}\left\{\left(u_{2}-u_{1}\right)+\left(u_{12}-u_{11}\right)\right\} \cdot \frac{1}{\Delta x} \\
& \varepsilon_{z}=\frac{1}{2}\left\{\left(w_{1}-w_{11}\right)+\left(w_{2}-w_{12}\right)\right\} \cdot \frac{1}{\Delta z} \\
& r_{x z}=\frac{1}{2}\left\{\left(u_{1}-u_{11}\right)+\left(u_{2}-u_{12}\right)\right\} \cdot \frac{1}{\Delta z}+\frac{1}{2}\left\{\left(w_{2}-w_{1}\right)+\left(w_{12}-w_{11}\right)\right\} \cdot \frac{1}{\Delta x}
\end{aligned}
$$

Bild 32 : Verzerrungsberechnung

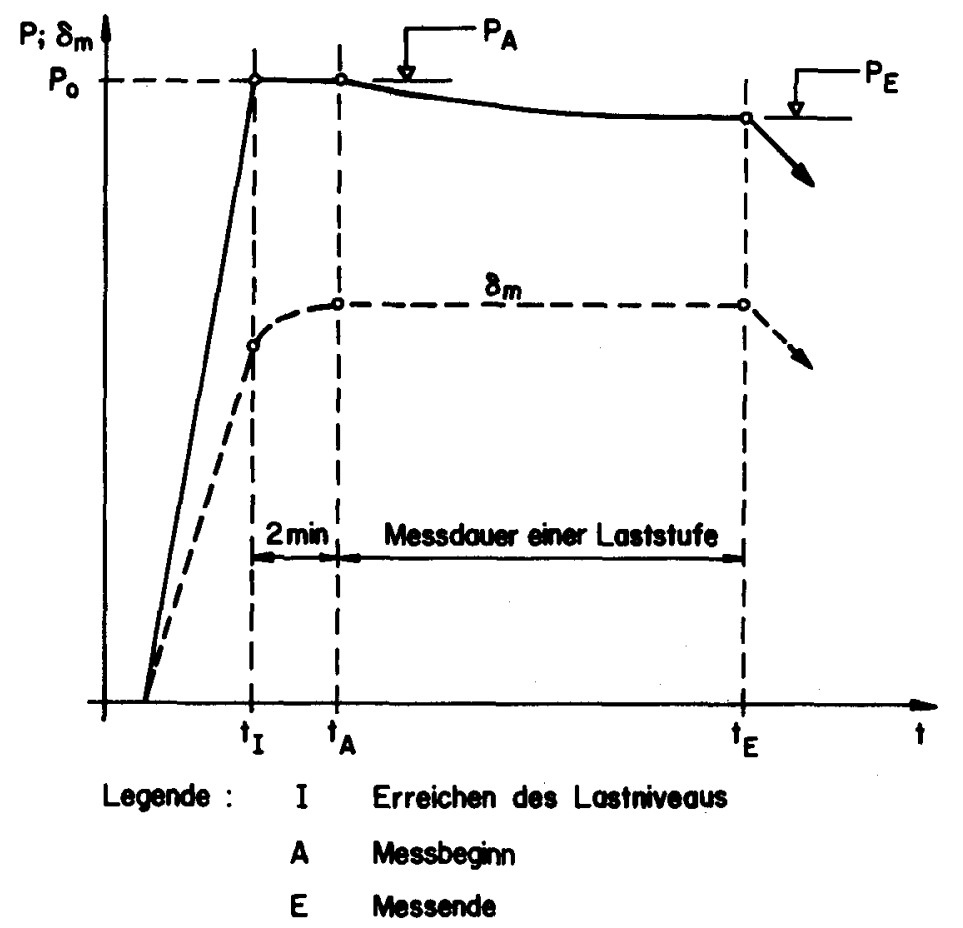




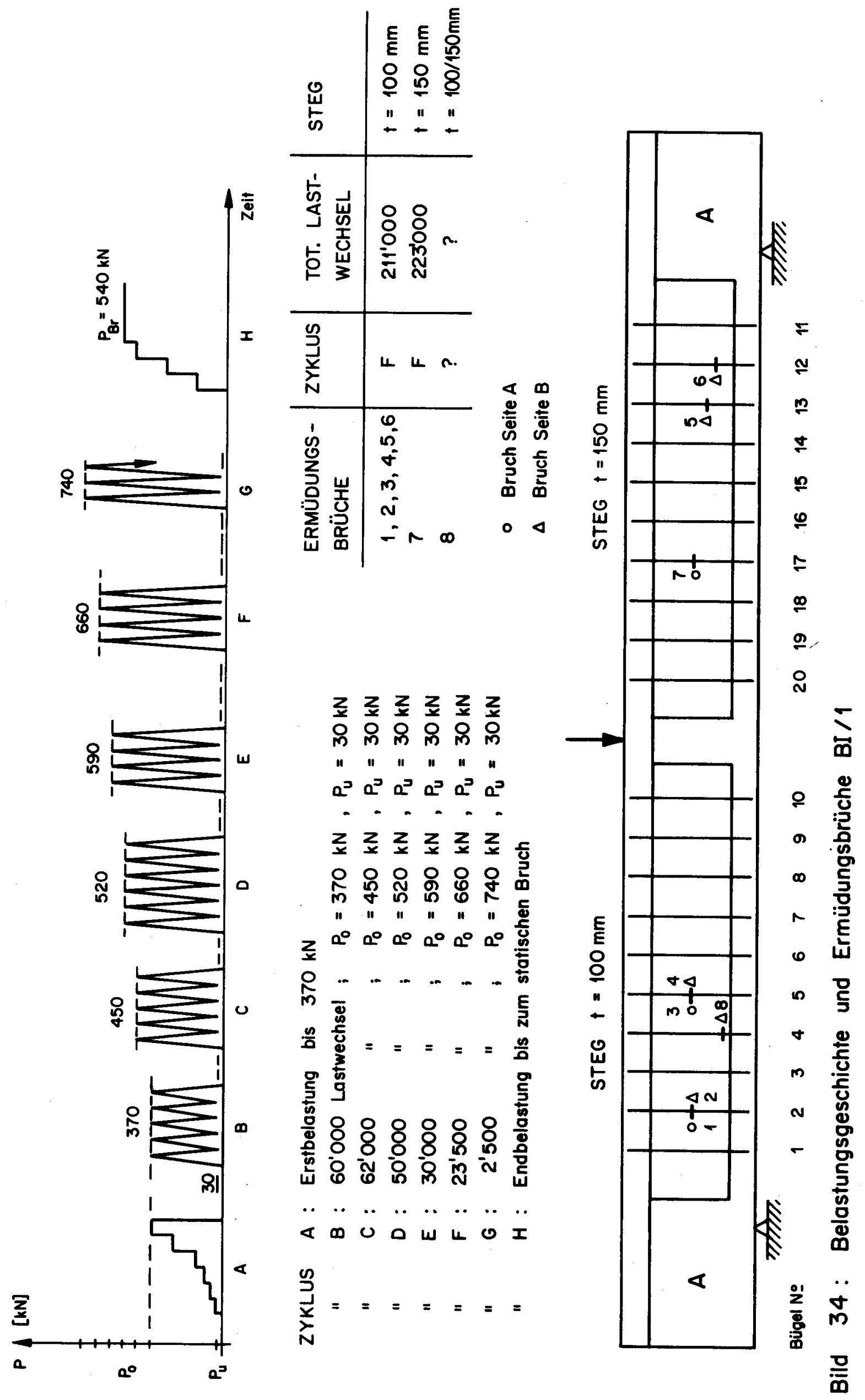



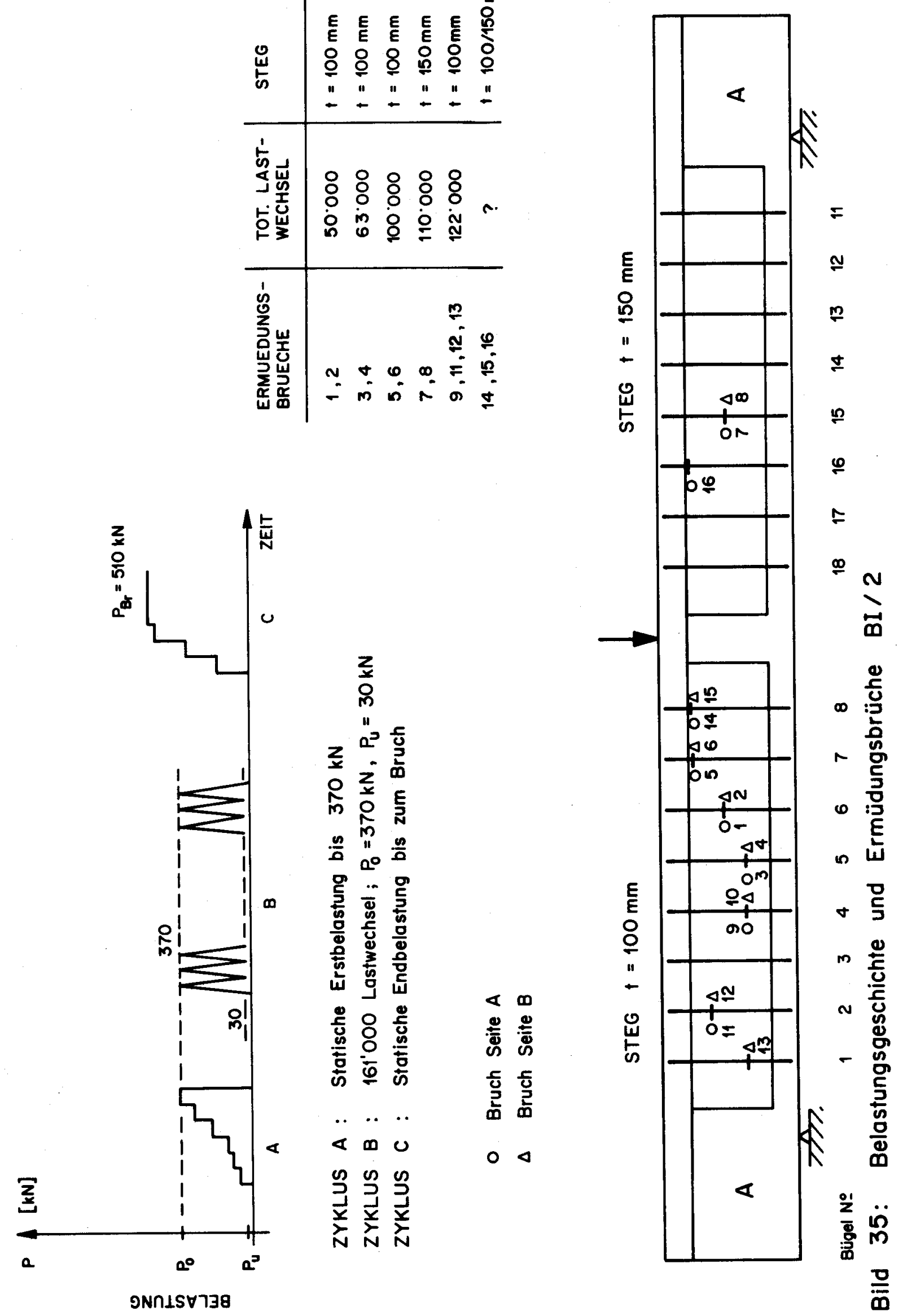


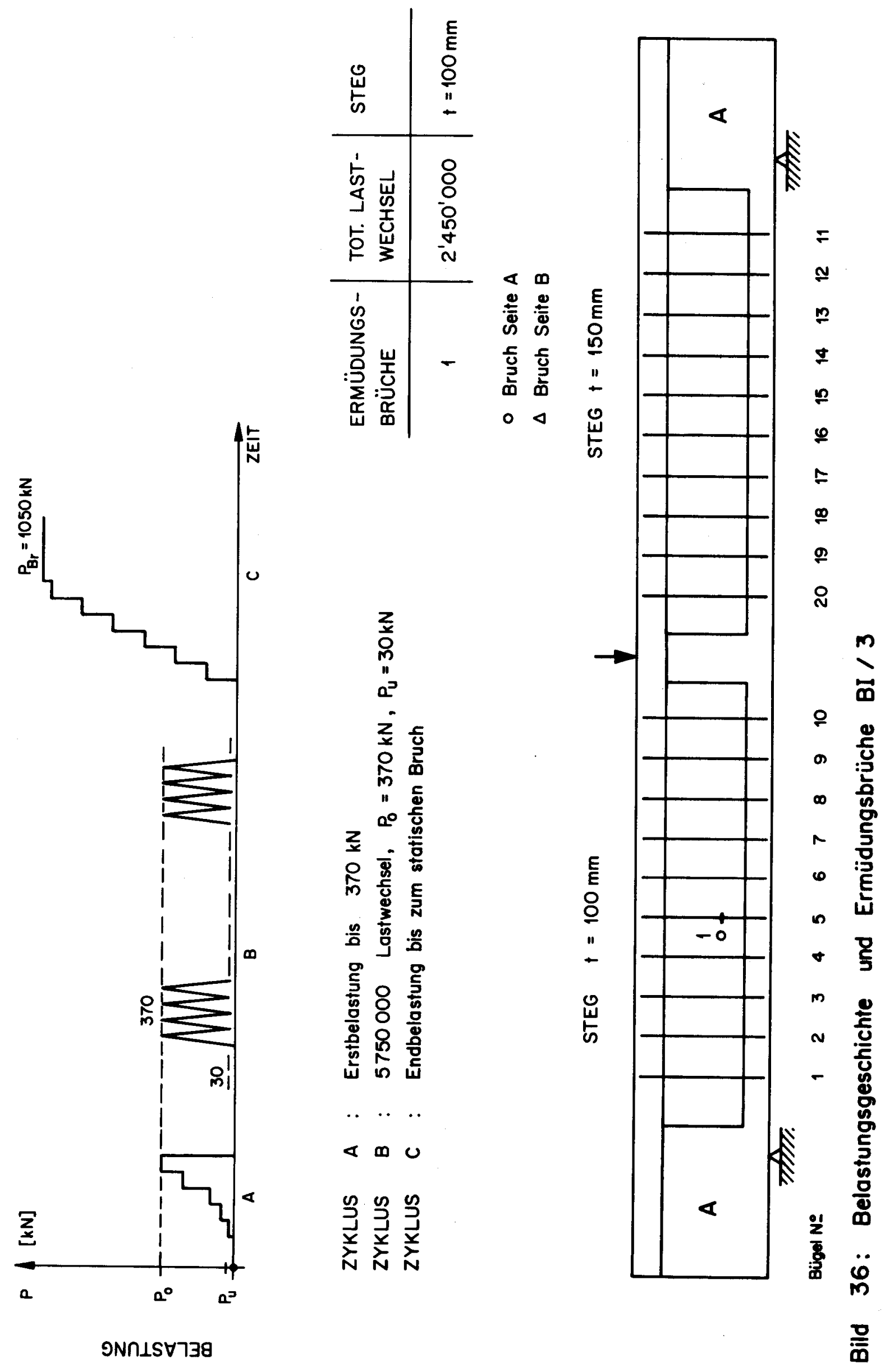



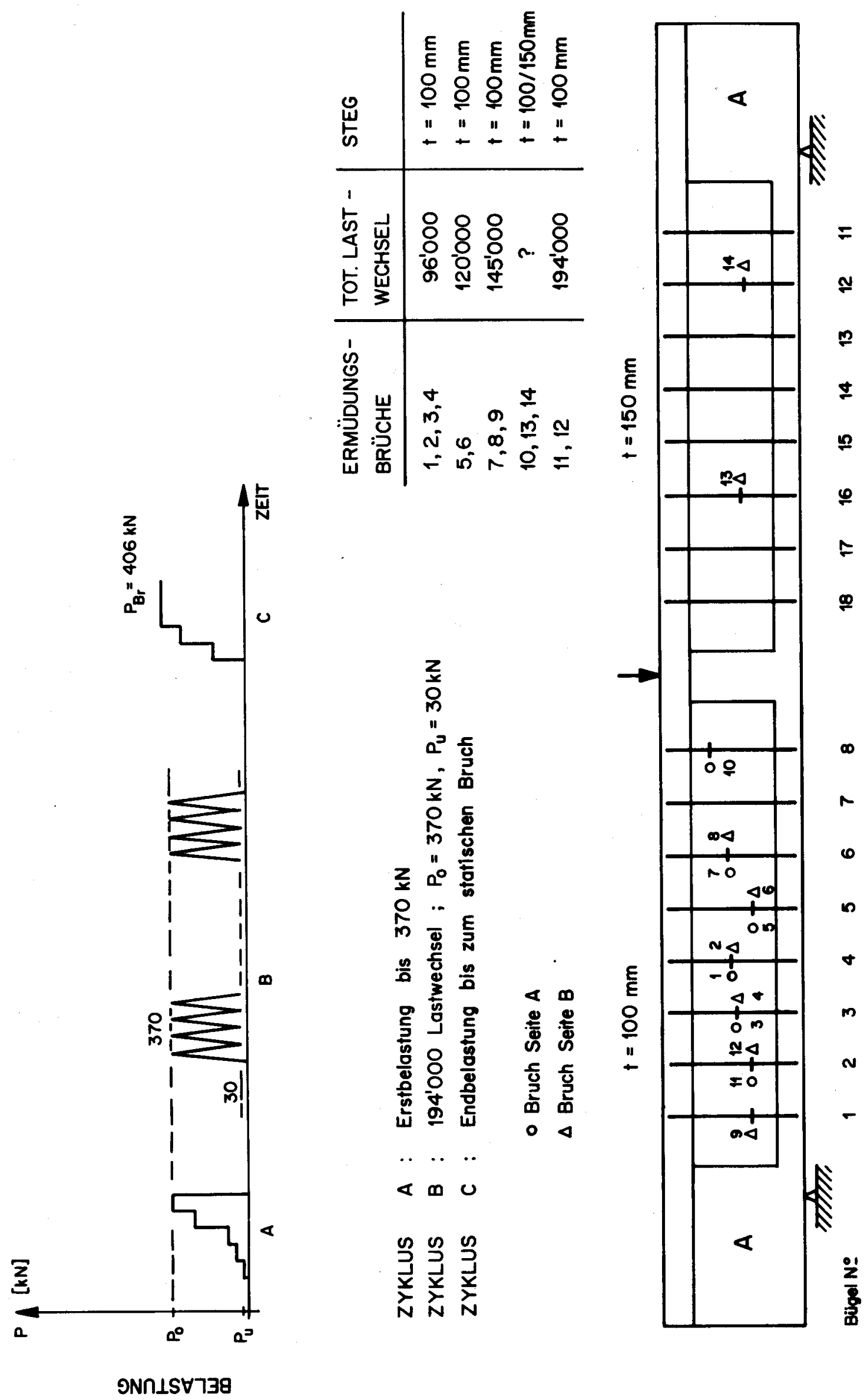

ธ

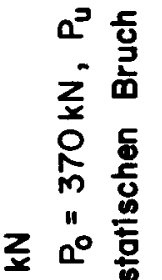

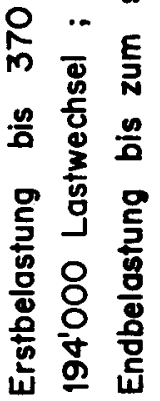

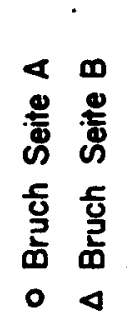

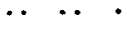

$\varangle \infty u$

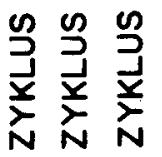

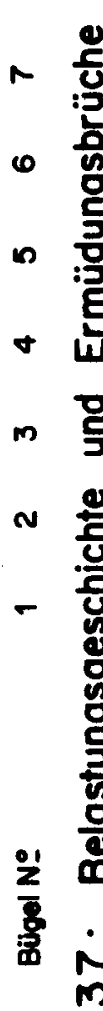

产 

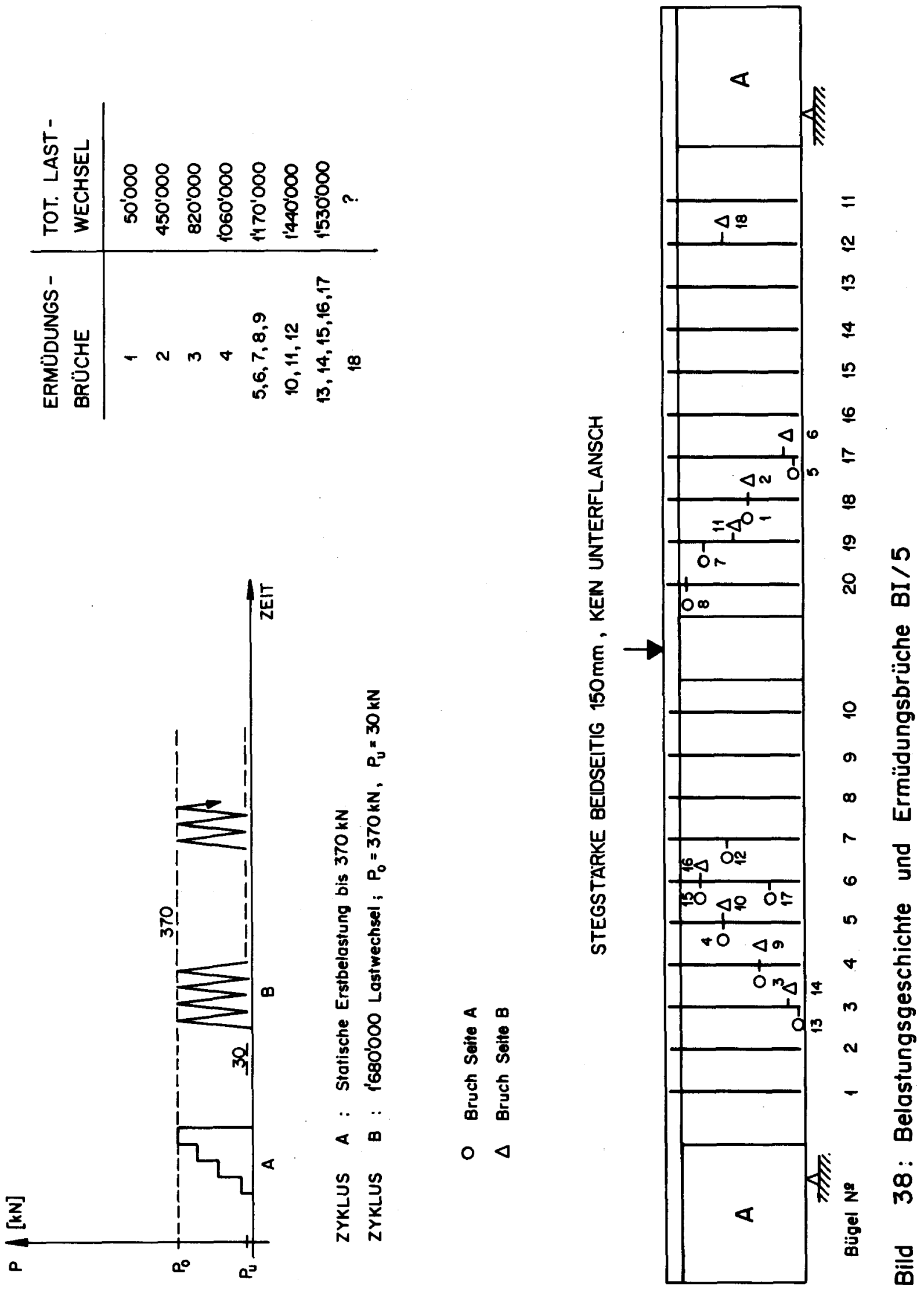

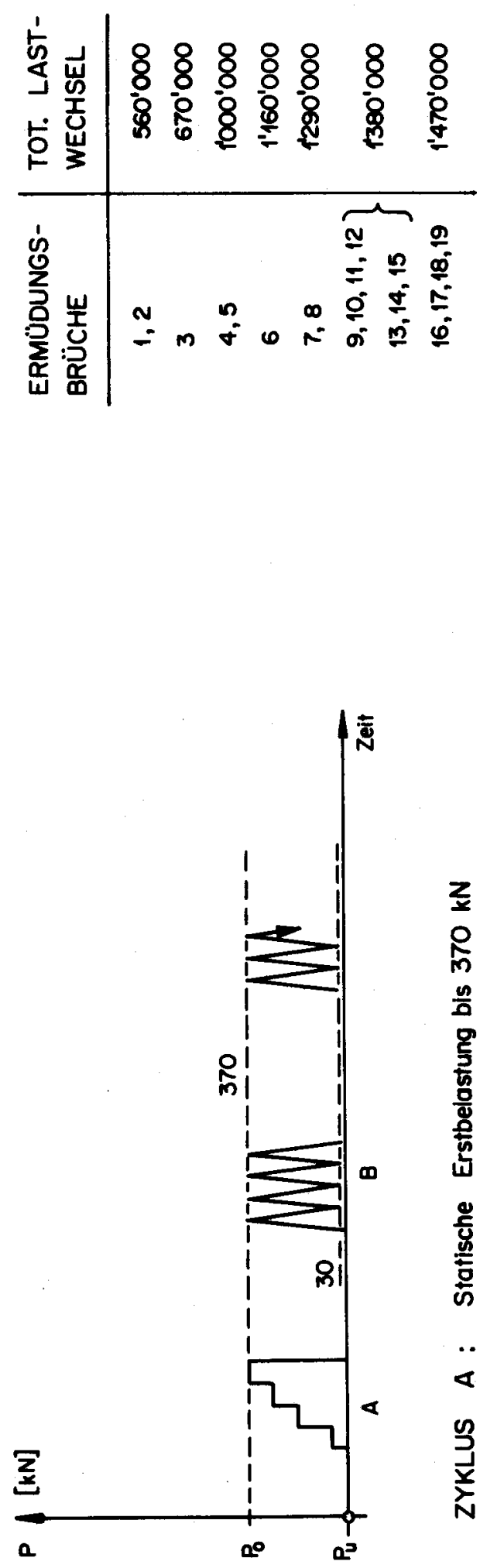

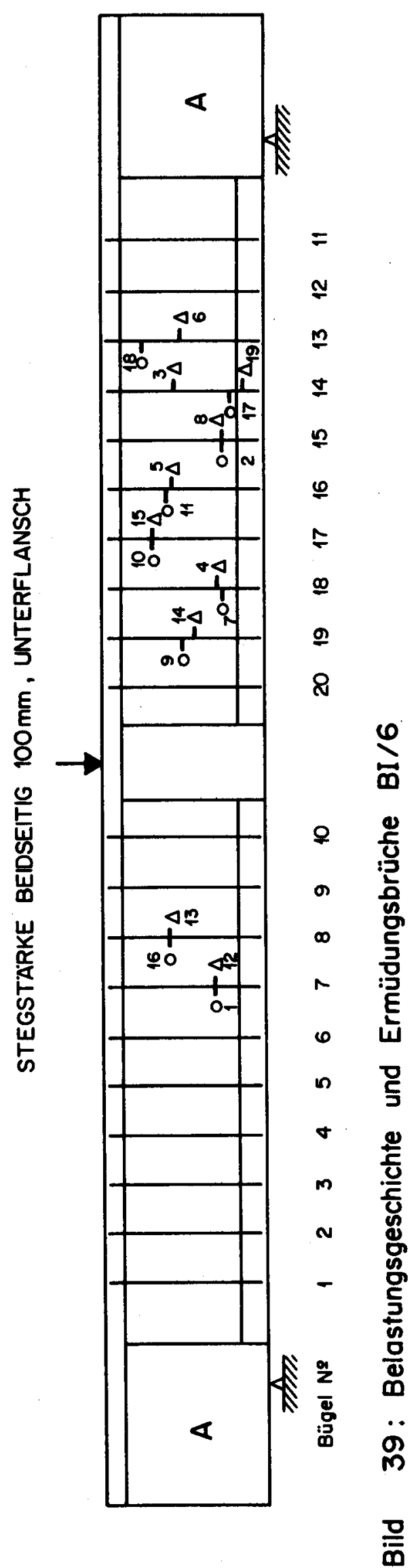




\begin{tabular}{|c|c|c|c|c|c|c|c|c|c|c|c|c|}
\hline $\begin{array}{l}\text { Bügel № } \\
\text { Bruch № }\end{array}$ & \multicolumn{2}{|c|}{$\begin{array}{l}4 \\
3\end{array}$} & \multicolumn{2}{|c|}{$\begin{array}{l}5 \\
4\end{array}$} & \multicolumn{2}{|c|}{$\begin{array}{l}6 \\
15\end{array}$} & \multicolumn{2}{|c|}{$\begin{array}{c}7 \\
12\end{array}$} & \multicolumn{2}{|c|}{$\begin{array}{l}19 \\
7\end{array}$} & \multicolumn{2}{|c|}{$\begin{array}{c}18 \\
1\end{array}$} \\
\hline $\begin{array}{l}\text { Anzahl Last- } \\
\text { wechsel } N\end{array}$ & $\begin{array}{r}\Delta \varepsilon \\
{[1]}\end{array}$ & $\varepsilon_{\text {mox }}$ & $\Delta \varepsilon$ & $\begin{array}{l}\mid \varepsilon_{\max } \\
\left.10^{-3}\right]\end{array}$ & $\begin{array}{r}\Delta \varepsilon \\
{[1}\end{array}$ & $\begin{array}{l}\mid \varepsilon_{\max } \\
\left.0^{-3}\right]\end{array}$ & $\begin{array}{r}\Delta \varepsilon \\
{[1}\end{array}$ & $\varepsilon_{\max }$ & & $\begin{array}{l}{\left[\varepsilon_{\operatorname{mox}}\right.} \\
\left.0^{-3}\right]\end{array}$ & & $\begin{array}{l}\mid \varepsilon_{\max } \\
\left.0^{-3}\right]\end{array}$ \\
\hline 1 & 0.90 & 1.38 & 0.67 & 1.05 & 0.25 & 0.50 & 0.71 & 1.07 & 0.82 & 1.25 & 1.27 & 1.75 \\
\hline $5 \cdot 10^{3}$ & 1.04 & 1.55 & 0.94 & 1.29 & 0.52 & 0.78 & 0.90 & 1.25 & 1.39 & 2.02 & 2.01 & 248 \\
\hline $50 \cdot 10^{3}$ & 1.05 & 1.57 & 0.99 & 1.42 & 0.55 & 1.54 & 0.90 & 1.30 & 1.42 & 2.28 & 1.43 & 1.97 \\
\hline $0.45 \cdot 10^{6}$ & 1.00 & 1.59 & 1.02 & 1.59 & 0.42 & 0.68 & 0.83 & 1.35 & 1.61 & 2.99 & & * \\
\hline $0.88 \cdot 10^{6}$ & 0.96 & 1.60 & 1.03 & 1.65 & 0.39 & 0.62 & 0.81 & 1.33 & 1.97 & 3.63 & & \\
\hline $1.06 \cdot 10^{6}$ & & ; & 1.31 & 2.14 & 0.51 & 0.75 & 0.90 & 1.49 & 1.98 & 3.91 & & \\
\hline $1.44 \cdot 10^{6}$ & & & & * & 0.85 & 1.26 & 0.99 & 1.73 & & * & & \\
\hline $1.53 \cdot 10^{6}$ & & & & & 1.35 & 1.78 & & * & & & & \\
\hline $1.68 \cdot 10^{8}$ & & & & & & & & & & & & \\
\hline
\end{tabular}

- Bügel gebrochen

Messbasis $100 \mathrm{~mm}$

Bild 40: BI/5, Gemessene max. Dehnungen und Dehnungsdifferenzen der Bügel bis zum Bruch im Lastzyklus $B$

\begin{tabular}{|c|c|c|c|c|c|c|c|c|c|c|c|c|c|c|}
\hline $\begin{array}{l}\text { Bügel № } \\
\text { Bruch № }\end{array}$ & & 7 & & 6 & & $\begin{array}{l}19 \\
9\end{array}$ & 1 & 8 & & $\begin{array}{l}7 \\
0\end{array}$ & & $\begin{array}{l}16 \\
11\end{array}$ & & $\begin{array}{l}13 \\
18\end{array}$ \\
\hline $\begin{array}{l}\text { Anzahl Lost- } \\
\text { wechsel N }\end{array}$ & $\begin{array}{r}\Delta \varepsilon \\
{[1}\end{array}$ & $\begin{array}{l}\varepsilon_{\max } \\
\left.0^{-3}\right]\end{array}$ & $\Delta \varepsilon$ & $\begin{array}{l}\varepsilon_{\max } \\
\left.0^{-3}\right]\end{array}$ & $\begin{array}{r}\Delta \varepsilon \\
{[1}\end{array}$ & $\begin{array}{l}\mid \varepsilon_{\max } \\
\left.0^{-3}\right]\end{array}$ & $\begin{array}{r}\Delta \varepsilon \\
{[x}\end{array}$ & $\begin{array}{l}\varepsilon_{\max } \\
\left.\partial^{-5}\right]\end{array}$ & $\begin{array}{r}\Delta \varepsilon \\
{[1}\end{array}$ & $\begin{array}{l}\mid \varepsilon_{\max } \\
\left.10^{-3}\right]\end{array}$ & $\begin{array}{r}\Delta \varepsilon \\
{[1}\end{array}$ & $\begin{array}{c}{\left[\varepsilon_{\max }\right.} \\
\left.10^{-3}\right]\end{array}$ & & $\begin{array}{l}{\left[\varepsilon_{\max }\right.} \\
\left.0^{-3}\right]\end{array}$ \\
\hline 1 & 1.03 & 1.59 & 0.85 & 1.35 & 0.11 & 0.25 & 0.13 & 0.23 & 0.40 & 0.78 & 0.59 & 0.96 & 0.82 & 1.29 \\
\hline $5 \cdot 10^{3}$ & 1.30 & 1.83 & 0.98 & 1.50 & 0.95 & 1.31 & 0.59 & 0.90 & 0.67 & 1.11 & 0.72 & \begin{tabular}{|l|} 
\\
\end{tabular} & 1.04 & 1.51 \\
\hline $5 \cdot 10^{3}$ & 1.22 & 1.86 & 0.87 & $\begin{array}{l}4.44 \\
\end{array}$ & 1.0 & 1.42 & 0.56 & 1.02 & 0.73 & 1.26 & 0.90 & 1.30 & 1.16 & 1.67 \\
\hline $0.15 \cdot 10^{6}$ & 1.26 & 1.90 & 0.89 & 1.49 & 1.0 & 1.41 & 0.61 & 1.05 & 0.76 & 1.27 & 0.89 & $\begin{array}{l}1.29 \\
\end{array}$ & 1.13 & 1.63 \\
\hline $0.56 \cdot 10^{6}$ & 1.25 & 2.07 & 0.91 & 1.58 & 4.0 & 1.48 & 0.65 & 1.14 & 0.85 & 1.38 & 0.94 & 1.49 & 1.17 & 1.73 \\
\hline $1 \cdot 10^{6}$ & & * & 1.19 & 1.99 & 1.10 & 1.55 & 0.64 & 1.22 & 0.93 & 1.55 & 1.31 & 2.31 & 1.17 & 1.73 \\
\hline $1.16 \cdot 10^{6}$ & & & 1.26 & 2.02 & 1.07 & 1.60 & 0.90 & 1.54 & 1.09 & 1.63 & 1.79 & 301 & 1.10 & 1.73 \\
\hline $1.38 \cdot 10^{6}$ & & & 1.19 & 2.01 & 1.46 & 2.09 & 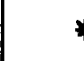 & * & 1.17 & 1.90 & 2.51 & 4.36 & 1.33 & 2.19 \\
\hline $1.47 \cdot 10^{8}$ & & & 1.97 & 3.51 & & * & & & 3 & * & & * & 1.46 & 2.40 \\
\hline $1.54 \cdot 10^{6}$ & & & & & & & & & & & & & & \\
\hline
\end{tabular}

Bild 41: BI/6, Gemessene max. Dehnungen und Dehnungsdifferenzen der Bügel bis zum Bruch im Lastzyklus B 

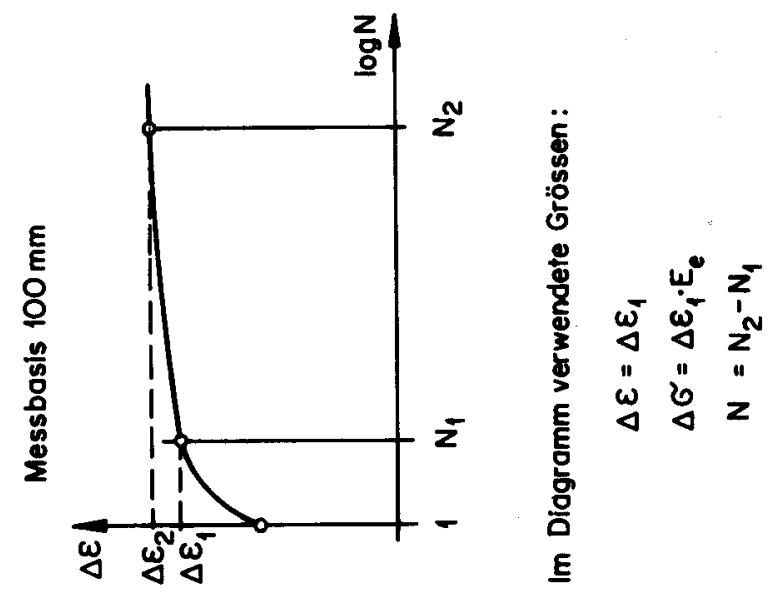

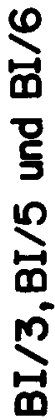

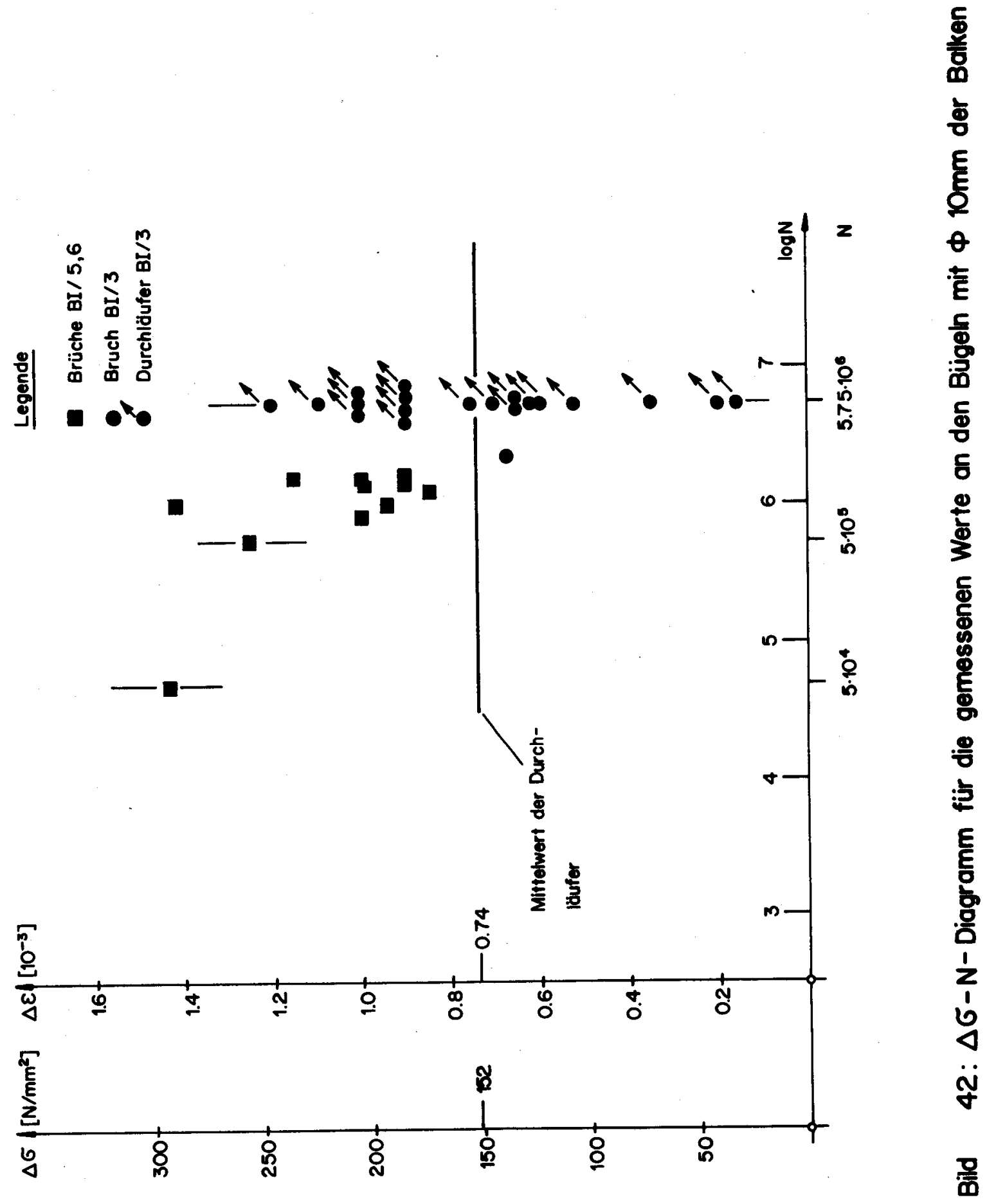




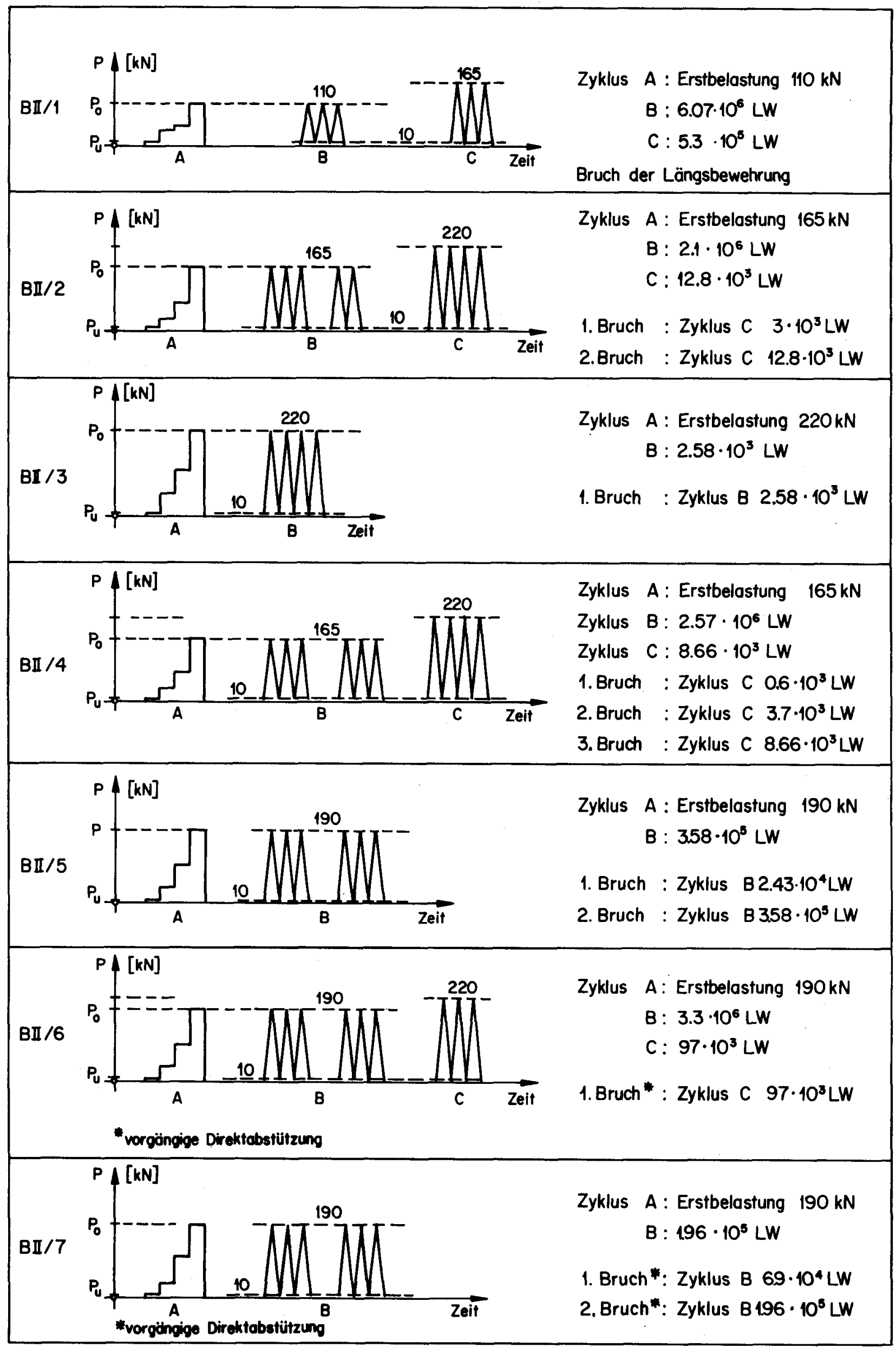

Bild 43: Belastungsgeschichte und Ermüdungsbrüche $\mathrm{BI} / 1$ bis $\mathrm{BI} / 7$ 


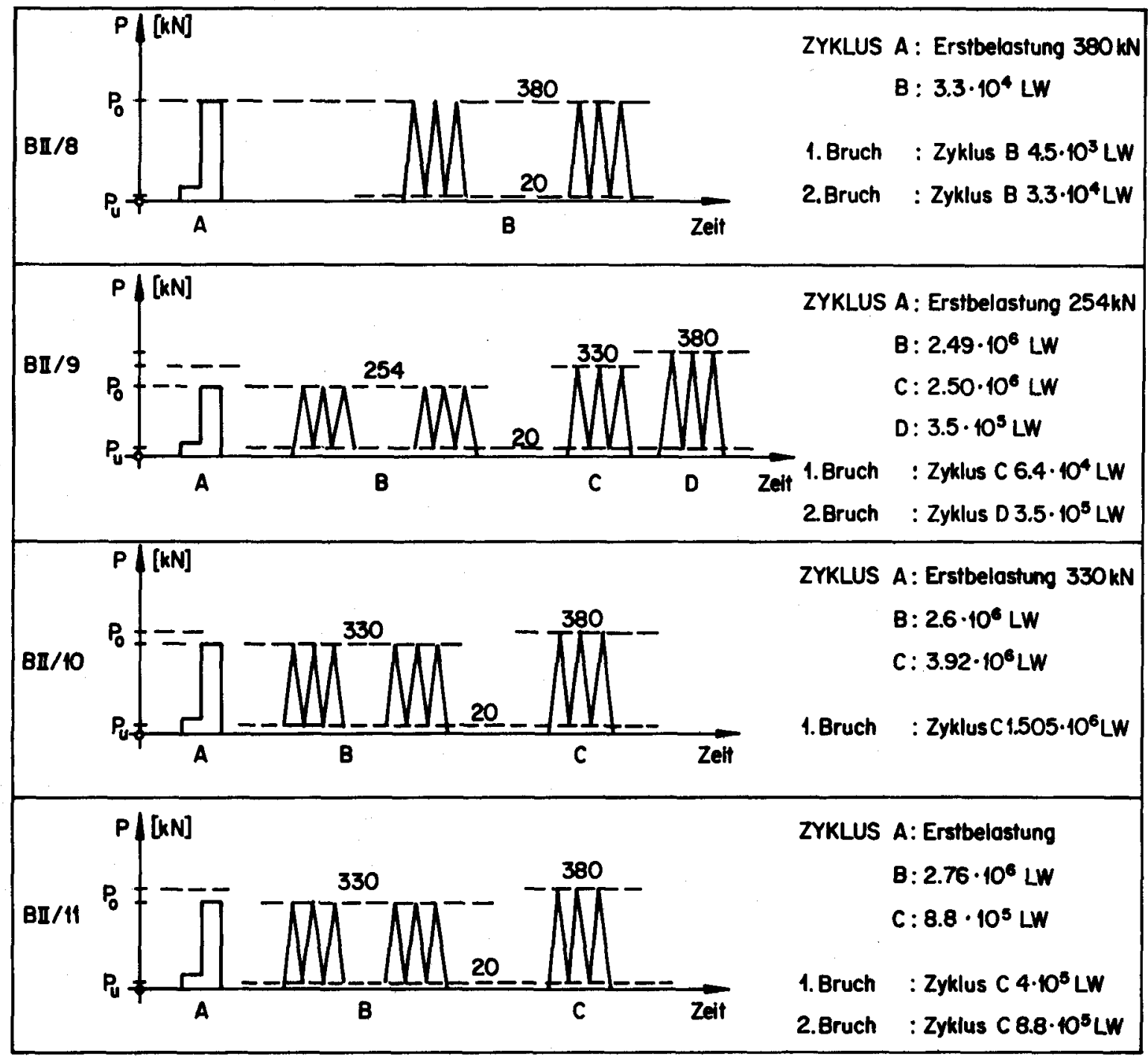

Bild 44 : Belastungsgeschichte und Ermüdungsbrüche $\mathrm{BII} / 8$ bis $\mathrm{BI} / 11$ 


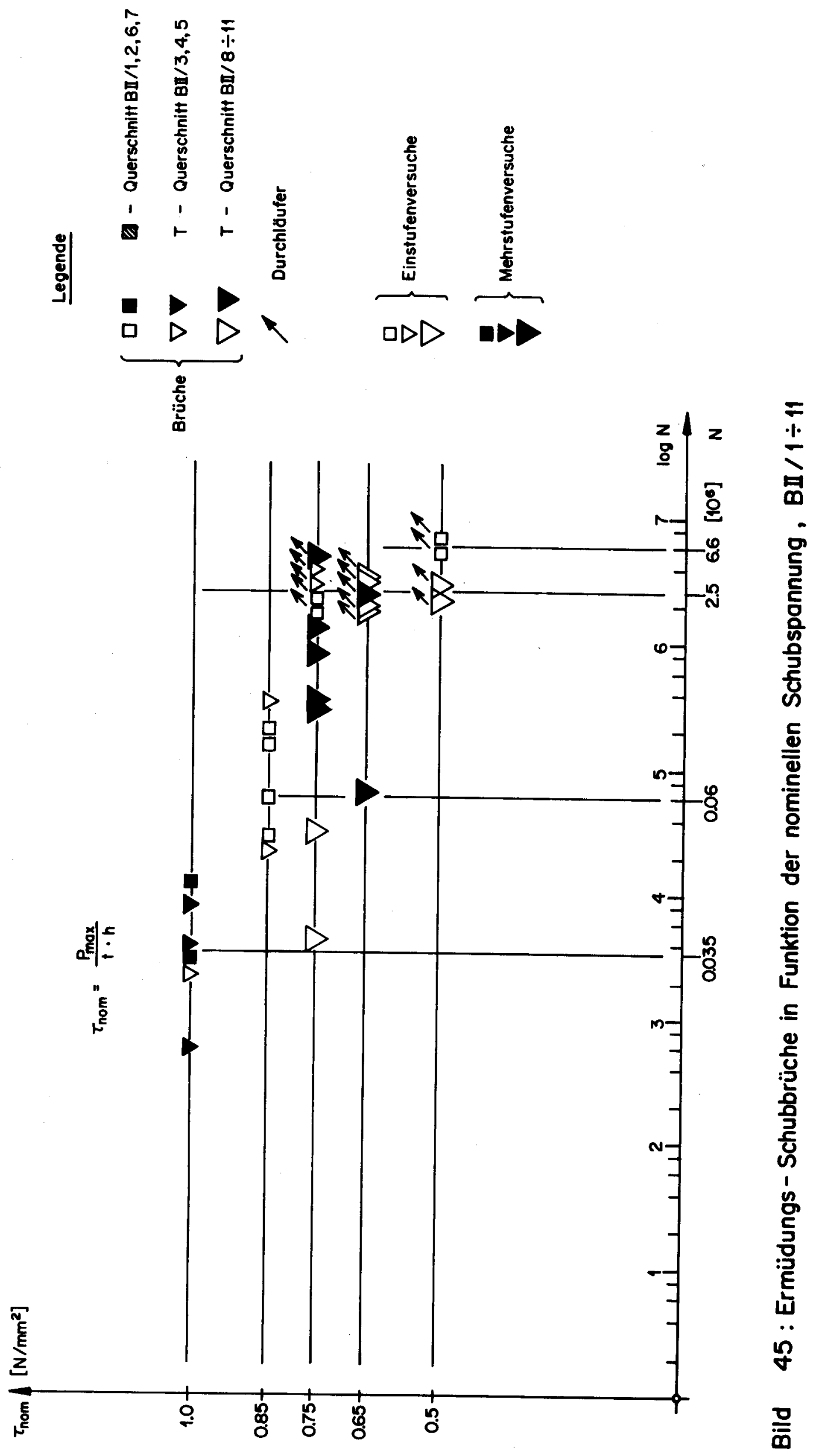



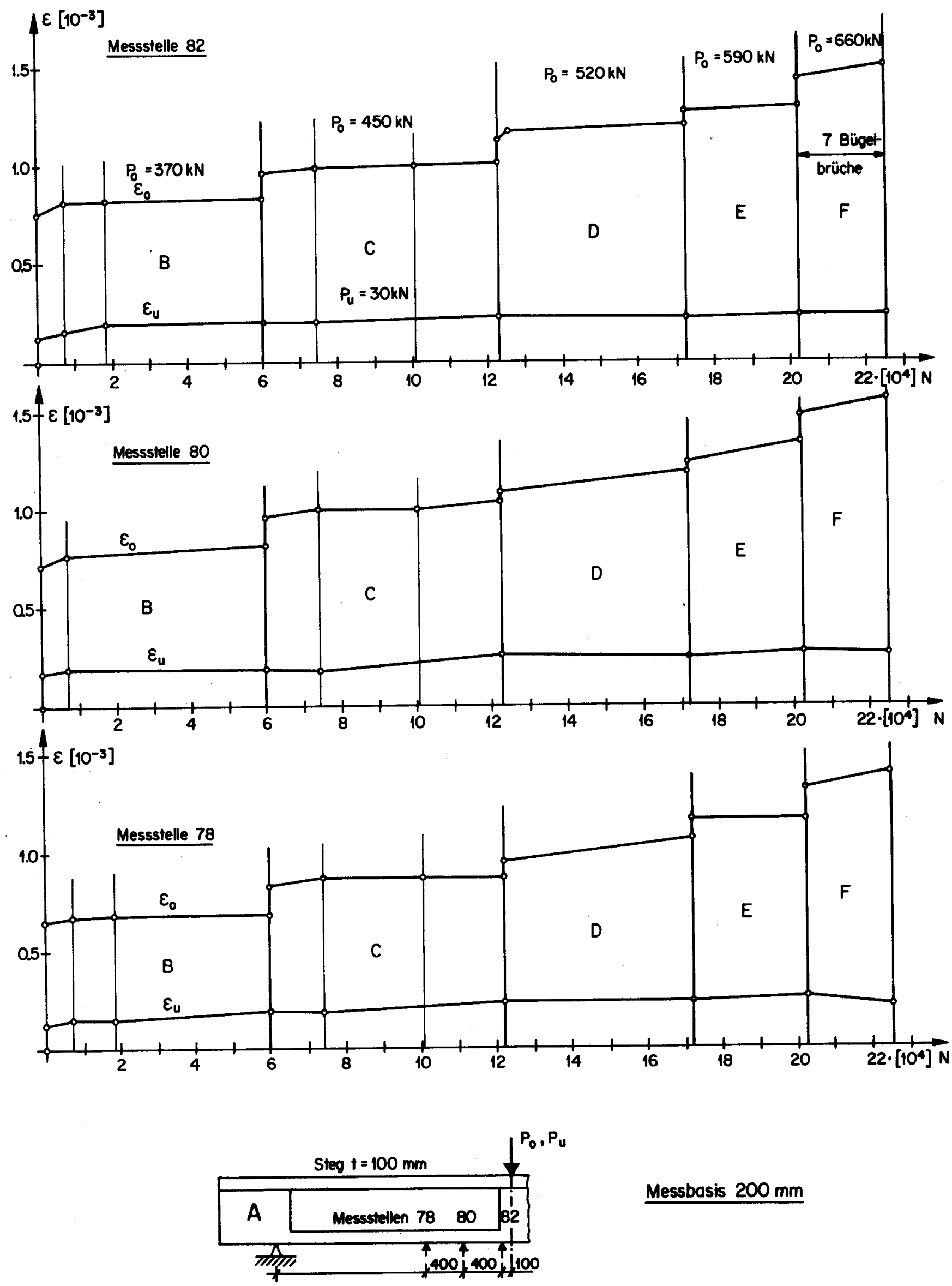

Messbasis $200 \mathrm{~mm}$

Bild 48: BI/1 Dehnungs - Lastwechsel- Diagramme für die Längsbewehrung in den Lastzyklen $B$ bis $F$ 


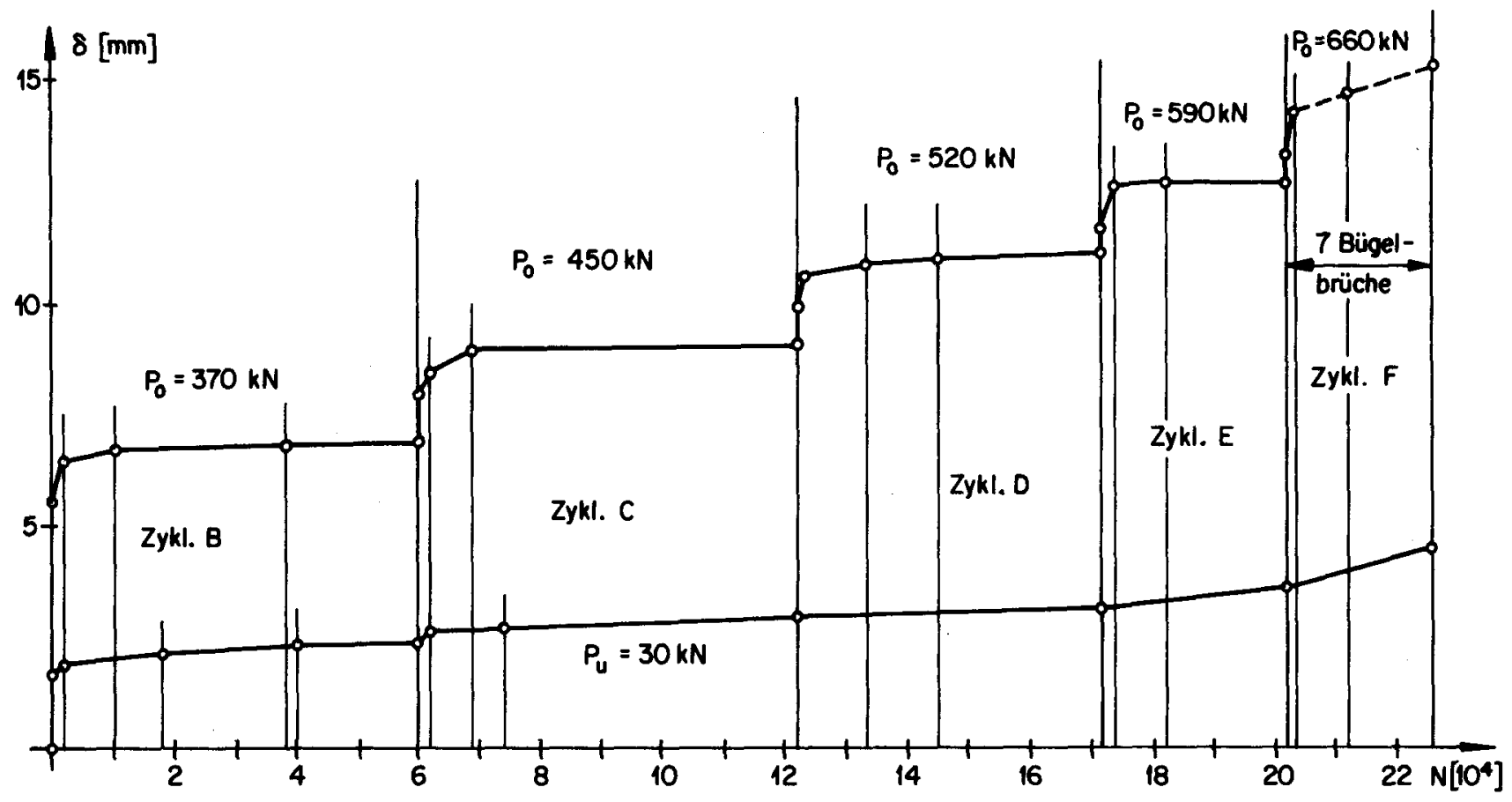

Bild 46 : BI/1 Durchbiegungs - Lastwechsel-Diagramm fïr die Trägermitte in den Lastzyklen B bis F.
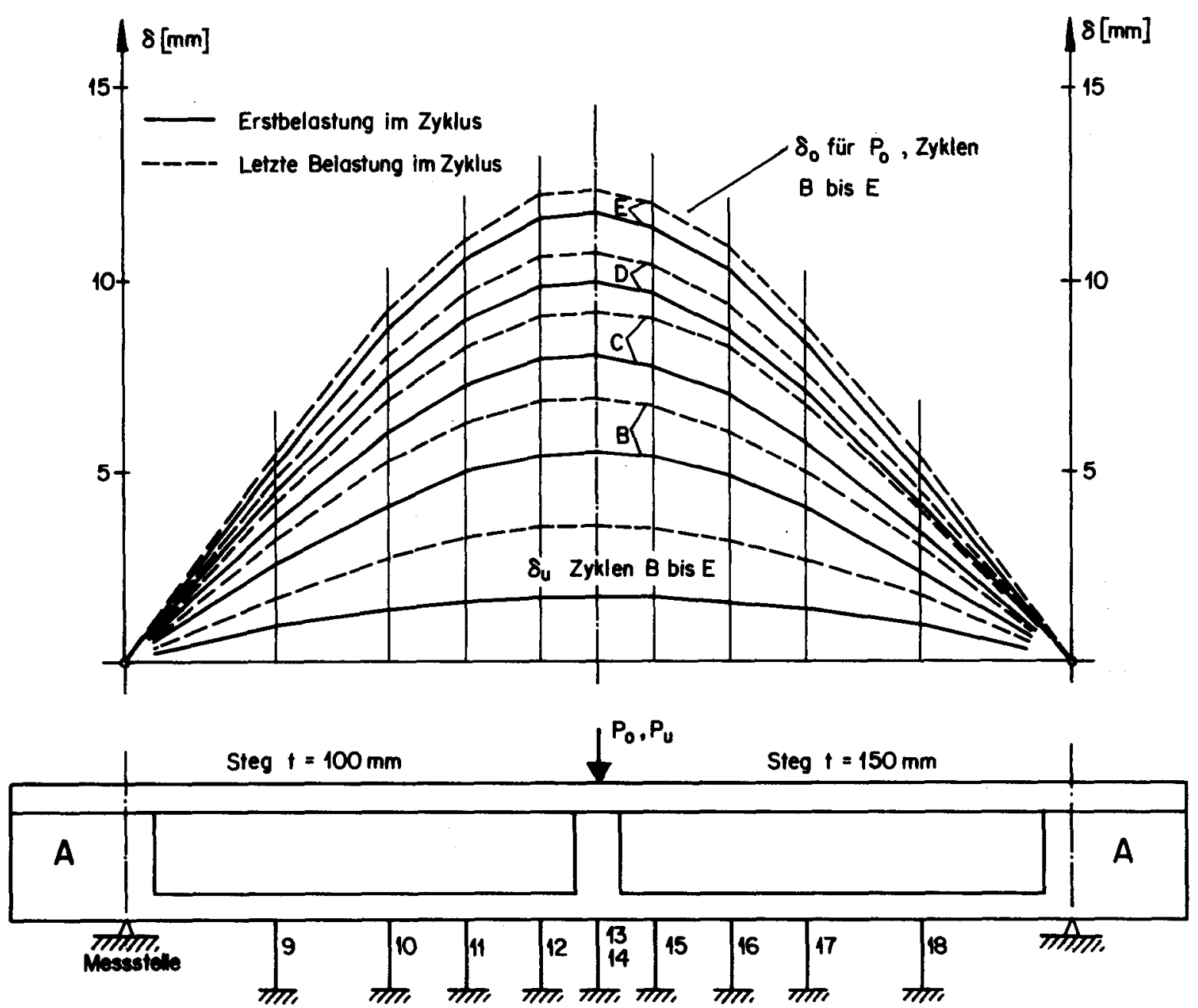

Bild 47 : BI/1, Verlouf der Durchbiegung während der Lastzyklen B bis E unter wachsender Oberlast und konstanter Unterlast 

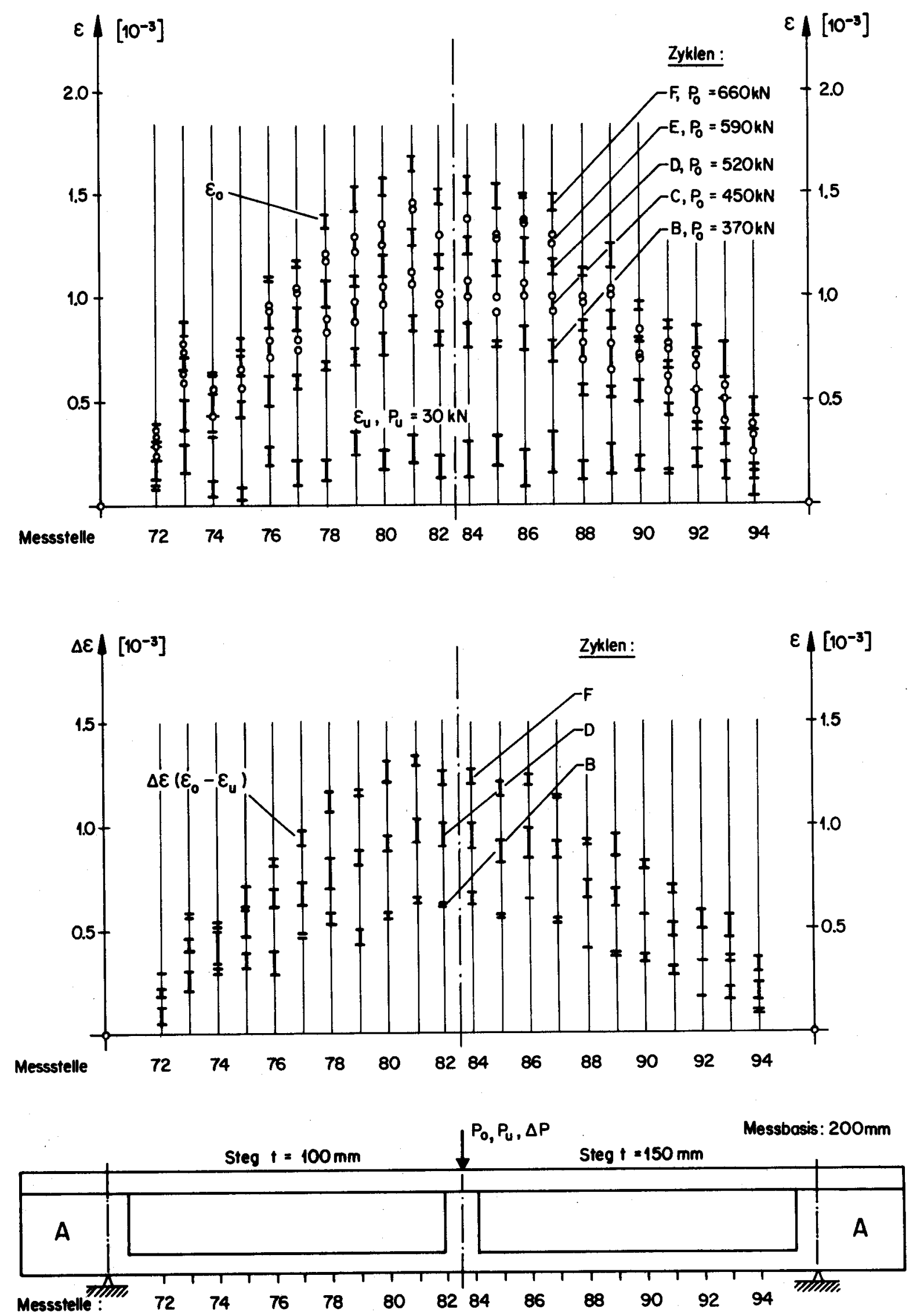

Bild 49 : BI/1, Dehnungen und Dehnungsdifferenzen in der Längsbewehrung in den Lastzyklen $B$ bis $F$ 

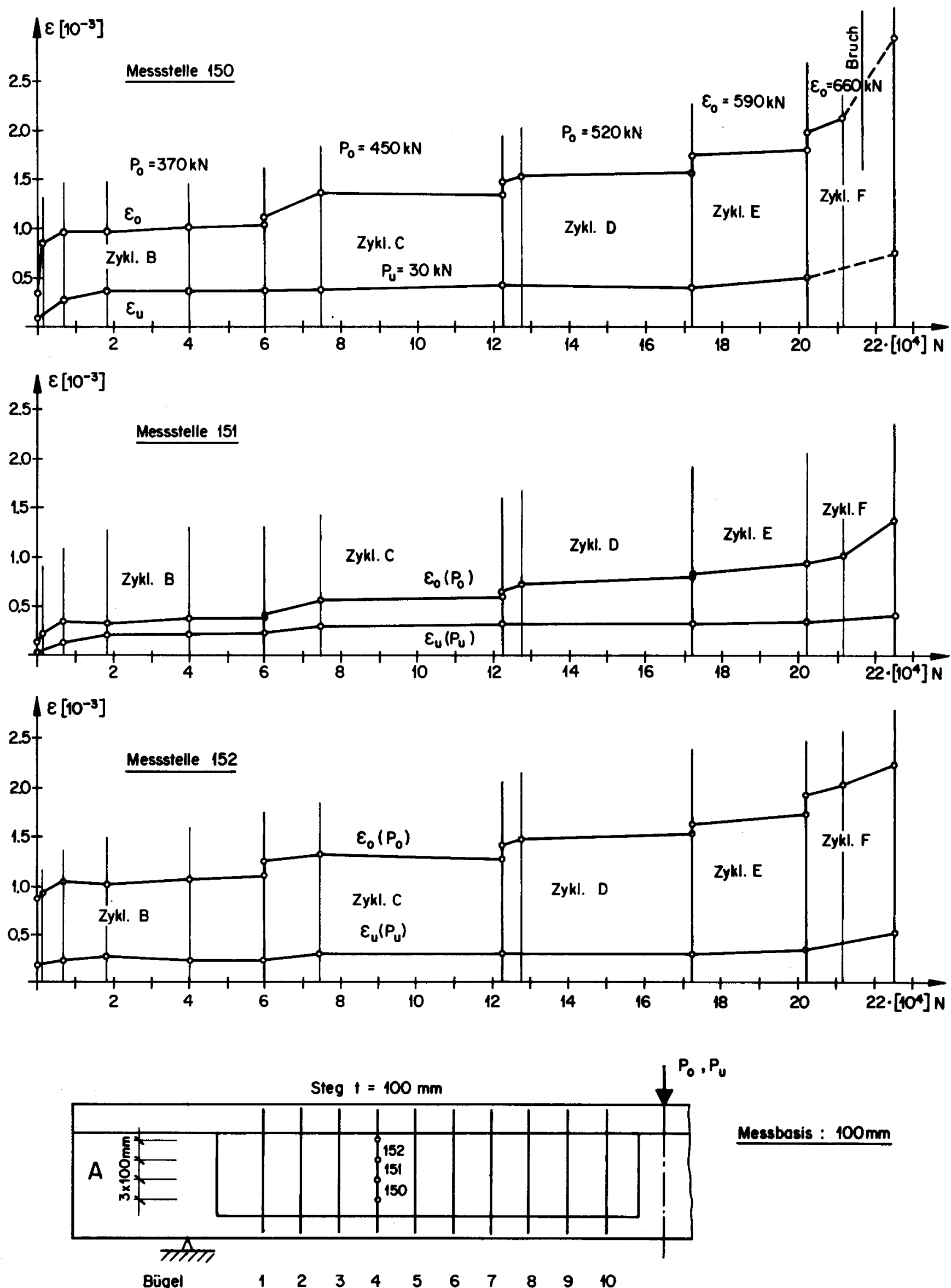

Messbasis : $100 \mathrm{~mm}$

Bild 50: BI/1, Dehnungs - Lastwechsel-Diagramme für den Bügel № 4 in den Lastzyklen $B$ bis $F$ 

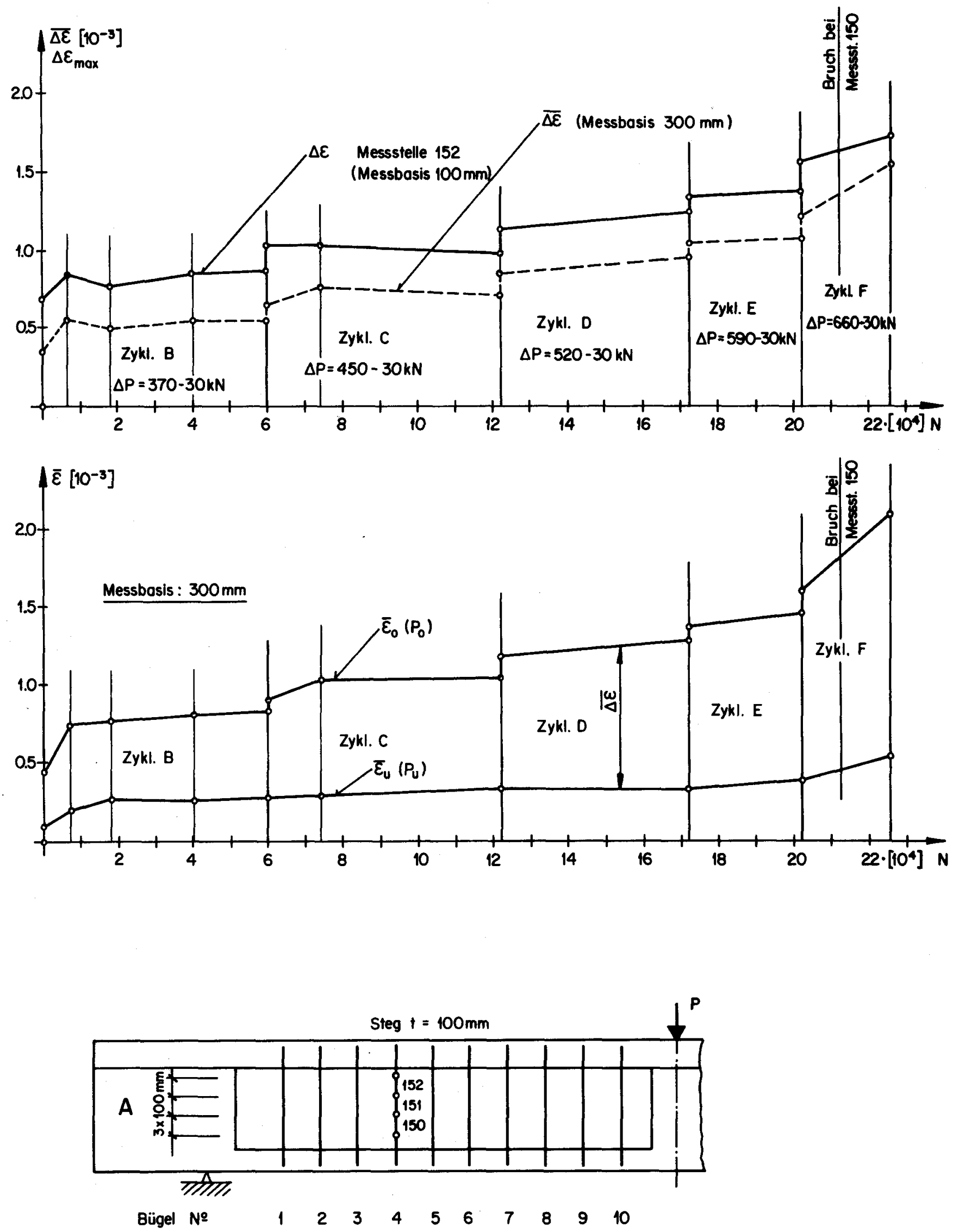

Bild 51: BI/1 Dehnungs - Lastwechsel-Diagramm für den Bügel № 4 in den Lastzyklen $B$ bis $F$, mittlere Bügeldehnung und Dehnungs differenzen 

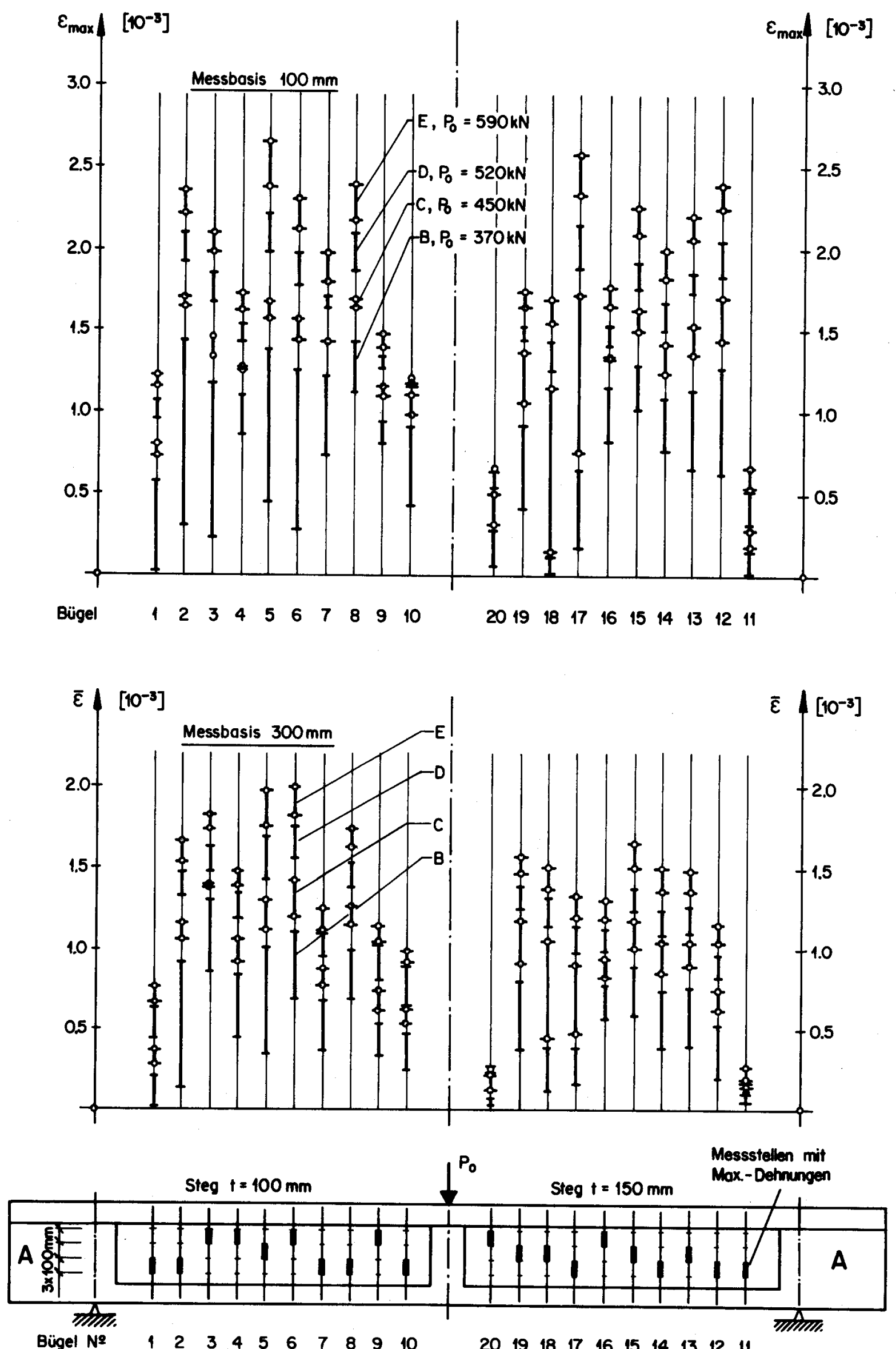

$\begin{array}{lllllllllllllllllllll}\text { Bügel N2 } & 1 & 2 & 3 & 4 & 5 & 6 & 7 & 8 & 9 & 10 & 20 & 19 & 18 & 17 & 16 & 15 & 14 & 13 & 12 & 11\end{array}$

Bild $52: B I / 1$, Bügeldehnungen unter Oberlast in den Lastzyklen $B$ bis $E$ 

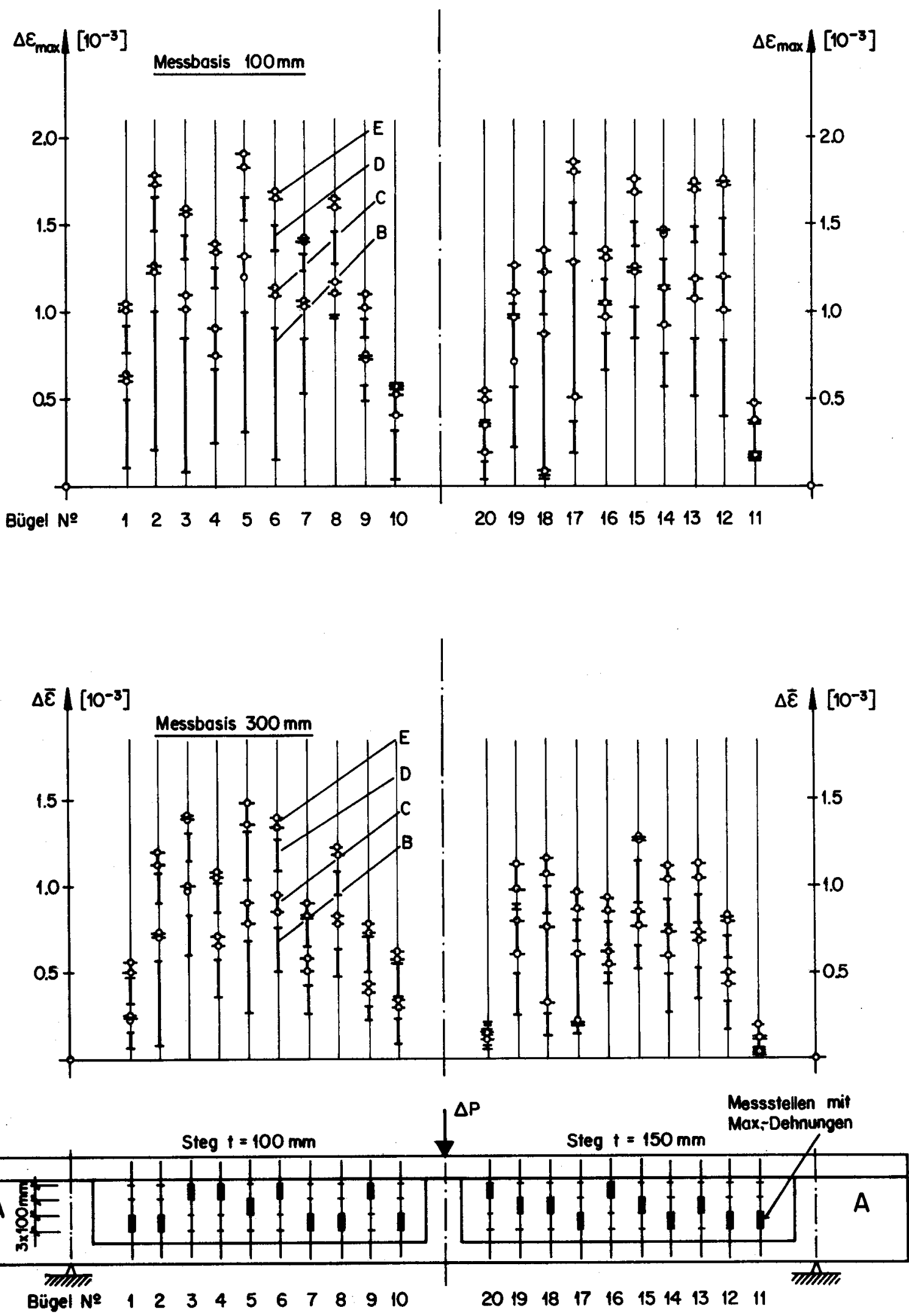

Bild 53: BI/1, Dehnungsdifferenzen in den Buigeln in den Lostzyklen B bis E 


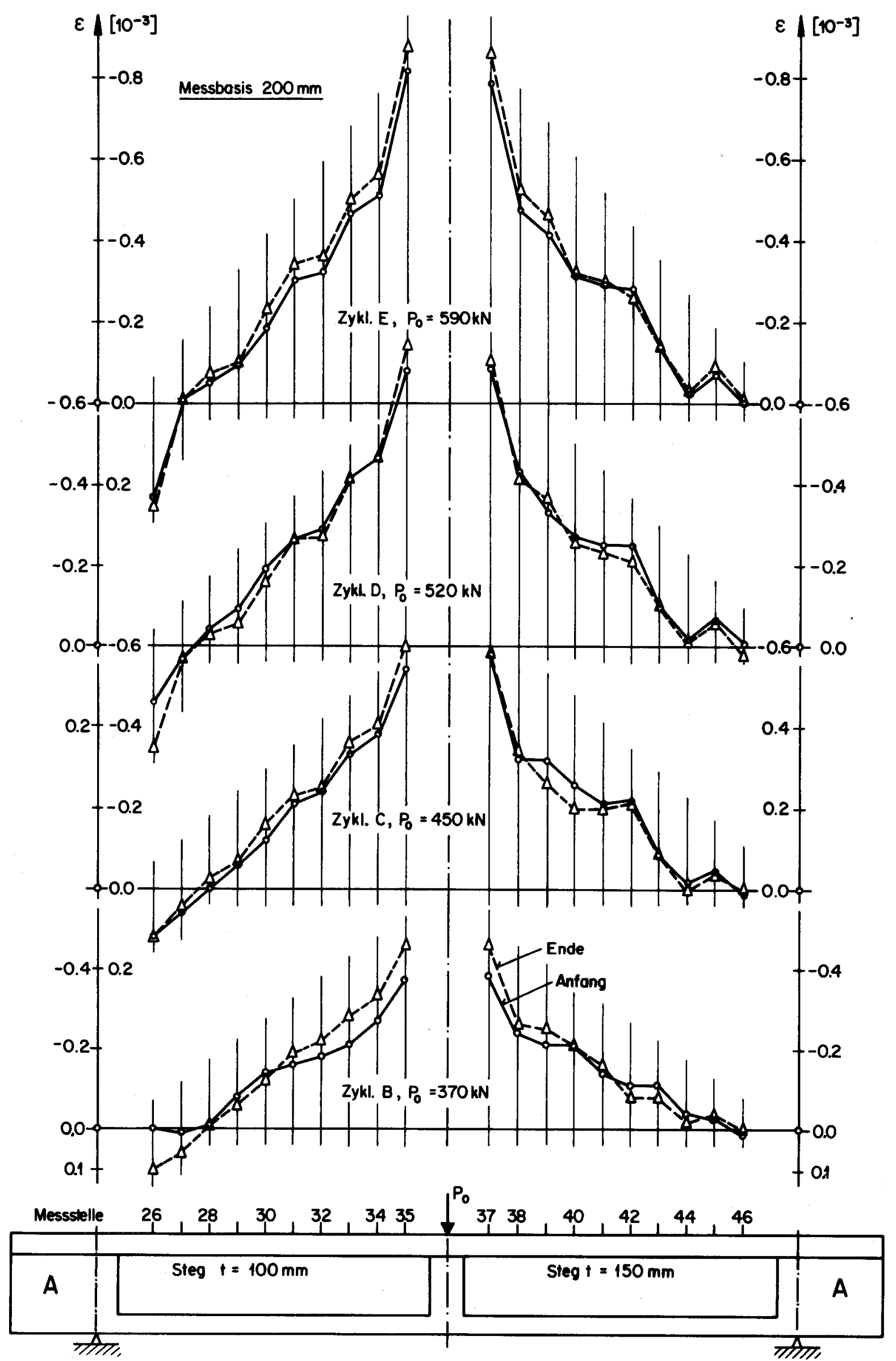

Bild 54: $B I / 1$, Betondehnungen unter Oberlast im Oberflensch in den Lastzyklen $B$ bis $E$ 


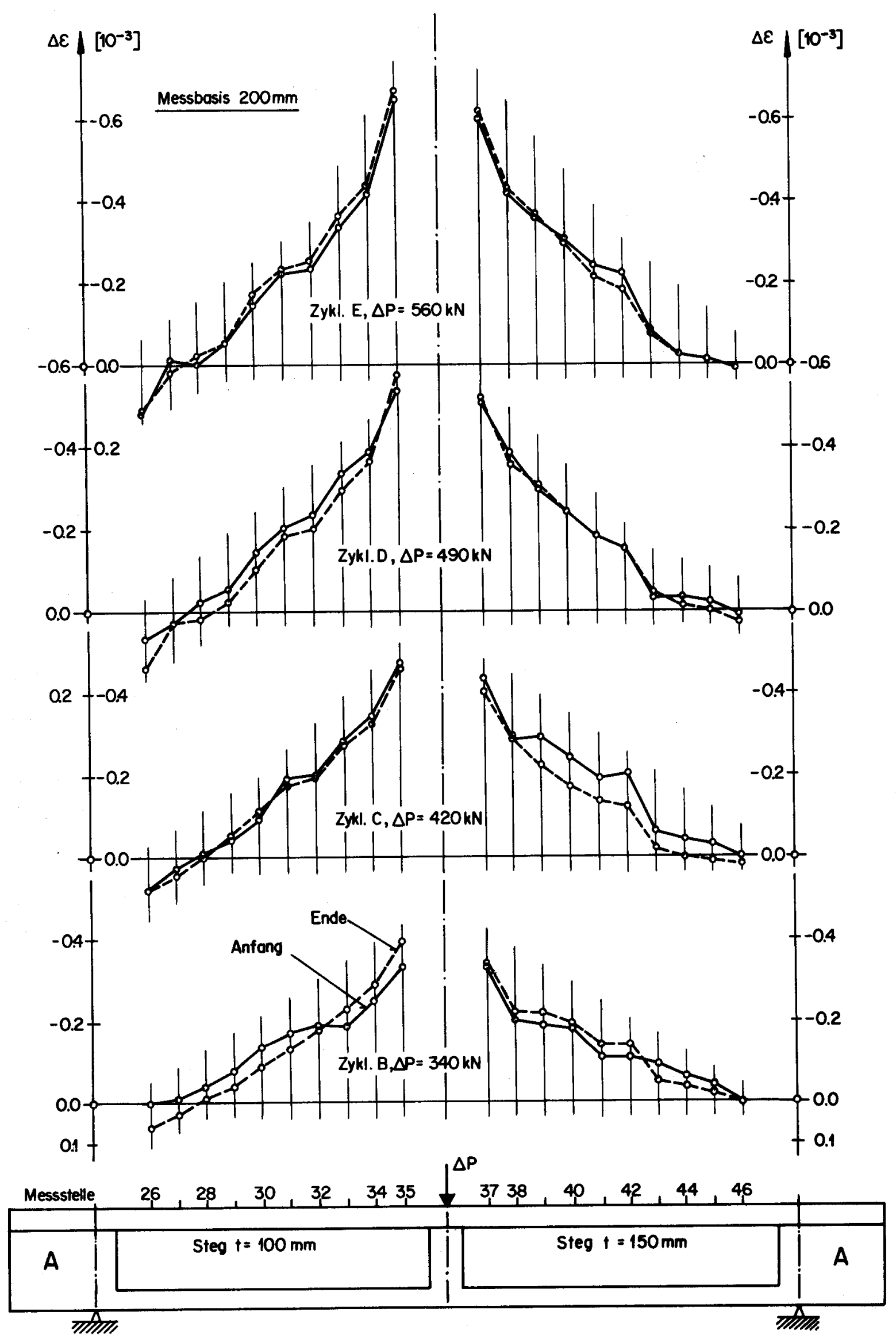

Bild 55: $\mathrm{BI} / 1$, Beton-Dehnungsdifferenzen im Oberflansch in den Lastzyklen $B$ bis $E$ 


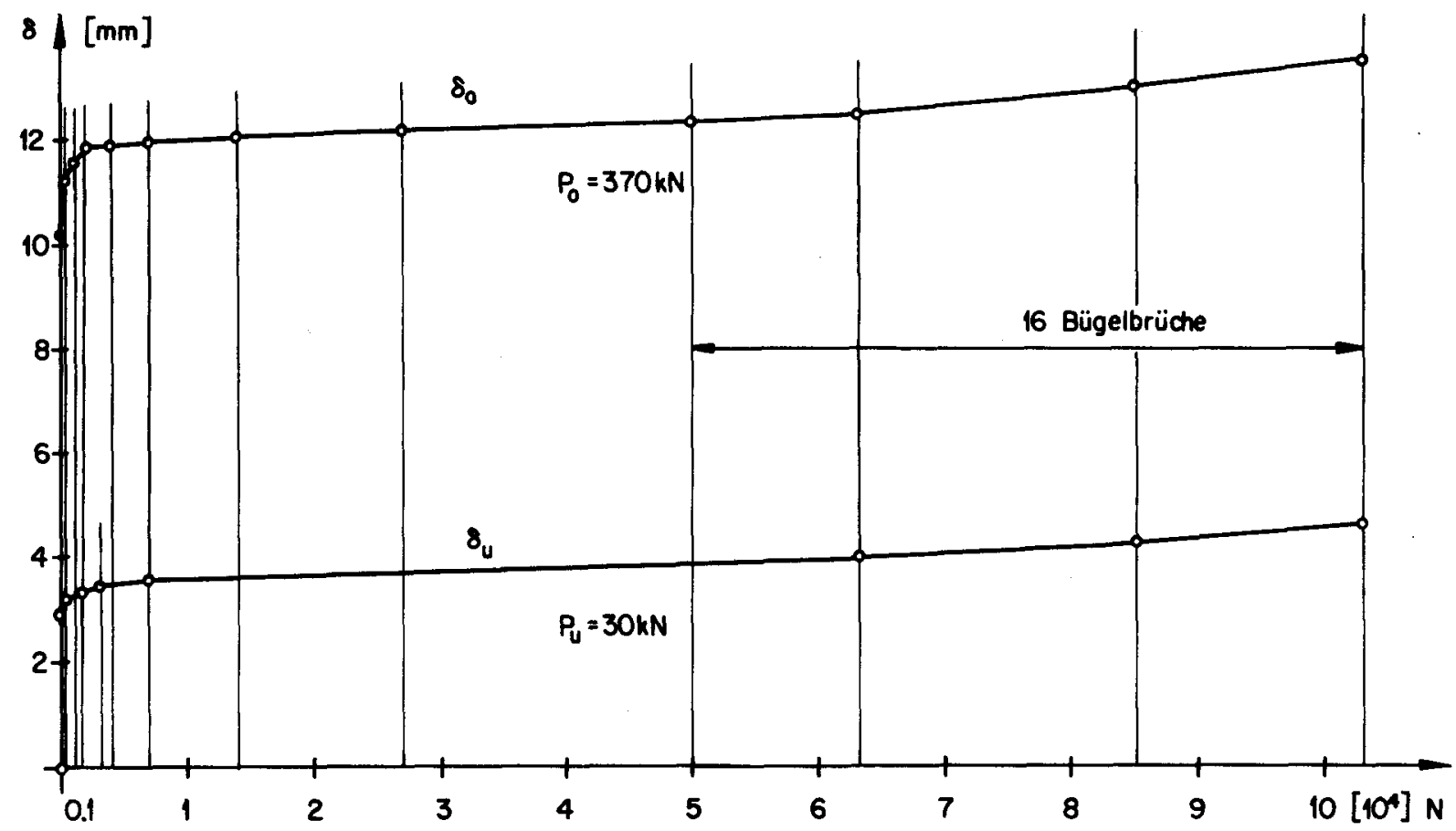

Bild 56: BI/2, Durchbiegungs-Lastwechsel-Diagramm für die Trägermitte im Lastzyklus B
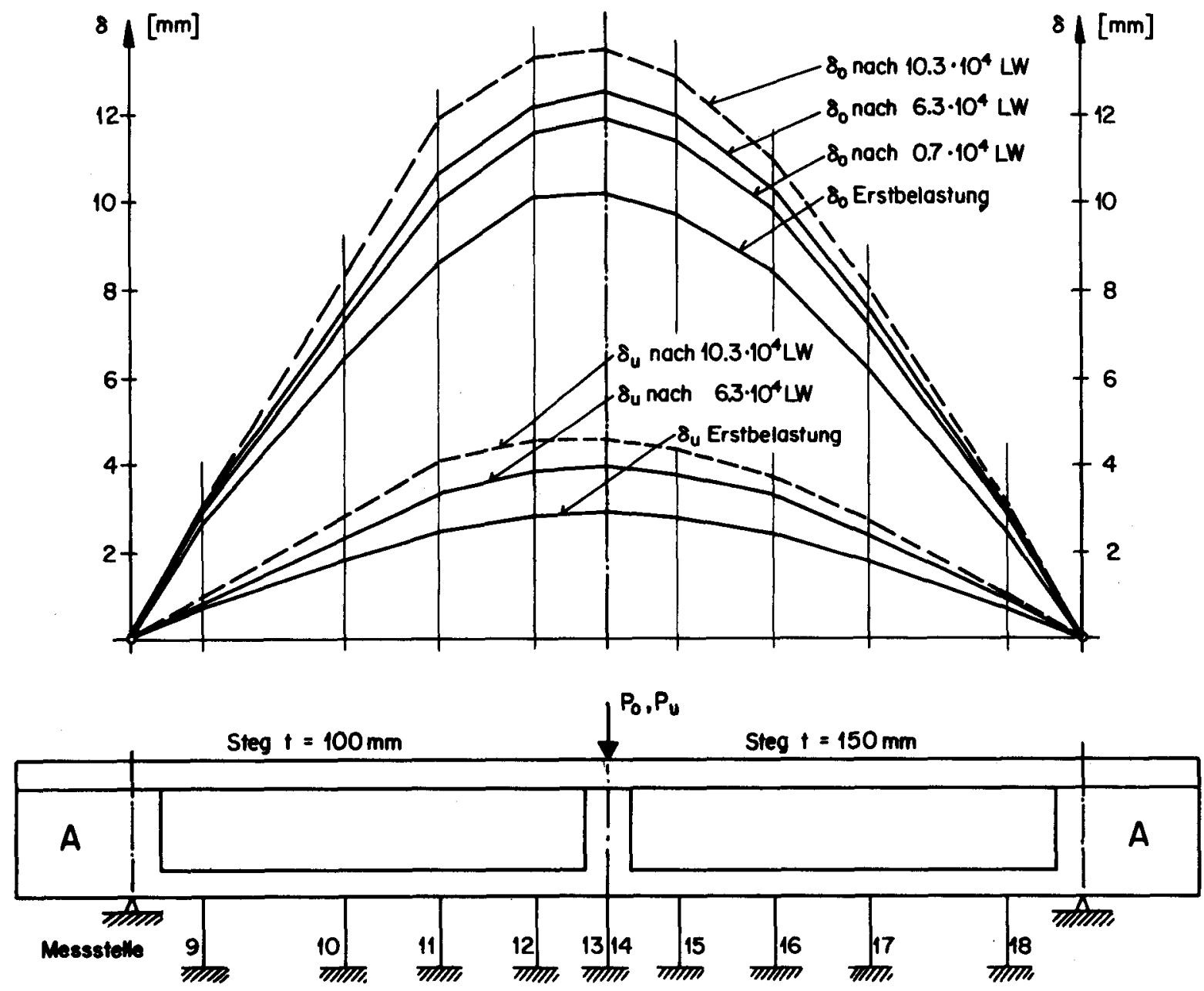

Bild 57: BI/2, Verlauf der Durchbiegung im Zyklus B 

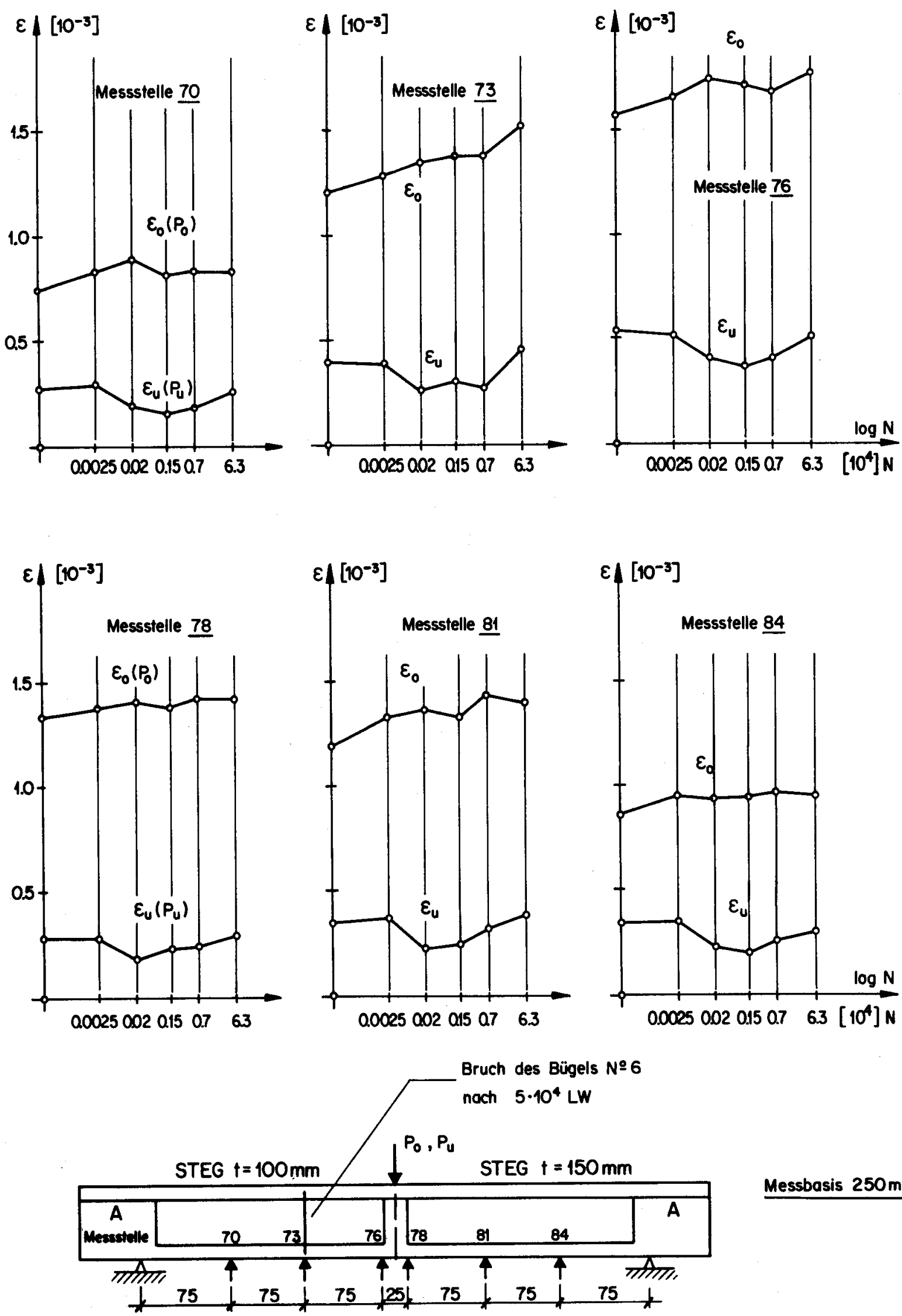

Messbasis $250 \mathrm{~mm}$

Bild $58:$ BI/2, Dehnungs-Lastwechsel-Diagramme für die Längsbewehrung im Lastzyklus B 

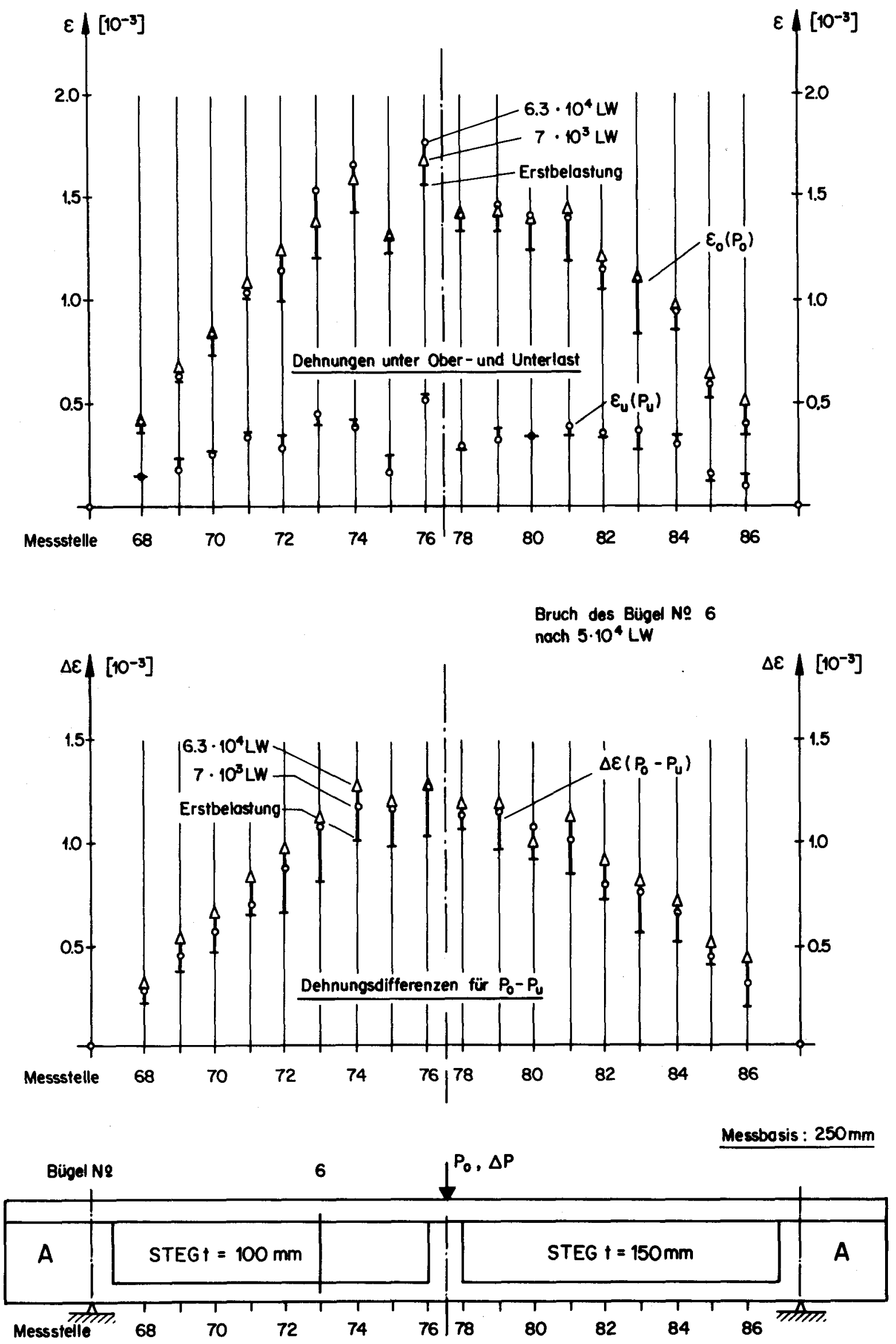

Bild 59: $\mathrm{BI} / 2$, Dehnungen und Dehnungsdifferenzen in der Längsbewetrung im Lastzyklus B 

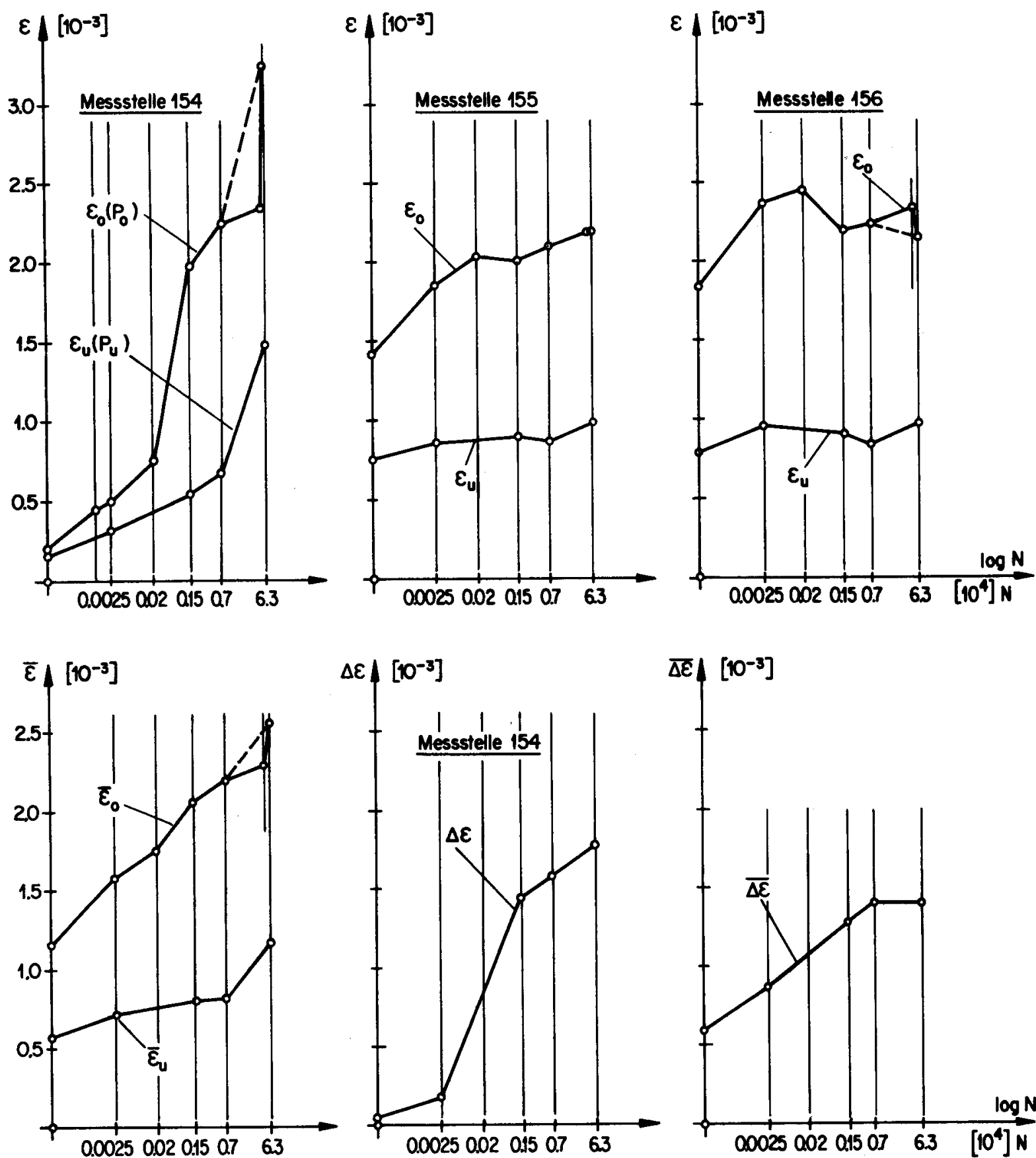

$\Delta \varepsilon \mid\left[10^{-3}\right]$

$\overline{\Delta \varepsilon}\left[10^{-3}\right]$

$\varepsilon=\frac{\sum \varepsilon}{3}$
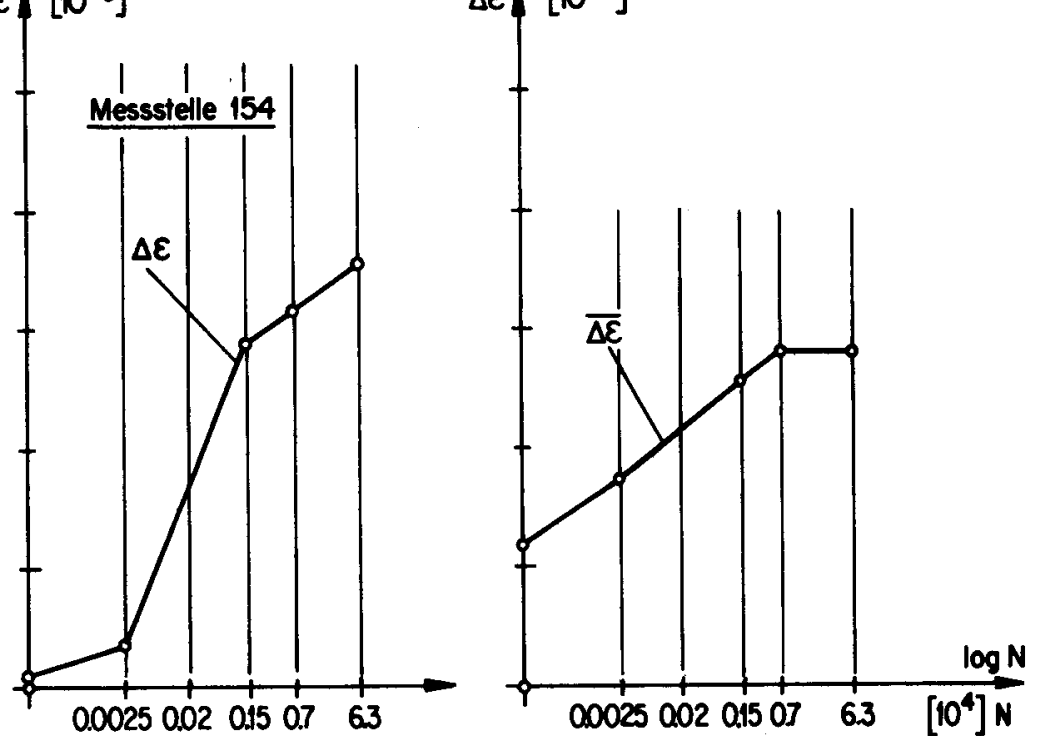

Maximalwert

$$
\overline{\Delta \varepsilon}=\frac{\sum \Delta \varepsilon}{3}
$$

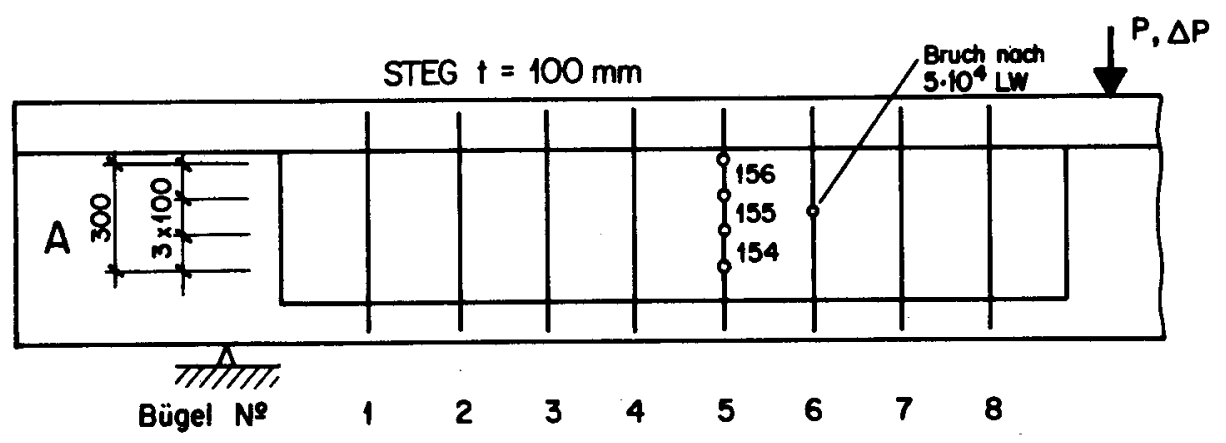

Bild 60: BI /2, Dehnungs - Lastwechsel - Diagramme für den

Bügel № 5 im Lastzyklus B 

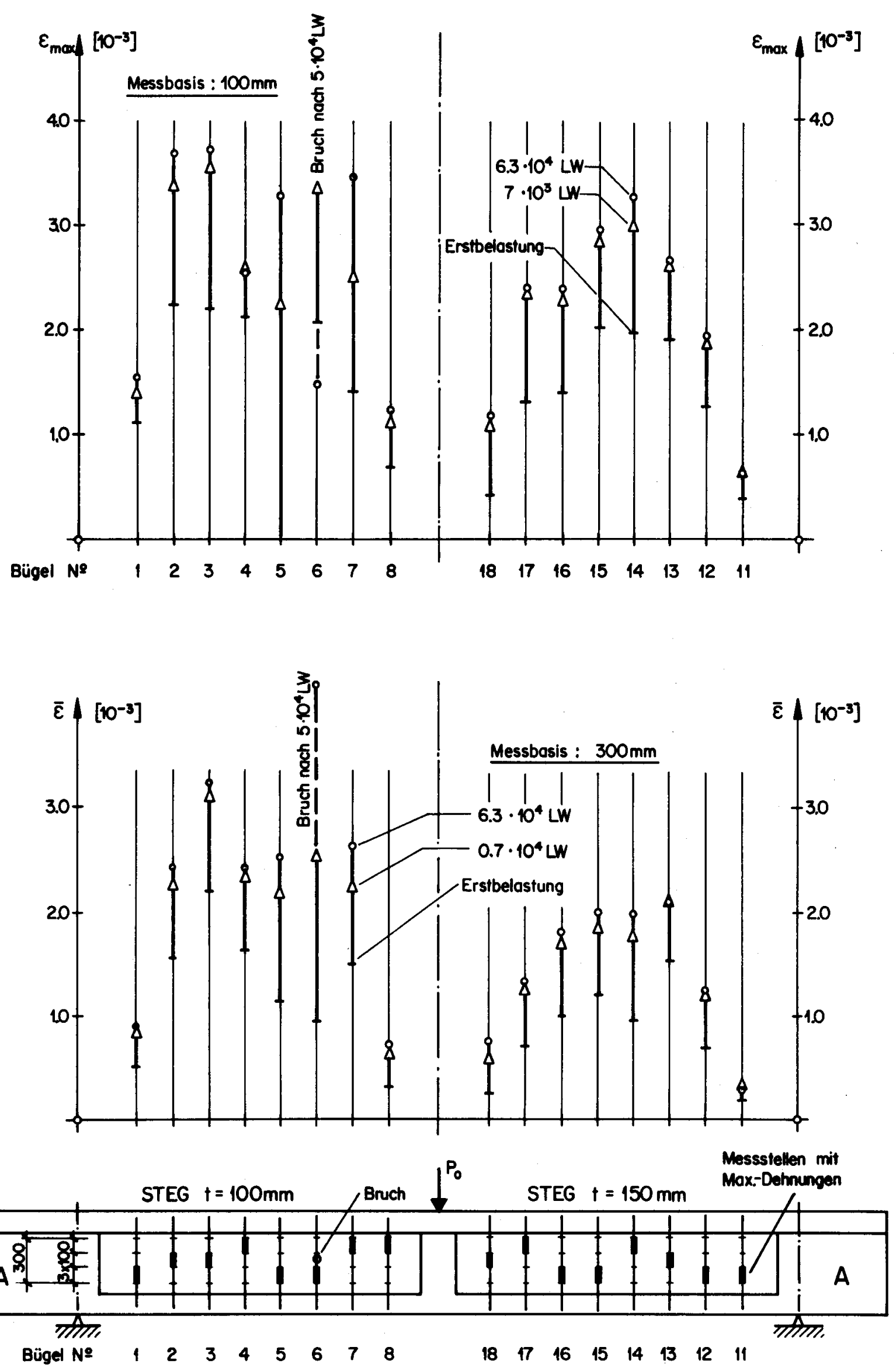

Bild 61: BI/2, Bügeldehnungen unter Oberlast im Lastzyklus B 

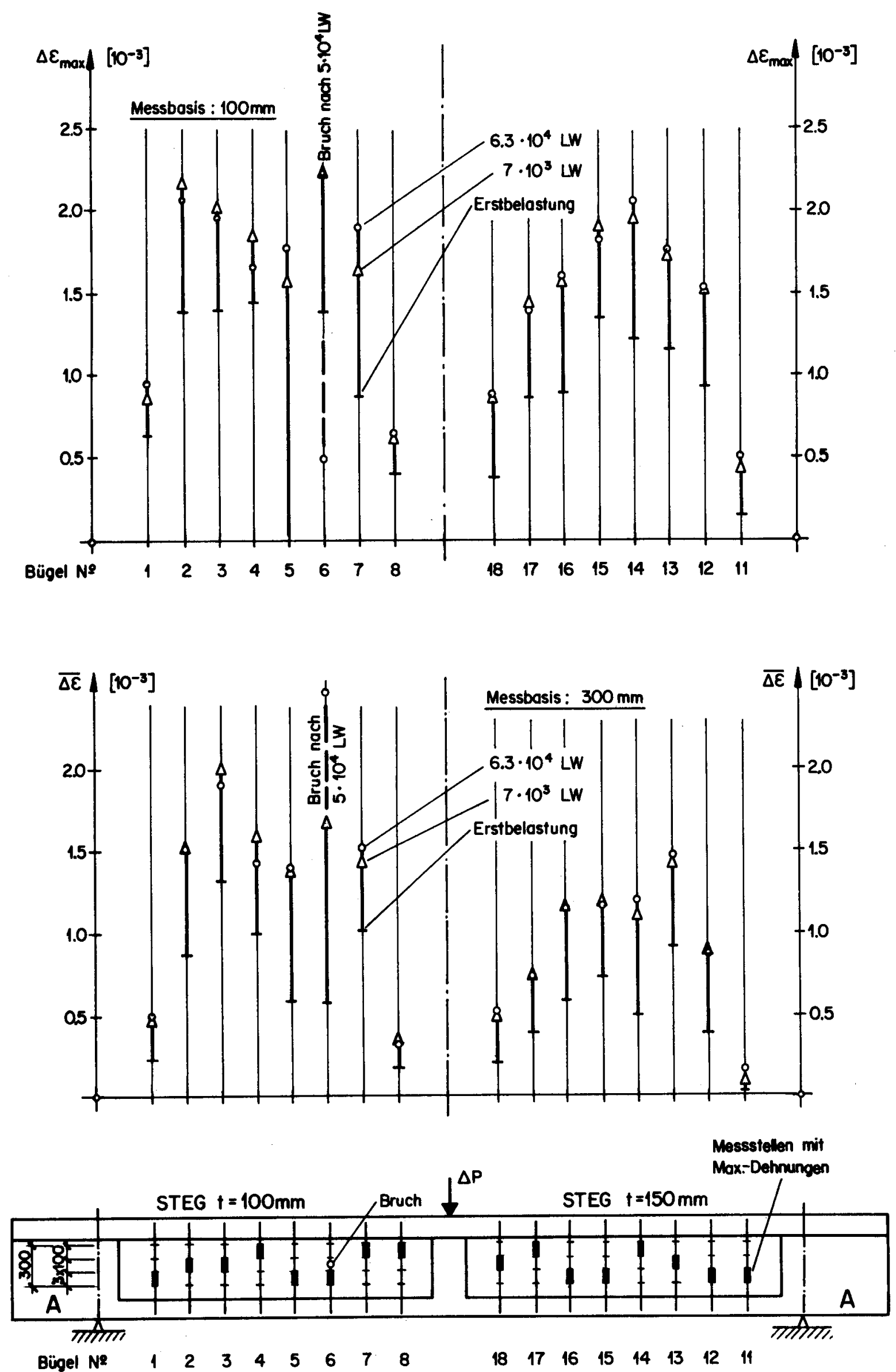

Bild 62: $\mathrm{BI} / 2$, Dehnungsdifferenzen in den Biigeh im Lastzyklus B 


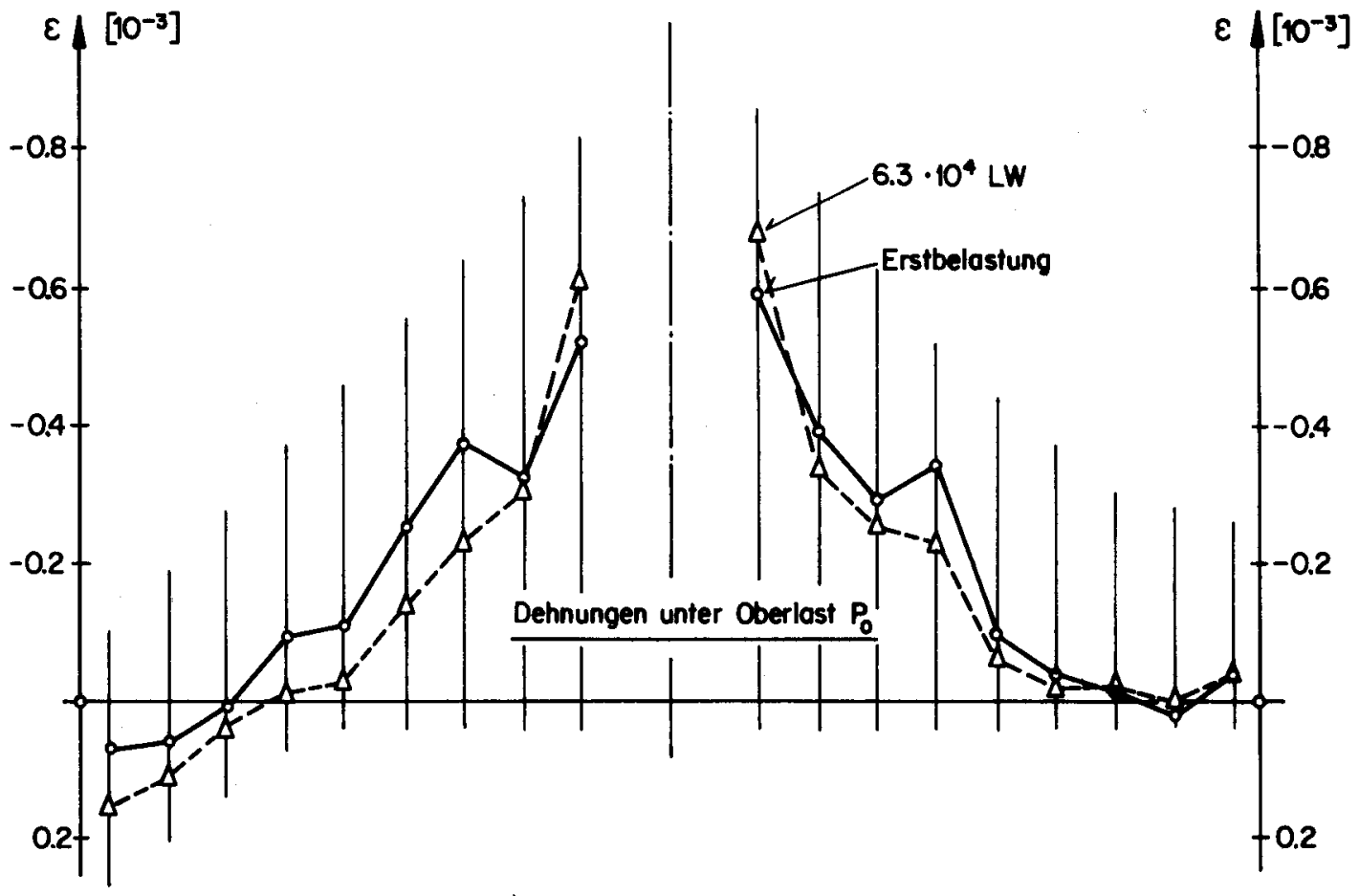

$\begin{array}{lllllllllll}\text { Messstelle } & 26 & 28 & 30 & 32 & 34 & 36 & 38 & 40 & 42 & 44\end{array}$
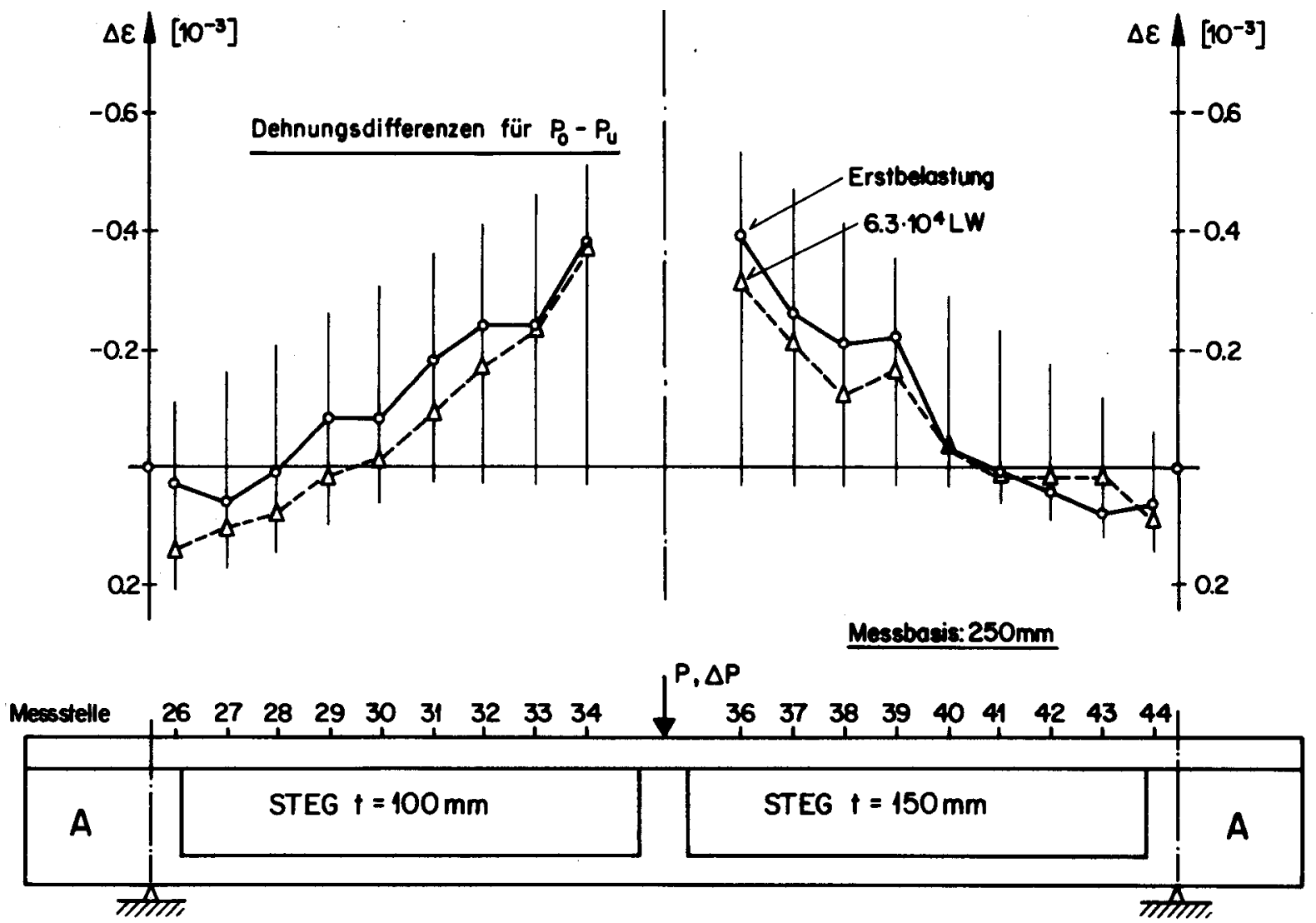

Bild 63: BI/2, Betondehnungen in Oberflansch im Lastzyklus B 


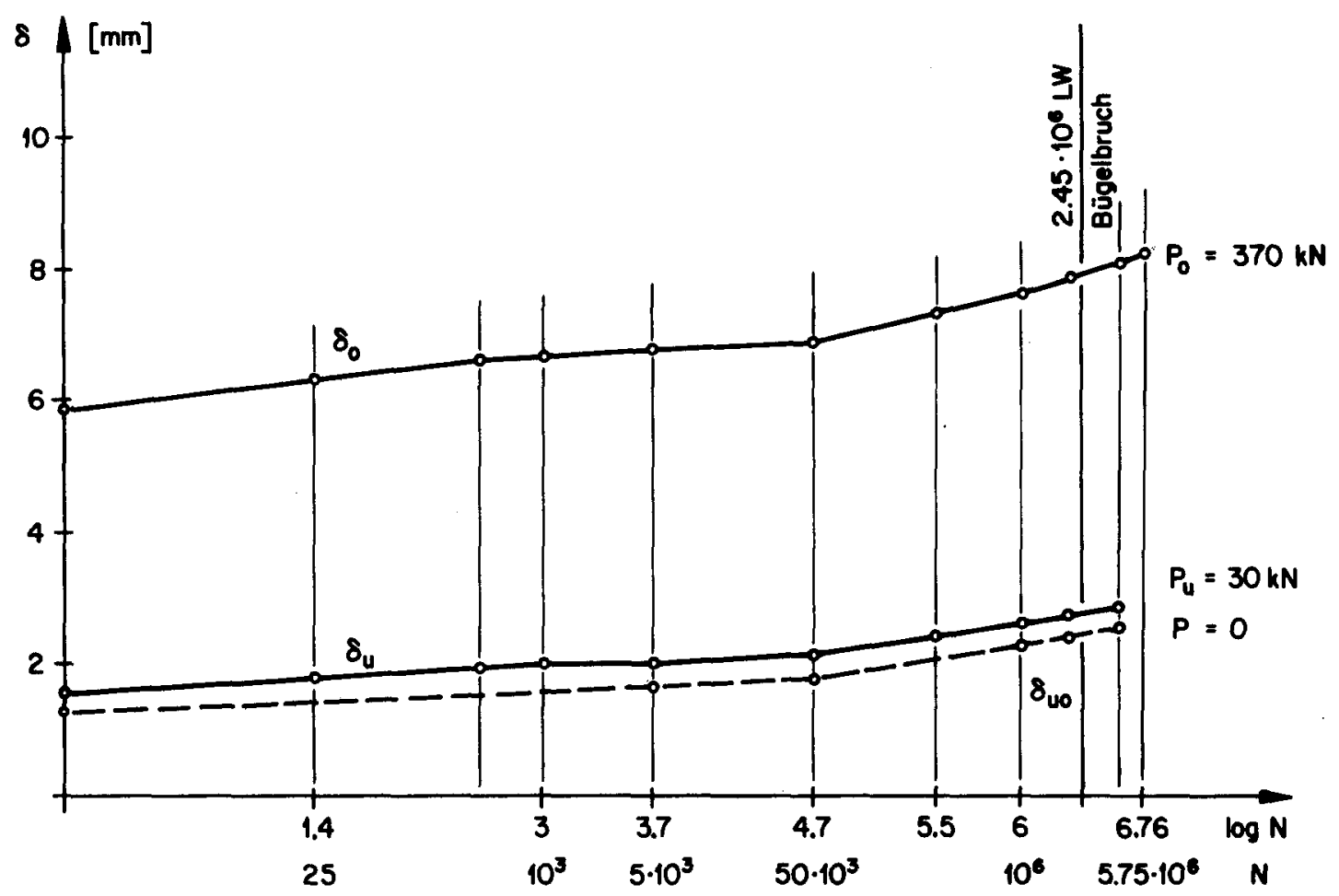

Bild 64: BI/3, Durchbiegungs - Lasłwechsel-Diagramm für die Trägermitte im Lastzyklus B

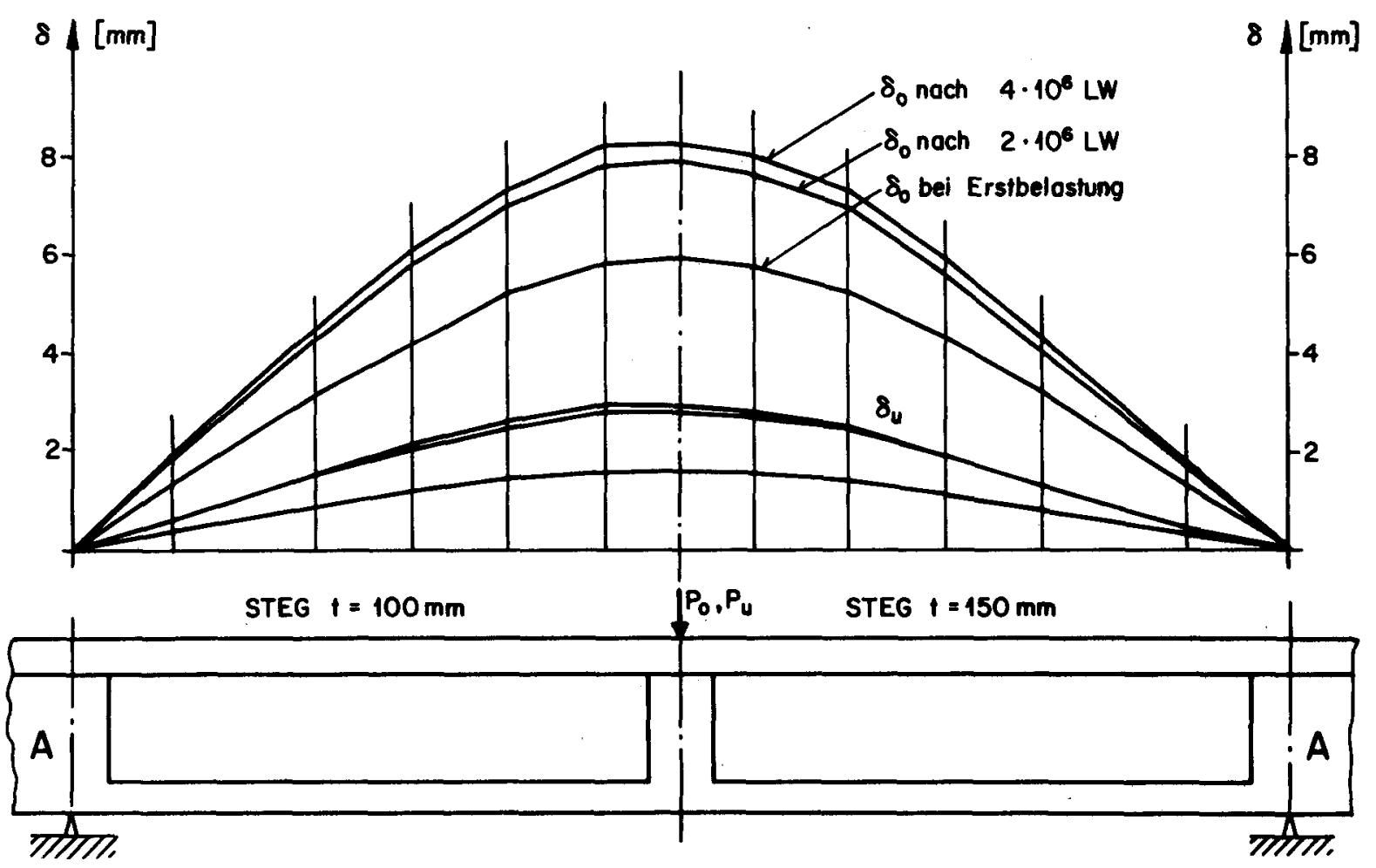

Bild 65: BI/3, Verlauf der Durchbiegung im Lastzyklus B 

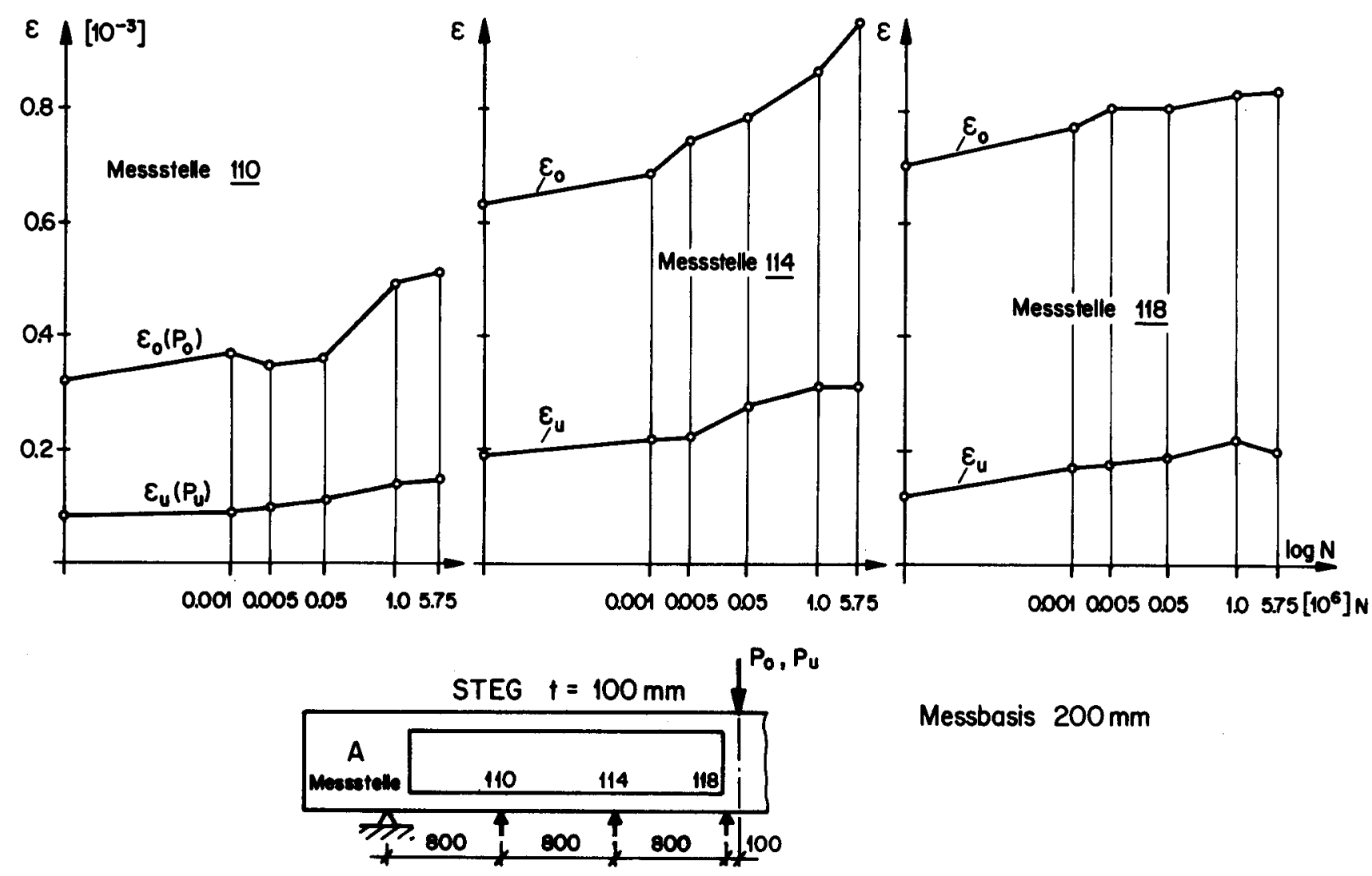

Messbasis $200 \mathrm{~mm}$
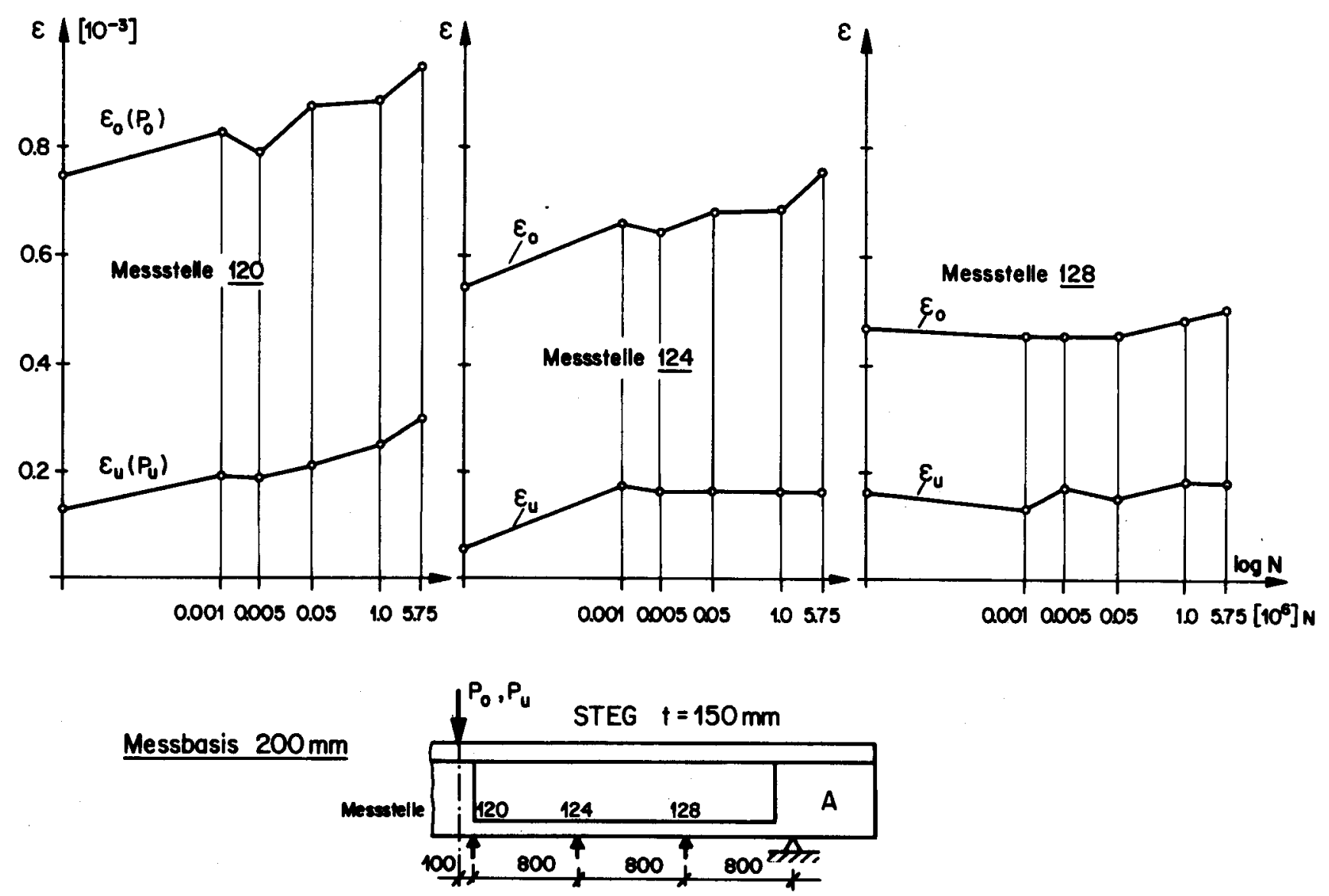

Bild 66: BI/3, Dehnungs - Lastwechsel - Diagramme für die Längsbewehrung im Lastzyklus B 

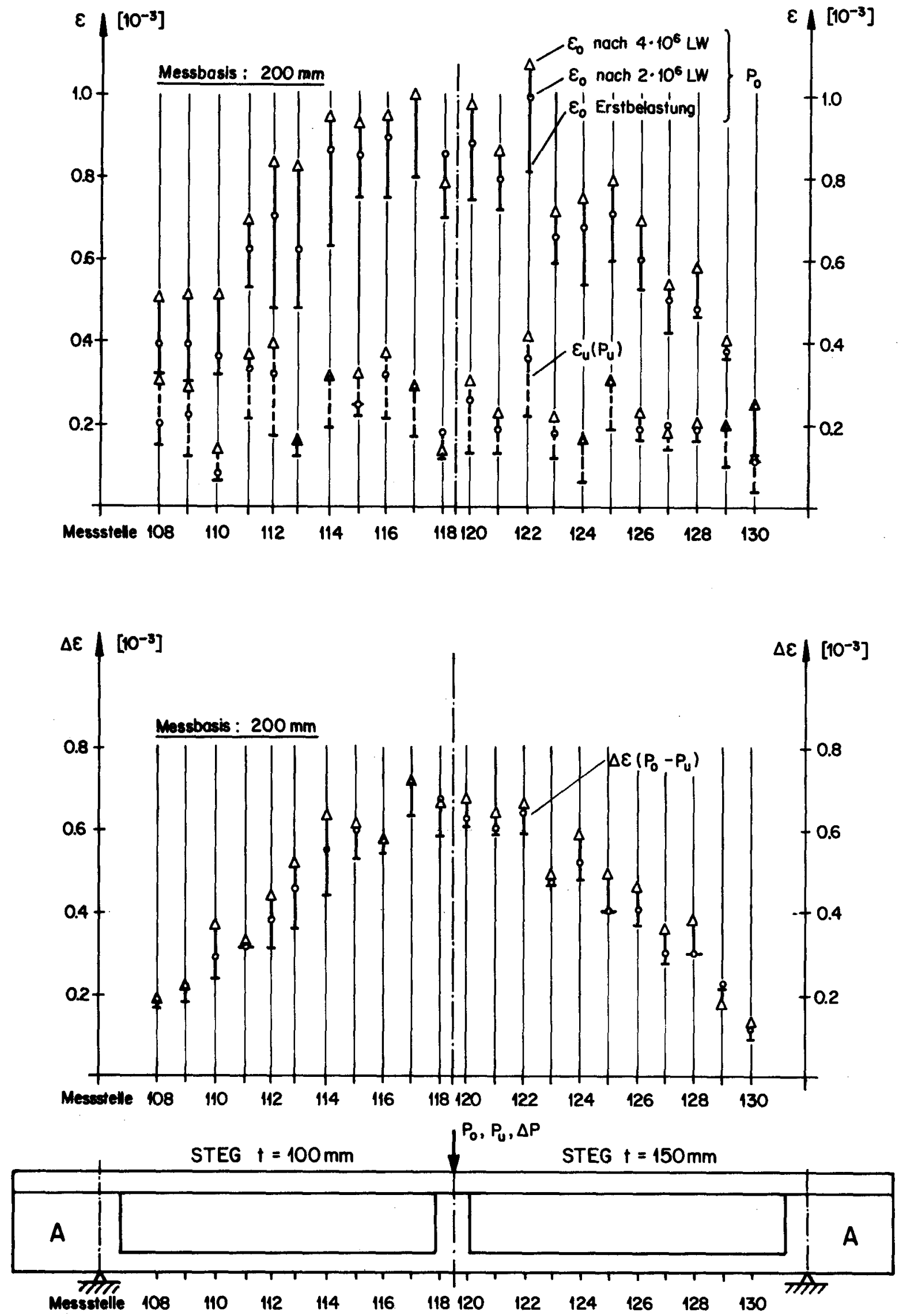

Bild $67: B I / 3$, Dehnungen und Dehnungdifferenzen in der Längsbewehrung im Lastzyklus B 

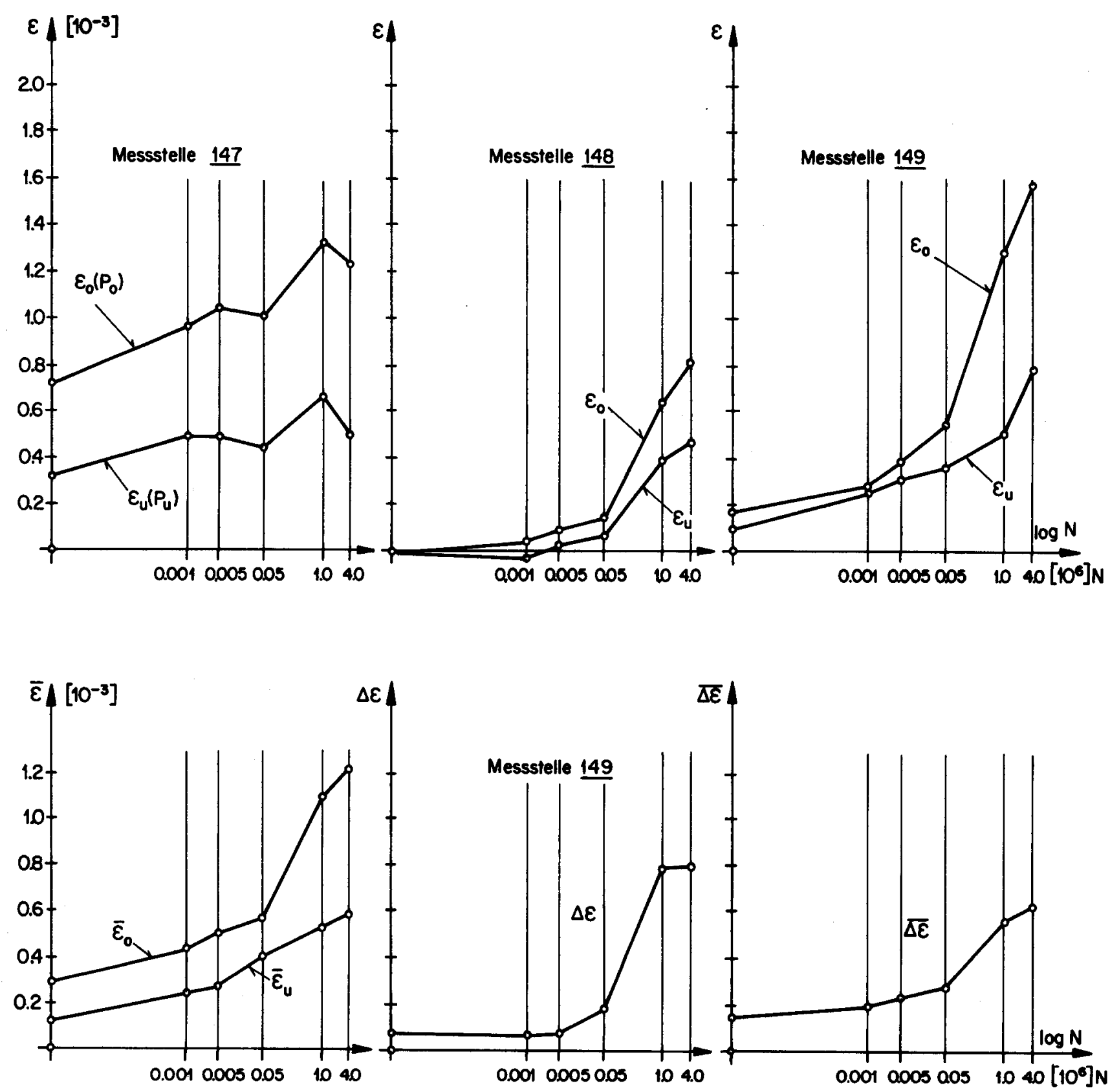

$\overline{\Delta \varepsilon}$

$\bar{\varepsilon}=\frac{\Sigma \varepsilon}{3}$

Maximalwert

$\overline{\Delta \varepsilon}=\frac{\sum \Delta \varepsilon}{3}$

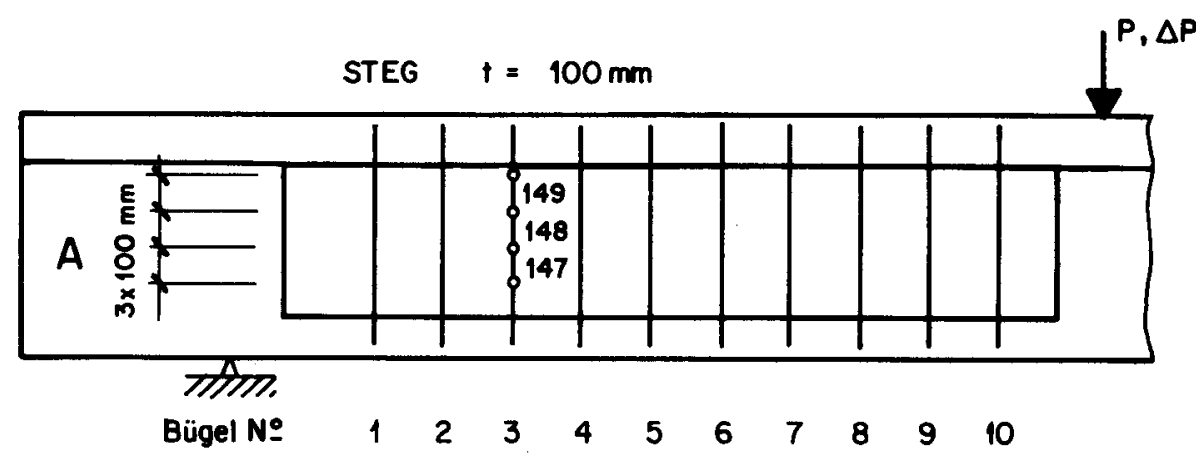

Bild 68: BI/3 Dehnungs - Lastwechsel-Diagramme für den Bügel № 3 im Lastzyklus B 

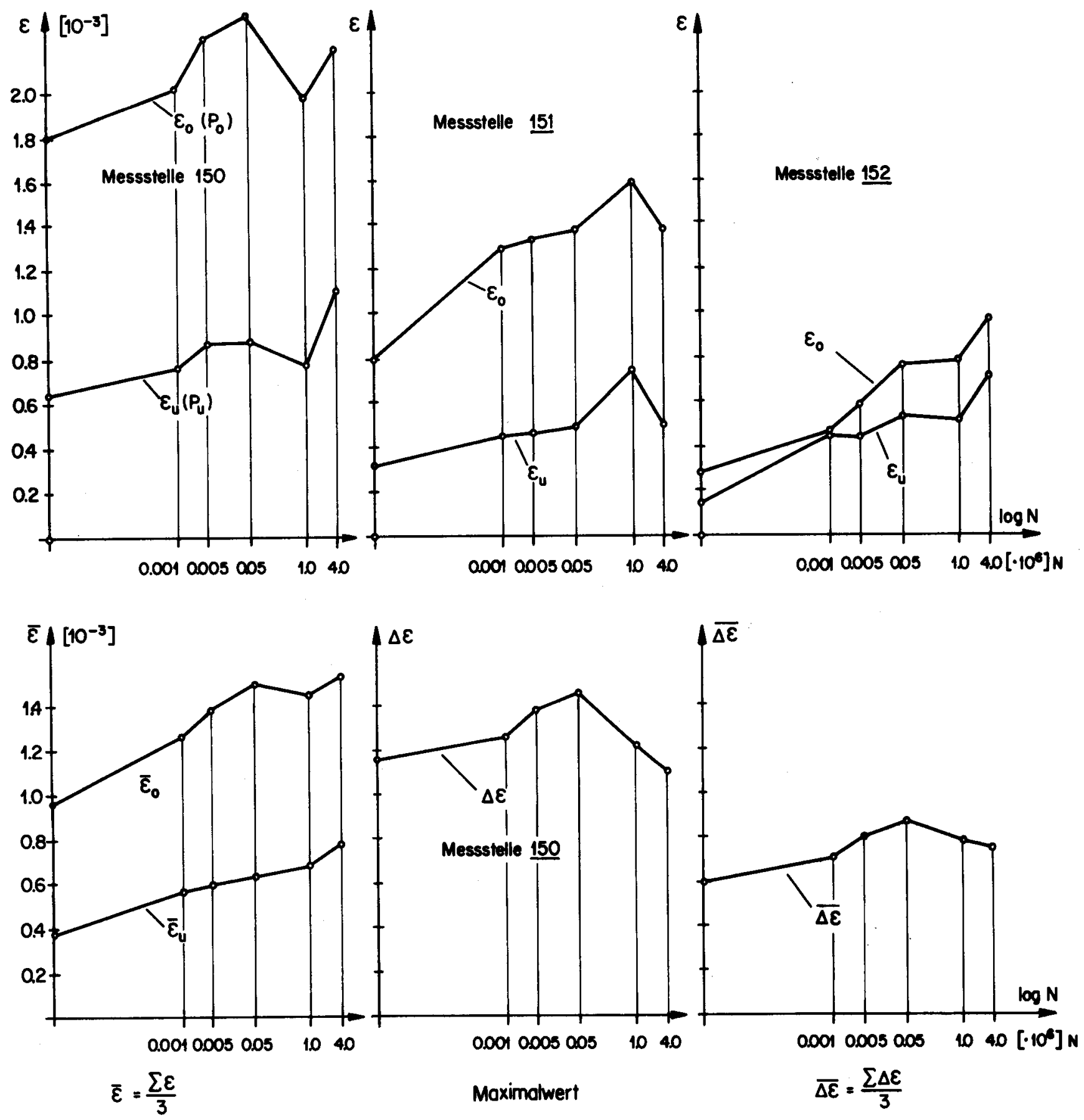

$\bar{\varepsilon}=\frac{\sum \varepsilon}{3}$

$$
\overline{\Delta \varepsilon}=\frac{\Sigma \Delta \varepsilon}{3}
$$

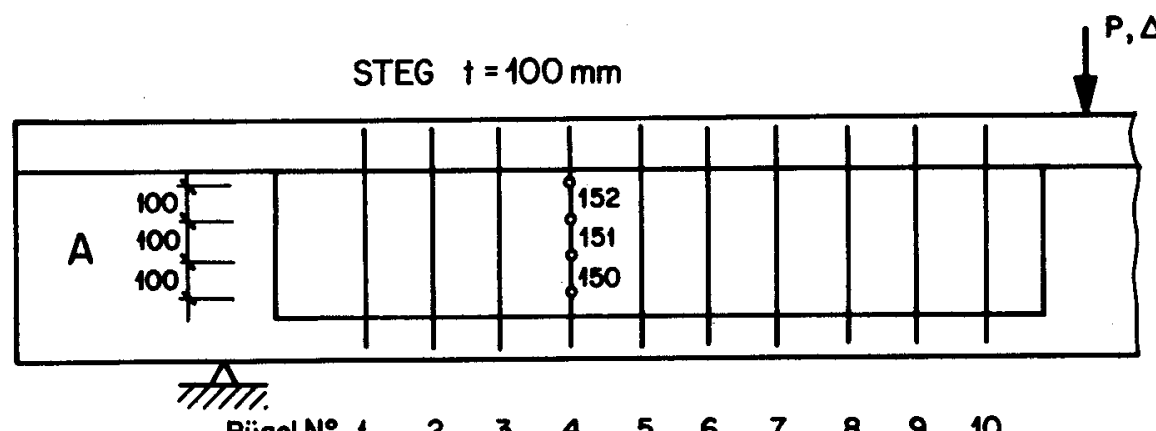

$\begin{array}{llllllllll}\text { Bügel № } 1 & 2 & 3 & 4 & 5 & 6 & 7 & 8 & 9 & 10\end{array}$

Bild $69:$ BI/3, Dehnungs - Lastwechsel - Diagramme für den Bügel № 4 im Lostzyklus B 

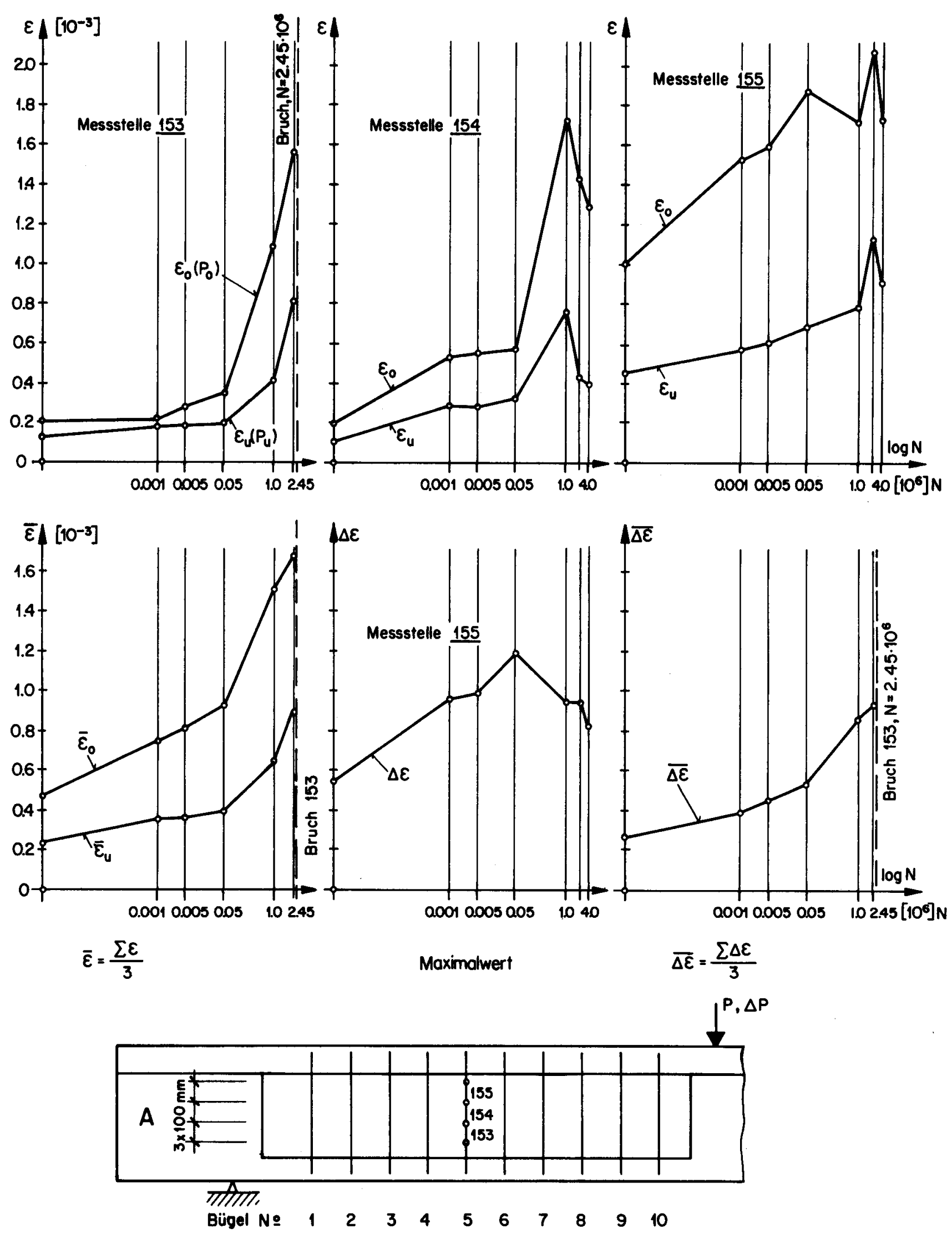

Bild 70: BI/3, Dehnungs - Lastwechsel-Diagramme für den Bügel № 5 im Lastzyklus B 


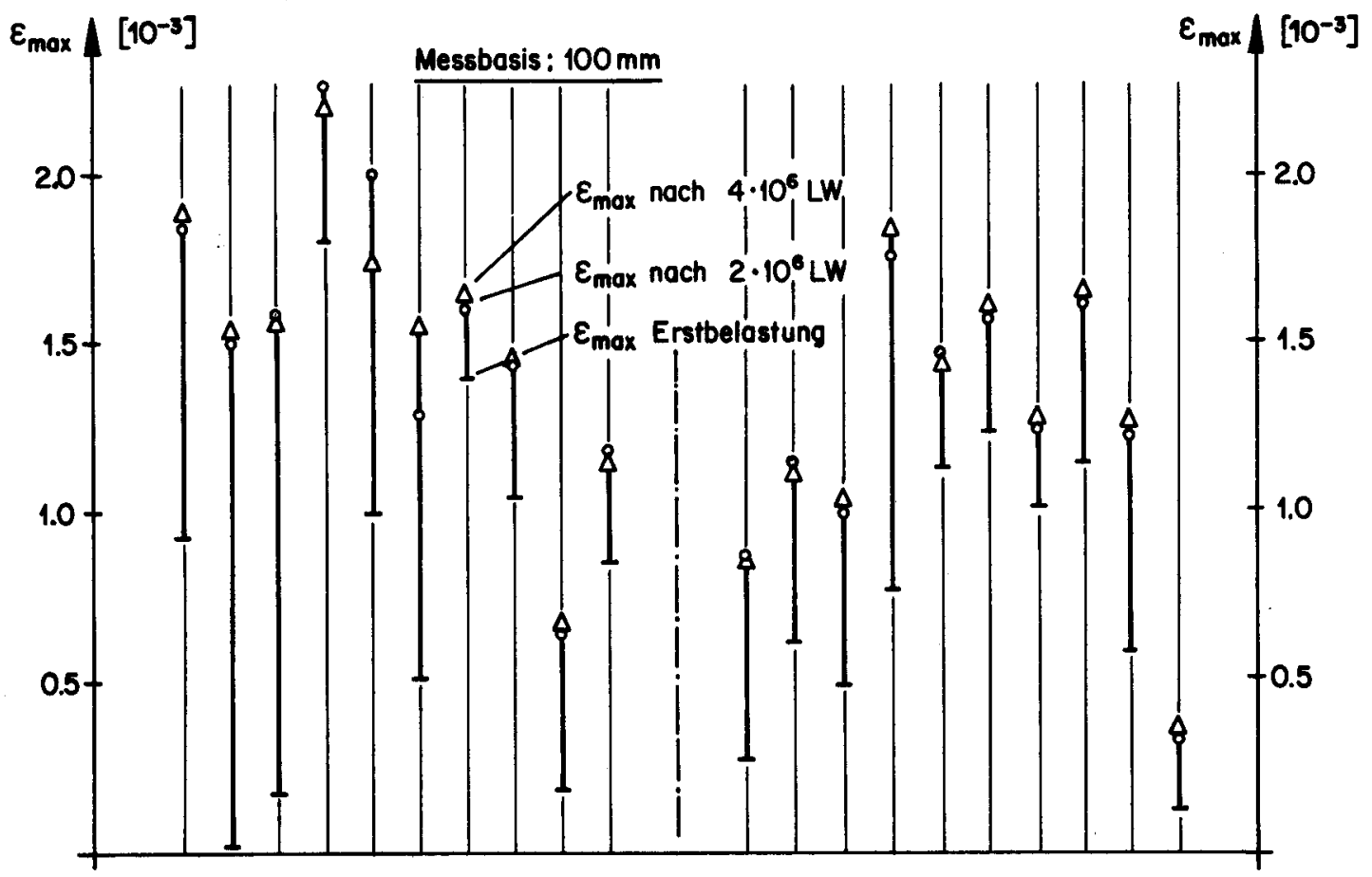

$\begin{array}{lllllllllllllllllllll}\text { Bügel N2 } 1 & 2 & 3 & 4 & 5 & 6 & 7 & 8 & 9 & 10 & & 20 & 19 & 18 & 17 & 16 & 15 & 14 & 13 & 12 & 11\end{array}$

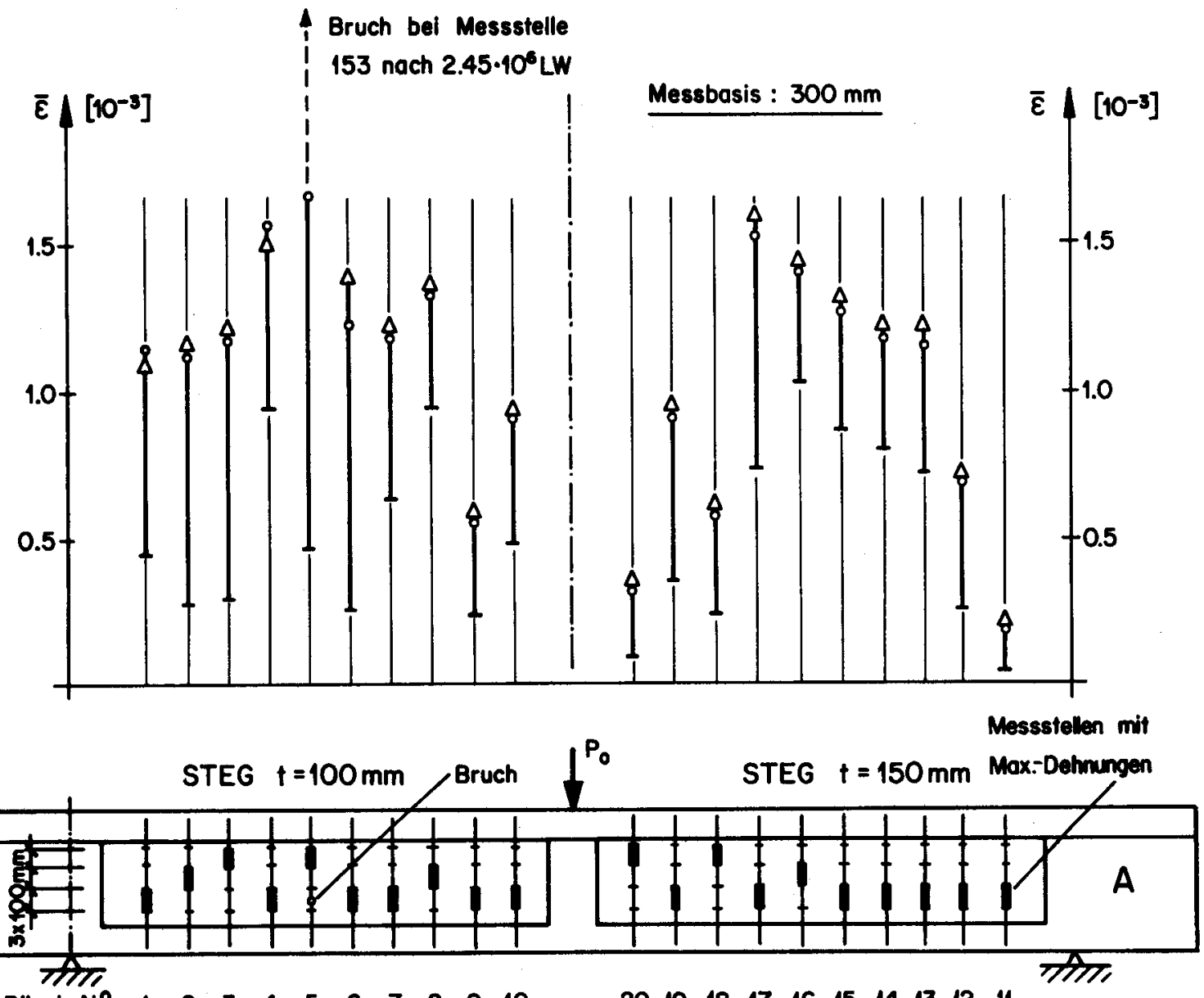

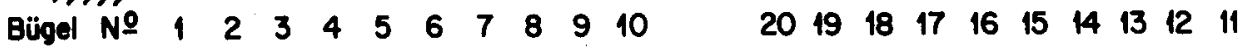

Bild 71: BI/3, Biggeldehrungen unter Oberlast im Lastzyklus B 


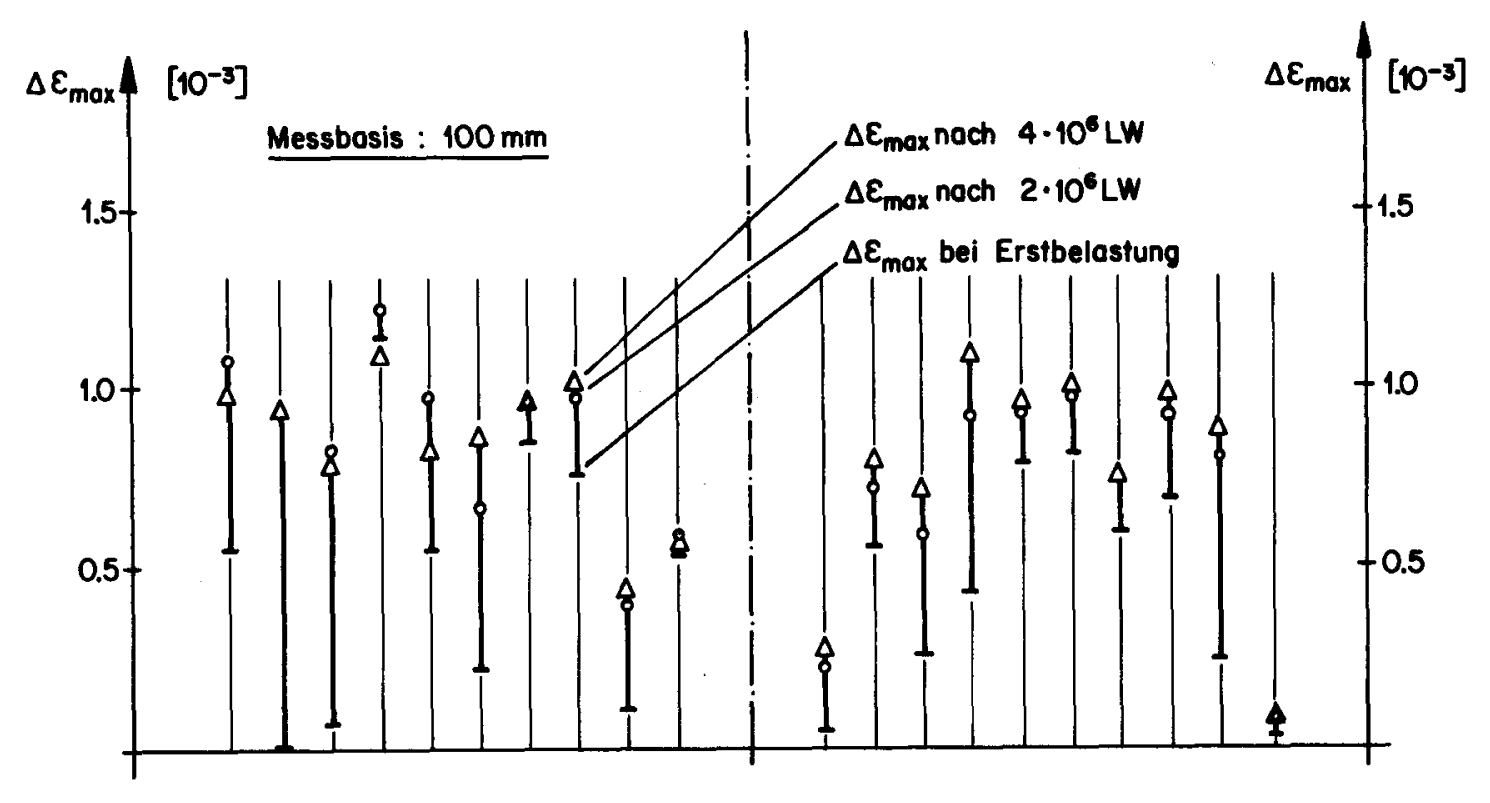

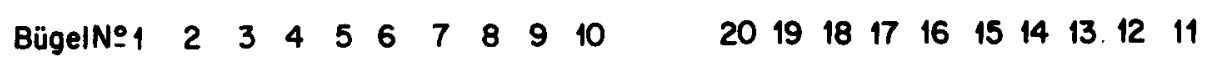

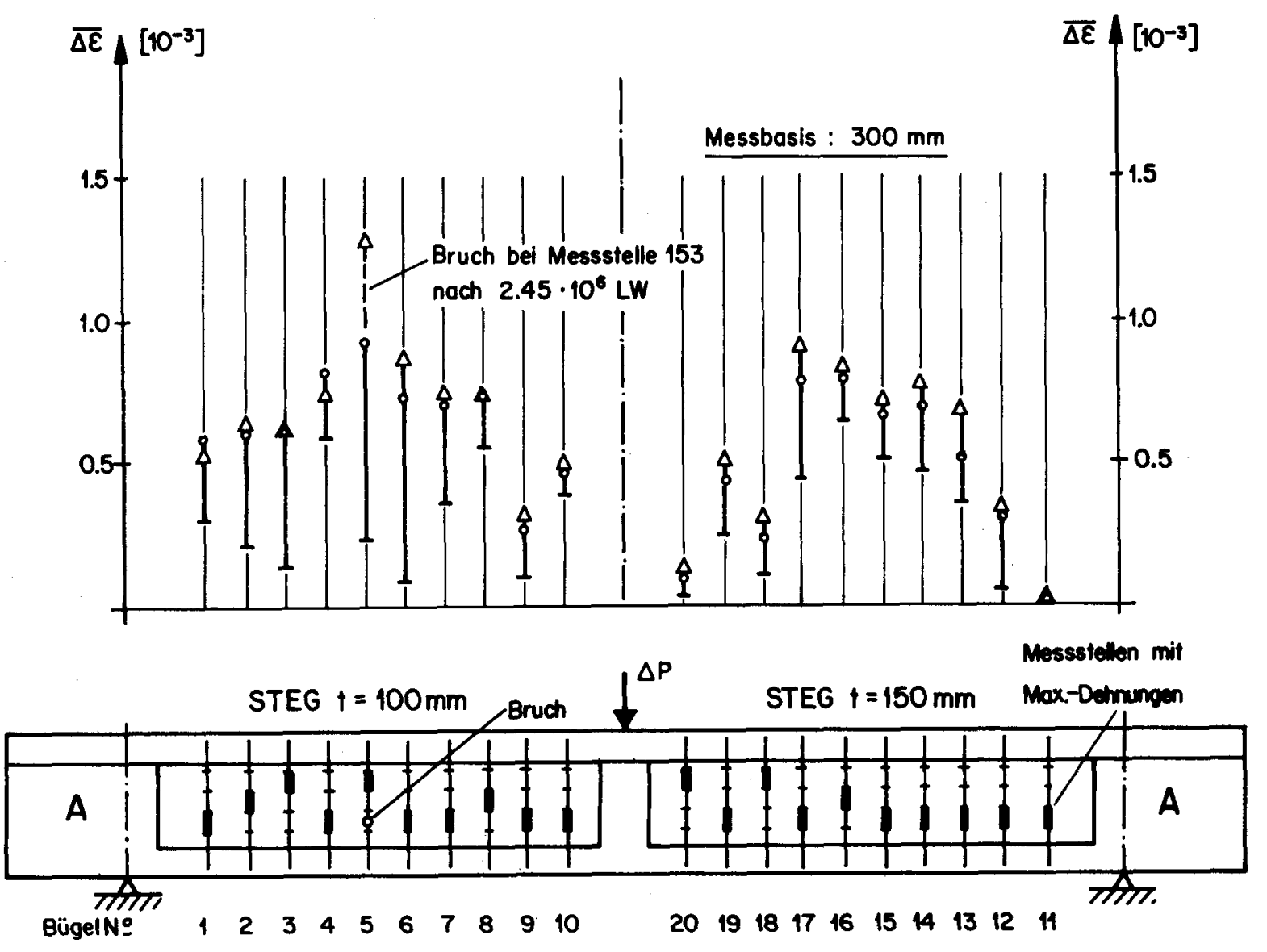

Bild 72: BI/3, Dehnungsdifferenzen in den Bügeln im Lastzyklus B 

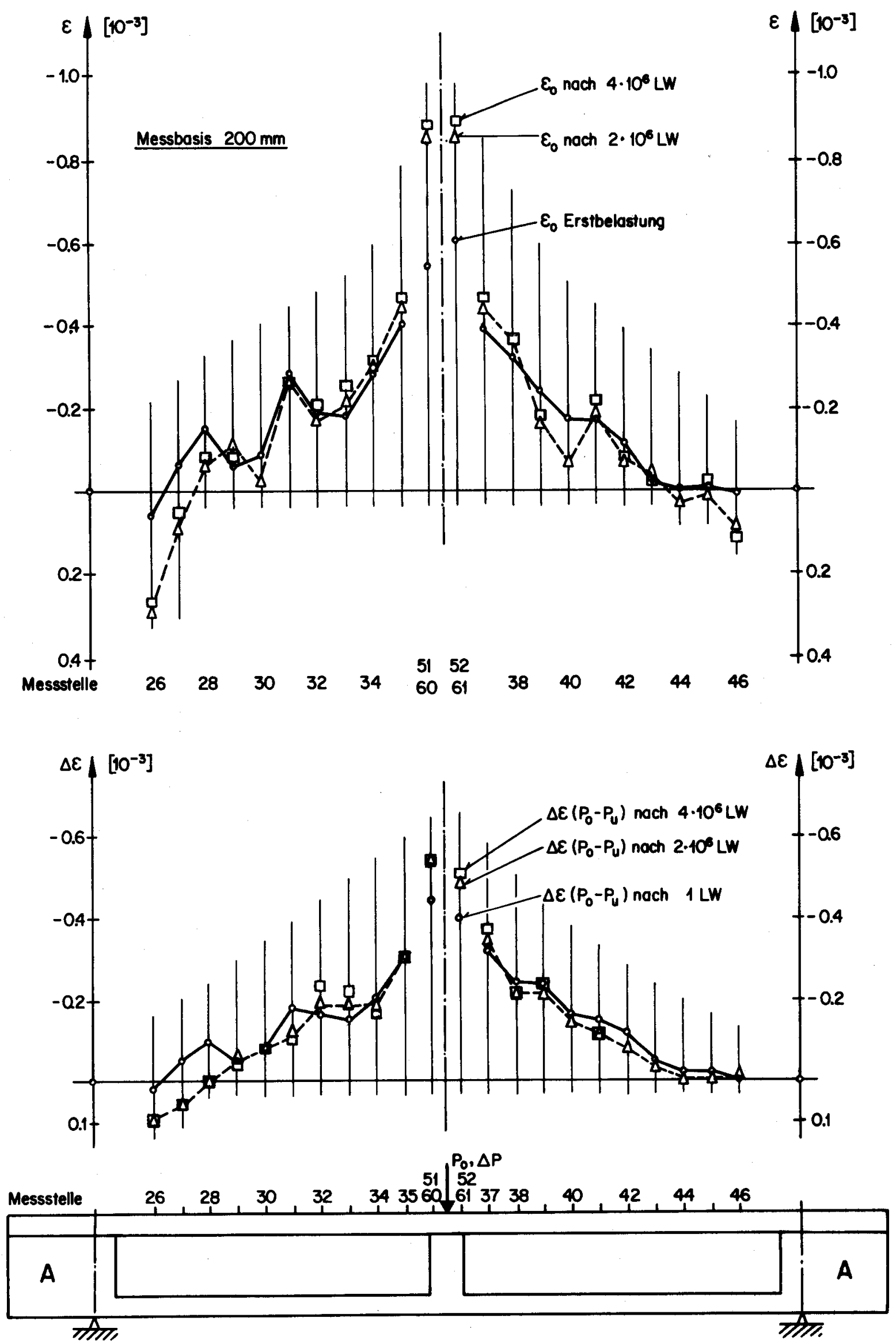

Bild 73: BI/3, Betondehnungen im Oberflansch im Lastzyklus B 


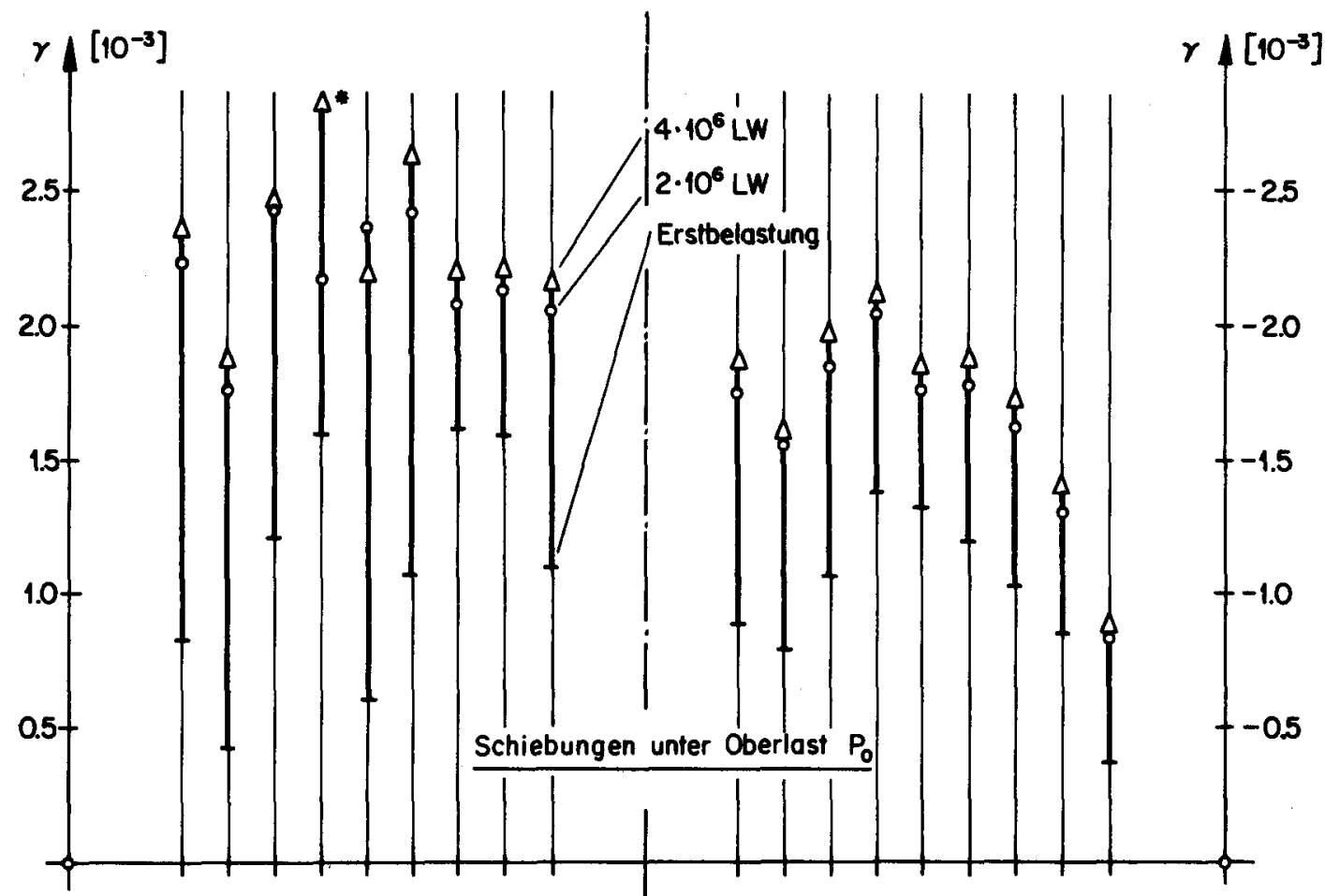

$\begin{array}{lllllllllllllllllllll}\text { Bügel } N^{9} & 1 & 2 & 3 & 4 & 5 & 6 & 7 & 8 & 9 & 10 & 20 & 19 & 18 & 17 & 16 & 15 & 14 & 13 & 12 & 11\end{array}$

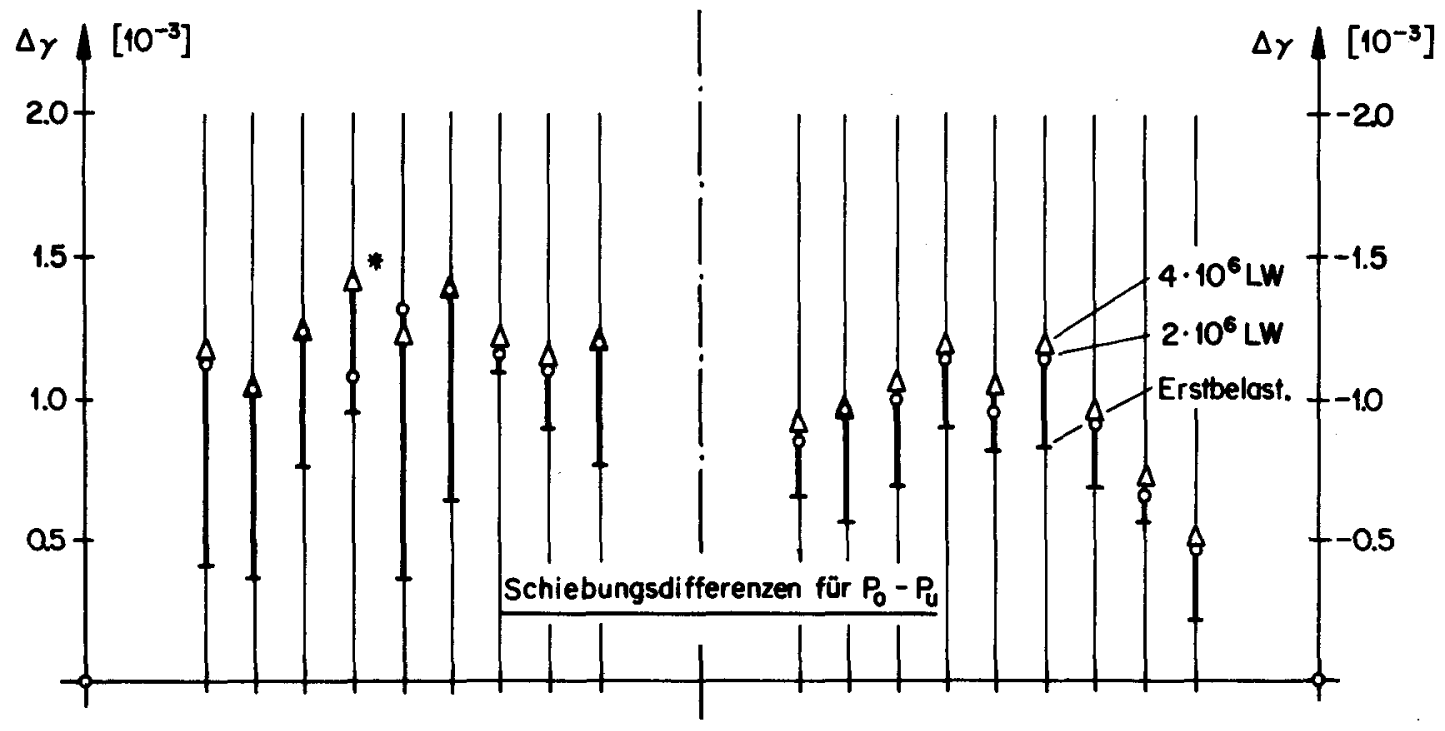

* Bruch des Bügels N2 5 nach $2.45 \cdot 10^{6} \mathrm{LW}$ (einseitig)

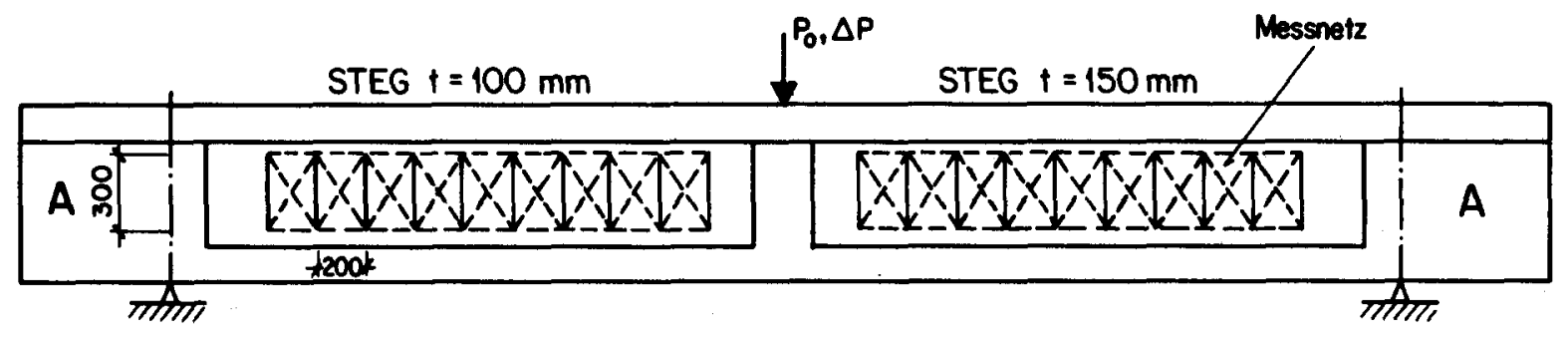

$\begin{array}{lllllllllllllllllllll}\text { Bügel № } & 1 & 2 & 3 & 4 & 5 & 6 & 7 & 8 & 9 & 10 & 20 & 19 & 18 & 17 & 46 & 15 & 14 & 13 & 12 & 11\end{array}$

Bild $74:$ BI/3, Schiebungen und Schiebungsdifferenzen im Lastzyklus $B$ 


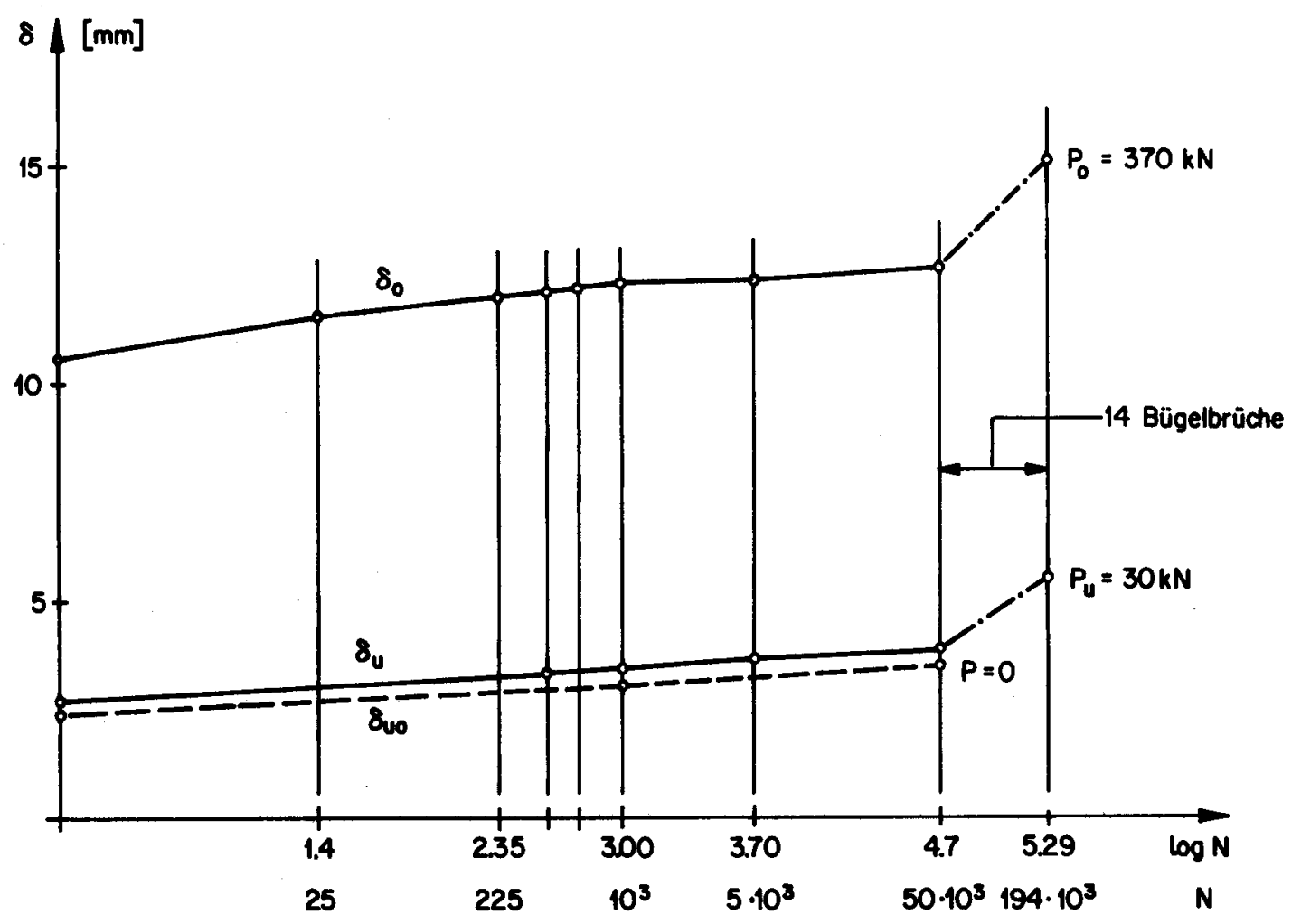

Bild 75 : BL/4, Durchbiegungs - Lastwechsel - Diagramm für die Trägermitte in Lastzyklus $B$

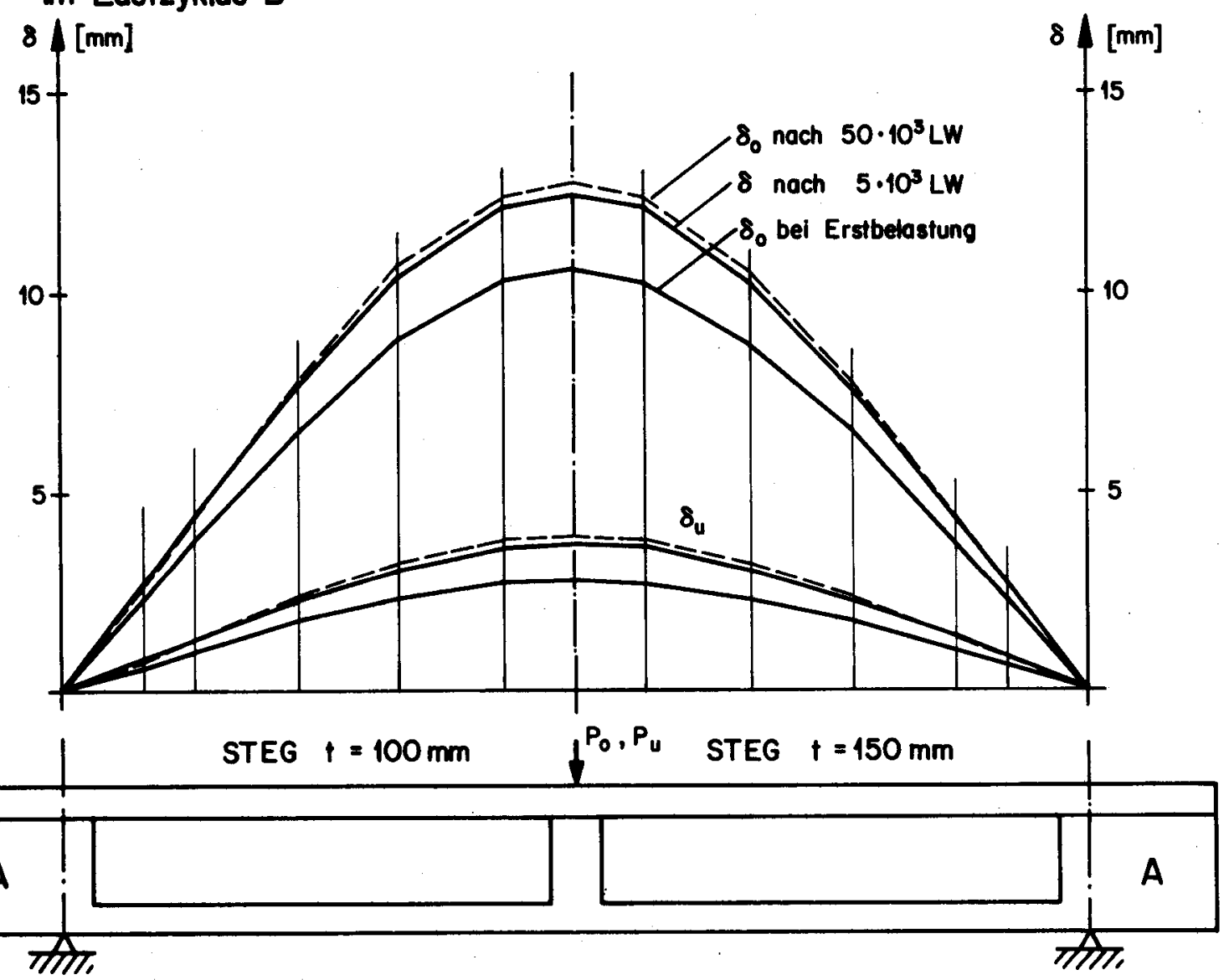

Bild 76: BI/4, Verlouf der Durchbiegung in Lastzyklus B 

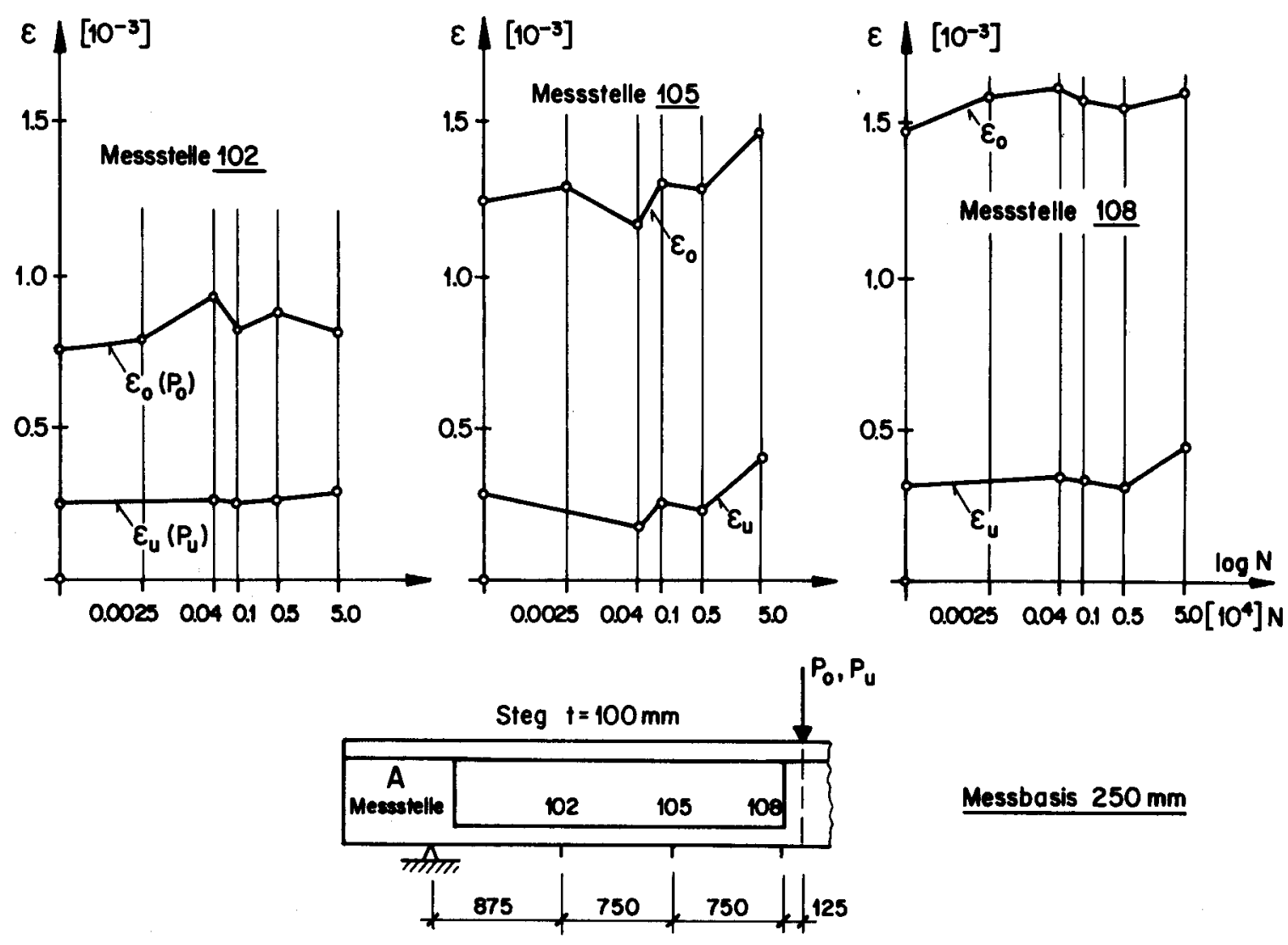

Messbasis $250 \mathrm{~mm}$
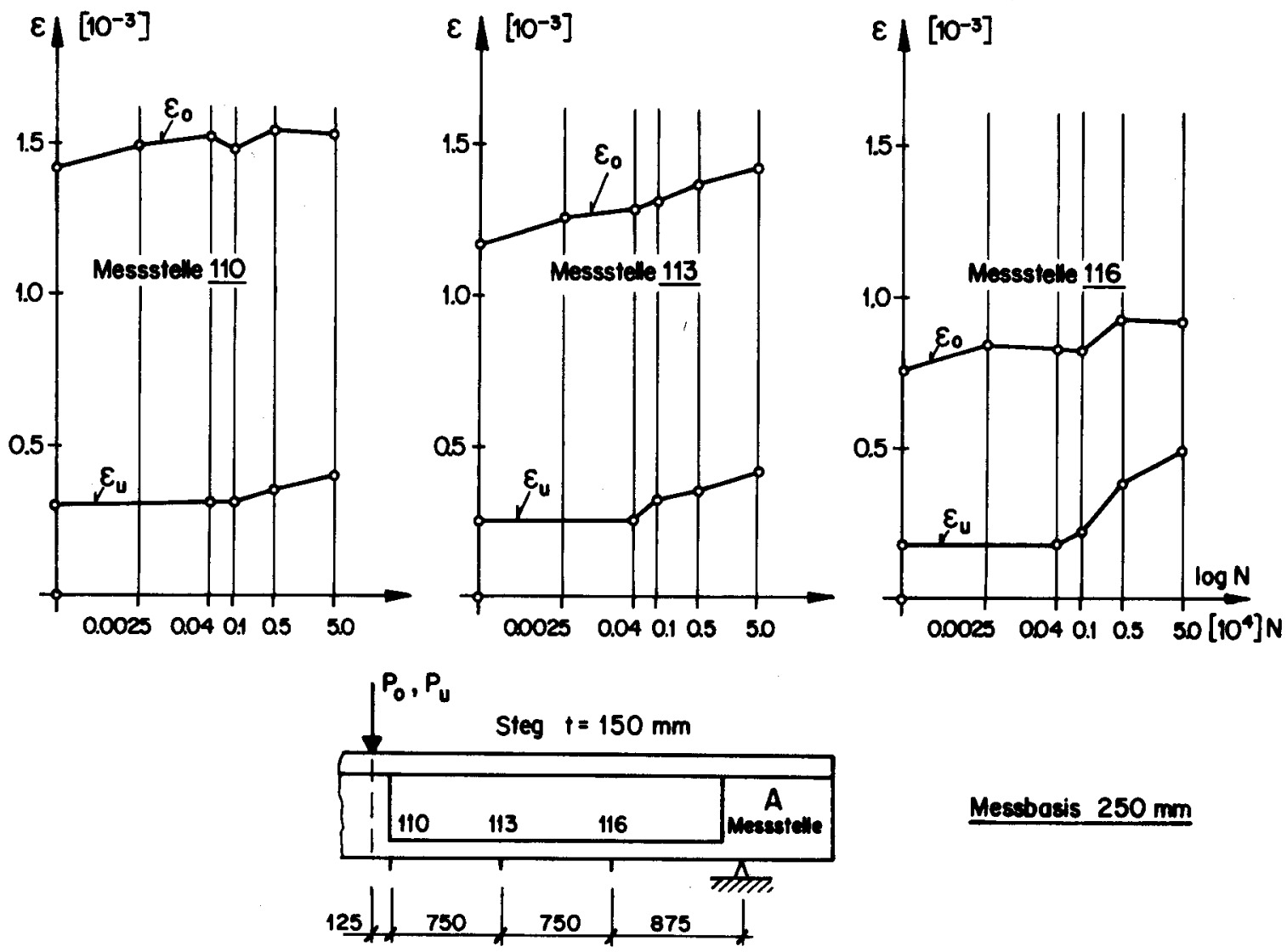

Mesebasis $250 \mathrm{~mm}$

Bild 77 : BI/4, Dehnungs- Lastwechsel-Diagramme - für die Längsbewehrung im Lastzyklus B 

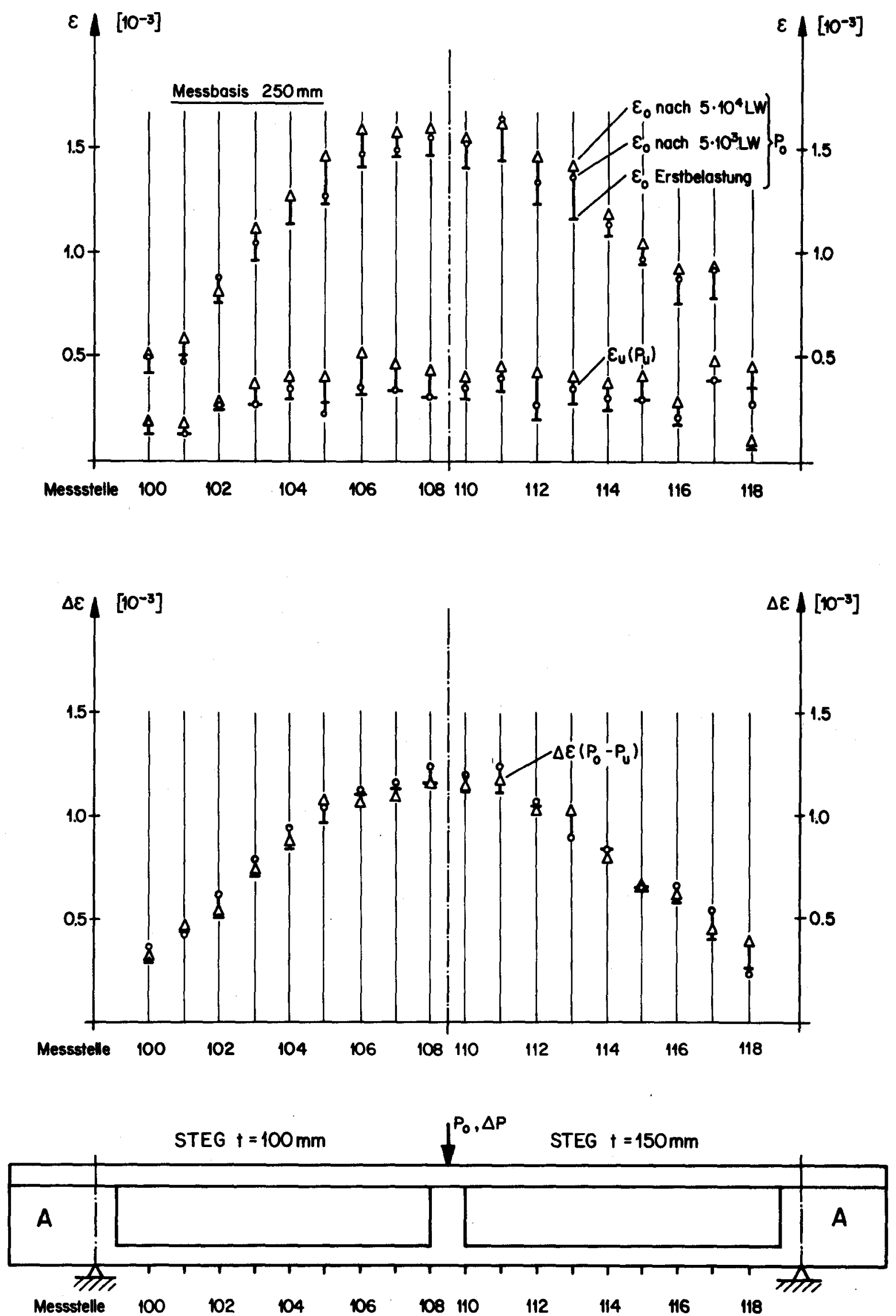

Bild $78: \mathrm{BI} / 4$, Dehnungen in der Längsbewehrung in Lastzyklus $\mathrm{B}$ 

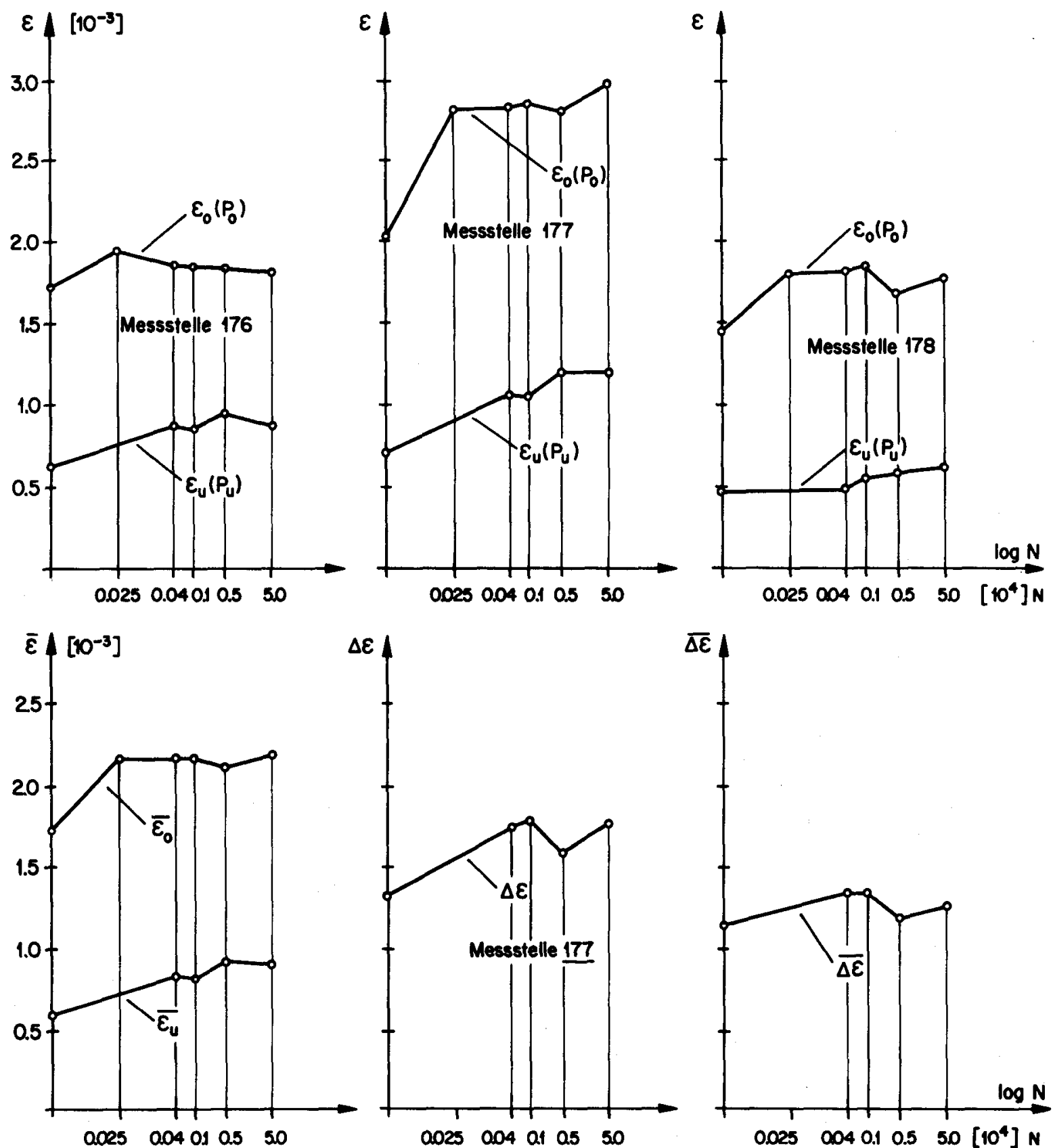

$\bar{\varepsilon}=\frac{\sum \varepsilon}{3}$

Maximatwert

$\overline{\Delta \varepsilon}$

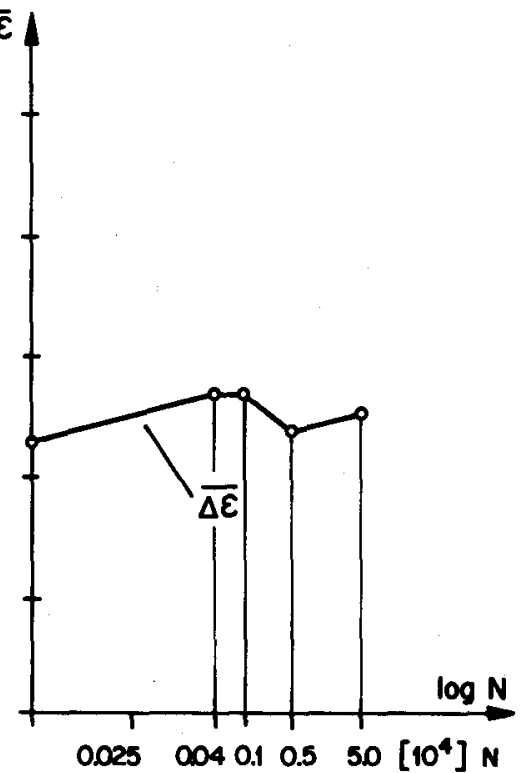

$\overline{\Delta \varepsilon}=\frac{\Sigma \Delta \varepsilon}{3}$

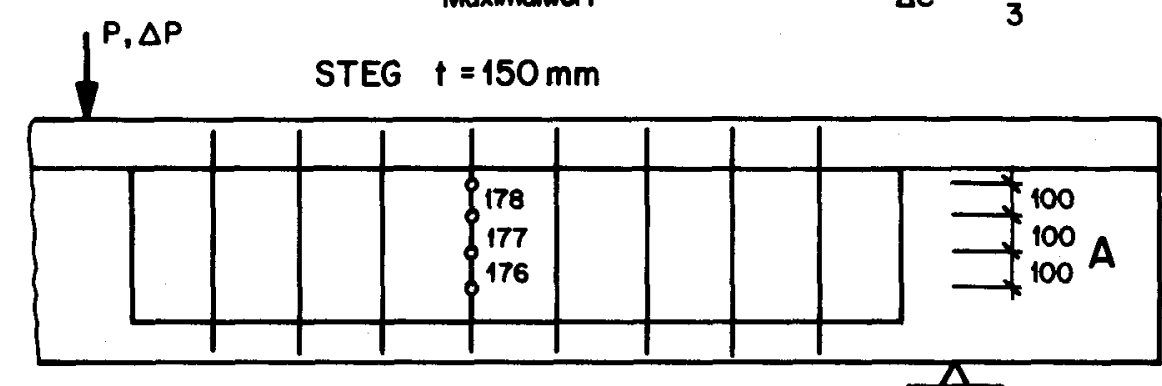

$\begin{array}{lllllllll}\text { Bügel N2 } & 18 & 17 & 16 & 15 & 14 & 13 & 12 & 11\end{array}$

Bild 79: BI/4 Dehnungs-Lostwechsel-Diagramme für den Bügel NN 15 im Lastzyklus B 

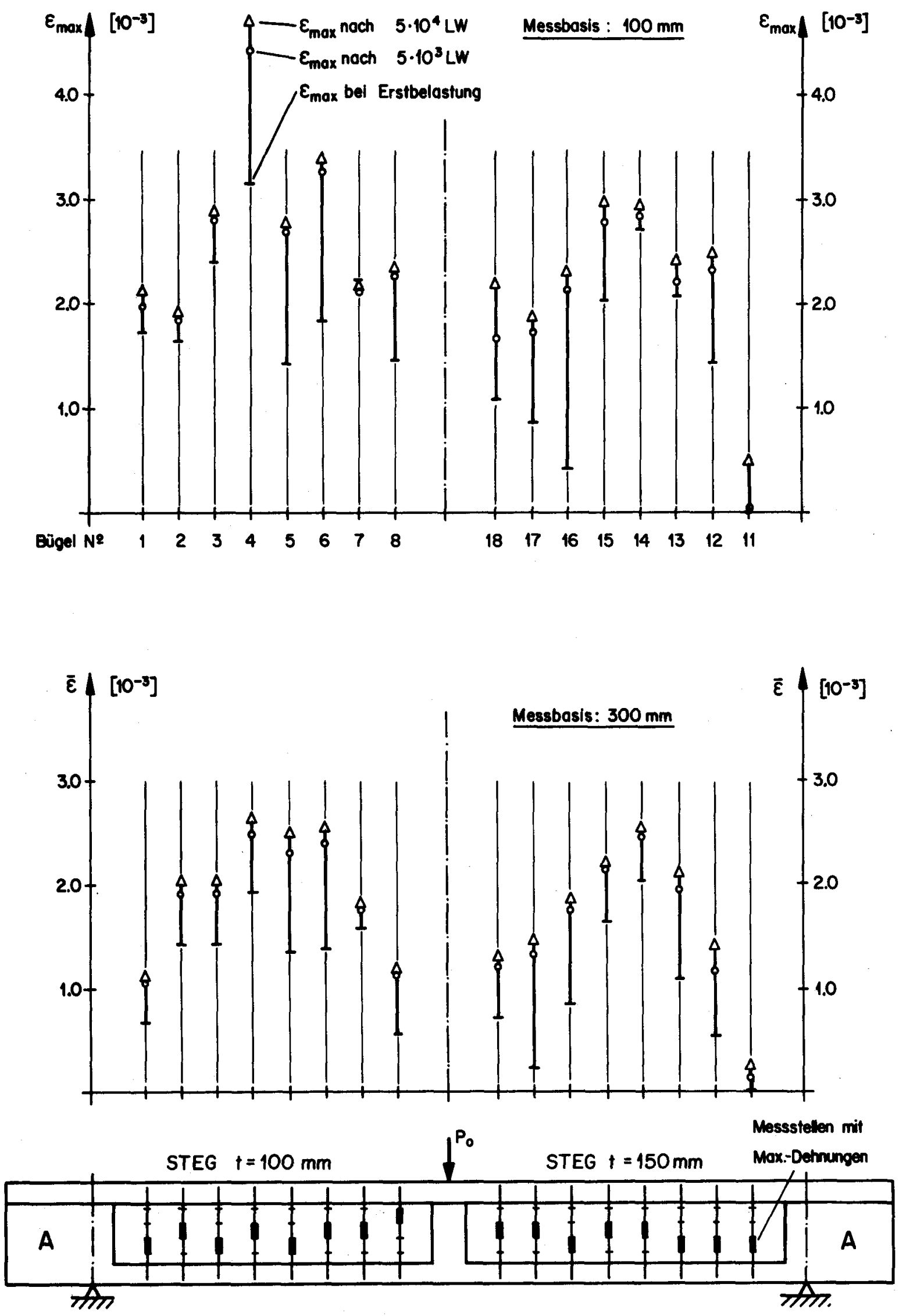

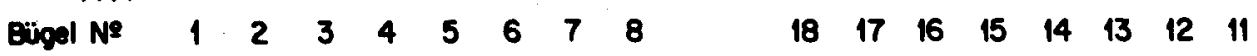

Bild 80: BI/4, Bügeldehnungen unter Oberlast im Lasłzyklus B 


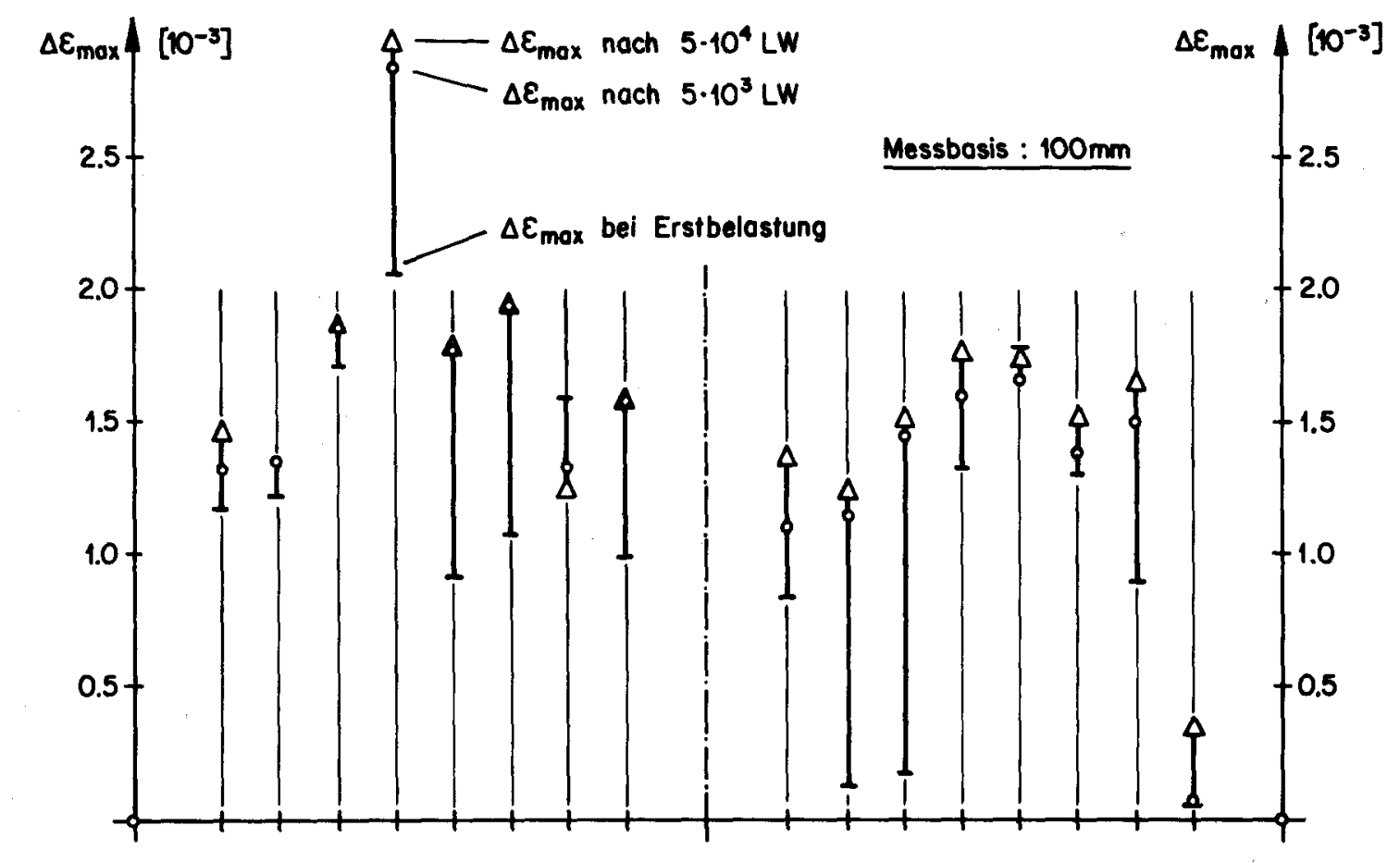

$\begin{array}{llllllllllllllllll}\text { Bügel № } & 1 & 2 & 3 & 4 & 5 & 6 & 7 & 8 & & 18 & 17 & 16 & 15 & 14 & 13 & 12 & 11\end{array}$
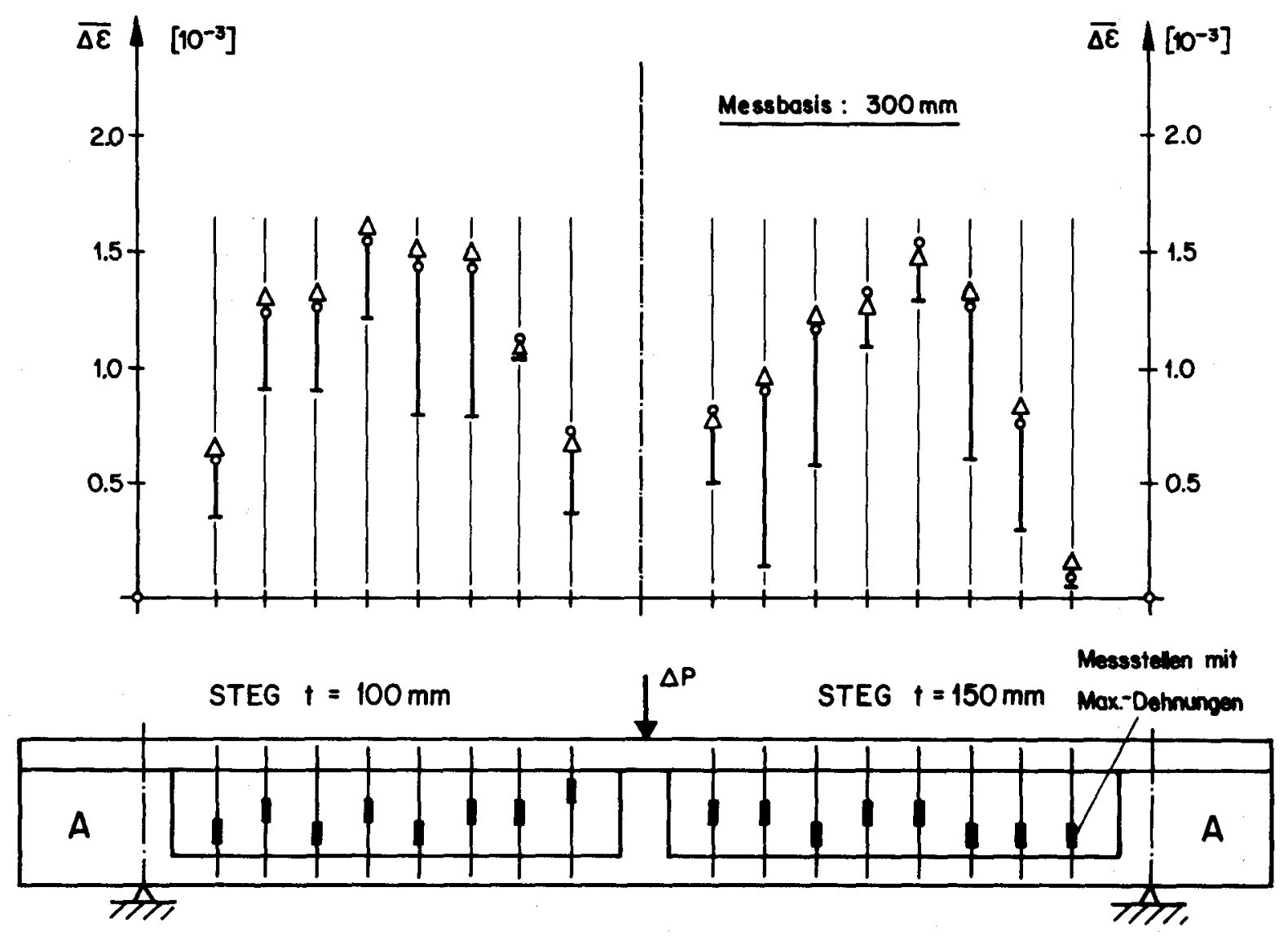

$\begin{array}{lllllllllllllllllll}\text { Bügel N } & 1 & 2 & 3 & 4 & 5 & 6 & 7 & 8 & & 18 & 17 & 16 & 15 & 14 & 13 & 12 & 11\end{array}$

Bild 81: $\mathrm{BI} / 4$, Dehnungsdfferenzen in den Bügeh im Lastzyklus $B$ 


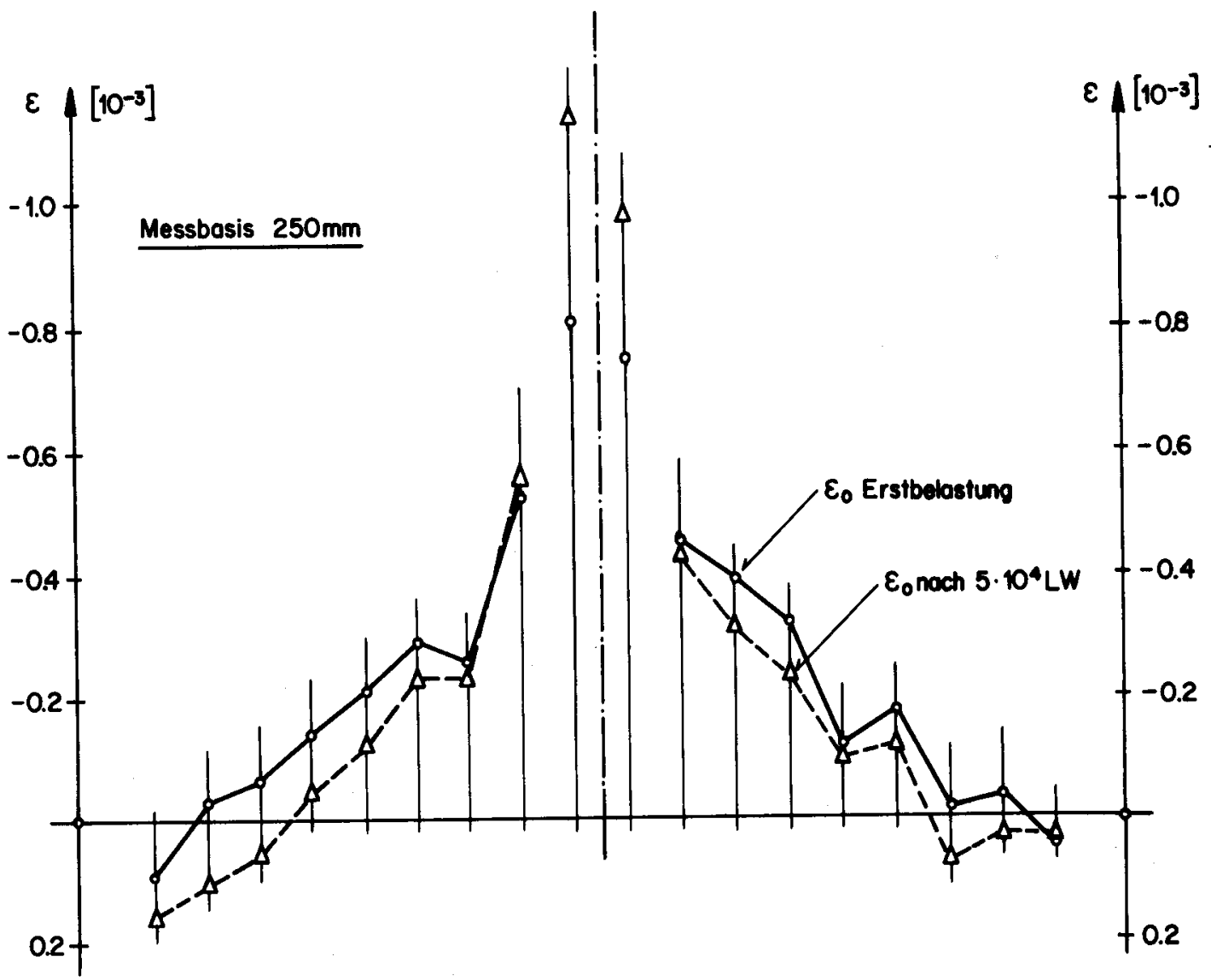

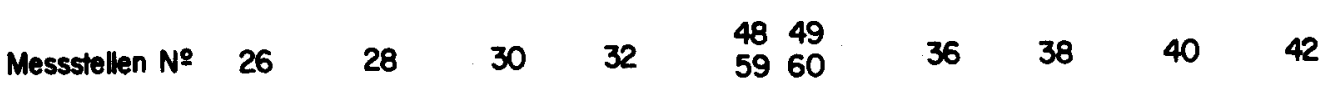
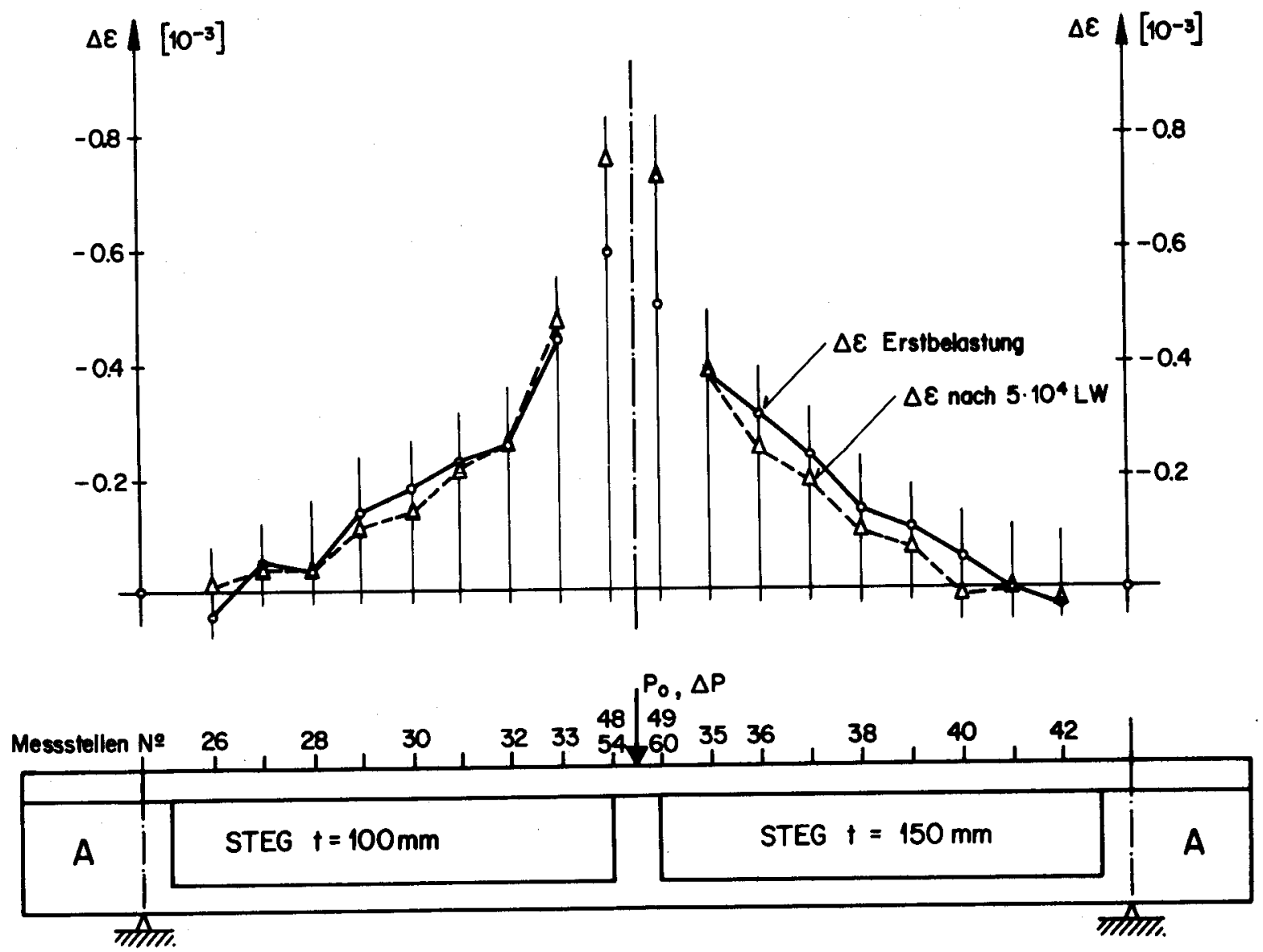

Bild 82: BI $/ 4$, Betondehwungen im Oberflansch im Lastzyklus B 


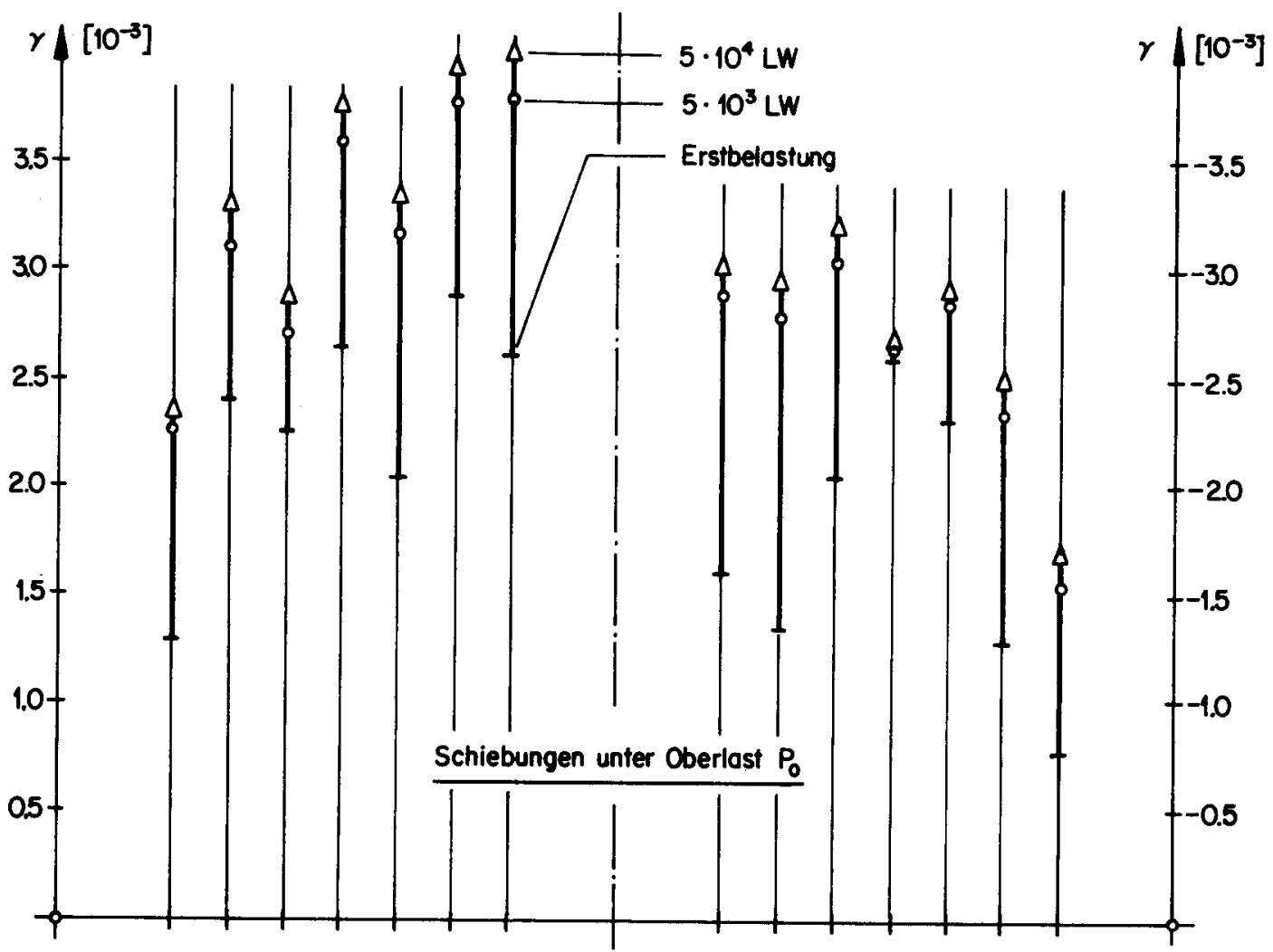

$\begin{array}{llllllllllllllllll}\text { Bügel № } & 1 & 2 & 3 & 4 & 5 & 6 & 7 & 8 & & 18 & 17 & 16 & 15 & 14 & 13 & 12 & 11\end{array}$
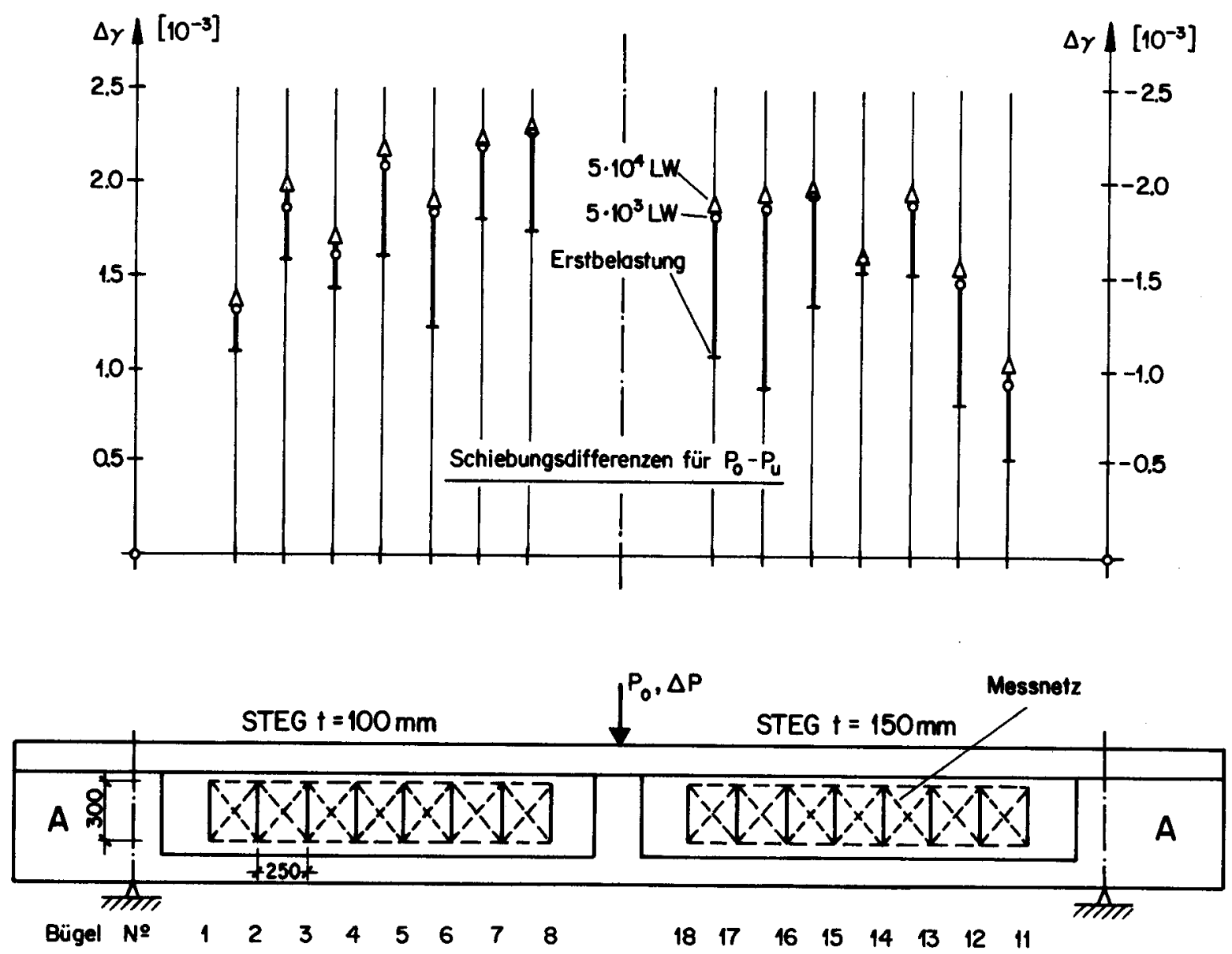

Bild 83: BI/4, Schiebungen und Schiebungsdifferenzen im Lastzyklus B 


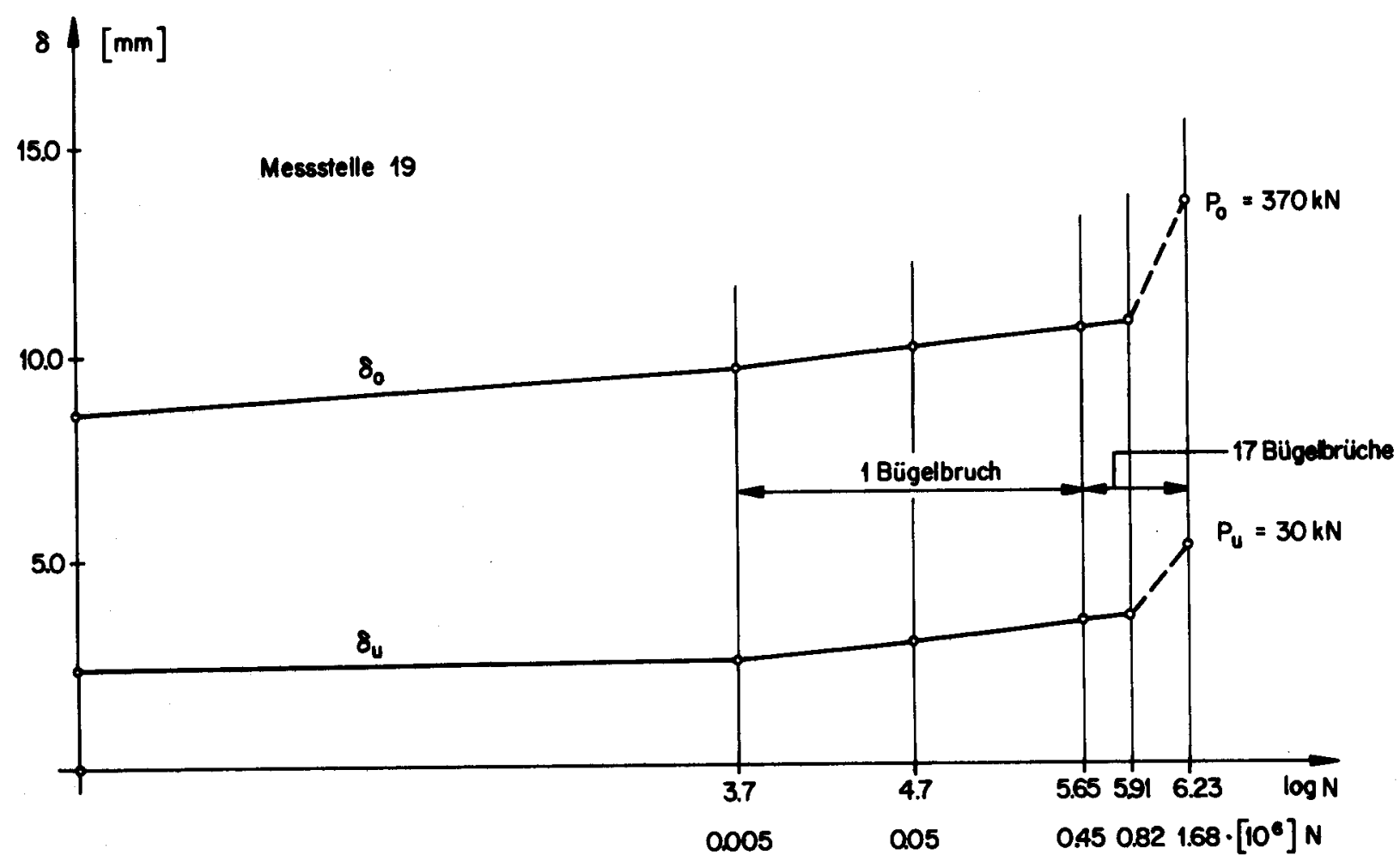

Bild $84: \mathrm{BI} / 5$, Durchbiegungs - Lastwechsel-Diagramm für die Trägermitte im Lastzyklus B

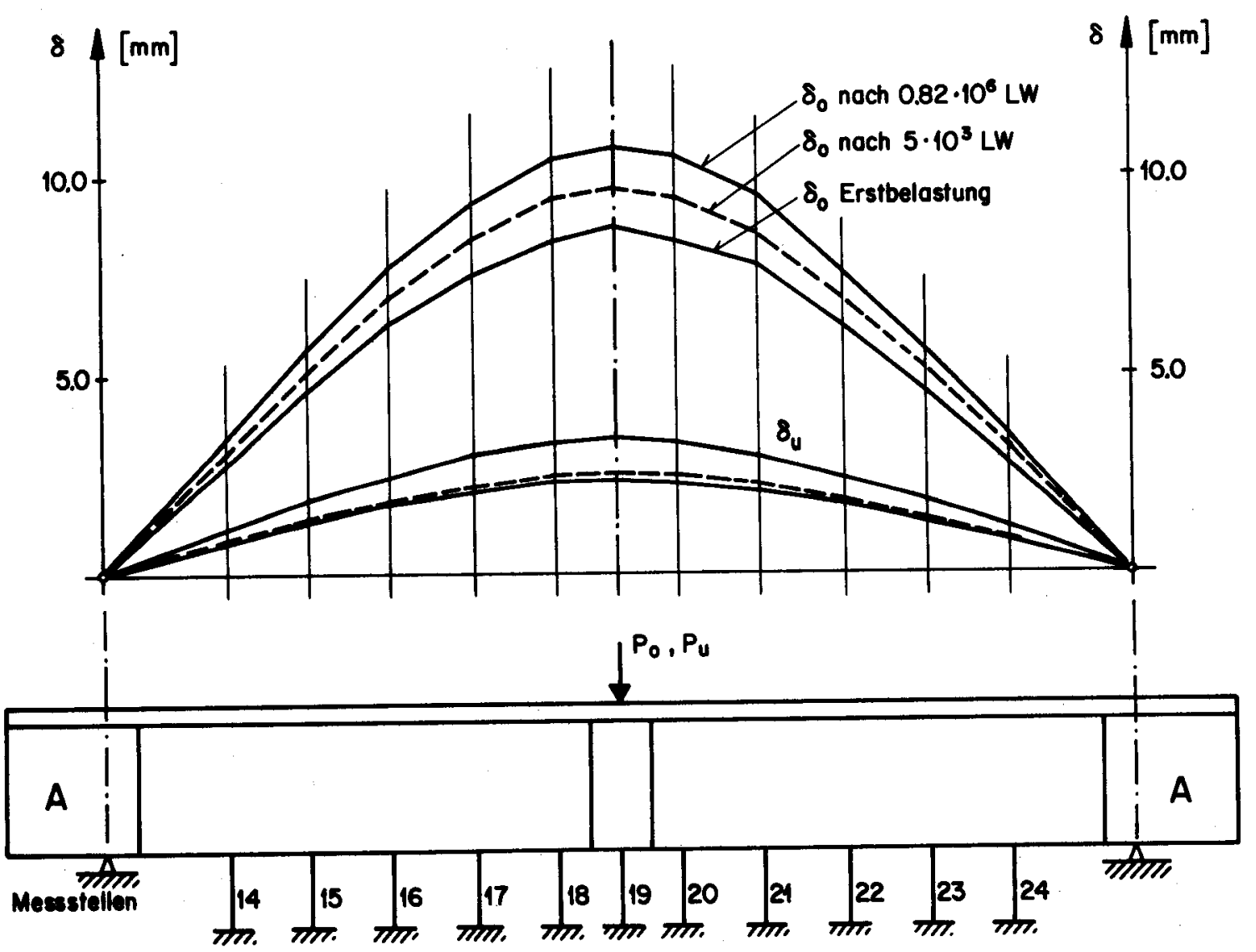

Bild 85: BI/5, Verlauf der Durchbiegung im Lastzyklus B 

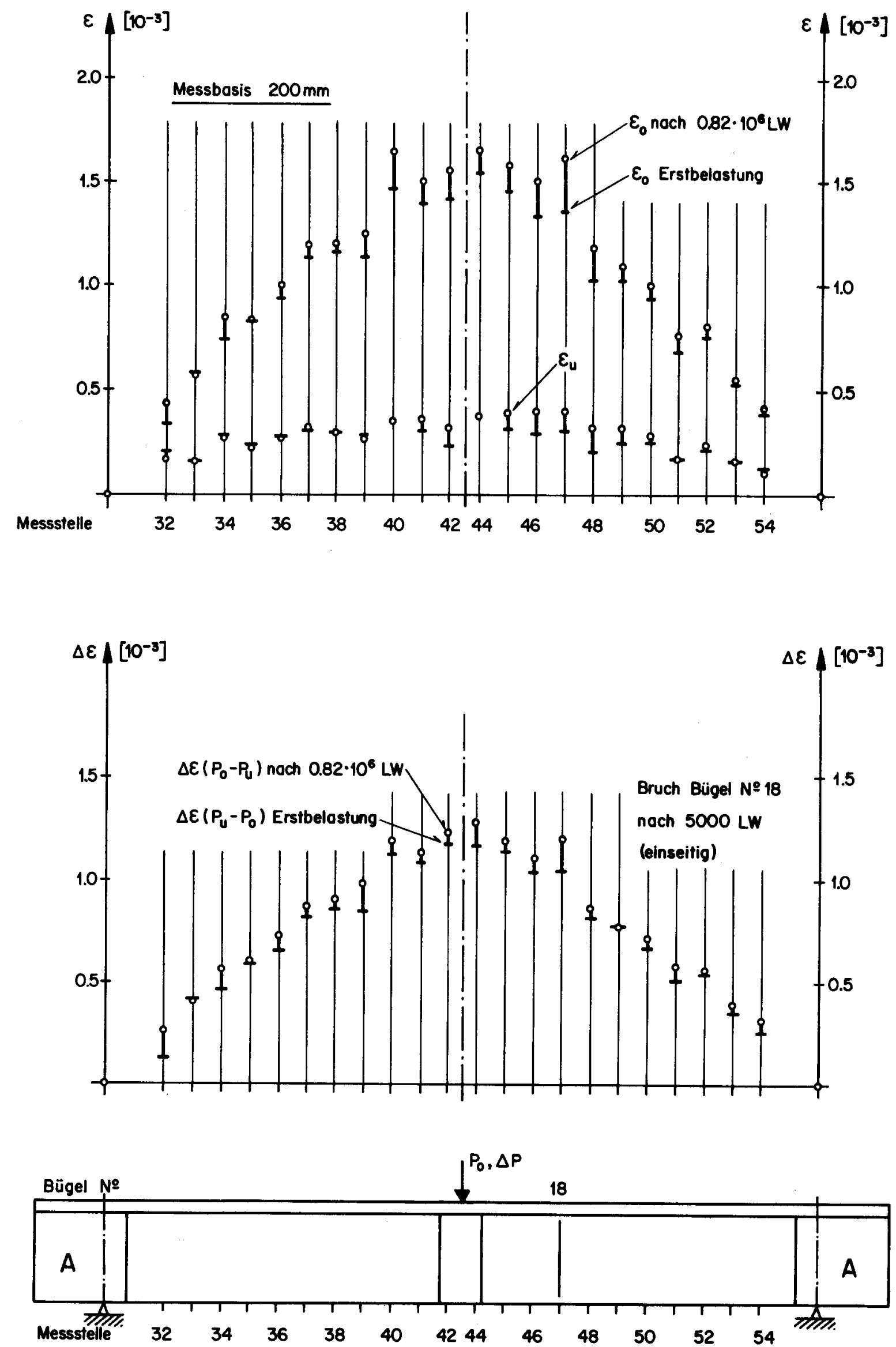

Bild 86: BI/5, Dehnungen in der Längsarmierung im Lastzyklus B 

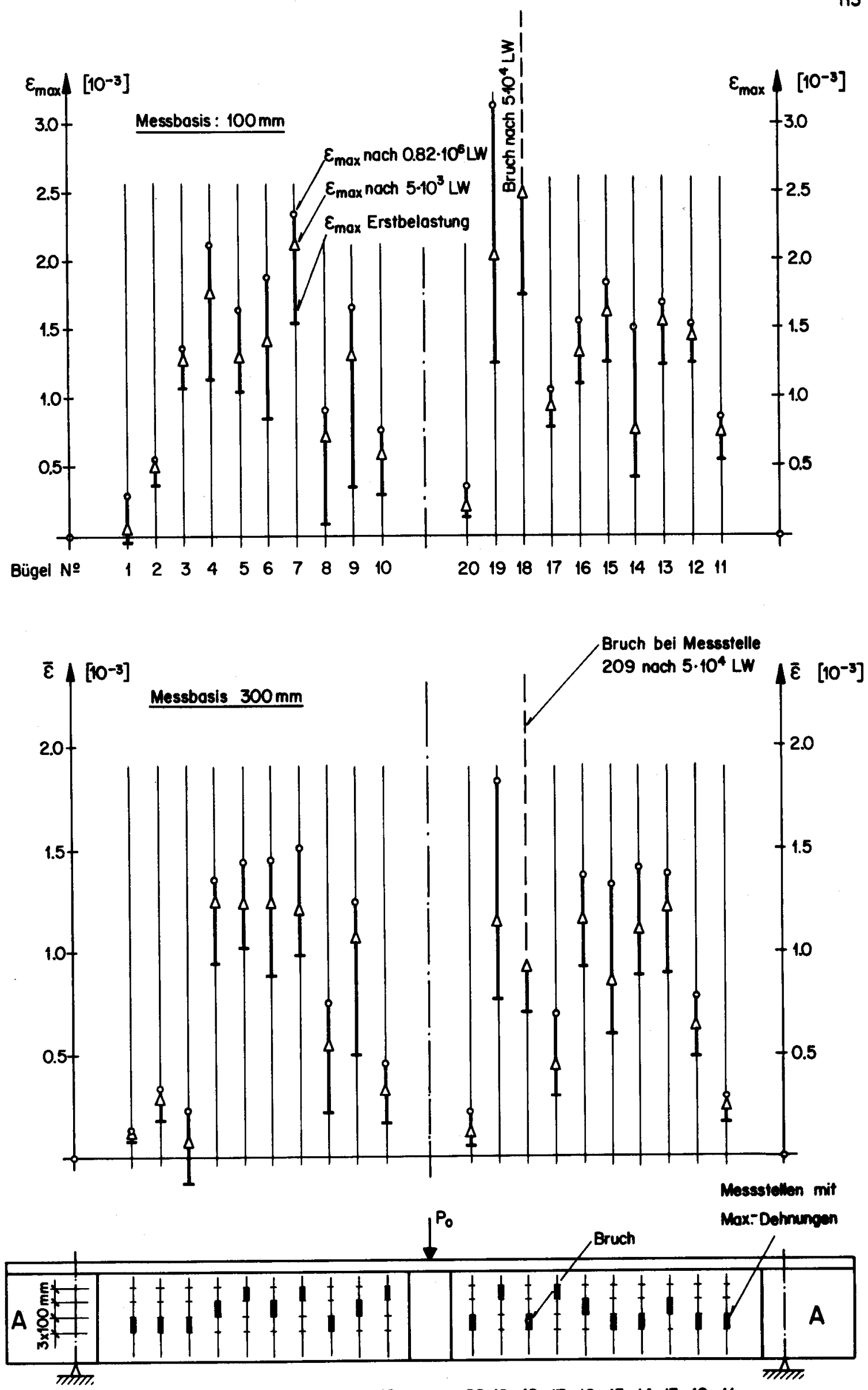

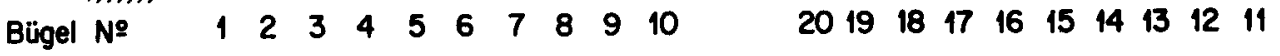

Bild 87: BI/5, Bügeldehnungen unter Oberlast im Lastzyklus B 


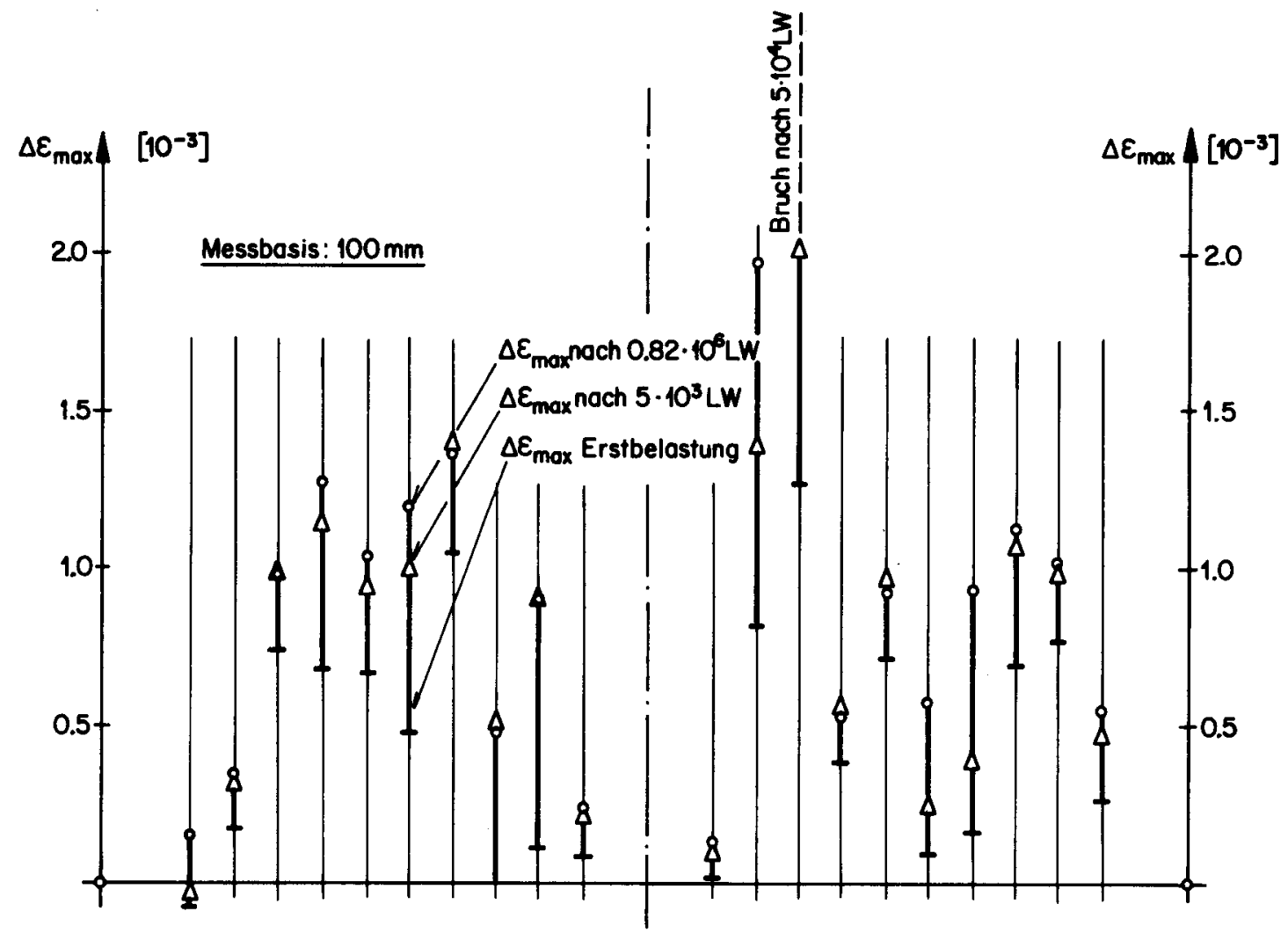

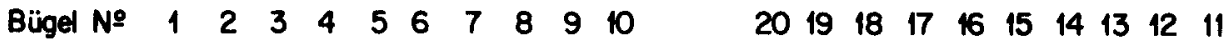
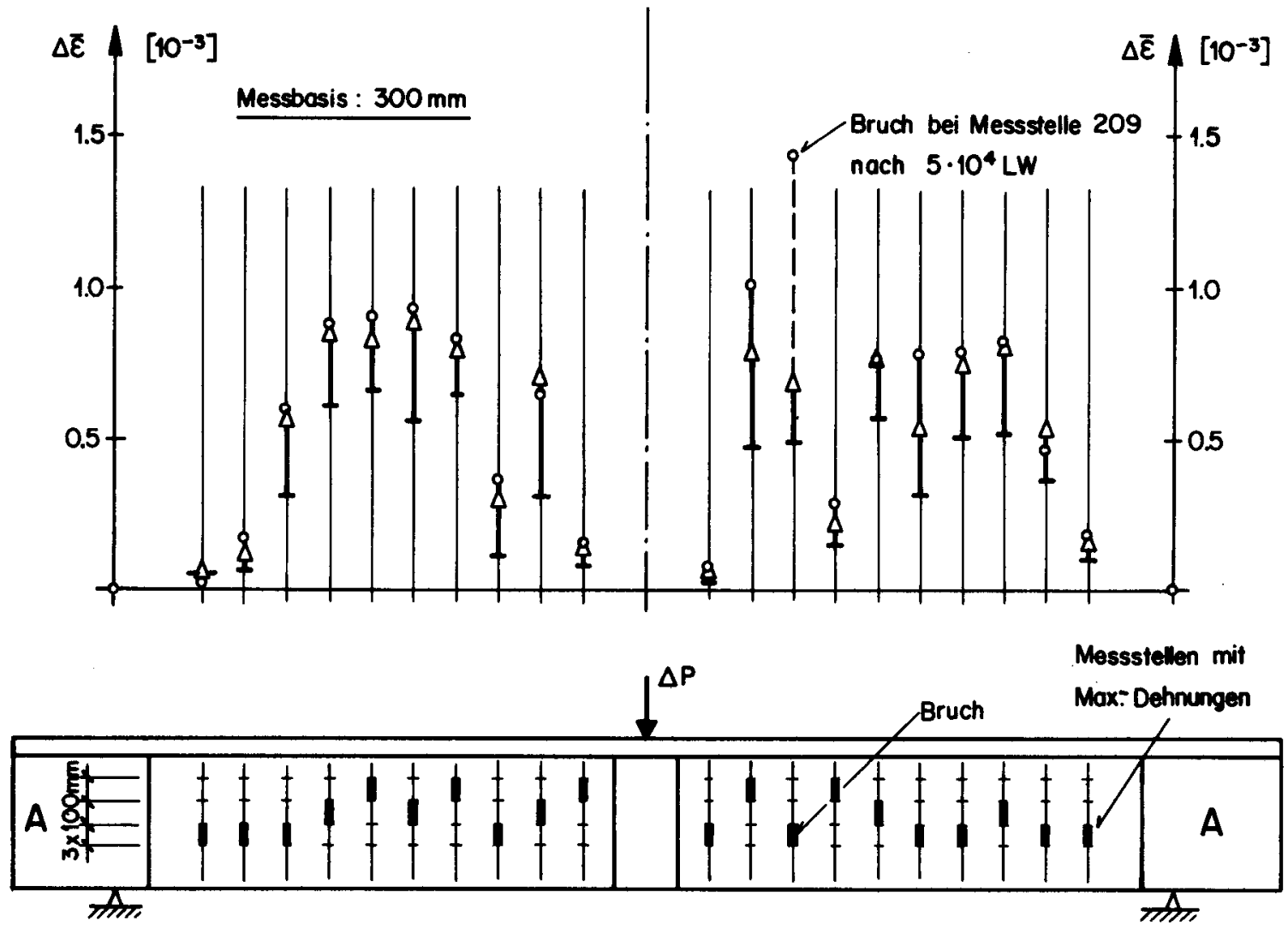

$\begin{array}{lllllllllllllllllllll}\text { Bügel Ne } & 1 & 2 & 3 & 4 & 5 & 6 & 7 & 8 & 9 & 10 & 20 & 19 & 18 & 17 & 16 & 15 & 14 & 13 & 12 & 11\end{array}$

Bild 88: BI /5, Dehnungsdifferenzen in den Bügeh im Lastzyklus B 


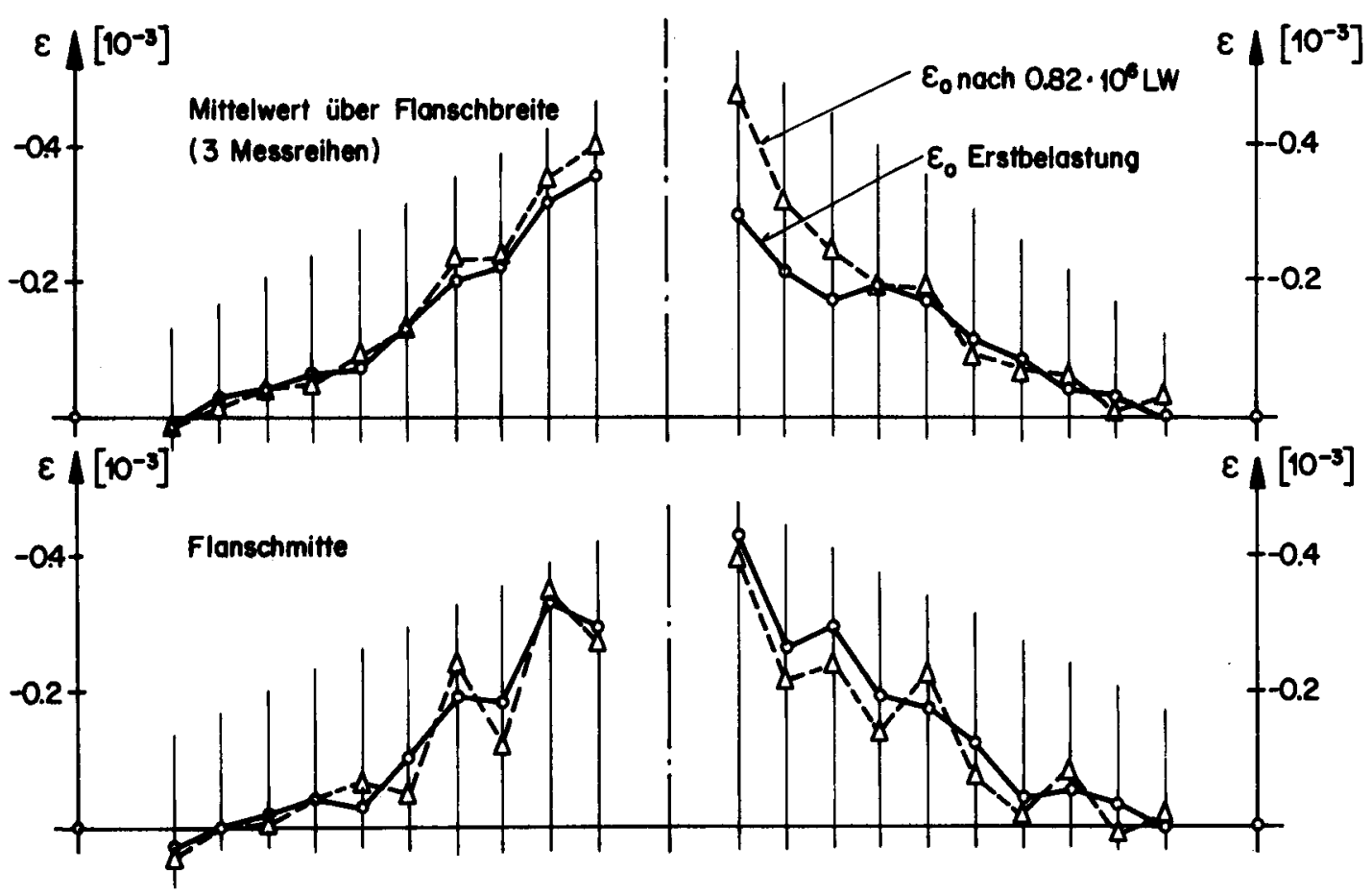

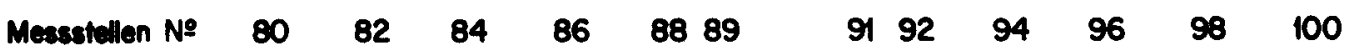
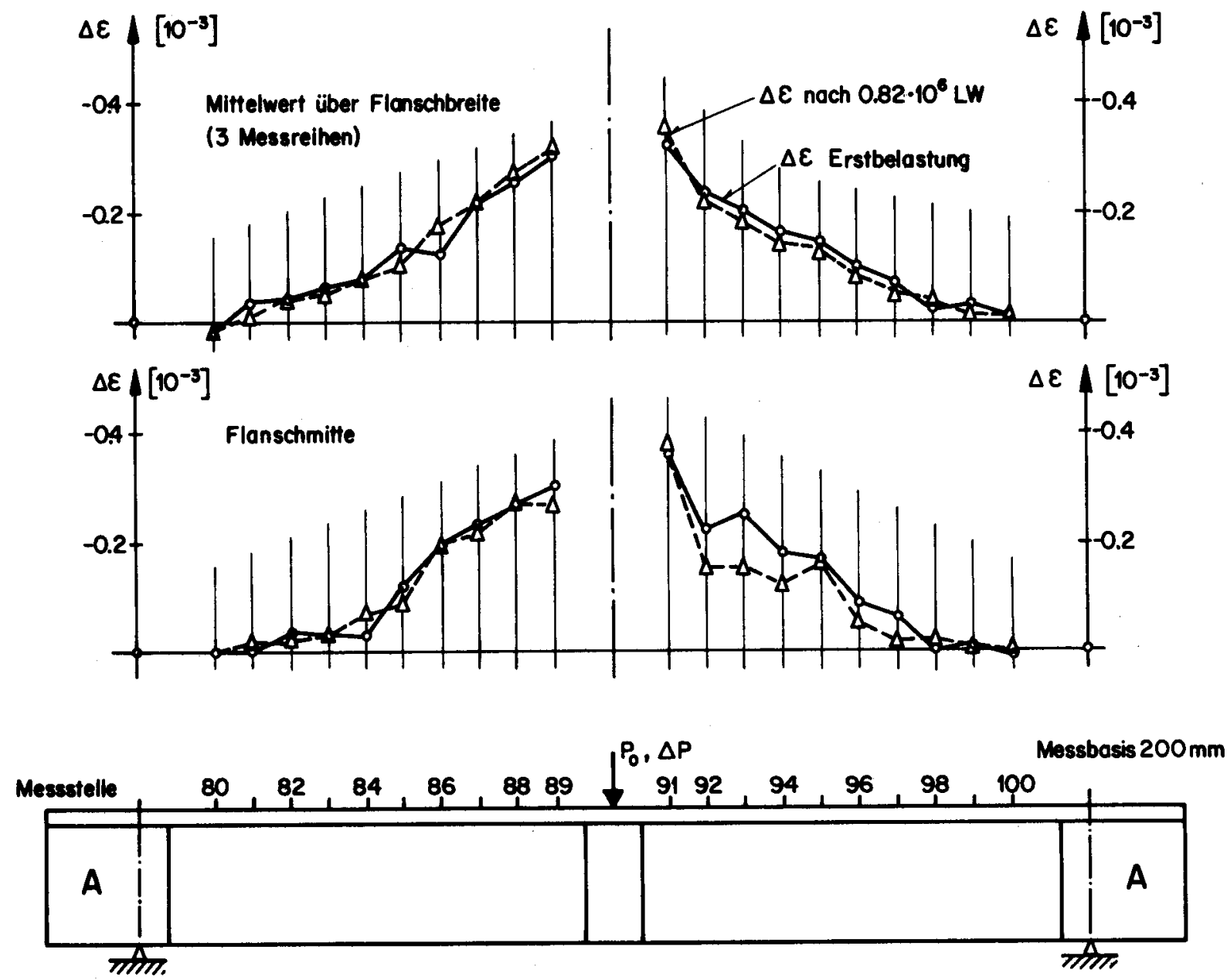

Bild 89: BI/5, Betondehnungen im Oberflansch im Lastzyklus B 

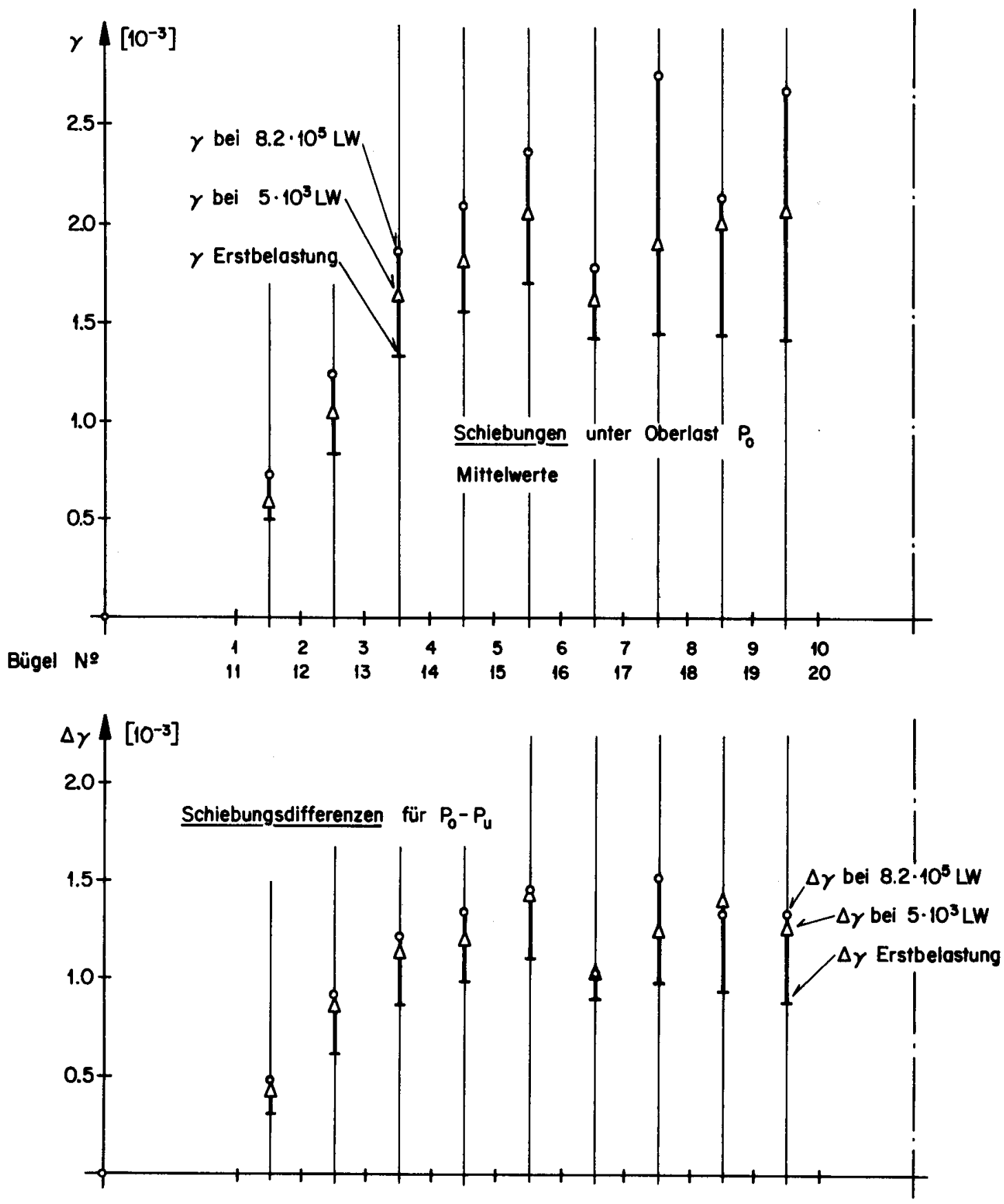

Nota: Bruch des Bügels № 18 nach $5 \cdot 10^{4}$ LW (einseitig)

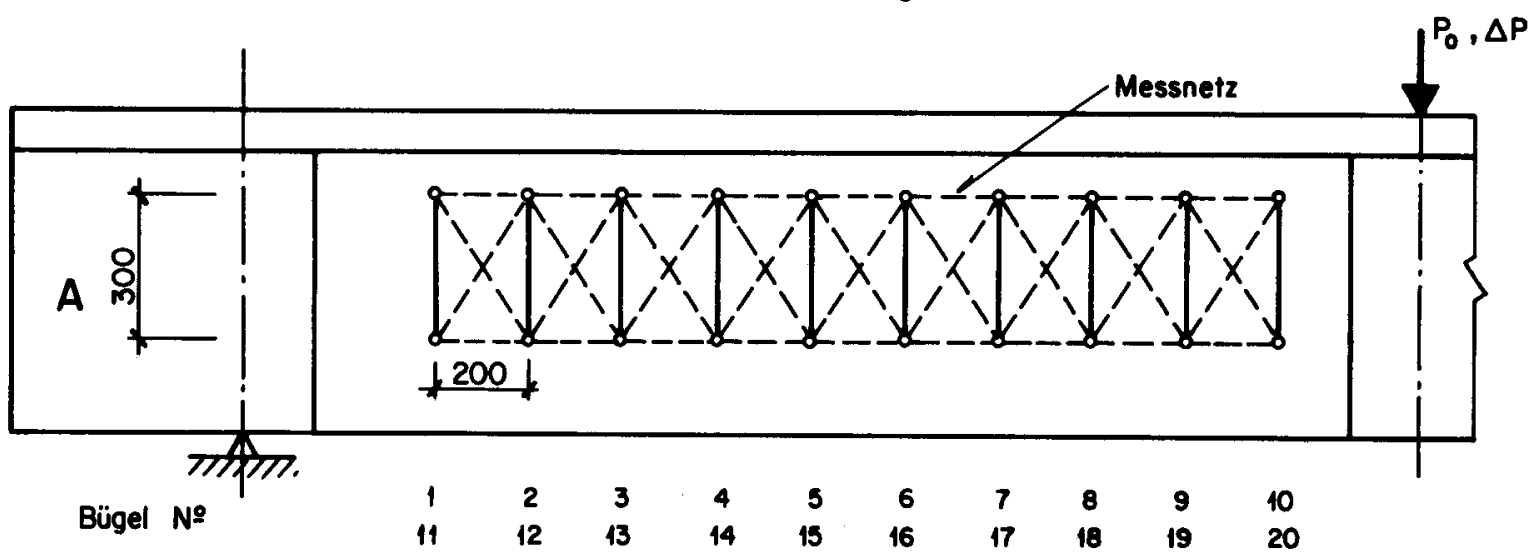

Bild 90 : Bl/5, Schiebungen und Schiebungsdifferenzen im Lastzyklus B, Mittelwerte aus linker und rechter Balkenseite 


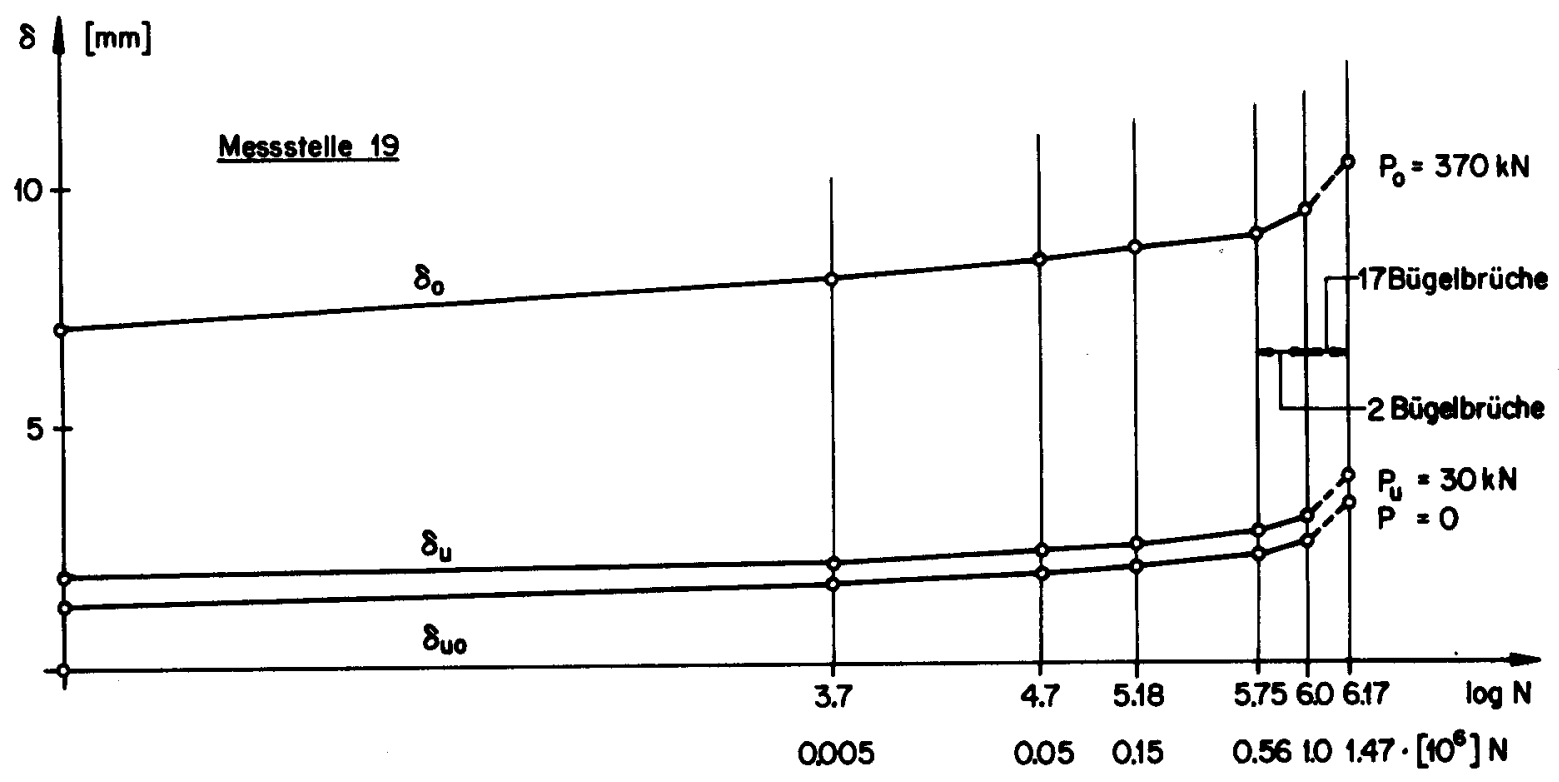

Bild 91: BI /6, Durchbiegungs - Lasłwechsel-Diagramm für die Trägermitte im Lastzyklus B

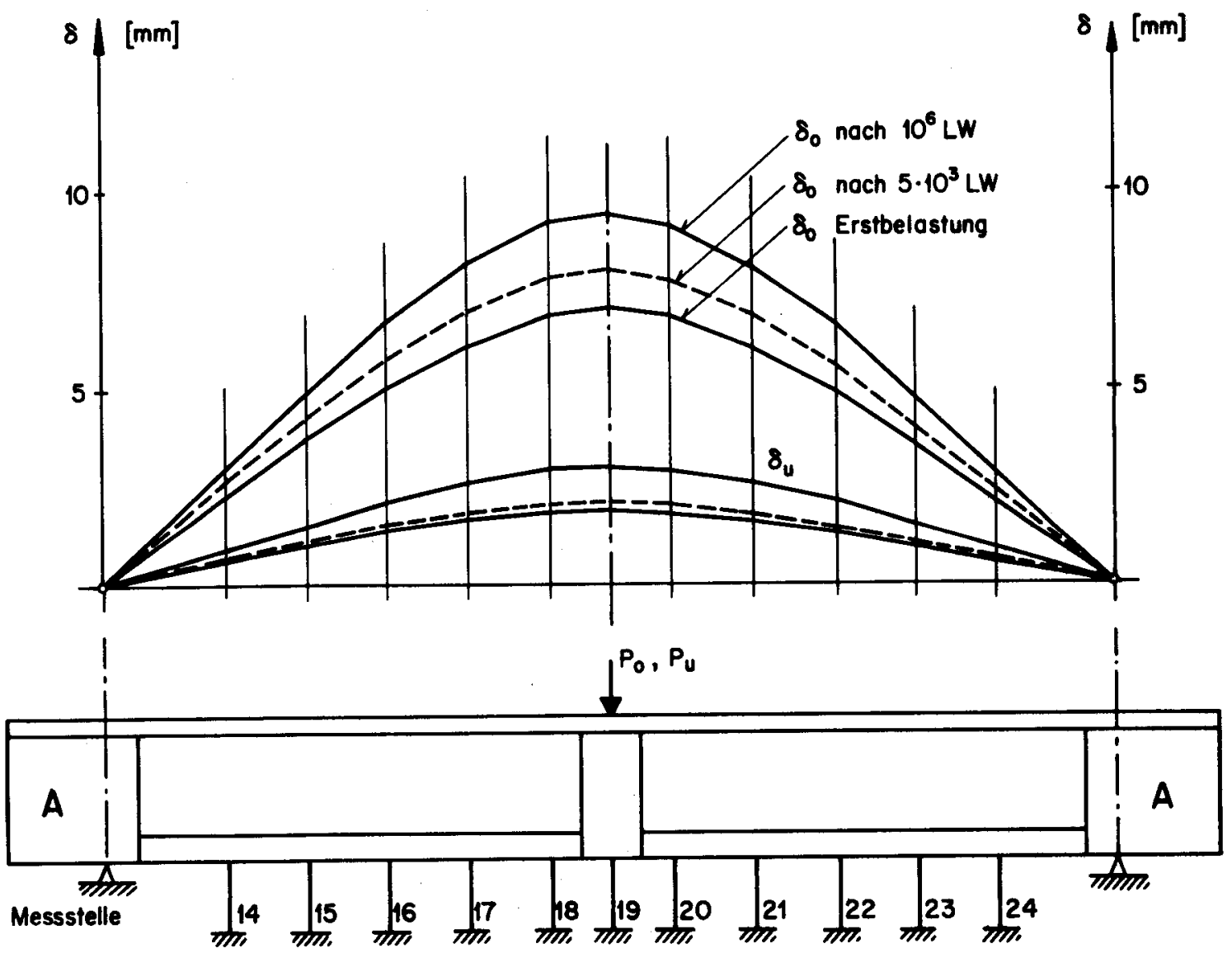

Bild 92: BI/6, Verlouf der Durchbiegung im Lastzyklus B 

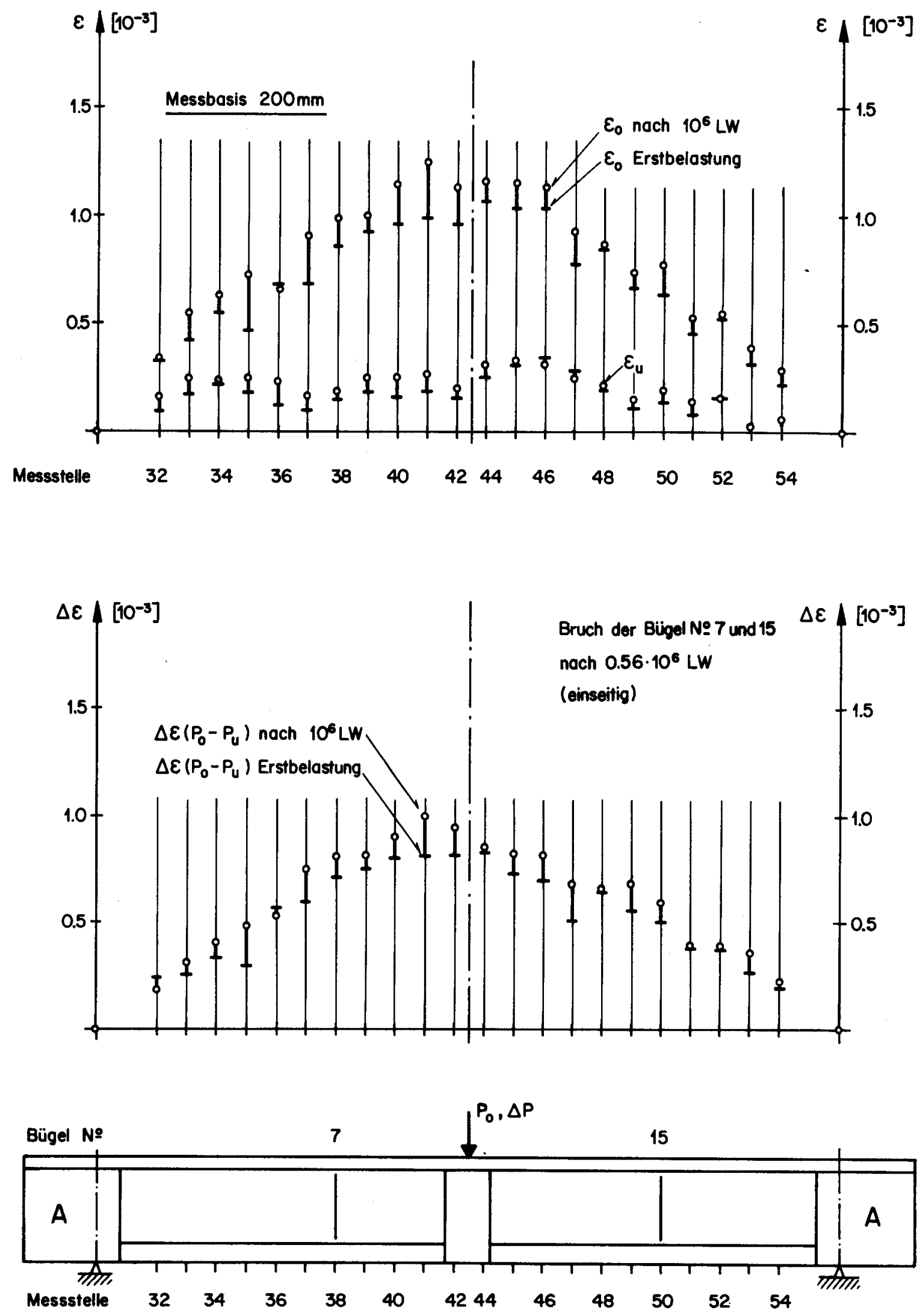

Bild 93: BI/6, Dehnungen in der Längsbewehrung im Lastzyklus $B$ 

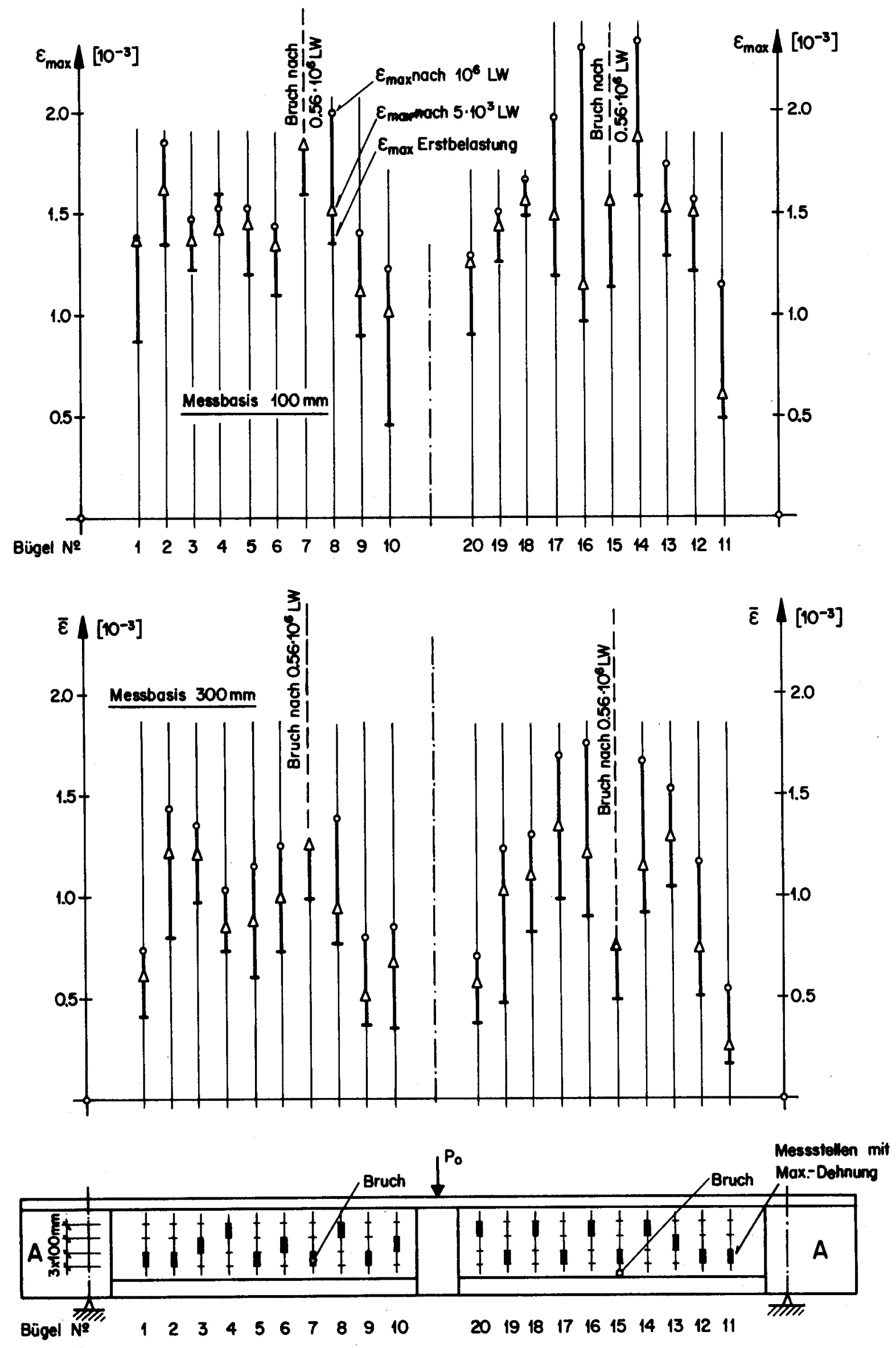

Bild 94: BI/6, Bügeldehnungen unter Oberlast im Lastzyklus B 

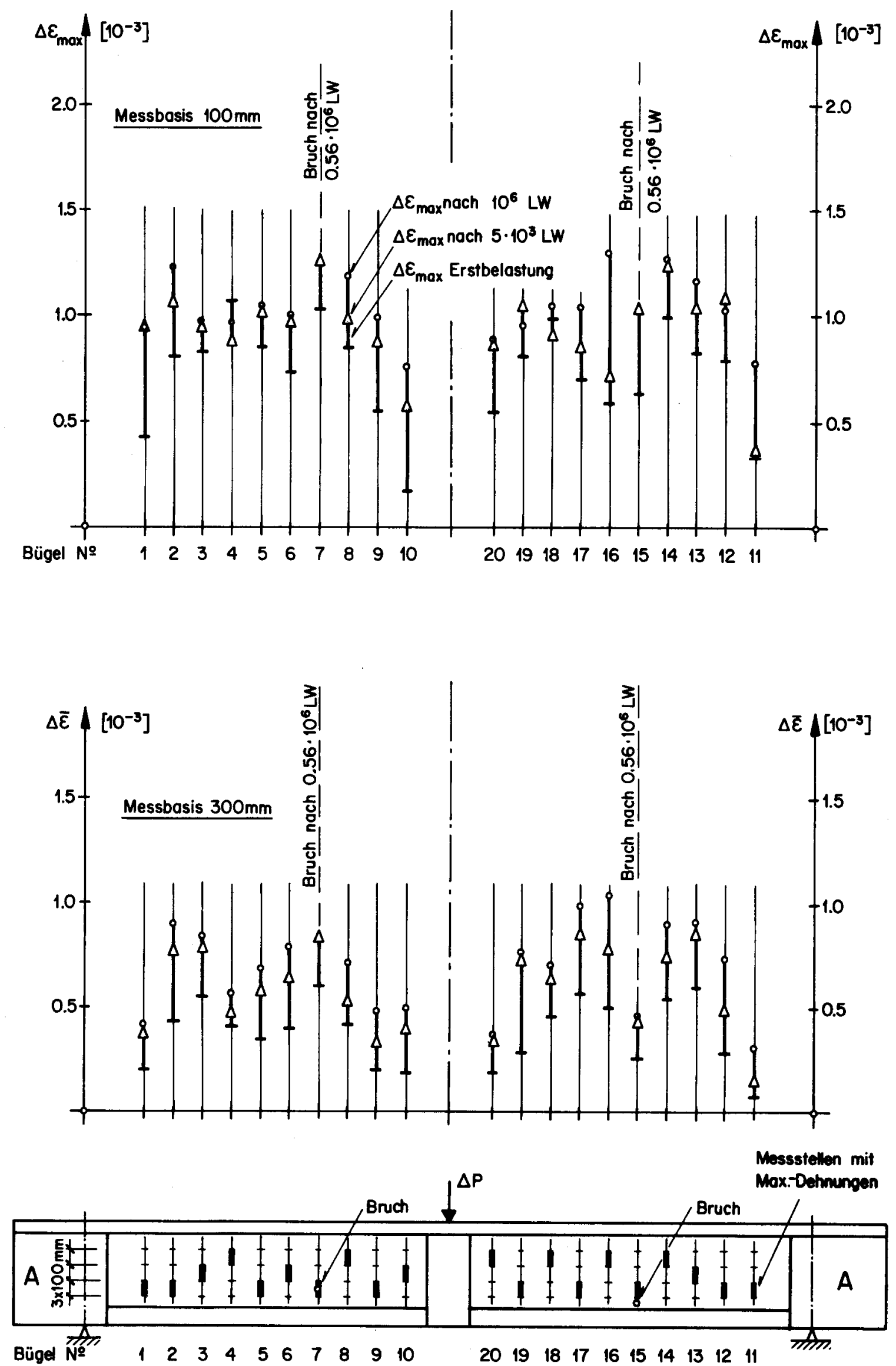

Bild $95:$ BI/6, Dehnungsdiffenzen in den Bügeln im Lastzyklus B 


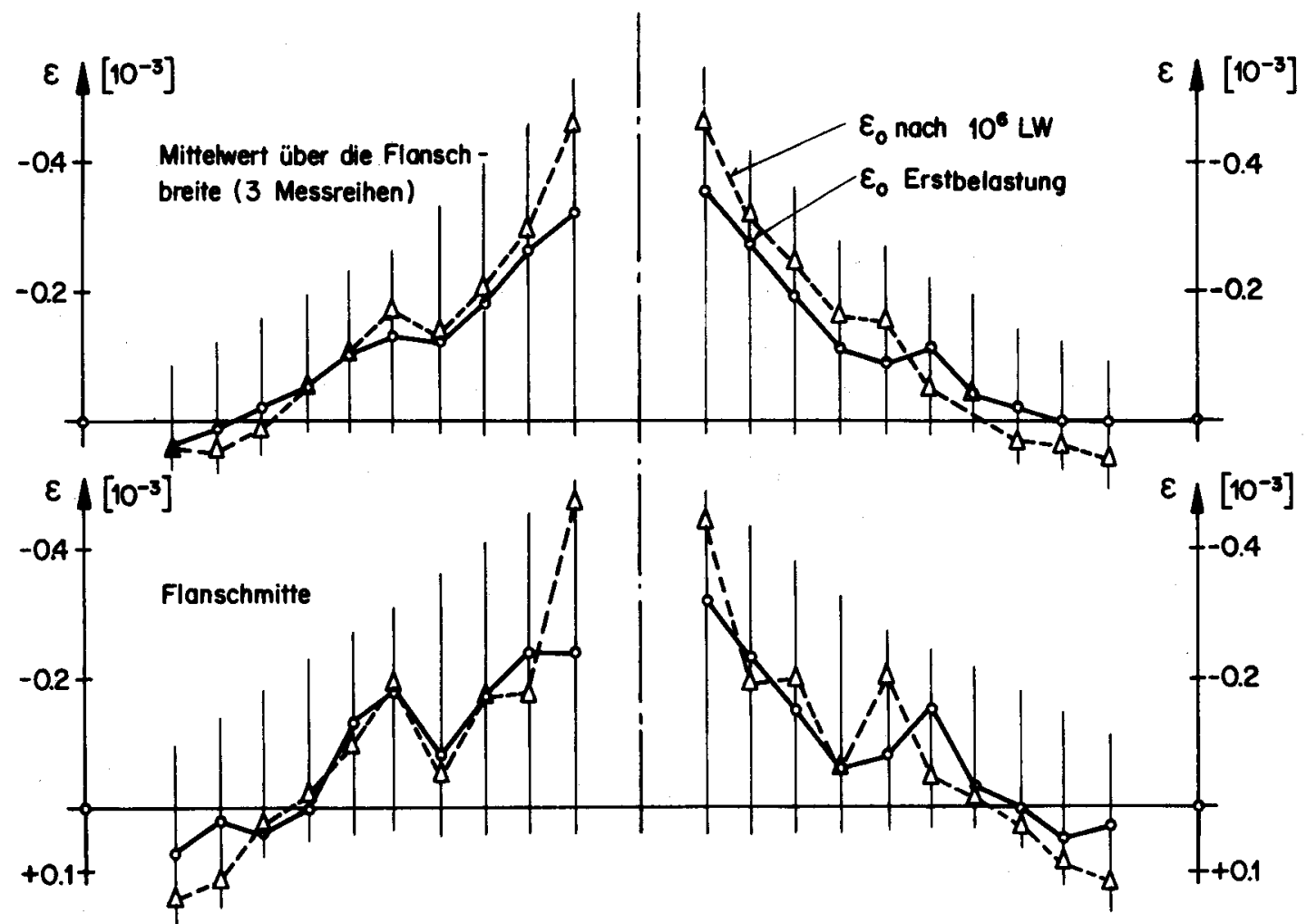

Messstelle

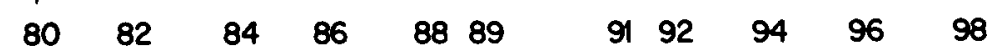
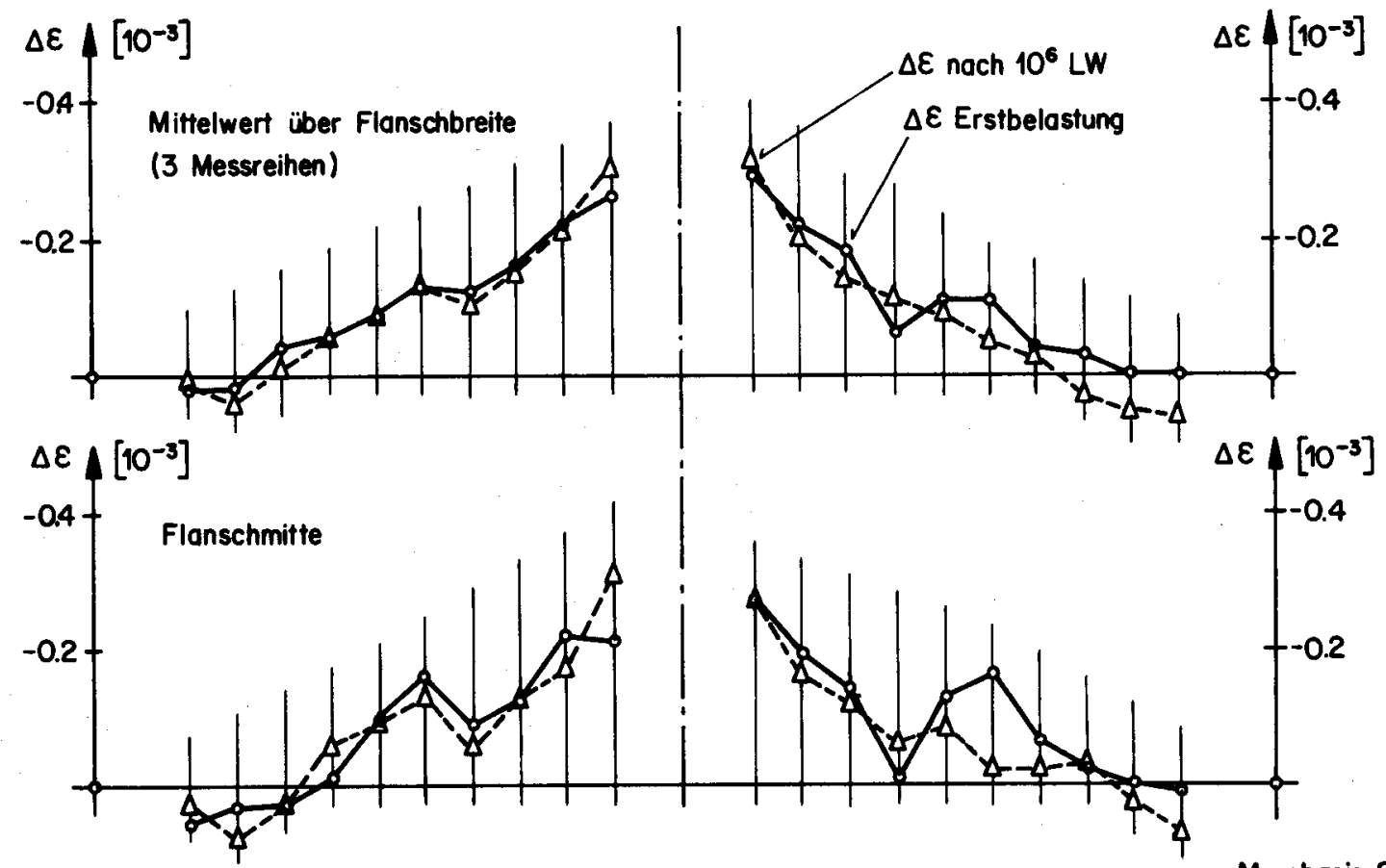

Messbosis $200 \mathrm{~mm}$

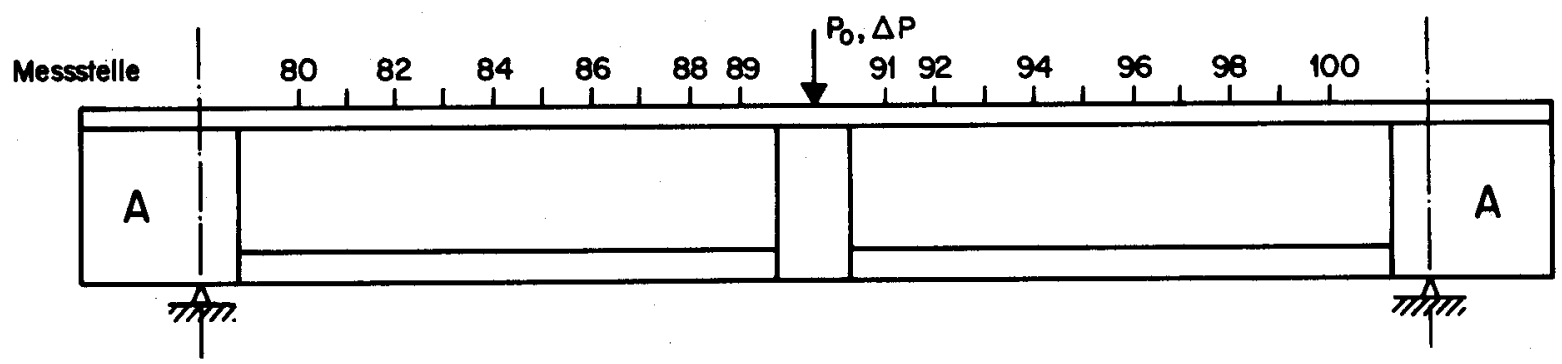

Bild 96: BI/6, Betondehnungen im Oberflansch im Lastzyklus B 


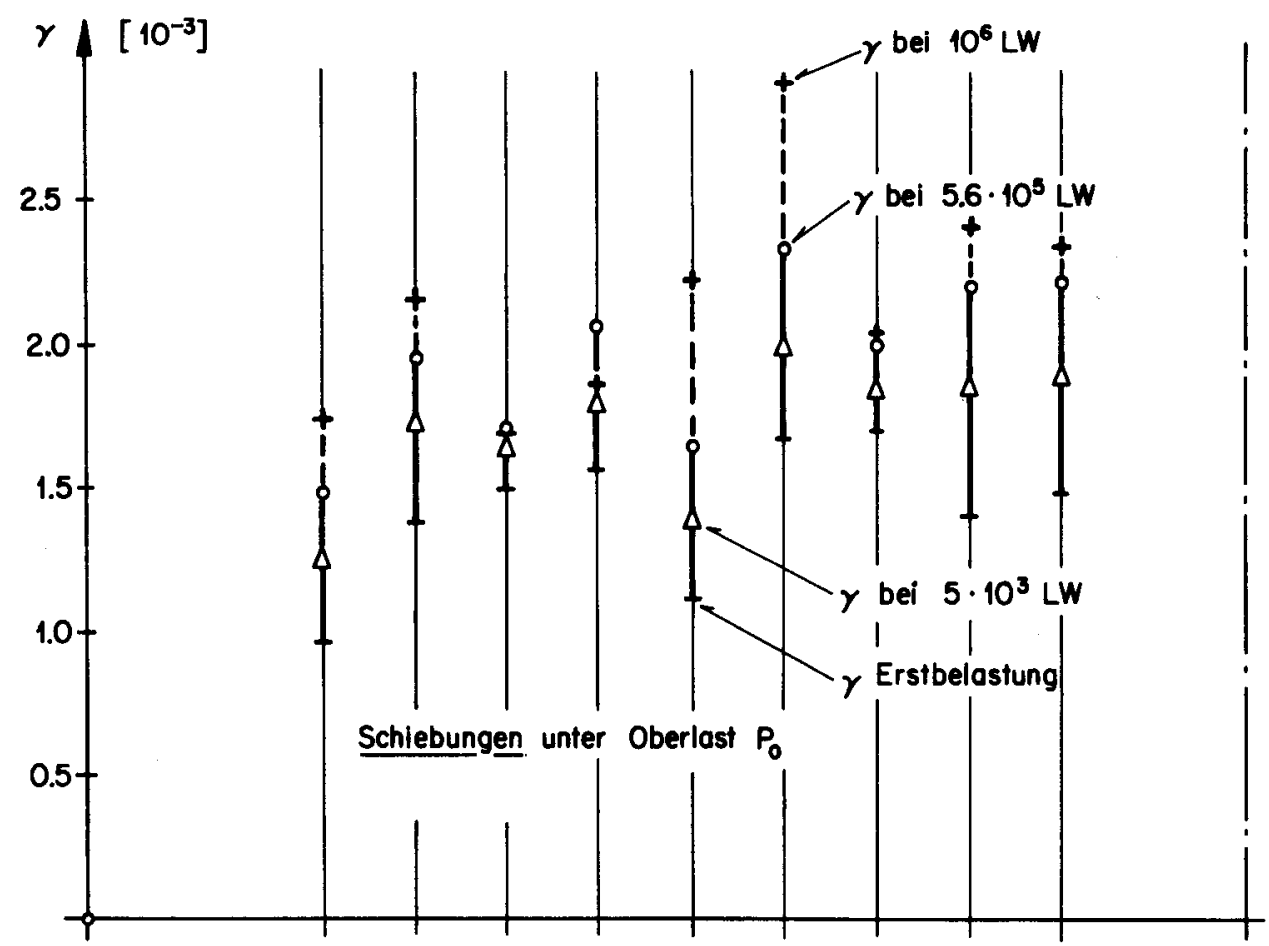

Bügel №

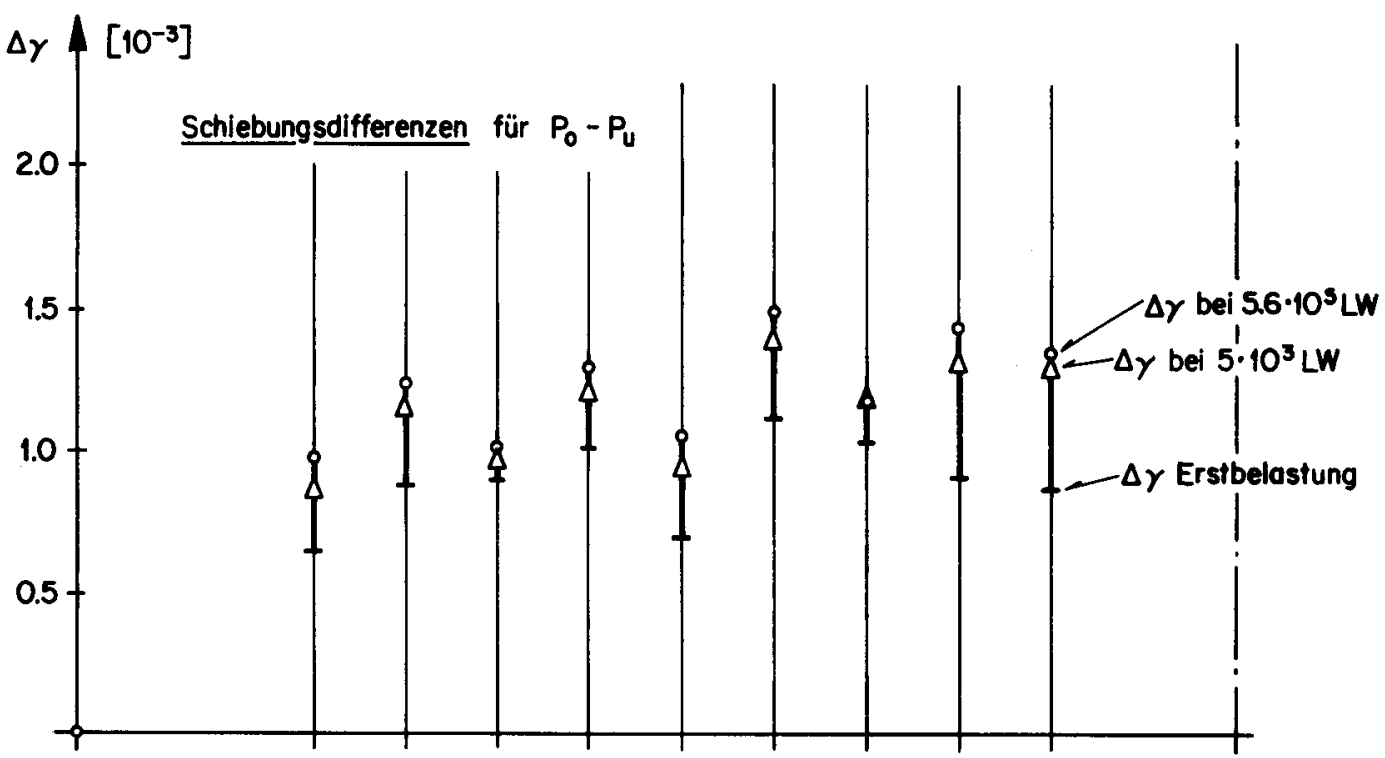

ruch der Bügel № 7 und 15 noch $5.6 \cdot 10^{4} \mathrm{LW}$

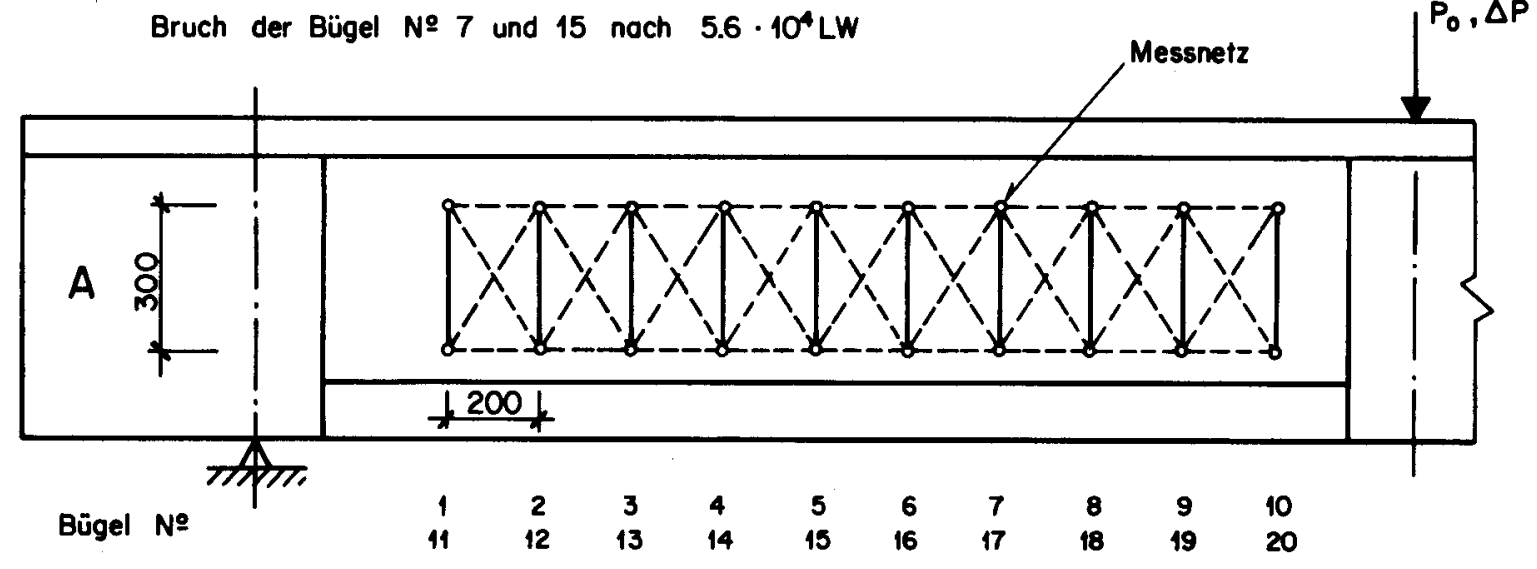

Bild 97: BI/6, Schiebungen und Schiebungsdifferenzen im Lastzyklus B, Mittelwerte aus linker und rechter Balkenseite 
Leer - Vide - Empty 


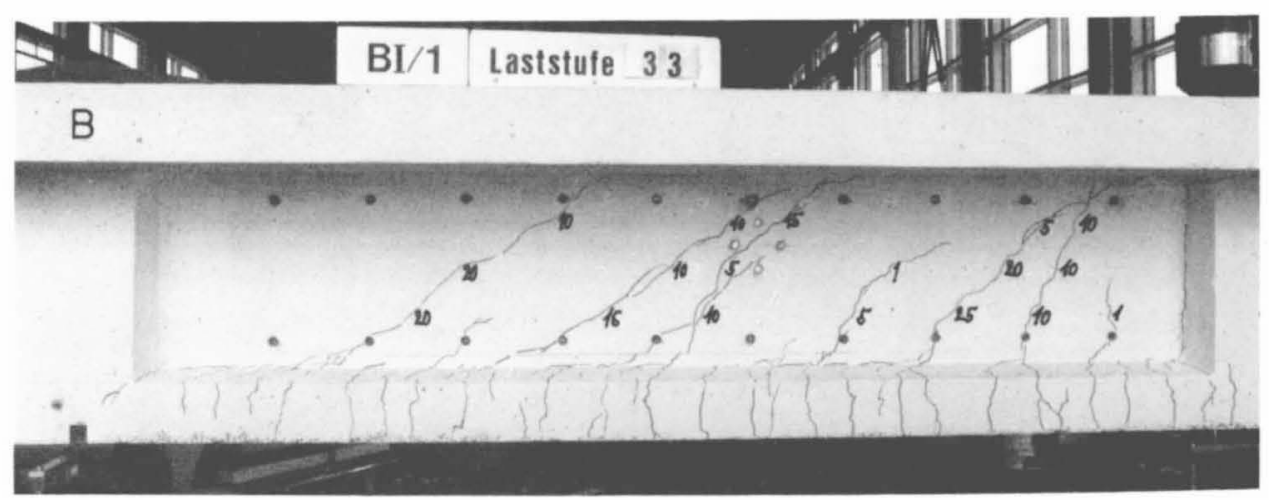

Lastzyklus B

$P_{0}=370 \mathrm{kN}$

$N_{B}=6 \cdot 10^{4} \mathrm{LW}$

$\left(N_{\text {tot }}=6 \cdot 10^{4} \mathrm{LW}\right)$

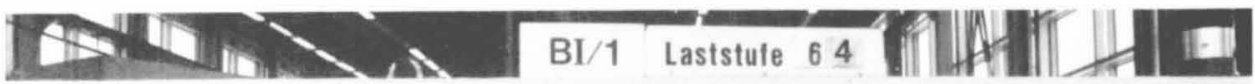

B
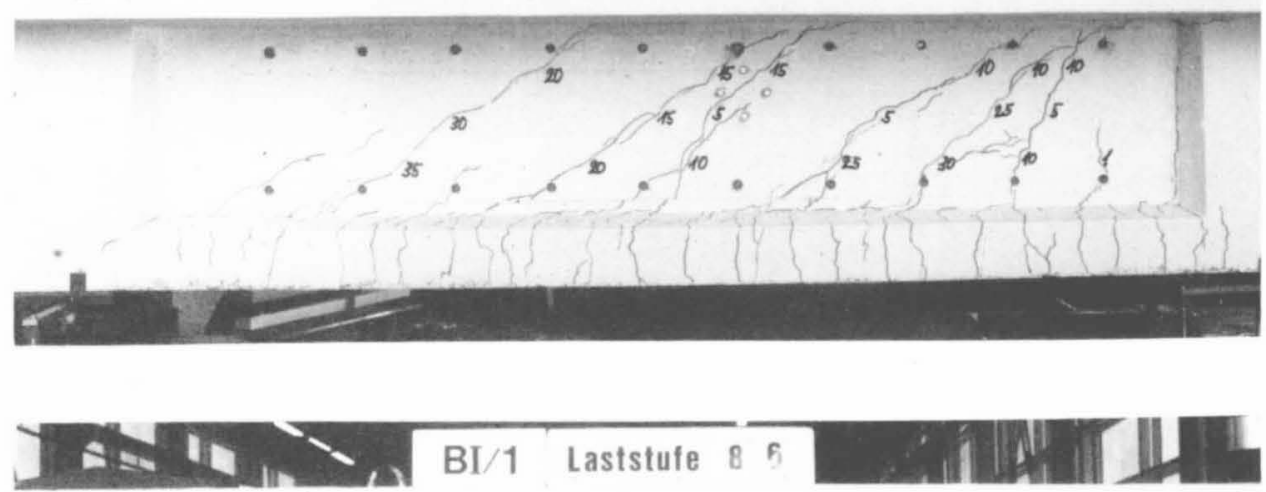

B

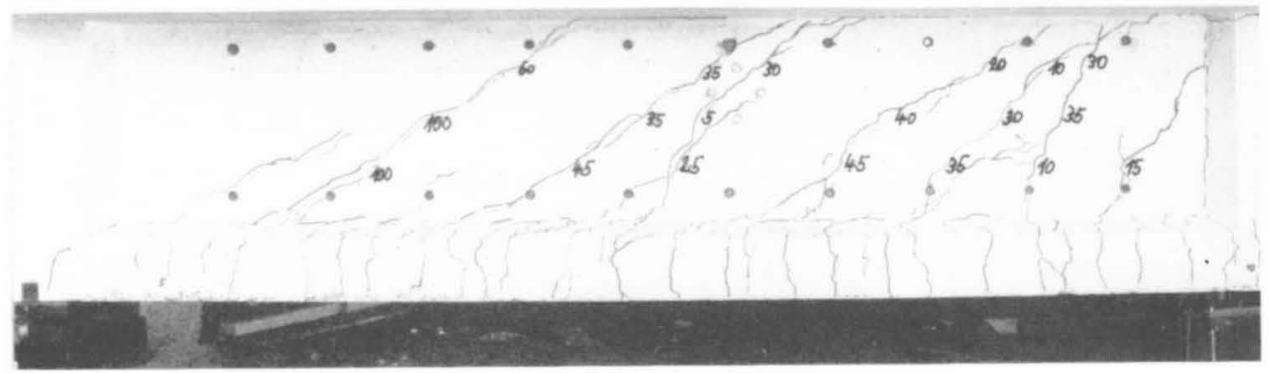

Rissweiten in $1 / 100 \mathrm{~mm}$
Lastzyklus D

$$
\begin{aligned}
P_{0} & =520 \mathrm{kN} \\
N_{D} & =5 \cdot 10^{4} \mathrm{LW} \\
\left(N_{\text {tot }}\right. & \left.=1.72 \cdot 10^{5} \mathrm{LW}\right)
\end{aligned}
$$

Lastzyklus F

$P_{0}=660 \mathrm{kN}$

$N_{F}=2.35 \cdot 10^{4} \mathrm{LW}$

$\left(N_{\text {tot }}=2.25 \cdot 10^{5} \mathrm{LW}\right)$

Bild 98: BI/1, Rissbilder in den Lastzyklen B, D und F, Steg $t=150 \mathrm{~mm}$ 


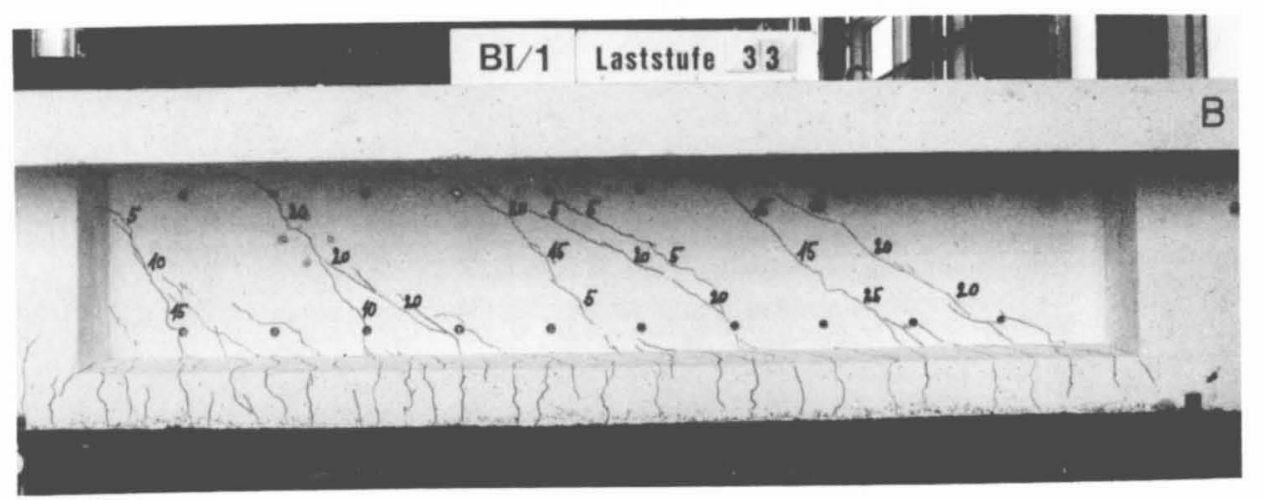

Lastzyklus B

$P_{0}=370 \mathrm{kN}$

$N_{B}=6 \cdot 10^{4} \mathrm{LW}$

$\left(N_{\text {tot }}=6 \cdot 10^{4} \mathrm{LW}\right)$

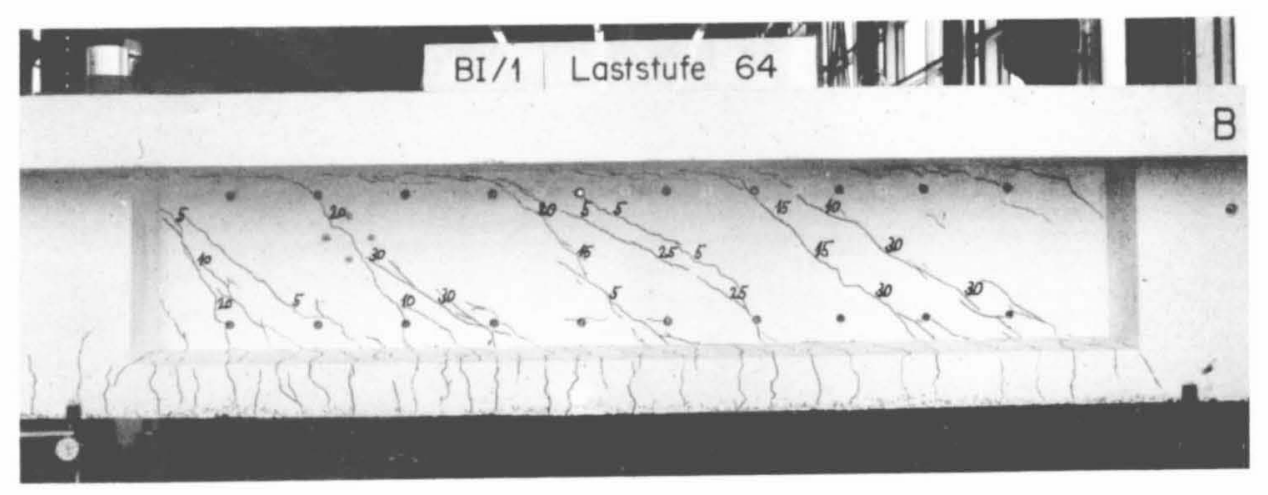

Lastzyklus D

$P_{0}=520 \mathrm{kN}$

$N_{D}=5 \cdot 10^{4} \mathrm{LW}$

$\left(N_{\text {tot }}=1.72 \cdot 10^{5} \mathrm{LW}\right)$

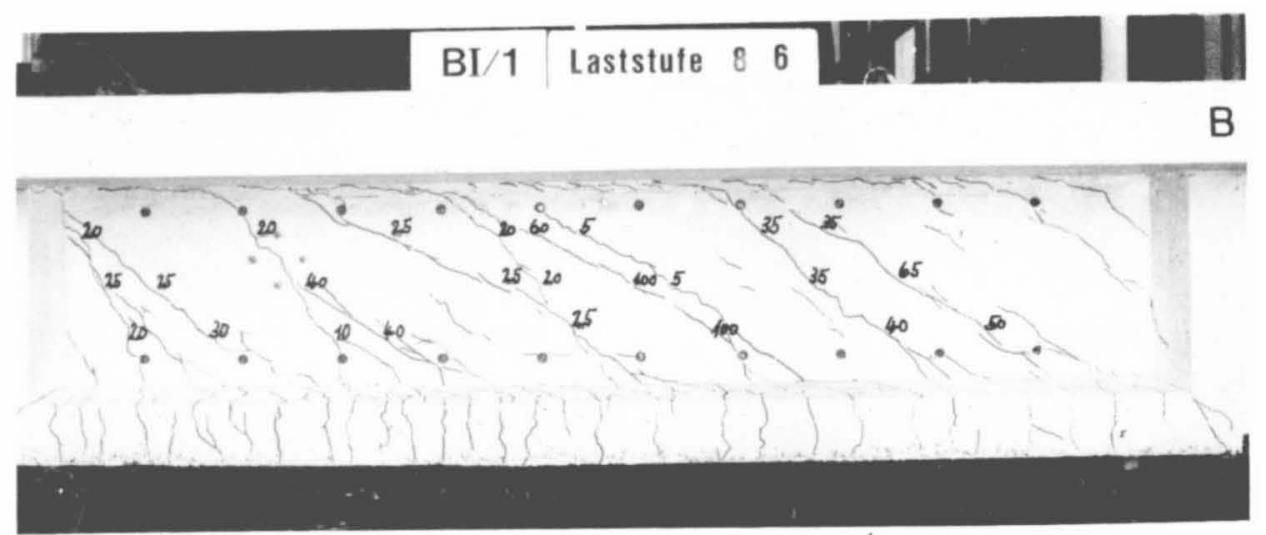

Lastzyklus F

$P_{0}=660 \mathrm{kN}$

$N_{F}=2.35 \cdot 10^{4} \mathrm{LW}$

$\left(N_{\text {tot }}=2.25 \cdot 10^{5} \mathrm{LW}\right)$

Rissweiten in $1 / 100 \mathrm{~mm}$

Bild 99: BI /1, Rissbilder in den Lastzyklen B, D und F, Steg $t=100 \mathrm{~mm}$

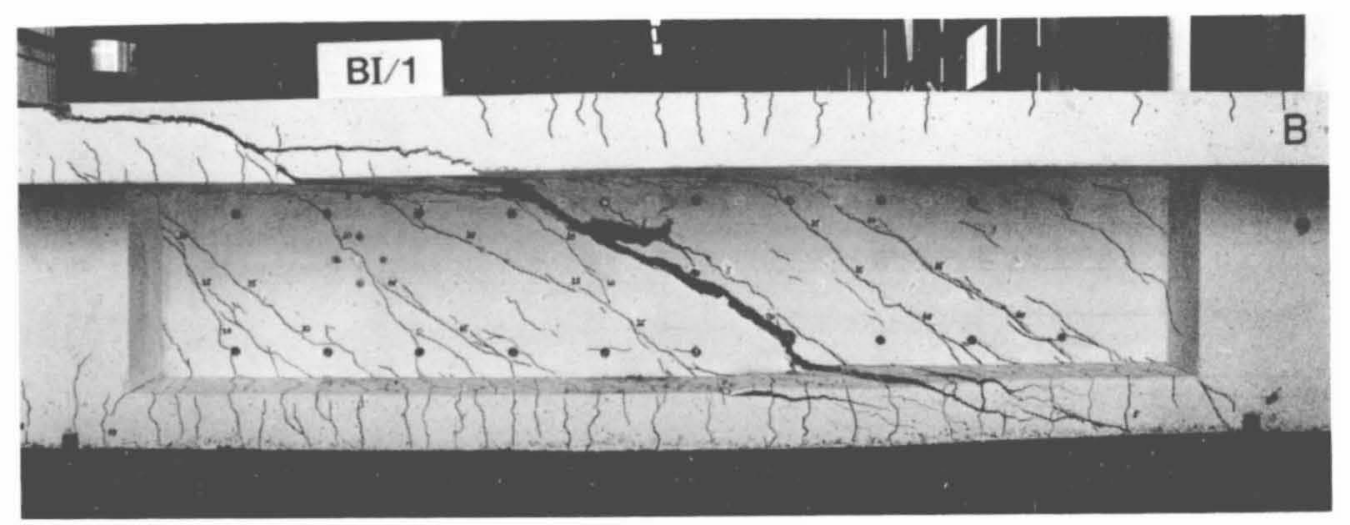

$P_{B r}=540 k N$

Bild 100 : BI/1, Bruch nach statischer Endbelastung im Lastzyklus $H$, Steg $\dagger=100 \mathrm{~mm}$ 

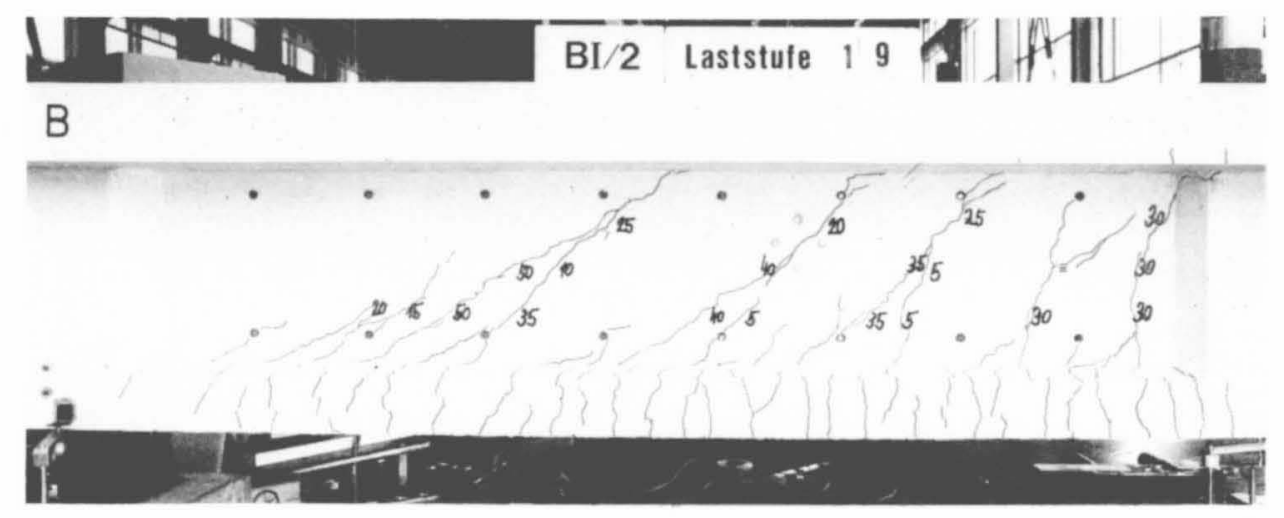

$P_{0}=370 \mathrm{kN}$
$N=1.5 \cdot 10^{3} \mathrm{LW}$

Steg $t=150 \mathrm{~mm}$
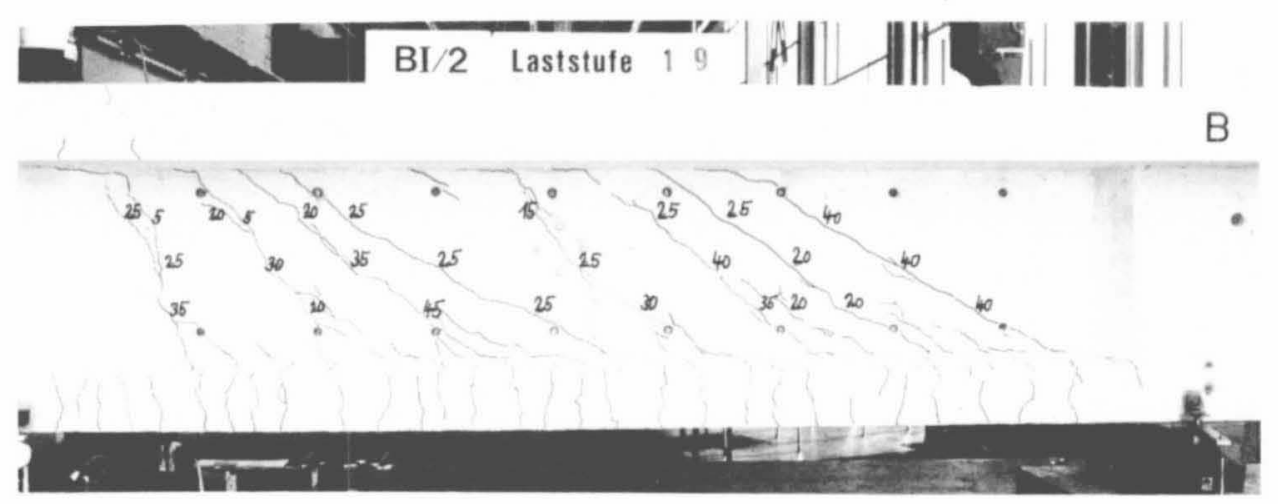

Rissweiten in $1 / 100 \mathrm{~mm}$

Bild 101 : BI/2, Rissbild im Lastzyklus B

Steg $t=150 \mathrm{~mm}$ Steg $t=100 \mathrm{~mm}$
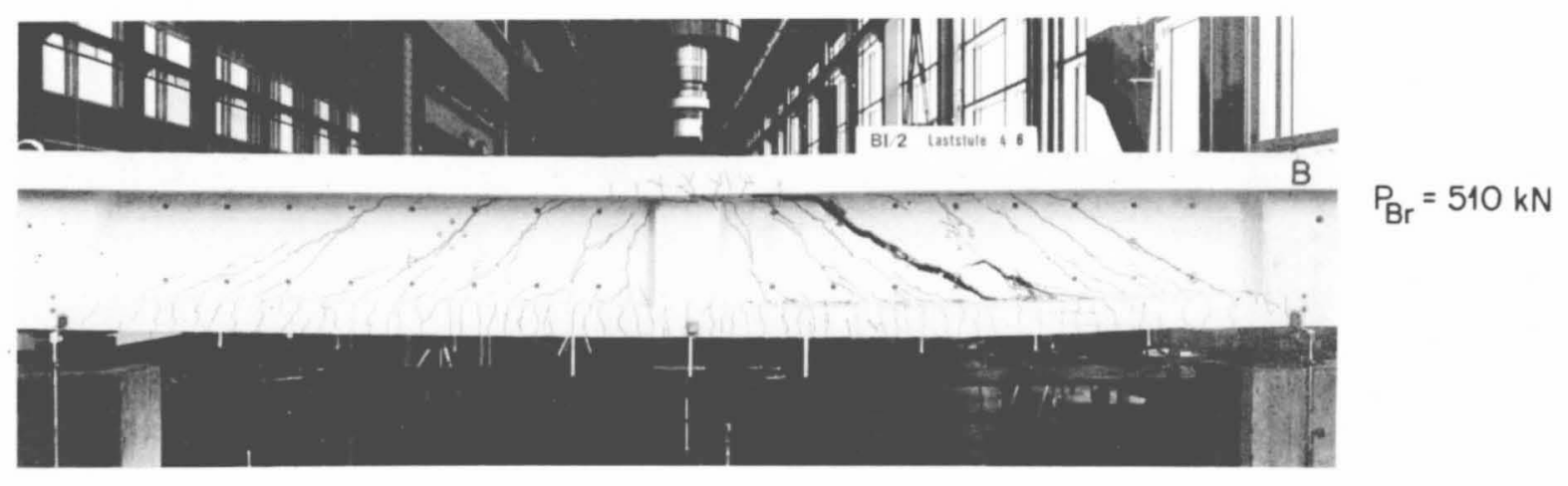

Bild 102 : BI/2, Bruch nach statischer Endbelastung im Lasłzyklus C 

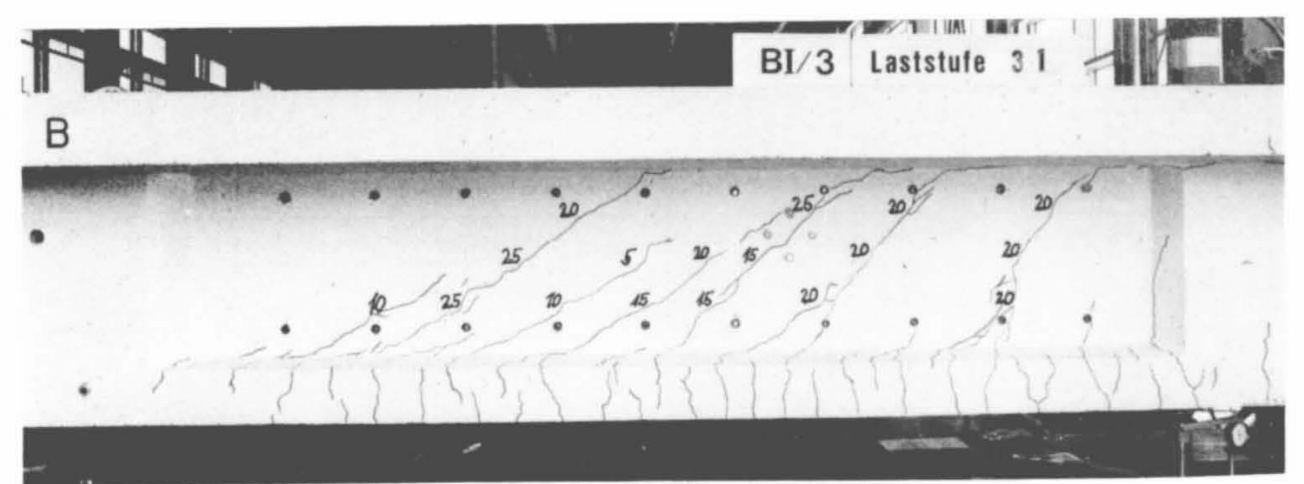

$\underline{P_{0}}=370 \mathrm{kN}$

$N=2.45 \cdot 10^{6} \mathrm{LW}$

Steg $t=150 \mathrm{~mm}$
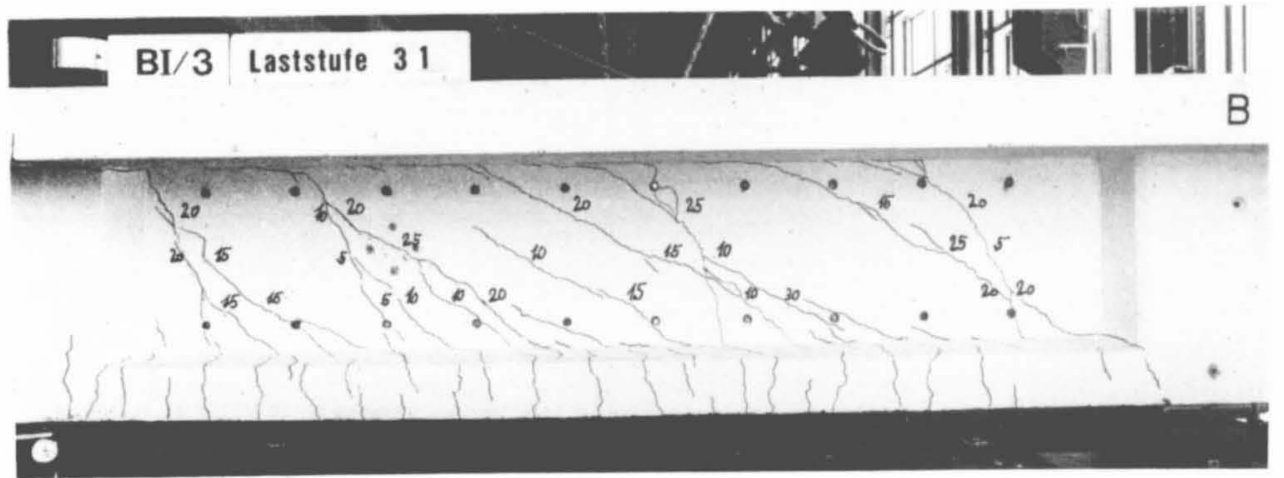

$\underline{P_{0}}=370 \mathrm{kN}$

$N=2.45 \cdot 10^{6} \mathrm{LW}$

Steg $t=100 \mathrm{~mm}$

Rissweiten in $1 / 100 \mathrm{~mm}$

Bild 103: BI/3, Rissbild im Lastzyklus B

\section{Not III $\mathrm{BI} / 3$ H}

$3 \mathrm{~B}$
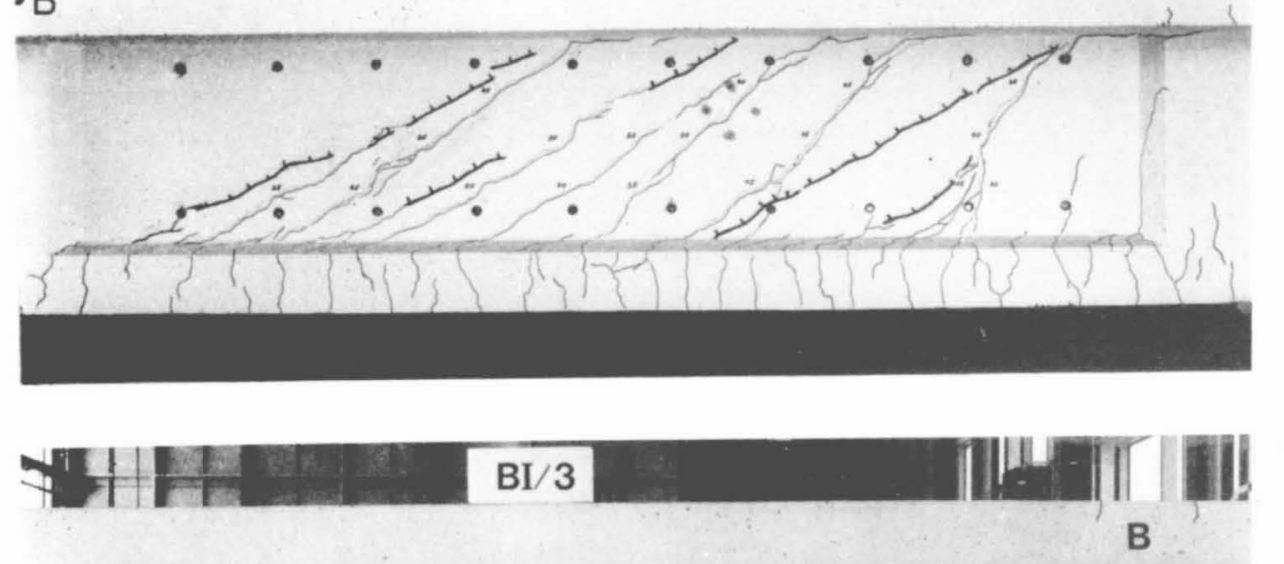

$\underline{\mathrm{P}_{\mathrm{Br}}=1050 \mathrm{kN}}$

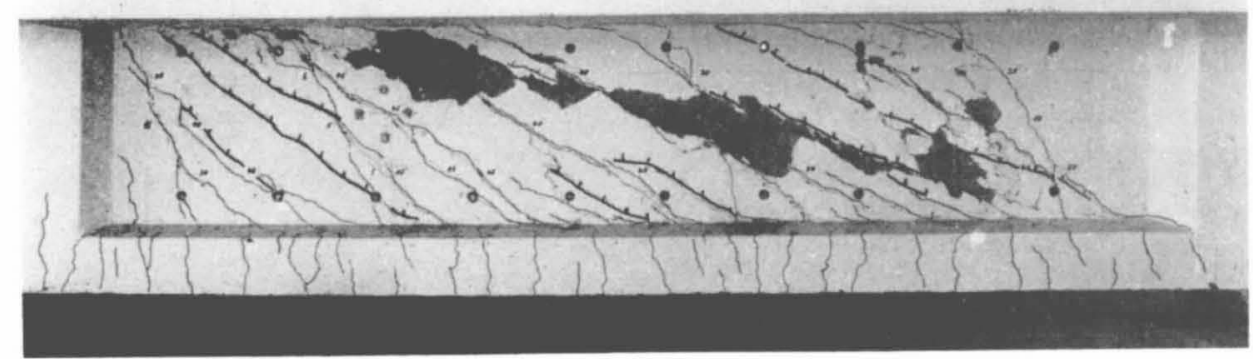

Steg $t=100 \mathrm{~mm}$

Bild 104 : BI/3, Bruch nach statischer Endbelastung im Lastzyklus C 


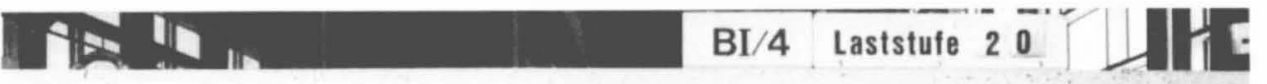 \\ B}

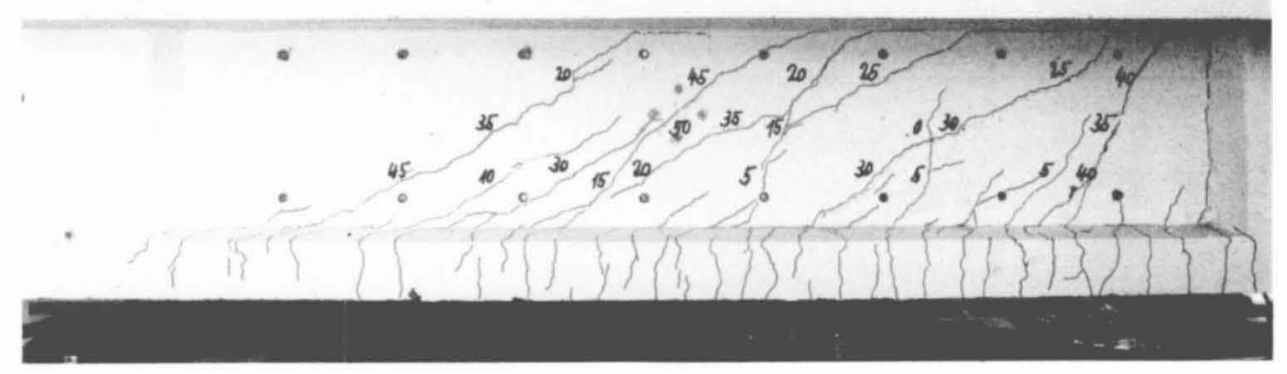

$\underline{P_{0}}=370 \mathrm{kN}$

$N=5 \cdot 10^{4} \mathrm{LW}$

Steg $\uparrow=150 \mathrm{~mm}$

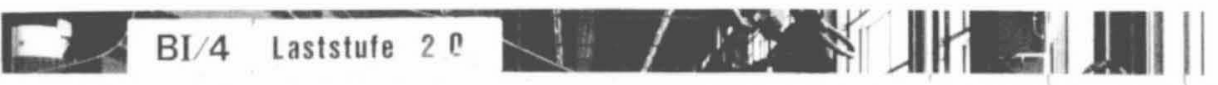

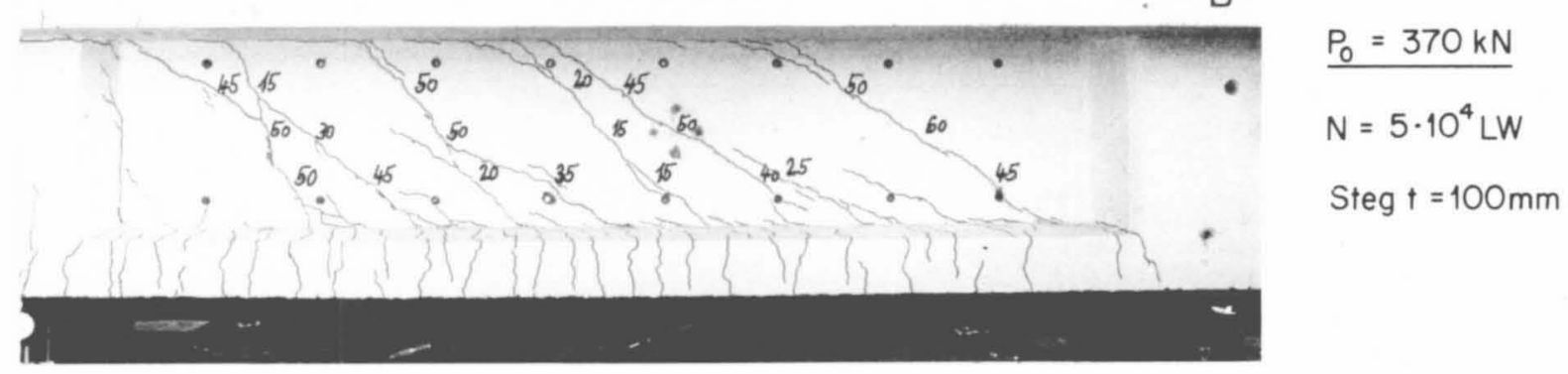

Rissweiten in $1 / 100 \mathrm{~mm}$

Bild 105: BI/4, Rissbild im Lastzyklus B
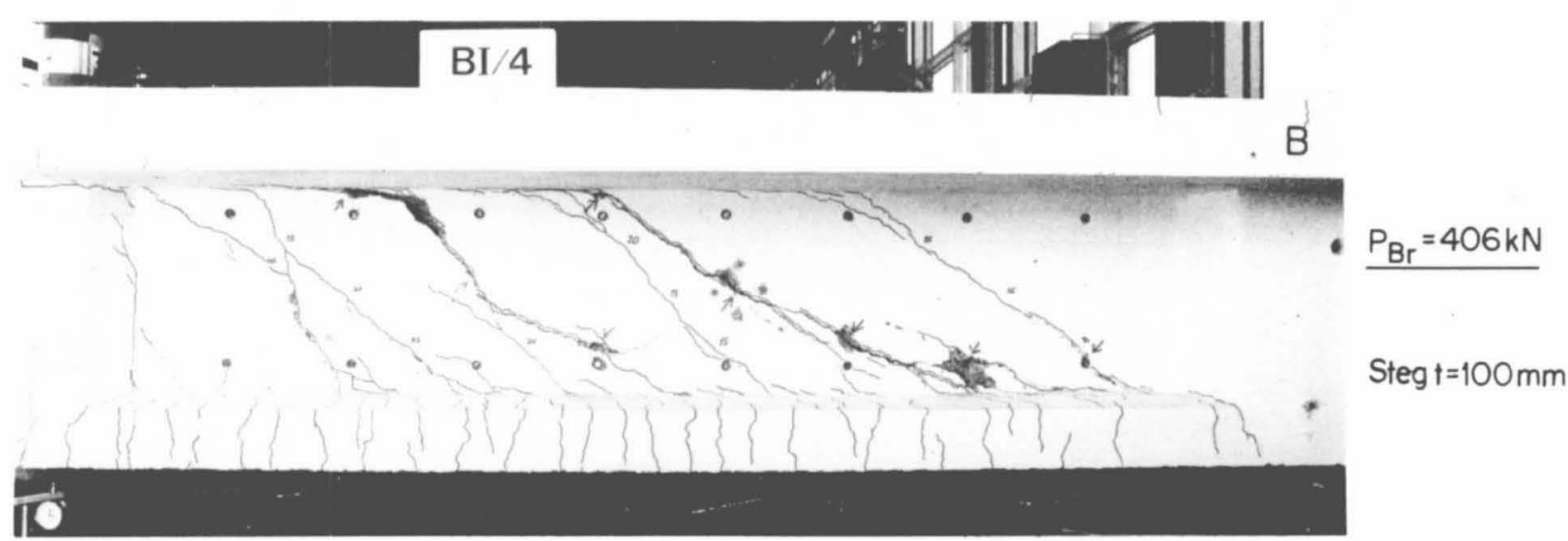

Bild 106: BI/4, Bruch nach statischer Endbelastung im Lastzyklus C 

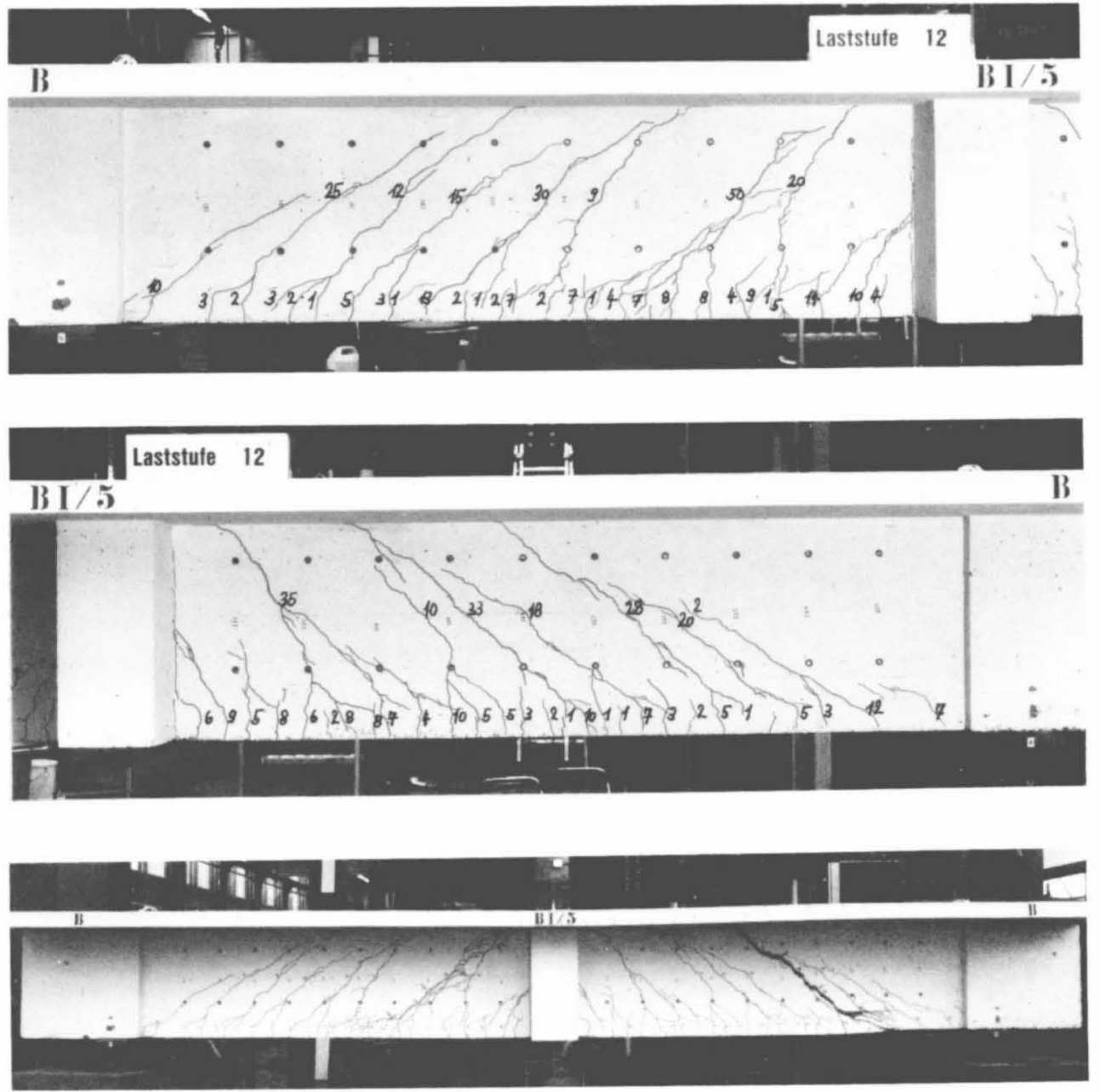

Dyn. Bruch

$\mathrm{N}=1.68 \cdot 10^{6} \mathrm{LW}$

Bild 107: BI/5, Riss - und Bruchbild im Lastzyklus B

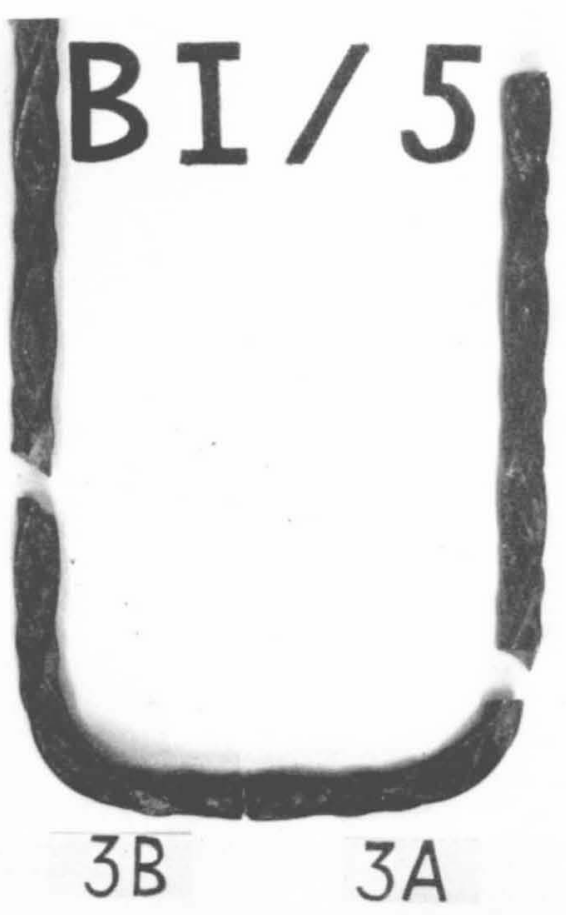

$3 B \quad 3 A$

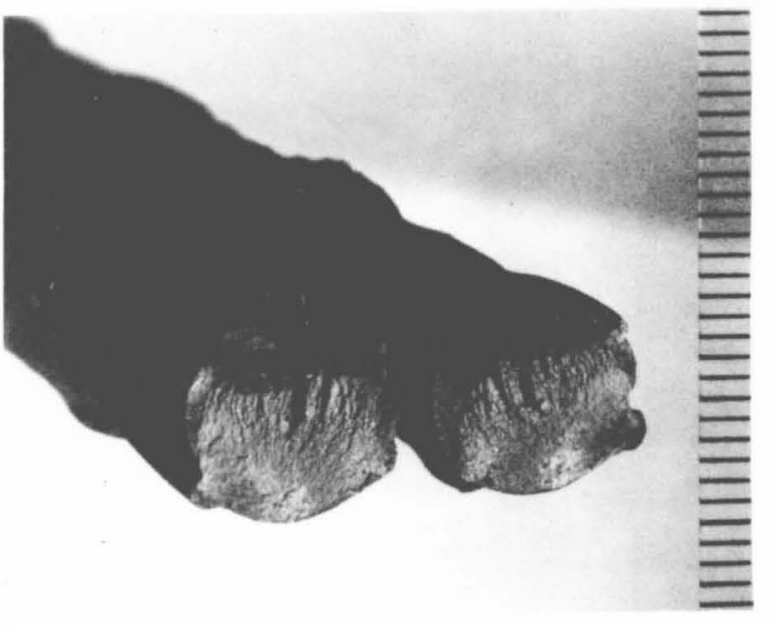

Bild 108: Bruchfläche Bügel №18, $\$ 10 \mathrm{~mm}$ 


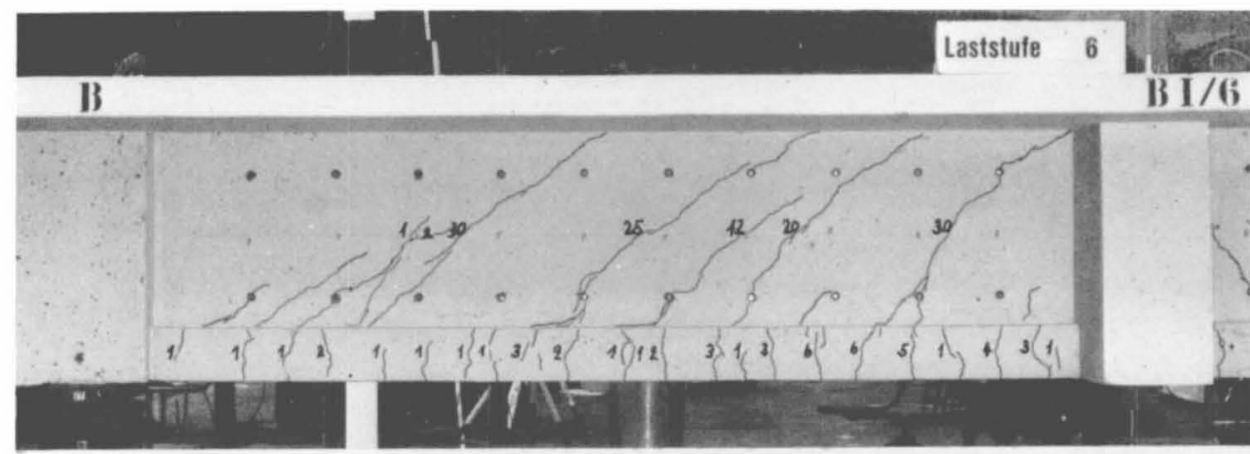

$\underline{P_{0}=370 \mathrm{kN}}$

Erstbelastung
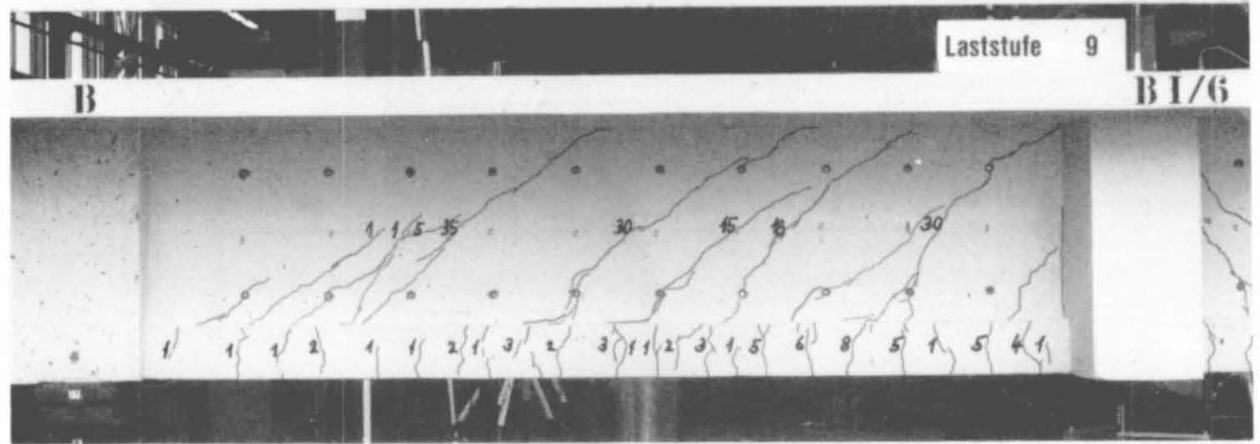

$P_{0}=370 \mathrm{kN}$

$N=5 \cdot 10^{3} \mathrm{LW}$
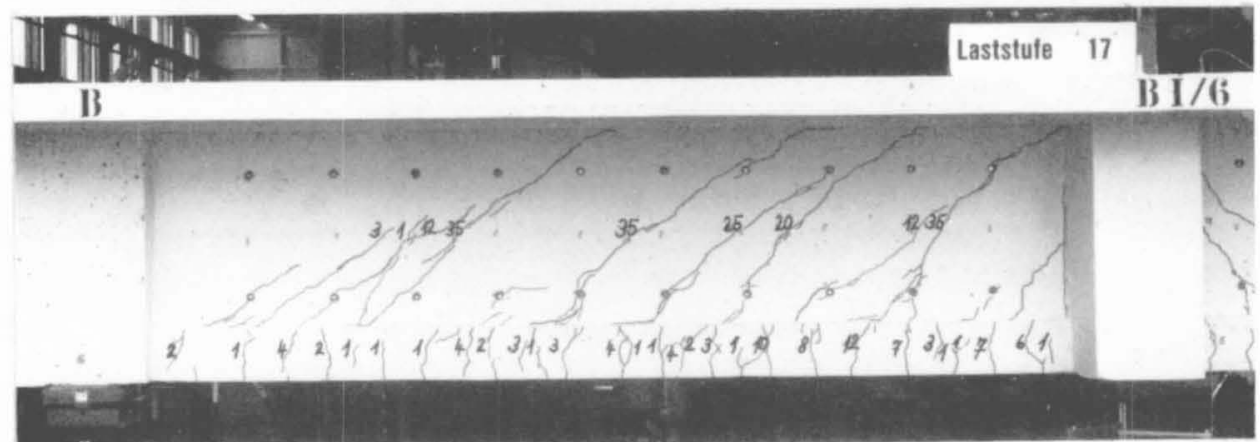

$\underline{P_{0}}=370 \mathrm{kN}$

$N=5.6 \cdot 10^{5} \mathrm{LW}$

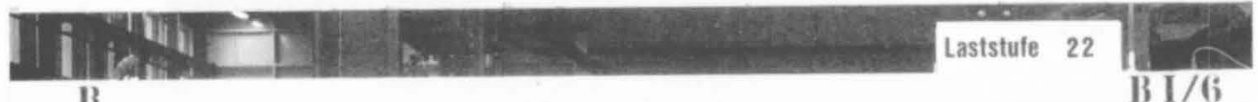

B

BI/6

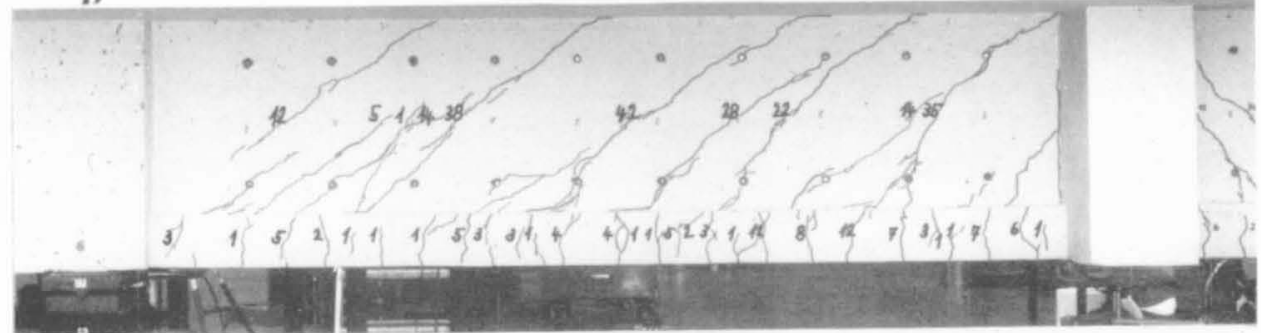

$P_{0}=370 \mathrm{kN}$

$N=1.0 \cdot 10^{6} \mathrm{LW}$

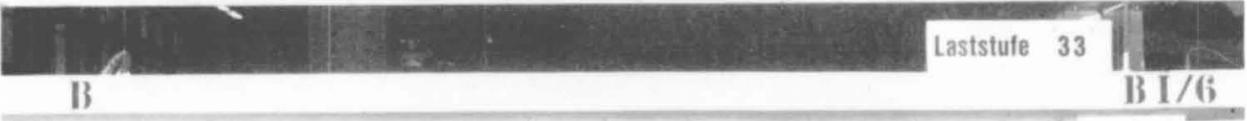

$P_{0}=370 \mathrm{kN}$
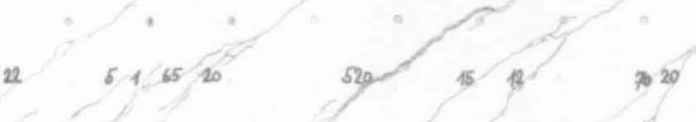

$\mathrm{N}=1.54 \cdot 10^{6} \mathrm{LW}$

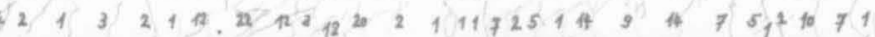

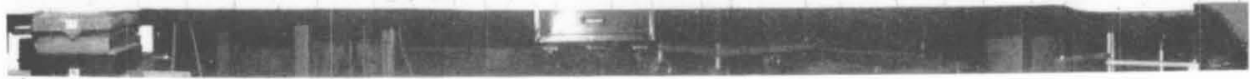

Rissweite in $1 / 100 \mathrm{~mm}$

Bild 110: BI/6, Rissentwicklung im Lastzyklus B, Steg $\dagger=100 \mathrm{~mm}$ 


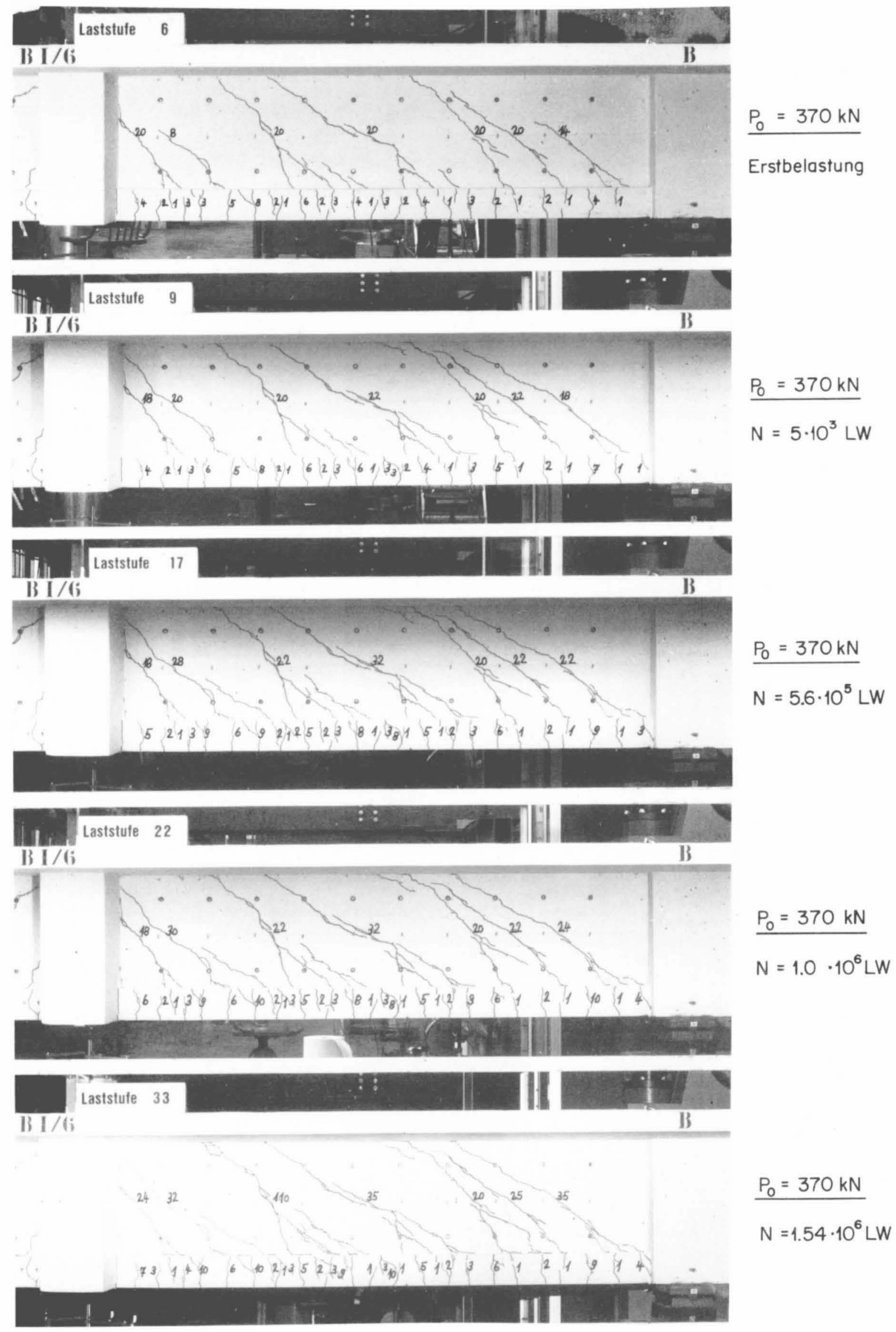

Rissweiten in $1 / 100 \mathrm{~mm}$

Bild 111: BI/6, Rissentwicklung im Lastzyklus B, Steg $\dagger=100 \mathrm{~mm}$ 


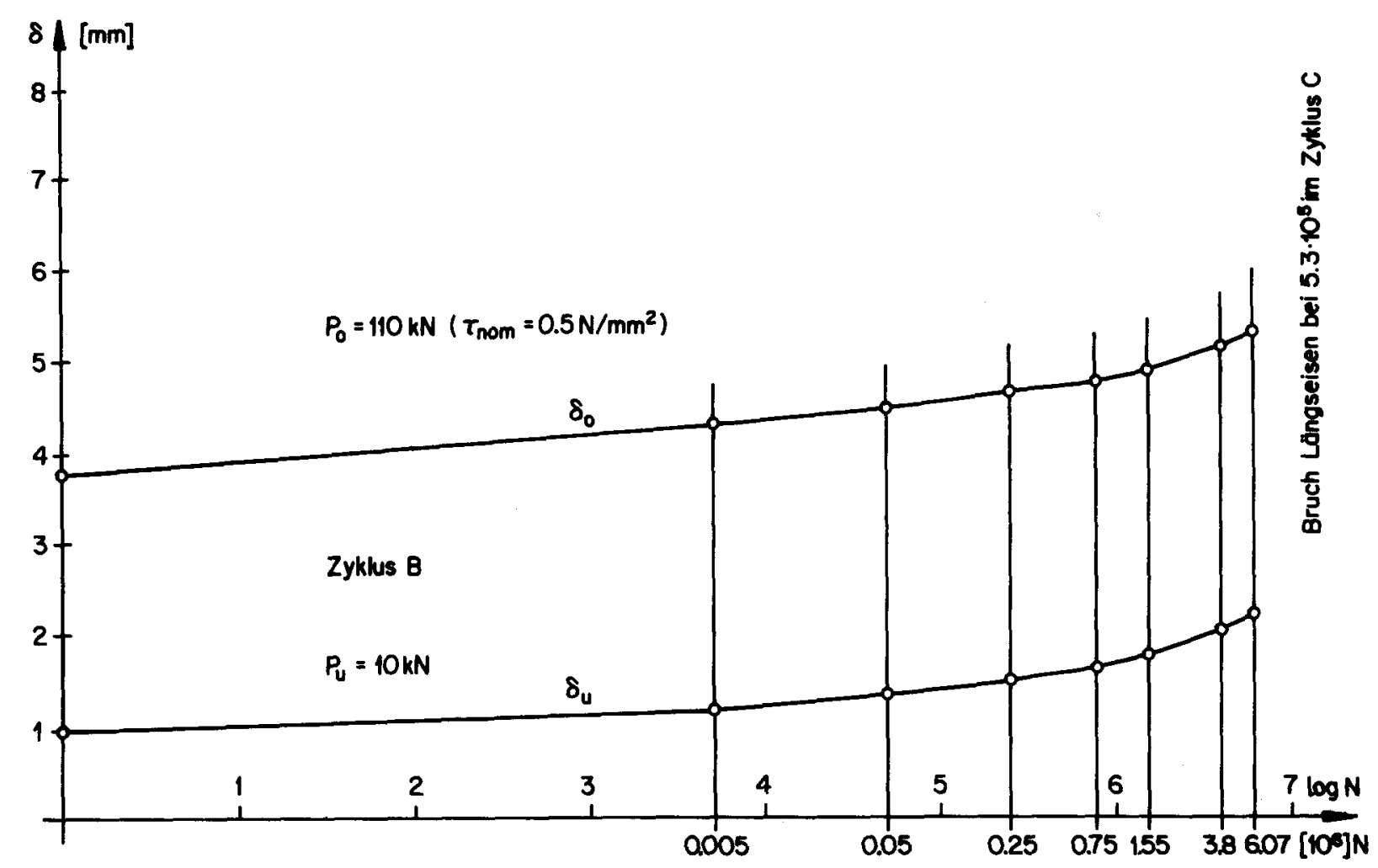

Bild $112:$ BI /1, Durchbiegung in Trägermitte in Funktion der Lastwechselzahl
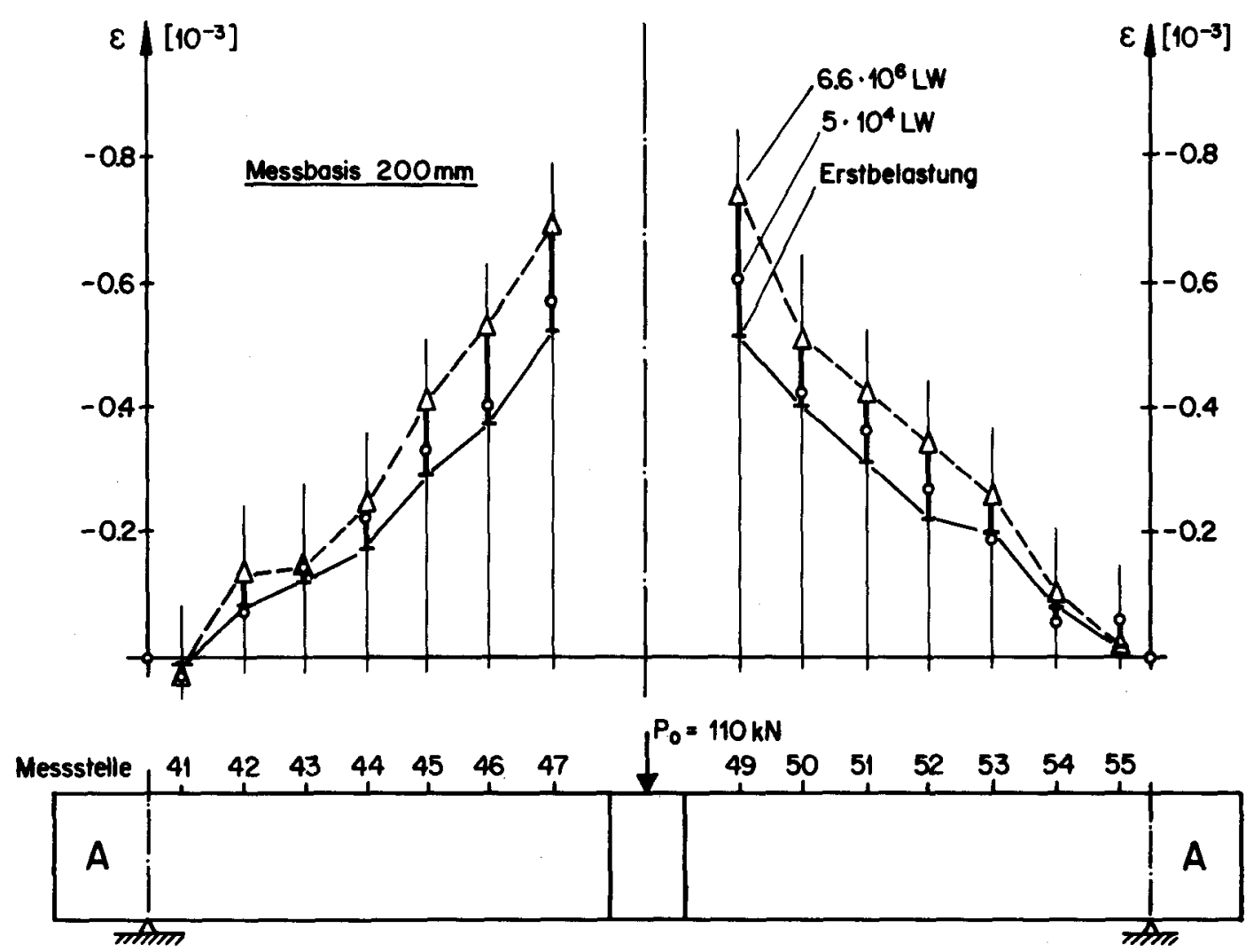

Bild 113 : BI/ , Betondehnungen unter Oberlast im Lastzyklus B 

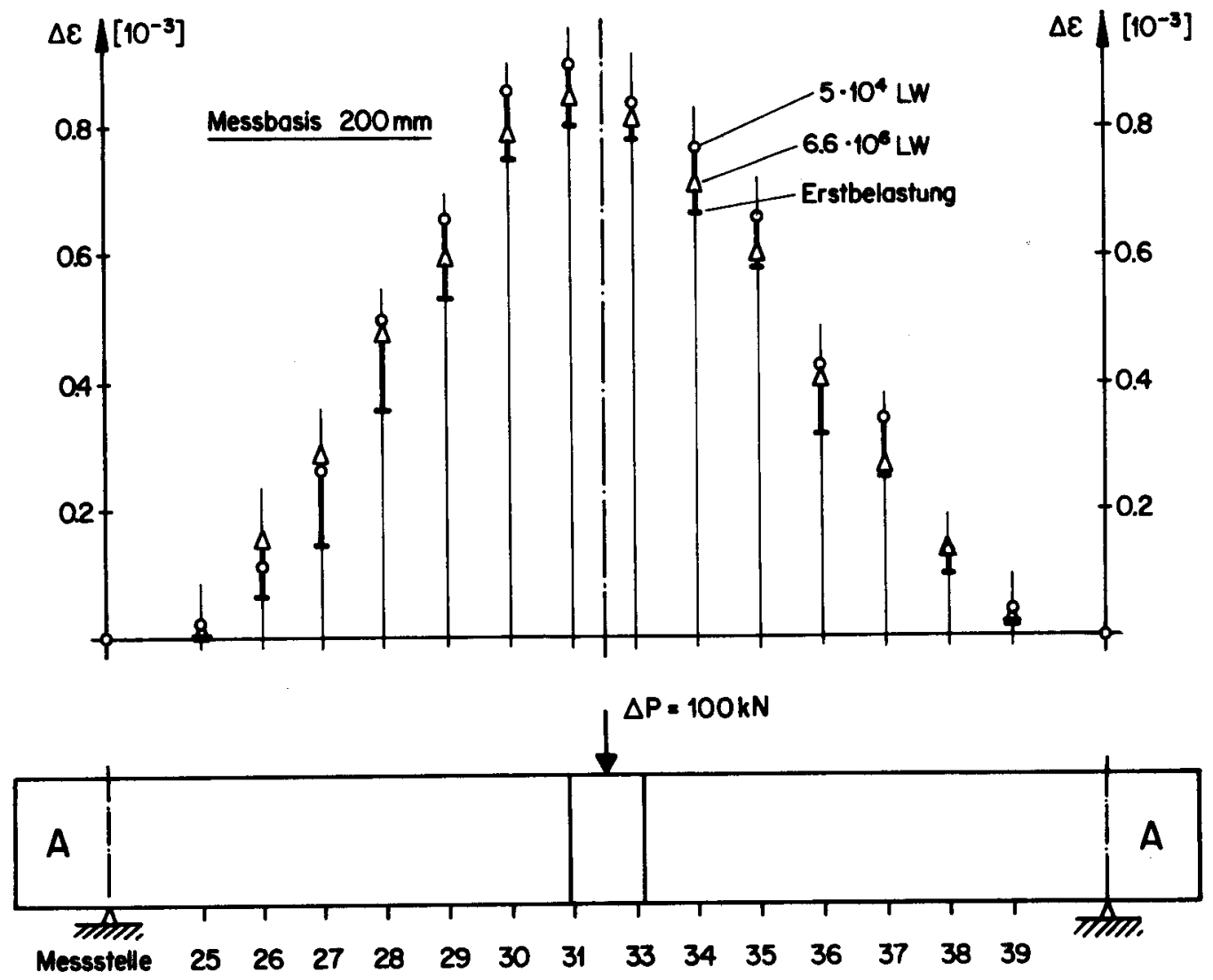

Bild $114:$ BII/1, Dehnungsdiffenzen in der Längsbewehrung in Lasłzyklus B
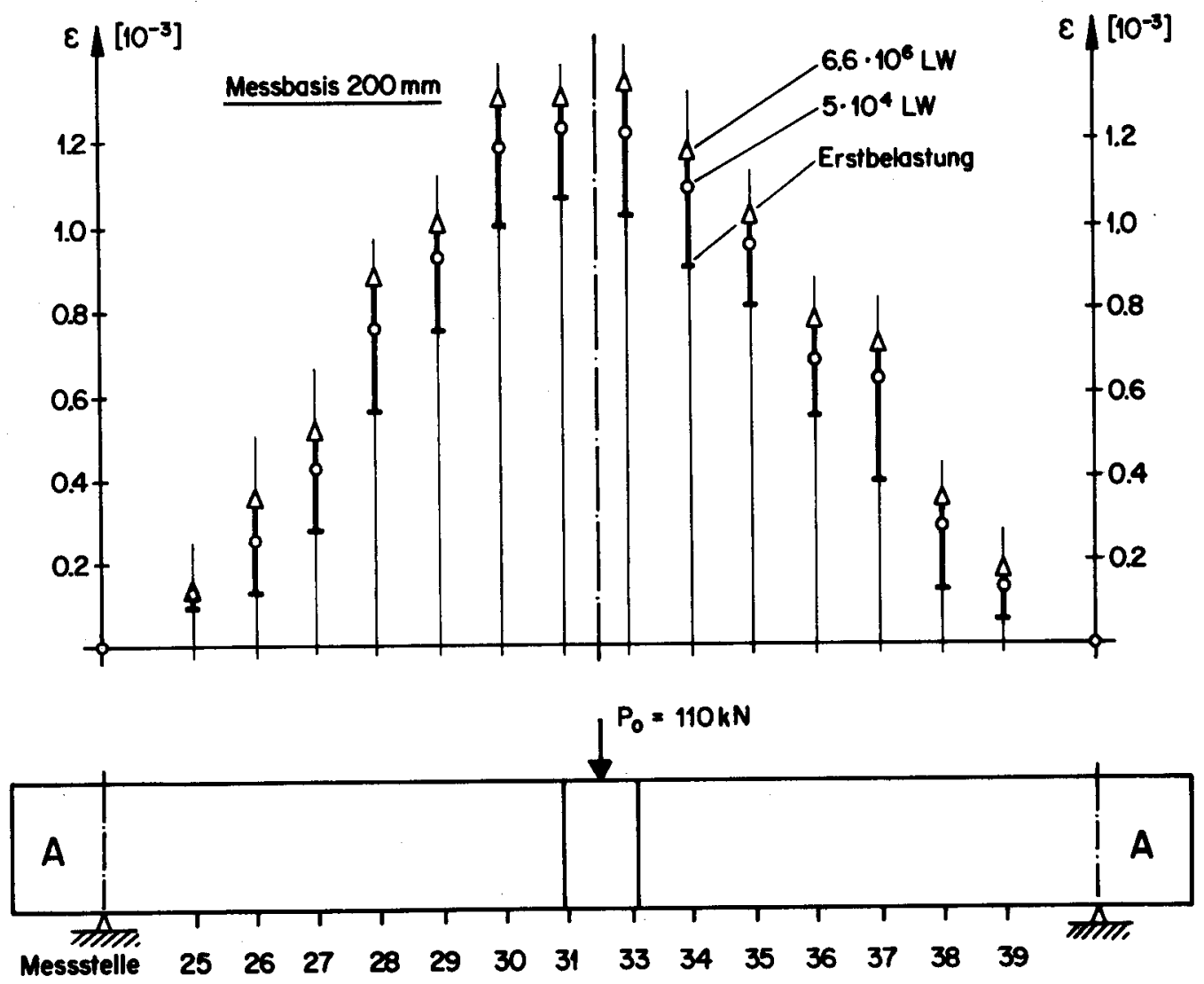

Bild $115:$ BI/1, Dehnungen in der Löngsbewehrung unter Oberlast im Lastzyklus B 


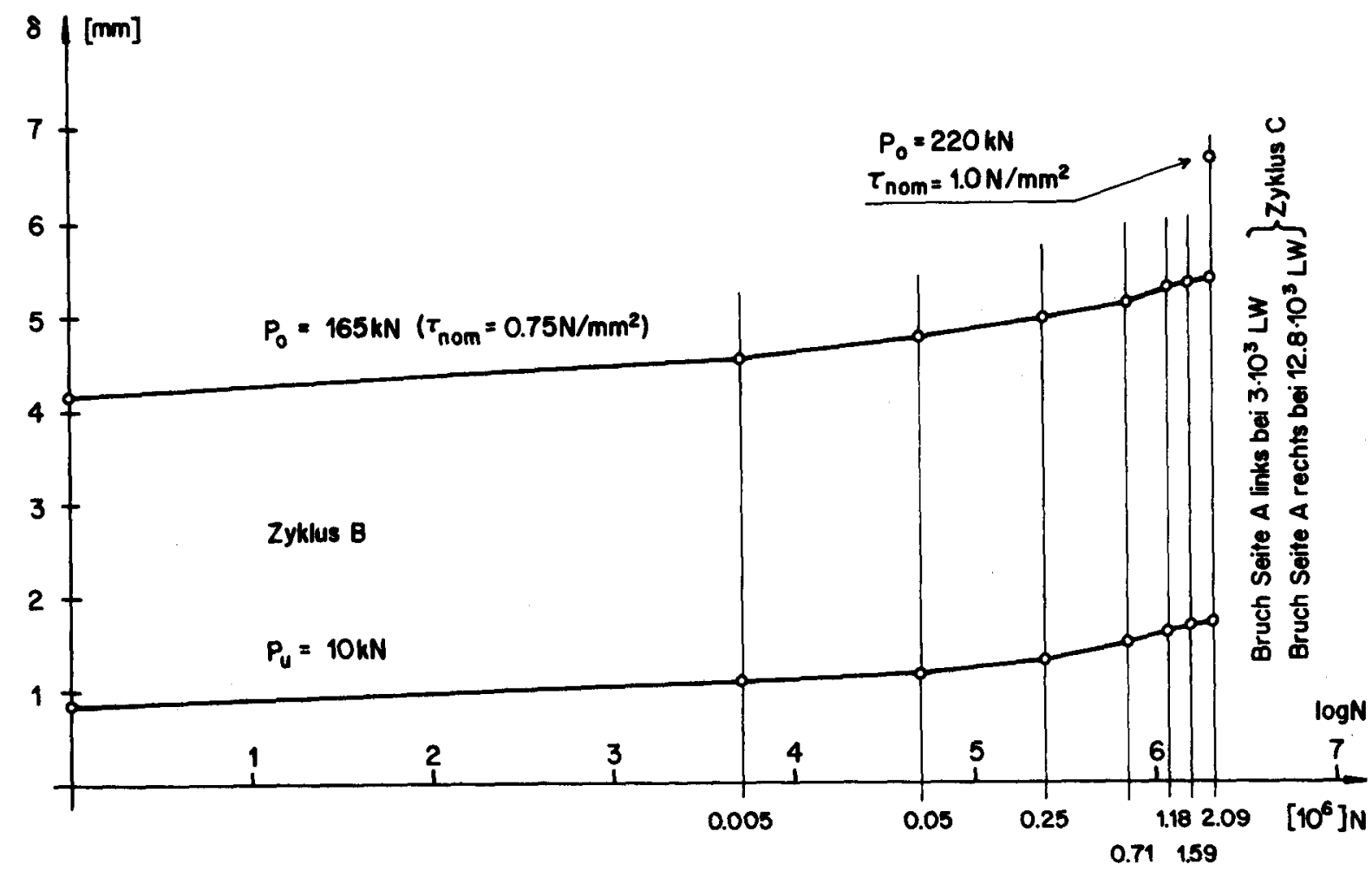

Bild 116: BI/2, Durchbiegung in Trägermitte in Funktion der Lastwechselzahl
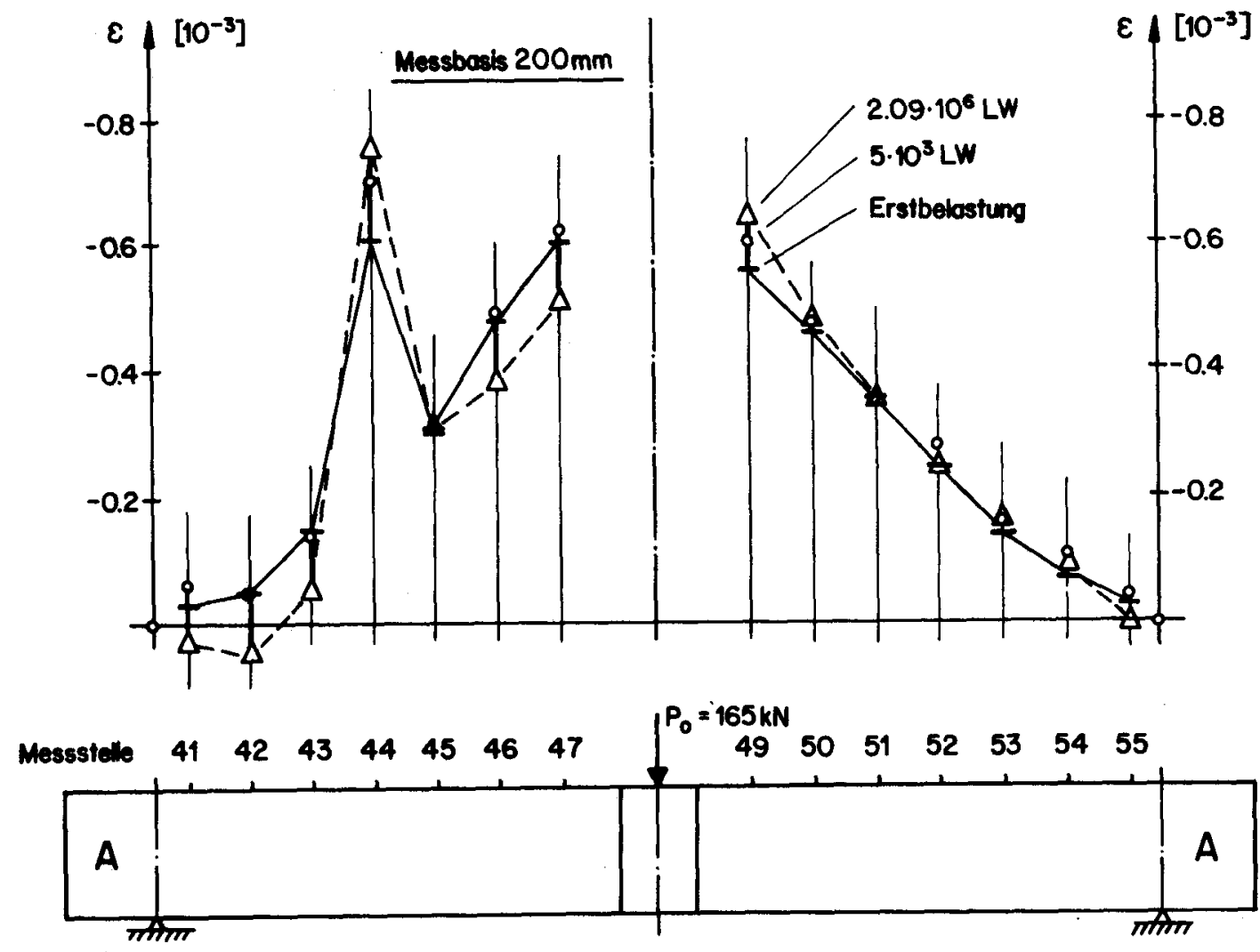

Bidd $117: B I / 2$, Betondehnungen unter Oberlast im Lastzyklus B 


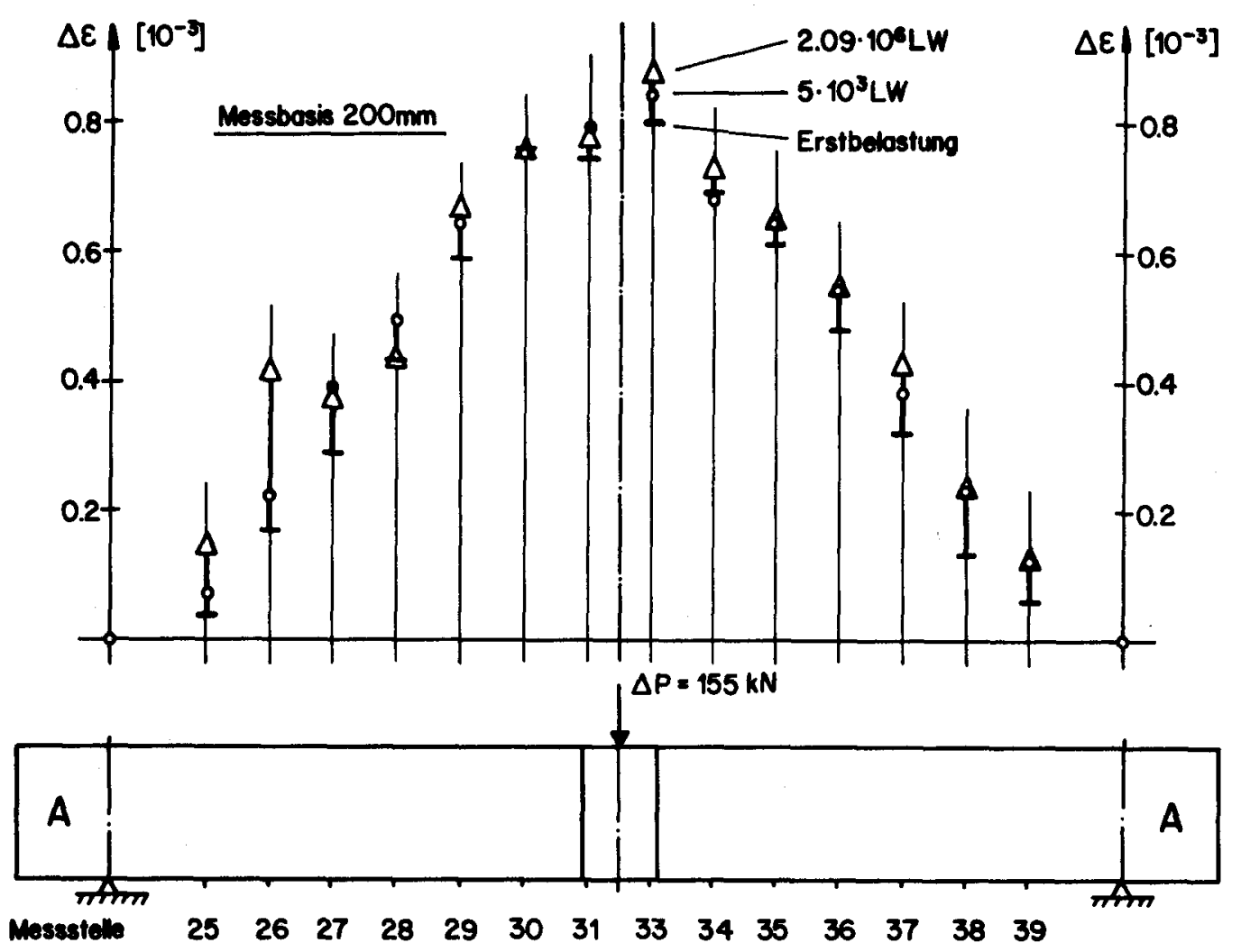

Bild 118: BI/2, Dehnungsdifferenzen in der Längsbewehrung im Lastzyklus B

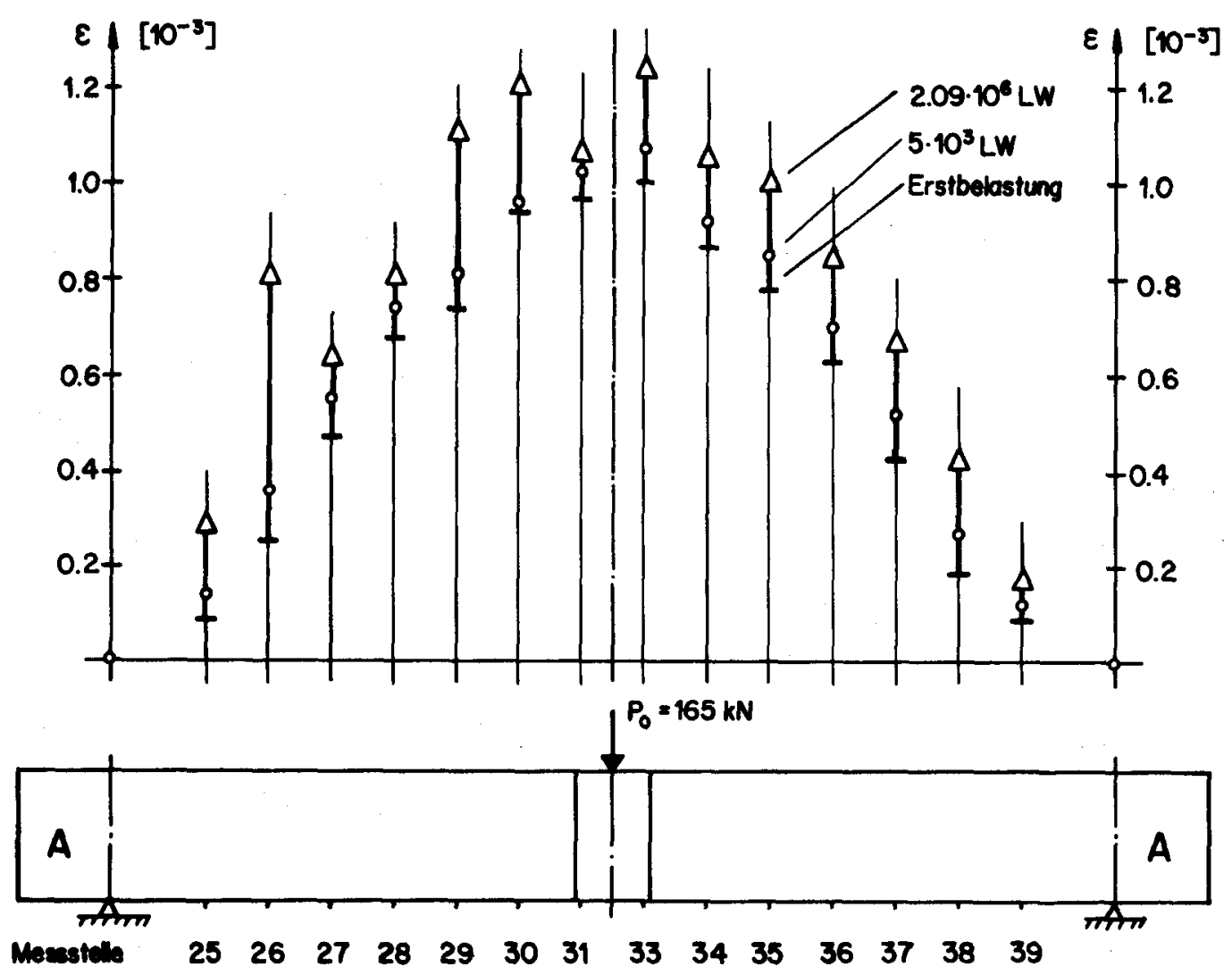

Bild 119: BII /2, Dehnungen in der Längsbewehrung unter Oberlast im Lastzyklus B 


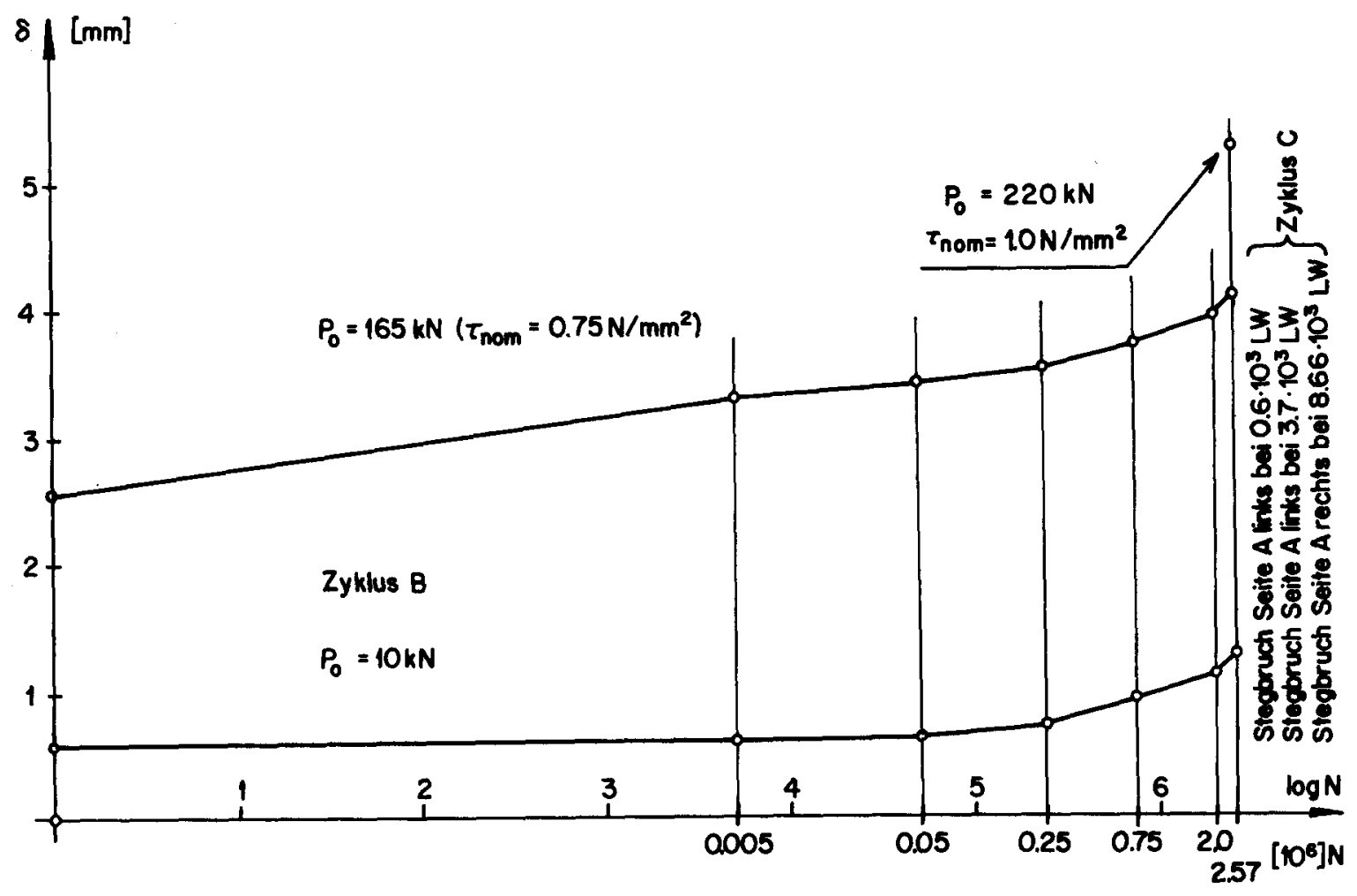

Bild 120: BII/4, Durchbiegung in Trögermitte in Funktion der Lastwechselzahl

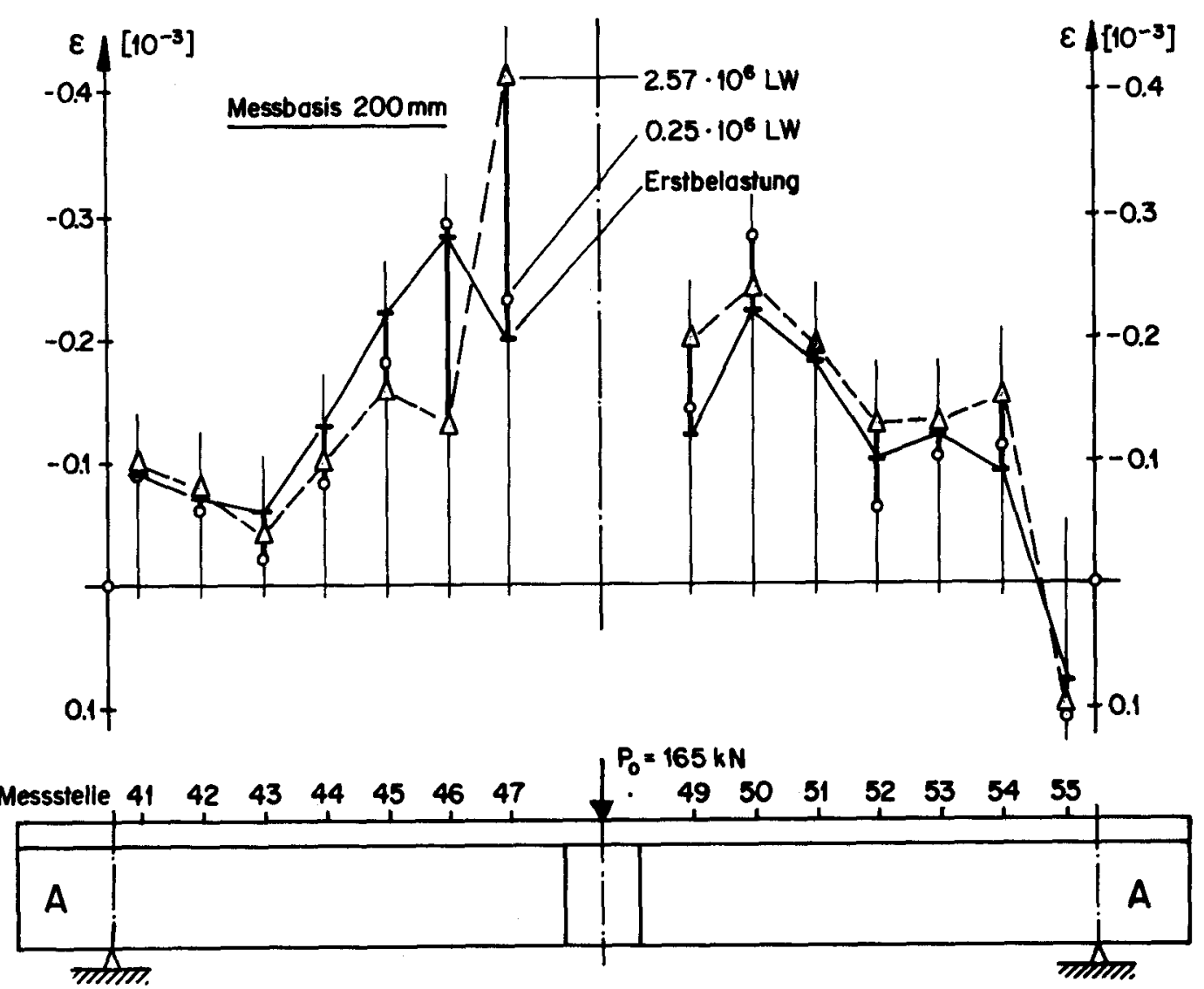

Bild 121: BII/4, Betondehnungen unter Oberlast im Lastzyklus B 

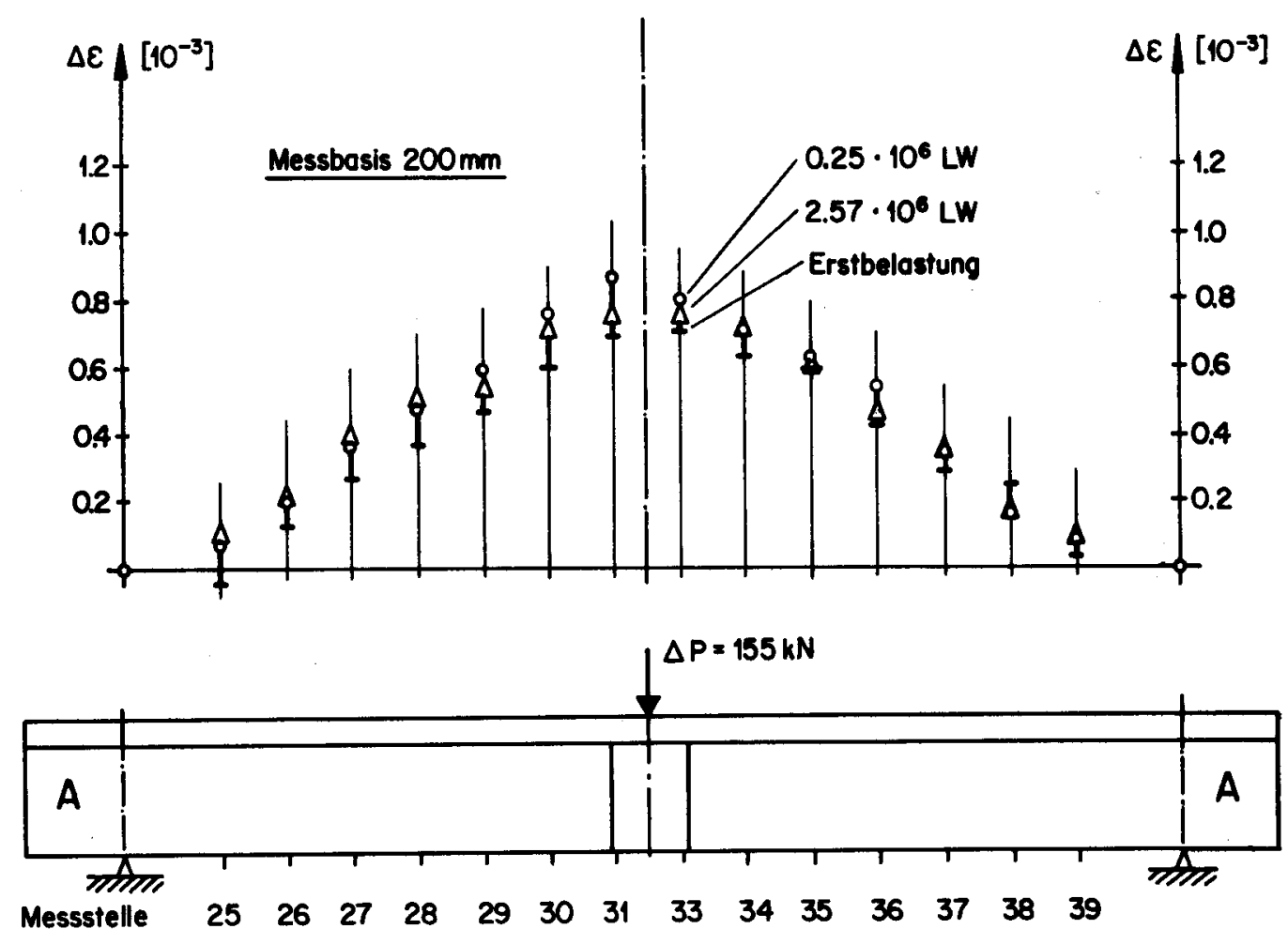

Bild $122: B I / 4$, Dehnungsdifferenzen in der Längsbewehrung im Lastzyklus B

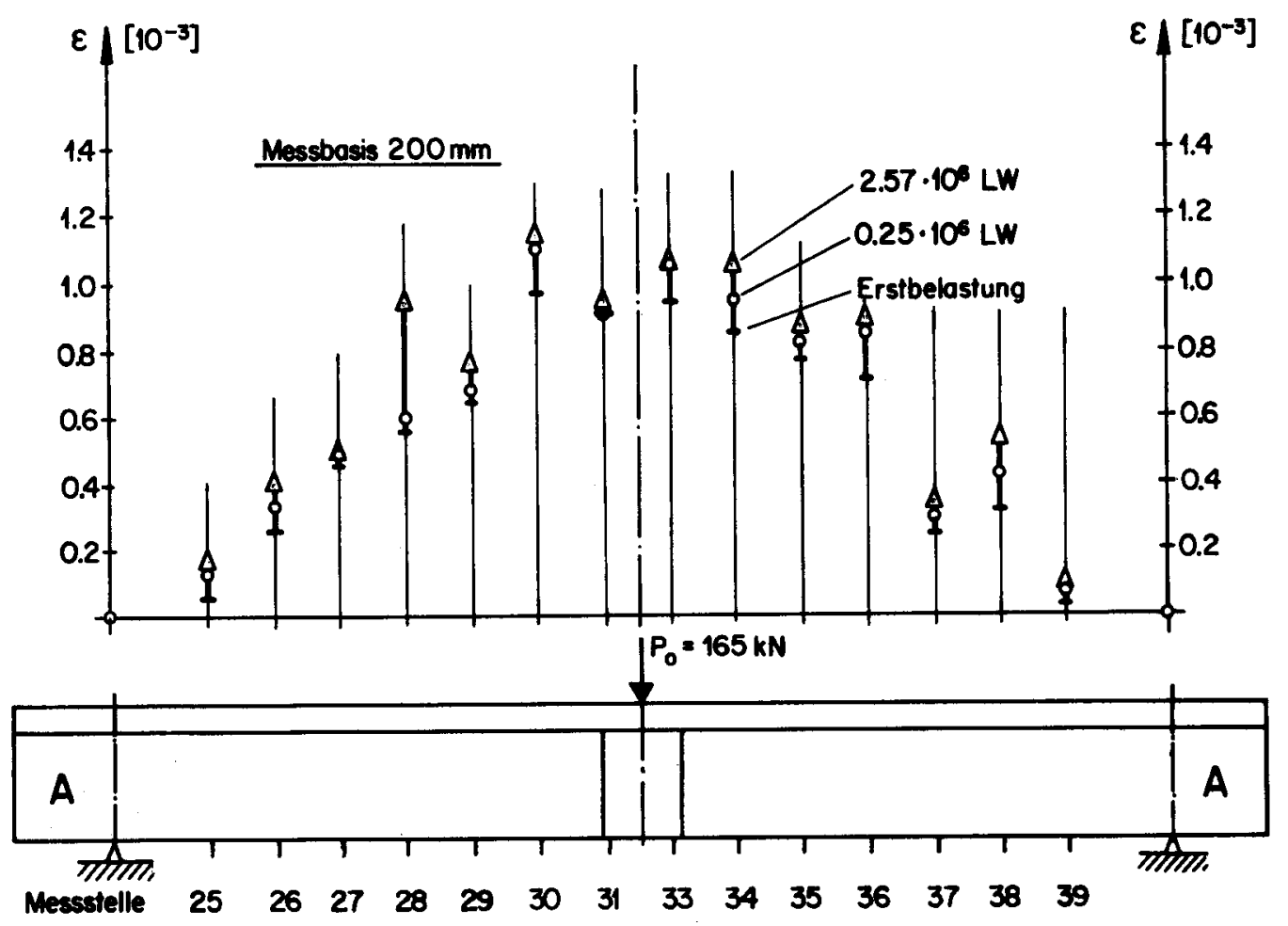

Bild 123: BII/4, Dehnungen in der Längsbewehrung unter Oberlast in Lastzyklus B 


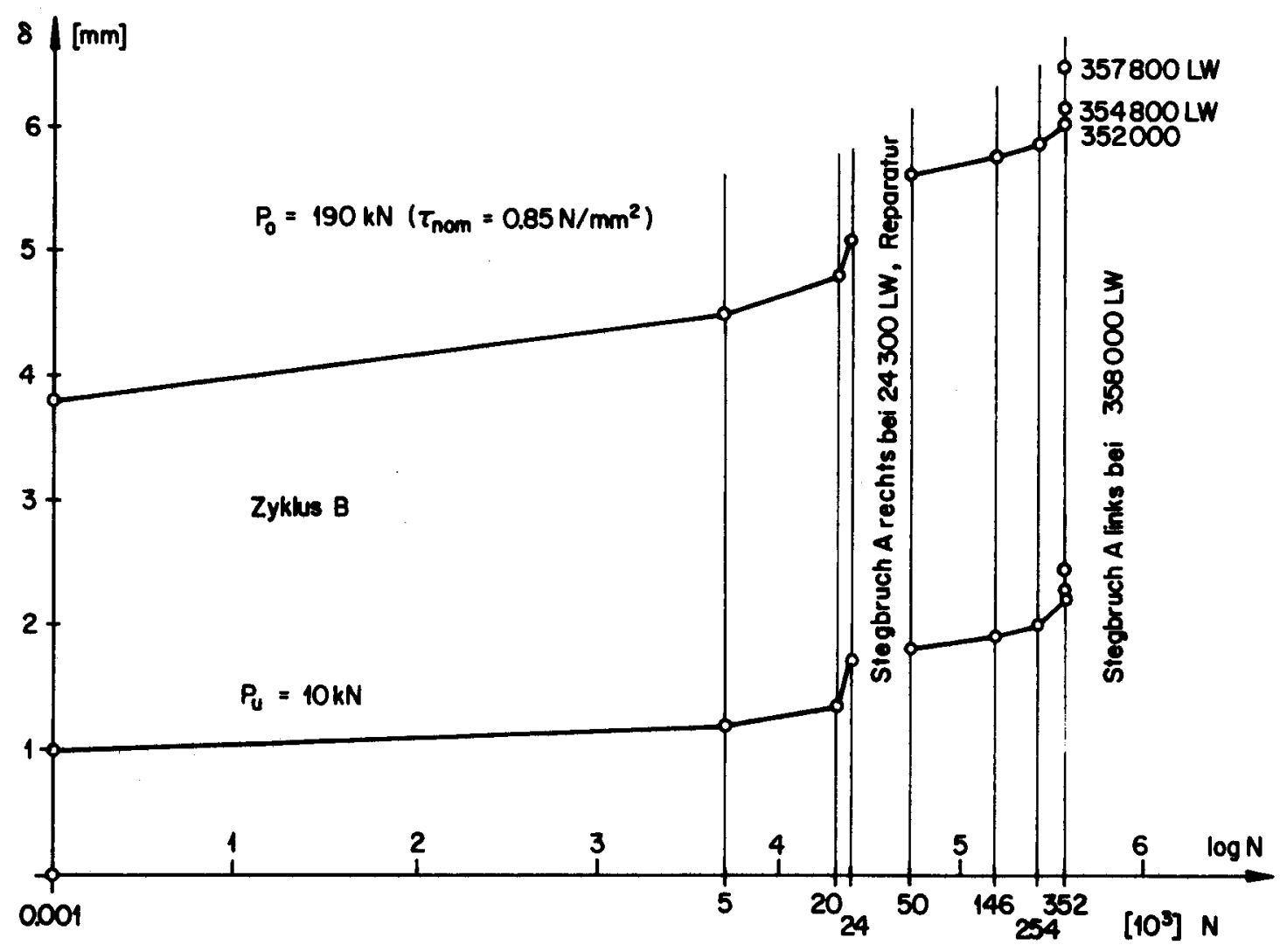

Bild 124:BI/5, Durchbiegung in Trägermitte in Funktion der Lastwechselzahl
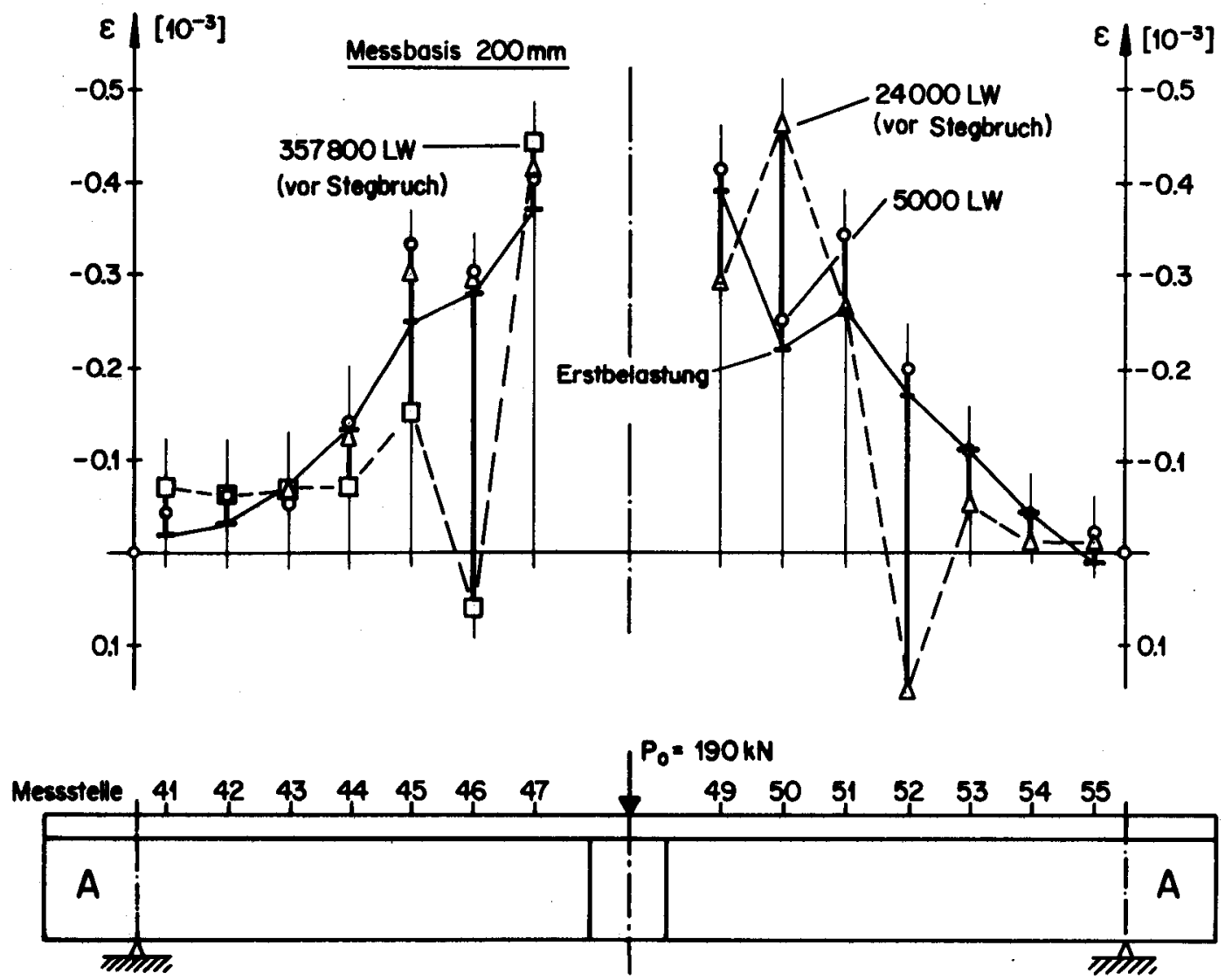

Bild 125: BI/5, Betondehnungen unter Oberlast im Lastzyklus B 

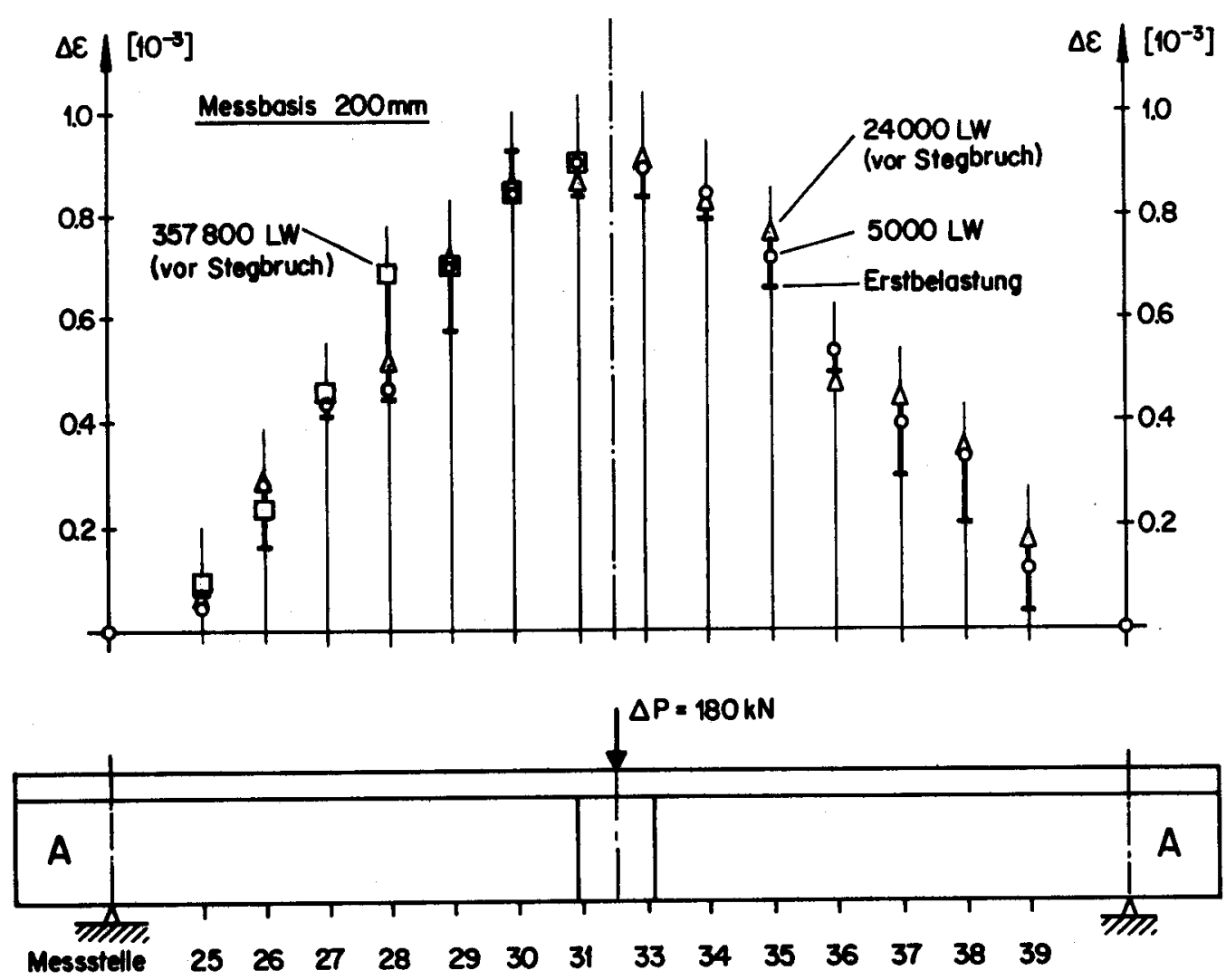

Bild $126: \mathrm{BII} / 5$, Dehnungsdifferenzen in der Löngsbewehrung im Lastzyklus B

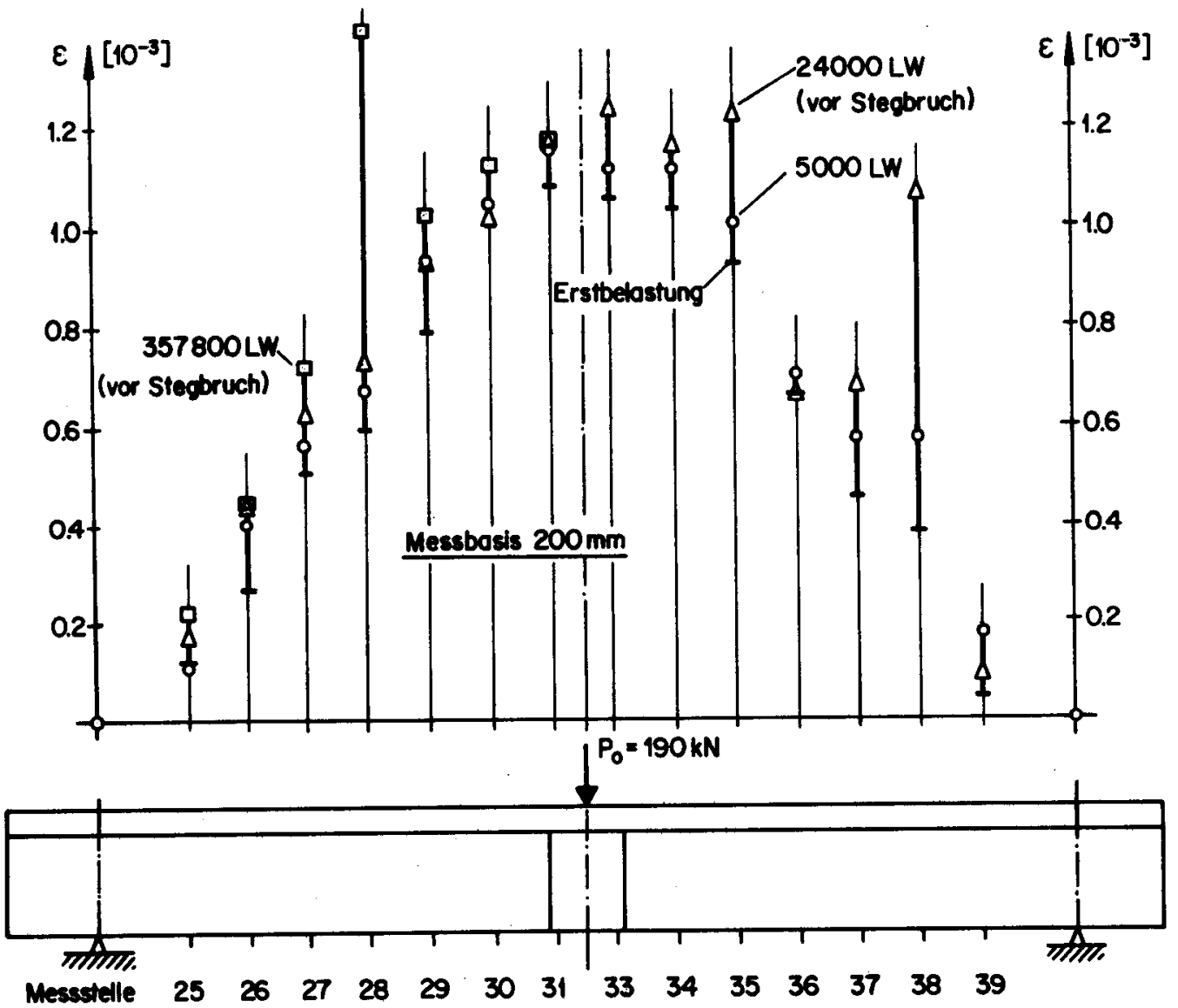

Bild 127 : BII/5, Dehnungen in der Löngsbewehrung unter Oberlast im Lastzykhus B 


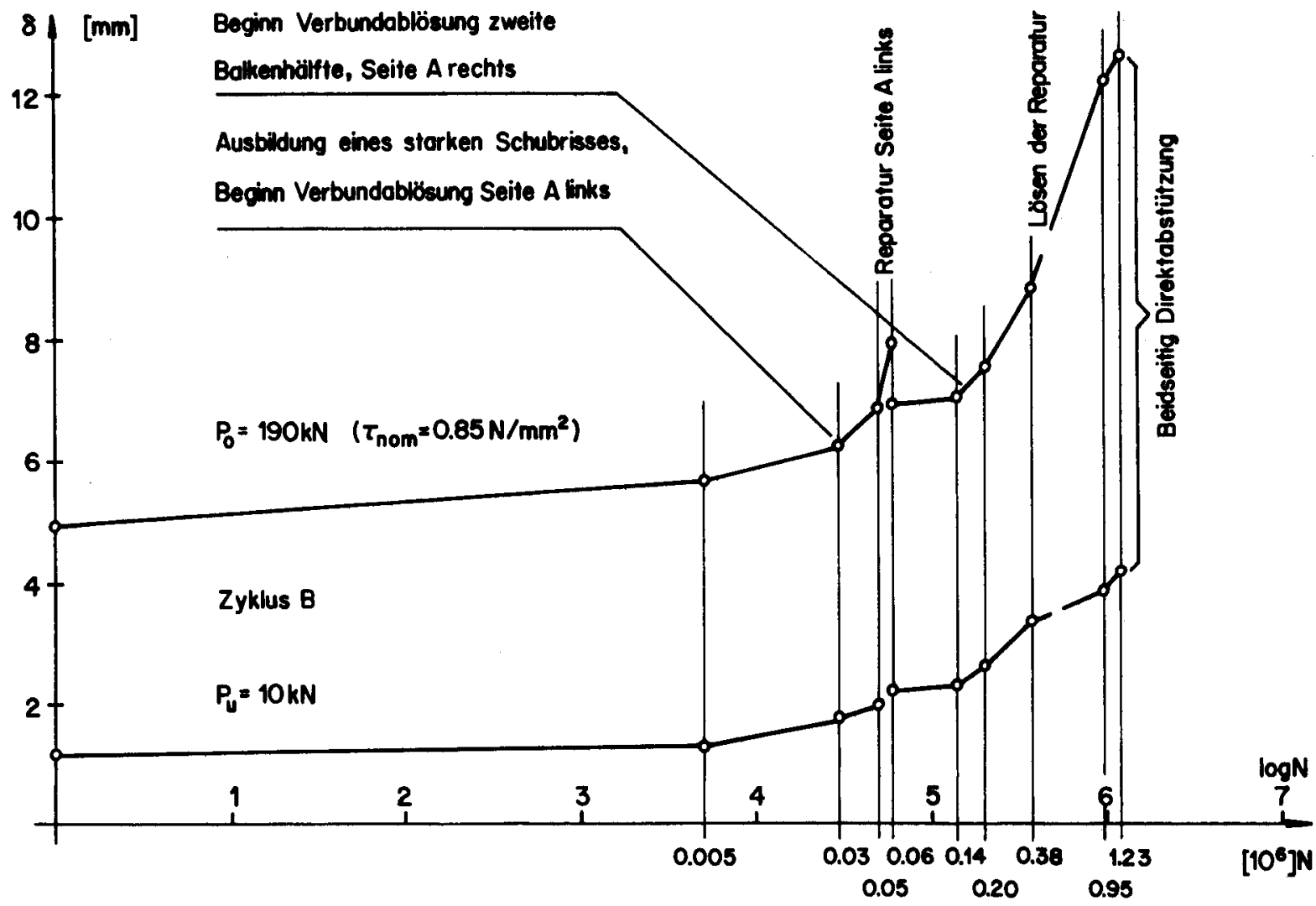

Bild 128: BI/6, Durchbiegung in Trägermitte in Funktion der Lastwechselzahl
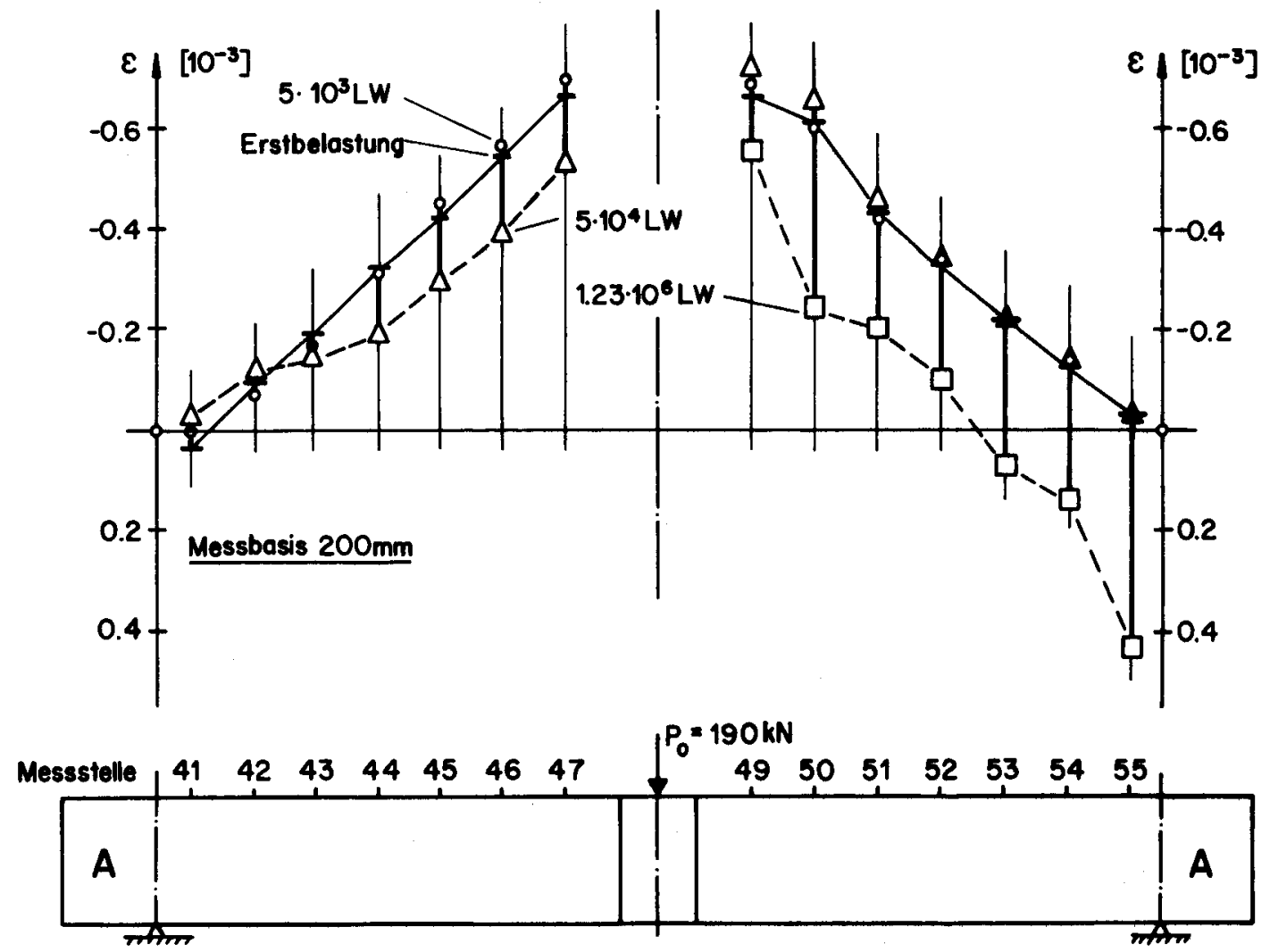

Bild 129: BII/6, Betondehnungen unter Oberlast im Lastzyklus B 

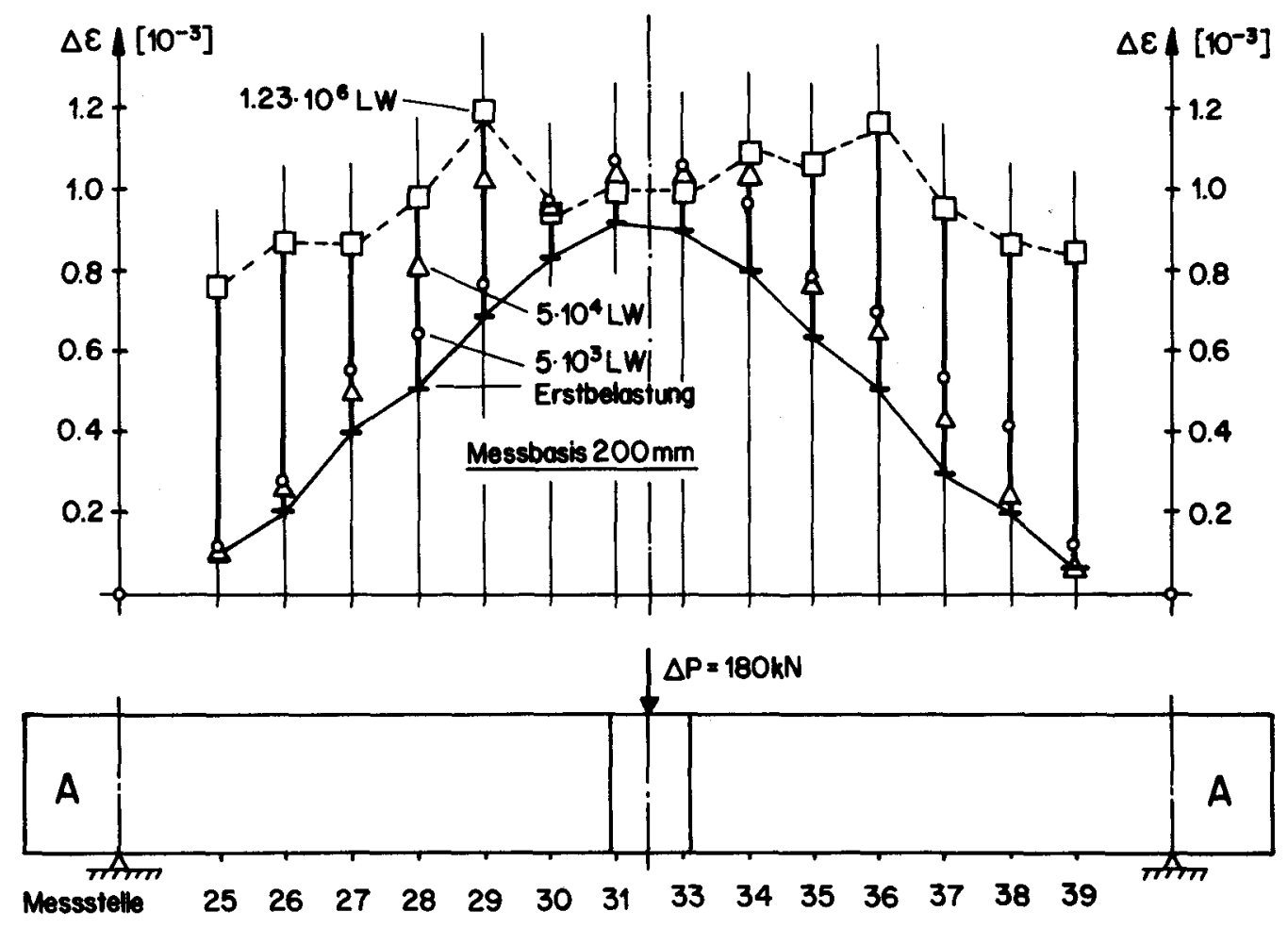

Bild 130: BI/6, Dehnungsdifferenzen in der Längsbewehrung im Lastzyklus B
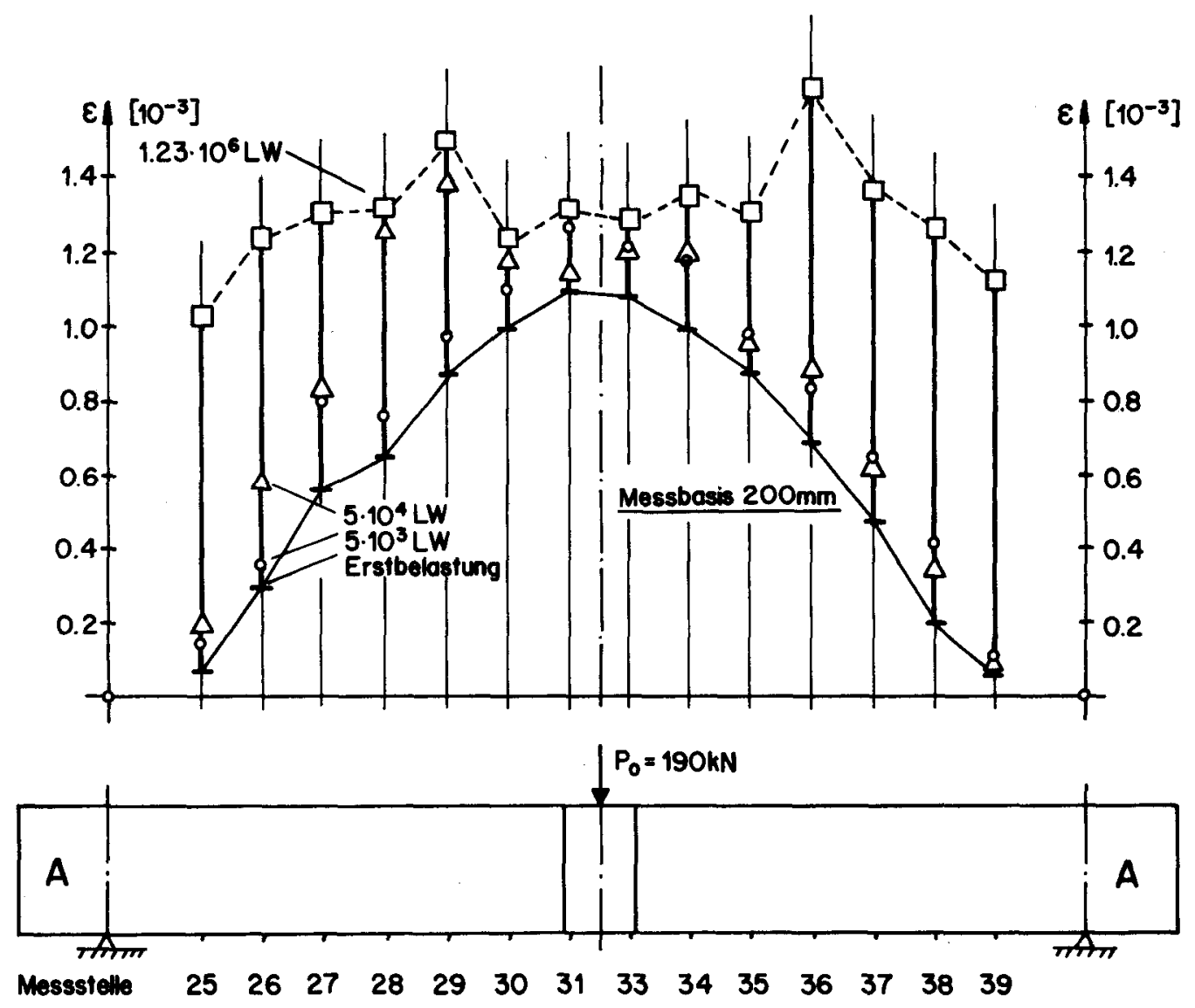

Bild 131: BI/6, Dehnungen in der Längsbewehrung unter Oberlast im Lastzyklus B 


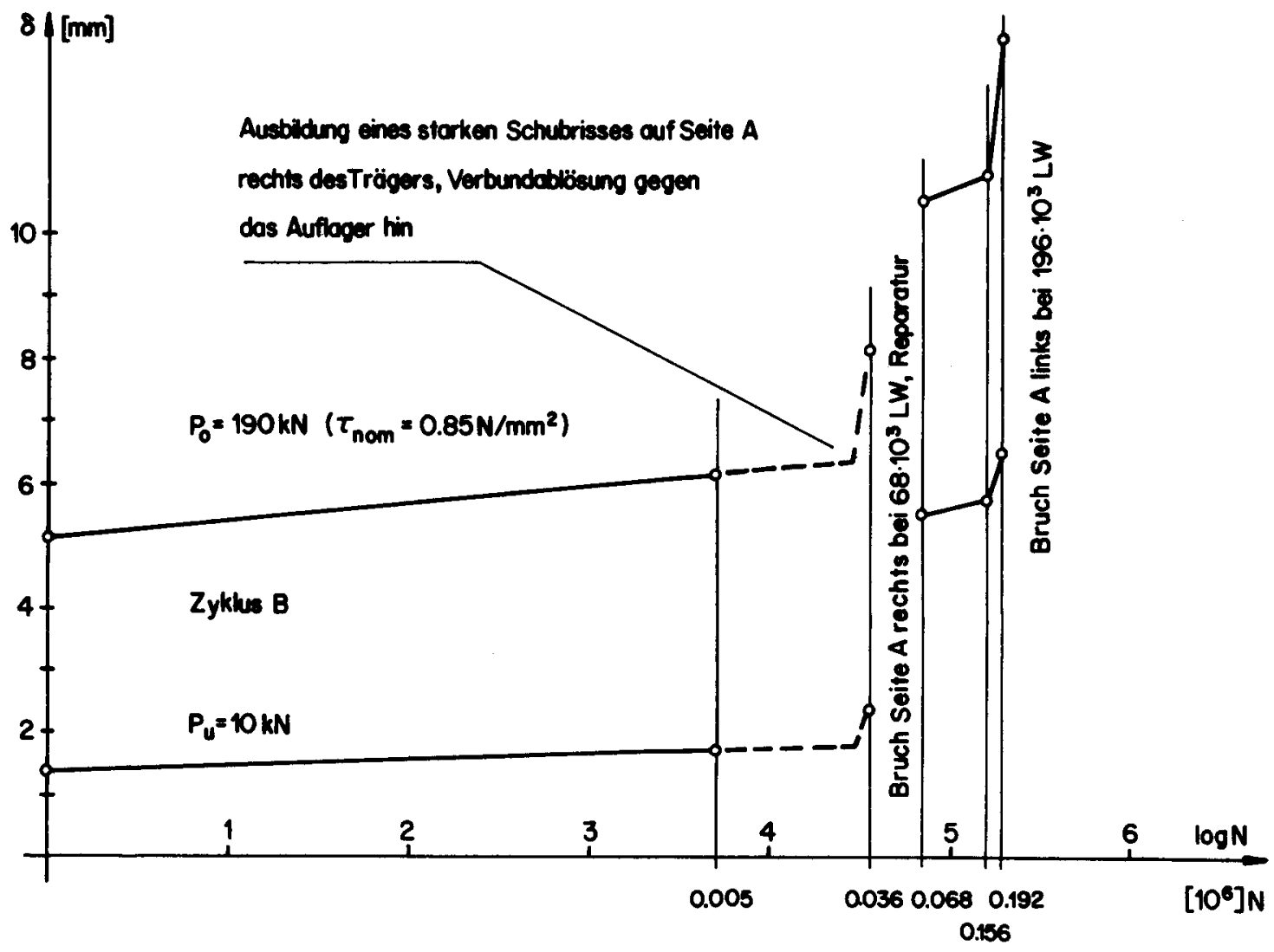

Bild 132: BI/7, Durchbiegung in Trägermitte in Funktion der Lastwechselzahl
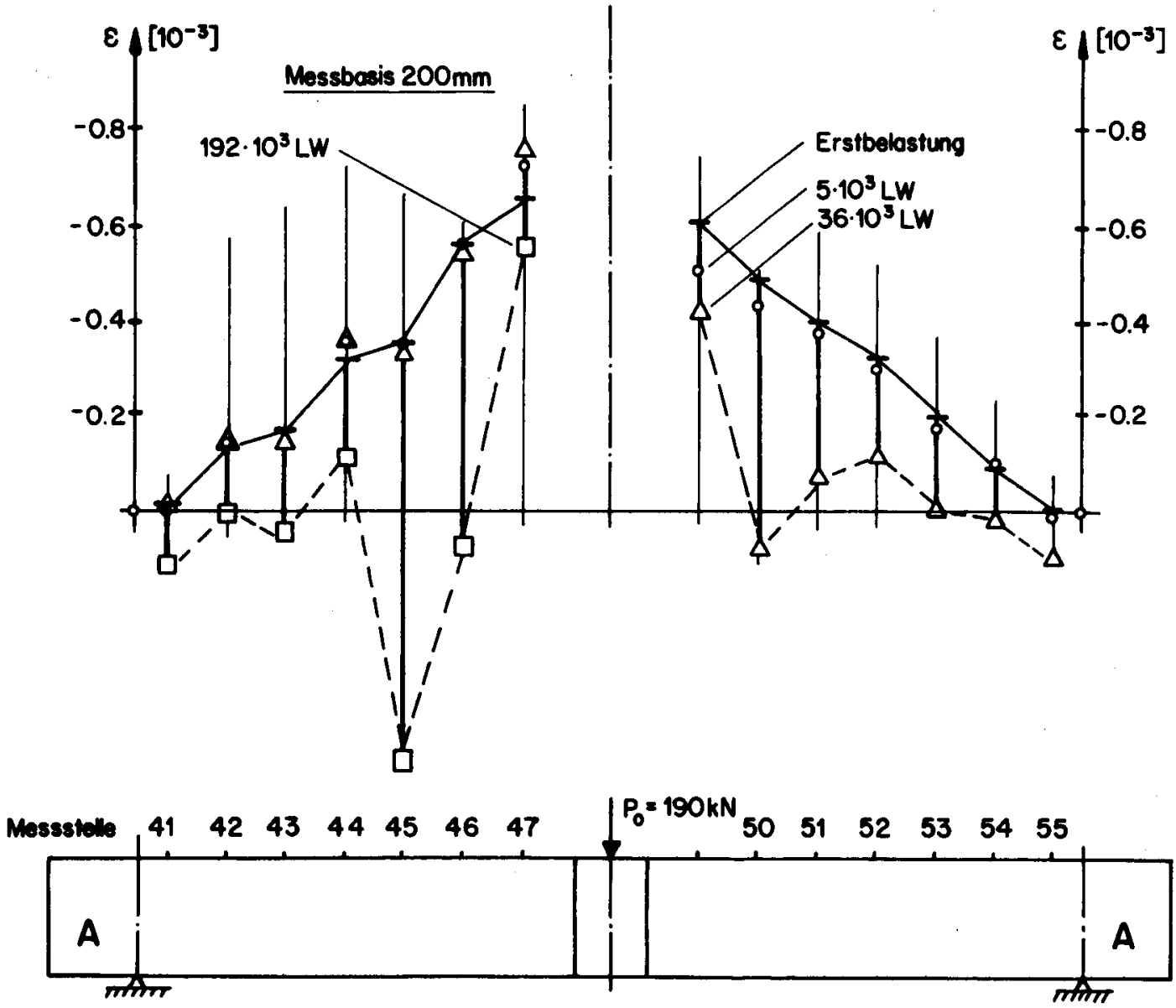

Bild $133:$ BII/7, Betondehnungen unter Oberlast in Lastzyklus B 


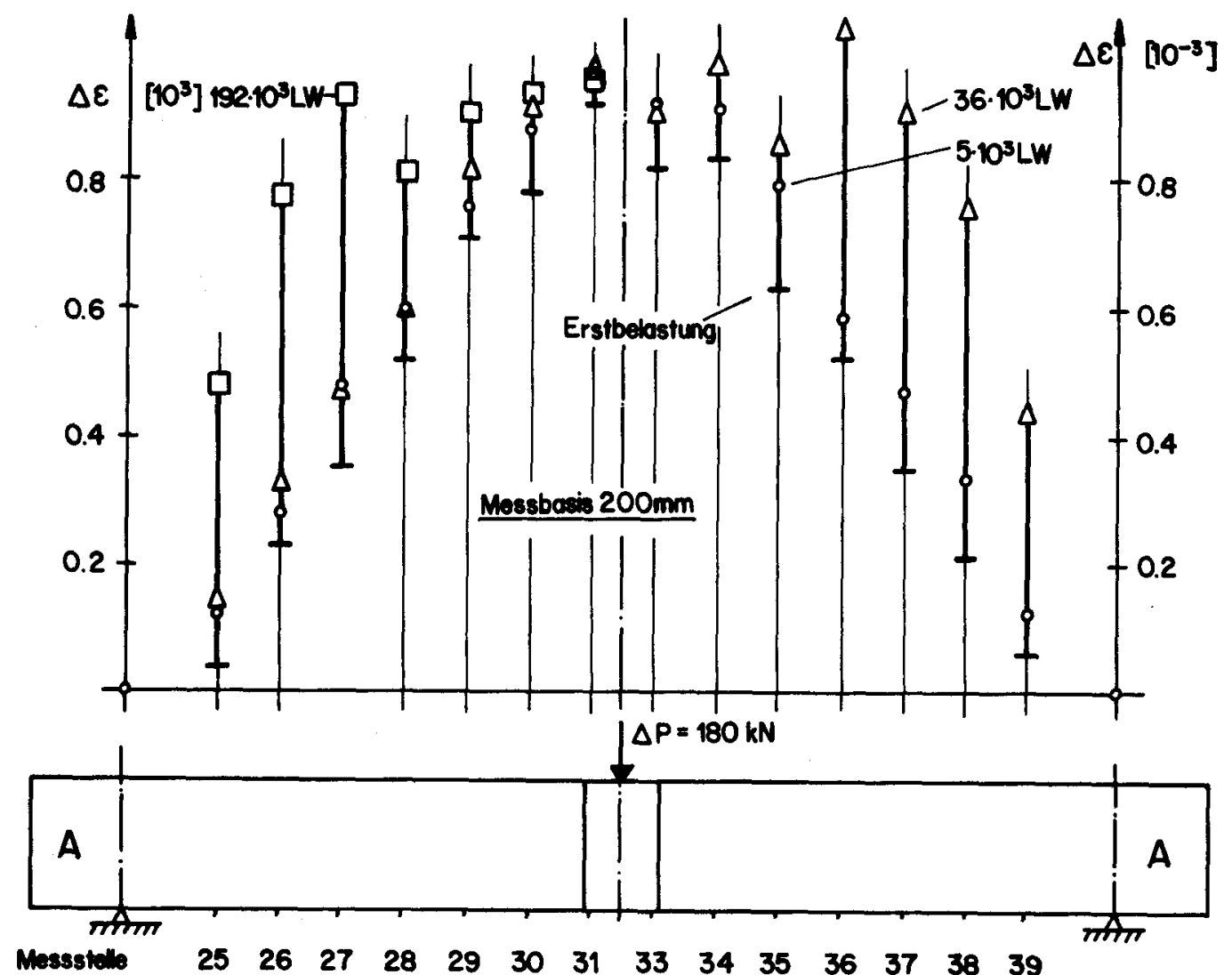

Bild $134:$ BII/7, Dehnungsdifferenzen in der Längsbewehrung im Lastzyklus B

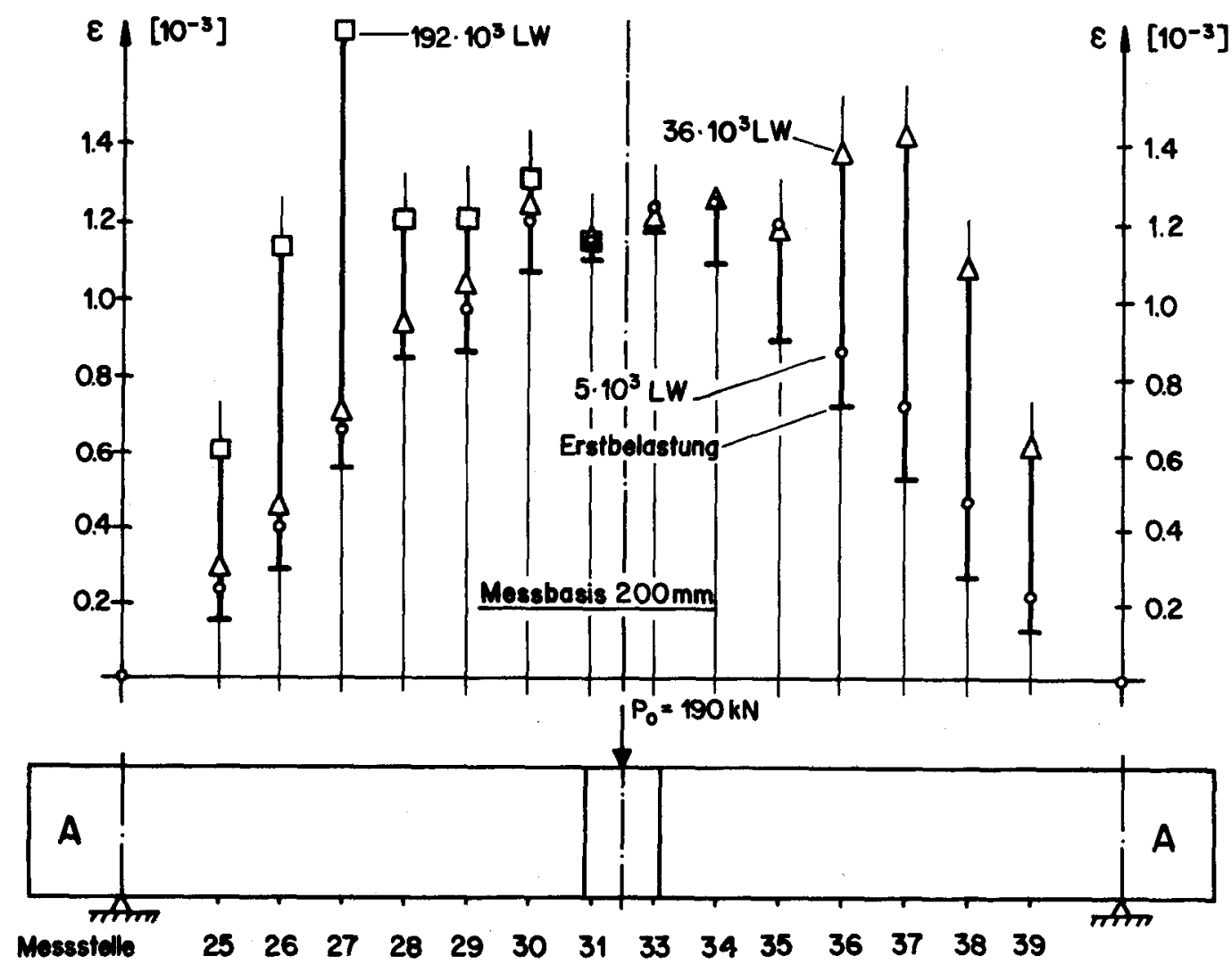

Bid 135: $B I / 7$, Dehnungen in der Löngsbewehrung unter Oberlast in Lastzyklus B 


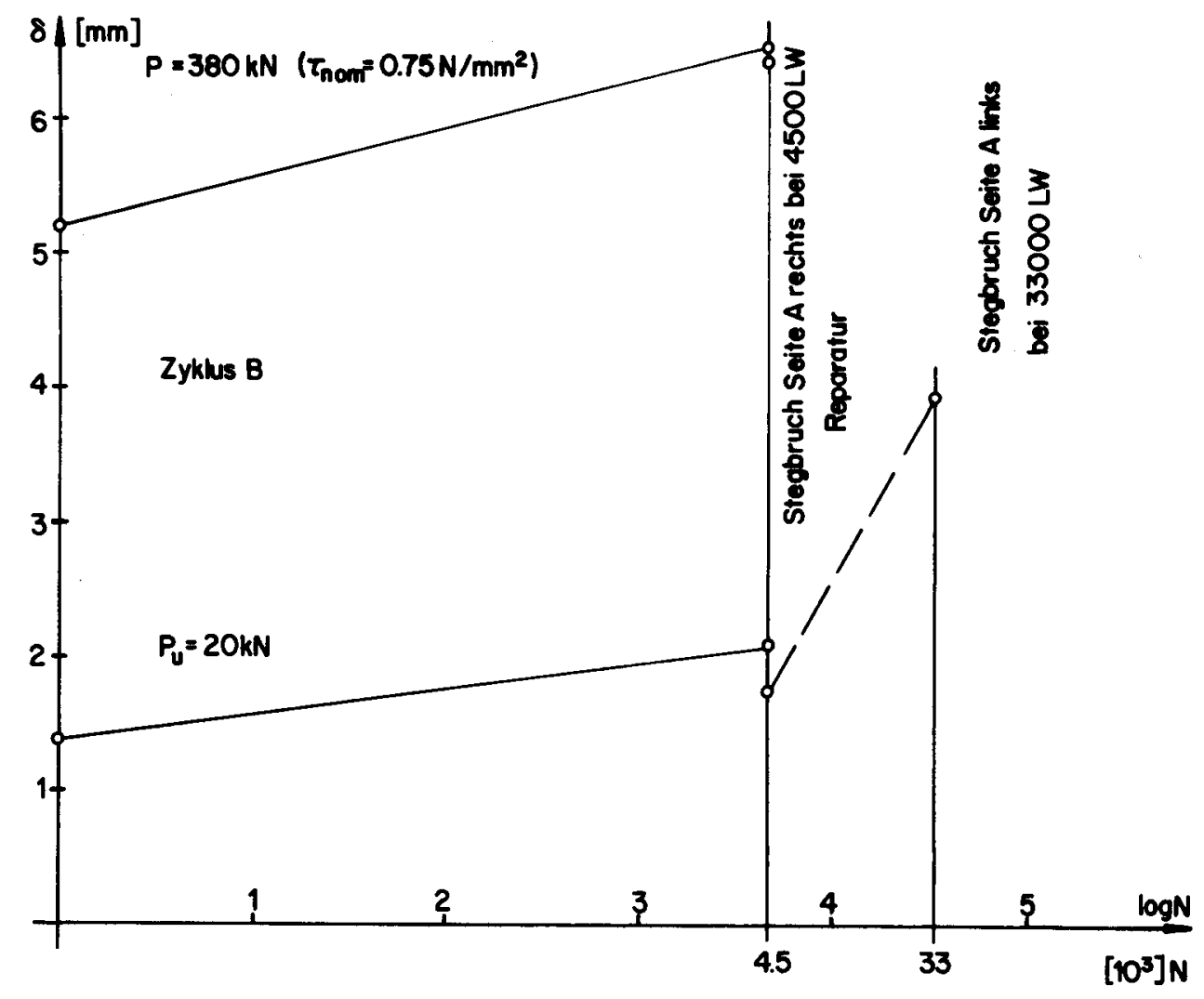

Bild 136: BI/8, Durchbiegung in Trögermitte in Funktion der Lastwechselzahl
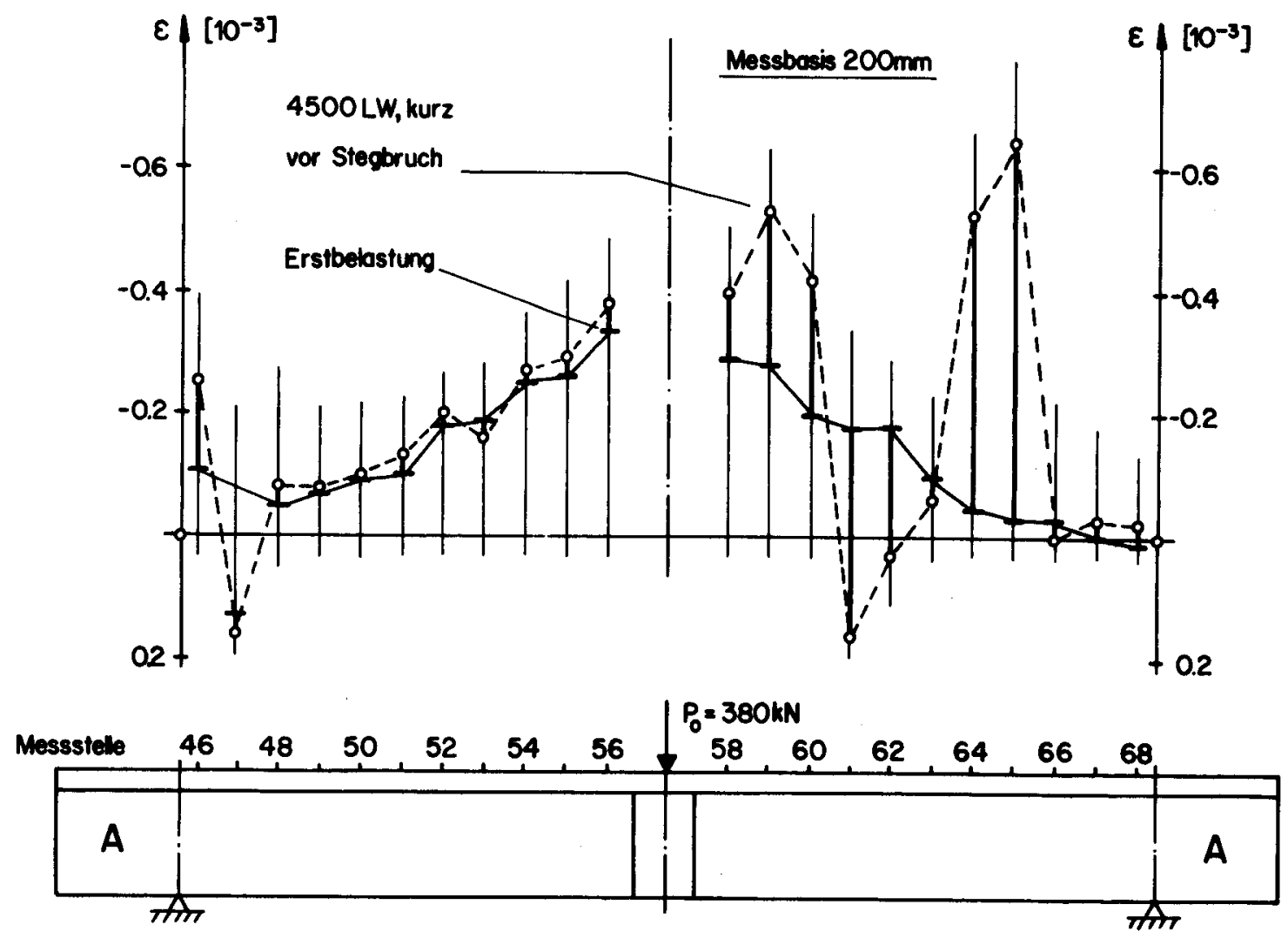

Bild 137: BI/8, Betondehnungen unter Oberlast in Lastzyklus B 


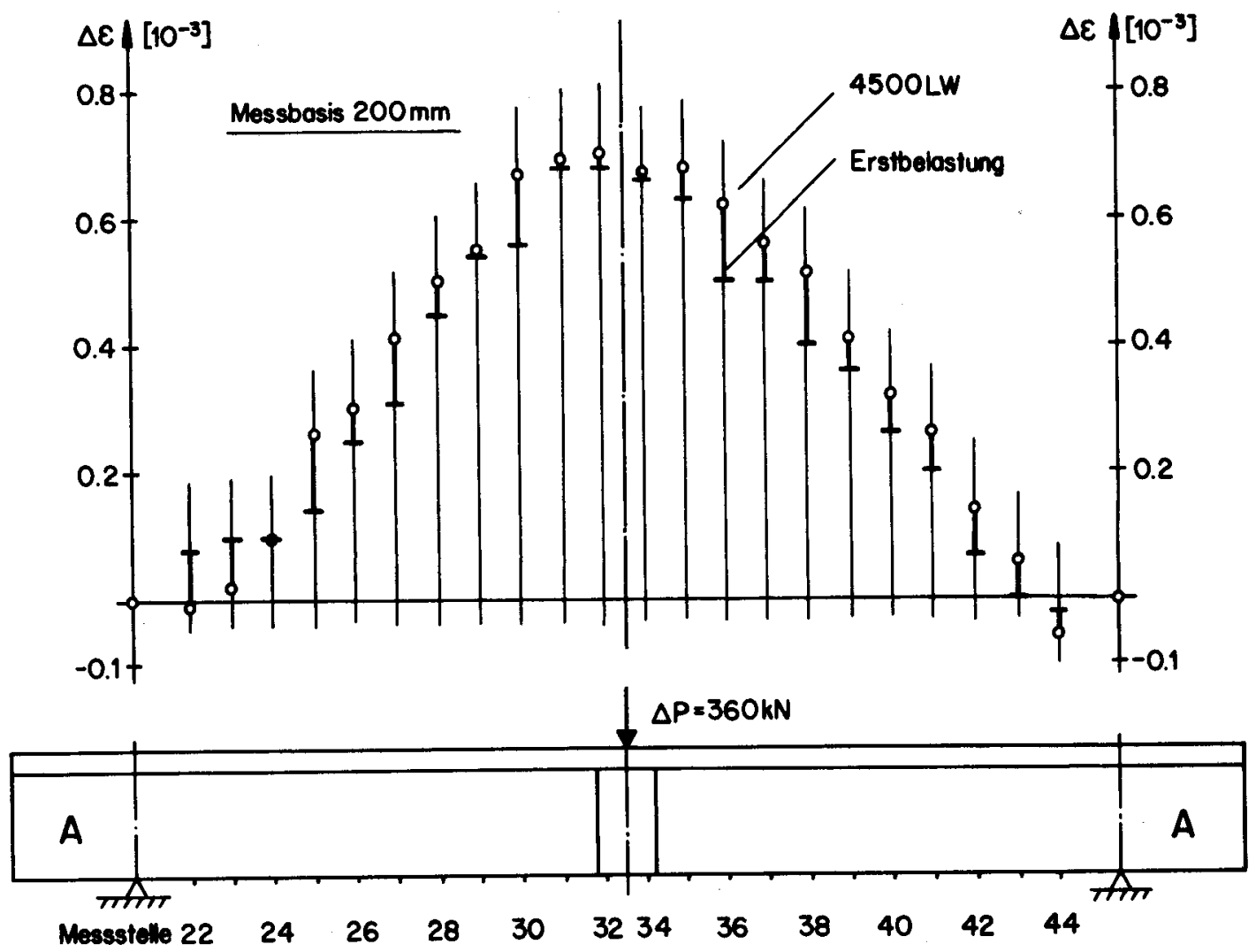

Bild 138 : BII/8, Dehnungsdifferenzen in der Längsbewehrung im Lasłzyklus B
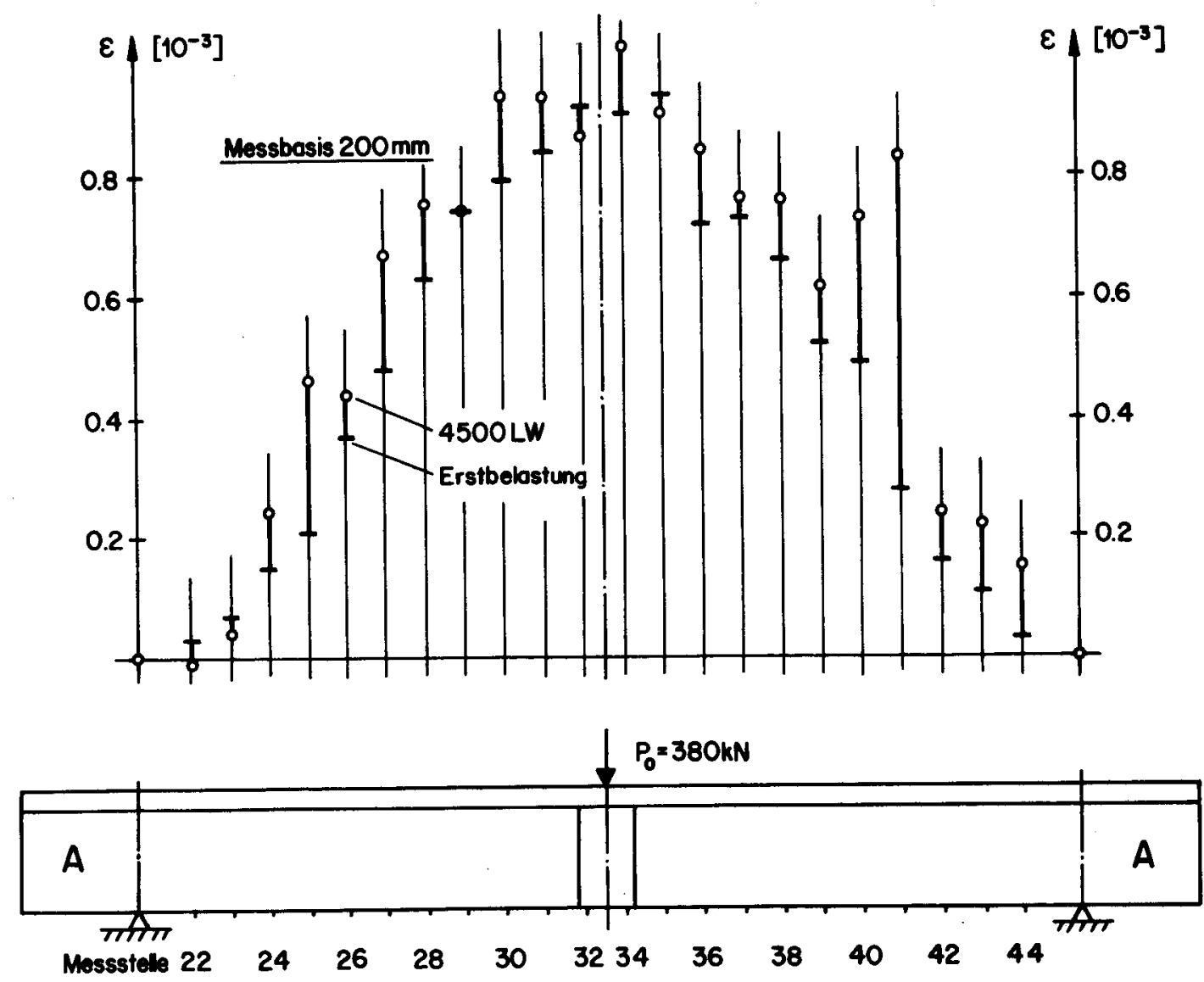

Bild $139: B I / 8$, Dehnungen in der Längsbewehrung unter Oberlast im Lastzyklus B 


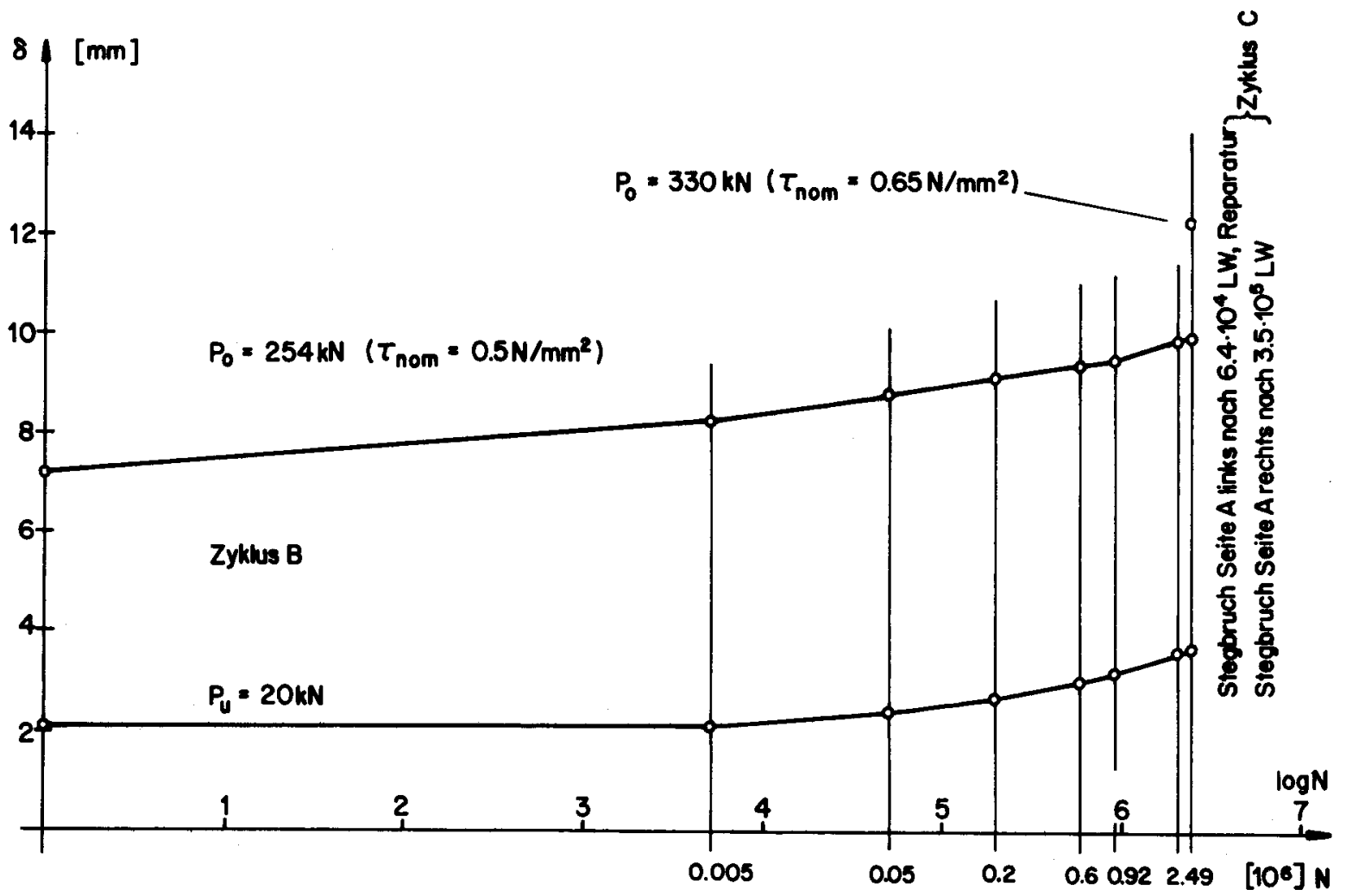

Bild 140: BII/9, Durchbiegung in Trägermitte in Funktion der Lasłwechselzahl
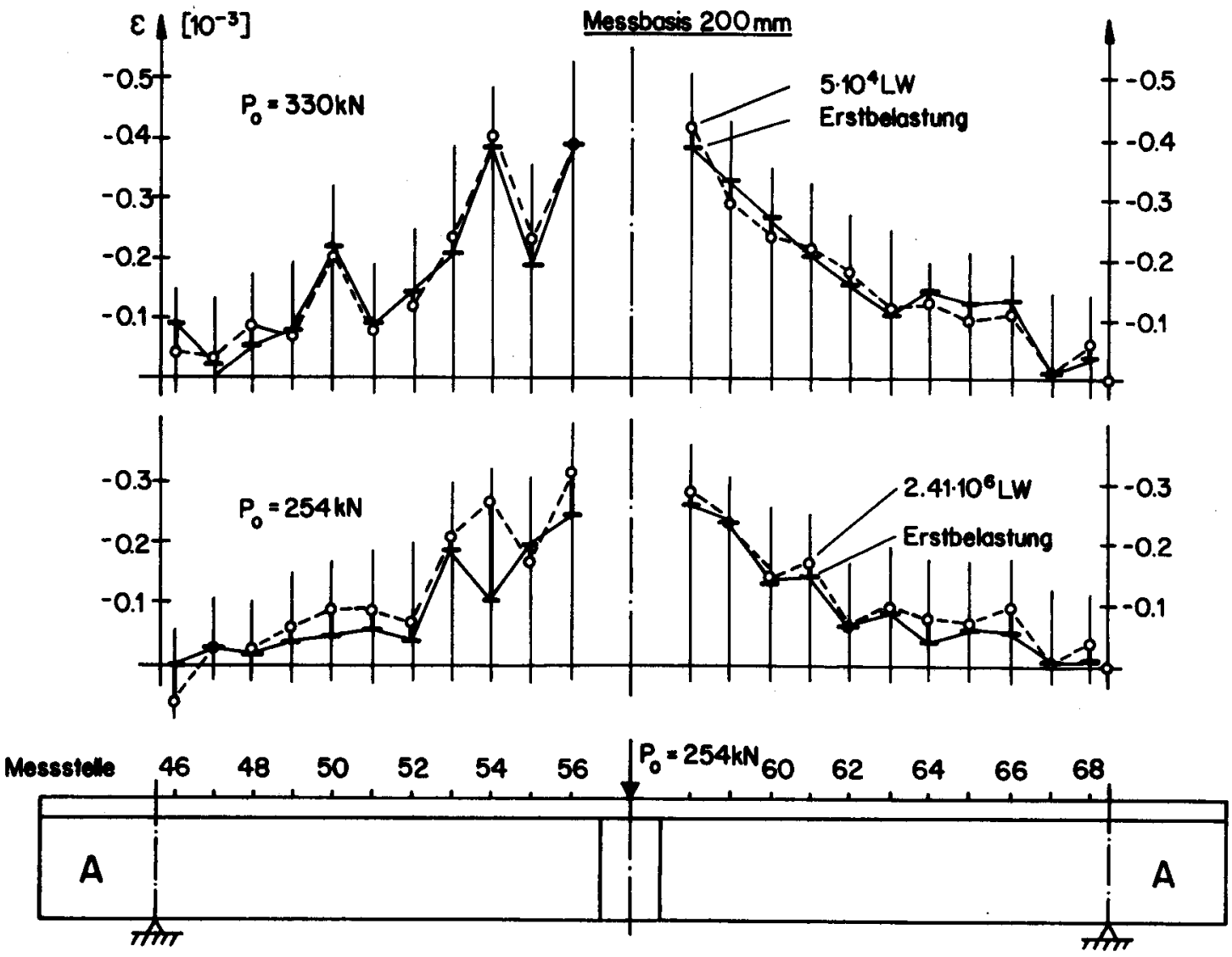

Bild 141: BII/9, Betondehnungen unter Oberlast in den Lastzyklen B und C 

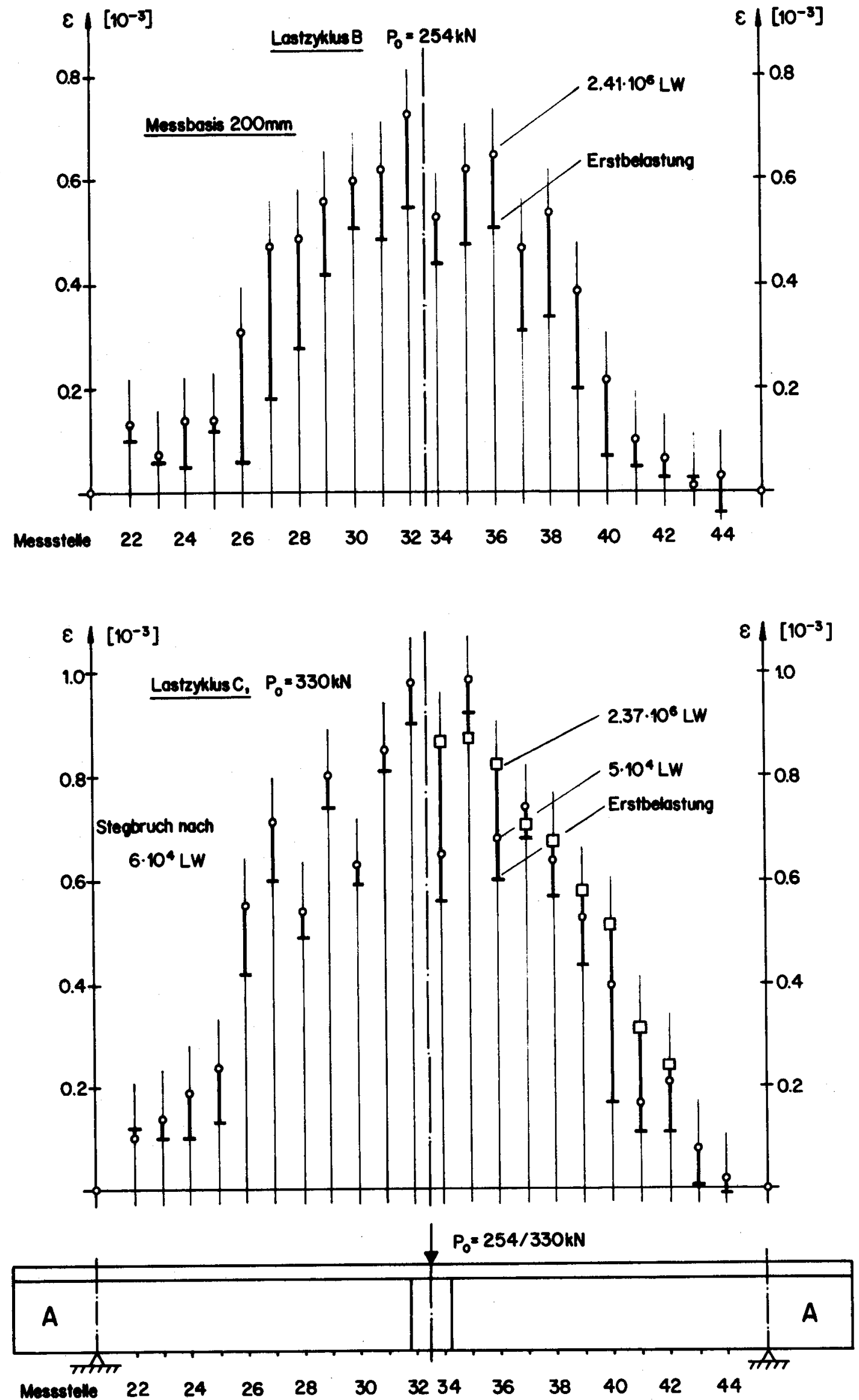

Bidd 142: BI/9, Dehnungen in der Löngsbewehrung unter Oberlast in den Lastzyklen $B$ und $C$ 


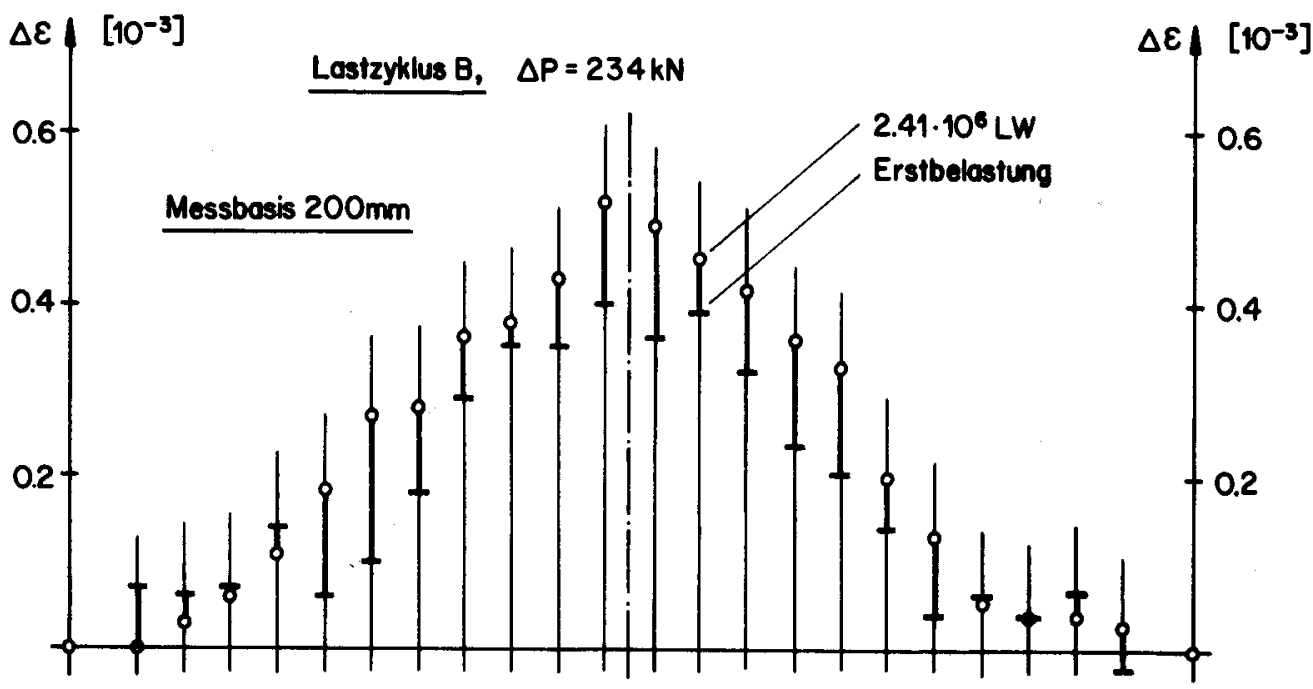

$\begin{array}{lllllllllllll}\text { Messstele } & 22 & 24 & 26 & 28 & 30 & 32 & 34 & 36 & 38 & 40 & 42 & 44\end{array}$
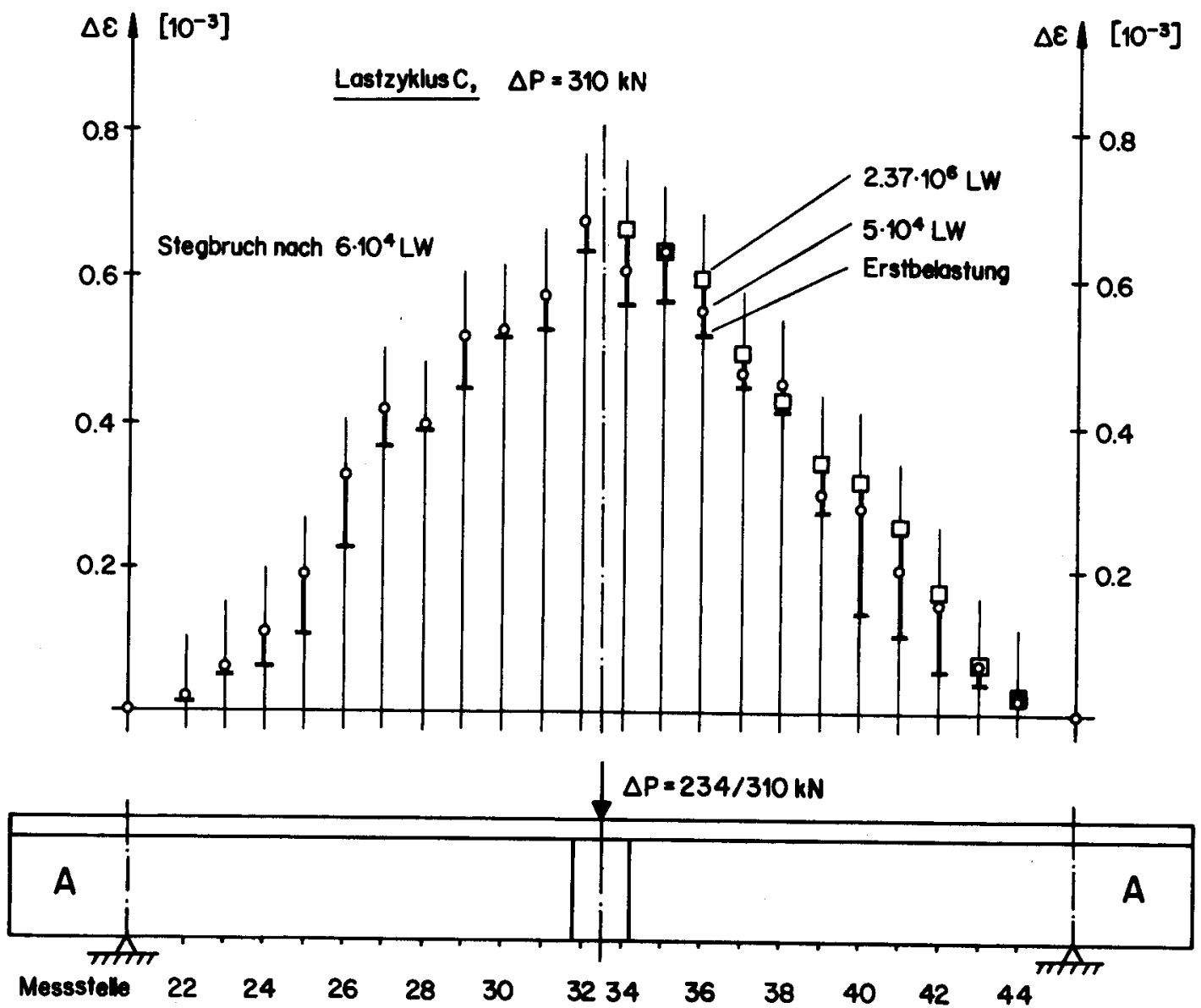

Bild 143: BI/9, Dehnungsdifferenzen in der Löngsbewehrung in den Lastzyklen $B$ und $C$ 


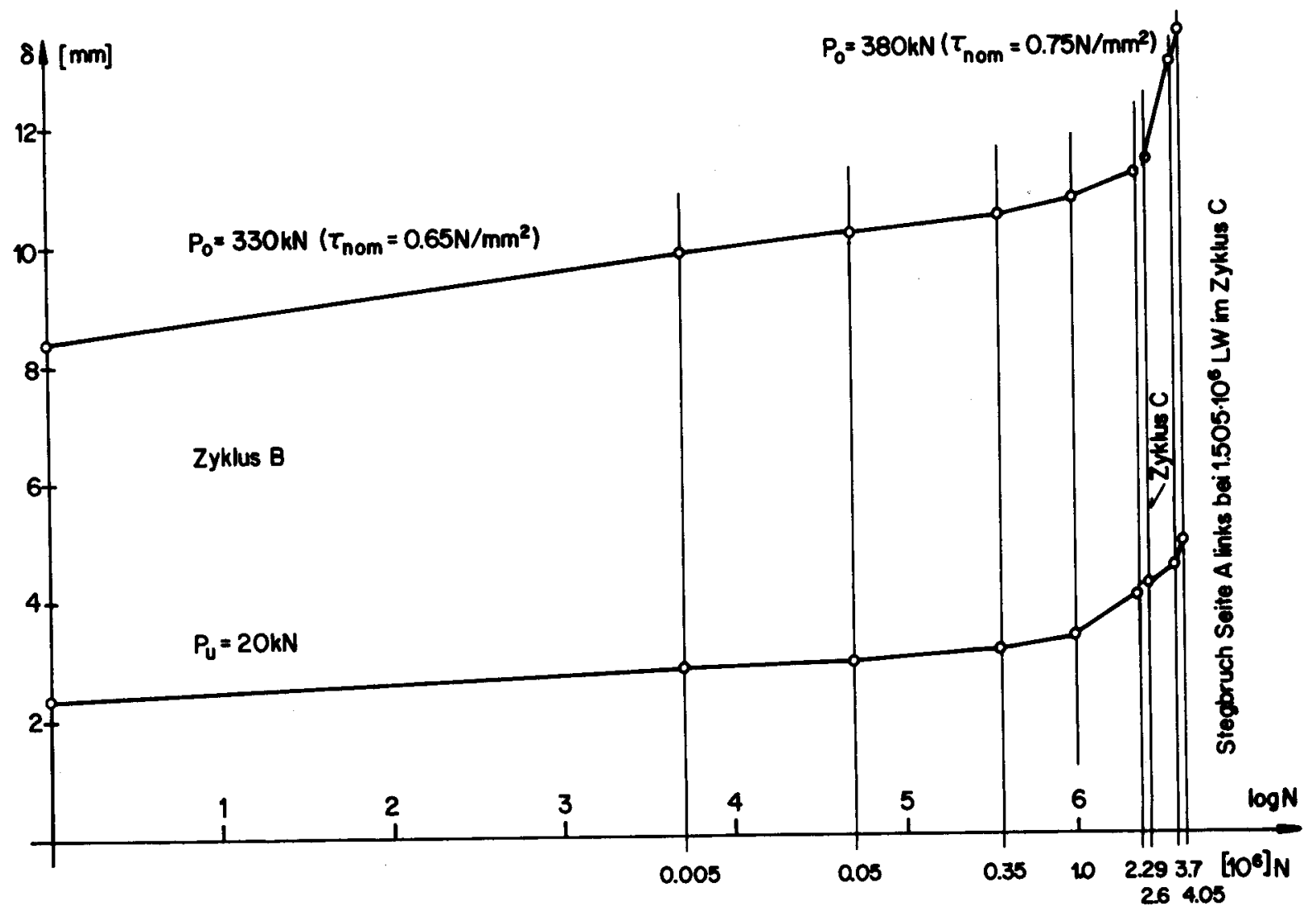

Bild 144: BI/10, Durchbiegung in Trägermitte in Funktion der Lastwechselzahl
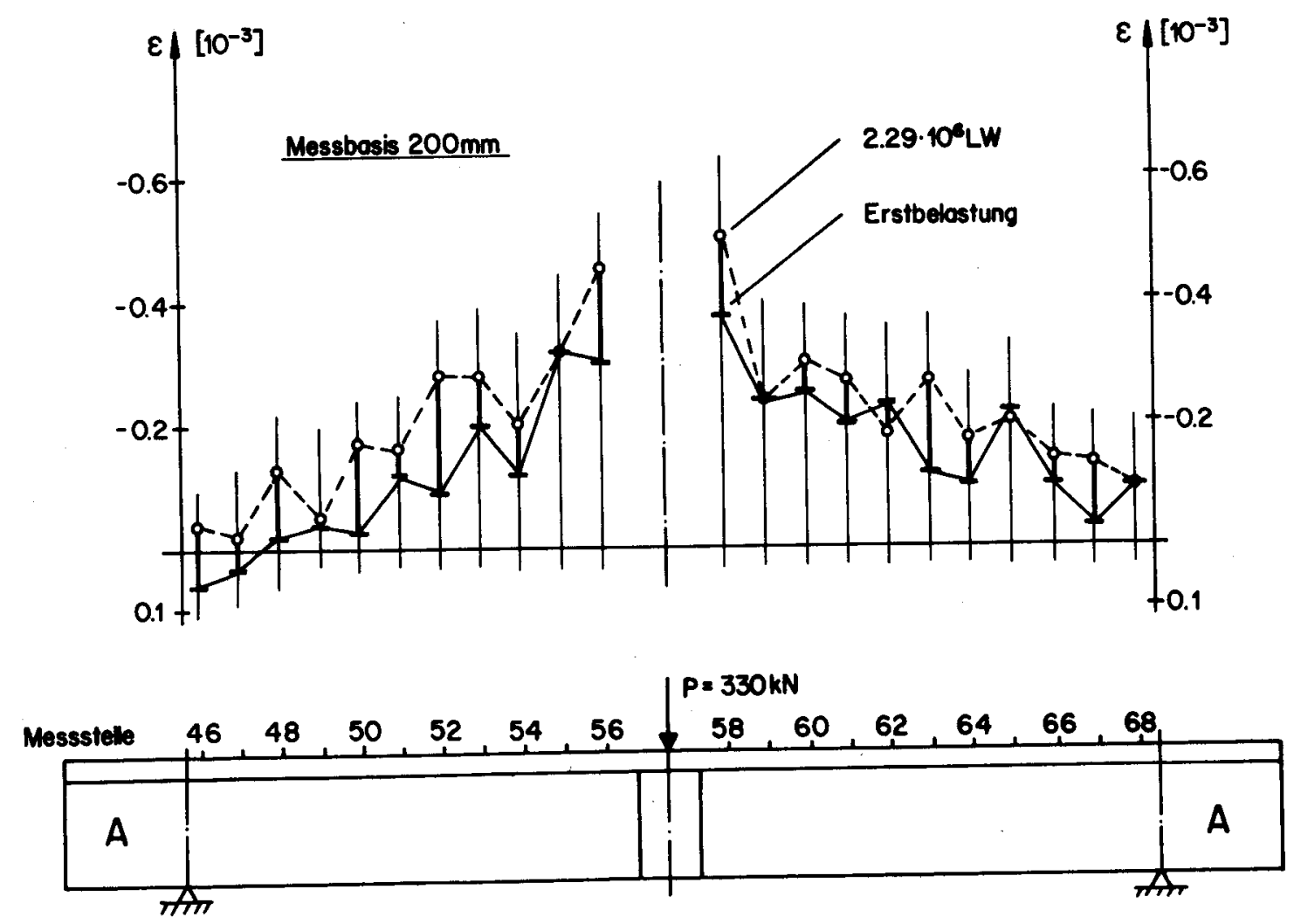

Bild 145: BI/10, Betondehnungen unter Oberlost im Lastzyklus B 


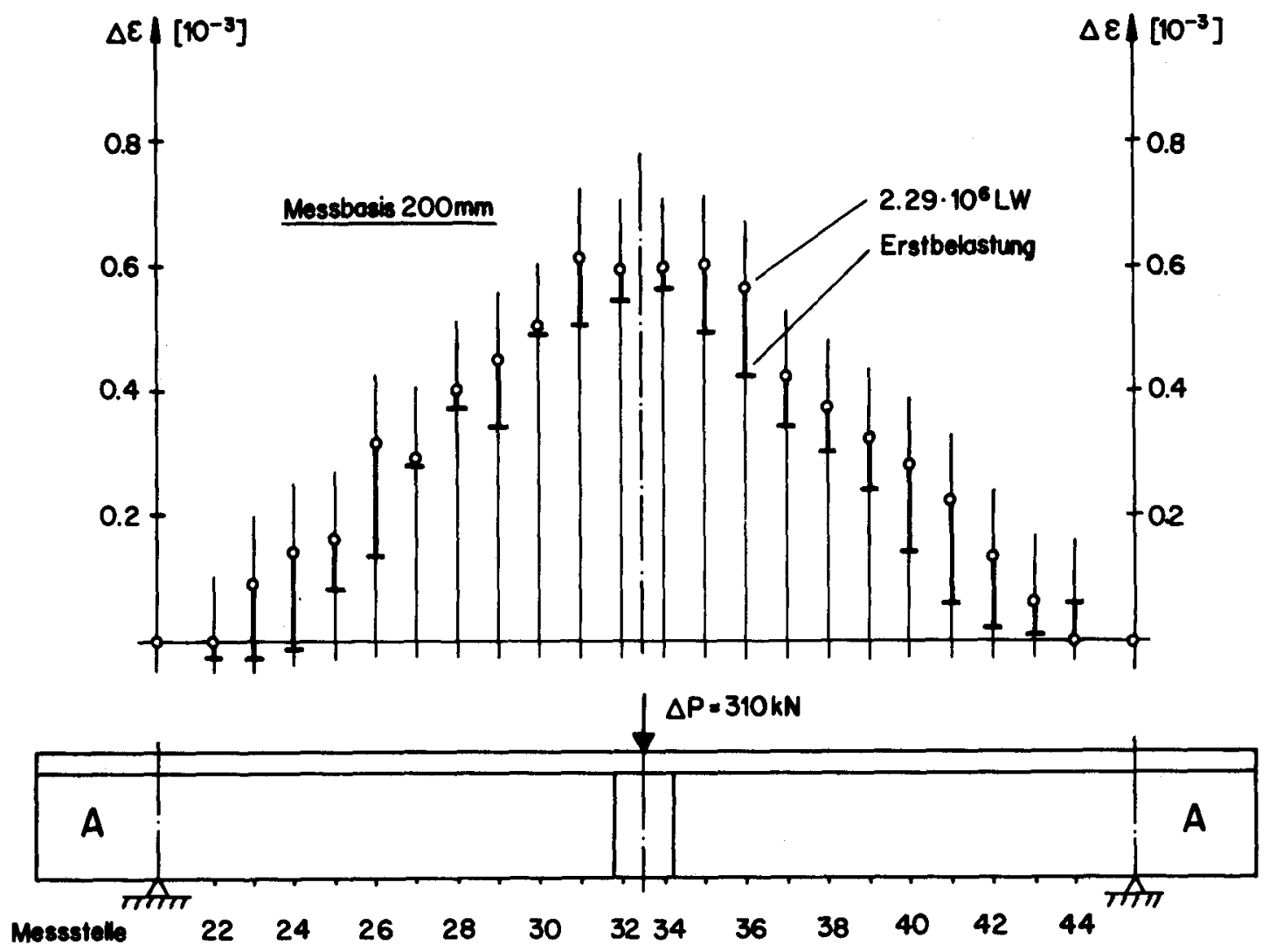

Bild 146: BI MO, Dehnungsdifferenzen in der Längsbewehrung im Lastzyklus B
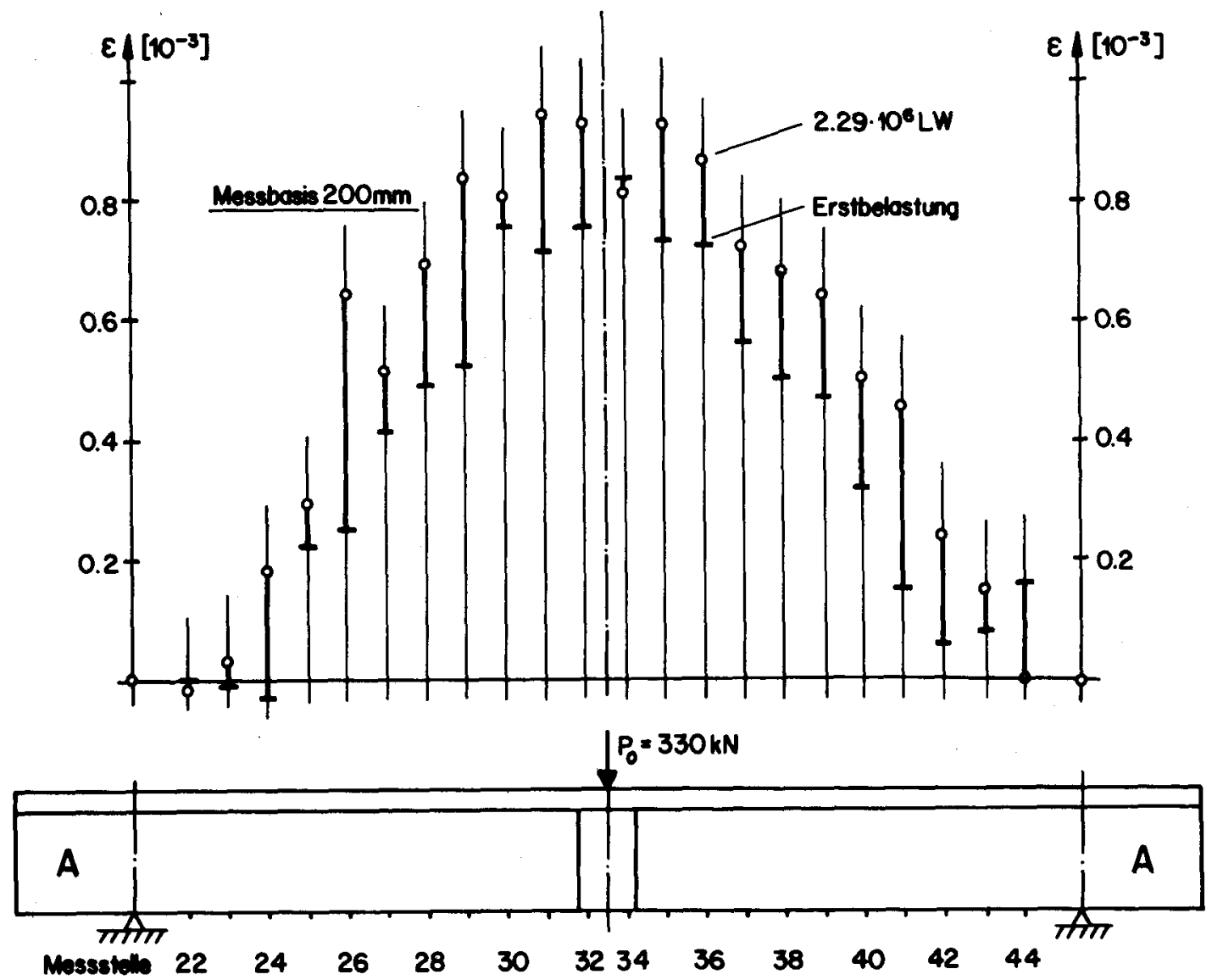

Bidd 147 : BII/10, Dehnungen in der Längsbewehrung unter Oberlast im Lastzyklus $B$ 


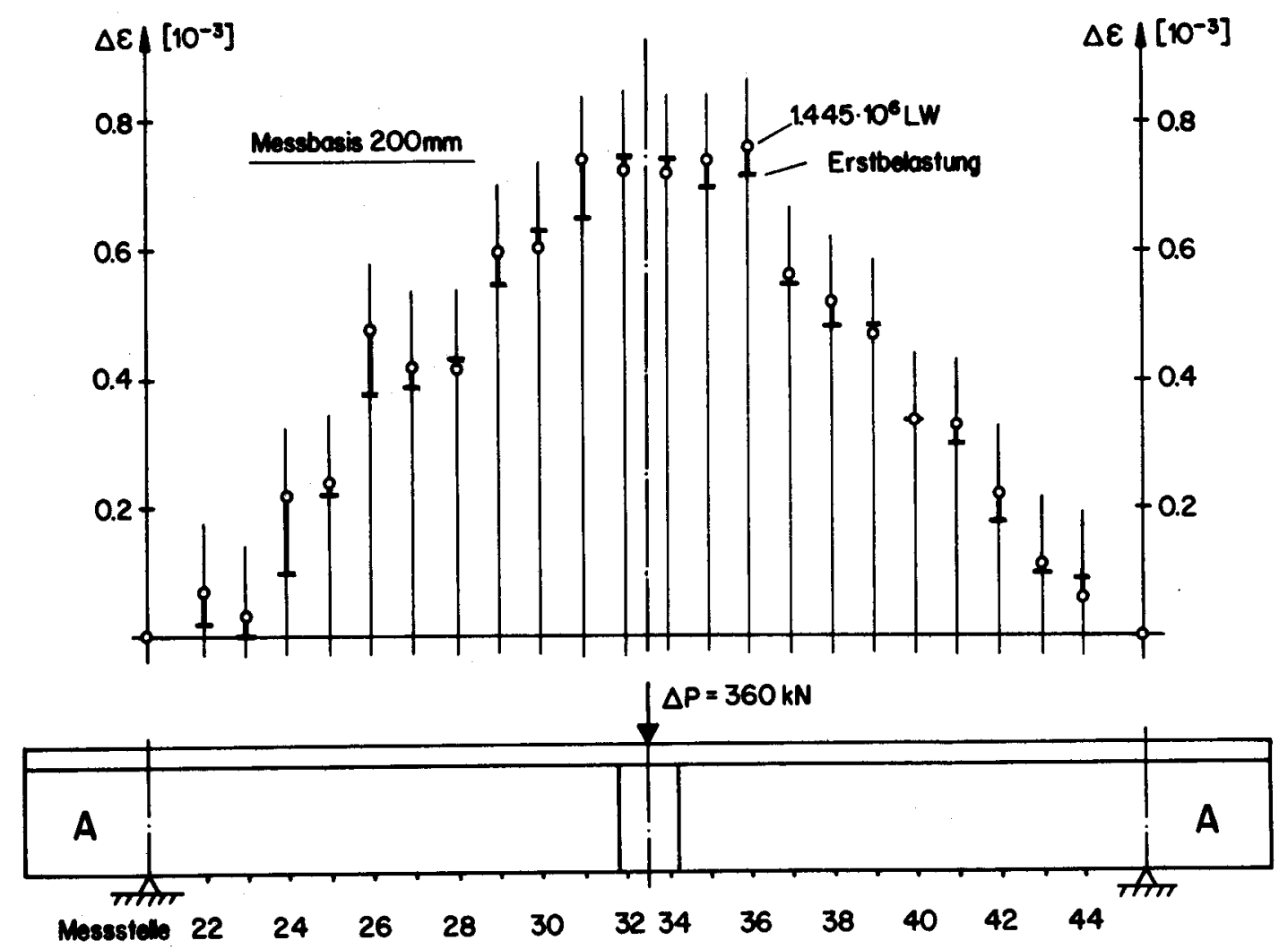

Bild 148: BI/10, Dehnungsdifferenzen in der Längsbewehrung im Lastzyklus C

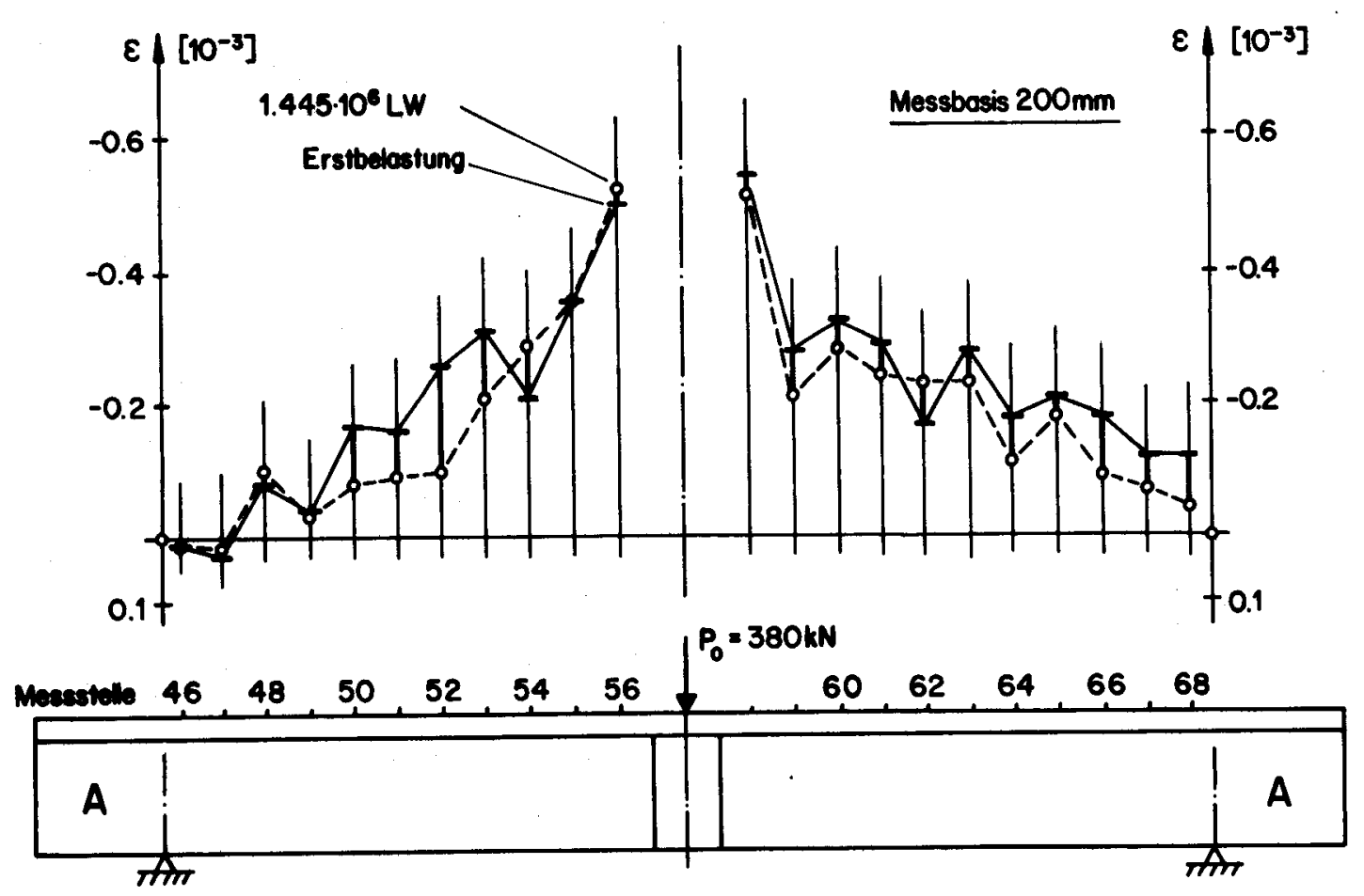

Bid 149: BI/10, Betondehnungen unter Oberlast in Lastzyklus C 


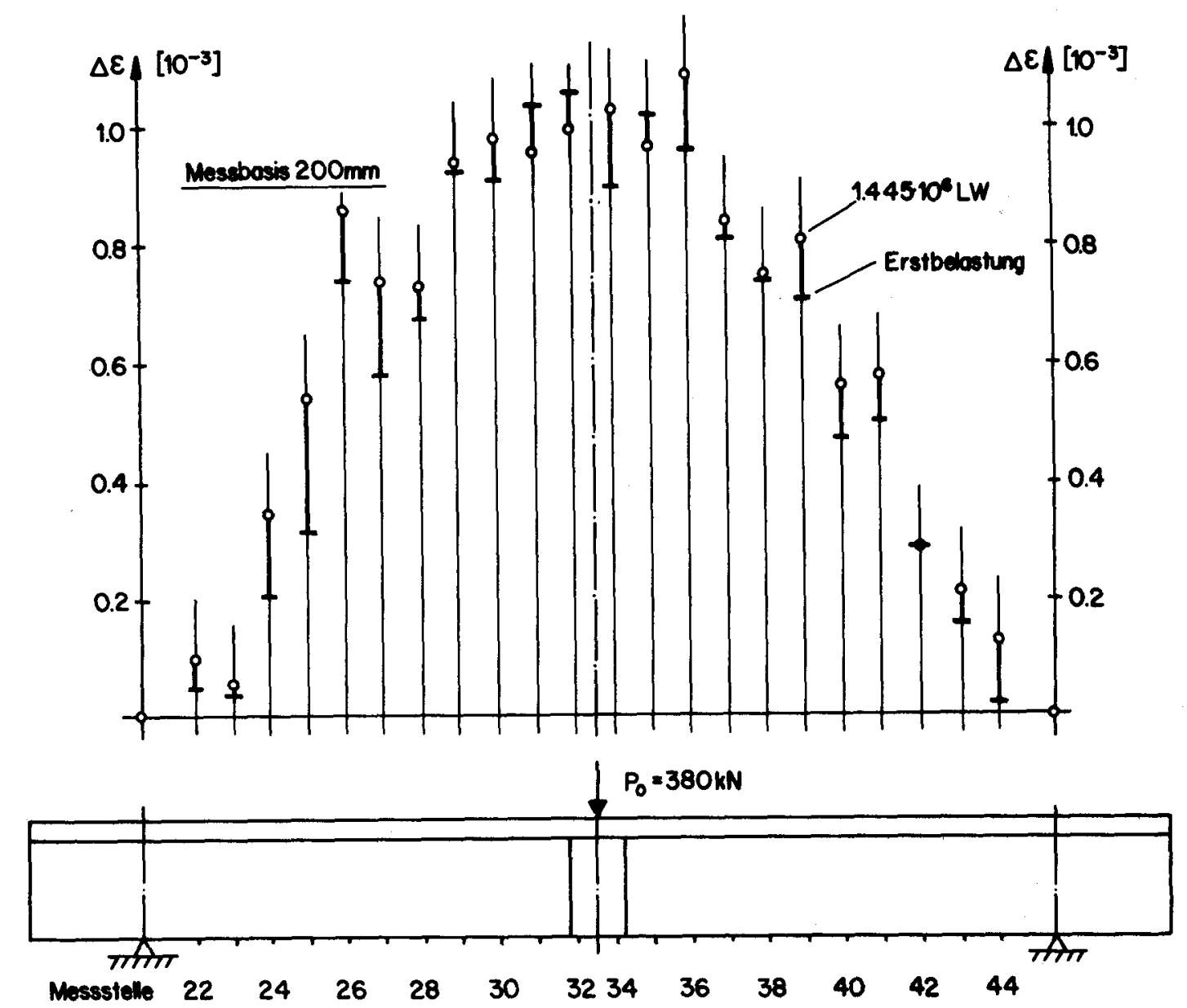

Bild 150: B1/10, Dehnungen in der Löngsbewehrung unter Oberlasł in Lastzyklus C 


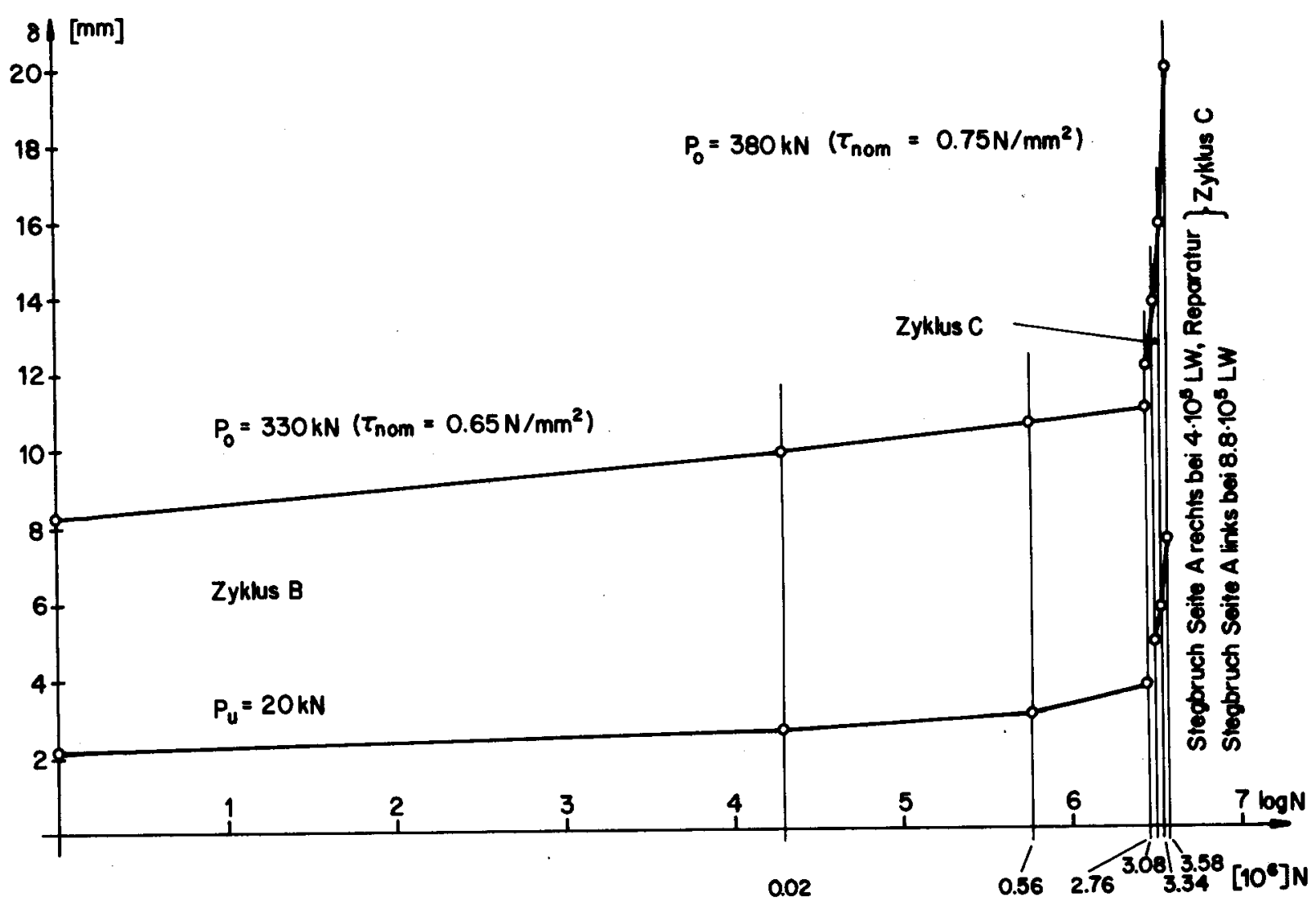

Bild 151: BI/11, Durchbiegung in Trägermitte in Funktion der Lastwechselzahl
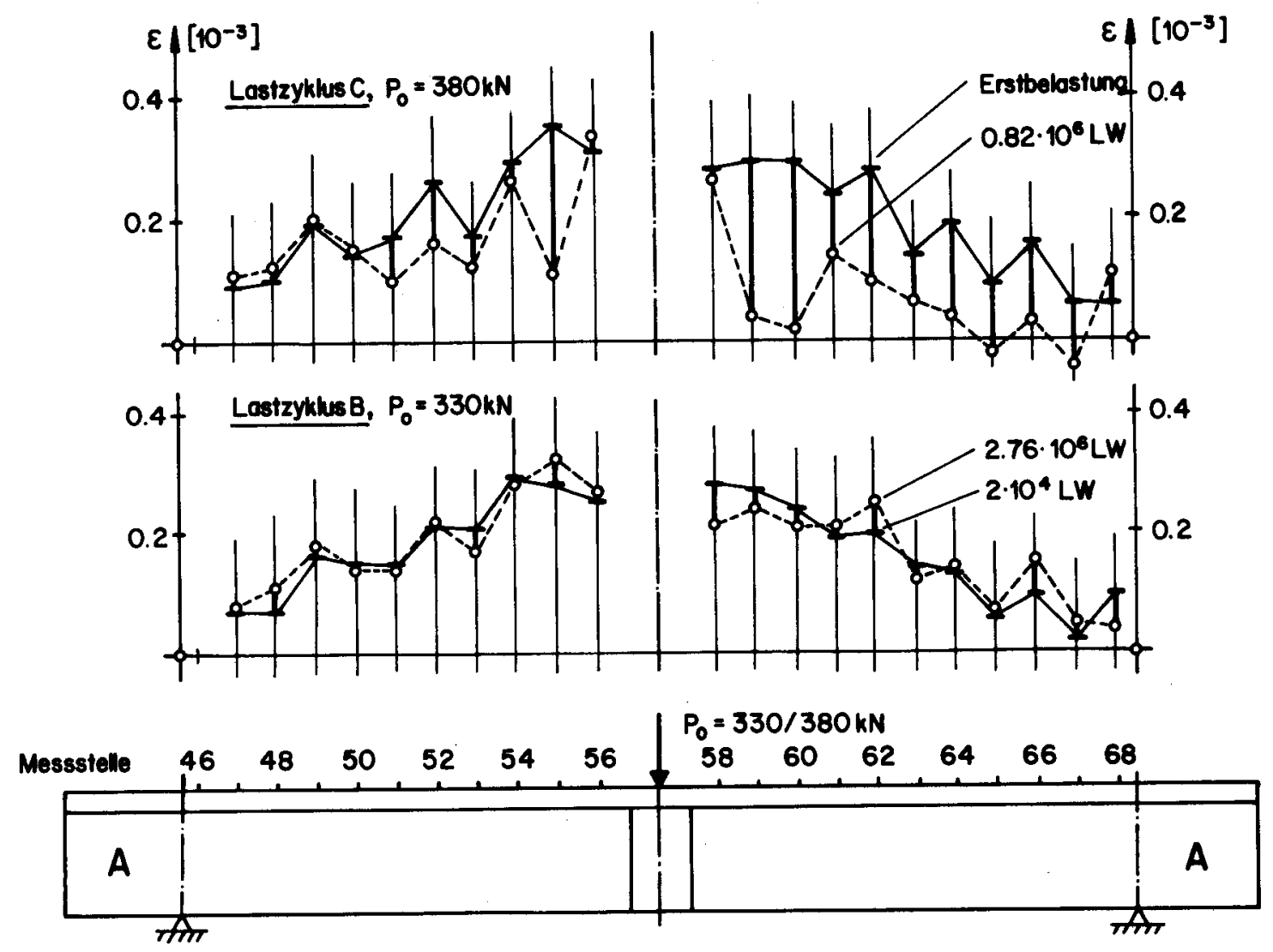

Bild 152: BII $/ 1$, Betondehnungen unter Oberlast in den Lastzyklen $B$ und $C$ 

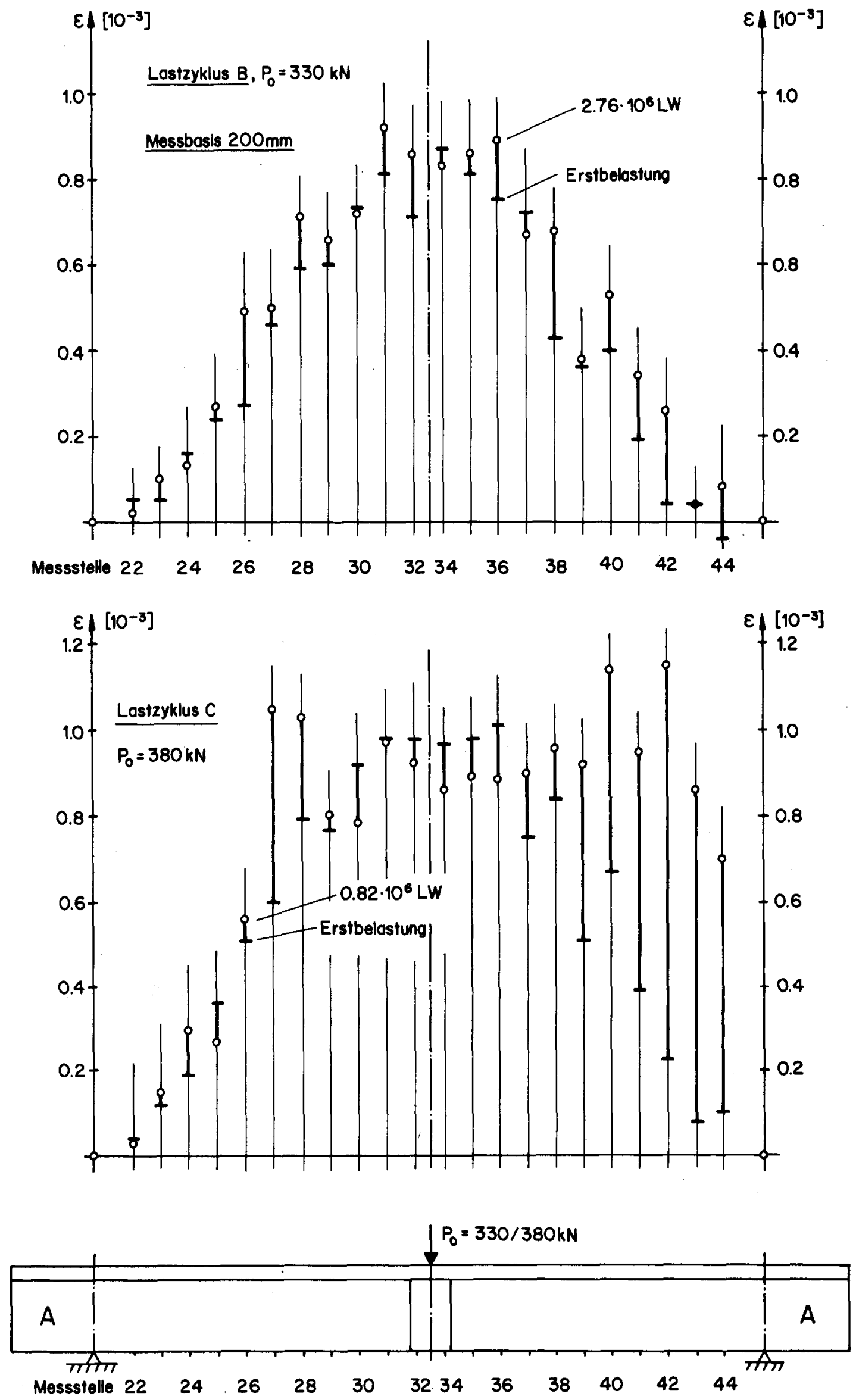

Bild 153: BII/11, Dehnungen in der Längsbewehrung unter Oberlast in den Lastzyklen $B$ und $C$ 

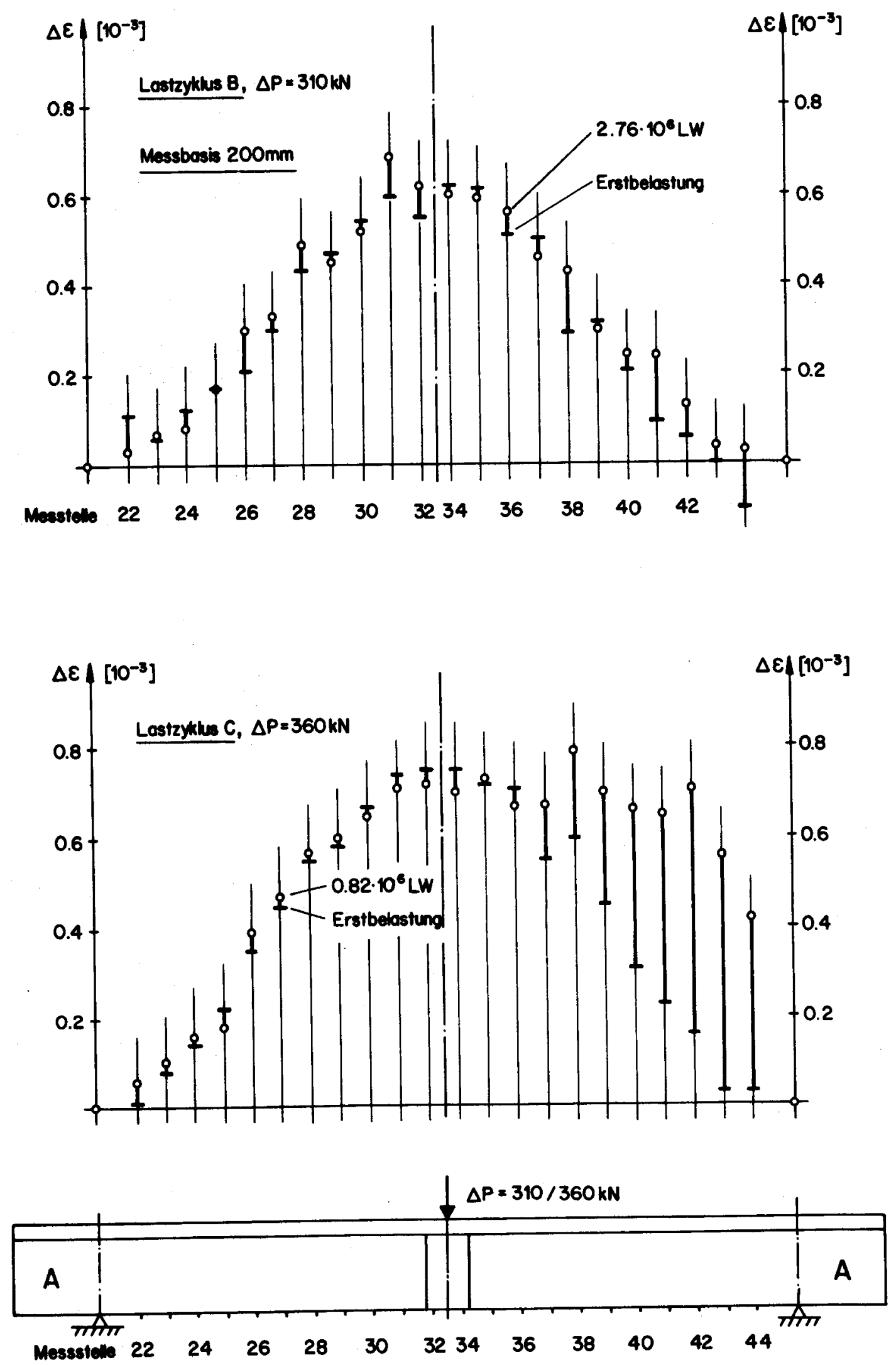

Bid 154: BI/11, Dehnungsdifferenzen in der Löngsbewehrung in den Lastzyklen $B$ und $C$ 

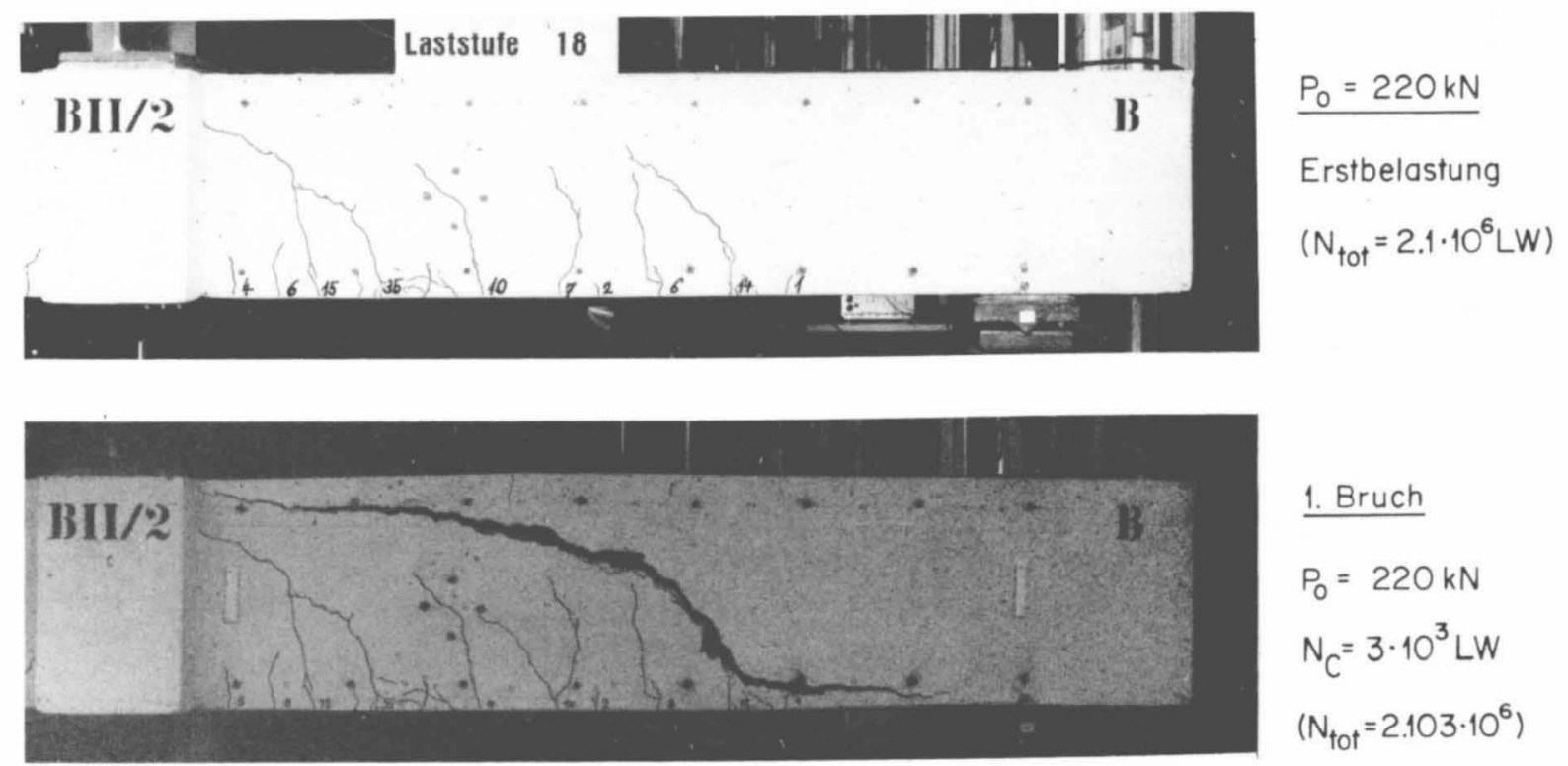

1. Bruch

$P_{0}=220 \mathrm{kN}$

$\mathrm{N}_{\mathrm{C}}=3 \cdot 10^{3} \mathrm{LW}$

$\left(N_{\text {tot }}=2.103 \cdot 10^{6}\right)$

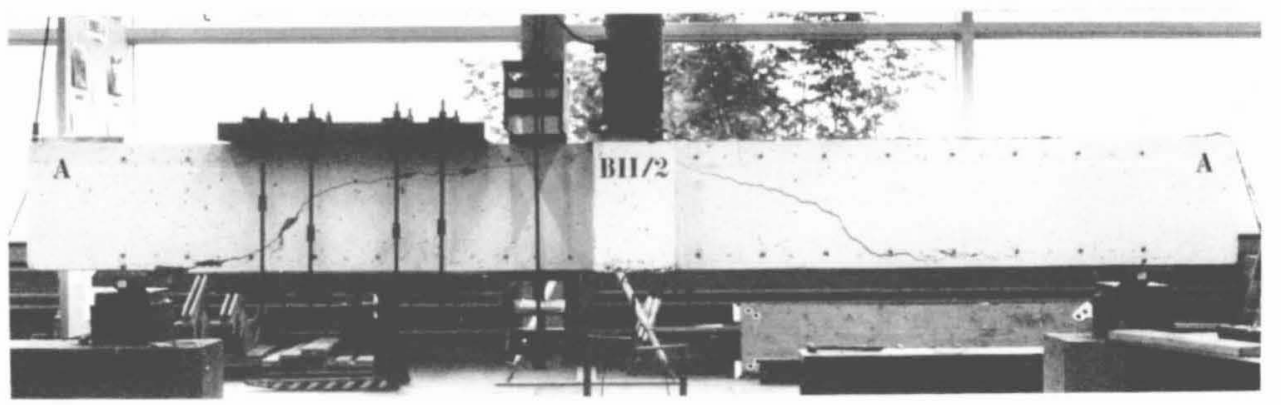

2. Bruch

$P_{0}=220 \mathrm{kN}$

$N_{C}=1.28 \cdot 10^{4} \mathrm{LW}$

$\left(N_{\text {tot }}=2.113 \cdot 10^{6} \mathrm{LW}\right)$

Bild 155: BII/2, Rissbild und Brüche im Lastzyklus C

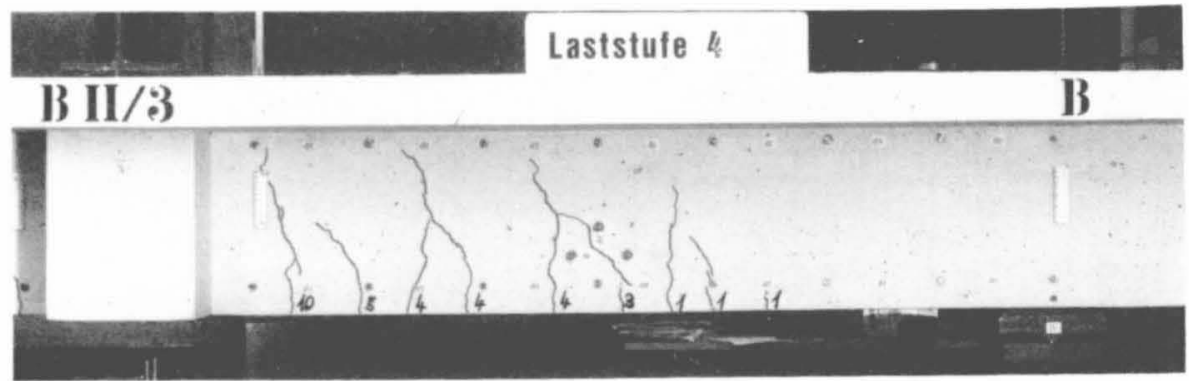

$\underline{P_{0}}=220 \mathrm{kN}$

Erstbelastung

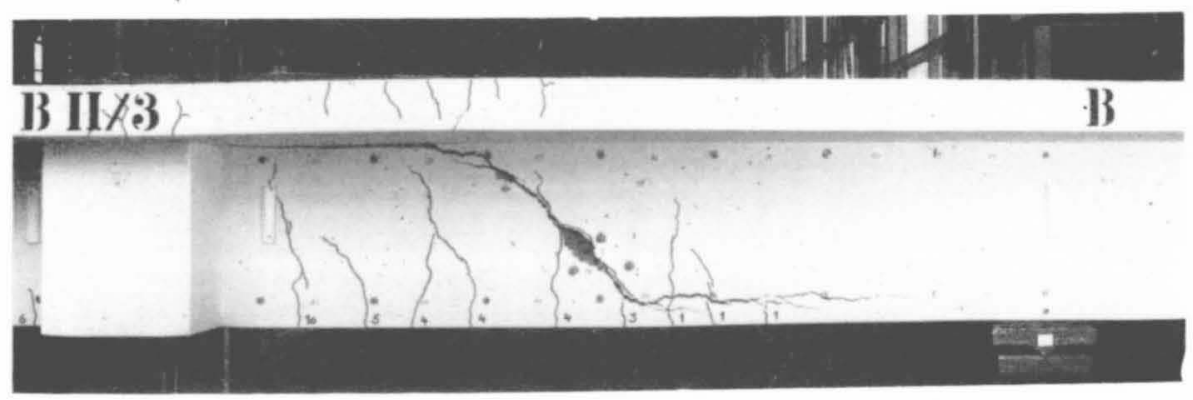

Stegbruch

$\mathrm{N}_{B}=2.58 \cdot 10^{3} \mathrm{LW}$

Rissweiten in $1 / 100 \mathrm{~mm}$

Bild 156: BI/3, Rissbild und Stegbruch im Lastzyklus B 


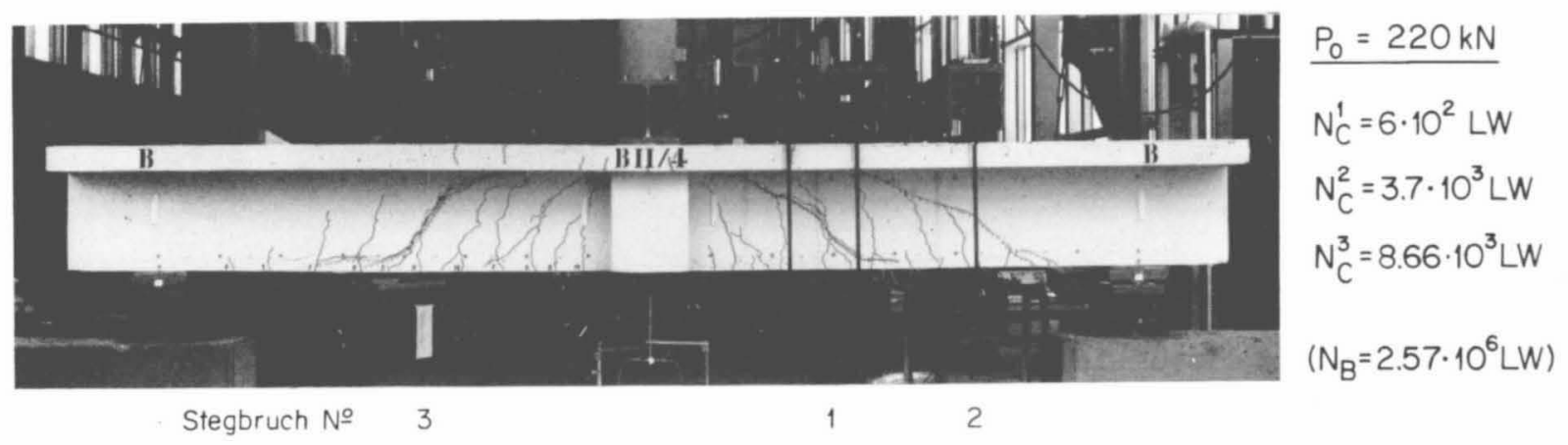

Bild 157: BI/4, Stegbrüche im Lastzyklus C
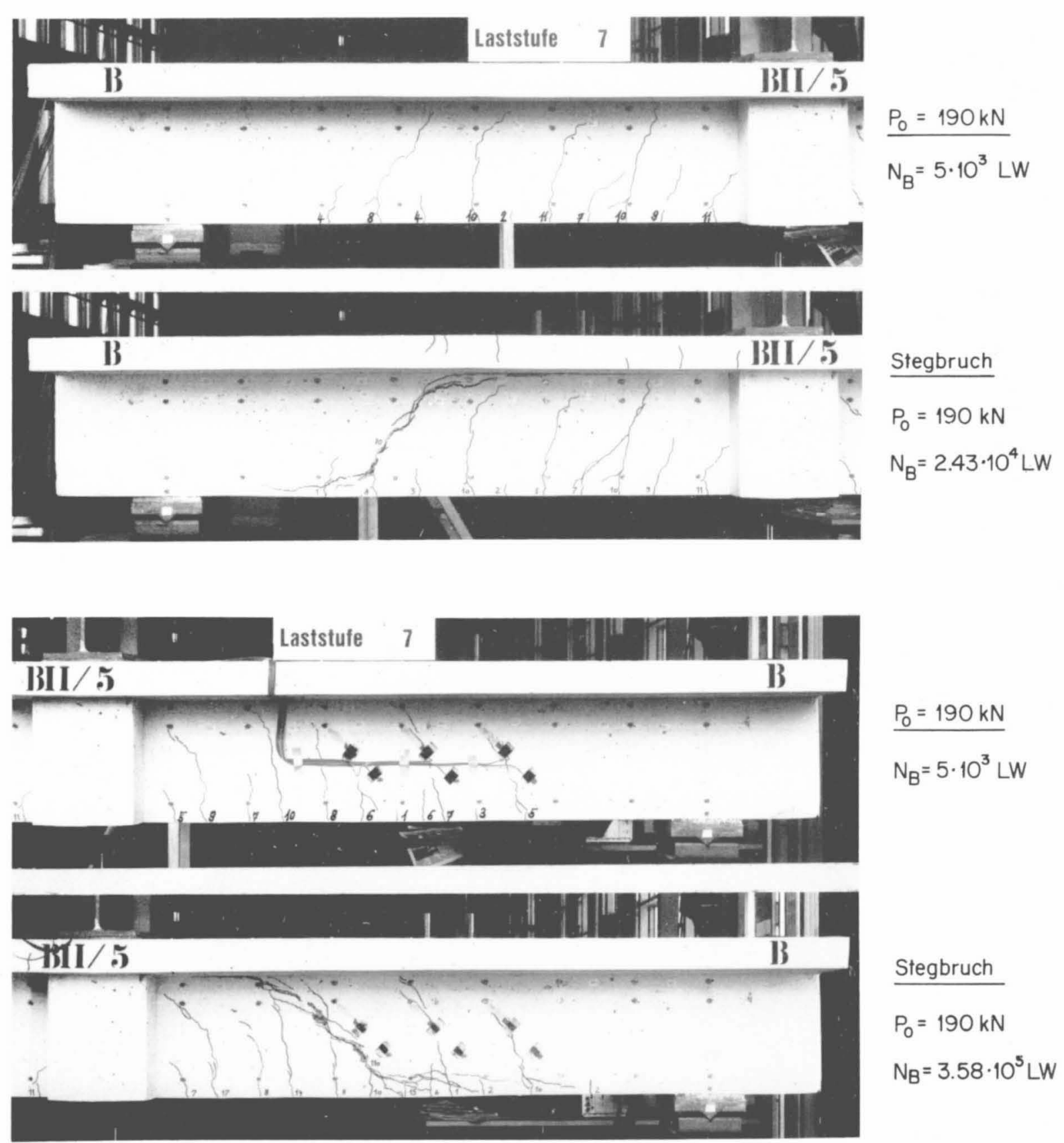

Rissweiten in $1 / 100 \mathrm{~mm}$

Bild 158: BI/5, Rissbilder und Stegbrüche im Lastzyklus B 


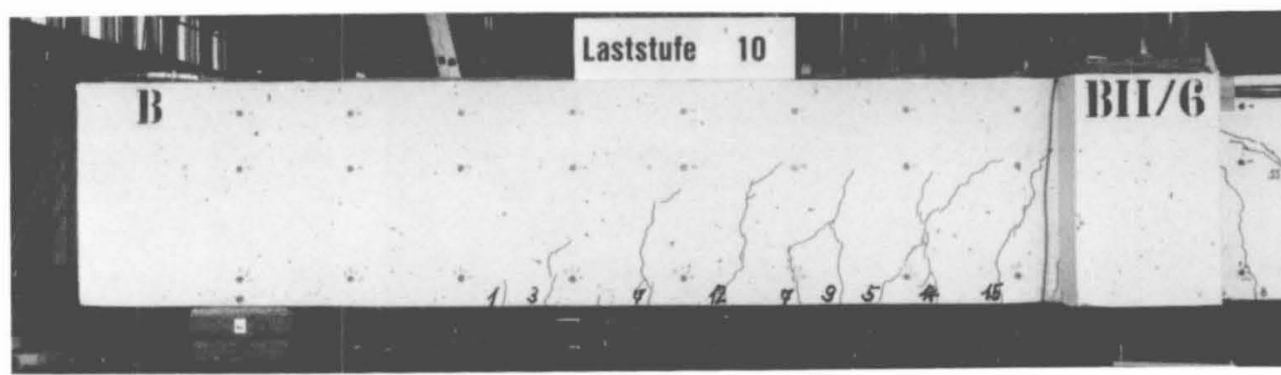

$$
\begin{aligned}
& P_{0}=190 \mathrm{kN} \\
& N_{B}=5 \cdot 10^{4} \mathrm{LW}
\end{aligned}
$$

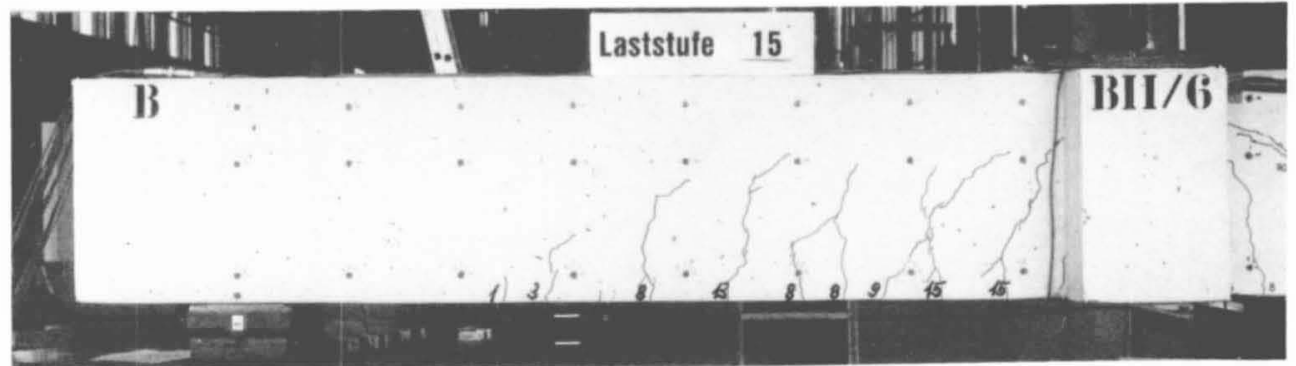

$\underline{P_{0}=190 \mathrm{kN}}$

$N_{B}=1.41 \cdot 10^{5} \mathrm{LW}$

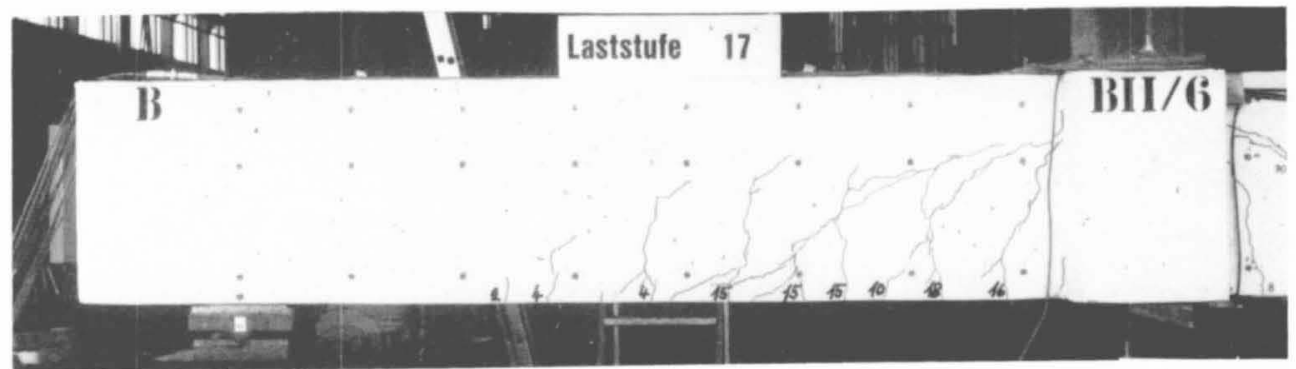

$\underline{P_{0}=190 \mathrm{kN}}$

$N_{B}=2.06 \cdot 10^{5} \mathrm{LW}$

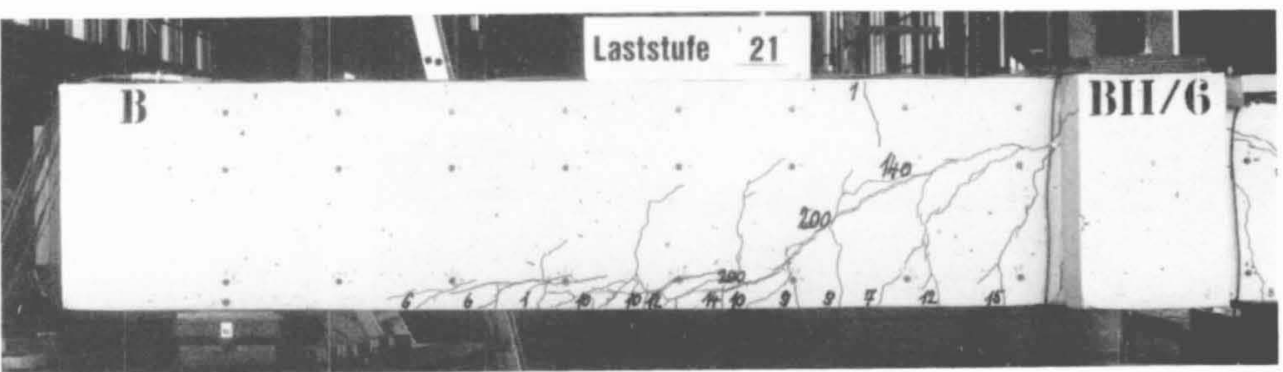

$P_{0}=190 \mathrm{kN}$

$N_{B}=2.35 \cdot 10^{5} \mathrm{LW}$

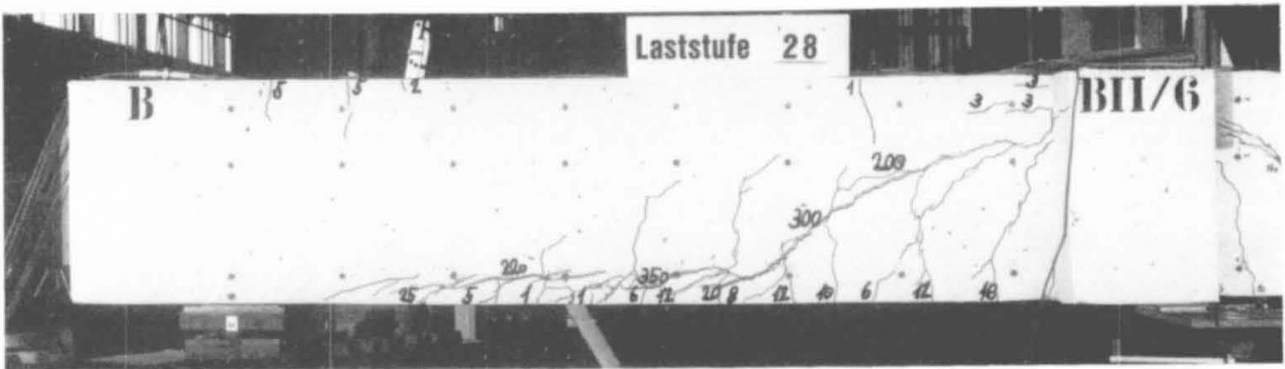

$\underline{P_{0}}=190 \mathrm{kN}$

$N_{B}=1.23 \cdot 10^{6} \mathrm{LW}$

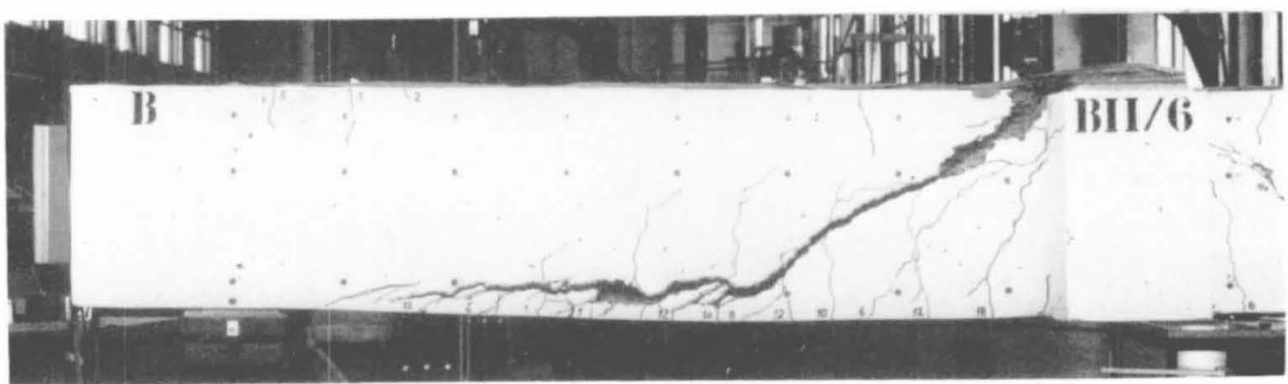

Bruch, Zykl.C

$P_{0}=220 \mathrm{kN}$

$N_{C}=9.7 \cdot 10^{4} \mathrm{LW}$

$\left(N_{\text {tot }}=3.397 \cdot 10^{6} \mathrm{LW}\right)$ 


\section{Leer - Vide - Empty}



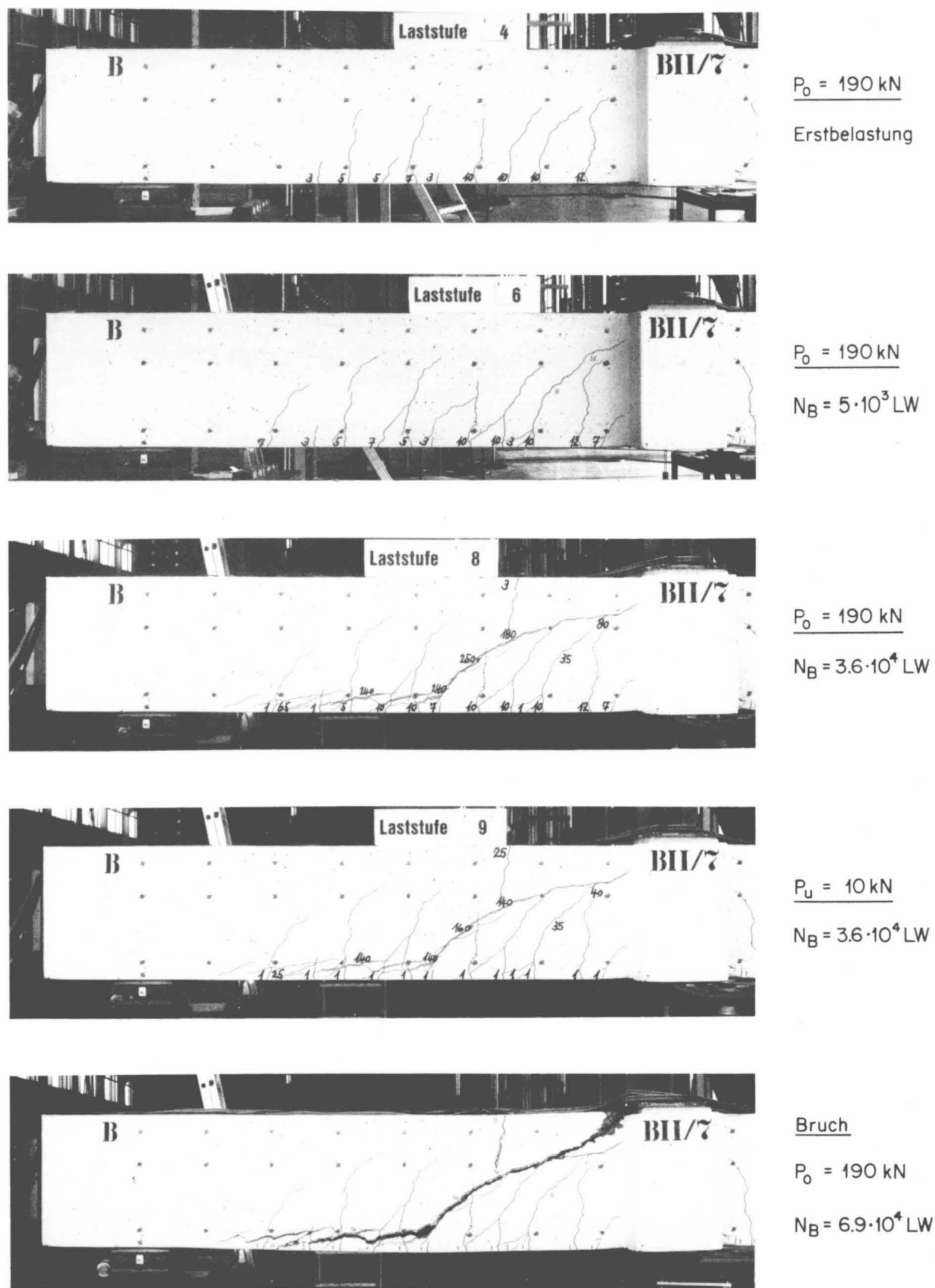

Rissweiten in $1 / 100 \mathrm{~mm}$

Bild 160: BI/7, Rissentwicklung bis zum Bruch im Lastzyklus B 


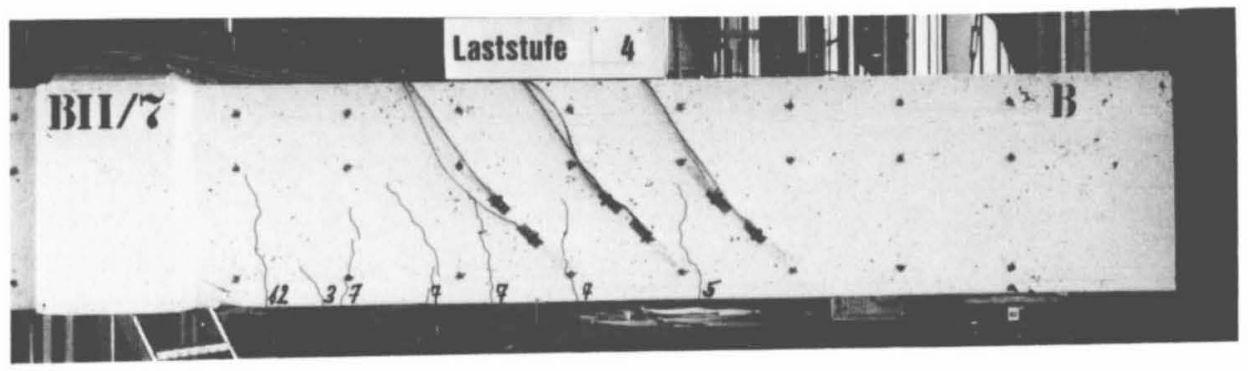

$\underline{P_{0}=190 \mathrm{kN}}$

Erstbelastung

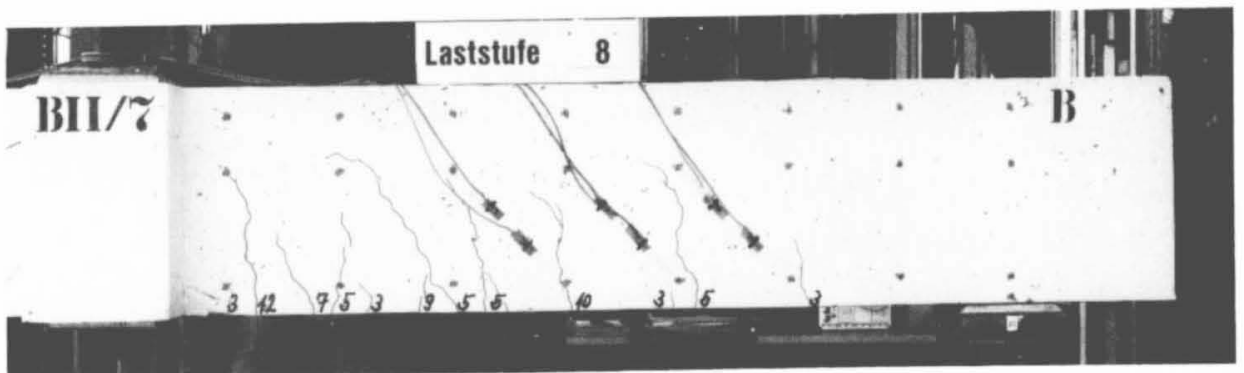

$\underline{P_{0}=190 \mathrm{kN}}$

$N_{B}=3.6 \cdot 10^{4} \cdot L W$

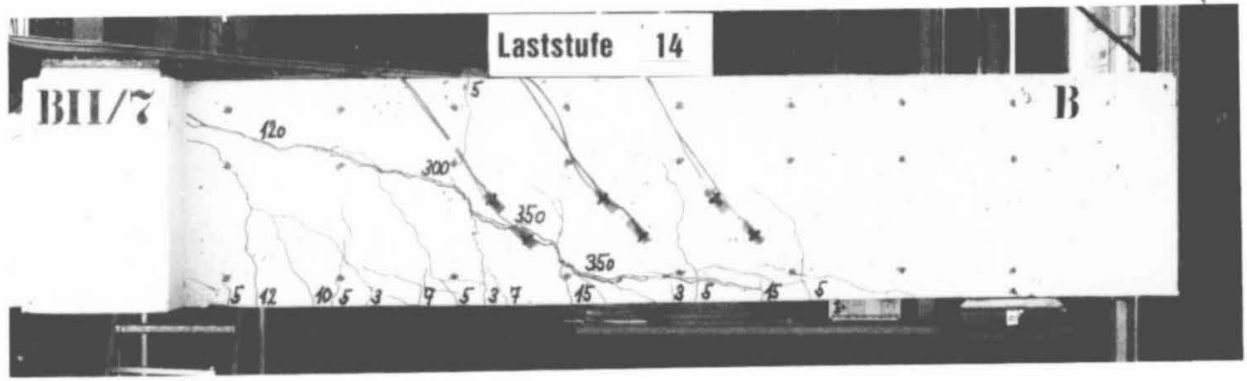

$\underline{P_{0}}=190 \mathrm{kN}$

$N_{B}=1.92 \cdot 10^{5} L W$

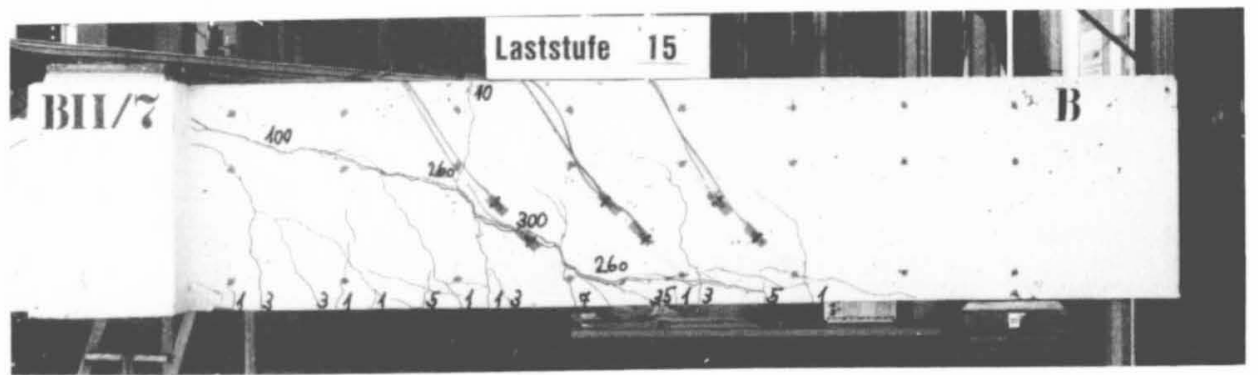

$\underline{P_{u}}=10 \mathrm{kN}$

$\mathrm{N}_{\mathrm{B}}=1.92 \cdot 10^{5} \mathrm{LW}$

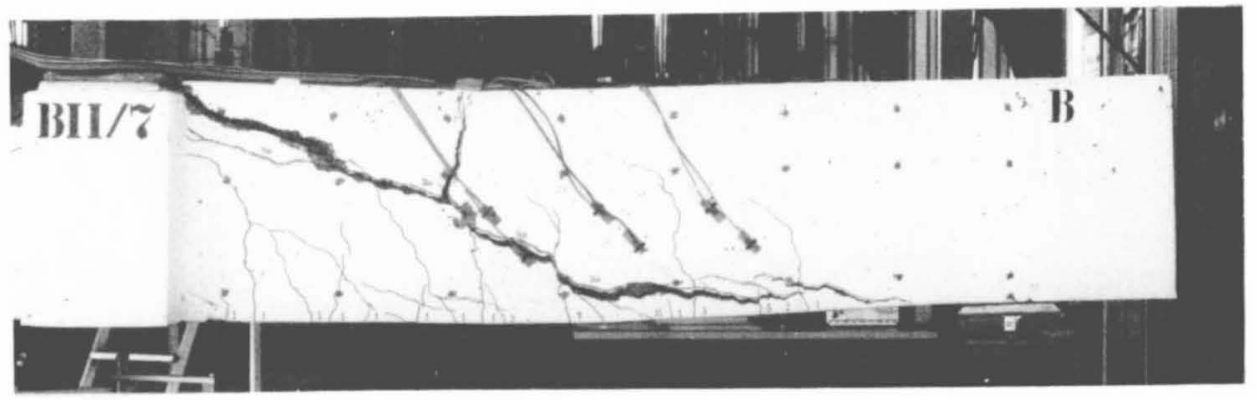

Bruch

$P_{0}=190 \mathrm{kN}$

$N_{B}=1.96 \cdot 10^{5} \mathrm{LW}$

Rissweiten in $1 / 100 \mathrm{~mm}$

Bild 161 : BII/7, Rissentwicklung bis zum Bruch im Lastzyklus B 


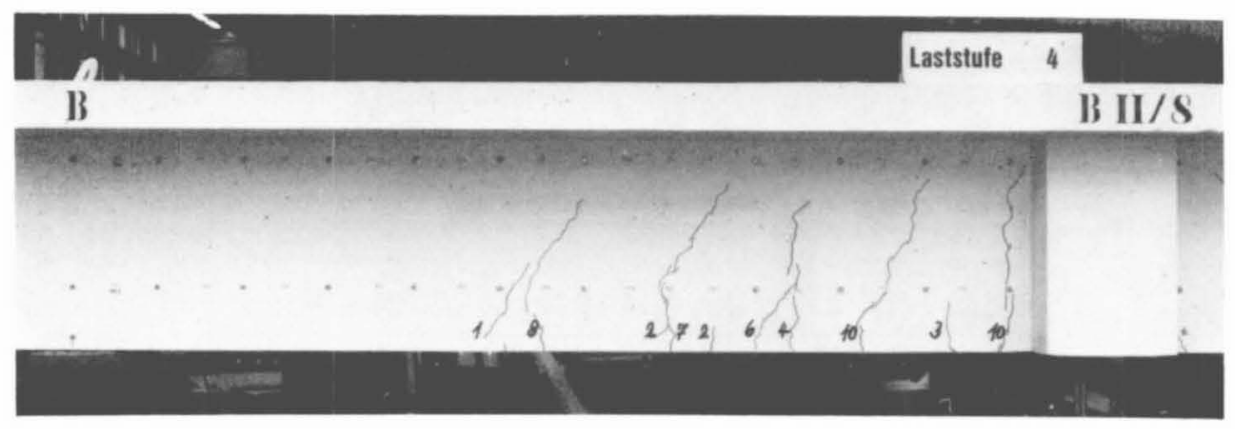

$P_{0}=380 \mathrm{kN}$

Erstbelastung

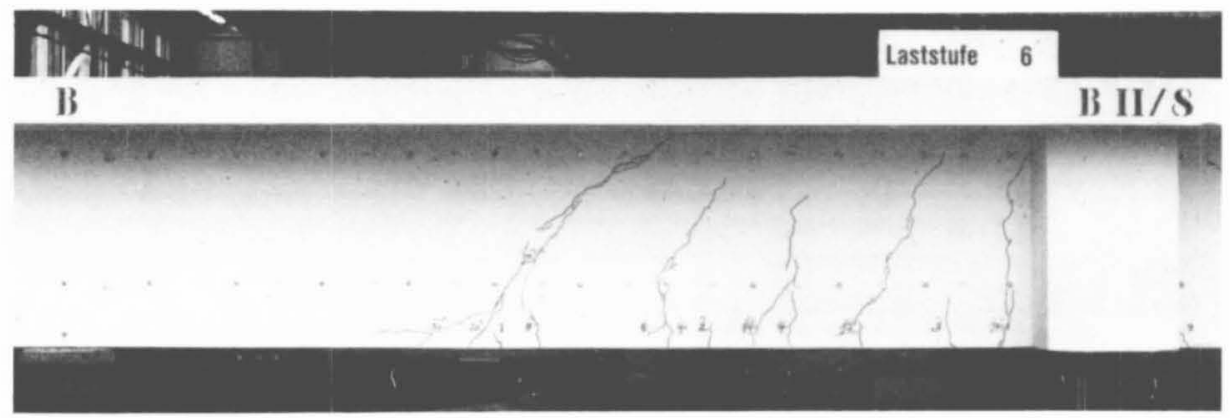

Stegbruch

$P_{0}=380 \mathrm{kN}$

$\mathrm{N}_{B}=4.5 \cdot 10^{3} \mathrm{LW}$

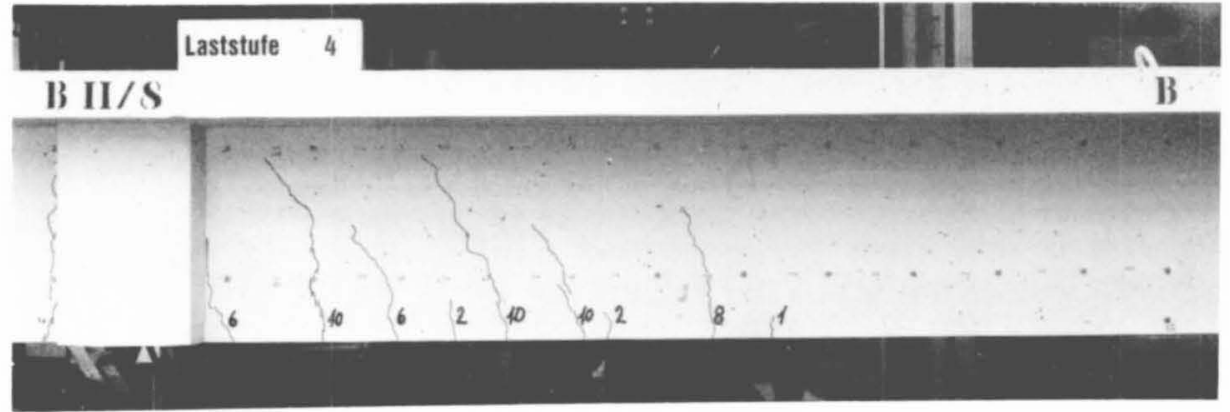

$P_{0}=380 \mathrm{kN}$

Erstbelastung
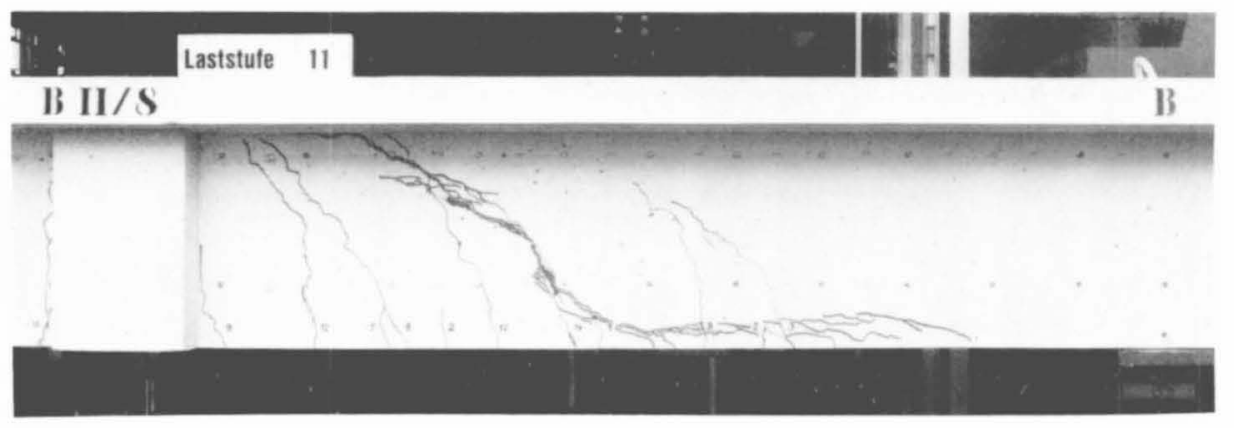

Stegbruch

$P_{0}=380 \mathrm{kN}$

$N=3.3 \cdot 10^{4} \mathrm{LW}$

Rissweiten in $1 / 100 \mathrm{~mm}$

Bild 162: BI/8, Rissbilder und Stegbrüche im Lasłzyklus B 

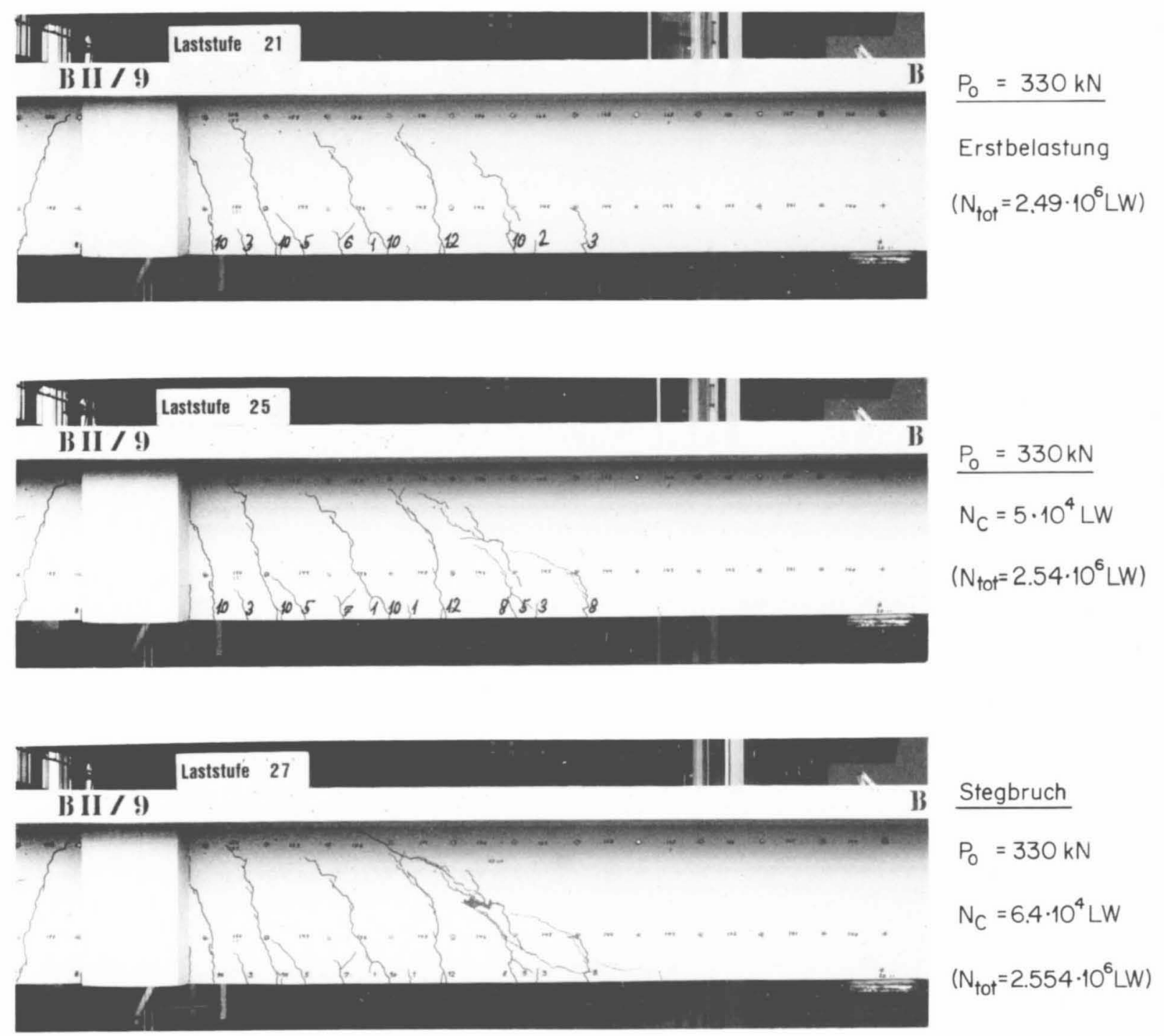

Stegbruch

$P_{0}=330 \mathrm{kN}$

$\mathrm{N}_{\mathrm{C}}=6.4 \cdot 10^{4} \mathrm{LW}$

$\left(N_{\text {tot }}=2.554 \cdot 10^{6} \mathrm{LW}\right)$

Bild 163: BII/9, Rissentwicklung bis zum Stegbruch im Lastzyklus C

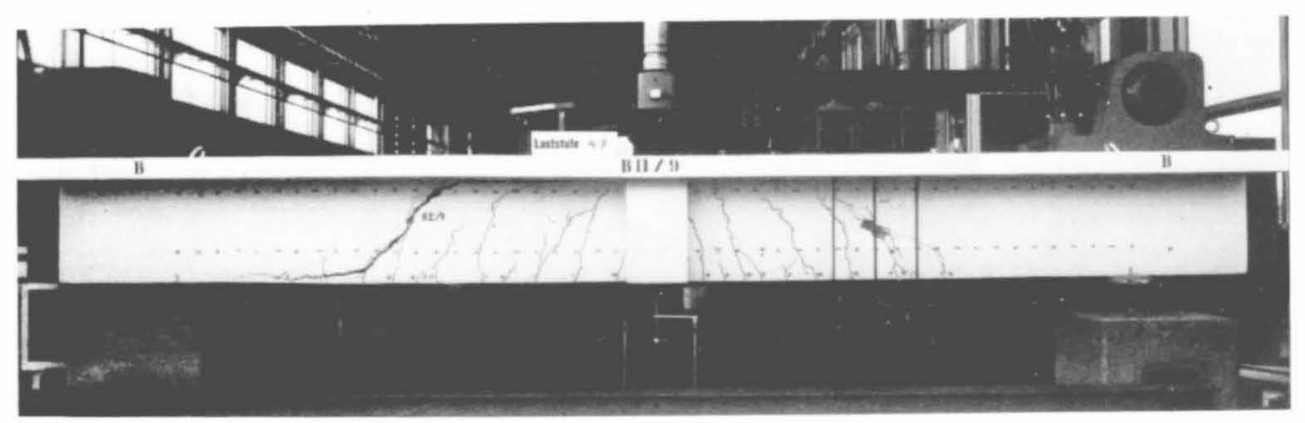

Stegbruch

$P_{0}=380 \mathrm{kN}$

$N_{D}=3.5 \cdot 10^{5} \mathrm{LW}$

$\left(N_{\text {tot }}=4.99 \cdot 10^{6} \mathrm{LW}\right)$

Rissweiten in $1 / 100 \mathrm{~mm}$

Bild 164: BII/9, Stegbruch im Lastzyklus D 


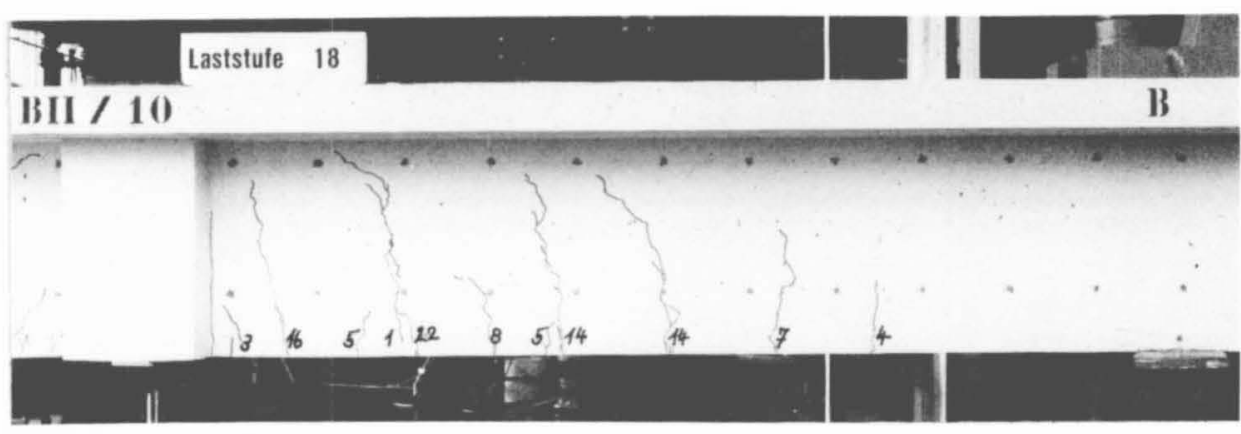

\section{$P_{0}=380 \mathrm{kN}$}

Erstbelastung

$\left(N_{\text {tof }}=2 \cdot 6 \cdot 10^{6} \mathrm{LW}\right)$

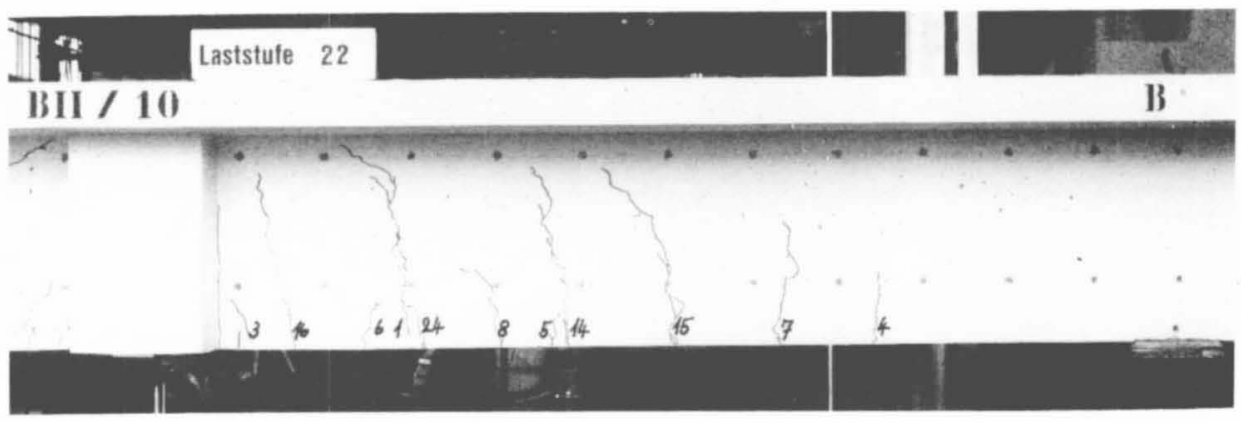

$$
\frac{P_{0}=380 \mathrm{kN}}{N_{C}=4 \cdot 10^{5} \mathrm{LW}}
$$$$
\left(N_{\text {tot }}=3.0 \cdot 10^{6} \mathrm{LW}\right)
$$

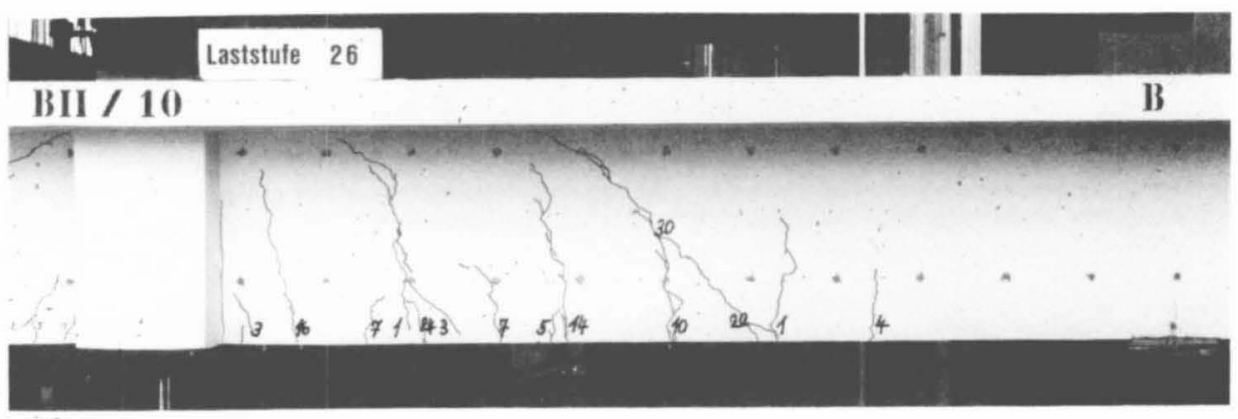

$$
\begin{aligned}
& P_{0}=380 \mathrm{kN} \\
& N_{C}=1.445 \cdot 10^{6} \mathrm{LW} \\
& \left(N_{\text {fof }}=4.045 \cdot 10^{6} \mathrm{LW}\right)
\end{aligned}
$$

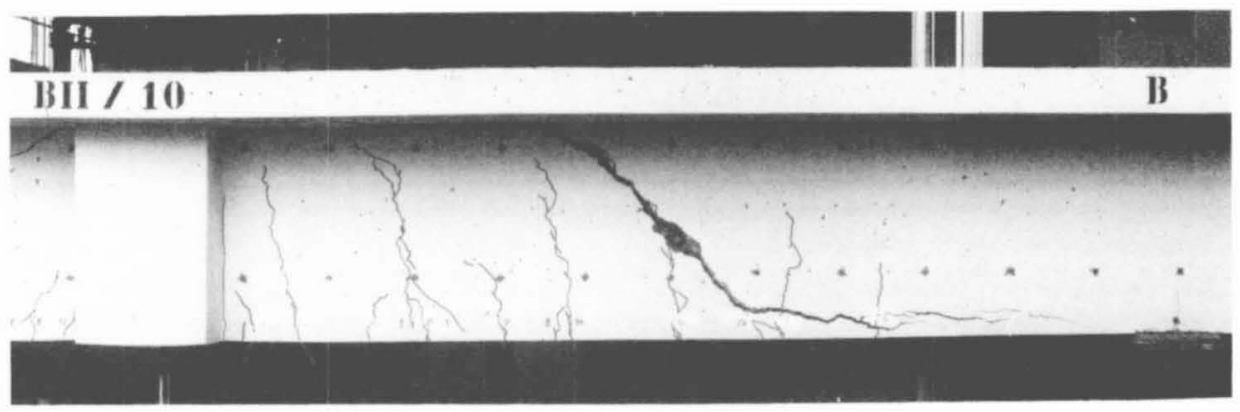

Stegbruch

$P_{0}=380 \mathrm{kN}$

$N_{C}=1.505 \cdot 10^{6} \mathrm{LW}$

$\left(N_{\text {tor }}=4.105 \cdot 10^{6} \mathrm{LW}\right)$

Rissweiten in $1 / 100 \mathrm{~mm}$

Bild 165: BI/10, Rissentwicklung bis zum Stegbruch im Lastzyklus C 


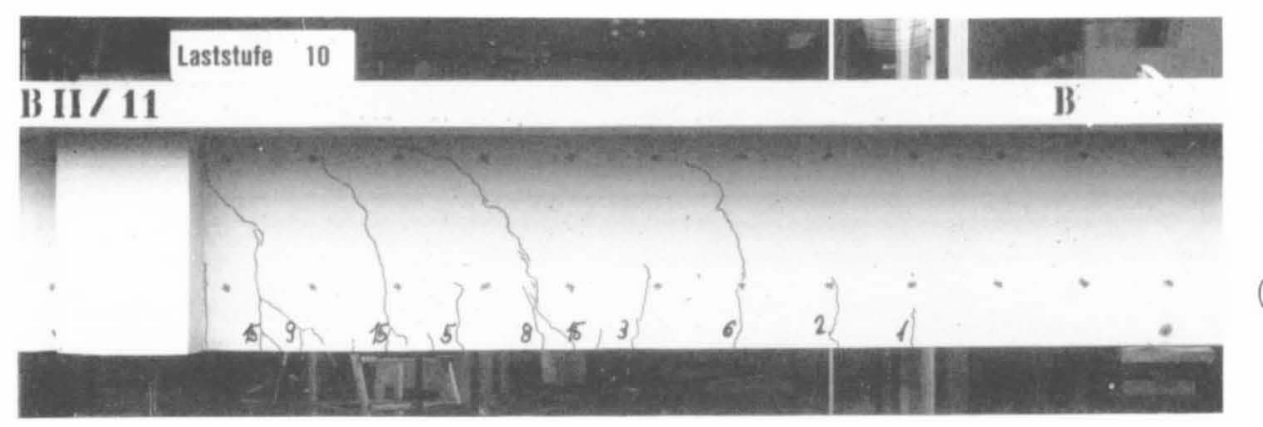

$\underline{P_{0}}=380 \mathrm{kN}$

Erstbelastung

$\left(N_{\text {tot }}=2.76 \cdot 10^{6} \mathrm{LW}\right)$

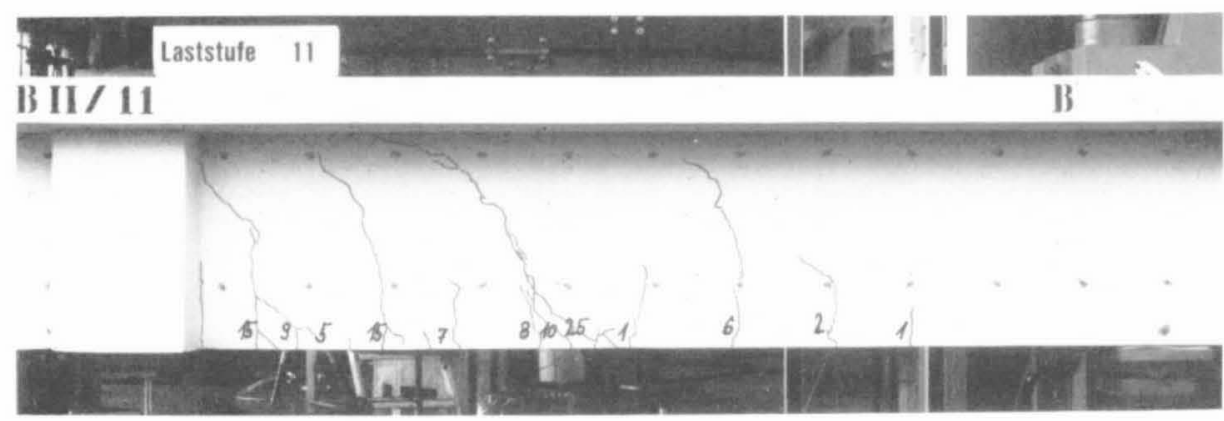

$$
\begin{aligned}
& P_{0}=380 \mathrm{kN} \\
& N_{C}=3.2 \cdot 10^{5} \mathrm{LW} \\
& \left(N_{\text {tot }}=3.08 \cdot 10^{6} \mathrm{LW}\right)
\end{aligned}
$$

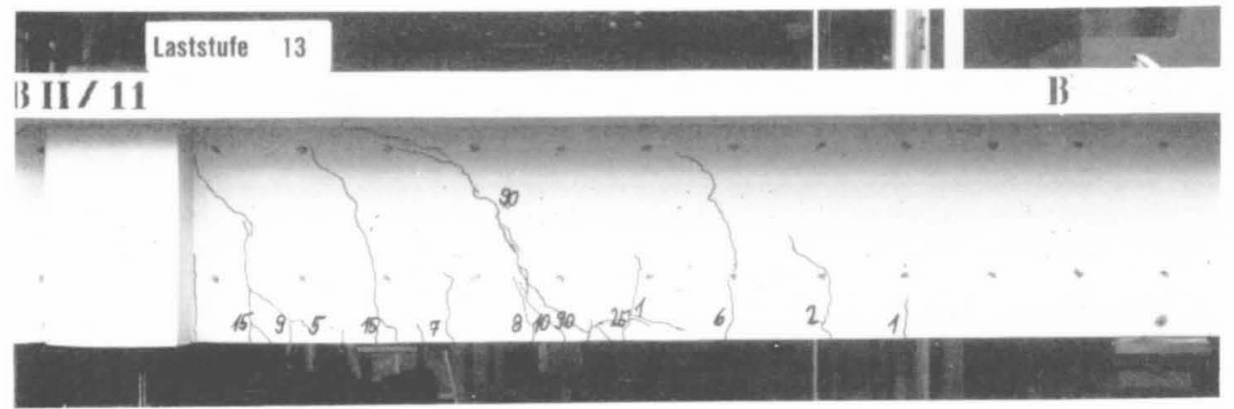

$$
\begin{aligned}
& P_{0}=380 \mathrm{kN} \\
& N_{C}=5.8 \cdot 10^{5} \mathrm{LW}
\end{aligned}
$$

$\left(N_{\text {tot }}=3.34 \cdot 10^{6} \mathrm{LW}\right)$

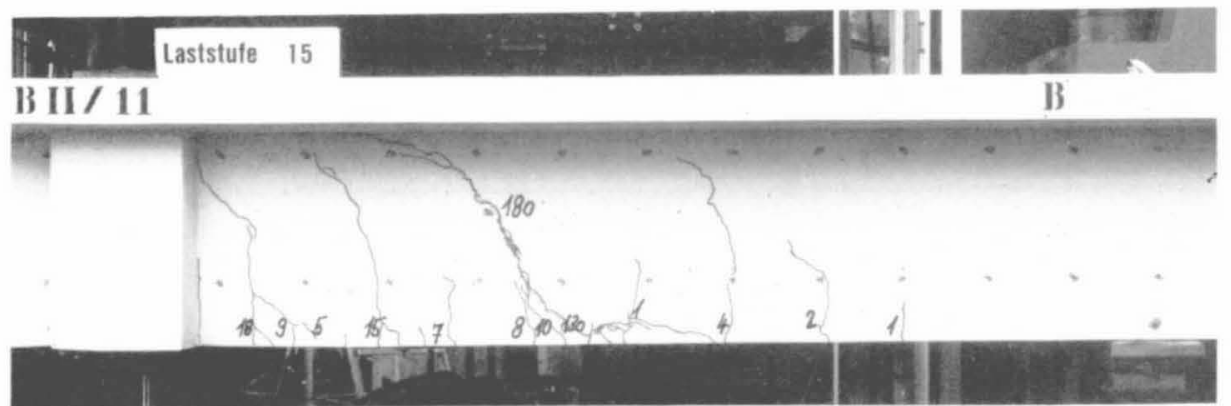

$$
\begin{aligned}
& P_{0}=380 \mathrm{kN} \\
& N_{C}=8.2 \cdot 10^{5} \mathrm{LW} \\
& \left(N_{\text {to }}=3.58 \cdot 10^{6} \mathrm{LW}\right)
\end{aligned}
$$

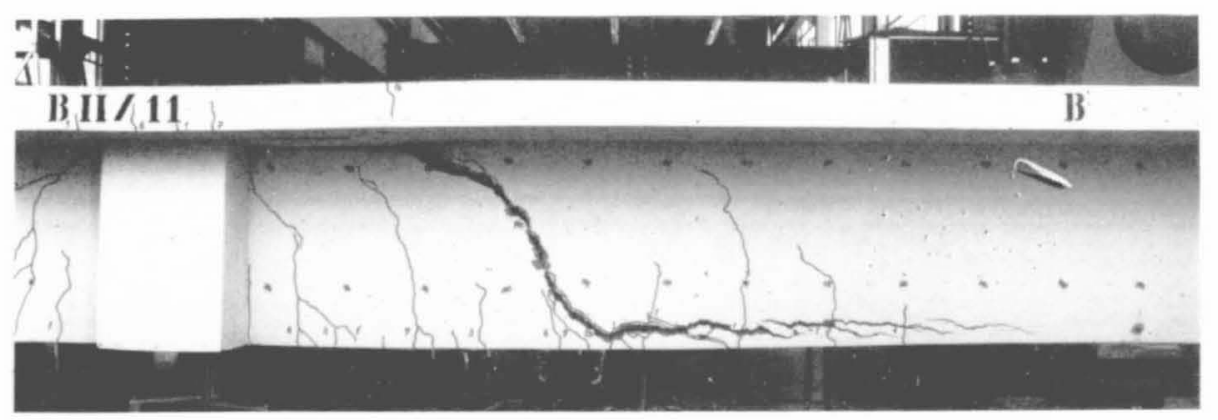

Stegbruch

$P_{0}=380 \mathrm{kN}$

$N_{C}=8.8 \cdot 10^{5} \mathrm{LW}$

$\left(N_{\text {tot }}=3.64 \cdot 10^{6}\right)$

Rissweiten in $1 / 100 \mathrm{~mm}$

Bild 166: BII/11, Rissentwicklung bis zum Stegbruch im Lastzyklus C 

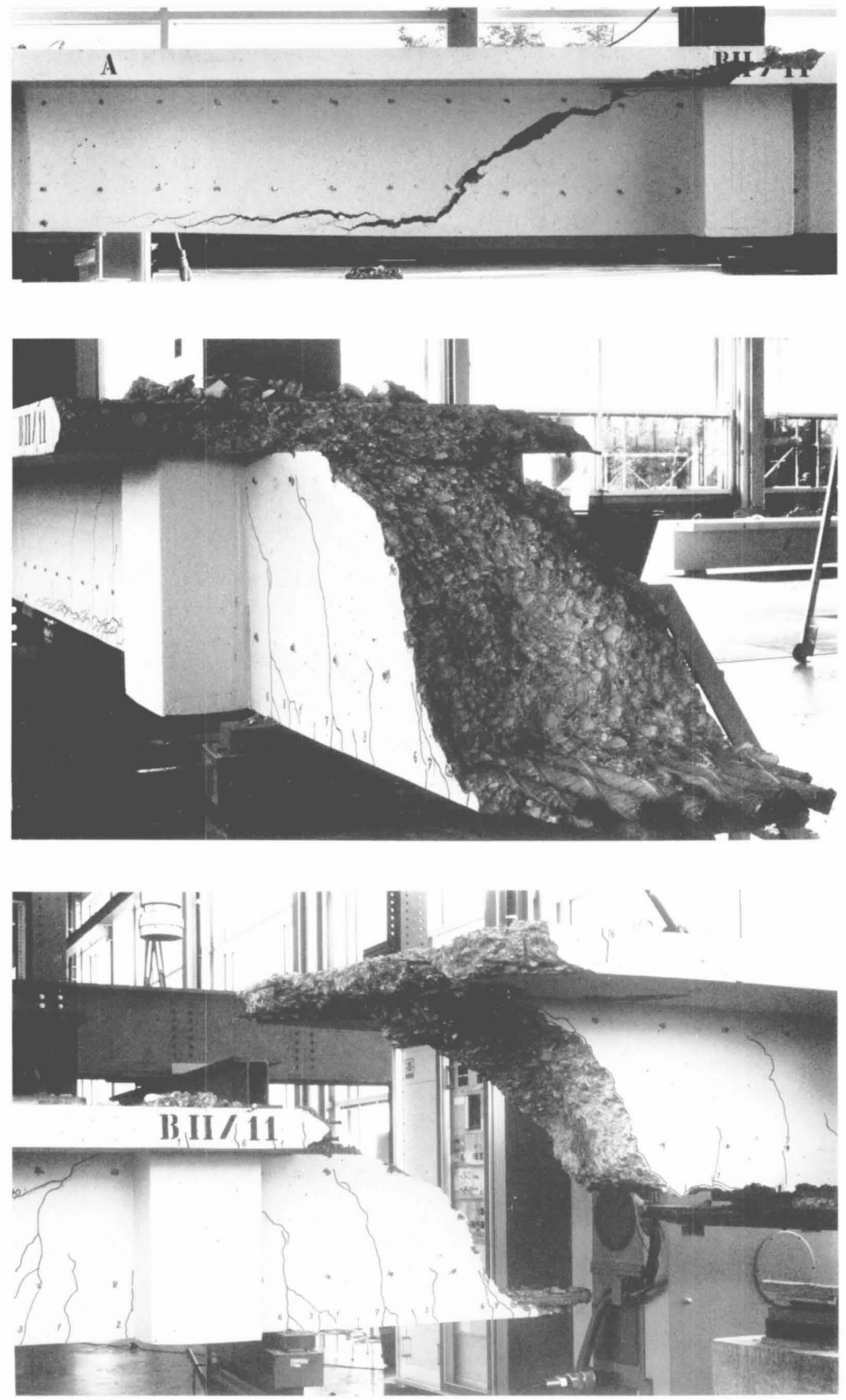

Rissweiten in $1 / 100 \mathrm{~mm}$

Bild 167: BII/11, Erschopfung der Tragfähigkeit, Bruchfläche 